i.S. DEPARTMENT OF ENERGY

RICHLAND OPRRATIONS OFFICE

MANAGEMENT \& OPERATINE (MEO) CONTRACT WORK AUTHORIZATION

1. Work Authorization No.

2. Initiator: D. E. KeKenney $\frac{\text { R\&UE } 376-1589}{\text { Name and Signature }}$ Org. Telephone No.

3. Program Title: Solid Weste Programp

4. Program wBS No.: $\ldots 1.2 .1$

5. Contractor: WEC (plus PAI, as descrtbed in MYPP)

6. Performance Period From:_10/1/95 To:_9/30/96

7. Start Date: $N / A$ Expected Completion Date: $N / A$

8. Description:

\title{
Direct Funding
}

\begin{tabular}{|c|c|c|c|c|c|}
\hline aDs & Expensen & $\begin{array}{l}\text { Capital } \\
\text { Equip. }\end{array}$ & $\underline{G P P}$ & Inine Item & TOTAI \\
\hline $\begin{array}{l}2200-0 \\
2320-0 \\
2200-1 \\
2220-1 \\
2320-02\end{array}$ & $\begin{array}{r}33,576 \\
21,384 \\
1,494 \\
11,275 \\
361\end{array}$ & $\begin{array}{l}754 \\
150 \\
0 \\
0 \\
0\end{array}$ & $\begin{array}{l}300 \\
1,000 \\
0 \\
0 \\
0\end{array}$ & $\begin{array}{l}0 \\
0 \\
6,606 \\
0 \\
2,100\end{array}$ & $\begin{array}{r}34,630 \\
22,534 \\
8,100 \\
11,275 \\
2,461\end{array}$ \\
\hline Stbtotal & \multicolumn{4}{|c|}{ (Direct FunAing) } & 79,000 \\
\hline Tndixect & \multicolumn{4}{|c|}{ Funding (MI) } & 8,996 \\
\hline A Fum & \multicolumn{4}{|l|}{$\mathrm{Ag}$ (MODOP) } & . 286 \\
\hline
\end{tabular}

I authorize work against the FY 1996 MYPP with the exception of the following area (state area, BCws, and funds).

Note 1: In the event of a continuing resolution or similar funding holdback, the CFO will address this issue under separate correspondence. Addressed by the RL 
CFO.

Note 2: The work authorizations can be for work scope that is less than that identified in the MYPP if there happens to be disagreement in any area. .

\section{Concurrence}

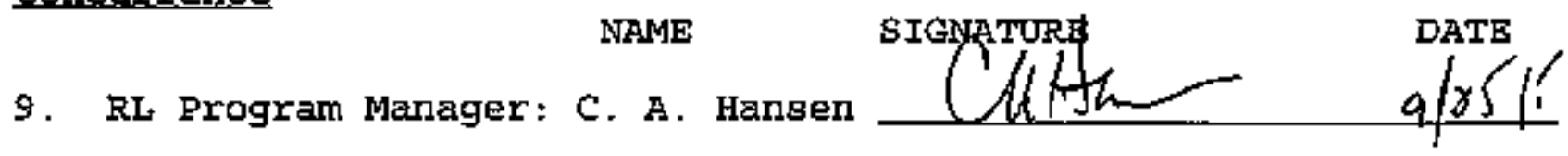

10. Contractor: 4. H. Hamilton, Jr. Livtamulta $9 / 25 / 55$

Approval

11. RL AM-COR:

c. A. Hensen $(1 / / 4 \pi$

Funds will be authorlzed via the FINPLAN in accordance with the authorized funding table.
c.C. OPI
CEO
M\&O Contractor 


\section{RELEASE AUTHORIZATION}

Document Number: $\quad$ MHC-SP-1114, Rev 1

Document Title: Solid Waste Program Fiscal Year 1996 Multi-Year Program PIan WBS 1.2.1

Release Date: $\quad 9 / 26 / 95$

This document was reviewed following the procedures described in WHC-CM-3-4 and is:

APPROVED FOR PUBLIC RELEASE

WHC Information Release Administration Speclalist:

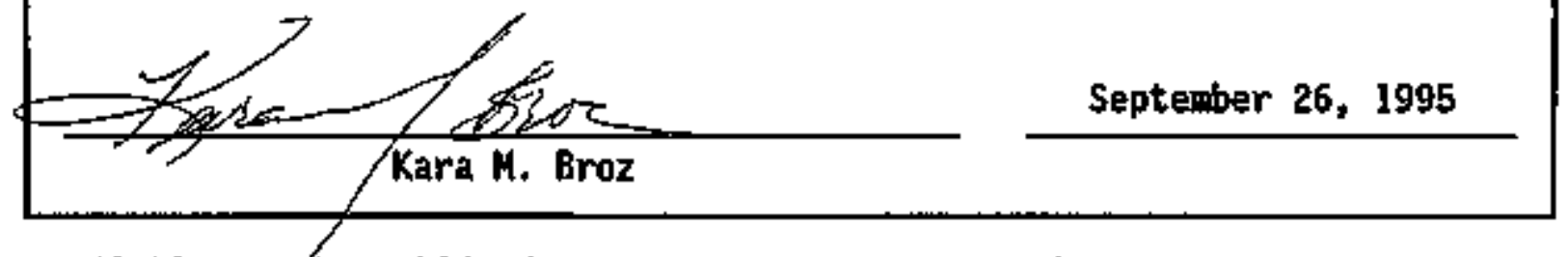

Available to the public from the U.S. Department of Commerce National Technical Information Service

5285 Port Royal Road

Springfield, VA 22161

(703) $487-4650$ 


\title{
Solid Waste Program Fiscal Year 1996 Multi-Year Program Plan WBS 1.2.1
}

\author{
J. G. Riddelle
}

Date Published

September 1995

Prepared for the U.S. Department of Energy Assistant Secretary for Environmental Management

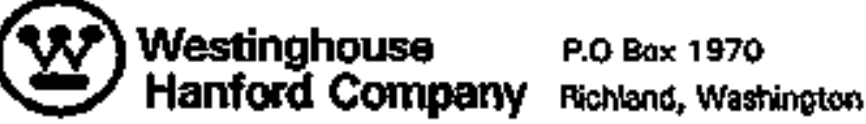

Nensganent and Operatians Contractor for the

U.S. Dapertmact of Enerov Undar Comtract DE-ACOS-87FL10e30

Approved for public release; distribution is unlimited 
IFCAL OISCLAINER

Thls report wos prepartod os an cooount of woik sponsored by en agency of the United States Govemment. Noither the Unīted States Govermment nor any agency thareof, nor ary ol thair employstes, nor any of thit contraction, eubeantraciors or their employees, makes amy warranty, express or implied. or assumas any legel liability or responsibility for the coouresy, completeness, or amy third party's use of the results of such use of envinformetion, apporatus, product, or process dischosed, or rapresents that lis use would not irfining: polvately owmed righte. Raference her tin to any sobeific

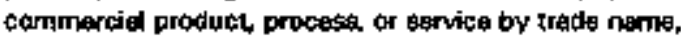
Iredemerk, mernefecturer, or otherwise, does not necessarily constitute or imply the endofsement, racommendetion, of teworing by the United States Government or ary agendy

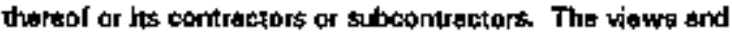
opinions of authors expressed hercin do not necossarily state of reflact those of the United States Govermment or amy agency thereot.

This repart has batr ceproduced from the begt avaliable coppy. Averable ho pepes copy and microficho.

Aysuble to the U.S. Depardinent of Eneror and its conkractors from

U.S. Dopartment of Energy

Office of Seienvifie and Teohniea Informarion IDSTH

P.o. Box 62

Osk Ridos, TN 37831

(6)15) 576-840)

Proted in the Untid 8trten of Amping

DISCLM-ACCHP (1-91) 


\section{DISCLAIMER}

Portions of this document may be illegible in electronic image products. Images are produced from the best available original document. 
SOLIO MASTE PROGRAH

HES 1.2.1

FY 1996 Program Plan

WHC-SP-1114, Rey. 1

Approva1s:

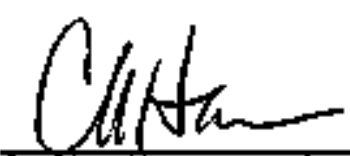

C. A. Hansen, Assistant Manager for Waste Management, DOE-RL

Mittamelita

T. H. Hamitzon, Jr. Sibager

Solid Waste Disposal, WHC

W/4tamulion of ta

E. E. NcKenney, hanajger

Restoration and Upgrade Drograms, WHC

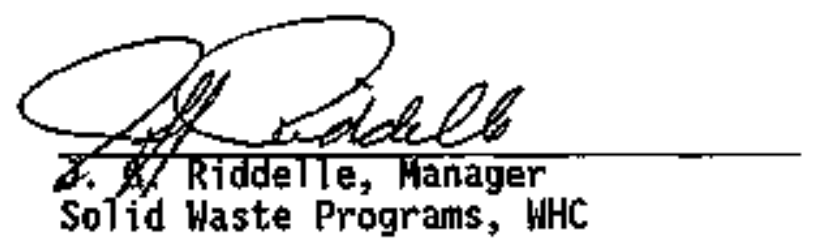

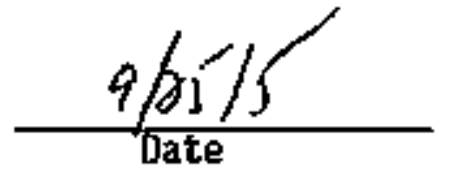
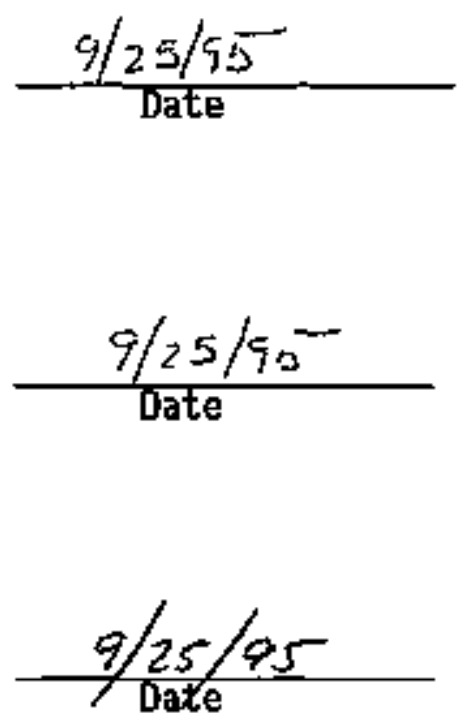

$-1.0-1-$ 


\section{SOLID HASTE PROGRAH}

WBS 1.2.1

Fy 1996 Program Pian

WHC-SP-1114, Rev. 1

\section{Table of Contents}

- 1. Program Overview

- 1.A Prograrn Visjon/Mission

- 1.B Program Strategy

- 1.B.1 Technical Functions and Requirements

- 1.B.2 Program Objectives

- 1.B.3 Pianning Assumptions

- 1.B.4 Program Constraints

- 2. Program Baseltnes

- 2.A Technical Base]ine

- 2.A.1 Technical Docaments Tree \& Products Structure

- 2.A.2 WBS Structure

- 2.A.3 WBS Djctionary

- 2.A.4 Responsibility Assignitent Matrix

- 2.A.5 Technology Requîrements

- 2.A.6 Waste Type Data

- 2.B Schedule Baseline

- 2.B.1 Program Logic Diagram

- 2.B.2 Program Master Baseline Schedule

- 2.B.3 Milestone List

- 2.B.4 Milestone Description Sheets

- 2.C Cost Baseline

- 2.C.1 Cost Baseline Summary by Year

- 2.C.2 Basis of Estimate

- 2.C.3 Planned Staffing Profiles

- 2.C.4 Performance Measures

- 3. Execution Vear

- 3.A Technical Objectives

- 3.B Program Perforwance Baselíne Schedule

- 3.c Cost Baseline by Month

- 3.D Prograta Funding Required 


\section{Program overview}

\section{A. Program Vision/Mission}

\section{VISION}

We vill be a Nationally Recognized Leader in Solid Waste Management and a Key Player in Achieving the Hanford Mission.

\section{MISSION}

The Hanford Mission Plan, Volume 1, Site Guidance identifies the need for the Soljd Waste Program to "treat, store, and dispose of a wide variety of solid material types consisting of multiple radioactive and hazardous waste classes". This includes "future Hanford Stte activities (which) will generate new wastes that must be handled as cleanup activitios are completed." Solid wastes are typically categorized as transuranic (TRU) waste, low-level waste (LLW), Jow-level mixed waste (LLHW), and non-radioactive hazardous waste.

To meet this need the Solid Waste Program has defined its mission as the following receive, store, treat, decontaminate, and dispose of solid radioactive and nonradioactive dangerous wastes in a safe, cost effective and environentally compliant manner, acconplished through:

- Interfacing with onsite and offsite generators to assist them in preparing waste for shipment to Solid Waste Facjitities.

- Field Verification and Certification Services; inciuding Containerized Waste Verification and Repackaging Services utilizing innovative NonDestructive Examtnation/Hon-Destructive Assay (NDE/NDA). Now developrents in this technology witl continue to be explored and utijized.

- Prepare for future (post FY2000) retrieval of transuranic waste from below grade storage to characterize the wastes and provide above ground monitored storage, and prepare the waste for disposal at the Waste Isolation Pilot Piant (WIPP). (Currently not funded for FY1996.)

- Waste Characterization, Repackaging and Treatment of Wastes, provided by the Waste Receiving and Processing (WRAP) Facility or commercially avallable processes.

- Hazardous and mixed waste treatgent integrated with private sector capabilities. (Partially funded for FY1996.)

- Recelpt, storage, treatment, packaging, and disposal of low level. transuran1c, mixed, and dangerous solid wastes as appropriate.

- Decontanination Services; utilizing $T$ Plant facilities or commercial services to decontaminate customer and legacy equipaent and structures for re-use, recycle, free release, storage or safe disposal. New decontasination technologies will be explored and utijized. 


\section{\$OLID HASTE PROGRAM}

HAS 1.2.1

WHC-SP-1114, Rev, 1

- Safe interim storage prior to the disposition of the 72 irradiated Pressurized Water Reactor (PWR) Core II fuel elements in the $T$ Plant Canyon pool and the one non-jrradiated fuel element on deck in the $T$ Plant Canyon.

- Liquid Waste Transfer Services; majntain certification for at least one government-owned liquid waste tank car (LWTC).

- Disposal of Category 3 Jow level wastes in high-integrity contatners (HICs).

- Tracking of Waste Contajner Inventories; in support of the site-wide electronic data base. The sjte-wide electronic data base will continue to be enhanced to improve useability and accessibility. 


\section{B. Program Strategy}

To accomplish the program mission, Solid Waste Programs strives to be the technical and operational experts in solid waste services at the Hanford Site through training, comnunication with other U.S. Department of Energy (DOE) sites, comtiercial operations, and operational experience. Solid Waste Programs will minifaize cost to the customer and maximize the efficiency of the Hanford Site Mission by careful allocation of resources through the following:

- Ensure compliance with safety and regutatory requirements by contínuous monitoring and review of activities and facilities.

- Meet waste minjmization goals by developing repackaging techniques, planning, and recycling.

- Maintaín as low as reasonably achievable (ALARA) goals by continued training, monitoring, and engineared radiological safety improvements.

- Continue waste forecasts, customer integration studies, shipment verifications, on-time processing/shipments.

- Assure cost effective service through value engineering, systems engineering and econontic analysis of facilities and processes.

* Operating within requirements established by the U.S. Environmental Protection Agency (EPA) Resources Conservation and Recovery Act (RCRA) and state of Washington Department of Ecology (Ecology) and the Department of Health (DOH) for treatment, storage, disposal and decontamination of waste.

- Utiizing best demonstrated available technologies.

- Assisting on-site and off-site waste generators to prepare waste for shipment to the Hanford site.

- Effectively utilize on-site and off-site treatment capabilities.

- Define specific alinimum requirements for performing Solid Waste activities and incorporating them into Standards and Requirements Identification Documents (SRIOS). 


\section{LB.1. Technical Functions/Requirements}

The technical functions and requjrements for the Solid Haste Program are delineated in the Solid Waste Program Technical Baseline Description (WHC-SD-hili-RPT-060, current revision).

The Solid Waste Program Technical Baseline Description employs a systen engineering approach to establish the program's techntcal baseline. The purpose of this document is to view the program as a singie system and document the external and internal interfaces necessary to successfuily manage a progran of this magnitude. By defining the program's baseline, the effects of uncertaintjes and changes (internally and externaliy driven) to key elements of the program can be understood. Impacts of these changes to the mission and the functions of the progran can be assessed and controlled.

Solid Waste Program external syster requirements are limits on the system design imposed from authority outside the mission. These requirements must be properiy alfocated to the functions to meet the mission. Externat system requirements cover a broad range of activities. Those standards and requirements that apply to the Sol id Waste Program are documented in the Solid Waste Disposa? Standards and Requirements Identification Document, WHC-IP-1120, Rev. 3.

Specific Solid Waste Program functions required to perform the mission include:

- Maintaín a safe and compliant solid waste operational enviroment by continualiy assessing and maintaining the sol id waste operatjonal environment in a safe and compliant condition.

- Maintain safe and compliant solid waste systems, equiprent and structures. Continually asșess and maintain the solid waste facility systems, equiprent, and structures, and their operations in a safe condition. MaIntain a qualiffed solid waste staff, and maintain required solid waste facility and operating documentation.

- Maintain safe and compljant solid waste documentation [Safety Analysis Reports (SAR), Operational Safety Requirements (OSR), Waste Acceptance Criterja, etc.]. Maintain recuired solid waste facility and operating documentation related to safe and compljant operations.

- Provide program control including scheduling, financial analysts, change control preparation, organizational financial/business management, programattc organtzational financial/schedule analysis and program/performing organizational business reporting activities. Scheduling activities include fiscal year and outyear development, cost account monitoring, and change control tasks and reporting. Financia] activities include fiscal year budget development and outyear requirements planning, cost account monftoring and change control tasks including variance analysis reports and estimate at completion report preparation.

- Retrieve suspect. TRU solid waste. This includes retrieval planning, solid waste access and removal, obtaining waste characterization samples, generating waste information, estabilishing retrieval capabiltty requirements, and forecasting future retrieval operations. (Currentiy not funded.) 
- Treat solid waste. Provide for the treatment of solid waste to convert the waste to an acceptable form for final disposition. (Some activities are restricted by funding levels.)

- Inspect container contents of newly generated waste to meet requirentents for waste verification and inspect contents of "unknown" or "backlog" wastes to provide segregation and compliant packaging of waste.

- Store solid waste in a safe and compliant manner prior to shipment to an approved treatment and/or disposal site.

- Receive' newiy generated solid waste generated during Hanford Cleanup mission operations and from other missions. Receipt of new waste includes solid waste receipt planning, characterization information verification for acceptance, establishing receipt capability requirements, and forecasting future receipt operations.

- Ship solid waste. Prepare the necessary shipment requests and docunentation. Coordinate the solid waste package shipment with shippers and receivers to verify that the waste transfer was accomplished. Document the solid waste shipment status.

- Dispose sotid waste. Schedule solid waste package disposal and prepare waste transport package and disposal documentation. Provide final inspection of the solid waste package, load, and place in disposal site.

- Through a systems engineering approach develop and maintain baseline documentation including defining the technical baseline of the program and interfaces with other site programs, deveiop alternatives and trade studies for materlals requiring a path-definition for management and djsposition (e.g., materials within the scope of TPA Milestone M-33). Provide records management and tracking of solid waste through the Solid Waste Information Tracking Systen (SWITS).

- Process high dose rate waste and contaminated equipment to meet applicable standards for djsposal, storage, re-use, or free release. The equilpment to be processed wil1 include Tank Farms augers, SHF casks, tank farms contaminated equipment, and contaminated equipment stored in the $T$ Plant canyon. In addition, other high dose rate contaminated equipnent and waste being stored at site facil1ties undergoing decommissioning and generated during the site cleanup will be treated. Treatment of high dose rate waste and equipment will take place in the 221-T canyon.

- Recejve, store, contain, perform decontafination, and repackage incoming wastes for reduction in radiation fieids and recategorization of wastes from mixed to non-mixed and/or Greater Than Category 3 low-level to Category 1 or 3 low-level or if possible to levels making the waste a releasable (nonradioactive) material.

- Package solid waste. Ensure solid waste is in a container suitable for the waste type. Provide solid waste packaging documentation including packaging certifjcations and solid waste feed stock traceability. Haintain appropriate labeting and inspection of the containers of solid waste. 
- Provide contaminated equipment storage and storage of spent fuel elements at T Plant.

- Perform all work necessary to maintain certification of at least one LWTC.

- Prepare solid waste disposition specifications. Generate specifications for solid waste operations including treattent, packaging, certiffcation, storage, shipping, disposition, and archiving samples and records.

- Disposition soljd waste. Provide packaging and shipping, treatment/storage and/ar iramobilization for packaging and certifying the solid waste, store or ship it as needed, treat and/or immobilize it, and accomplish final disposition.

- When necessary identify, inventory, and characterize the waste received from generators by reviewing the process operations (current and historical), by reviewing new waste information, and by sampling and analyzing the waste inventory. Assess the current condition of the waste. Archive the results of these investigations. (Some activities are restrfeted by funding lovels.)

- Assess solid waste characterization information. Evaluate the solid waste characterization information consisting of generator request information, process knowledge information, and waste sample characterization data, against the solid waste disposition requirements and provide validation of meeting the disposition requirements.

- Determine solid waste disposition requirements by compiling and sorting imposed requirements, defining requirements that result from proposed solutions and required capabilities, and assessing constraints under which the function must operate.

- Certify and verify solid waste. Certify that solid waste is treated according to the disposition requirements and that the documentation is in order to a] low the solid waste package to be dispositioned.

- Assess the solid waste treatment, storage, and disposal (TSD) capability needs and. resources based on required versus design throughput and capacities.

- Provide archtval storage and retrieval operations for the analyzed solid waste process sample analysis data. 


\section{B.2. Proqram Objectives}

When execution of the Soljd Haste Program mission is completed and the waste treatment and storage facilities are declared surplus, the facilities will be deactivated and ultimately turned over to the Decontamination and Decorumissioning Program. Disposal facilities will be closed in accordance with applicable regulations. The following provides the description of the final state of the Solid Waste Program.

\begin{tabular}{|c|c|c|}
\hline Iopic Description & Eina] Condition & Basis \\
\hline \multicolumn{3}{|l|}{$\begin{array}{l}\text { Dispositioned } \\
\text { Soldd Waste }\end{array}$} \\
\hline TRU Waste & $\begin{array}{l}\text { Processed to meet } \\
\text { W1Pp Waste } \\
\text { Acceptance } \\
\text { Criteria, Shipping } \\
\text { Requirerents }\end{array}$ & $\begin{array}{l}\text { Hanford Defense } \\
\text { Waste- } \\
\text { Environmental } \\
\text { Impact Statemen } \\
\text { Record of } \\
\text { Decisjon }\end{array}$ \\
\hline $\begin{array}{l}\text { Low Leve1 } \\
\text { Hixed Waste }\end{array}$ & $\begin{array}{l}\text { Processed to meet } \\
\text { Land DisposaT } \\
\text { Restrictions under } \\
\text { RCRA and Hanford } \\
\text { Site Sol id Waste } \\
\text { Acceptance Criteria }\end{array}$ & $\begin{array}{l}\text { Washington } \\
\text { Administrative } \\
\text { Code Section } \\
173-303\end{array}$ \\
\hline
\end{tabular}

Sol jd Waste Program Strategy

Low Leve1 Waste

$\begin{array}{ll}\text { Category 1 } & \text { Near surface } \\ \text { Waste } & \text { disposa1 of } \\ & \text { Category 1 low } \\ & \text { level waste }\end{array}$

Category 3 Waste

Greater Than Category 3 Waste Hazardous Waste
Near surface disposal of Category 3 low level waste

Final Environmental Statement Hanford Waste Management. Operations; $\mathrm{DOE}$ Order 5820.2A;

Performance Assessment

Performance Assessment
Waste shipped to WIPP for disposal

Hear surface disposal in radioactive mixed waste disposal trenches. Burial ground will be closed in compli ance with RCRA perrait.

Near surface disposal in low Tevel burial grounds. Burial ground will be clased in compl i ance with RCRA permit.
Near surface disposal in low level burial grounds using Jigh integrity containers. Burial ground will be closed in compliance with RCRA permit.

Provide continued storage awaiting determination of disposal requirements.

Waste shipped off site for

Treatment, storage, Washington and disposal in Administrative accordance with Code Section RCRA and Washington 173-303 Administrative Code reoulations 


\begin{tabular}{|c|c|c|c|}
\hline Iopic Description & Final Condition & Basts & Solid Waste Program Strategy \\
\hline - & $\begin{array}{l}\text { Removed from Solid } \\
\text { Waste managed }\end{array}$ & Not Applicable & $\begin{array}{l}\text { Spent Fuel transferred to the } \\
\text { Spent Fuel Project. }\end{array}$ \\
\hline
\end{tabular}

Facilities

Treatment and Storage

Buildings

Burial Grounds
Transfer to the Hanford Surplus Facilities Program (Decontamination and Decomatissioning Program)

RCRA C1osure
Not Applicable

Clasure under requi rements of RCPA. Deactivated facilities turned over to the Hanford Surplus Facilities Program.

Closure under the requirements of RCRA.

\author{
Burfal Ground \\ Dangerous Waste \\ Permit \\ Application
}


- Design, construct, and operate required treatment, storage, disposal, and related projects.

- Provide compliant collection, storage, transfer, treatment, and disposal systems as required under operating permits.

Program objectives in the area of treatment are:

- Plan for and implenent the retrieval of TRU waste stored in burial ground trenches and aipha caissons since may 1, 1970. Since that date, waste suspected or known to contain TRU constituents have been segregated and retrievably stored pending a decision on management of the waste. The Record of Decision for the Disposal of Hanford Defense High-Leve1, TRU, and Tank Wastes - Environmental Impact Statement states that retrievably stored solid waste will be retrieved, and newly generated TRU-contaminated solid waste will be processed as necessary, and sent to WIPP for disposal. (No TRU processing is funded within the current planning window.)

- Process high dose rate contaminated equipment and/or waste to meet applicable standards for reuse, storage, or disposal. Process contaminated equipment and/or waste for reuse or disposal in accordance with D0-020004, "Perform Beta-Gamma Decontamination", (or as superseded by an approved work Plan/procedure) and the WHC-SD-CP-SAR-007, Rev. 1, (including Engineering Change Notices in effect as of September 30, 1995.) "T Plant Safety Analysis Report". The processing will' be perforned from 0ctober 1, 1995, through September 30, 1996.

- Process low dose rate LL.H to meet debris rule standards for disposal or free release. Process low dose rate contaminated equipment for repair and return to service.

- Verify LLW and LLMN packages to confirm generator characterizations.

- Process contact-handled (CH) TRU waste in drunts and NDE/NDA standard waste boxes at the WRAP Facility for acceptance at WIPP. The Disposal of Hanford Defense High-Level. Transuranic, and Tank Wastes, Hanford Site, Richland, Hashington; Record of Dectsion states "A fac11ity will be designed, constructed and operated at Hanford to sort, process, and repackage retrievably stored and newly generated TRU sol id waste for shipment to the Haste Isolation Pllot Plant (WIPP)". Orums will be inspected, assayed, and opened if necessary. Waste contents can be sorted, segregated, treated for certain characteristics, repackaged, certified, and shipped to final disposal. SWB's will be passed through NDE/NDA stations only.

- Provide treatment for CH-LLHㄴ. Treatment is necessary to provide an acceptable form for disposal in an approved mixed waste disposal trench. Treatment needs are separated into themal treatment needs and nonthermal treatinent needs due to the distinction in the processing. Waste must be treated to meet land disposal restrictions. For a portfon of the CH-LLMW this can be accomplished through stabilization and other 
non-thermal techniques. The remaining $\mathrm{CH}$-LLN either requires thermal treatment as a technology-based standard or would benefit from thermal treatment. (constituent destruction, volume reduction, etc.).

- Provide treatment for remote-handled (RH) LLMW and RH-TRU waste and oversized CH-LLNW and TRI waste. Treatment is necessary to provide an acceptable form for disposal in efther an approved mixed waste disposa] trench (for LLMi) or at WIPP (TRI waste). The specific scope of these needs is being addressed as part of Tri-Party Agreenent milestone M-33.

- Provide for disposition of contaminated (radioactive) sodium under the control of the Solid Waste Progran. The first option is transfer to an off-site vendor through either procurement for beneficial use or offsfte RCRA compliant treatalent for disposal. The second option will evaluate the potential for on-site use of sodium byproducts. The third option is to react the sodium in the FFTF Sodium Reaction Factlity when it. is available.

- Provide for disposition of PUREX and B PJant contaminated tri-butyi phosphate (TBP) through transfer to an off-site vendor.

- Obtain treatment variances where applicabie.

Program objectives in the area of storage are:

- Provide compliant storage in the 616 Mon-Radioactive Dangerous Waste Storage Facility (NRDWSF) for acceptable non-radioactive dangerous waste containers offered by generators.

- Provide interim storage of contaminated equipment al ready existing in the 221-T canyon.

- Provide compliant storage in the 224-T Transuranic 5torage and Assay Facility (TRUSAF) for acceptable transuranic druns offered by generators.

- Provide compliant storage in the Hanford Central Waste Complex for acceptable radjoactive mixed waste (druns and boxes) and other waste requtring long-term storage offered by generators.

- Provide for safe, monitored storage of the 72 spent fuel elements from Shippingport Pressurized Water Reactor Core II at T Plant.

- Perform a11 work necessary to maintain certification for at least one government-owned LHTC.

Program objectives in the area of disposal are:

- Complete active burial ground closure activities in accordance with permitting requirements. 
- Maintain the low level active and new bur1al grounds (218-E-10, 218-E$12 B, 218-W-3,218-W-3 A E, 218-W-4 B, 218-W-4 C, 218-W-5,218-W-6)$.

- Receive and dispose the acceptable L.LW offered by approved generators.

- Receive and dispose the acceptable LLMH offered by generators.

- Maintajn the radioactive mixed waste burial trenches (trenches T-31 and T+34 of burial ground 218-W-5). Operation is dependent on identification of mission need in Fr1996.

Specific FY1996 objectives required to accomplish the above general objectives are contained in the Sol id Waste Disposal contract. (NOTE: at the time of document publication the product i ine deliverables were still under negotiation.) 


\section{B.3 Progran_Planning Assumptions}

The Multi-Year Program Plan is based on the following key assumptions:

- Start up and operation of the LLW line in WRAP Module 1 will meet TriParty Agreement milestone M-18 on March 31, 1997.

- Contact-handled LLWW stabilization treatment will consist of direct. disposal, onsite treatment, and commercial treatment. Transfer of waste to the vendor awarded the CH-LLMW stabilization treatment contract will meet Tri-Party Agreement mflestone M-19 on September 30, 1999.

- The WIPP will be abie to receive waste from Hanford until a7l Hanford TRU waste is transported to WIPP. Assuming the current funding profile, Hanford will begin WIPP shipments no sooner than FY2006. (Ho Hanford TRU processing is planned within the current planning window.)

- Trenches 31 and 34 of burial ground 218-W-5-will be heid in a state of readiness to receive waste, but will not be operated or receive waste in FY1996.

- Processing required for oversized TRU and LLMW and RH-TRU and RH-LLMH will be determined under Tri-Party Agreement Milestone $\mathrm{M}-33$, and funded in addition to current. FY1996-FY2002 funding levels.

- T Plant will maintain its TSD facility status through continued compliance with the T Plant Part A permit stipulations and through approval of the Part B permit application.

- No retrieval or processing of IRU waste is required before the year 2005.

- Hazardous waste will continue to be shipped offsite for disposal.

- Waste volume receipts do not change significantly from FY 1995 forecasts.

- Environmental Restoration Disposal Facility (ERDF) is operated by Environmental Restoration.

- The DOE waTk-in work account (lal...) is contract approved workthrough the MYPP and Work Authorization signatures. Work scope will be detailed throughout the year by any FDS approved method (work orders, new task packages, etc.) 
- Fiscal Year 1995 carryover workscope contained in this MYPP is authorized to start October $1,1995$.

- Per DOE-HQ, the Solid Waste minimum case operations costs are EM-30 direct funded via the ADS stomittal. The remainder of the Solid Waste Program, in support of waste rece1pts, will be assessed to the waste generators. The EM-30 direct funding allows Sol id waste management to be ready to remain in cold staniby to receive solid wastes.

- The scope of the performance assessment for the 200 West Burial Grounds has been expanded to include only some of the requirements of DNFSB recommendation 94-2 for FY 1996. By utilizing HIC5, the performance assessment supports contfnued disposal of higher activity LLW or category 3 wastes.

- Low-level waste disposal at Hanford will continue without a new Solid Waste Program Environmenta] Impact Statement.

- Part B' perinit applications for Solid Waste facilities have been submitted to Ecology. Hew facility construction will be accomplished under interim status. It is assumed that final status and approval of all required permits will be obtained to support facilfty operation. The low level burial grounds closure schedule will be renegotiated prior to approval of the Part B permit.

- Continued indefinite storage of mixed wastes requiring incineration is acceptable to regulators. (Funding identifted for FY 1996 to FY 2002 is inadequate to impTement the present contract.)

- For waste/equipment to be decontaminated at T Plant, funding for special lab work, transportation packaging, transportation to and from T Plant, interim storage needs, and disposal charges will be the responsibility of the waste generator. Decontamination activities above and beyond those defined in the Multi-Year Program Plan (MYPP) will be generatorfunded.

- The environmental protective coverings over the 291-T sand filter and the soi7 area east of 221-T, will remain intact unt $11 \mathrm{FY} 1997$ when funding is available to re-coat the areas ta ensure the prevention of plant growth and the spread of contamination.

- The T Plant head end contains mtscellaneous LLI, excess chemtcals, and abandoned process equipment. This waste poses an Industrial and radiological hazard to $T$ Plant personnel during administrative controlled entries for surveillance of the area. Sol id Waste Program assunes that the Nuclear Energy program (EM-60) retains responsibility for those chemicals and dispositions them properly.

- No, or minimal, offsite mixed wastes are received with the exception of Havy SRCs. 
- No additional regulatory or DOE requfrements are placed upon the SWD program, beyond those defined in the SWO SRIOS.

* No major unexpected facility degradation is experienced.

- The pricing for FY1997-2002 was compiled from the Project/2 Series $X$ scheduling software utilizing Financial Data System (FDS) rates in affect as of September 12, 1995. 


\section{B.4 Program Constraints}

Various Program constraints are applicable to the MYPP. The following apply:

Uncertafaties in waste data impact effective planning for treatment. storage, disposal

Volume and characterization data for some waste types are uncertain and add to planning risks. These uncertainties affect waste identified for disposition by the \$oljd Waste Program. Future National Environmental Policy Act (NEPA) and Federal Facjility Corapliance Act (FFCAct) decisions may impact the future volumes and types of waste received. In addition, high-level waste is not currently received by Solid Waste, although alternatives are being sought regarding disposftion of waste from the Spent Nuclear Fuels organization and the PAL hot cells.

Many factors contribute to uncertainties in waste data. Data on older waste (circa post-1970 stored TRU waste) are less detailed and less specific than data on new waste. Volume forecasting remains uncertain due to funding availability, programmatic impacts, and decisions awaiting approval through the NEPA process and FFCAct planning.

\section{Insufficient TRU Anal ytical Laboratory Sugport at Hanford}

Emerging requirements regarding WRAP process support and WIPP waste acceptance criteria have outpaced avaflable analytical capabilities, both within the DOE complex and in the commercial sector. Specialized laboratory facilities are required to analyze TRU contaminated samples to environmental protocols for waste processing and shipment to the WIPP. Facilities at the Hanford site and capacity on a national scale [commercia] and DOE) is extremely limited. The Hanford site needs these services to support the WRAP facility. Commercial 1aboratories may provide interia services for lower-activity services ( $<200 \mathrm{nCi} / \mathrm{gm}$ ). Hanford Analytical Labs are working with offsite DOE facilities to obtain services for $>200 \mathrm{nCi} / \mathrm{gm}$ ) samples.

\section{Plant Related Issues}

Liquid Waste Tank Car (LWTC) numbers H0-18581 and HO-18582 currently in use to transport liquid waste fron Hanford site locations to Tank Farms will be replaced in FY 1996. These LHTCs will nost likely be declared waste once they are retired. If the LWTCs are declared waste, regulations require they be stored in a RCRA conpliant storage area or be dispositioned within 90 days. FY 1995 carryover funding wij] be used to make the raflcars RCRA conpliant for disposai. 
Since line item funding is not provtded in FY 1996 for T Plant ventilation upgrade, project $\mathrm{C}-077$, then there will be a year-for-year sip in the completion date of June, 2001 , resulting in risk in personnel working conditions related to temperature control, and work delays.

\section{Sodiun Storage}

Solid Waste Operations maintains indefinite storage of radioactively contaminated metallic sodium materjal (Sodium Reactor Experiment and Hallam Sodium). The OOE plan to shutdown PUREX operations has resulted in an indeternisate delay in the use of converted sodium hydroxide. Alternate users such as the Savannah River Site were evaluated, but not found viable. Current1y, it remains in non-RCRA storage classified as materia1. Solid Waste is going through the excess process to deternine a beneficial use, however, if no use is identified, this material will be designated as RCRA nixed waste. Once sodium material has been declared as waste, it must be placed in RCRA compliant storage and/or properly disposed. At this time, construction of storage facilities for tanks of Hallam sodium has not been initiated while commercial treatment is pursued. (Ful1 funding for neither RCRA-compliant storage facilities nor comiercial treatment are identified.)

\section{Thermal treatment}

Alpha contaminated polychlorinated biphenyl (PCB) waste remains stored in the Centra? Waste Complex (CNC), years past the one year limit a llowed for PCB storage to meet state and Federal regulations and Toxic Substances ControT Act (TSCA) compliance. To date, no facility exists to treat and dispose of this PCB waste. This waste nust be incinerated to satisfy regulatory requirenents.

A portion of Radioactive Hixed Waste in storage at Hanford is mandated to be treated by incineration. The most cost-effective near term option is to transport waste to an offsite pernitted treatment facijity. If offsite capacity is not provided and funded, thermal treatment. capability on site would not be avaliable in this planning periad.

\section{NEPA Documentation}

Current Hanford NEPA documentation does not fully address LLW disposal on the Hanford site. The Solid Waste Performance Assessment (PA) Wi11 qualify the radionuclides of interest that will need to be addressed regarding the iong-term environmental inpacts of Solid Waste Disposal. An EA/EIS may be required on Hanford Solid LLW Disposal. 


\section{WIPP Operation}

The current projected capacity of the CWC is based on WIPP receiving certified TRU waste from Hanford. If MIPP contínues to be delayed, additional RCRA-compliant mixed waste storage-capacity will be required.

\section{Facility closure}

Institutional control and the future mission of the Hanford site remain an issue concerning the Low Level Waste Burial Ground closure. Due to the proximity of faciljties in the 200 Areas, utilization of land for facilittes such as WRAP will impact the current closure schedule. 


\section{A Technical Baseline}

This section of the Fiscal Year 1996 Solid Waste Program Multi-Year Prograri P1an contains the following elements:

- 2.A.1 - Technical Documents Tree and Product Structure

- 2.A.2 - WB5 structure

- 2.A.3 - WBS Dictionary

- 2.A.4 - Respons1b1lity Assignment Matrix

- 2.A.5 - Technology Requirements

- 2.A.6 - Waste Type Data 


\section{Solid Waste Program}

WBS 1.2.1

FYTg96 AYPP

A,1 Thehritiol Doeuments Thee

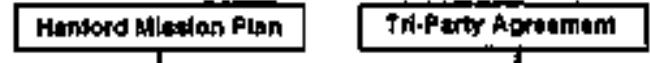

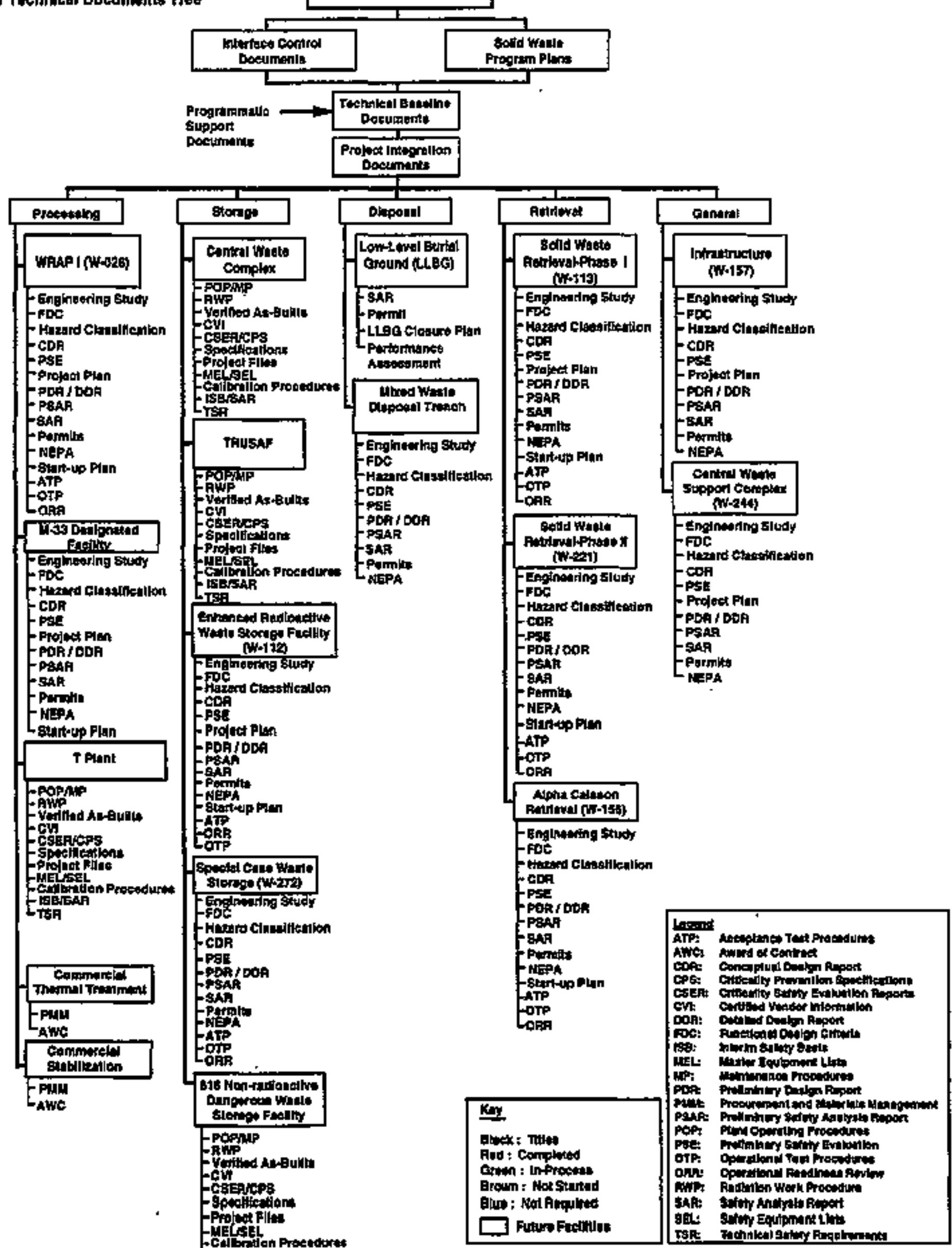

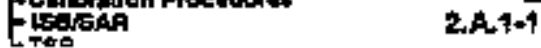




\section{Solid Waste Program}

WBS 1.2.1

FY 1996 GYPP

\section{A.1 Products Structure}

\section{Product Groups}

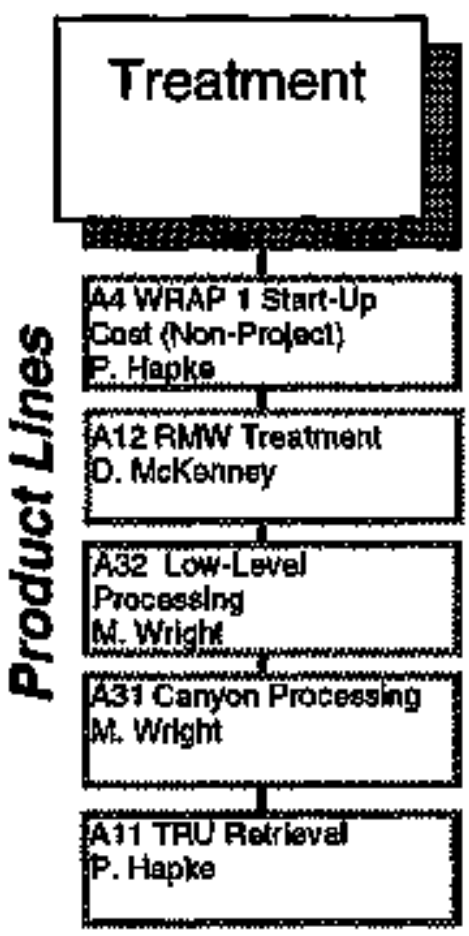

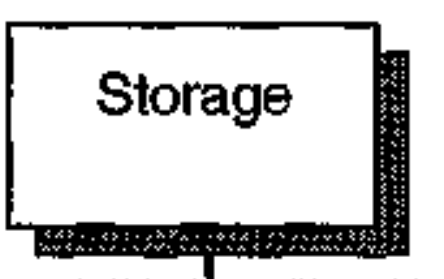

\begin{tabular}{|c|c|}
\hline $\begin{array}{l}\text { A15 Hazardous Waste } \\
\text { Storage } \\
\text { A. Piorce } \\
\text { MoDop Flazardous - } \\
\text { Waste Analysts }\end{array}$ & 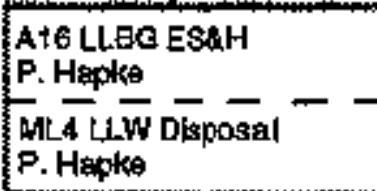 \\
\hline 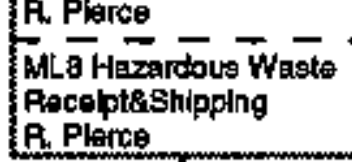 & 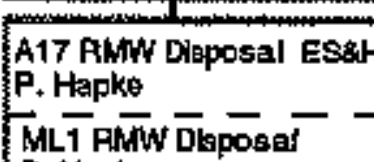 \\
\hline & \\
\hline
\end{tabular}
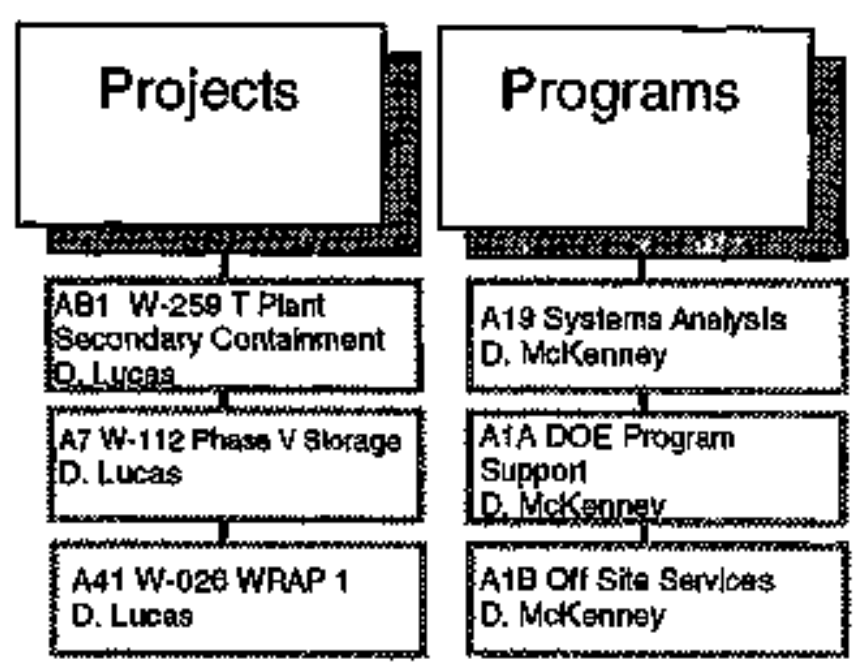

P. Hapke

ML5 Transuranio Recuet P. Hapke

A14 RHW/CWC Storage P. Hapko

MLL R RWW/CWC Receipt P. Hapke

As3 Contaminaied Equo Siorage M. Wright

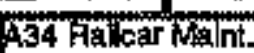

Certillcation

4. Wingh

सेख्यन W-112 startup Cost (Non-Profect)

D. McKenney 


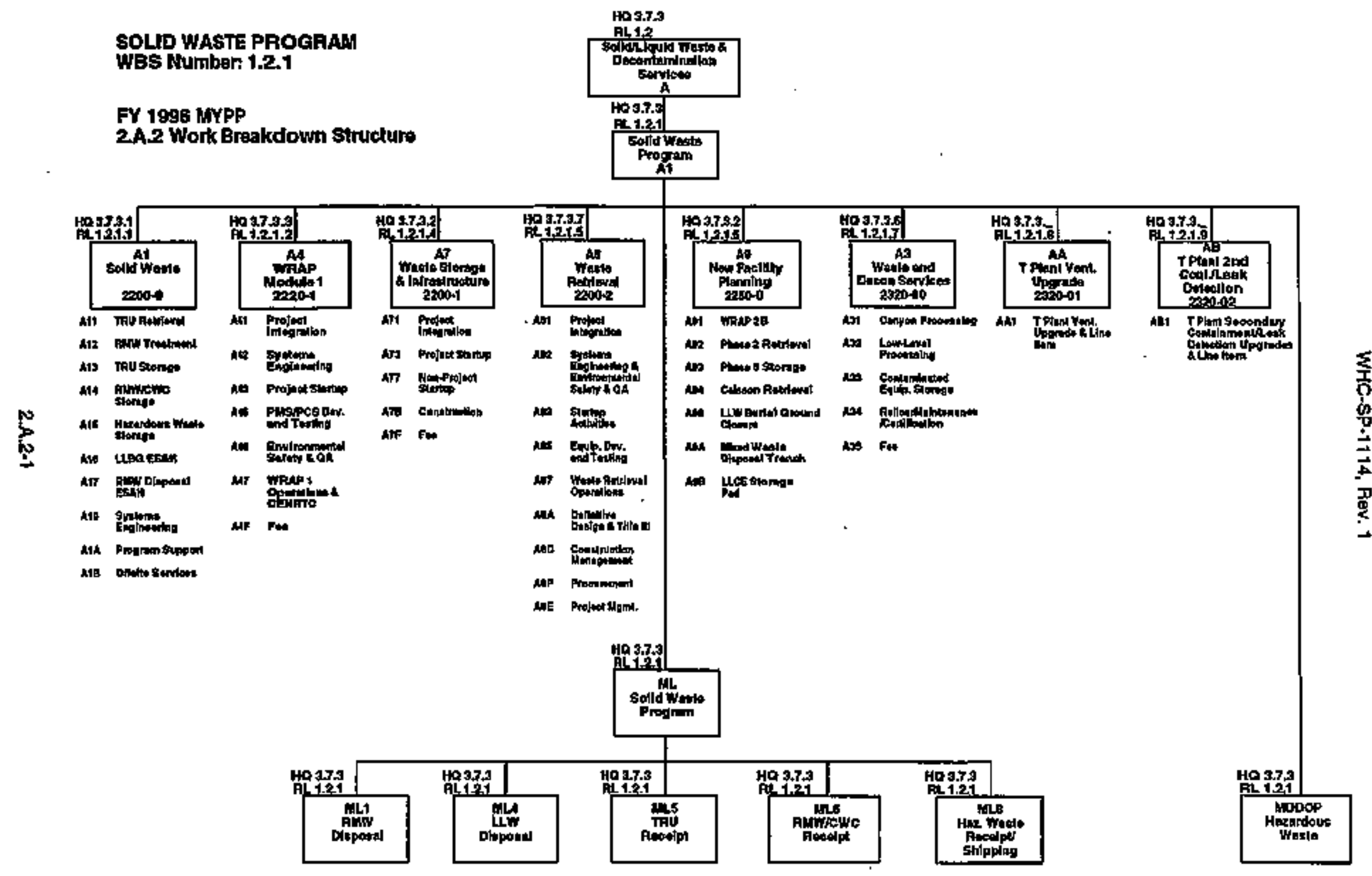




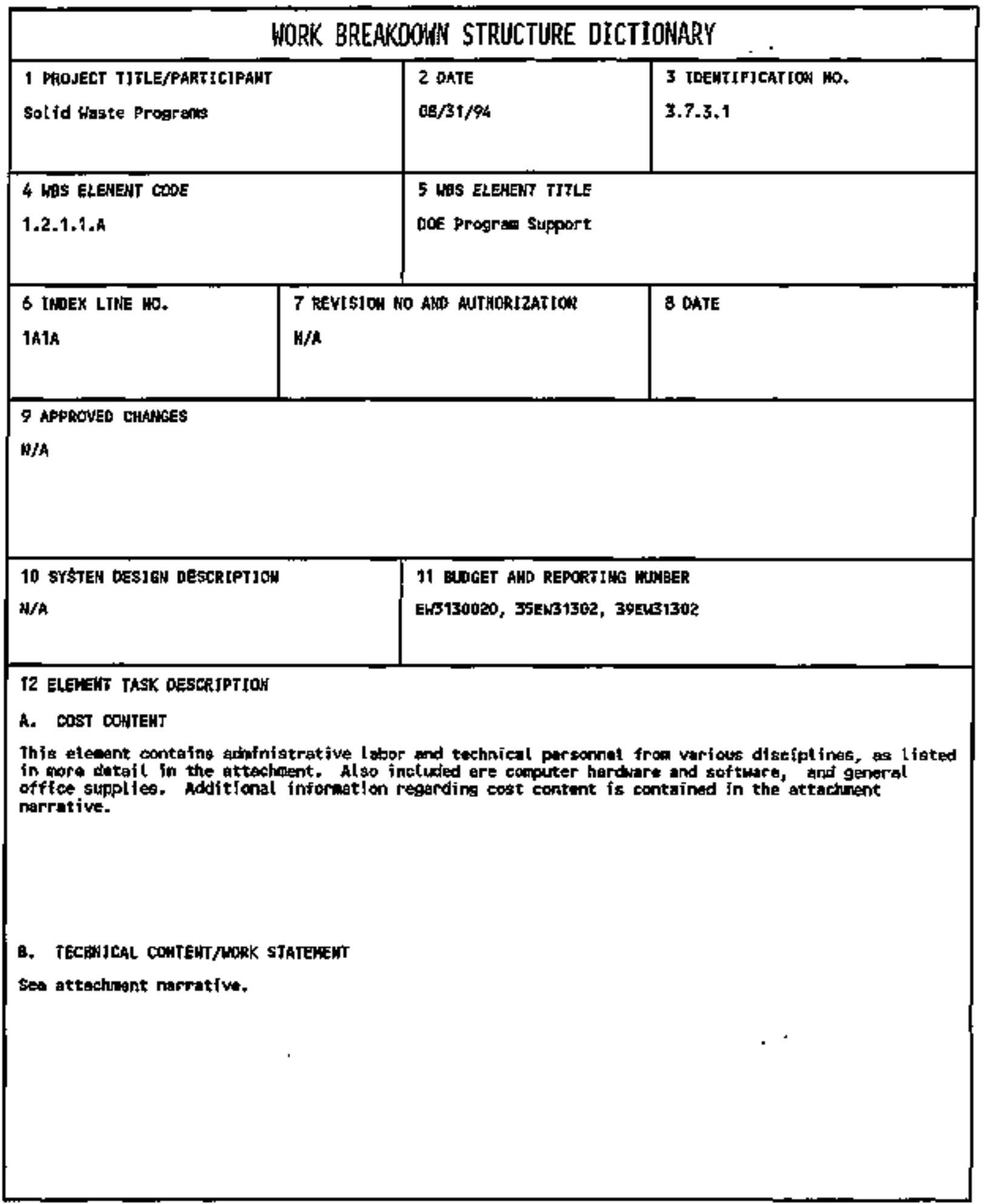




\section{SOLIO WASTE PROGRAM}

HBS 1.2.1

This activity provides for the on-going program management portion of the Solid Waste Program. The prograns management responsibilities include the management of spent fue $\{$, low level, transuranic, mixed, and non-radjoactive hazardous wastes.

This actvity also supports specia] requests of RL for support-related actívities.

Included in program management responsibilities are the activities to support short and Tong range planning such as the Hanford Strategic Plan, Hanford Mjssion Plan, Hanford Mult t-Year Program Plan, and the U.S. Department of Energy, Headquarters (DOE-HO) activity data sheets budget development.

Program manageant is responsible for a variety of monthiy reporting activities to U.S. Department of Energy, Richland Operations Office including support to the site Maragement System and the DOE-HQ monthly Progress Tracking System.

Also included in this activity are the budgeting and scheduling functions required to support program management.

\section{Program Support}

- The program management responsibilities include the management of low levei, transuranic, mixed, and non-radioactive hazardous wastes. Program support. includes scheduiting, financial, records management and program business reporting activities. Scheduling activities include fiscal year and long-term development; program nonitoring and control functions; and reportíng support as defined in 4 Hic-CM-5-34, Section 1.31. Financial activities include fiscal year budget development and long-term requirements pianning; program monitoring and control functions to include Variance Analysis Report and estimate at completion preparation; and reporting support as requested. Records management support wil7 include assistance in assessment of all documents on-hand and preparation for necessary storage of these documents. Records/documents will be prepared, indexed, processed through the optical scanner system and forwarded to the Records Holding Area as necessary. The Boeing Computer Services, Richl and Records Management group wi11 provide training to Solid Waste Disposal personnel on preparation of records for scanning systern. The Records Inventory and Disposition Schedules and Report of Records Holdings process are included in this work scope. 


\section{SOLID WASTE PROGRAM}

WBS 1.2.1

FY 1996 Program Plan

WHC-SP-1114, Rev, 1

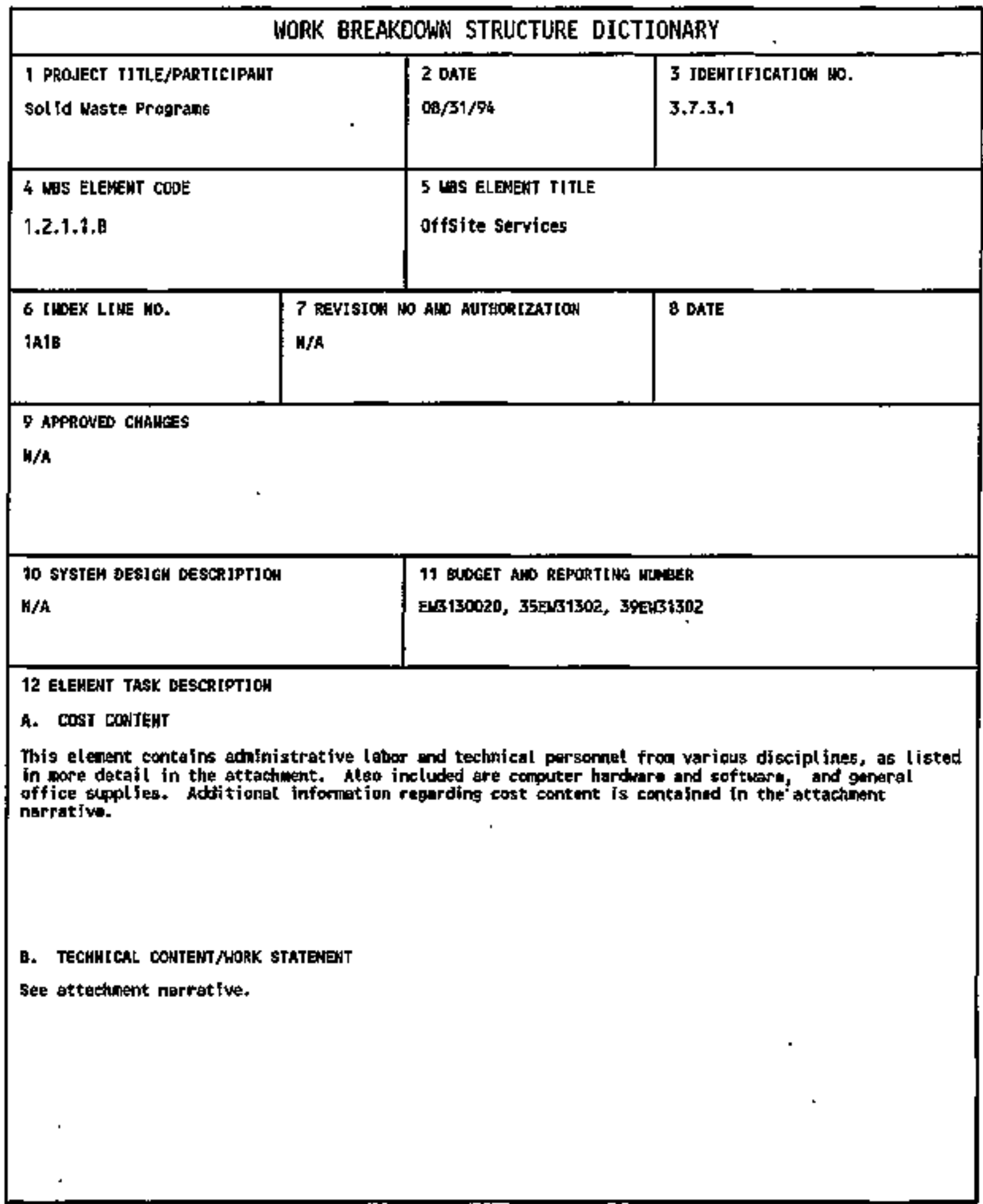


This product line represents work for off-site contractors (other $\mathbf{U} . \mathbf{S}$. Department of Energy offices and spectalized agencies, i.e., PSNS) provided by Soljd Waste Management. Four key areas are encompassed by this product 1 ine activity. These areas are waste receipt and handing of offsite low-level and low-level mixed wastes, off-site generator assistance, acceptance of the Navy's Deftueled Subuarine Reactor Compartments (SRC), and Federal Facilities Compliance Act (FFCA) technical support to the U.S. Department of Energy Richland office (RL). Funding is provided via DOE interworks, MPO, funding (fin plan), or other approved funding mechanism, and is performed on an as requested/needed basis.

Offsite Generator Assistance is provided via the Generator Assistance Progran (GAP). This program is responsible for direct point of contact support to all offsite generators shipping low-Tevel and radioactive mixed waste to the Hanford Site CWC and Burial Grounds. The GAP assists generators in a11 developmental phases of waste certification including program planning, Quality Assurance, working level procedures, training, and complete waste characterization and designation. Further support to offsite generators is provided to rec]assify and maintain their program status from a liaited to full approval status.

This product line further provides for the acceptance and disposal of the Navy's defueled Submarine Reactor Compartments (SRC) and other ancillary DOE wastes such as KAPL pumps, KAPL core baskets, Bettis pumps, and other Bettis wastes. Act1vities include preparation of waste acceptance criteria and pronulgation of this criteria to the Navy via approved Starage/Dfsposal Approval Record (SDAR) documentation, providing a]1 estimates and schedules, and obtaining funding from DOE or the Navy in support of SRC disposition. operations and maintenance of the Navy Trench (218-E-12B, Trench 94) is provided under this activity through trench preparation and construction of SRC foundations in accordance with the Havy design and applicable Hanford procedures.

Prior to receipt of waste, all necessary permits and completed documentation (e.g., Acceptance and Transport Movenent Checklist) are prepared and approved. Inspection and acceptance of waste packages as they are received to verify they meet the SDAR defined waste acceptance criteria is a1so performed. For SRC disposal a Transport Manager to represent RL and the Navy during waste movements is provided under this activity to coordinate onsite organizations and offsite contractors for the safe and efficient off-loading and transport of these large waste packages for disposal at their final destination.

Technical support to Federal Facjlities Compliance Act. (FFCA) activities is provided through this product line. The FFCA activities require continued support from Hanford to the Policy Coordination Group and associated work groups for the U.S. Department of Energy complex-wide system. This work involves the provision of an updated Mixed Waste Inventory Report (including five year volume projection, waste stream name, waste description, characterization data, 1 ikely preferred waste streati treatment option, quantities stored that are Land Disposa) Restriction (LDR) and non-LDR, waste 
minimization, Best Demonstrated Available Technology (BDAT) used for developing LDR requirements, and radionuclide content) and the developrent of the Final Site Treatment Plan Crosswalk document representing Hanford's Site Treatment Plan. The FFCA requires identification of existing site mixed waste treatment capabilities, planned treatment facilities including capacities, and planned technology development activities. This effort further supports various data management tasks, including techaical support for DOE complex wide systems development and integration, management of data calls, and DOE requested report preparations. Site interface tasks include review and comment on off-site and various DOE-HO FFCA draft documents. Participation in the DOE data management group meetings and technology development working group meetings is further provided. 


\section{SOLID WASTE PROGRAM}

WBS 1.2 .1

FY 1996 Program Plan

WHC-\$P-1114, Rev. 1

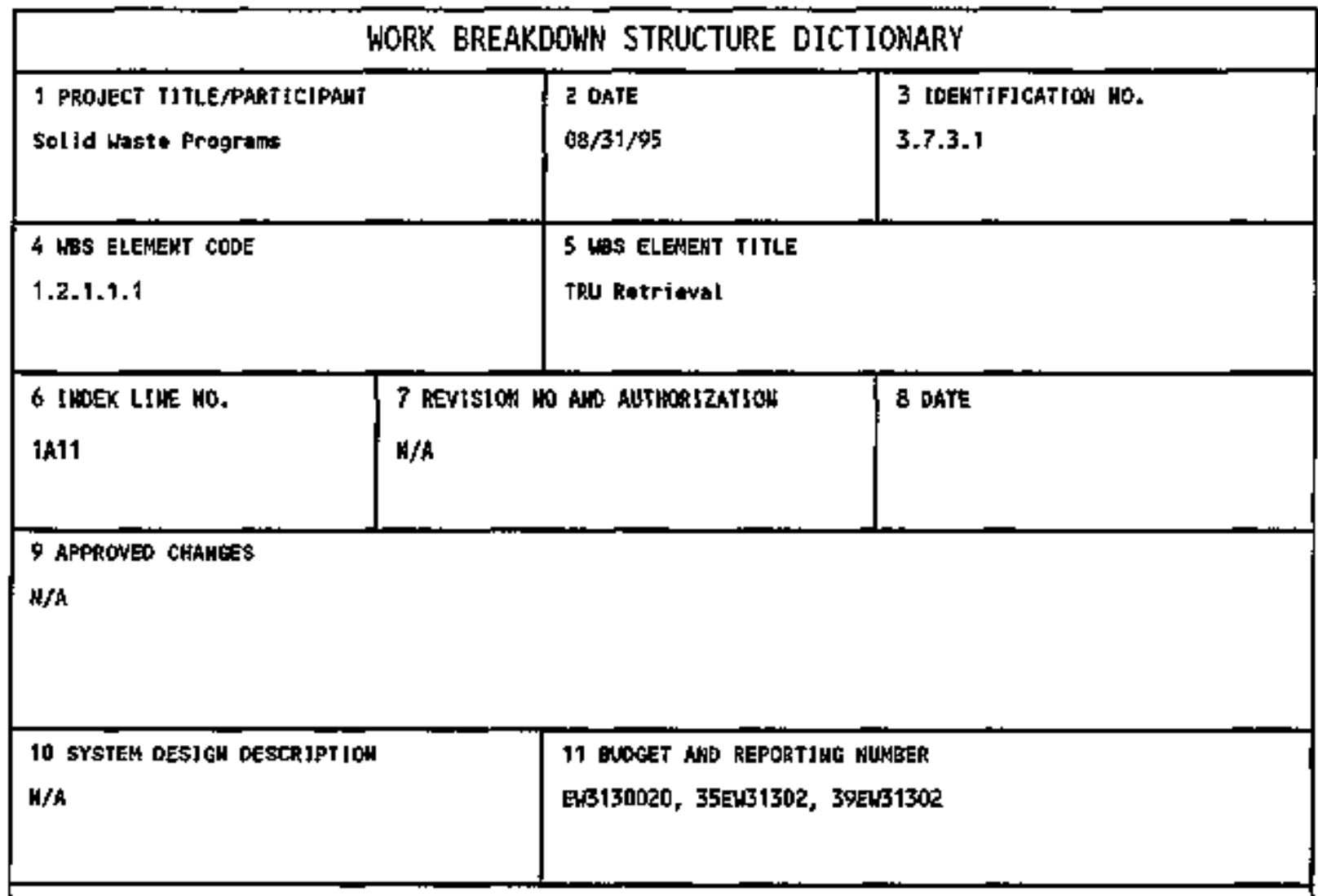

12 EEEHEHT TASK DESCRIPTIOW

A. COST CONTENT

This elenent provides for the pi lot retrieval of retrievably stored TRU. This iten is currently not funded.

B. TECAMICAL COWTENT/WORK STATEMENT 


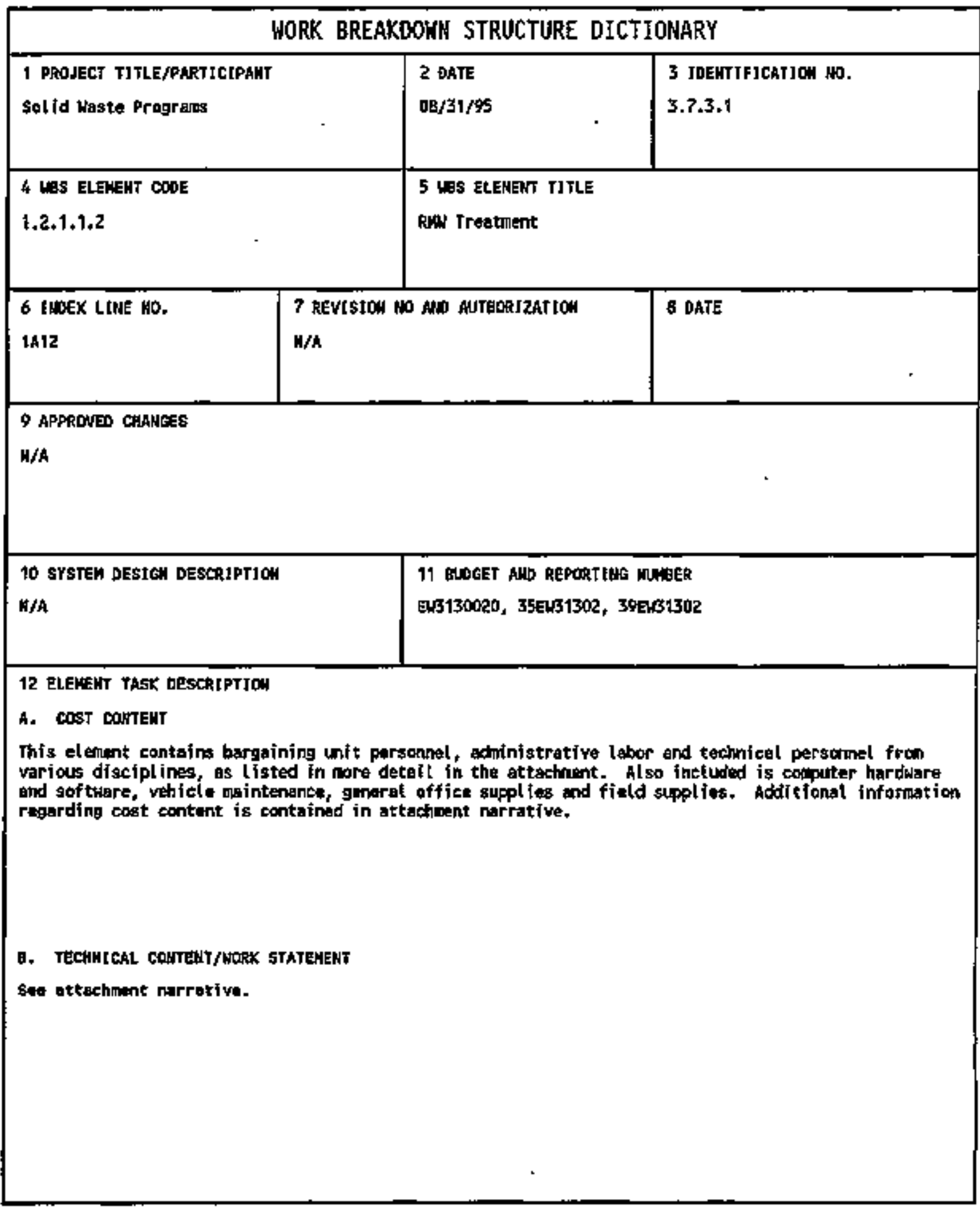


Operations

- Prior to disposition of RWN for disposal, treatment, or shipment, characterization of the waste is required to determine the dangerous waste constituents. Once the waste constituents are known, then the appropriate labeling, handling, treatment, and disposal measures may be applied per the Washington Administrative Code (WAC), the Code of Federal Regisations (CFR), the Resource Conservation and Recovery Act (RCRA), the Toxic Substances Control ACt (TSCA), and DOE Orders. It is a requirement of the waste generator to properily characterize the waste prior to shipment from the generating facility and to a treatment, storage, and disposal facility. However, there is an inventory of RHW that has not fully been characterized. Th1s waste includes polychiorinated bi-pheny1 (PCB) contaminated RWH. Before further actions (treatment, storage, disposal) may be taken with this waste, final characterization will be required pursuant to regulatory reçuirements.

Solid Waste wlll utflize a waste inventory control system that employs analytical characterization equipment which interacts with data management hardware and software to provide on-1ine waste analysis.

\section{Program control and Administration}

Progran support includes scheduling, financial, records management and program business reporting activities. Scheduling activities include FY and Tong-term development; program monitoring and control functions; and reporting support as defined in WHC-CH-5.34, Section 1.31. Financial activities inciude FY budget development and long-term requirements planning; program monitoring and control functions to include variance analysis report and estimate at completion preparation; and reporting support as requested. Records management support wilר include assistance in assessment of all documents onhand and preparation for necessary storage of these documents. Records/documents wil] be prepared, indexed, processed through the optical scanner system and forwarded to the Records Holding Area as necessary. The Boeing Computer Services Richland Records Managenent group will provide training to Solid Waste Disposal personnel on preparation of records for scanning system. The Records Inventory and Disposition Schedules and Report of Records Holdings process is included in this work scope.

\section{IBP Treatment Shipment Activities}

- This work package provides for the support of the shipment of 24,000 gallons of Tributyl Phosphate, currently stored at PUREX, to a commercial treatment contractor for destruction via thermal treatment. It al so funds support for the characterization and shiprent of 26,000 gallons of TBP stored at B P1ant.

A Waste Sampiing and Analysis plan will be prepared to deal with the specific requirements for waste characterization of waste to be shipped off the Hanford site for treatment by a commercia] vendor, and for veriftcation that the returned treated waste meets LDR and other applicable requirements. This Waste Sampling and Analysis Plan wiT stress cost effectiveness in meeting these requirements. The pian wi]] be subnitted to Ecology/EPA for review and comment, and for recommendations on ways to minitize costs while meeting applicable regulatory requirenents. 


\section{LLWH Stabilization}

- This activity provides for conarcialization efforts to secure and utjljze contract services for the stabilization of contact handled (CH) radioactive low level mixed wastes (LLMN) that are currently stored at the Hanford Central Waste Complex awaiting a treatment capability to permit permanent disposal compliant with the Land Dtsposal Restrjctions and LLFi that is forecasted to be generated by cleanup work at the Hanford site. The services will provide for non-therma] treatment, processing, packaging, and certification for permanent burial at the Hanford Site disposal facilities per the requirements of the Resource Conservation and Recovery Act (RCRA) and the State of Washington Administrative Code (WAC) 173-303.

\section{Sodium.Treatment}

- This work package provides funding to complete the procurement of the treatment services contract for SRE and HALLAM sodium presently stored in CWC, and for the treatment of this sodjum.

\section{Direct Disposa]}

- This work package provides for the preparation and implementation of the direct disposal of $183 \mathrm{H}$ solidified liquids and backiog soils. Activities will jnclude preparing a petition for the $183 \mathrm{H}$ formic acid waiver and a position paper regarding the acceptability of $183 \mathrm{H}$ solidified liquids, and submitting the docutients to Ecology/EPA for approval. Direct disposal of backlog soils will tnciude comp?etion of sampling and analysis to estabijsh that the soils are LDR compljant, preparation of a summary report, and submitting the report to Ecology/EPA for concurrence.

\section{ChC Operations plan}

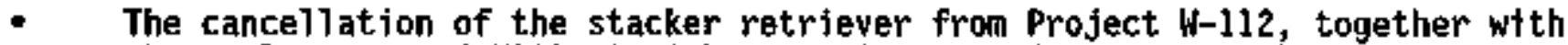
the replacement of WRAP 2A with a combjnation of a connercial treatment. contract, on site treatment and direct disposal, wij] significantly change the baseline operating plan for CHC. This work package provides for a value engineering study, and the participation of SWD management, technical support. and bargaining unit personnel to prepare a new CWC Operations plan based on these changes.

\section{1-5Y=101 Air Lances and oil treatment}

- This work package provides for the macroencapsulation and preparation for disposal of 241-\$Y-101 air lances, and for procuring treatment services for approximately 210 drums of low level waste oil presently stored in CHC. 


\section{SOLID WASTE PROGRAM}

WBS 1.2 .1

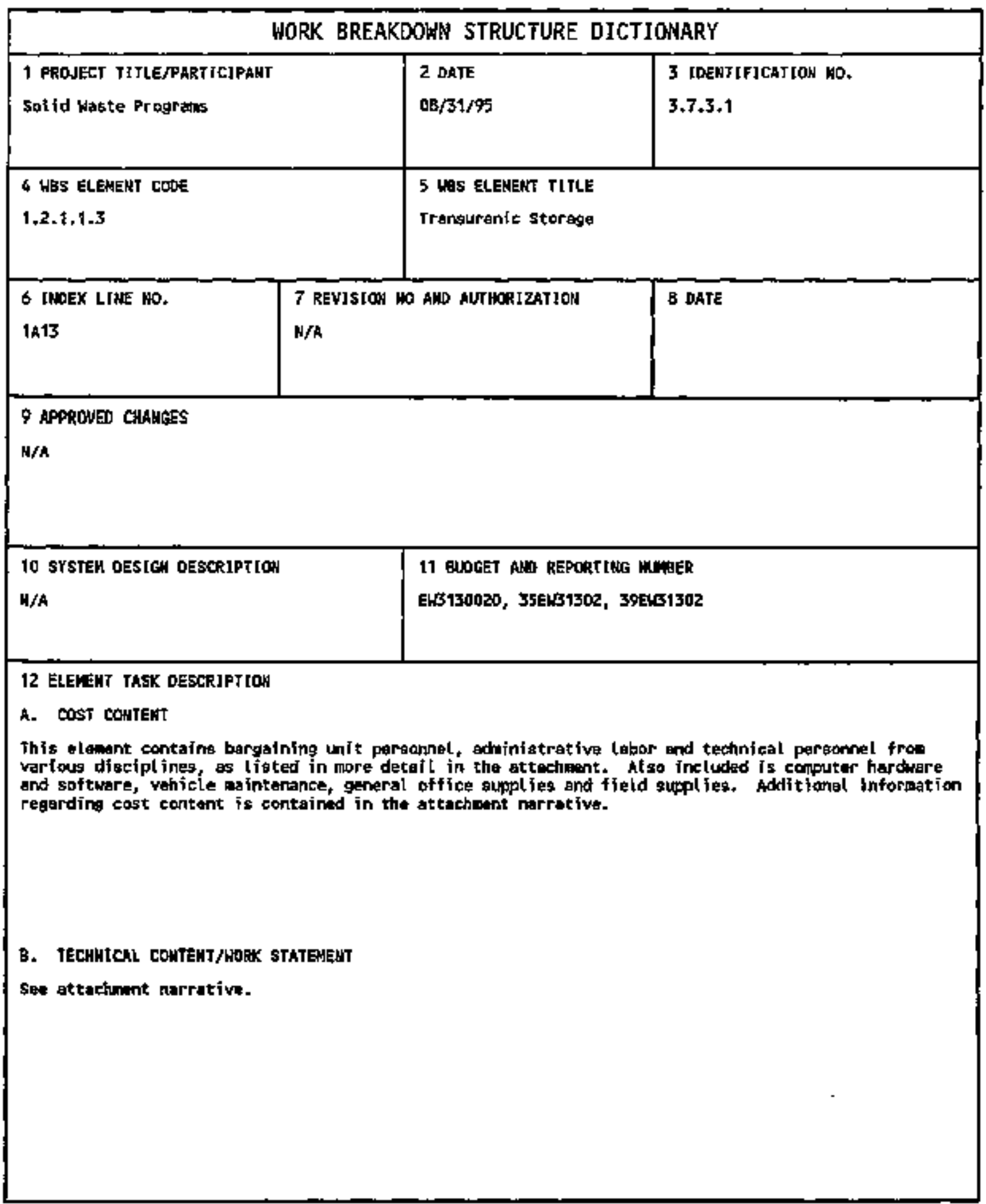


Transuranic (TRU) storage is the overall operation and maintenance of the Iransuranic Storage and Assay Facility (TRISAF). TRUSAF provides Resource Conservation and Recovery Act (RCRA) compliant storage of TRU mixed wastes and certified TRU wastes. Newly generated Contact-Handled (CH) TRU waste druns are received at TRUSAF for x-ray examinations and TRU content assay. The TRUSAF (224-T Bullding) processes up to 3,500 $\mathrm{ft}^{3}$ of $\mathrm{CH}-\mathrm{TRt}$ per year and provides the final quality assurance oversight necessary to assure that the waste complies with the Waste Isolation Pjiot Plants (WIPP) Waste Acceptance Criteria (WAC). The assay system is capable of distinguishing whether the TRU content is above or below the $100 \mathrm{nC} 1 / \mathrm{g} 1 \mathrm{imit}$ for Low Level Waste (LLW), thereby reducing the number of drums requiring interiat storage. JRUSAF also provides mixed waste storage in accordance with WAC 173-303. If drums of waste are below the TQU 11mit they are sent to the LLW Burial Grounds for disposai or Central Waste Complex for further interim storage. Additionally, it provides funding for engineering and design support for final closure; as well as, required engineering and certification review committee support for TRU waste management activities. Funding to perform actual receipt activities is funded, by the chargeback/assessment program ML5.

These support funettons include engineering analysis, building surveillance and nonitoring, facility maintenance and equipment calibration, personnel training and certification, database management, quality assurance and quality contro?, facility utility costs, design and procurement of support equipment, Ireatment, Storage, and Disposa1 (TSD) statutory/regulatory compliance, faciltty upgrades, engineering studies through development of functional design criteria, waste certification activities, waste acceptance criteria developalent, TSD spi11 prevention, RCRA dangerous waste periuit appTication activities, safety documentation activities, National Environmental Protection Agency documentation activities, operations support, work control, facility cognizant engineering, operations, lab support, technical support, RCRA closure plan activities, sampling activities, health physics, and safety and environmental engineering support activities. These support functions include Operational Readiness, Requirements Hanagement, and Defense Nuclear Facility Safety Board problem resolution support.

\section{Operat.tons}

- Operations activities include operations suppoirt to perform required facility routines and inspections; housekeeptng, tours, inventory, personnel training and certification, utility costs, logbook/records imput; Quality Assurance (QA)/Qualtty control support to perform periodic facility surveillances, procedure reviews and work plan approvals; Environmental Safety and Health Support includes safety evaluations and operating document reviews.

Health Phys1cs provides a, system to verify employees medical and training status prior to entry into radiological areas. Radiological data from both dose rates and contapination levels found in the fac1lities is tracked through the completion of routine surveys, documentation of results, and follow-up surveys. Support is also provided to effluent sampling and monitoring, radiological alarm response, off normal conditions, and energency response actions. A minimum baseline is required to provide support to operations, maintenance, and laboratory activities. These requirements include Operationa1 Safety Document. and Operational Safety Report surveillances, 
radioactive source control, air sampling tracking and trending, training certifications/recertification/on-the-job-training, emergency response capabilities, and absence relief personne?.

Managers provide oversight and managenent of the entire radiological protection program and TRUSAF Operations. AnaTyst/engineers provfde support to budgeting, purchasing, tracking and trending, scheduling, Job control System (ICS), As Low As Reasonable Achievable (ALARA) implementation, radiologica] controls, safety compliance, and emergency response. Clerical support includes typing correspondence, manual revisions, ensuring office and radiological suppties are maintained, answering phones, maintaining filing systems of administrative and radiological support and processing (A/legal record documents to the file custodian.

\section{Maintenance}

- Majntenance activities include the corrective maintenance work package activities, preventative majntenance, component-based recall calibrations, facility upgrades, fire systems maintenance, carpenter support, fabrication shop support, maintenance engineering support for the developnent of maintenance procedures, and the requjred training to support this activity.

\section{Hork Control}

- Work control provides the support for the scheduling of organizational manpower and equipment for this activity. Hork package planning utilizing the JCS wiTl also be provided by work control to ensure the required release of work to be performed on a day-to-day basis. This support will include any procurement needed to meet the schedule commitments for the support of this activity.

\section{Engineerina Support}

- Cognizant engineering support provides the technical approvals and assistance for review of the Safety Analysis Reports (SAR), work plans, procedures, JC5 work packages, facility drawings, engineering change notjces, and other supporting documents that relate to the facility operations. Engineering in this work package is responsible for the facllity configuration control and the required training to support the function of this operation.

\section{Safety Documentatjon}

- This work package provides the safety analysis documentation to define the safe operating envelope for the Transuran1c Storage and Assay Facility (TRUSAF) as a Mon-Reactor Nuclear Factlity. Preparation and review of the Solid Haste Storage Master SAR's in support of the facility is further provided. Tasks included in this work package are hazard classiffication, hazard identification and evaluation, and preliminary safety evaluations. This work package also provides the funding to maintain training in support of this activity. 


\section{Environmental Operations}

- This work package provides engineering support to the TRUSAF Facility including air quality permitting documentation, packaging and shipping interface, sampling and analysis, and storage requirements. This work package also provides for the required training needed to support this activity.

Further support is provided to continue permitting efforts associated with the TRUSAF Facility Part B Permit, resolve notice of deficiencies as negotiated with Ecology and the Environmental Protection Agency, prepare and issue unit manager's meeting minutes and provide perlodic status reports to the solid waste program offtce, and interface with other Hestinghouse Hanford company programs and regulators regarding the site wide permit. This work package also provides the funding to maintain training in support of this activity.

\section{Operations.Support}

- This work package provides for certification training of operatjons and Maintenance. Operations support activities including procedures, Readiness Review activities, waste minimization, ALARA, environmental compliance supports, Solid Waste Information Tracking System administration, Building Energency Plans, and occurrence reports will also be provideo in this work package. Work control and the operations support functions receive the required training needed to support the operations activities through this work package.

\section{Technical Support}

- Provide the technical engineering support to the TRUSAF facility and for compliance with the Waste Isolation Pilot Program (WIPP) Waste Acceptance criteria. This work package also provides the funding to maintain training in support of this activity.

\section{Operationa] Readiness}

- This work package provides Operational Readiness support for new or modjfied operations, processes or facilittes as requested. It further establishes and maintains the requirement's baseline, and assists with implementations or exemptions, as necessary. Defense Nuclear Facility Safety Board problem resolution support is al so provided.

\section{IRU Certification}

- This work package provides technicai support to the Hanford site generators for TRU certification to the WIPP criteria. TRU certification is required prior to shipment of waste to the WIPP. TRU certiftcation requirements are dissemtnated to the generators through regularly scheduled meetings, generator assessments, and incorporated into the Hanford Solid Haste Acceptance Criteria (내C-EP-0063).

This work package aiso provides the funding to matntain training in support of this activity. 


\section{Program Support}

- Program support includes scheduling, financial, records managenent and pragram business reporting activities. Scheduling activities include FY and Jong-term development; program monitoring and control functions; and reporting support as defined in $\mathrm{WHC}-\mathrm{CH}-5-34$, Section 1.31. Financial activities include $\mathrm{FY}$ budget development and long-term requirements planning; program monitoring and control functions to include Variance Analysis Report and estimate at completion preparation; and reporting support as requested. Records management support will inctude assistance in assessment of all documents onhand and preparation for necessary storage of these documents. Records/documents will be prepared, indexed, processed through the optical scanner system and forwarded to the Records Holding Area as necessary. The Boejing Computer Services Richland Records Managenent group will provide training to Solid Waste Disposal personnel on preparation of records for scanning syster. The Records Inventory and Disposition Schedules and Report. of Records Holdings process is included in this work scope. 


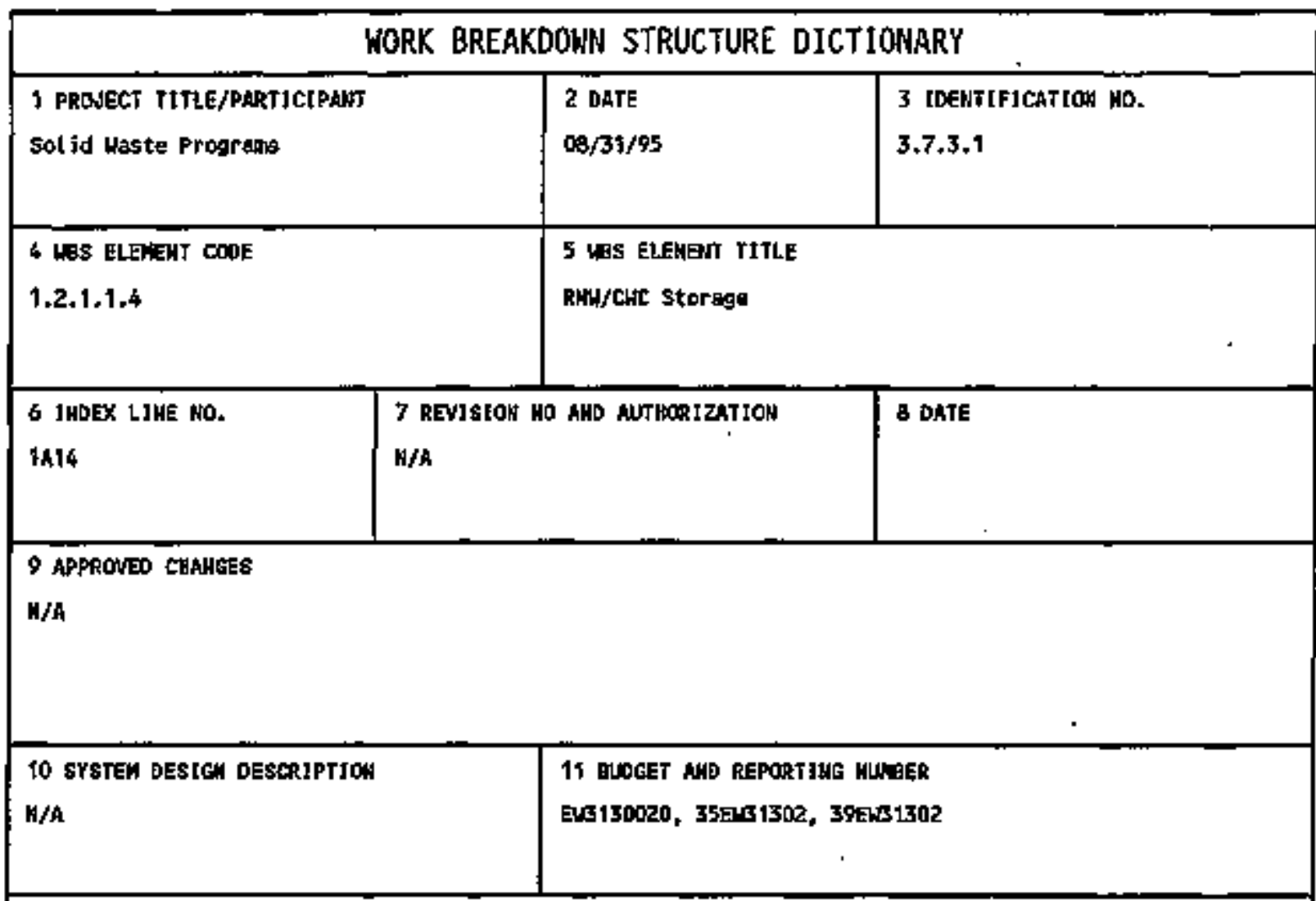

12 ELEMEMT TASK DESThaptItoH

A. cost CONTENT

This element centaing bergatining unt personnel, odwinistrstive labor and tochnlcal personnel from varfous discipl fnes, as tisted in wore datail in the attachent. Alsa included is canputer herdurare and software, vohicle maintenence, general office suppties and field stpplies. hdditional inforation regarding cost content is containgd in the attachuont narrative.

B. TECAKJGAL CONTEHT/LORK STATENEMT

See ottachment narrative. 
Radjoactive Mixed Haste (RMDi)/Central Haste Complex (CNC) storage is the overall operation and maintenance of the CWC which provides permitted Resource Conservation and Recovery Act (RCRA) and Toxic Substance and Control Act (TSCA) compliant mixed waste storage for the Hanford site. Mixed waste includes radjoactive iow level waste and Transuranic (TRU) mixed waste. The CWC also stores other wastes such as contaminated 0 \%1 s, chelating agents and certain other TRU and greater than Class 3 Hastes. Approximately 12,000 cubic feet of mixed waste is received annually. The Cuc consists of a series of metal above ground RMW buildings (16 total), the mixed waste storage pad, polychlorinated biphenyls storage facility, low flash point storage modules (12 total) and alkal 1 metal waste storage (12 total). Additionaliy it provides funding for engineering and design support for final closure; as well as, required engineering and certification review cormittee support for Batl management activities. These support functions include engineering analysis, building survell1ance and monitoring, factlity maintenance and equipment calibration, personnel tralning and certification, data base management, quality assurance and quality control, facility utility costs, design and procurement of support equipment, Treatment, Storage, and Disposa? (TSD) statutory/regutiatory complance, facllity upgrades, engineering studies through develepment of functional design criteria, waste certification activities, waste acceptance criteria development, TSD spinj prevention, RCRA dangerous waste permit application activities, safety documentation activities, National Environmental Protection Agency documentation activities, operations support, work control, facility cognizant engineering, operations, lab support, technical support, RCRA closure plan activitíes, sampling activities, health physics, and safety/environmental engineering support activities.

\section{Operations Activities}

- Operations activities include operations support to perform requíred facility routines and inspections, housekeeping, quarterly program reporting, tours, inventory, personnel training and certifícation, uti]ity costs, logbook/records input; Quality Assurance (QA)/Qualjty Control support to perform periodic facility survelllances, procedure reviews and work plan approvais; Environmental Safety and Health Support includes safety evaluations and operating document reviews.

Health Physics provides a system to verify employees medical and training status prior to entry into radiological areas. Radiological data from both dose rates and contamination levels found in the faciljties is tracked through the completion of routine surveys, documentation of results, and follow-up surveys. Support is a]so provided to effluent sampling and monitoring, radiological alarm response, off-normal conditions, and emergency response actions. A minimum baseline is required to provide support to operations, maintenance, and laboratory activities. These requirements include Operational Safety Document and Operationa] Safety Report survel11ances, radioactive source control, air sampling tracking and trending, training certifications/recertification/on-the-job-training, emergency response capabilities, and absence relief personnel.

Managers provide oversight and management of the entire radiological protection pragram and RAW/CWC Storage Operations. Analyst/engineers provide 
support to budgeting, purchasing, tracking and trending, scheduling, Job Contro] Systen (JCS), As Low As Reasonable Achievable (ALARA) implementation, radiological controls, safety compliance, and emergency response. Clerical support includes typing correspondence, manual revistons, ensuring office and radiological supplies are maintained, answering phones, abintaining filing systems of admintstrative and radiological sapport and processing QA/Tegai record documents to the file custodian.

\section{Majintenançe}

- Maintenance activities include the corrective maintenance work package activities, preventative maintenance, component-based recall cal1brations, facility upgrades, fire systems mafntenance, carpenter/painter support. fabrication shop support, maintenance engineering support for the development of mainterance procedures, and the required training to support this activity.

\section{Work Contro?}

- Work control provides the support for the scheduling of organizational manpower and equipment for this activity. Work package planning utilizing the JCS will also be provided by work control to ensure the required release of work to be performed on a day-to-day basjs. This support will include any procurement needed to meet the schedule commitnents for the support of this activity. Maintenance procedure development and control is provided by this work package.

\section{Coanizant Engineering Support - Cuc}

- Cognizant engineering support provides the technical approvals and assistance for review of the Safety Anajys is Reports (SAR), work plans, procedures, JCS work packages, facil ity drawings, engineering change notices, and other supporting documents that relate to the facility operations. Engineering in this work package ts responsible for the facility configuration control, project support, and the reguired training to support the funct.lon of this operation.

\section{Safety Documentation}

- This work package provides the safety analysis documentation to define the safe operating envelope for the $c t i c$ as a Hon-Reactor Nuclear Facijity. Preparation and review of the Solid Waste Storage Master SAR's in support of the facility is further. provided. Tasks included in this work package are hazard classtfjcation, hazard identification and evaluation, and preliminary safety evaluations. This work package also provides the funding to maintain tratning in support of this activity.

\section{Environmental Operations}

- This work package supports the cuc facilities including air quality perjitting documentation, packaging and shipping interface, sampling and analysis, and storage requirements. This work package also provides for the required training needed to support this activity. 


\section{SOLID WASTE PROGRAM}

WBS 1.2.1

Further support is provided to continue permitting efforts associated with the CWC facilities Part B Permit, resolve Notice of Deficiencies as negotiated with Ecology and the Environinenta] Protection Agency, prepare and issue unit manager's meeting minutes and provide periodic status reports to the soif id waste program office, and interface with other liestinghouse Hanford Company programs and regulators regarding the site wide permit. This work package also provides the funding to majntain training in support of this activity.

Overall program planning and integration for solid waste Nationat Environmental Protection Agency (KEPA) documentation is provided. In addition, this activity provides for a point of contact with regulators for Solid haste Programs, and maintains awareness of evolving regulations and inpacts to current operations and planning. Requests for determination of NEPA documentation for solid waste activities and fac17ities is also supported.

\section{Qperations Support}

- This work package provides for certification training of Operations and Maintenance. Operations support activities including procedures, Readiness Review activities, waste minimization, ALARA, environmental compliance support, Sol id Waste Information Tracking System administration, Building Emergency Plans, and occurrence reports is also provided in this work package. Work control and the operations support functions receive the required training needed to support the operations activities through this work package.

\section{Technical Support}

- This work package supports the CHC facilities for compliance with Resource Conservation and Recovery Act (RCRA), TSCA, and state regulations. This work package also provides the funding to maintaln training in support of this activity. Support activities also include recommending changes to sample and analysis requirements for generators of solid waste, ensuring any required changes are integrated into waste acceptance criteria documents, taking and analyzing samples for RCRA/state criteria, and issufing reports of sampie analytical results. The $183 \mathrm{H}$ Basin waste sample analysis and data evaluations are supported by this work package.

\section{Operational Readiness}

- This work package provides Dperational Readiness support for new or modified operations, processes or facilities as requested. It further establishes and maintains the requirement's baseline, and assists with implementations or exemptions, as necessary. Defense Nuclear Facility Safety Board problem resolution support is al so provided.

\section{TRU Certfficatton}

- This work package provides interface between Hanford and WIPP for TRU waste disposal and 5 torage issues and activities. TRU certification is required prior to shipment of waste to the WIPP. TRU certification requirements are disseminated to the generators and incorporated into the Hanford Solid Waste 
Acceptance Criteria (bHC-EP-0063).

This work package also provides the funding to maintain training in support of this activity.

\section{Program Support}

- Program suppori includes scheduling, financfal, records management and program business reporting activities. Scheduling activities include $F Y$ and long-term development; program monitoring and control functions; and reporting support as defined in WHC-CH-5-34, Section 1.31. Financial activities include Fy budget development and long-term requireaents planning; prograro monitoring and control functions to include Variance Analys1s Report and estinate at. completion preparation; and reporting support as requested. Records management support will include assistance in assessment of al1 documents onhand and preparatton for necessary storage of these documents.

Records/documents will be prepared, indexed, processed through the optical scanner systern and forwarded to the Records Holding Area as necessary. The Boeing Computer Services Richland Records Management group will provide training to Solid Waste Disposal personnel on preparation of recoris for scanning system. The Records Inventory and Disposftion Schedules and Report of Records Holdings process is included in this work scope. 


\section{SOLID WASTE PROGRAM}

WBS 1.2 .1

\begin{tabular}{|c|c|c|}
\hline \multicolumn{3}{|c|}{ NORK BREAKDOHE STRUCTURE DICTIONARY } \\
\hline $\begin{array}{l}1 \text { PROJECT TITLE/PARTICIPAHT } \\
\text { Solid Waste Progran: }\end{array}$ & $\begin{array}{l}2 \text { OATE } \\
0 \mathrm{OB} / 31 / 94\end{array}$ & $\begin{array}{l}3 \text { SOENTIELCATLOH NO. } \\
3.7 .3 .1\end{array}$ \\
\hline $\begin{array}{l}\text { \& UAS Eleirent toof } \\
1.2 .1 .1 .5\end{array}$ & \multicolumn{2}{|c|}{$\begin{array}{l}5 \text { HBS ELEMENT TITLE } \\
\text { Hazardotse haste storage }\end{array}$} \\
\hline $\begin{array}{l}6 \text { JMDEX LJME NO. } \\
\text { IA15 }\end{array}$ & $\begin{array}{l}7 \text { REVISION NO ND AUTHORJZATION } \\
\text { K/A }\end{array}$ & 8 DATE \\
\hline \multicolumn{3}{|l|}{$\begin{array}{l}9 \text { APPRONEO CHANGES } \\
\text { W/A }\end{array}$} \\
\hline $\begin{array}{l}\text { } 10 \text { SYSTEN DESIGH DESCRIPTLON } \\
\text { M/A }\end{array}$ & \multicolumn{2}{|c|}{ 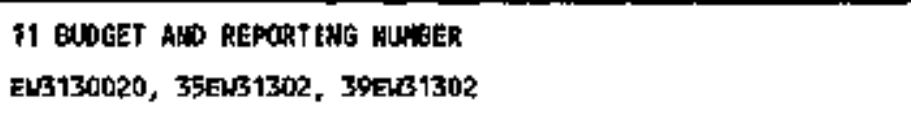 } \\
\hline \multicolumn{3}{|c|}{ 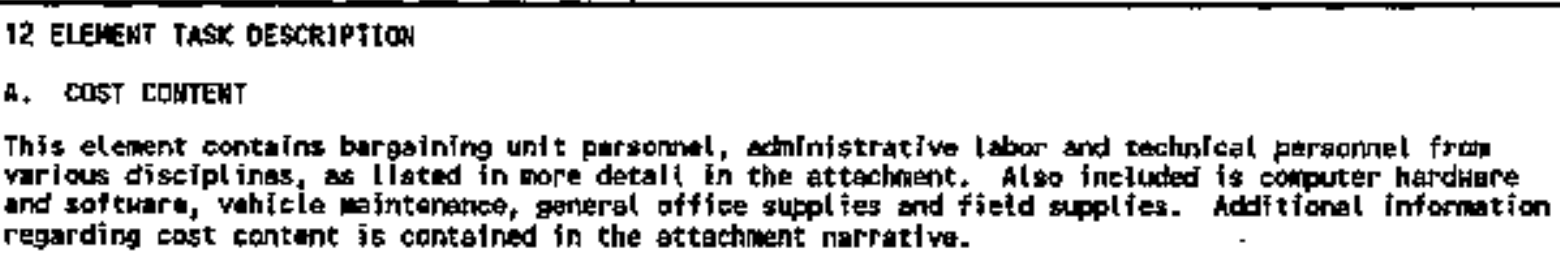 } \\
\hline \multicolumn{3}{|c|}{ 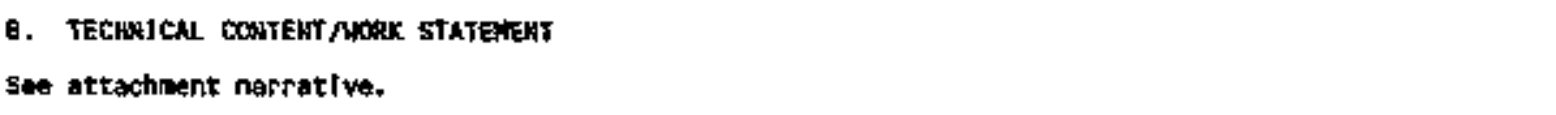 } \\
\hline
\end{tabular}


Hazardous Waste Storage Operations is the overall operation and malntenance of the 616 Non-radioactive Dangerous Waste Storage Facitity (NRDSF). The 616 Building serves as a centrailized Hanford facility to segregate and store nonradioactive dangerous wastes prior to offsite shipment for treatment and disposal. This building provides Toxic Substances Control Act (TSCA) and Resource Conservation and Recovery Act (RCRA) compliant storage of Nonradioactive Dangerous Waste per WAC 173-303. Operations typically receives approximately 1,200 containers of waste each year. Then approximately 12 to 15 times per year, the waste is manifested, inspected, and shipped offsite for treatment/disposal. Additionally, funding of administrative support to the Hanford Central Landfill is included. Funding to perform actual receipt activities is funded by the chargeback/assessment program ML8.

\section{pperations Activitjes}

- Operations activities include operations support to perform required facility routines and inspections, housekeeping, quarterly program reporting, tours, inventory, personnel trajning and certification, utility costs, logbook/records input; Quality Assurance (QA)/Quality Control support to perform periodic facility surveillances, procedure reviews and work plan approvals; Environmental Safety and Health Support includes safety evaluations and operating document reviews.

Managers provide oversight and managenent of the MRDWSF Operations. Analyst/engineers provide support to budgeting, purchasing, tracking and trending, scheduling, Job Control Systen (JCS), safety compliance, and emergency response. Clerical support includes typing correspondence, manua] revisions, ensuring office supplies are maibtained, answering phones, maintatning f1ling systens of adrijistrative support, and processing QA/legal record documents to the fille custodian.

\section{Matntenance}

- Maintenance activities include the correcttve maintenance work package activities, preventative maintenance, component-based recail caljbrations, factilty upgrades, fire systems maintenance, carpenter support, fabrication shop support, maintenance engineering support for the developenent of raintenance procedures, and the required training to support this activity.

\section{Hork Control}

- Hork control provides the support for the scheduling of organizational manpower and equipment for this activity. Work package planning utjlizing the JCS will also be provided by work control to ensure the required release of work to be performed on a day-to-day basis. This support will include any procurement needed to meet the schedule coalinipents for the support of this activity.

\section{Engineering Support}

- Cognizant engineering stpport provides the technica] approvals and assistance for review of Safety Assessments, work plans, procedures, JCS work packages, facility drawings, engineering change notices, and other supporting documents 
that relate to the facility operations. Engineering in this work package is responsibie for the facility configuration control and the required training to support the function of this operation. Included is funding for Reengineering review of 616 operations and transition planning if the facility is shutdown.

\section{Safety Documentation}

- This work package provides the safety analysis documentation to define the safe operating envelope for the NRDWSF. Tasks included in this work package are hazard classification, hazard identification and evaluation, and preliminary safety evaluations. This work package also provides the funding to maintain training in support of this activity.

\section{Environmenta] Operations}

- Support is provided to continue permitting efforts associated with the NRDWSF Part B Permit, as negotiated with Ecology and the Environmental Protection Agency, prepare and issue unit manager"s meeting minutes and provide periodic status reparts to the solid waste progrard office, and interface wth other Westinghouse Hanford company programs and regutators regarding the site wide permit. Planning for tasks necessary to complete clean closure of the 200 West Area Ashpit, 218-E-8 Borrow Pit, and Hanford Patrol Acadend Demolition (HPAD) sites is further provided. This work package also provides the funding to maintain training in support of this activity.

\section{Operations Support}

- This work package provides for certification training of operations and Maintenance. Operations support activities including procedures, Readiness Review activities, waste minimization, environmental compliance supports, Solid liaste Information Tracking System administration, Bujlding Emergency Plans, and occurrence reports will also be provided in this work package. Work control and the operations support functions receive the required training needed to support the operations activities through this work package.

\section{Technjcal Support}

- This work package provides for the technjcal engineering support to the NRDWSF facility for offsite shipments of waste for treatment/disposal. It further ensures that the shipments are in accordance with WAC 173-303. Activities iaclude procurement of offsite services, reports, moratorium waste release, land disposal restrictions notification to TSDs, and support to the 212-P Facility for PCB's.

\section{Dperational Readiness}

- This work package provides Operational Readiness support for new or modified operations, processes or facitities as requested. It further establishes and maintains the requirement's baseline, and assists with implementations or exemptjons, as necessary. 


\section{Program Support}

- Program support includes scheduling, financial, records management and program business reporting activities. Schedulting activities include $F Y$ and long-term development; program monitoring and control functions; and reporting support as defined in $\mathrm{WHC}-\mathrm{CH}-5-34$, Section 1.31. Financial activities include $\mathrm{FY}$ budget development and long-term requirements planning; program monitoring and control functions to include Variance Analysis Report and estimate at completion preparation; and reporting support as requested. Records management support wi11 inciude. assistance in assessment of all documients onhand and preparation for necessary storage of these documents. Records/doctuments will be prepared, indexed, processed through the optical scanner system and forwarded to the Records Holding Area as necessary. The Boeing Computer Services Richland Records Management group will provide training to Solid Waste Disposal personnel on preparation of records for scanning system. The Records Inventory and Disposition Schedules and Report of Records Holdings process is included in this work scope. 


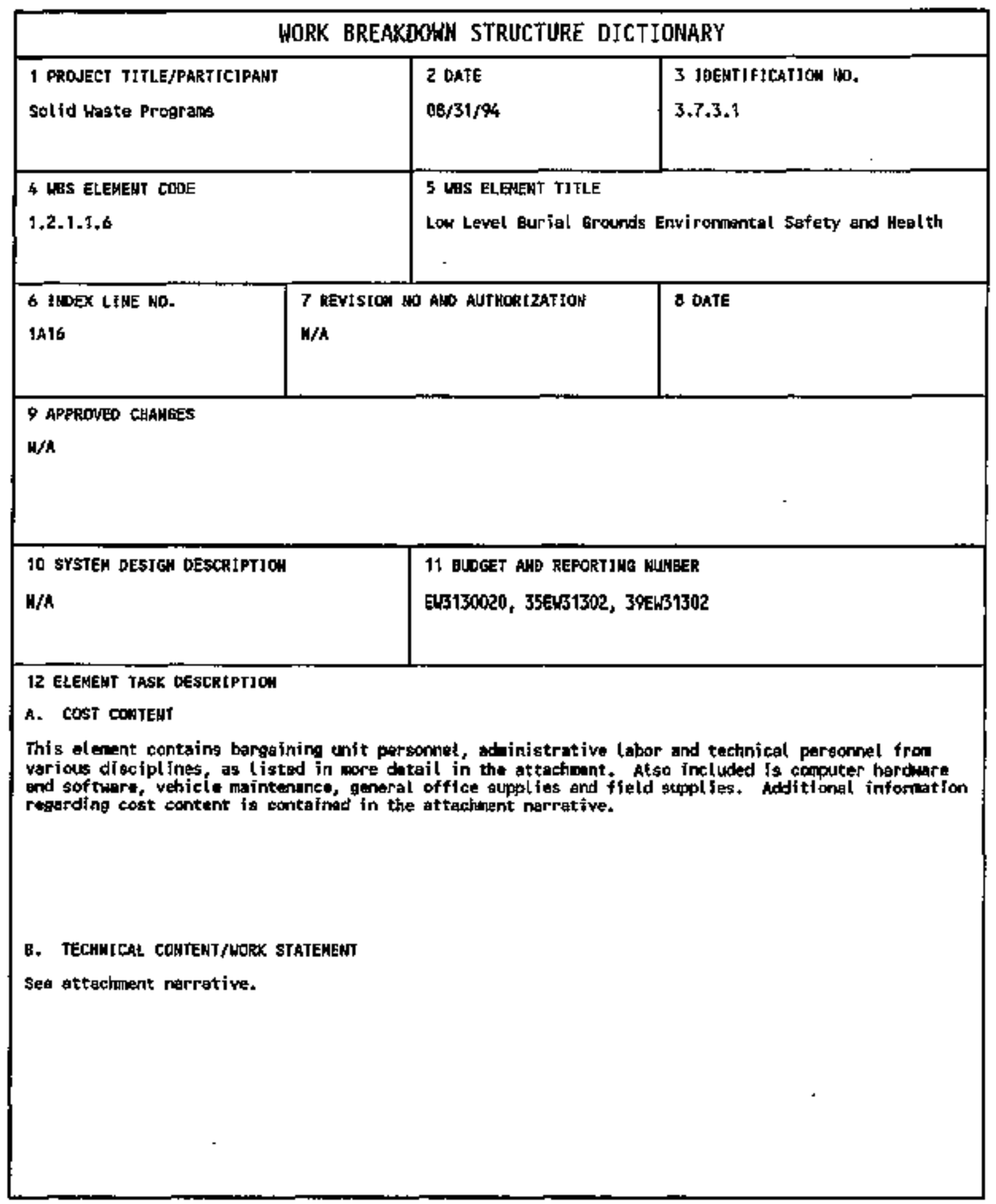


Low Level Waste Burial Ground (LLBG) Operations is the overall operation and maintenance of Hanford's Low Level Waste (LLW) near-surface disposal trenches located in 200 East and 200 West Areas. The main purpose of the LLBG operations is to receive and dispose of LLH from onsite and offsite generators. It serves as disposal area for drums and ttems which cannot be contained by drums due to size or configuration. It receives for disposal approximately 200,000 cubic feet of contact-handled and remote-handled waste on an annual basis. The burial grounds are composed of active (unfilled) and new trenches. Active burial grounds include 218-W-3A, 218-W-3AE, 218-W-4C, 218-E-10 and 218-E-12B, (sub reactor compartments), and 218-W-4B (caissons). Future low level buria] grounds will include 218-w-6.

This activity funds managerial, operational, technical, and administrative support to set up and operate the LLBGs. Additionally it provides funding for engineering and design support for final closure; as well as, required engineering and certification review committee support for LLW management activities. These support functions inclade engineering analysis, facility surveillance and monitoring, facility majntenance and equipment calforation, personnel training and certification, database management, quality assurance and quality control, facility utility costs, design and procurement of support equipment, Treatment, Storage, Disposal (TSD) Facility statutory/regtuatory compliance, waste certification activittes, waste acceptance criteria development, TSD spil1 prevention, Resource Conservation and Recovery Act (RCRA) dangerous waste permit application activities, safety documentation activities, Hational Envíronmental Policy Act (HEPA) documentation activities, operations support, work control, fac1lity cognizant engineering, operations, lab support, technical support, RCRA closure plan activities, sampling activities, heal th physics, and safety and environmental engineering support activities. These support functions include Operational Readiness, Requirements Management, and Defense Naclear Facility Safety Board prob]en resoiution support.

\section{Operations}

- Operations activities include operations support to perform required facility routines and inspections, housekeeping, quarterly program reporting, tours, inventory, personnel trajning and certification, utility costs, logbook/records input; Quality Assurance (QA)/Qual ity Control support to perform periodic facility surveillances, procedure reviews and work plan approvals; Environmental Safety and Health Support includes safety evaluations and operating document reviews.

Health Physics provides a system to verify employees medical and training status prior to entry into radiological areas. Radiological data from both dose rates and contamination levels found in the factijties is tracked through the completion of routine surveys, documentation of resuits, and follow-up surveys. Support 1s also provided to effluent sampling and monitoring, radiological alarm response, off normal conditions, and energency response actions. A minimur baseline is required to provide support to operations, maintenance, and laboratory activities. These requirements include Operational Safety Document and 0perational Safety Report surveiliances, radioactive source control, air sampling tracking and trending, training certifications/recertification/on-the-job-training, eimergency response 
capabitities, and absence reitef personnel.

Managers provide oversight and management of the entire radiologfcal protection program and LLBG operations. Analyst/engineers provide support to budgeting, purchasing, tracking and trending, scheduling, Job Control 5ystem (JCS), As Low As Reasonable Achievable (ALARA) implementation, radjologicai controls, safety comptiance, and emergency response. Clerical support includes typing correspondence, manuai revisions, ensuring office and radiological supplies are maintained, answering phones, maintaining filing systems of administrative and radiological support and processing QA/legal record documents to the file custodian.

\section{Maintenance}

- Maintenance activities inciude the corrective maintenance work package activities, preventative maintenance, component-based recal? caltbrations, fire systems alaintenance, carpenter/painter support, fabrication ship support, maintenance engineering support for the developtent of maintenance procedures, and the required training to support this activity.

\section{Hork Control}

- Hork control provides the support for the scheduling of organizational manpower and equipment for this activity. Hork package planning utilizing the JCS will also be provided by work control to ensure the required release of work to be performed on a day-to-day basis. This support will include any procurement needed to meet the schedule comoitments for the support of this activity.

\section{Engineering Supoort.}

- Cognizant engineering support provides the technical approvals and assistance for review of the Safety Analysis Reports (SAR), work plans, procedures, JCS work packages, facility drawings, engineering change notices, and other supporting documents that relate to the facility operations. Engineering in this work package is responsible for the facitity configuration control and the required training to support the function of this operation.

\section{Safety Documertation}

- This work package provides the safety analysis documentation to define the safe operating envelope for the LLBG as a Non-Reactor Nuclear Facility. Preparation and review of the Soljd Waste Disposal Master SAR's in support of the facility is further provided. Tasks included in this work package are hazard classification, hazard identification and evaluation, pre]imfnary safety evaluations, and LLBG Performance Assessment preparation. This work package a] so provides the funding to maintain training in support of this activity.

\section{Environmente] 0perations}

- This work package provides engineering support to the LLBG facilities including air quality permitting documentation, packaging and shipping 
interface, sampiing and analysis, and storage requirements. This work package also provides for the required training needed to support this activity.

Further support is provided to continue permitting efforts associated with the LLBG facilities Part 8 Permit, resolve Notice of Deficiencies as negotiated with Ecology and the Environinental Protection Agency, prepare and issue unjt manager's meeting minutes and provide periodic status reports to the solid waste program office, and interface with other Westinghouse Hanford Company programs and regulators regarding the site wide permit. This work package also provides the funding to maintain training in support of this activity.

This activity develops a strategy to integrate the operation and ultimate closure of the LLBG's. This includes optimization of the existing burial trenches; development of closure caps; monitoring, waste quantification, and development of capital projects; identification of MEPA process requirements; and preparation of appropriate plans and schedules. In addition, this activity provides for a point of contact with regulators for solid Waste Programs, and maintains awareness of evolving regulations and impacts to current operations and planning.

\section{Operations_Support}

- This wark package provides for certification training of Operations and Maintenance. Operations support activitjes inciuding procedures, Readiness Review activities, waste minimization, ALARA, environmenta] compliance support, Solid Waste Information Tracking System administration, Building Emergency Plans, and occurrence reports is also provided in this work package. Work control and the operations support functions recefve the required training needed to support the operations activities through this work package.

\section{Iechnical Support}

- This work package provides the technical engineering support to the LIBG facilities for compliance with Resource Conservation and Recovery Act (RCRA) and state regulations. This work package also provides the funding to majntafn training in support of this activity. Support activities also include recommending changes to sample and analysis requírements for generators of solid waste, ensuring any required changes are integrated into waste acceptance critería documents, taking and analyzing samples for RCRA/state criteria, and issuing reports of sample analytical results.

\section{0perational Readiness}

- This work package provides Operational Readiness support for new or modified operations, processes or facilities as requested. It further establishes and maintains the requirement's baseline, and assists with implementations or exemptions, as necessary. Defense Nuclear Facjlity Safety Board support is al so provided. 
Program Sunport

- Program support includes scheduling, financial, records management and program business reporting actlvities. Scheduling activities include FY and long-terth development; program monttoring and controi functions; and reporting support as defined in WHC-CM-5-34, 5ection 1.31. Financial activities include FY budget development and long-term requirements planning; program monitoring and control functions to include Variance Analysis Report and estimate at completion preparation; and reporting support as requested. Records management support wij include assistance in assessment of a]l documents onhand and preparation for necessary storage of these documents.

Records/documents will be prepared, Indexed, processed through the optical scanner system and forwarded to the Records Holding Area as necessary. The Boeing Computer Services Richland Records Management group w111 provide training to Solid Waste Disposal personnel on preparation of records for scanning system. The Records Inventory and Disposttian Schedules and Report of Records Holdings process is included in this work scope. 


\section{SOLID WASTE PROGRAM}

WBS 1.2.1

FY 1996 Program Plan

WHC-SP-1114, Rev, 1

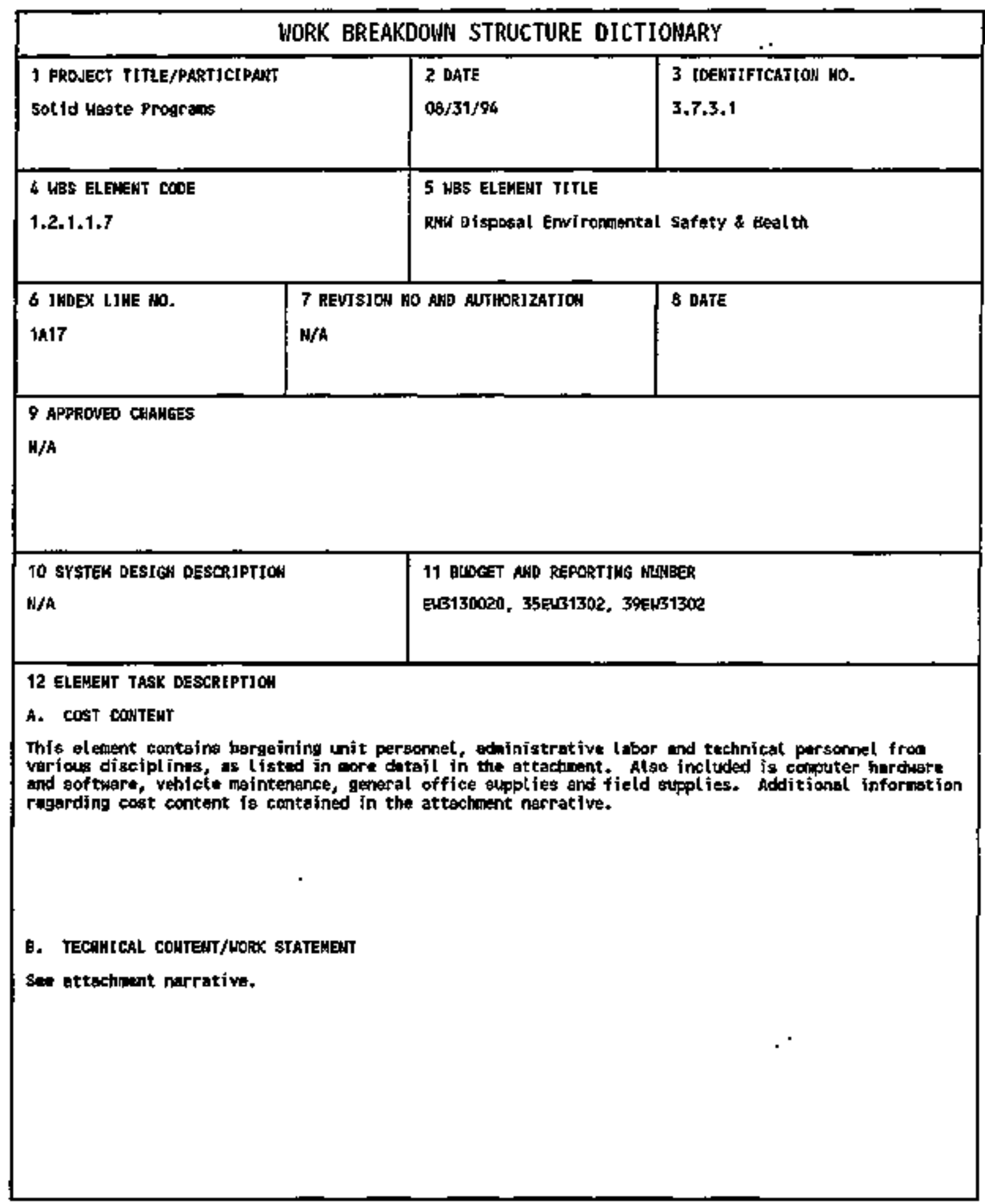


Radioactive Mixed Waste (RMW) disposal is the overail operation and maintenance of the two RHW trenches provided by Project $W=025$. These two trenches are RCRA compliant and 7 andfilis which contain double lined leachate collections systems and are located in 218-W-5 Burial Ground. They provide disposal capacity for 270,000 cubic feet of low level Rrat per trench. The landfill design will allow for operation of heavy equipment and placement of heavy waste loads. The waste is expected to come mainly from onsite waste generators, particularly the Environmental Restoration Program. The major planning assumption is that the trench will not be operated until large volumes of waste are ready for disposal.

This activity funds managerial, operational, technical, and administrative support to prepare operations of the RMW Disposal Trenches. Additionaliy, it provides funding for engineering and design support for final closure; as wel] as, required engineering and certification review committee support for RMW management activitfes. These support functions include engineering analysis, facijity surveillance and monitoring, facility maintenance and equipment calibration, personnel training and certification, database management, qual jty assurance and quality control, facility utility costs, desfon and procurement of support equipment, Treatment, Storage and 01sposal (TSD) statutory/regulatory complifance, facility upgrades, waste certification activities, waste acceptance criterla development, Resource Conservation and Recovery Act dangerous waste permit application activities, safety documentation activities, operations support, work control, facility cognizant. engineering, operations, lab support, technical support, RCRA closure pian activities, sampling activities, health physics, and safety and environmental engineering support activities. These support functions inciude Operationa? Readiness, Requirements Management, and Defense Nuclear Faci7ity Safety Board prablem resolution support.

\section{Operations}

Operations activities include operations support to start-up activities and to perform required facility routines and inspections, housekeeping, tours, personnel training and certification, logbook/records input; Quaj1ty Assurance (QA)/Quality Control support to perform periodic facility surveiliances, procedure reviews and work plan approvals; Environmental Safety and Health Support includes safety evaluations and operating document reviews.

Health Physics provides support to start-up activities inciuoing support to readiness evaluation. Support is also provided to effluent sampling and monitoring.

Managers provide oversight and management of the RNN Disposal Operations start-up activities. Ana]yst/engineers provide support to budgeting, purchasing, tracking and trending, scheduling, Job control system (JCS), As Low As Reasonable Achievable (ALARA) implementation, radiological contro?s, safety compliance, and emergency response. Clerical support includes typing correspondence, manual revisions, ensuring office and radiological supplies are maintained, answering phones, maintaining filing systems of adminjstrative and radiological support and processing $\mathrm{QA} /$ legal record documents to the file custodian. 
Maintenance

- Majntenance activities include the corrective maintenance work package activities and maintenance engineering support for the development of maintenance procedures.

\section{Work Control}

- Work control provides the support for the scheduting of organizational manpower and equipment for this activity. Work package planning utilizing the JCS will also be provided by work control to enstre the required release of work to be performed on a day-to-day basis. Thts support will include any procurement needed to meet the schedule commitments for the support of this activity. Procedure development/control is provided in parallel with Maintenance Engineering efforts.

\section{Enqineering_Support}

- Cognizant engineering support provides the technical approvals and assistance for review of the Safety Analysis Reports (SAR), work plans, procedures, JCS work packages, facility drawings, engineering change notices, and other supporting documents that relate to the facility operations. Engineering in this work package is responsible for the facility configuration control and the required training to support the function of this operation.

\section{Safety Documentation}

- This work package provides the safaty analysis dacumentation to define the safe operating envelope for the RAW Disposa] trenches as Mon-Reactor Nuclear Facilities, Preparation and review of the Solid Waste Disposal Master SAR's in support of the facility is further provided. Tasks included in this work package are hazard classification, hazard identification and eva]uation, and preliminary safety evaluations. This work package also provides the funding to maintain trajning in support of this activity.

\section{Environmental pperations}

This work package provides support to continue permitting efforts associated with the low level burial ground facilities Part B Permit, to resolve Notice of Deficiencies as negotiated with Ecology and the Environmental Protection Agency, to prepare and issue unit manager's meeting minutes and provide periodic status reports to the solid waste progran office, and to interface with other Westinghouse Hanford Company programs and regulators regarding the site wide permit. This work package also provides the funding to maintain training in support of this activity.

\section{Operations Sunport}

- This work package provides for certjfication training of Operations and Haintenance. Operations support activities including procedures, Readiness Review activities, waste mínimization, ALARA, environmenta] compiliance supports, Solid Waste Information Tracking System adrinistration, Building Emergency P]ans, and occurrence reports will also be provtded in this work 
package. Work contro? and the operations support functions receive the required training needed to support the operations activities through this work package.

\section{Technical Support.}

- This work package provides the technical engineering support to the RMN Disposal trenches for compliance with the Resource Conservation and Recovery Act (RCRA) and State Regulations, recommends changes to sample and analysis requirements for generators of RHW waste, and ensures any required changes are integrated into waste acceptance criteria documents. This work package also provides the funding to maintain training in support of this activity.

\section{Operationa? Readiness}

- This work package provides Operational Readiness support for new or modified operations, processes or faciljties as requested. It further estabiishes and maintains the requirement's baseline, and assists with implementations or exemptions, as necessary. Defense Nuclear Facility Safety Board support is also provided. 


\section{SOLID HASTE PROGRAM}

WBS 1.2.1

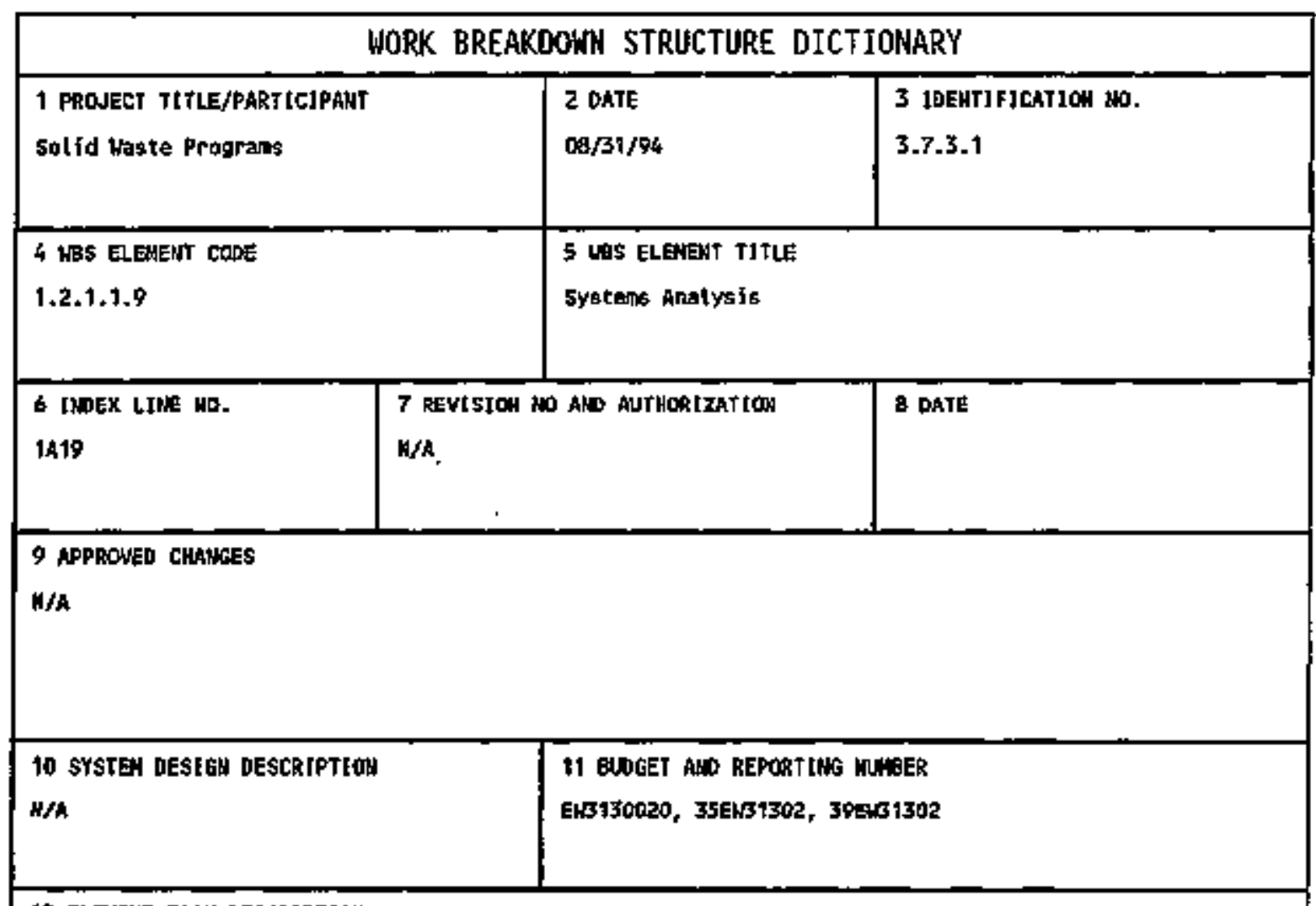

12 ELEEATT TASK DESGRIPTIOMN

A. cost LONTEWT

This eteoent contains adninistratiwe labor and technical parsonnal fron variouls disciplinge, as litoted in more dataft in the attachnent. Al so included ere cantulter hardware and saftware, vahiclit

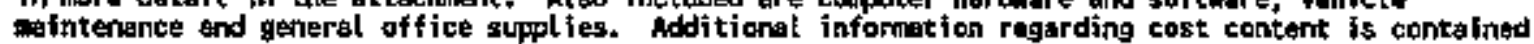
in the attachment norrative.

B. TECHKICAL COHTEHT/MORK STATEHEHT

soe attachment narrative. 


\section{SOLID WASTE PROGRAN}

WBS 1.2 .1

Systems Engineering analysis activities include continuance and development of the So] id Waste Technical Base7 ine Document (MHC-SD-WHA-RPT-060), the Waste Acceptance Criteria (HHC-EP-0063), Remote Handled (RH) soJid waste processing alternatives engineering study, and the Solid Waste Information and Tracking Systems (SWITS). Development and implementation of the Interface Control Documents with generators is also included in this cost account.

The Solid Waste Technical Baseline Document (WHC-SO-WM-RPT-060) forms the bases for the entfre Solid Waste Program and is the key Solid Waste program level systems engineering document. It consists of a Mission analysis, Functions and Requirements analysis, System Description, Program Documentation, Facility and Projects bases, and Uncertainties. To keep the baseline current, several sections of the document must be updated.

The Interface Control Documents (ICDs) serve as the vehicie to formally document the feed requirements imposed on Solid Waste by the major generators. The ICDs form a technical baseline for waste to be received by Solid Waste or waste and material to be sent by Solfd Waste to other prograns. The ICDS also satisfy the requirement of annual waste forecast and must be revisited annually to determine if change is warranted.

Support to the Site Wide Systems Engineering effort is key in satisfying the Tri-Party Agreement M-33 milestone for determination of site requirements for future Remote Handled waste and material facilities. This effort is also the current mechanism to achieve integration across the site to support the clean up mission.

The SWITS is an interactive, on-7ine data management systent that provides records management and tracking of solid waste data. The SWITS database is used to integrate Hanford waste generators with the Treatment, Storage, and Disposal (TSO) facility personnel. Haste designation, bar coding, and inventory tracking are also provided by SWITS.

Data from the SHITS database is used to provide Input to the DOE Integrated Database, Federal Facilities Compliance Act deliverables and other reparts required by DOE and other Federal and State agencles. DOE Orders and environmental regulations such as Resource Conservation and Recovery Act and the State Dangerous Waste Regulations requjire data management for TSD fac1lities.

The "Hanford Site Solid Waste Acceptance Criteria," (WHC-EP-0063) is reviewed anntally to incorporate any updated requirements for all waste type receiving requirements.

\section{work control}

- This work package provides for management oversight of the Systems Engineering Analysis efforts. Clerical support is also funded under this work package.

- This work package provides for the Solid Waste Systems Engineering

Alternatives Evaluation consistent with the Tri-Party Agreement $\mathrm{M}-33$ milestone. This activity includes an analys is of solid materials and waste requirements, and the development of new and revision of existing ICDs acting 
as a contractual agreement between Solid waste and the appropriate waste generator. This work package further provides the funding to maintain trajning in support of this activity.

Envfronmenta] Operations

- This work package provides overail progran planning and integration for soljd waste National Environmental Protection Agency (NEPA) documentation. Specific NEPA on new or planned Solid Haste facilities or activities will be required. These NEPA reviews may include categorical exclusions, Envíronmental Assessments (EA), supplemental analyses, and Environmental Impact Statements (EIS). An evaluation must be made and reported to U.S. Department of Energy, Headquarters annually, which identifies projects which nay require EAs or EISs in the next 12 or 24 months, respect1vely. This work package also provides requests for determination of NEPA documentation for solid waste activities and facilitles. Provision of a point of contact with regulators for solid waste programs in order to maintain awareness of evolving regilations and Impacts to current operations and planning is also made. This work package further provides the funding to maintain training in support of this activity.

\section{Techntcal Support}

- This work package provides technical support to Systems Engineering Analysis. The current Simscript Simulation model will be upgraded to include probabil1stic forecasting capability. other activities provided include maintenance of the Solid Haste Disposal Division Technical Baseline Description Document (WHC-SD-WIM-RPT-060); revfew and update of the "Hanford Soljd Waste Acceptance Criteria," $\mathrm{HHC}+\mathrm{EP}-0063$; and funding to matntain training in support of this activity. In addition, support for the developent of alternatives for processing Remote Handled (RH) solid materials and waste is provided.

\section{Program_Control Adginistration}

- Program Control Adninistration includes schedu]ing, financiai, records management and progran business reporting activities. Scheduing activities include FY and long-term development; program monjtoring and control functions; and reporting support as defined in $4 \mathrm{HC}-\mathrm{CH}-5.34$, Section 1.31 . Financlal activittes include $\mathrm{FY}$ budget development and iong-term requirements planning; program monitoring and control functions to include Variance Analysis Report and estimate at completion preparation; and reporting support. as requested. Records management support will include assistance in assessment of all documents on-hand and preparation for necessary storage of these documents. Records/documents witt be prepared, indexed, processed through the optical scanner system and forwarded to the Records Holdfng Area as necessary. The Boeing Computer Services Richland Records Managenent group will provide training to solid Waste Disposal personnel on preparation of records for scanning system. The Records Inventary and Disposition Schedules and Report of Records Holdings process is included in this work scope. 
SHITS Program Development.

- This work package provides continued developnent of the Solid WasteInformation Tracking System (SWITS). The SWITS is an interactive, on-1ine, user friendiy data management system that provides records managenent and tracking of solid waste data. The SWITS database is used to integrate Hanford waste generators with the Treatment, Storage, and Disposal (TSD) facility personnel. Waste designation, bar coding, and inventory tracking are also provided by SWITS. PI anned upgrades inciude development to convert waste generator tracking system users, automate waste acceptance services recejpt forms, add a geographic information operation, and trench radionuclide tracking. The enhancement of the capability to respond to ad hoc report requests and the deveiopment of HRAP data management interface will al so be provided. 


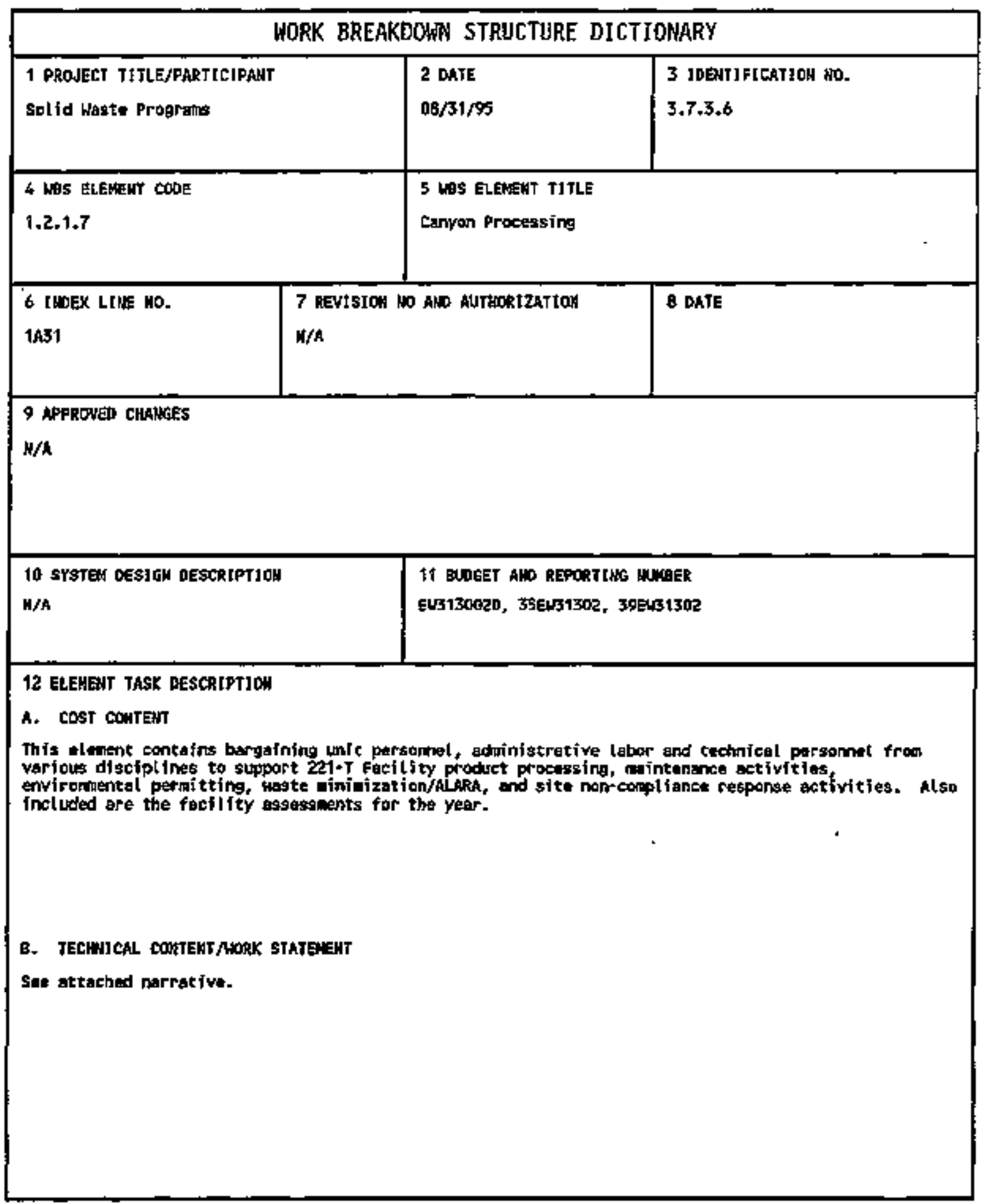




\subsubsection{7/1A31 CANYON PROCESSING (CONTINUED)}

\section{B. TECHNICAL CONEENT/HORK STATENENT}

Canyon processing includes processing of high dose rate waste and contaninated equipment in accordance with the Hanford Site Solid Waste Acceptance Criteria, HHCEP-0063-4, for disposal, re-use, or free release. The 221-T canyon processing activities include:

- Provide support for required permits.

- Support piant modifications necessary for product processing in $221-T$.

- Identify and purchase equipment required for size reduction and decontamination.

- Provide waste stream forecasting.

- Utjijze a vartety of decontamination techniques to reduce the volume of the contaminated component of the waste.

- Reduce the size of large pieces of waste and equipment to fit in burial containers.

- Package waste for burial.

- Decontaninate, repair, and return to service reusabie failed equipment.

- The equipment to be processed will consist of Tank Farms augers and contaminated equipisent stored in the $\mathrm{T}$ Plant canyon. In additfon, other high dose rate contarinated equipment and waste being stored at site facilities undergoing decomissioning and generated during the site cleanup will be treated. Treatment of high dose rate waste and equipment will take place in the 221-T canyon. 


\section{SOLID WASTE PROGRAH}

W.S 1.2.1

FY 1996 Program Plan

WHC-SP-1114, Rev. 1

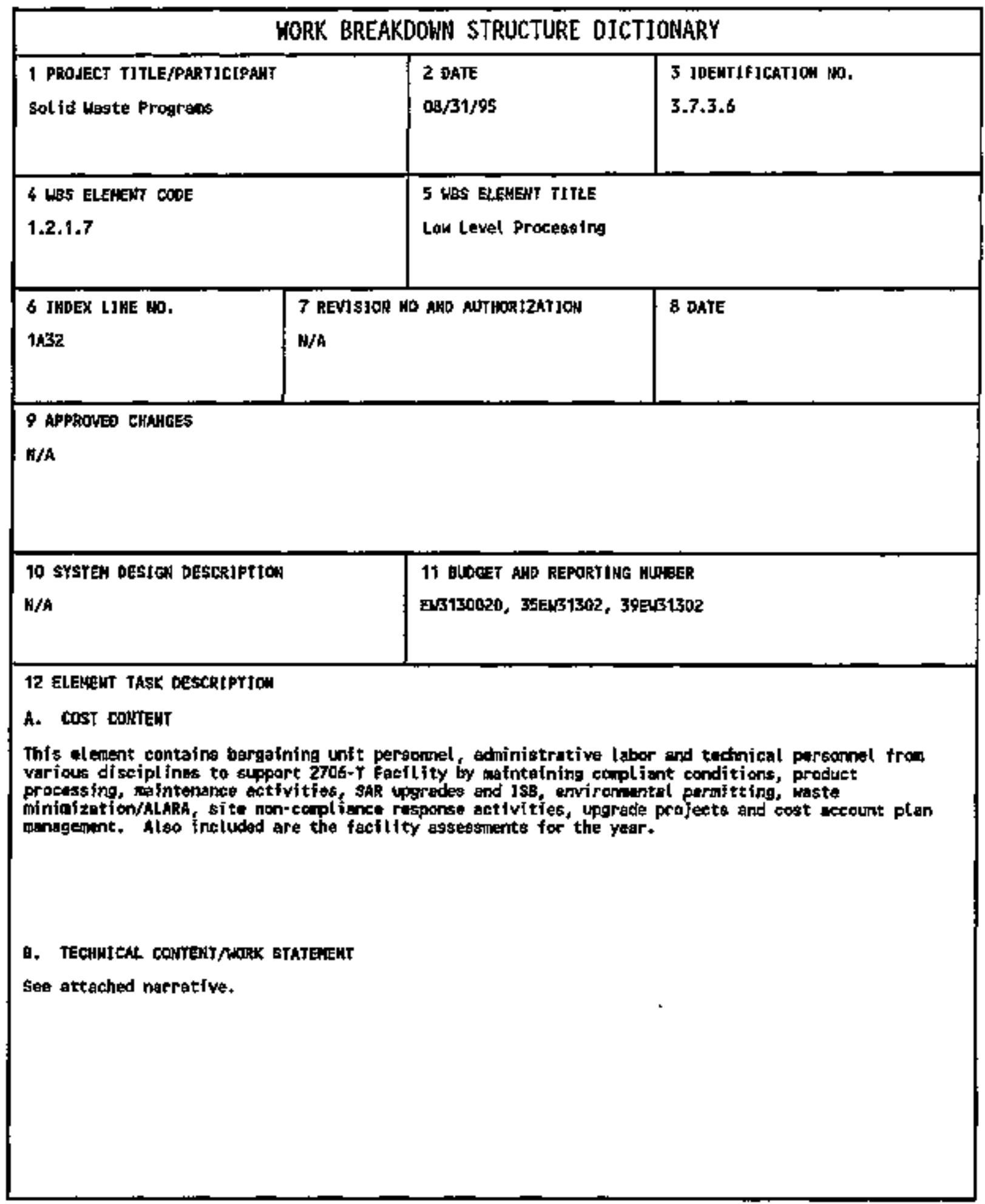




\subsubsection{7/1A32 LOM LEVEL PROCESSING (CONTINUED)}

\section{B. TECHNICAL CONTEHT/WORK STATEMENT}

Low leve] processing includes $2706-T$ hot standby activities and $2706-T$ product processing activities.

The hot standby work wi17 provide safe, monjtored storage for contaminated equipment located in the 2706-T facility. This work reflects the ginimum scope needed to ensure publ ic safety and environmental protectfon unti门 contamínated equipment/ materials are removed from 2706-T. The 2706-T hot standby activities include:

- Provide technical support for DOE Order 5480.21 review.

- Inspection, operation, and maintenance of equipment needed to maintain the 2706-T facility in a safe configuration, including radiation mont toring equipment, fire protection and other safety equipment.

- Operation, surveillance, and maintenance of liquid waste handi ing equipment and ventilation systems.

- Administration and training associated with maintaining a safe, compliant facility.

- Preparation of T P1ant SAR, echs, PSARs, fsars.

The 2706-T product processing work will include processing of low dose rate waste and contaminated equipment in accordance with the Hanford Site Solid haste Acceptance Criteria, WHC-EP-0063-4, for disposal, re-use, or free release per 10CFR835. The $2706-T$ product processing activities w111 1nclude:

- Provide support for required peraits.

- Support 2706-T plant modifications necessary for product processing.

- Identify and purchase equipment required for size reduction and decontamination.

- Provide waste stream forecasting.

- Utilize a variety of decontamination techniques to reduce the volume of the contaminated component of the waste. Reduce the size of large pieces of waste and equipment to fit in burial containers.

- Package waste for burial.

- Decontaminate, repair, and return to service reusabie failed equipment.

- The equipment to be processed will consist of customers cranes, vehicles, rail cars, trucks, and tools. In addition, other facility services include the sampling, inspection of low level waste and radioactive mixed waste/equipment, inventory/verification of constituents and repackaging for disposal. 


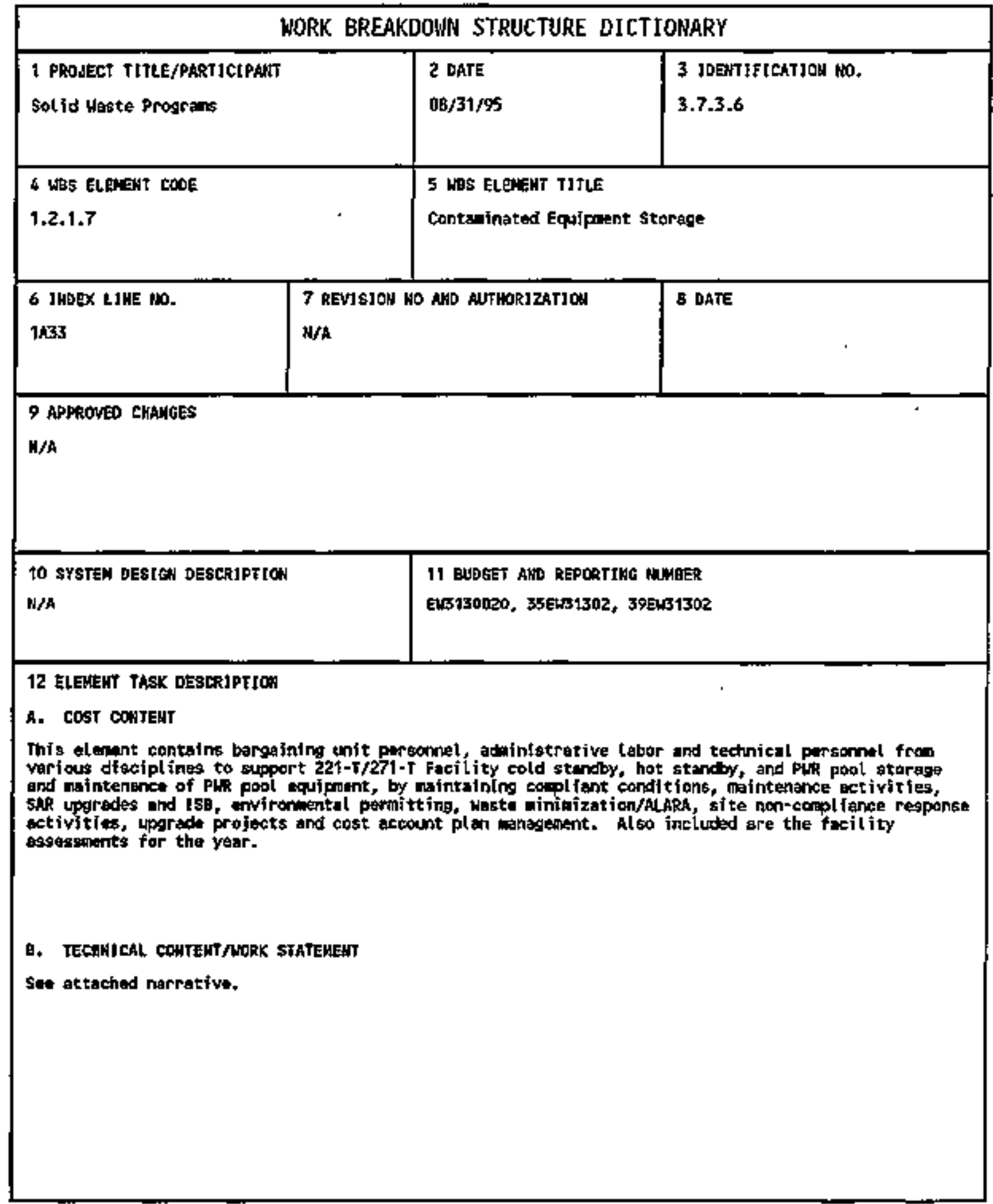




\subsubsection{7/1A33 CONTAMIMATED EQUIPHENT STORAGE (CONTIMUED)}

\section{B. TECHNICAL CONTENT/WORK STATEMENT}

Contaminated equipment storage includes 221-T/271- $\uparrow$ cold standby activities, hot standby activities, and PWR pool storage and maintenance of PWR pool equipment.

The 221-T cold standby work and the hot standby work wi1l include providing safe, monitored storage for contamfnated equipment located in the 221-T facility. This activity reflects the minfmum scope reeded to ensure pubicic safety and environmental protection unt $\{1$ contaminated equipment and materials are removed from $221-T$. This scope includes Project C-157, Electrical Upgrades.

The PWR pool work includes the storage of 72 spent fuel elements, and one nonirradiated elenent at $T$ Plant fn accordance $w$ ith the 1 atest revision of WHC-SD-CPSAR-007, "T p]ant Safety Analysis Report". Also included is the storage of the pieces of contaminated equipment shown on figure 1 of the "T Plant Canyon Towers Work Plan", SH-PE-94-001 (Apri1 1994) in accordance with the most recent revision of WHC-SD-CP-SAR-007. This activity reflects the minimum scope needed to ensure public safety and environmental protection until Spent Huclear fueis and al contaminated equipment and materials are removed from $T$ Plant.

The contaminated equipment storage activities include:

- Provide technical support for DOE Order 5480.21 review.

- Inspection, operation, and maintenance of equipment needed to maintain the 221-T factitity and the Phe pool in a safe configuration, inciuding radiatjon monitoring equipment, fire protection and other safety equipment.

- Operation, surveillance, and maintenance of liquid waste handiling equiprent and ventilation systems.

- Administration and training associated with waintaining a safe, compliant facility.

- Preparation of $T$ Plant SAR, ECHs, PSARs, FSARs.

- Support plant modifications necessary for the profuct line.

- Prepare for steam removal in the facility by replacing all systens that are dependant on steam. 


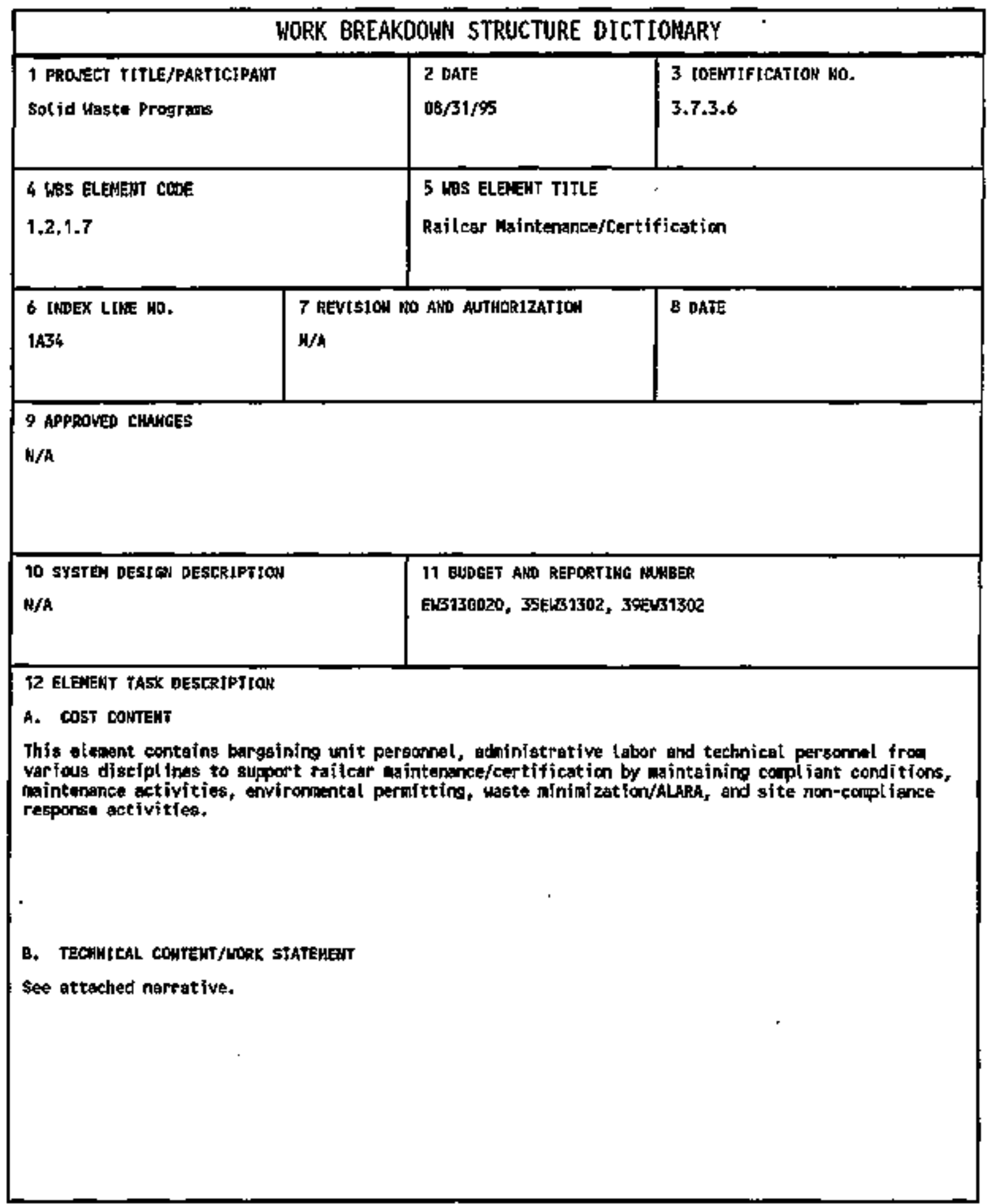




\subsubsection{7/1A34 RAILCAR MAINTENANCE/CERTIFICATION (CONTINUED)}

\section{B. TECHNICAL CONTENT/WORK STATEMENT}

This activity assures the availability of at least one liquid waste tank car (LWTC) for the transport of liquid waste, generally from T Plant, the 300 area labs, and the 400 area to Tank Farms. The LWTCS also serve as site backup liquid waste transfer for Hanford sfte faciljties currently utilizing hard piping. LWTC services are essential to maintain < 90 day storage requirements for facilities that do not have liquid waste storage permits, and for storage tank liquid level control. Raflcar activities include:

- The performance of required corrective and preventive maintenance on the LUTCs.

- Certffication of the LWTCS on a regular basis to assure they meet safety requirements as specifled in T Plant's Safety Analys is Report (SAR) for continued service.

- The LWTCS now in use will be retired as their replacements are placed in service. In FY1996, they will require decontamination and disposal. 


\begin{tabular}{|c|c|c|c|}
\hline \multicolumn{4}{|c|}{ HORK BREAKDOWN STRUICTURE DICTIONARY } \\
\hline $\begin{array}{l}1 \text { PROJECT YITLE/EARTICIPANT } \\
\text { Sol fd Waste Programs }\end{array}$ & & $\begin{array}{l}2 \text { DATE } \\
08 / 31 / 95\end{array}$ & $\begin{array}{l}3 \text { JDENTIFICATIOH M. } \\
3.7 .3 .6\end{array}$ \\
\hline $\begin{array}{l}4 \text { Was ELEYUAT COOE } \\
1.2,1.7\end{array}$ & & \multicolumn{2}{|l|}{$\begin{array}{l}5 \text { YKS BLEHENT TITLE } \\
\text { FEe }\end{array}$} \\
\hline $\begin{array}{l}6 \text { JMDEX LIME NO. } \\
\text { IA3S }\end{array}$ & \multicolumn{2}{|c|}{$\begin{array}{l}7 \text { REVISIOW WO AID AUTHORIZATION } \\
\text { H/R }\end{array}$} & 8 DATE \\
\hline \multicolumn{4}{|l|}{$\begin{array}{l}9 \text { APPROVED CHAMGES } \\
\text { H/A }\end{array}$} \\
\hline $\begin{array}{l}\text { 10 SYSTEM DESIGW DESCRLPTIOH } \\
\text { H/A }\end{array}$ & & $\begin{array}{l}\text { 1) BLAGET AHD REPOR: } \\
\text { EUS130020, 35EU3130: }\end{array}$ & $\begin{array}{l}\text { DABER } \\
\text { BSt302 }\end{array}$ \\
\hline \multicolumn{4}{|c|}{ 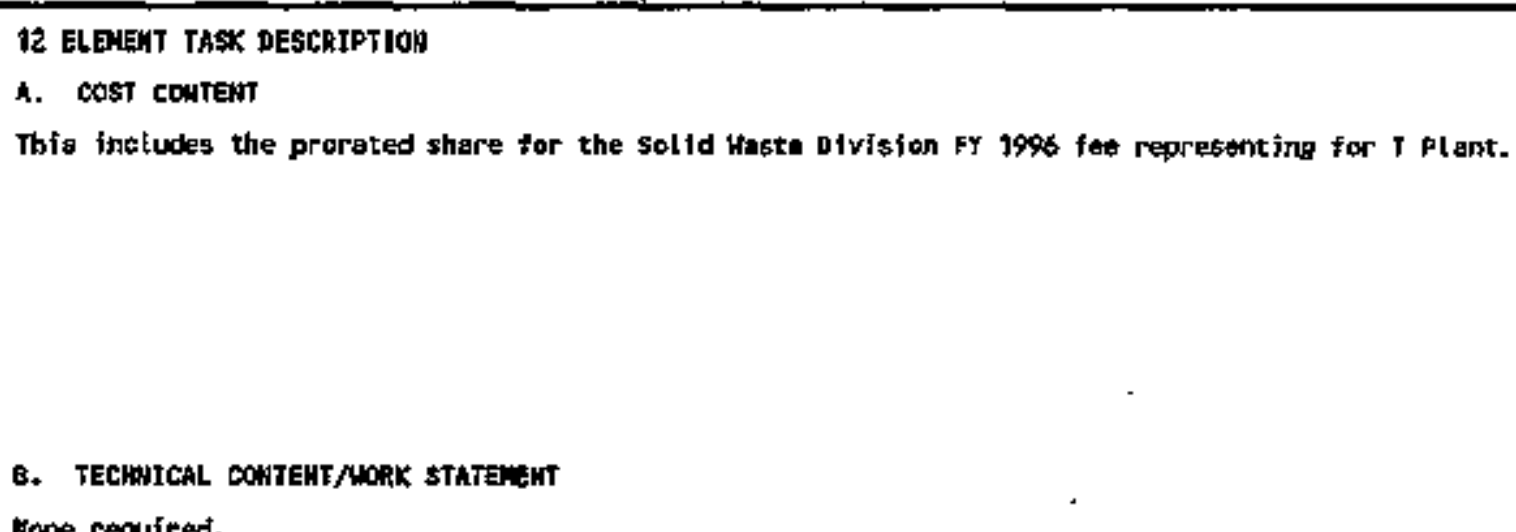 } \\
\hline
\end{tabular}




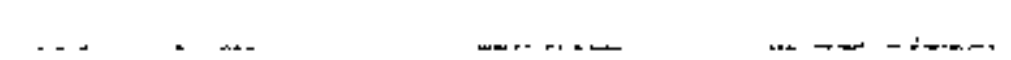

SOLIJ WASTE PROGRAY

WBS 1.2.1

FY 1996 Program Plan

WHC-\$P-1114, Rev. I

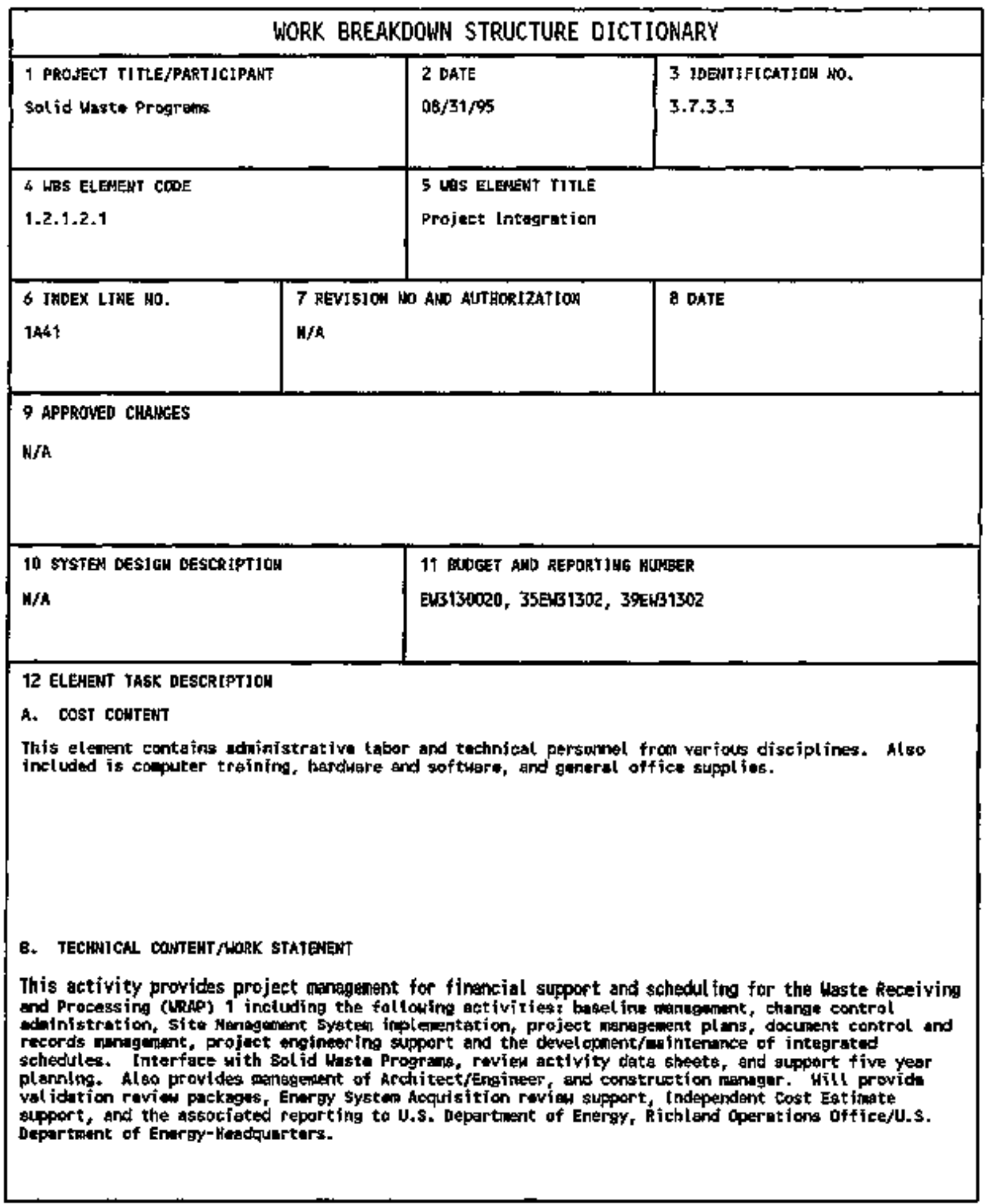




\section{SOLIO WASTE PROGRAF}

WBS 1.2.1

FY 1996 Program Plan

WHC-5P-1114, Rev. 1

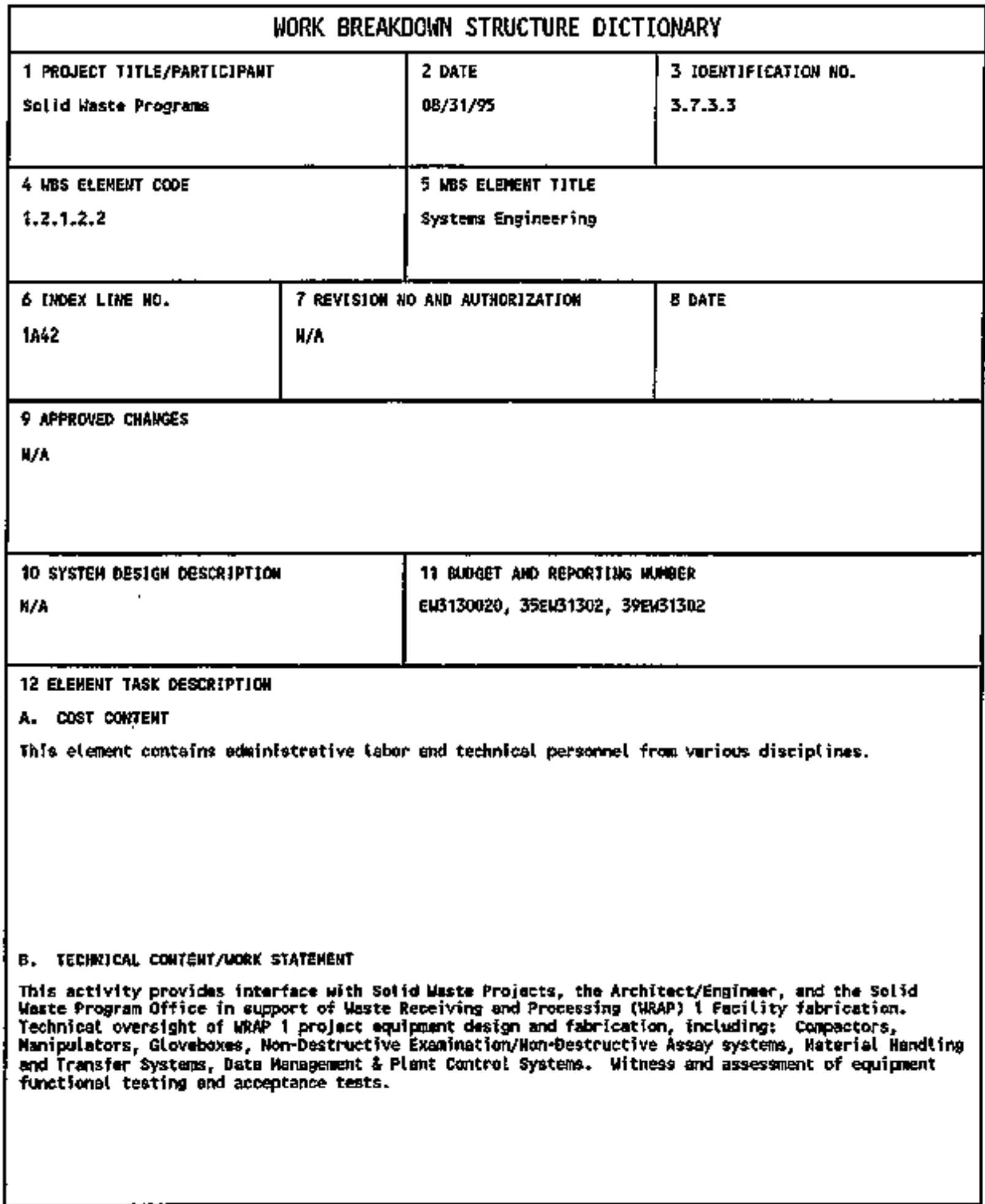


SOLID HASTE PROGRAM

NBS 1.2.1

FY 1996 Program Plan

WHC-SP-1114, Rev, I

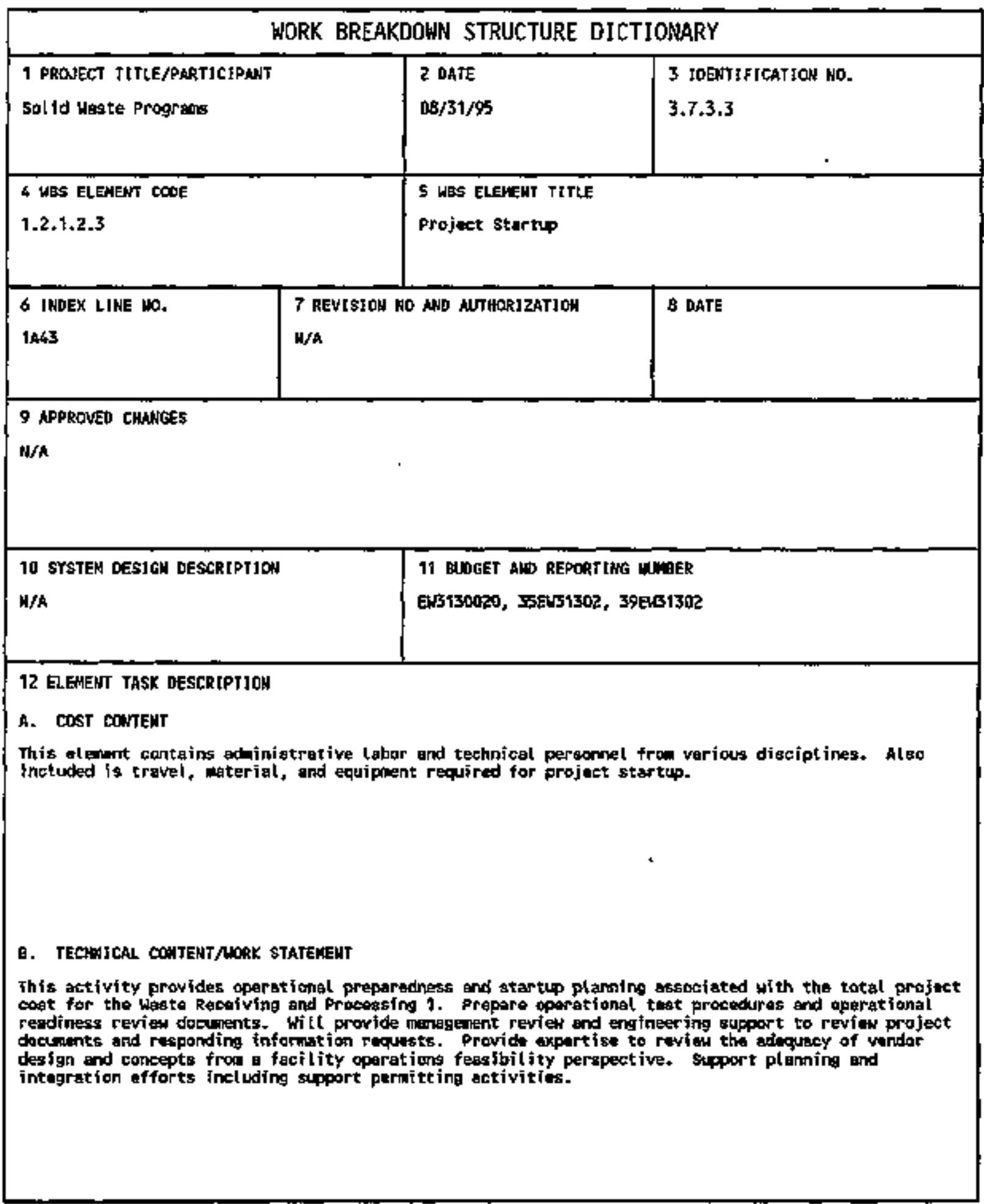




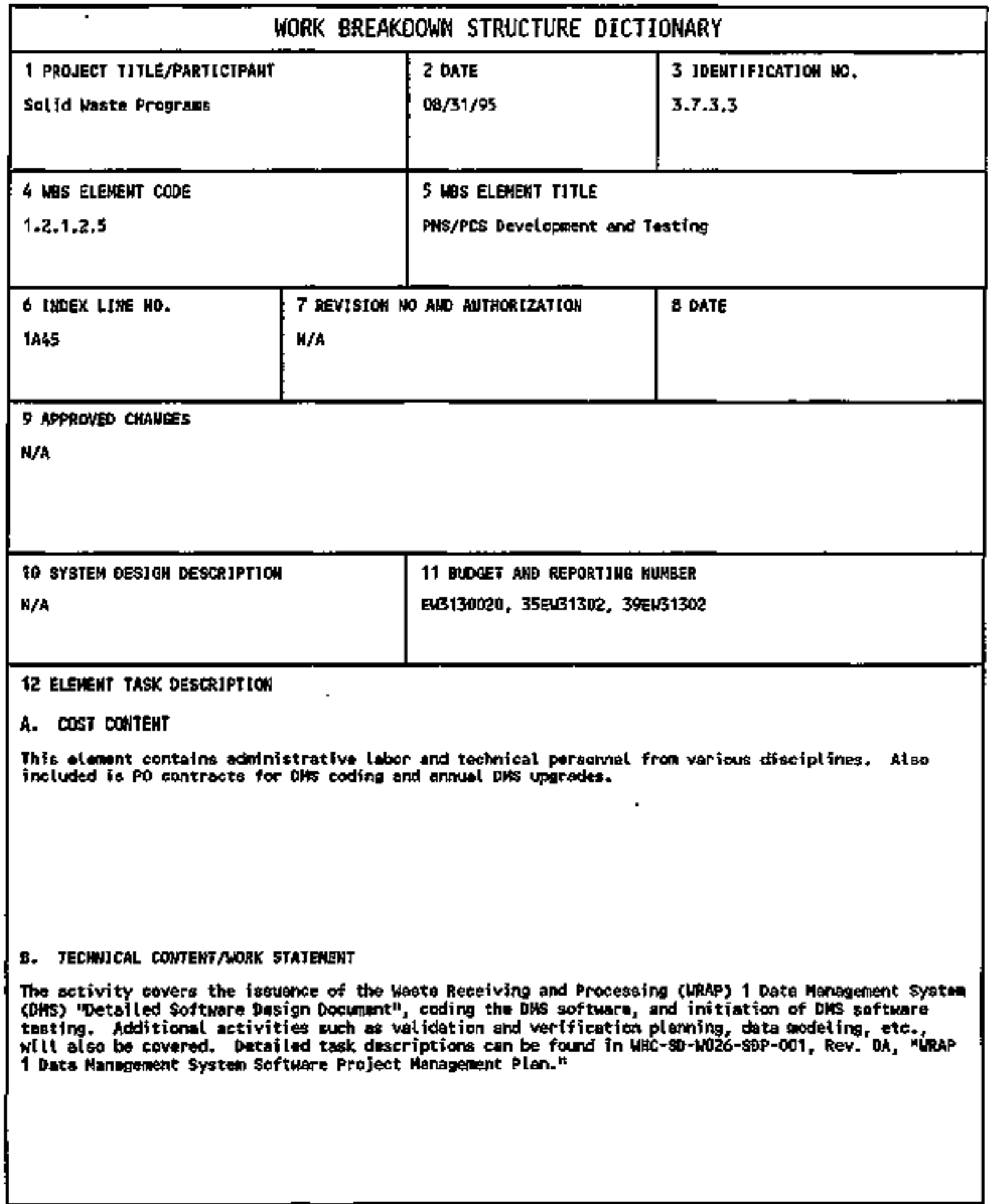




\section{SOLID WASTE PROGRAM}

HBS 1.2.1

\begin{tabular}{|c|c|c|}
\hline \multicolumn{3}{|c|}{ WORK BREAKDOWN STRUCTURE DICTIONARY } \\
\hline $\begin{array}{l}1 \text { PROJECT TITLE/PARTICIPANT } \\
\text { sol td watte Programa }\end{array}$ & $\begin{array}{l}2 \text { OATE } \\
\text { OB/31/9S }\end{array}$ & $\begin{array}{l}3 \text { JDEHTIFICATION MO. } \\
3.7 .3 .3\end{array}$ \\
\hline $\begin{array}{l}4 \text { WBS ELEKEKT COOE } \\
1.2 .1 .2 .6\end{array}$ & \multicolumn{2}{|c|}{$\begin{array}{l}5 \text { WAS ELENEAT TJTLE } \\
\text { Environnental Sofety and oA }\end{array}$} \\
\hline $\begin{array}{l}6 \text { IUOEX LIME NO. } \\
1446\end{array}$ & $\begin{array}{l}7 \text { REVISION HO AHO AUTHORIZATION } \\
\mathrm{H} / \mathrm{h}\end{array}$ & 8 DATE \\
\hline \multicolumn{3}{|l|}{$\begin{array}{l}9 \text { APPHOWED CKAHGES } \\
\text { H/A }\end{array}$} \\
\hline $\begin{array}{l}10 \text { STSTEM OESIGM DESCRIPTIOH } \\
\mathrm{H} / \mathrm{A}\end{array}$ & $\begin{array}{l}11 \text { BUDEET AND REPOI } \\
\text { MUS130000, 35ENS13 }\end{array}$ & 31302 \\
\hline \multicolumn{3}{|c|}{ 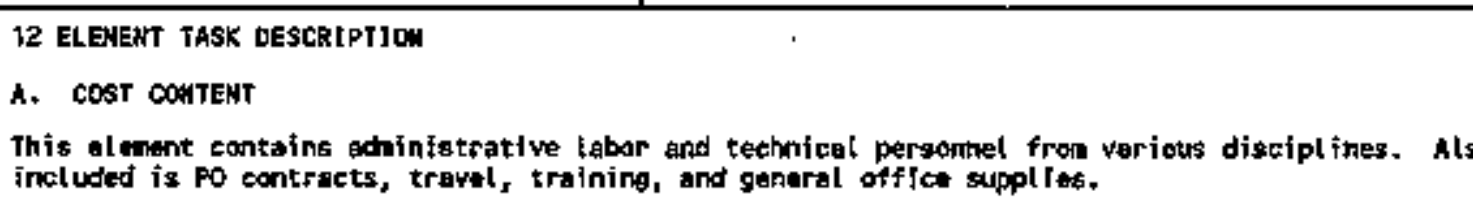 } \\
\hline \multicolumn{3}{|c|}{ 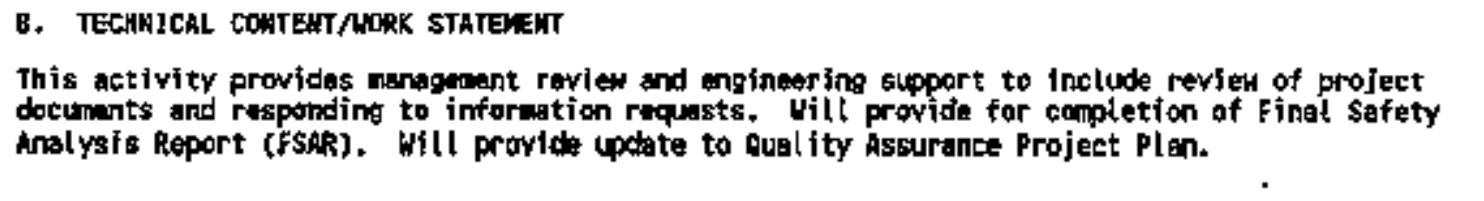 } \\
\hline
\end{tabular}




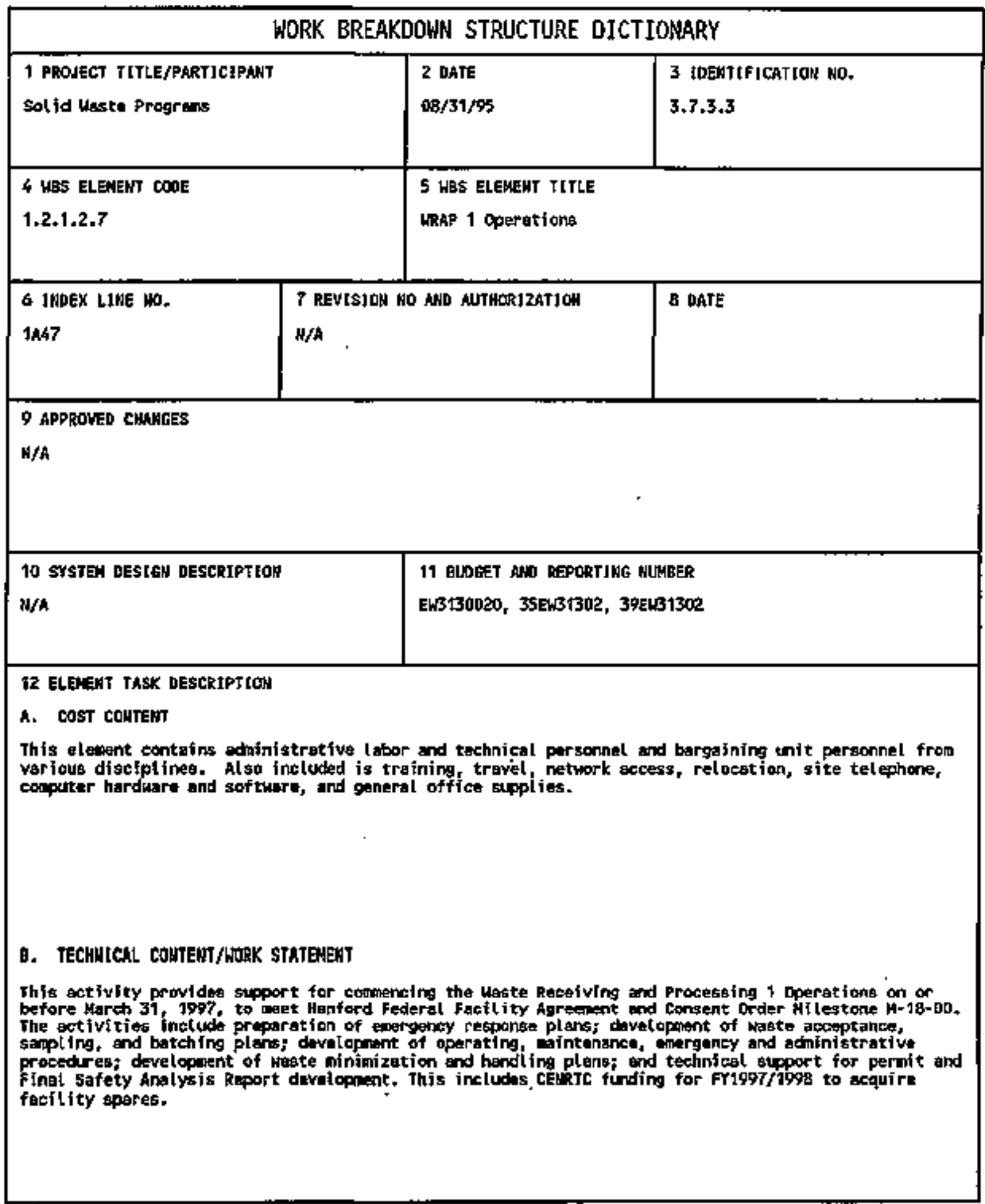


WHC-SP-1114, Rev. 1

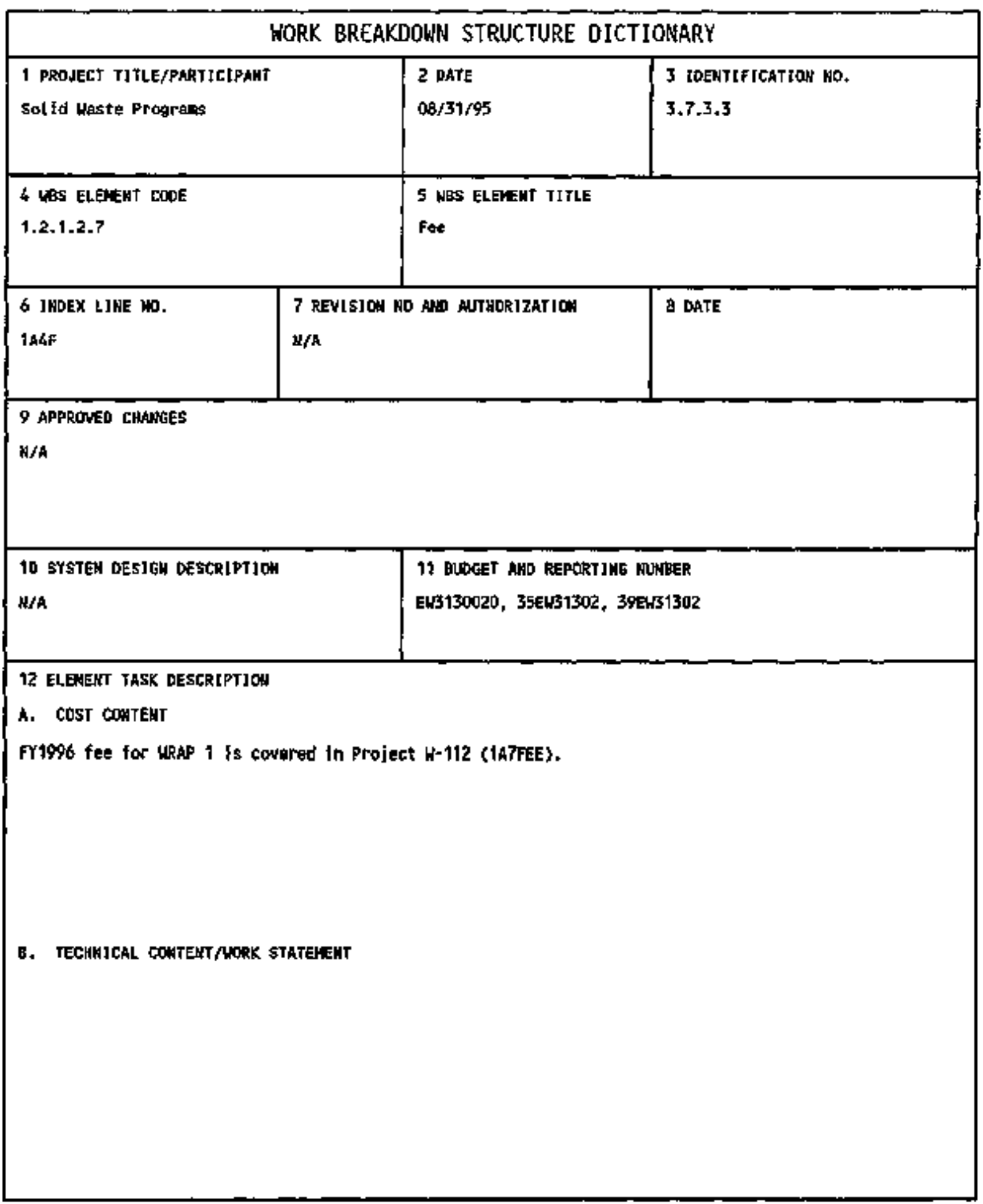




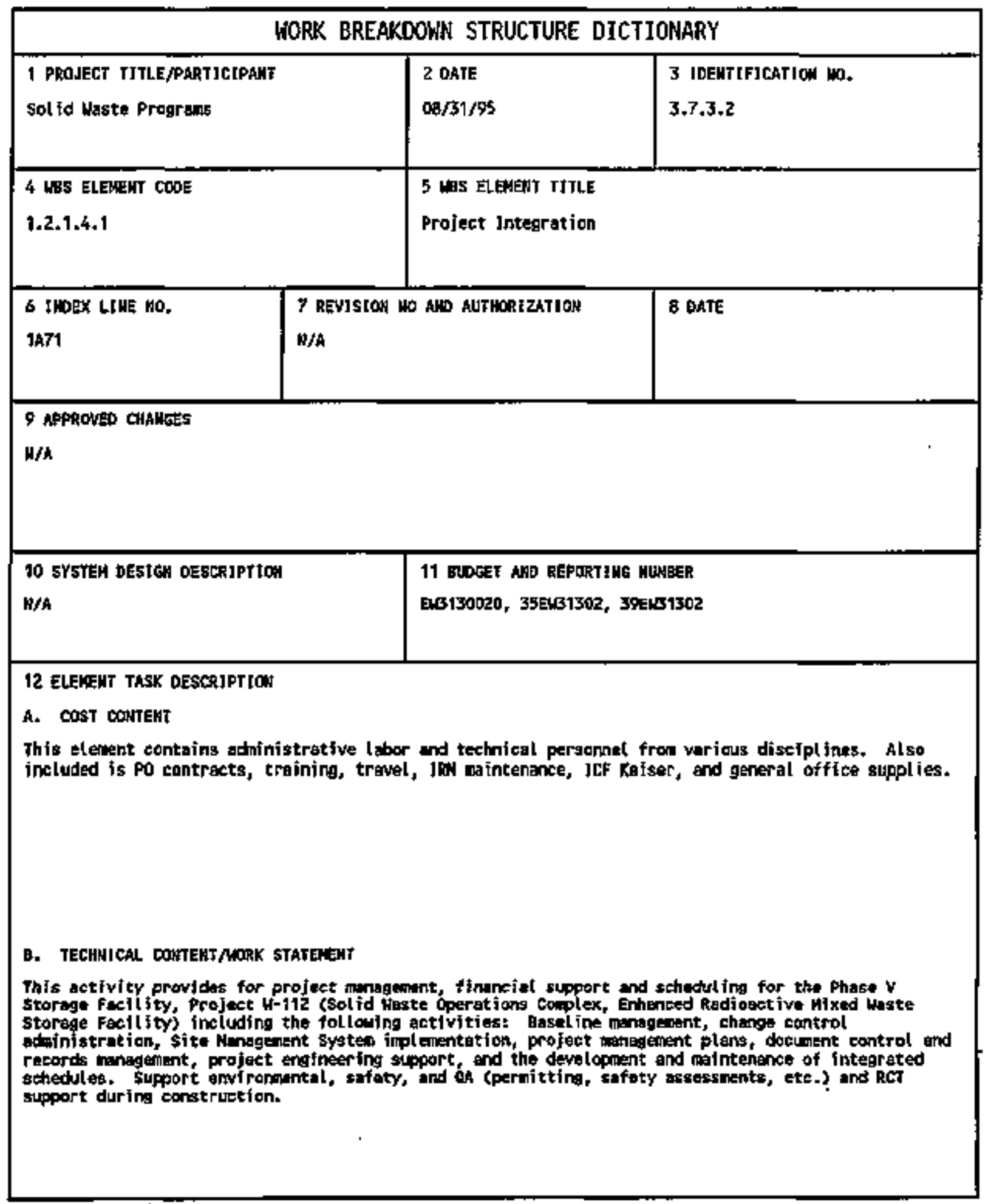




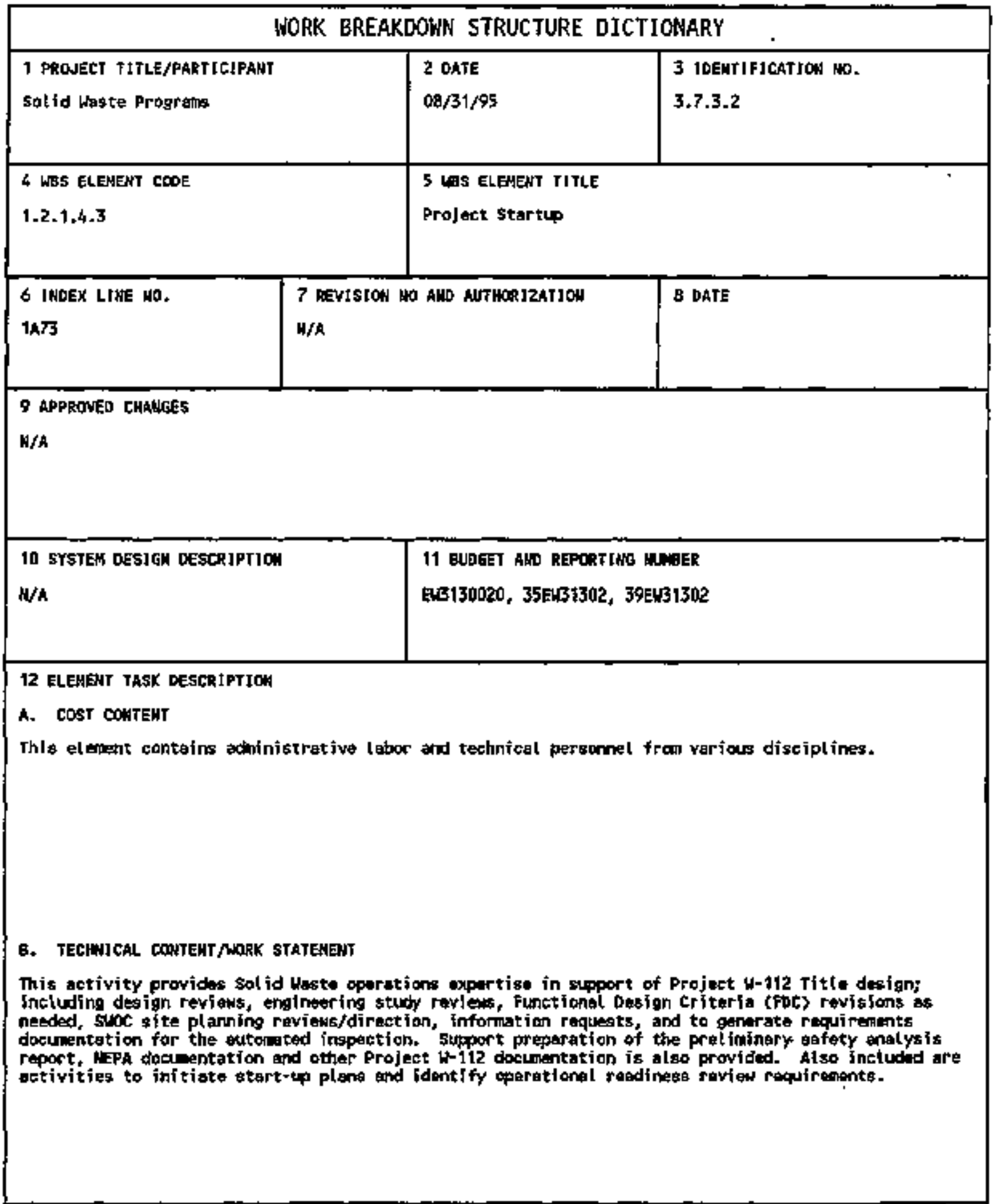




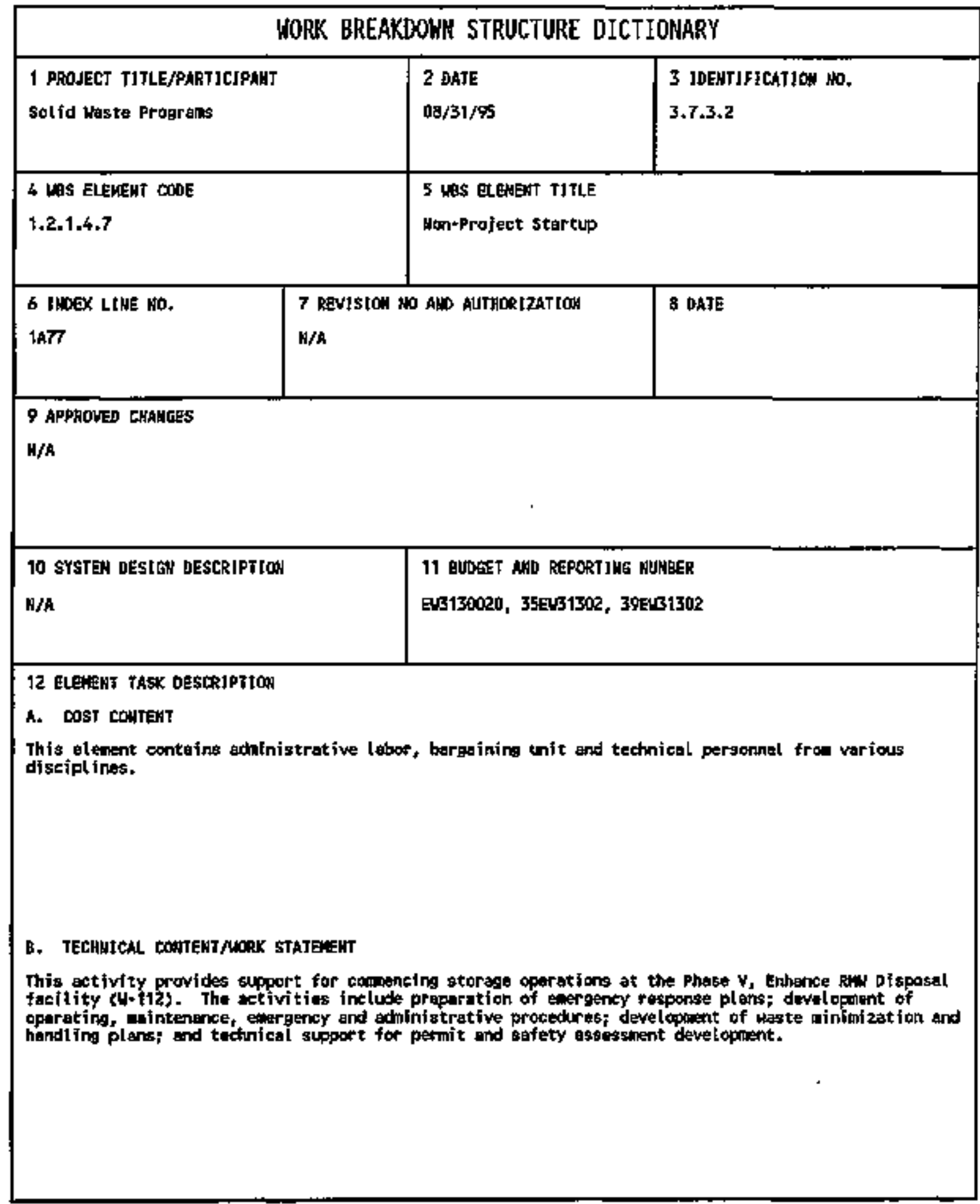


FY 1996 Program Plan

SOLID HASTE PROGRAH

WBS 1.2.1

WHC-SP-1114, Rev. 1

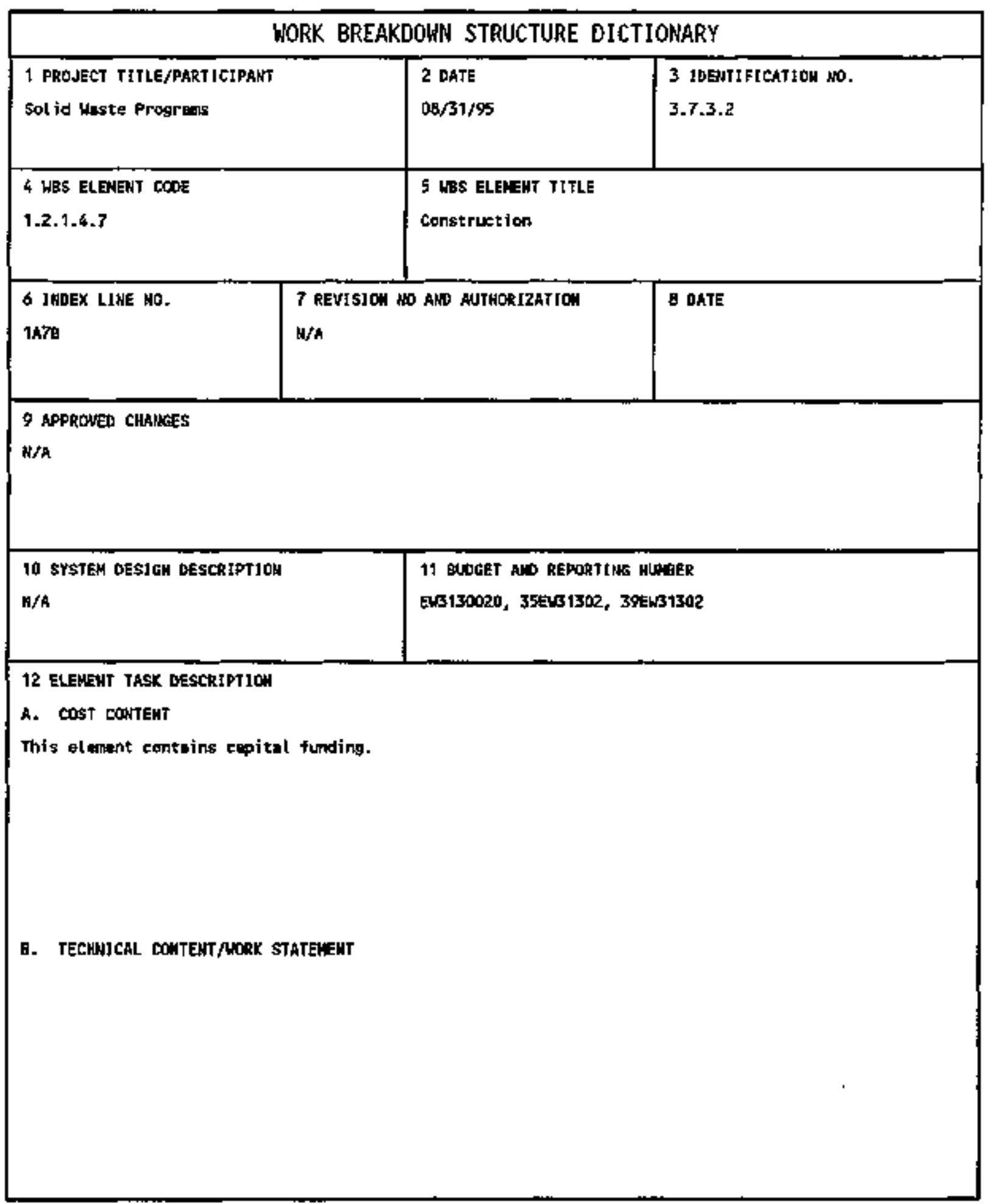




\section{SOLID MASTE PROGRAM}

WBS 1.2.1

Fy 1996 Program Plan

WHC-SP-1114, Rev, 1

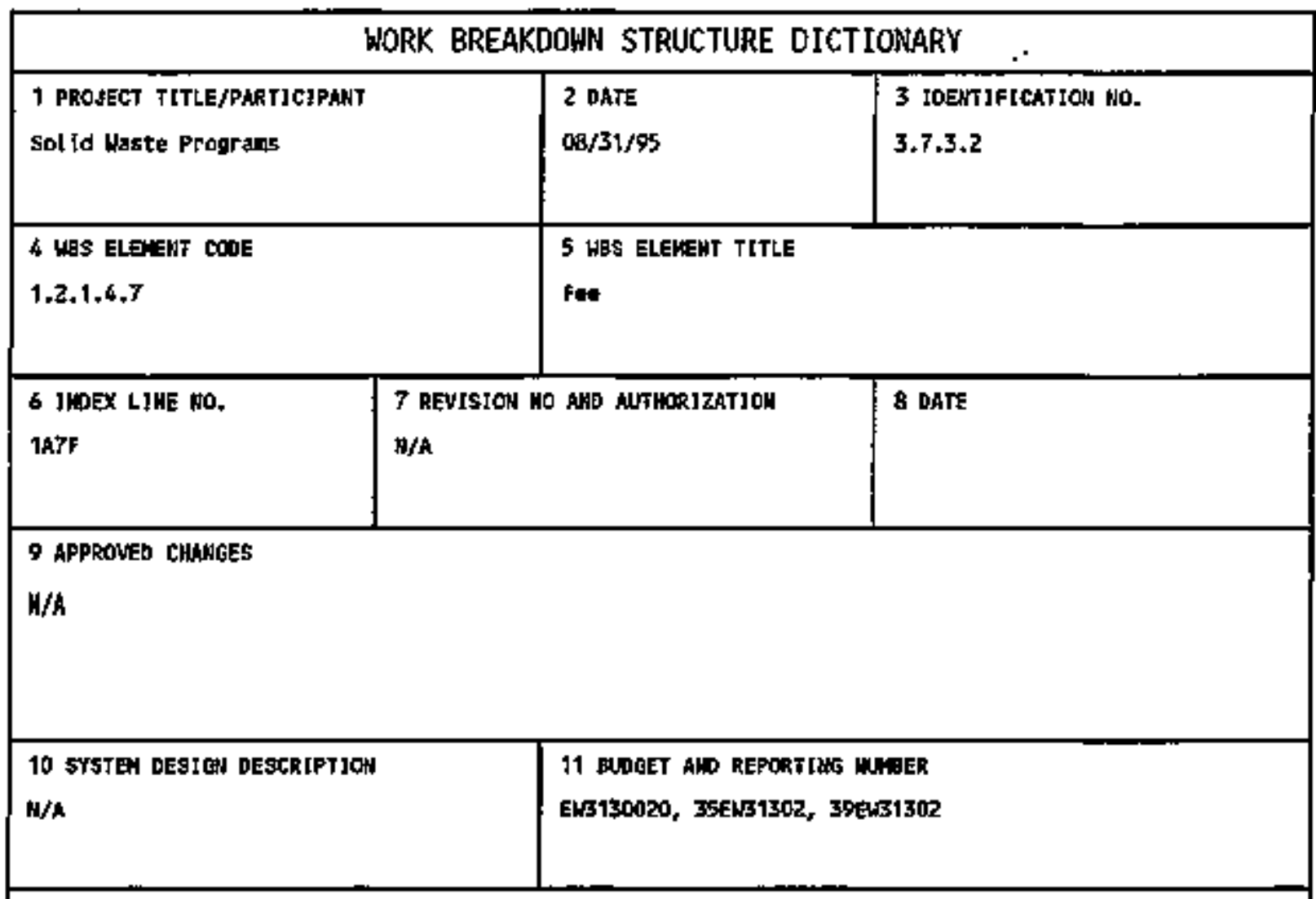

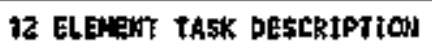

A. COST COHTENT

FY19g6 foe for WAP1 and H-t12 is covered within Project W-112 fee actount (LATFEE).

B. TECHH:PAL COUTENT/NOAK STATEMENT 


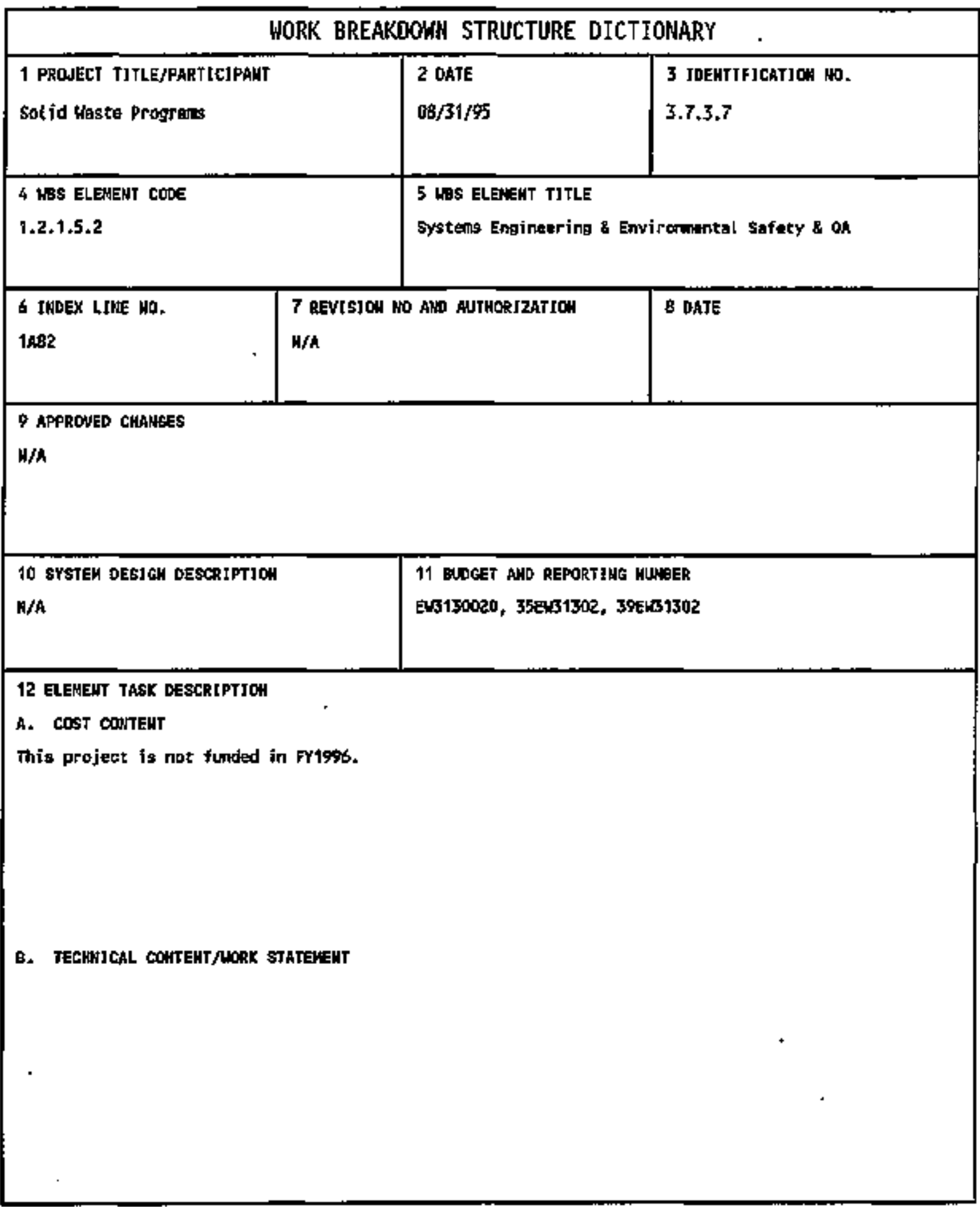




\section{SOLID WASTE PROGRAM}

HBS 1.2 .1

FY 1996 Program Plan

WHC-SP-1114, Rev. 1

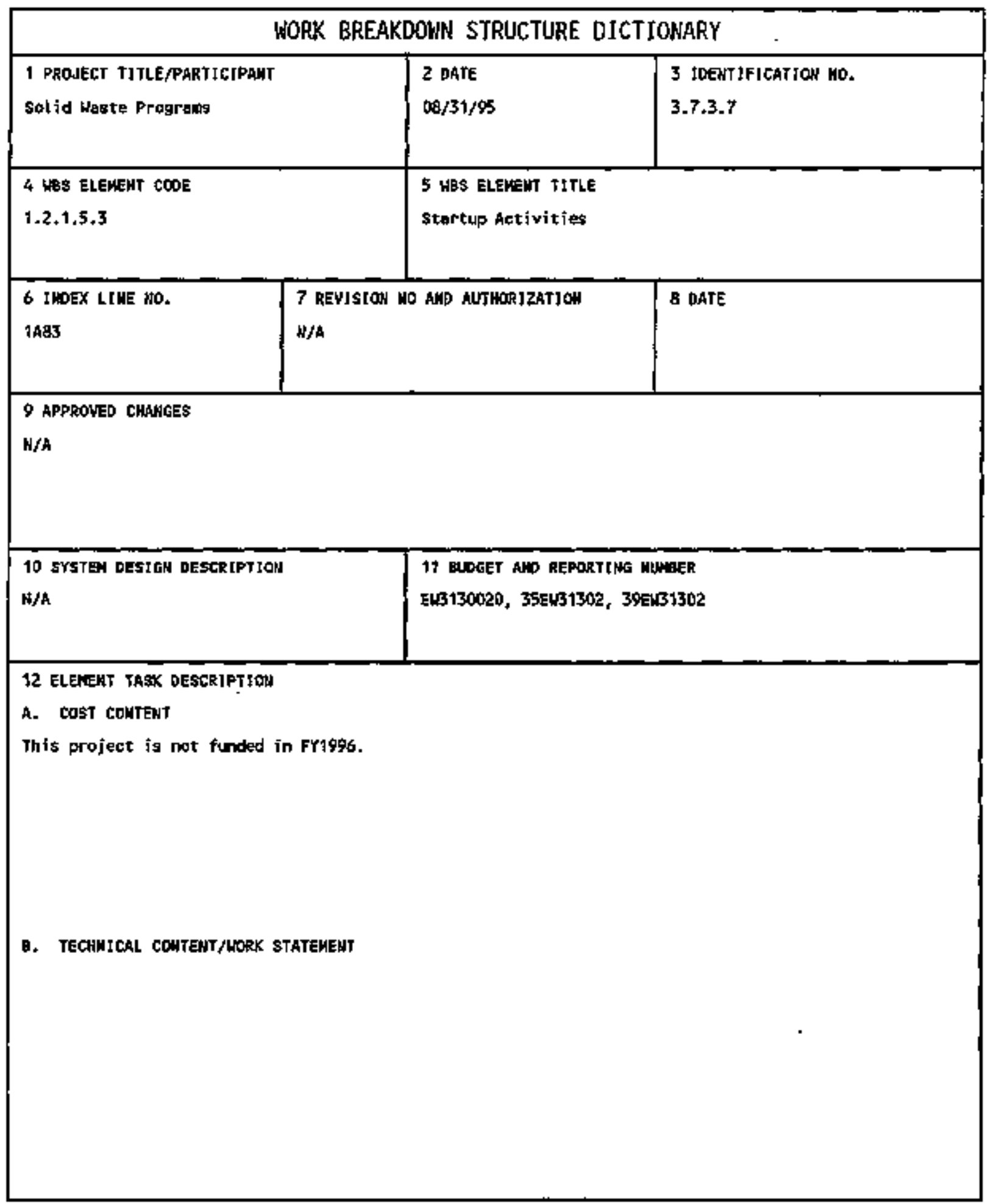




\begin{tabular}{|c|c|c|c|}
\hline \multicolumn{4}{|c|}{ WORK BREAKDOWN STRUCTURE DICTIONARY } \\
\hline $\begin{array}{l}1 \text { PRONECT TJTLE/PARTJCIPAHT } \\
\text { Solfid Waste Programs }\end{array}$ & & $\begin{array}{l}2 \text { OATE } \\
08 / 31 / 55\end{array}$ & $\begin{array}{l}3 \text { JDEHTIF ICATIOW WO, } \\
3.7 .3 .7\end{array}$ \\
\hline $\begin{array}{l}4 \text { Wig ELEREHT TOOE } \\
1.2 .1 .5 .5\end{array}$ & & \multicolumn{2}{|c|}{$\begin{array}{l}5 \text { UBS ELEMERT TITLE } \\
\text { Equipment Development and Testing }\end{array}$} \\
\hline $\begin{array}{l}6 \text { IWOEX LiNE ND. } \\
\text { TABS }\end{array}$ & \multicolumn{2}{|c|}{$\begin{array}{l}7 \text { REVISIOH HO AUE AUTHORTZATIOH } \\
\text { H/A }\end{array}$} & 8 DATE \\
\hline \multicolumn{4}{|l|}{$\begin{array}{l}9 \text { APARONED CEAHGES } \\
\text { H/A }\end{array}$} \\
\hline \multicolumn{2}{|l|}{$\begin{array}{l}10 \text { SYSTEN DESIG OESCRJPTIOH } \\
\text { W/A }\end{array}$} & \multicolumn{2}{|c|}{ 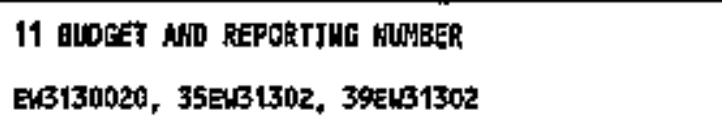 } \\
\hline \multicolumn{4}{|c|}{$\begin{array}{l}\text { T2 ELEEEHT TASK OESLRIPTLOW } \\
\text { A. DOST ConTERT } \\
\text { This projuct is not funded in Frigos. }\end{array}$} \\
\hline 9. TECHEICAL COMTEFT / & ATanent & & . \\
\hline
\end{tabular}




\section{SOLID WASTE PROGRAH \\ WBS 1.2 .1}

Fy 1995 Progran Plan

WHC-SP-1114, Rev. I

\begin{tabular}{|c|c|c|c|}
\hline \multicolumn{4}{|c|}{ WORK BREAKDOWN STRUCTURE DICTIONARY } \\
\hline $\begin{array}{l}\text { I PROJECT TITLE/PARTICIPANT } \\
\text { Solid Haste Programs }\end{array}$ & & $\begin{array}{l}2 \text { DATE } \\
08 / 31 / 95\end{array}$ & $\begin{array}{l}3 \text { TOENT TFICATION HO. } \\
3.7 .3 .7\end{array}$ \\
\hline $\begin{array}{l}4 \text { WS ELEKEHT SODE } \\
1.2 .1 .5 .7\end{array}$ & & \multicolumn{2}{|c|}{$\begin{array}{l}5 \text { hBS ELEYE\$T TITLE } \\
\text { Waste Retrifeval Operatione }\end{array}$} \\
\hline $\begin{array}{l}6 \text { [WOEX LINE HO. } \\
\text { IAg7 }\end{array}$ & $\begin{array}{l}7 \text { REVISLON } \\
\text { M/A }\end{array}$ & ALO AUTHORLZKTIOH & 8 DATE \\
\hline \multicolumn{4}{|l|}{$\begin{array}{l}9 \text { APPRONGD CHAHESS } \\
\text { W/h }\end{array}$} \\
\hline \multicolumn{2}{|l|}{$\begin{array}{l}\text { 10 SYSTEM DESION DESGRIPTION } \\
\text { W/A }\end{array}$} & \multicolumn{2}{|c|}{ 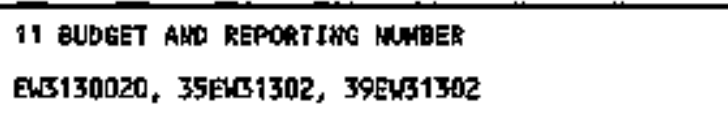 } \\
\hline \multicolumn{4}{|c|}{$\begin{array}{l}\text { T2 ELEMENT TASK DESCRIPTION } \\
\text { A. COST CONTEAT } \\
\text { This project is not funded in Fr1996. }\end{array}$} \\
\hline \multicolumn{4}{|c|}{ B. TECHNJCAL CONTENTMORK STATENEAT } \\
\hline
\end{tabular}




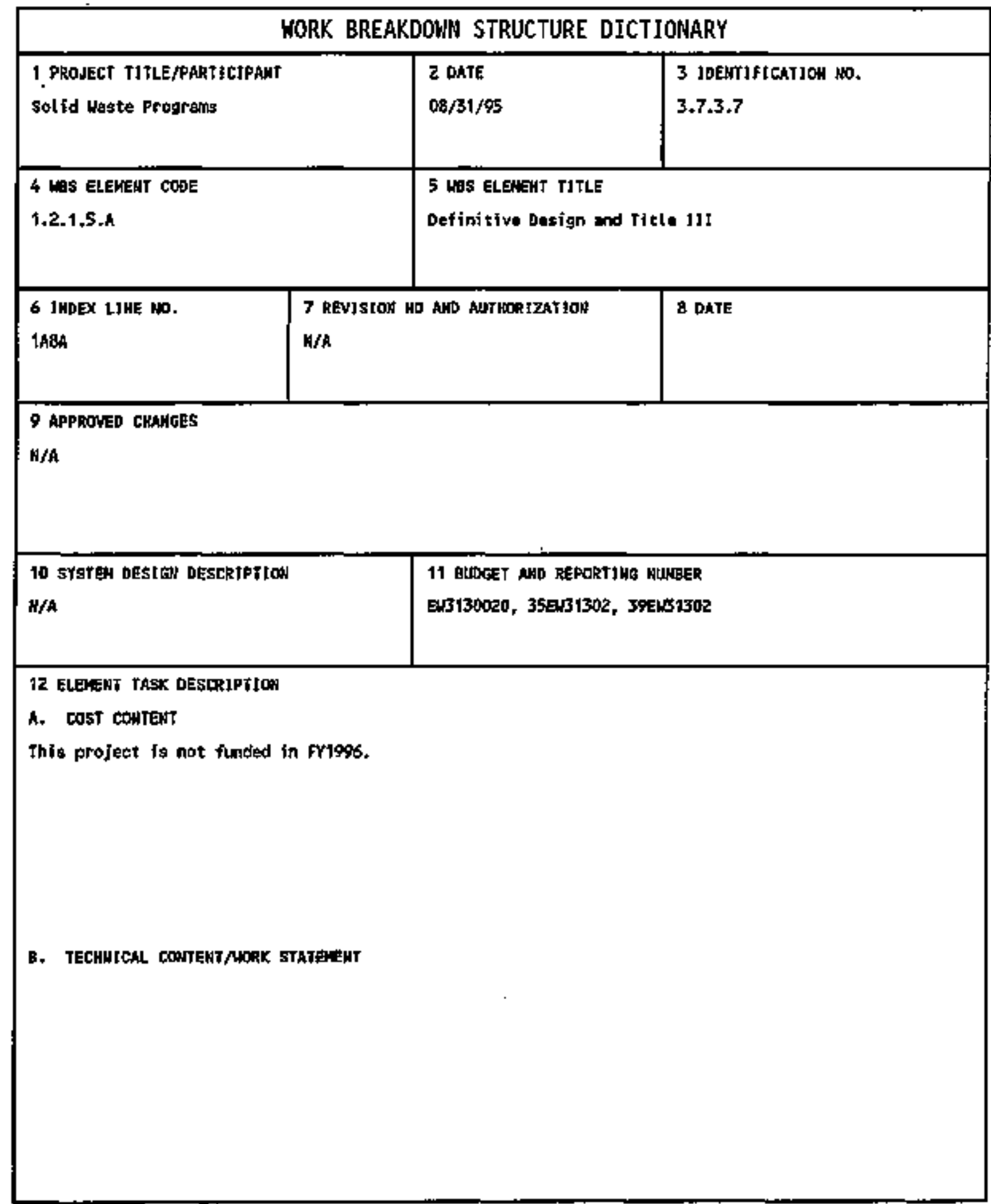


SOLID WASTE PROGRAM

HBS 1.2.1

FY 1996 Program Plan

WHC-SP-1114, Rev. 1

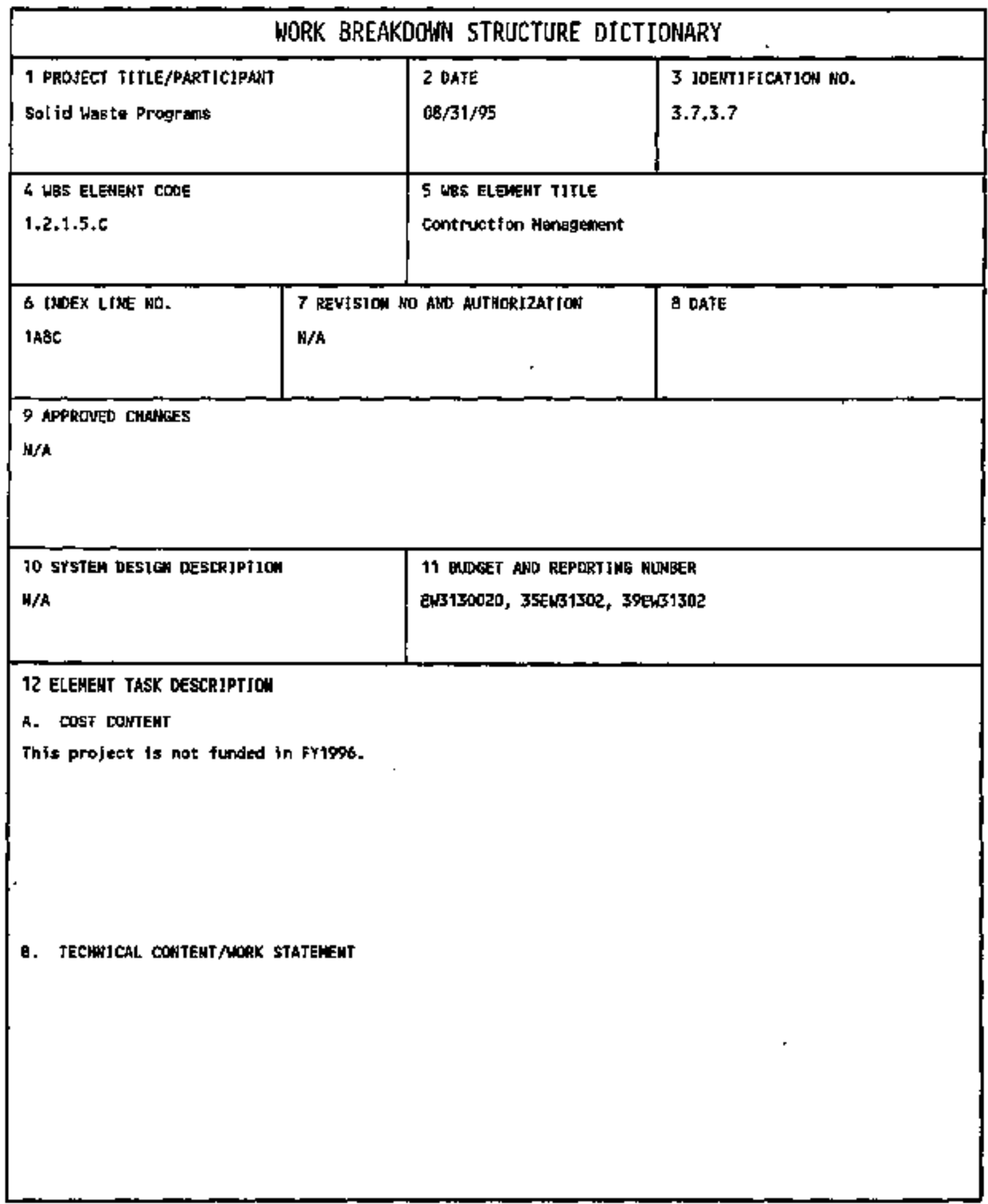




\begin{tabular}{|c|c|c|c|}
\hline \multicolumn{4}{|c|}{ WORK BREAKDOWN STRUCTURE OICTIONARY } \\
\hline $\begin{array}{l}1 \text { PROJECT TITLE/PARTICLPANT } \\
\text { Solfd Wasta Prograns }\end{array}$ & & $\begin{array}{l}2 \text { OATE } \\
08 / 31 / 95\end{array}$ & $\begin{array}{l}3 \text { LDENTIF LCATLOH MO. } \\
3.7 .3 .7\end{array}$ \\
\hline $\begin{array}{l}4 \text { WES ELELEHT COOE } \\
1.2 .1 .5 . F\end{array}$ & & $\begin{array}{l}5 \text { HBS ELEHEMT TLTLE } \\
\text { Protarement }\end{array}$ & \\
\hline $\begin{array}{l}6 \text { JHDEX LIME NO. } \\
\text { LAPP }\end{array}$ & \multicolumn{2}{|c|}{$\begin{array}{l}7 \text { REVISION HO AHD AUTHORJZATJOH } \\
\text { W/A }\end{array}$} & 8 OATE \\
\hline \multicolumn{4}{|l|}{$\begin{array}{l}9 \text { APPRONED CHHHGE5 } \\
\text { H/A }\end{array}$} \\
\hline $\begin{array}{l}\text { 10 SYSTEN OESLGH DESCRIPTIOOK } \\
\mathrm{W} / \mathrm{A}\end{array}$ & & $\begin{array}{l}11 \text { GUDGET ANO REPORT } \\
\text { EN31300RO, 35EV31302 }\end{array}$ & 1302 \\
\hline \multicolumn{4}{|c|}{$\begin{array}{l}\text { 12 ELENGNT TASK DESCR TPTIDU } \\
\text { A. COST Courent } \\
\text { This project is not funded in FYt9g6. }\end{array}$} \\
\hline \multicolumn{4}{|c|}{ 日. TECHNICAL COHTENT/MORK STATENENT } \\
\hline
\end{tabular}




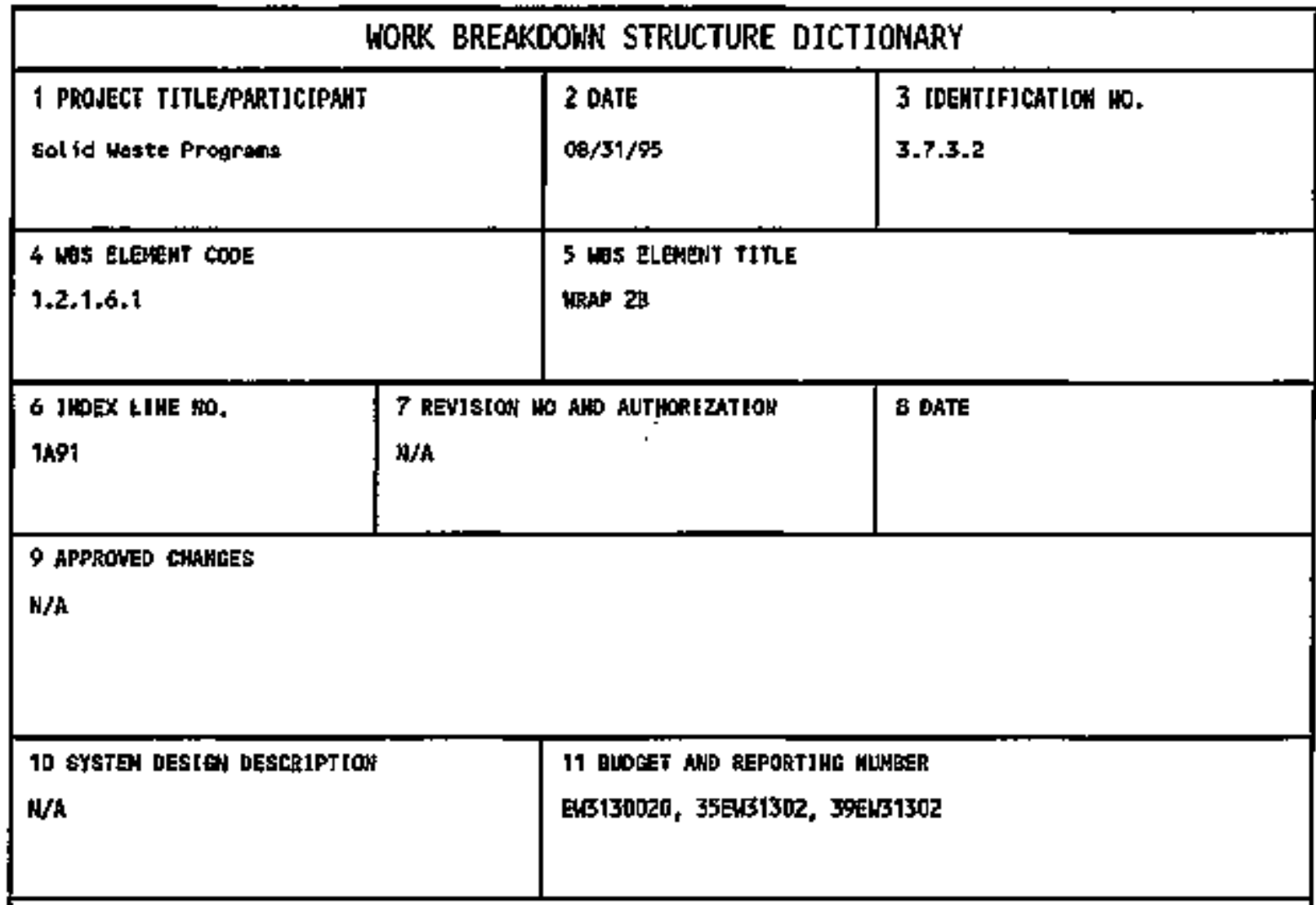

12 ELENENT TASK DESGRIPTION

A. COST CONTENT

There is curremtly no ptanued activity for fiscal Year $19 \%$.

\section{B. TECHHICAL CONTENT/NORK STATEMENT}

The Future Projects Product Líne provfdes for early project development furding of Engineering Studfes and Functionsl Desion Criteria for activities wose technological nied has bein identilied in wic-sbWM-RPT-060, "Solfd Uaste Disposal Technicat Bnselfino Deserfption Docunent." Futture chenges in Sol id Waste bisposal nethodology fmoked by *ither technologicel of regulstory chenges will be incorporeted into the Technical Besel ine Description Dacunent. As these changes are andyzed, future project noeds ote Identjffed and Incorporated as a part of this product line. This specific product îne witl make

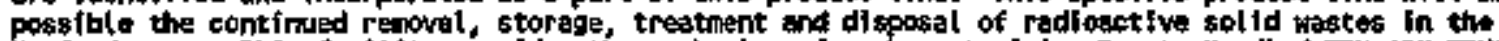
ifanford areas. this factitity provides the technol ogy for charactetizing Renote Hendled TRU (RH-TRU)

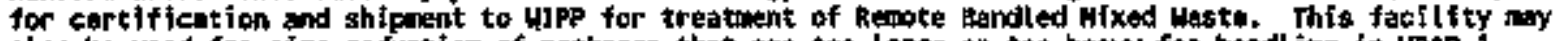
also be used for size reduction of packages that are too large or too heery for handl ing in WRAP 9. 


\begin{tabular}{|c|c|c|}
\hline \multicolumn{3}{|c|}{ HORK BREAKOONN STRUCTURE DICTIONARY } \\
\hline $\begin{array}{l}1 \text { DFOJECT TIFLE/PARTICIPANT } \\
\text { Solid uaste Prograns }\end{array}$ & $\begin{array}{l}2 \text { OATE } \\
08 / 31 / 95\end{array}$ & $\begin{array}{l}3 \text { lo的IF[CATION ND. } \\
3.7 .3 .2\end{array}$ \\
\hline $\begin{array}{l}4 \text { YWS ELEKEKT COOE } \\
1.2 .1 .6 .2\end{array}$ & \multicolumn{2}{|l|}{$\begin{array}{l}5 \text { HES ELeAEHT TITLE } \\
\text { Phase } 2 \text { Retriteval }\end{array}$} \\
\hline $\begin{array}{l}6 \text { JHDEX LIHE NO. } \\
1492\end{array}$ & $\begin{array}{l}7 \text { REVISION HO NWD AUTHLRJZATIOH } \\
\text { M/A }\end{array}$ & B DATE \\
\hline \multicolumn{3}{|l|}{$\begin{array}{l}9 \text { APPROMED CHAHEES } \\
\text { W/A }\end{array}$} \\
\hline $\begin{array}{l}10 \text { SYSTEN DESIGN DESCRIPTIOH } \\
\text { H/A }\end{array}$ & \multicolumn{2}{|c|}{ 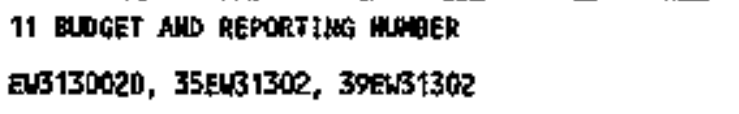 } \\
\hline \multicolumn{3}{|c|}{$\begin{array}{l}12 \text { ELEMENT TASK DESCREPTION } \\
\text { h. COST COATEMT } \\
\text { There is currantly no planned activity for fiseal Year } 1996 .\end{array}$} \\
\hline \multicolumn{3}{|c|}{ 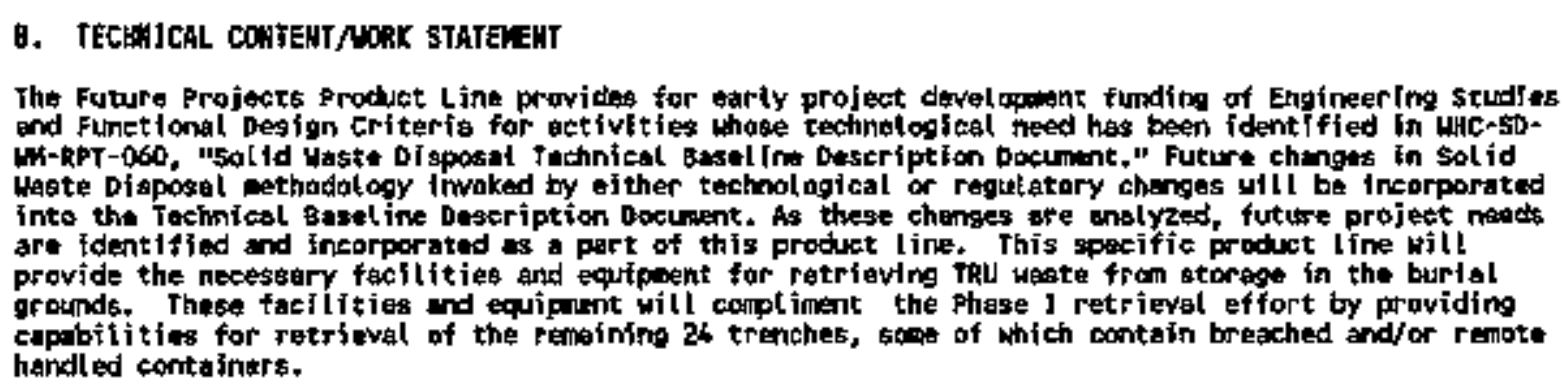 } \\
\hline
\end{tabular}




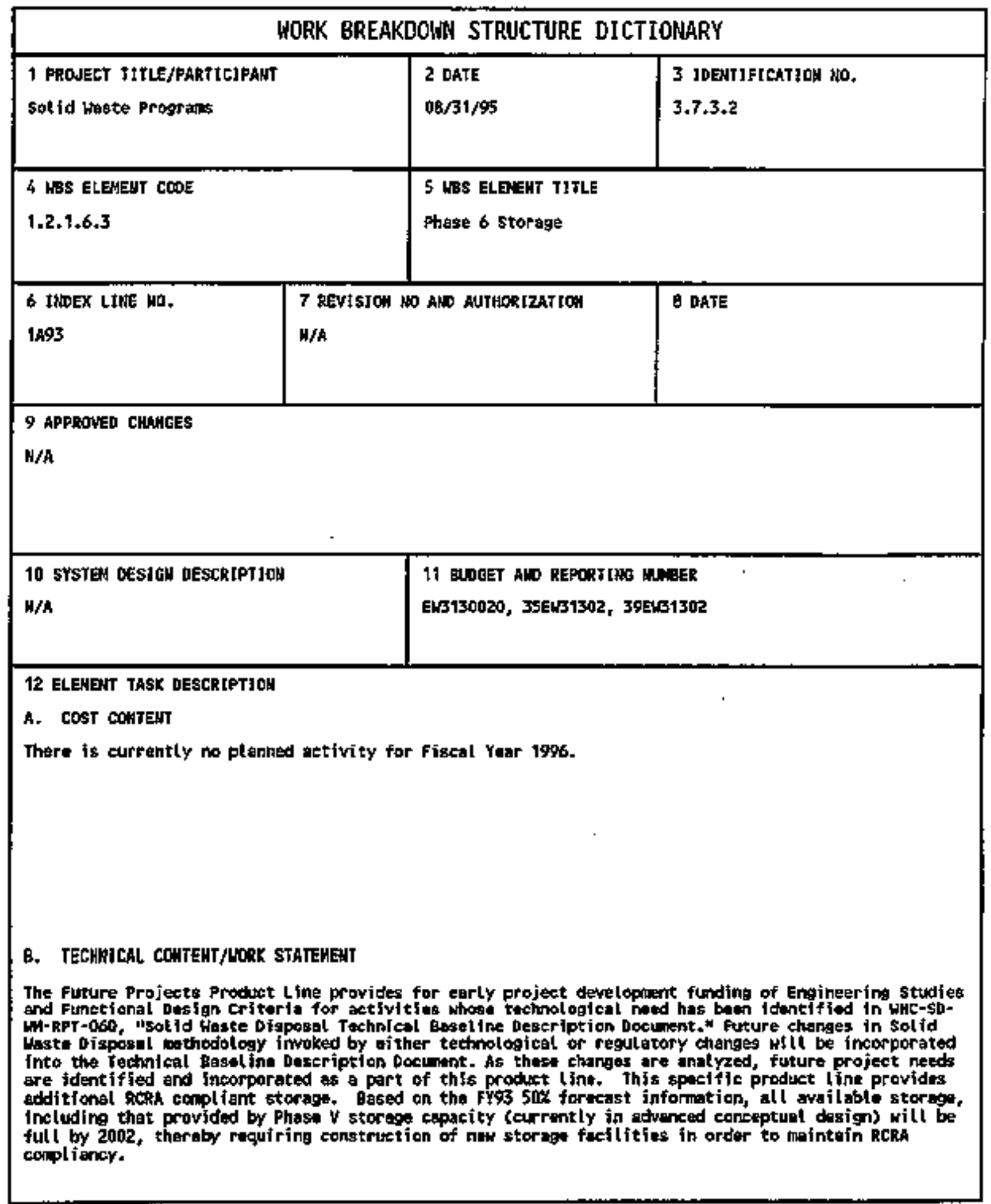




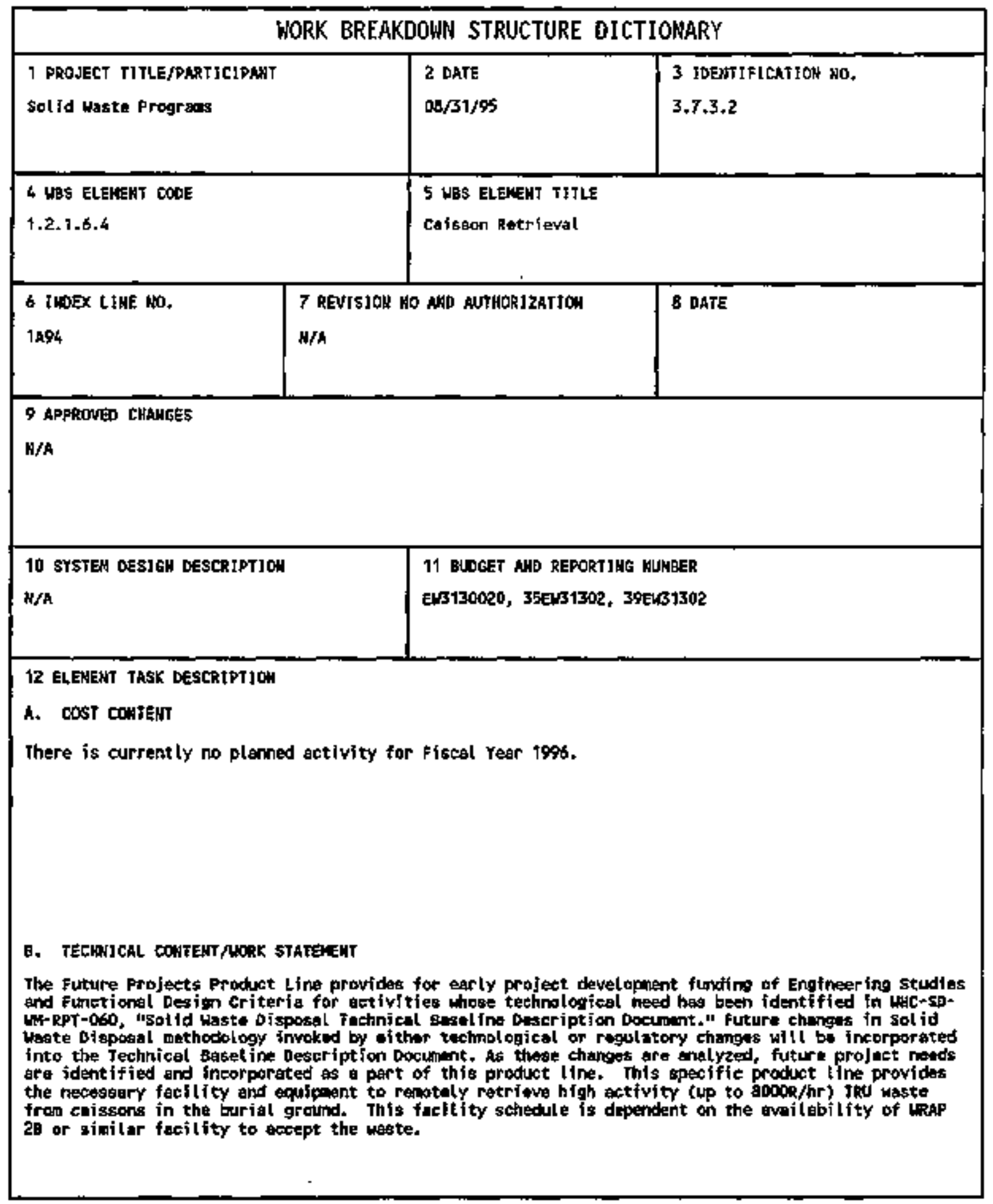




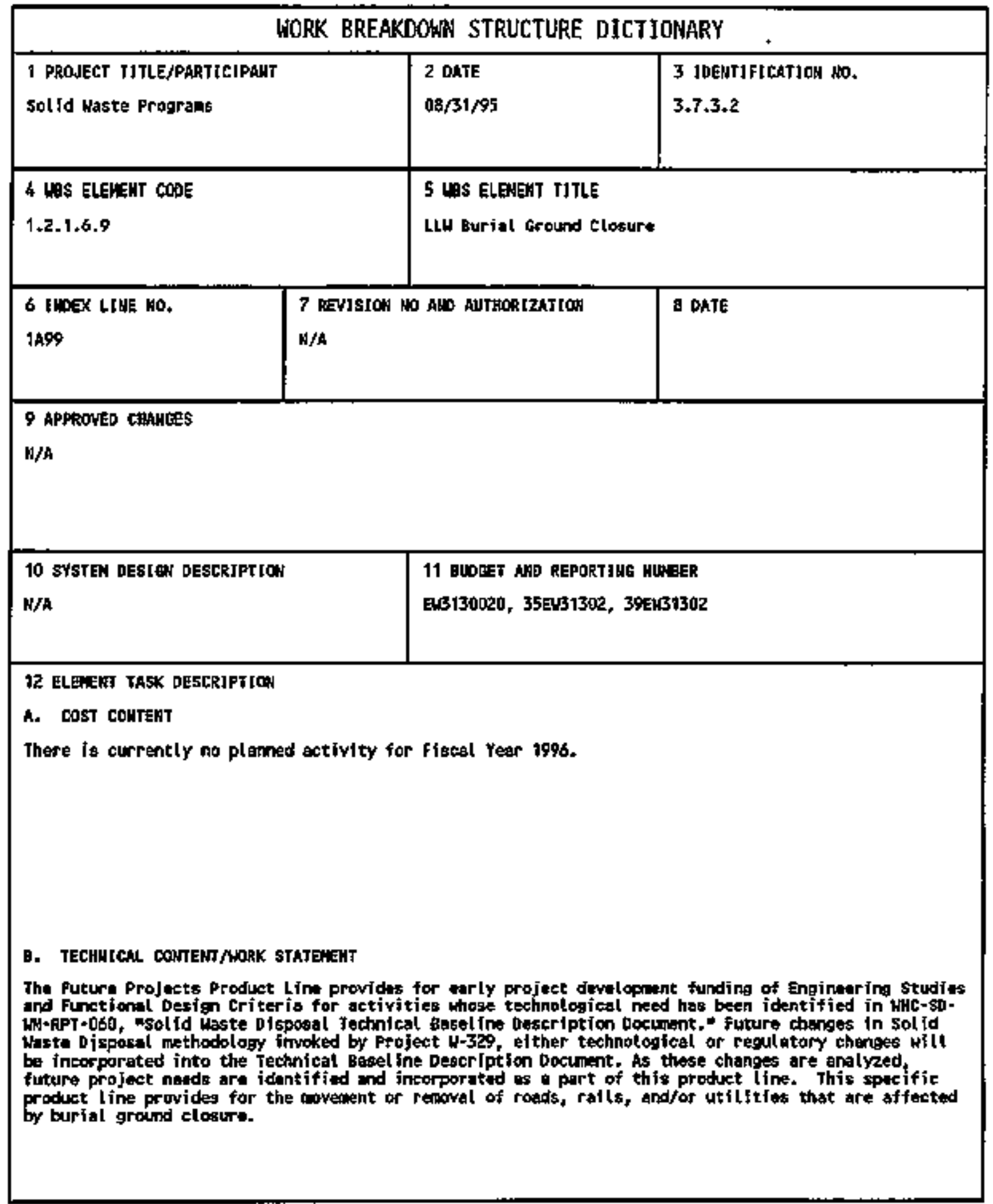


SOLID HASTE PROGRAM

UBS 1.2.1

FY 1996 Program Plan

WHC-SP-1114, Rev. 1

\begin{tabular}{|c|c|c|c|}
\hline \multicolumn{4}{|c|}{ WORK BREAKOOHWN STRUCTURE DICTIONARY } \\
\hline \multicolumn{2}{|l|}{$\begin{array}{l}1 \text { PROJECT TITLE/PARTLCIPANT } \\
\text { Solíd Wasto Proprams }\end{array}$} & $\begin{array}{l}2 \text { OATE } \\
08 / 31 / 95\end{array}$ & $\begin{array}{l}3 \text { 1DEHTIFICATIOH MO. } \\
3.7 .3 .2\end{array}$ \\
\hline \multicolumn{2}{|l|}{$\begin{array}{l}4 \text { LBS ELEMENT COPF } \\
1.2 .1 .6 .9\end{array}$} & \multicolumn{2}{|c|}{$\begin{array}{l}5 \text { LBS ELEEERT TITLE } \\
\text { Mixed thaste bisposal Trench }\end{array}$} \\
\hline $\begin{array}{l}\text { INOEX LINE HO. } \\
\text { 1AFA }\end{array}$ & \multicolumn{2}{|c|}{$\begin{array}{l}7 \text { REVISIOW MO ANO AUTHORIZATIOY } \\
\text { W/A }\end{array}$} & 8 DATE \\
\hline \multicolumn{4}{|l|}{$\begin{array}{l}9 \text { APPROVEO CHAHGES } \\
\text { H/A }\end{array}$} \\
\hline \multicolumn{2}{|l|}{$\begin{array}{l}10 \text { SYSTEM DESIES DESERIPTIOH } \\
\mathrm{H} / \mathrm{A}\end{array}$} & \multicolumn{2}{|c|}{ 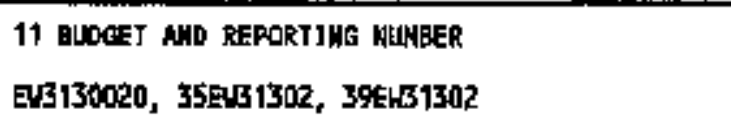 } \\
\hline \multicolumn{4}{|c|}{ 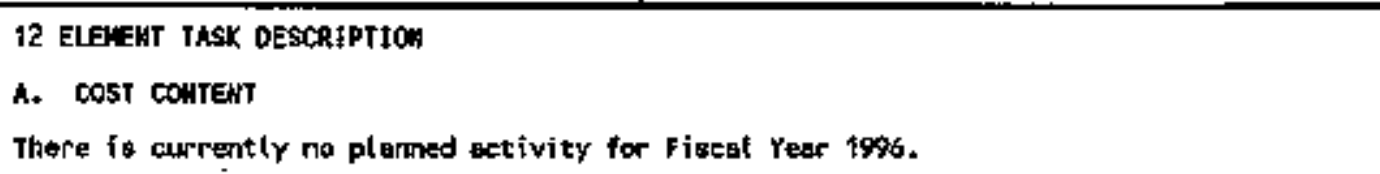 } \\
\hline \multicolumn{4}{|c|}{ B. TECHUTCAL CONTEHJ/HORK STATEREHT } \\
\hline
\end{tabular}




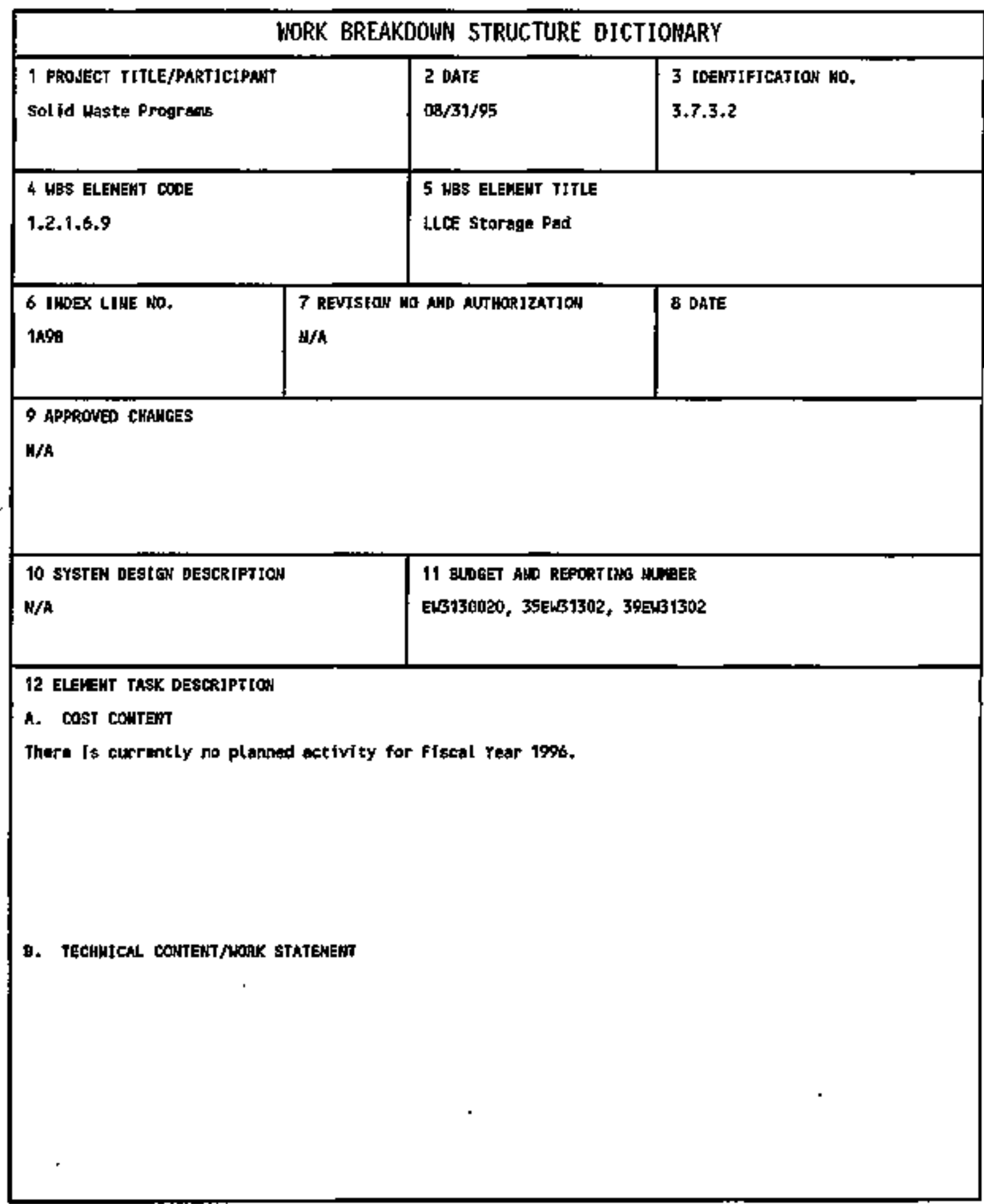




\section{COST CONTENT DETAIL}

\section{L-259 Project Integration}

Administration-staff \& safety meetings, HGET, and Site general training.

Routine Performance Reports/Schedules-EAC, VAR, Mid-year, Functtonal Revfew Schedule Updates, and associated meetings.

- Project Support-manager, secretary, CAM, fjnancial \& scheduling, support on upkeep activities for project: contract negotiations, ECN review, change control, and CAP/schedule maintenance.

\section{Document Control \& Records Management}

- Project Plans \& Validation-manager, CAM, financial \& scheduling, support on: PHP, CPOS, SORD, validation, ICE review.

- ADS/MYPP Development-manager, CAM, financial scheduling, support.

\section{W-259 Environgenta?, Safety \& OA}

Environmental Oversight-permitting plan, Environmental Peratit Application, project document oversight, project close-out \& record review.

- Safety oversight-PSE review, SAR/ISB analysis, SAR revision, project document oversight, project close-out \& record review.

- Qua]ity Assurance Oversight-QA Prograr P]an, project document oversight, project close-out \& record review.

- W-259 Design/Construction Support

- Definitive Design Support

- Title III Support

- Construction Support

- ATP Development \& Performance Support

- W-259 Project Startup

- $\quad$ OTP Development \& Performance

- Procedures Deve1opment Update

- As-bui1t Drawings Support

- Operator Training

- ORR Activities 


\begin{tabular}{|c|c|c|c|c|c|}
\hline PAOLRAN ELENEUT & ACTJVtTY & $\cos T$ ACCoLAT & TITEE & $\begin{array}{c}\text { RESPOOHSTEEE } \\
\text { WWAGER }\end{array}$ & $\begin{array}{l}\text { RESPONSTELE } \\
\text { OREAHIZAT ION }\end{array}$ \\
\hline \multirow[t]{11}{*}{$1.2 .1 .1 / \mathrm{Al}$} & & $\cdot$ & Solid Naste & & \\
\hline & A11 & $N / A$ & TRU Retrieval & P.L. Hapke & $\begin{array}{l}\text { \$ol id Waste } \\
\text { Nanagenant }\end{array}$ \\
\hline & $A 12$ & 1A1201 & Baw Treetment & D. E. Mckenney & $\begin{array}{l}\text { Solid Wostete } \\
\text { Prograns }\end{array}$ \\
\hline & 113 & 1A1301 & $\begin{array}{l}\text { Transuranic } \\
\text { storage }\end{array}$ & P. L, Hapke & $\begin{array}{l}\text { Solid Waste } \\
\text { Merregeiment }\end{array}$ \\
\hline & 114 & 1A1401 & RWH/CWC Storags & P. L. Hapke & $\begin{array}{l}\text { Solid Waste } \\
\text { Managerlotht }\end{array}$ \\
\hline & A15 & $1 A 15 B 1$ & $\begin{array}{l}\text { Hazerdous Haxto } \\
\text { Storege }\end{array}$ & R. D. Pierce & $\begin{array}{l}\text { Generator and } \\
\text { Waste } \\
\text { Acceptance } \\
\text { Services }\end{array}$ \\
\hline & A16 & $1 \mathrm{A1601}$ & $\begin{array}{l}\text { Low-Level } \\
\text { Suriel tround } \\
\text { Emviraninental } \\
\text { Sufaty and } \\
\text { Heal to }\end{array}$ & P. L. Hapke & $\begin{array}{l}\text { Sol fd Haste } \\
\text { Haragelient }\end{array}$ \\
\hline & A17 & TA1701 & $\begin{array}{l}\text { RHW Disposal } \\
\text { Emvirompontal } \\
\text { Sofety and } \\
\text { Bealth }\end{array}$ & P. L. Hapks & $\begin{array}{l}\text { Solid Waste } \\
\text { Hanagetnent }\end{array}$ \\
\hline & A19 & $1 \times 1901$ & $\begin{array}{l}\text { Systems } \\
\text { Enginetring }\end{array}$ & D. E. MeKennmy & $\begin{array}{l}\text { Solid Waste } \\
\text { Prograns }\end{array}$ \\
\hline & AlA & IA1AO1 & Progrem Support & D. E. Hekenney & $\begin{array}{l}\text { Solid Naste } \\
\text { Prograns }\end{array}$ \\
\hline & A1B & 1A180t & $\begin{array}{l}\text { Off-Situ } \\
\text { Services }\end{array}$ & D. E. HcKesney & $\begin{array}{l}\text { Sol id waste } \\
\text { Prograps }\end{array}$ \\
\hline \multirow[t]{8}{*}{$1.2 .1 .2 / \mathrm{A4}$} & & & MRAP Moctule 1 & & \\
\hline & $M 1$ & 1A4101 & $\begin{array}{l}\text { Project } \\
\text { Integration }\end{array}$ & D.R. Luces & $\begin{array}{l}\text { Solid Haste } \\
\text { Prajects }\end{array}$ \\
\hline & 142 & $1 A 4201$ & $\begin{array}{l}\text { Systens } \\
\text { Enginuering }\end{array}$ & P.L. Hapke & $\begin{array}{l}\text { Solid Weste } \\
\text { Wenagenent }\end{array}$ \\
\hline & $M 43$. & $1 A 4301$ & $\begin{array}{l}\text { Project stmrt- } \\
\text { Up }\end{array}$ & P.L. Hapke & $\begin{array}{l}\text { Sol id Waste } \\
\text { Managowant }\end{array}$ \\
\hline & 145 & - 144501 & $\begin{array}{l}\text { PHS/PCS } \\
\text { Devetopment and } \\
\text { Tasting }\end{array}$ & P,L. Hapke & $\begin{array}{l}\text { Sol ial Waste } \\
\text { Managenant }\end{array}$ \\
\hline & 146 & $1 A 4601$ & $\begin{array}{l}\text { Enwirnimental } \\
\text { Safety and of }\end{array}$ & P.L. Haptce & $\begin{array}{l}\text { Sol id unste } \\
\text { Hanugenent }\end{array}$ \\
\hline & 147 & 144720 & $\begin{array}{l}\text { Map } 1 \\
\text { Operattons \& } \\
\text { Cantic }\end{array}$ & P.L. Hajke & $\begin{array}{l}\text { Solid Woste } \\
\text { Nerragewent }\end{array}$ \\
\hline & MAF & UAFEE & Fee & D.E. Hekernoy & $\begin{array}{l}\text { Solid Uaste } \\
\text { Prograns }\end{array}$ \\
\hline $0.2 .1 .4 / A 7$ & & & $\begin{array}{l}\text { Waste storage } \\
\text { lnfrestructure }\end{array}$ & & \\
\hline
\end{tabular}




\begin{tabular}{|c|c|c|c|c|c|}
\hline & 171 & 1A7101 & $\begin{array}{l}\text { Project } \\
\text { Integretion }\end{array}$ & $0, R$, Lunes & $\begin{array}{l}\text { Sol id Waste } \\
\text { Project }\end{array}$ \\
\hline & A73 & IAT301 & $\begin{array}{l}\text { Project start. } \\
\text { up }\end{array}$ & D.E. HcKenney & $\begin{array}{l}\text { Sol fd waste } \\
\text { Programs }\end{array}$ \\
\hline & $A 77$ & 147720 & $\begin{array}{l}\text { Nan-Project } \\
\text { Startup }\end{array}$ & D.E. McKenney & $\begin{array}{l}\text { Sol id Wasto } \\
\text { Programs }\end{array}$ \\
\hline & Are & H/A & Construtetian & D,R. Lucas & $\begin{array}{l}\text { 5olid Waste } \\
\text { Profects }\end{array}$ \\
\hline & $A 7 F$ & MAPFE & Fee & D.E. McKenney & $\begin{array}{l}\text { Solid woste } \\
\text { Prog̣rams }\end{array}$ \\
\hline \multirow[t]{10}{*}{$1.2 .1 .5 / A B$} & & & Wagte Retrifral & & \\
\hline & A81 & TAB101 & $\begin{array}{l}\text { Project } \\
\text { Integration }\end{array}$ & D.R+ Lucas & $\begin{array}{l}\text { sol id Uaste } \\
\text { Projecta }\end{array}$ \\
\hline & 182 & 1AB201 & $\begin{array}{l}\text { Systens } \\
\text { Enginearing and } \\
\text { Emyironnental } \\
\text { Safuty and of }\end{array}$ & O.R. Lueas & $\begin{array}{l}\text { Sol id Uaste } \\
\text { projects }\end{array}$ \\
\hline & AB3 & 1AB301 & $\begin{array}{l}\text { start-Up } \\
\text { Activitís }\end{array}$ & D.R. Lucas & $\begin{array}{l}\text { 5ol Id Waste } \\
\text { Projects }\end{array}$ \\
\hline & $A B 5$ & $1 A B 501$ & $\begin{array}{l}\text { Equipmant } \\
\text { Doveloprent and } \\
\text { Testing }\end{array}$ & D.R. Lutes & $\begin{array}{l}\text { sol id Wasto } \\
\text { Projects }\end{array}$ \\
\hline & AB7 & 1A8701 & $\begin{array}{l}\text { Waste Retrieval } \\
\text { Oprrations }\end{array}$ & D.R. LUteAs & $\begin{array}{l}\text { Sol 1d waste } \\
\text { Prograns }\end{array}$ \\
\hline & $\boldsymbol{\mu B A}$ & $H / \lambda$ & $\begin{array}{l}\text { Definitive } \\
\text { Design end } \\
\text { Title I I }\end{array}$ & D.R. Lueas & $\begin{array}{l}\text { Solid Weste } \\
\text { Projects }\end{array}$ \\
\hline & Aac & $H / A$ & $\begin{array}{l}\text { Const ruction } \\
\text { Kansoment }\end{array}$ & D.R. Lucas & $\begin{array}{l}\text { Sot id Was te } \\
\text { Profects }\end{array}$ \\
\hline & $A B$ & $N / h$ & Praturenent & D.R. Lucas & $\begin{array}{l}\text { Solld waste } \\
\text { Projects }\end{array}$ \\
\hline & AgE & $M / A$ & $\begin{array}{l}\text { Project } \\
\text { Mandsement }\end{array}$ & D,R, Luces & $\begin{array}{l}\text { Solid Waste } \\
\text { Projects }\end{array}$ \\
\hline \multirow[t]{7}{*}{$1.2 .1 .6 / A 9$} & & & $\begin{array}{l}\text { Kew Factlity } \\
\text { Plamning }\end{array}$ & & \\
\hline & A91 & $M / A$ & NRAP $2 B$ & K.L. HIadak & $\begin{array}{l}\text { sol id Weate } \\
\text { systems } \\
\text { Enginesing }\end{array}$ \\
\hline & $A 92$ & $N / A$ & $\begin{array}{l}\text { Phose } 2 \\
\text { Retrieval }\end{array}$ & K.L. HIadak & $\begin{array}{l}\text { Solfd Uaste } \\
\text { systems } \\
\text { Engineering }\end{array}$ \\
\hline & A93 & $N / A$ & Phase 6 storage & K.L. Hladek & $\begin{array}{l}\text { Sol id Losta } \\
\text { syrttems } \\
\text { Engineering }\end{array}$ \\
\hline & $A 94$ & $N / A$ & $\begin{array}{l}\text { Caişş̣n } \\
\text { Retrieval }\end{array}$ & K.L. HIadak & $\begin{array}{l}\text { Solid Waste } \\
\text { systems } \\
\text { Engineering }\end{array}$ \\
\hline & A99 & $M / A$ & $\begin{array}{l}\text { Low-Level } \\
\text { Burial Ground } \\
\text { clasure }\end{array}$ & K.t. HLadek & $\begin{array}{l}\text { Sol ji unte } \\
\text { systems } \\
\text { Engineting }\end{array}$ \\
\hline & $M 9$ & $M / A$ & $\begin{array}{l}\text { Hixed Uagte } \\
\text { oisposal Trench }\end{array}$ & K. 1. HIadak & $\begin{array}{l}\text { sol id Waste } \\
\text { systems } \\
\text { Ensineering }\end{array}$ \\
\hline
\end{tabular}




\begin{tabular}{|c|c|c|c|c|c|}
\hline & A9g & $M / A$ & $\begin{array}{l}\text { LLaE storage } \\
\text { Pad }\end{array}$ & K. L. Hladek & $\begin{array}{l}\text { Solid Waste } \\
\text { Systwas } \\
\text { Enginaering }\end{array}$ \\
\hline \multirow[t]{6}{*}{$1.2 .1 .7 / A 3$} & & & $\begin{array}{l}\text { Waste and } \\
\text { Gecontwination } \\
\text { Services }\end{array}$ & & \\
\hline & $\mathbf{A} 3 \mathbf{1}$ & $1 / 3104$ & $\begin{array}{l}\text { Cenyon } \\
\text { Processing }\end{array}$ & N. S, Wright & $\begin{array}{l}\text { Waste and } \\
\text { Oecontarinat io } \\
\text { n Services }\end{array}$ \\
\hline & $13 z$ & $\begin{array}{l}1 \mathrm{A3202} \\
1 \mathrm{A3204}\end{array}$ & $\begin{array}{l}\text { Low-Lwral } \\
\text { Frocessing }\end{array}$ & и. S. Wright & $\begin{array}{l}\text { Waste and } \\
\text { Decontaminatio } \\
\text { n Serviers }\end{array}$ \\
\hline & A33 & $\begin{array}{l}1 A 3301 \\
1 A 3302 \\
1 A 3303 \\
2 A 3363 \\
4 A 3363\end{array}$ & $\begin{array}{l}\text { Contanianated } \\
\text { Equlpnent: } \\
\text { Storage, GP中, } \\
\text { and CELRTC }\end{array}$ & M. S. Wright & $\begin{array}{l}\text { Uaste and } \\
\text { Deconteninatio } \\
\text { n Sorvices }\end{array}$ \\
\hline & A34 & $1 A 3602$ & $\begin{array}{l}\text { Rail car } \\
\text { Nointenance/ } \\
\text { Gartification }\end{array}$ & M. S. Wright & $\begin{array}{l}\text { Waste and } \\
\text { Decontaninakio } \\
\text { n Servichs }\end{array}$ \\
\hline & A35 & 1A3501 & Fee support & D. E. MeKennay & $\begin{array}{l}\text { Solid Was to } \\
\text { Prograps }\end{array}$ \\
\hline \multirow[t]{2}{*}{$1.2 .1 .8 / M$} & & & $\begin{array}{l}\text { T Plant } \\
\text { Ventilation } \\
\text { Lpgrades }\end{array}$ & & \\
\hline & 4h1 & $W / 4$ & 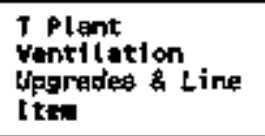 & D.R. Lucas & $\begin{array}{l}\text { solid waste } \\
\text { Projects }\end{array}$ \\
\hline \multirow[t]{2}{*}{$1.2 .1 .9 / A B$} & & & $\begin{array}{l}\text { T Plant } \\
\text { Secondary } \\
\text { Conteinnent and } \\
\text { Leak Detection } \\
\text { Upgrades }\end{array}$ & & \\
\hline & AB1 & $\begin{array}{l}1 \mathrm{kB101} \\
.\end{array}$ & $\begin{array}{l}\text { T Plant } \\
\text { Stcondary } \\
\text { Contaiment and } \\
\text { Leak Detection } \\
\text { Upgrades \& L fne } \\
\text { Jten }\end{array}$ & D.R. Lucas & $\begin{array}{l}\text { solid waste } \\
\text { Projects }\end{array}$ \\
\hline \multirow[t]{6}{*}{ HL } & & & $\begin{array}{l}\text { Solfd Haste } \\
\text { Assessanents }\end{array}$ & & \\
\hline & HL1 & thl101 & RHH Disposel & P. L. Hspke & $\begin{array}{l}\text { Sol id Waste } \\
\text { Dperations }\end{array}$ \\
\hline & NL4 & THL40e & แพ Disposal & P. 1. Hapke & $\begin{array}{l}\text { solid Weste } \\
\text { operations }\end{array}$ \\
\hline & HLS & TKLSOI & TRU Receipt & P. L. Haple & $\begin{array}{l}\text { Solid waste } \\
\text { Oparations }\end{array}$ \\
\hline & int 6 & 내 603 & RhW/CWC Receipt & P. L. Hapke & $\begin{array}{l}\text { Sol id Uaste } \\
\text { Operations }\end{array}$ \\
\hline & HLE & tHLAd & $\begin{array}{l}\text { Yuzurdous Waste } \\
\text { Receipt \& } \\
\text { shipping }\end{array}$ & R, D. Piarca & $\begin{array}{l}\text { Oantrecor and } \\
\text { Wazte } \\
\text { Accoptance } \\
\text { Servicas }\end{array}$ \\
\hline no & & & Hezardous Waste & & \\
\hline
\end{tabular}


SOLID WASTE PROGRAH

WBS 1.2.1

FY 1996 Program Plan

WHC-SP-1114, ReV. 1

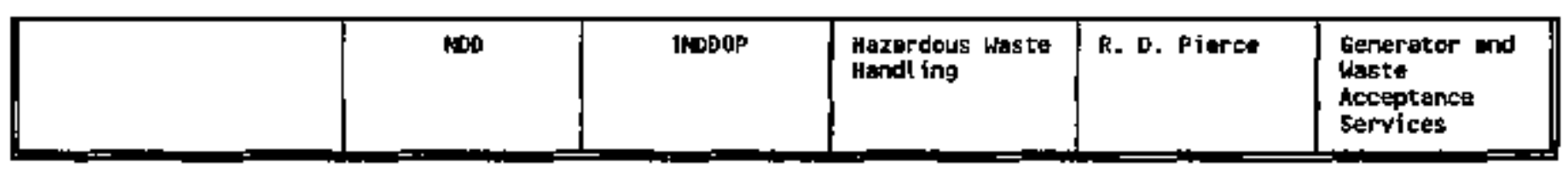




\section{A.5 Technology Requirements}

Due to the commercialization of all outyear Solid Waste Program projects, the technology needs of the program have diminished. AIT future needs have been based on the private sector being able to meet the need with existing technology or be able to develop the needed technology based on a statement of work. 
14tic-\$p-1114, Rev. 1

SOLID WASTE MYPP DATA ASSUMMPTIONS

The following wble lists the assumptions used in the Soltd Waste Projection Model for startiend dates and anmul capacites of treament facilizies.

\begin{tabular}{|c|c|c|c|}
\hline Treatment Facility & Start/End Date & Waste Sireamis & $\begin{array}{l}\text { Anmual } \\
\text { Capacity }\end{array}$ \\
\hline \multirow[t]{2}{*}{$\begin{array}{l}\text { Waste Rectiving and Processing } \\
\text { (WRAP) Module } 1\end{array}$} & \multirow[t]{2}{*}{$\begin{array}{l}\text { 1997-2024 LLW } \\
\text { 1998-2022 TRU_TRUM }\end{array}$} & $\begin{array}{l}\text { LLW in dnums } \\
\text { LLMW in drums } \\
\text { TRU_TRUM in drums }\end{array}$ & $\begin{array}{l}319 \mathrm{~m}^{3} \\
319 \mathrm{~m}^{3}\end{array}$ \\
\hline & & TRU_TRUM in SWBs & $119 \mathrm{~m}^{3}$ \\
\hline $\begin{array}{l}\text { Themal Trearment Fraility } \\
\text { (TTF) }\end{array}$ & $1997-2102$ & LLMW in đrums & $152 \mathrm{~m}^{3}$ \\
\hline $\begin{array}{l}\text { Stabilization Treatoment Program } \\
\text { (STP) }\end{array}$ & $1999-2102$ & $\begin{array}{l}\text { LJWW in drums } \\
\text { LLMW in boxes }\end{array}$ & $\begin{array}{l}985 \mathrm{~m}^{3} \\
400 \mathrm{~m}^{1}\end{array}$ \\
\hline M-33 Facility & $\begin{array}{l}2006-2022 \text { for } \\
\text { TRU/TRUM } \\
2006-2102 \text { for all other } \\
\text { streams }\end{array}$ & $\begin{array}{l}\text { waste in doums } \\
\text { waste in boxes }\end{array}$ & $\begin{array}{l}4,075 \mathrm{~m}^{3} \\
416 \mathrm{~m}^{3}\end{array}$ \\
\hline WIPP & $2002-2020$ & $\begin{array}{l}\text { waste in druors } \\
\text { waste in boxes }\end{array}$ & INF \\
\hline $\begin{array}{l}\text { Low Level Waste Burial Ground } \\
\text { (ILEG) }\end{array}$ & $\begin{array}{l}\text { Provided as needed. } \\
\text { Corrently open. }\end{array}$ & $\begin{array}{l}\text { waste in drums } \\
\text { waste in boxes }\end{array}$ & INF \\
\hline $\begin{array}{l}\text { Mixed Waste (MW) Disposal } \\
\text { Trench }\end{array}$ & $\begin{array}{l}\text { Provided as needed. } \\
\text { Open in } 1999 .\end{array}$ & $\begin{array}{l}\text { waste in dunfes } \\
\text { waste in boxes }\end{array}$ & INF \\
\hline
\end{tabular}

Volume splits for the waste streams were based upon the 1995 Baseline Solid Warte Management System Description (Anderson, et.al.). For volume splits which required waste volume information suth as fazardons constituents and physical waste forms, the 1993. Baseline Solid Waste Maragenotin System Description (Armacost, et. al.) was referenced to provide percentages. The following summary is not a complete description of the waste routings, but provides a bigh tevel overview of the assumptions that were used in the Solid Waste Projection Model to obtain results for the MYPP data call.

\section{CH_LLW_II IMd_RH_LLW_III}

- 5\% of CH_LLW_I in drums will go to WRAP I; 95\% will go to LIBG.

- $100 \%$ of CH_LLW in boxes, CH_LLW_II in drums and boxes, and RH_LLW in dinus and boxes will go to LLBG.

\section{CH LLLMW}

- $\quad 87 \%$ of CH_LLMW in drums will go to STP; 13 \% will go to TTF. All treated wohmes will be sent to the MW Disposal Trench 
MAC-5P-1114, Rev. 1

- Boxes cmaller than 6' $\times 8^{\prime} \times 12^{\prime}$ will be treated in the STP; larger boxes and LECs will go to M-33

Facitity, Of those seat to the M-33 faciliry, $87 \%$ will be semt to the STP and $13 \%$ will go the TTF.

- No voinwe adjustments sesult from treament at the TTF; a 2:1 increase resulis from treamient by the STP.

\section{RPILIMW}

- 99\% of RH_LLMW in drums and boxes will be treated in the M-33 Facility; 1\% will go wo Long Terw Storage becanse it requires thermal treacment. All RH_LLMW in LECs will go the M-33 facility.

- A $2+1$ waste volume increase results from processing in the M-33 facility.

\section{CH_TRU_SUSPECT}

- 40\% of the CH_TRU_SUS in drums will be teclassified as CH_LLW_Il in drums and go to WRAP 1.

- $\quad 5 \%$ of $\mathrm{CH}$ _TRU_SUS in drums will be reciassifed as CH_LLMW in drums and go to WRAP 1.

- $5 \%$ of $\mathrm{CH}_{-}$TRU_SUS in drums will be reclassified as CH_LLMW in drums and go to TTF.

- $\quad 30 \%$ of $\mathrm{CH}_{-}$TRU SUS in drwns will be reclassified as CH_TRU in dnums; $80 \%$ of this waste will go to WRAP 1; $5 \%$ of the $50 \%$ will go to Long Term Starage; and 15\% of the $50 \%$ will go to the $M-33$ facility.

- 100\% of $\mathrm{CH}$ TRU_SUS in boxes will go to M-33 Facility. $3.2 \%$ of the treated CH_TRU_SUS will be reclassified as CH L LNMW in truots. Half of that volume will go to STP and the other haif will go to the TTE. 12.8\% of the treated CH_TRU_SUS will be reclassified as CH_LIW_II and shipped to the LLBG. $84 \%$ of the treated CH_TRU_SUS will be reclassified as CH_TRU_TRUM and shipped to WIPP via storge.

- A 2:1 waste volume increase resules from processing in the M-33 facility.

\section{BH_TRU_SUSPECT}

- $100 \%$ of RH_TRU_SUS will go to the M-33 facility for treament and certification prior to shipment to WIPP.

- A 2:1 waste voluane increase results from processing in the M-33 factitity.

\section{CH TRUTRUM}

- $90 \%$ of CH_TRU in drums witl go to WRAP 1; $5 \%$ will go to Long-term Storage; $5 \%$ will go to the M-33 facility.

- 64\% of CH_TRU in bozes will go to M-33; 36\% will go to WRAP 1.

- A 2:1 paste volume intrease results from processing in the M-33 facility.

\section{RH_TRU/TRUM}

- 100\% of RH_TRU/TRUMA will go to the M-39 facility for treatment and certification prior to shipment to WIPP.

- A 2:1 waste volume increase results from processing in the M-33 facility. 
NHC-SP-11]4, Rev. 1

FY 1996 Weste Type Volum Projactions for W9S: 1.2.1

Program Hente: solfid Weste

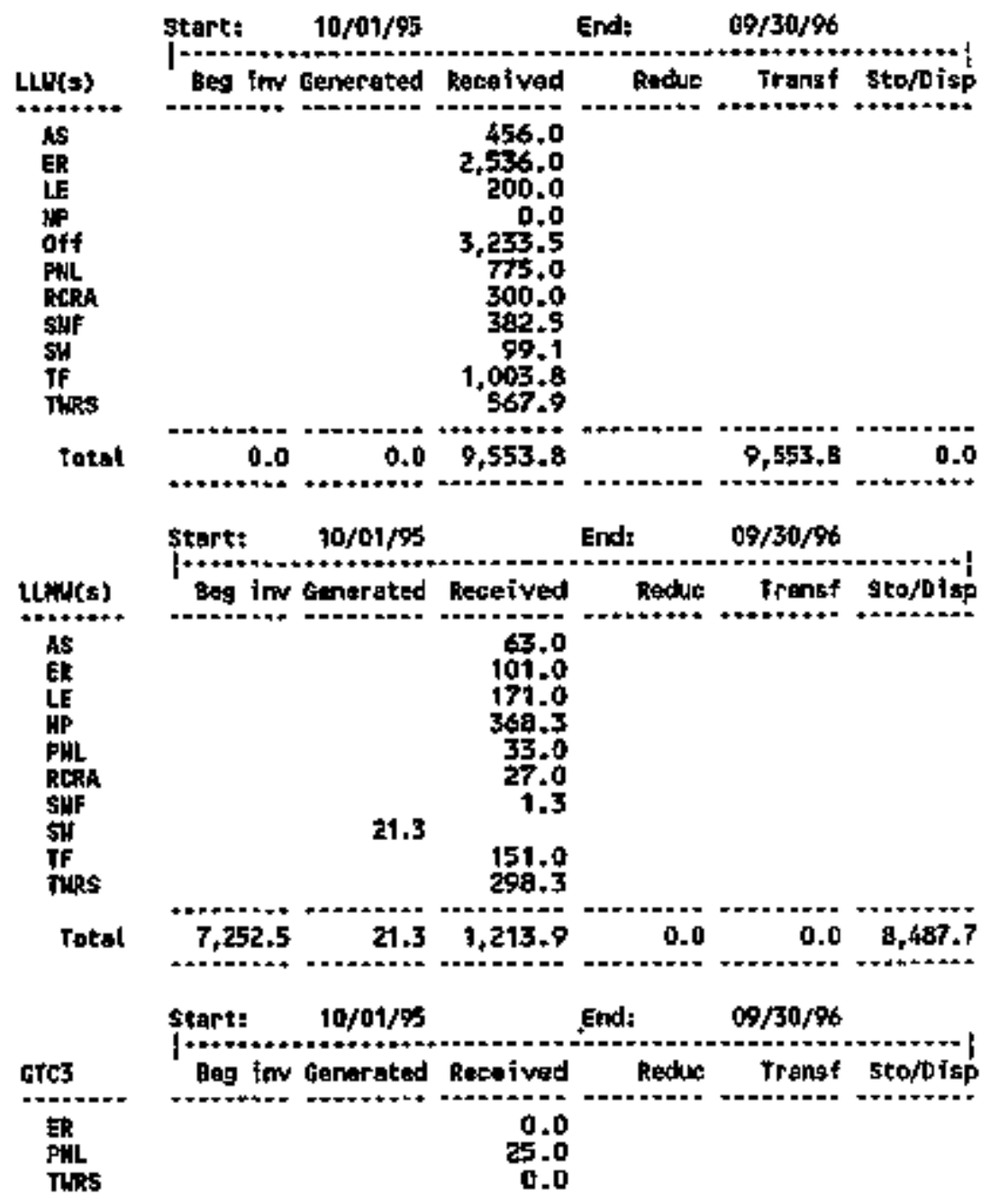

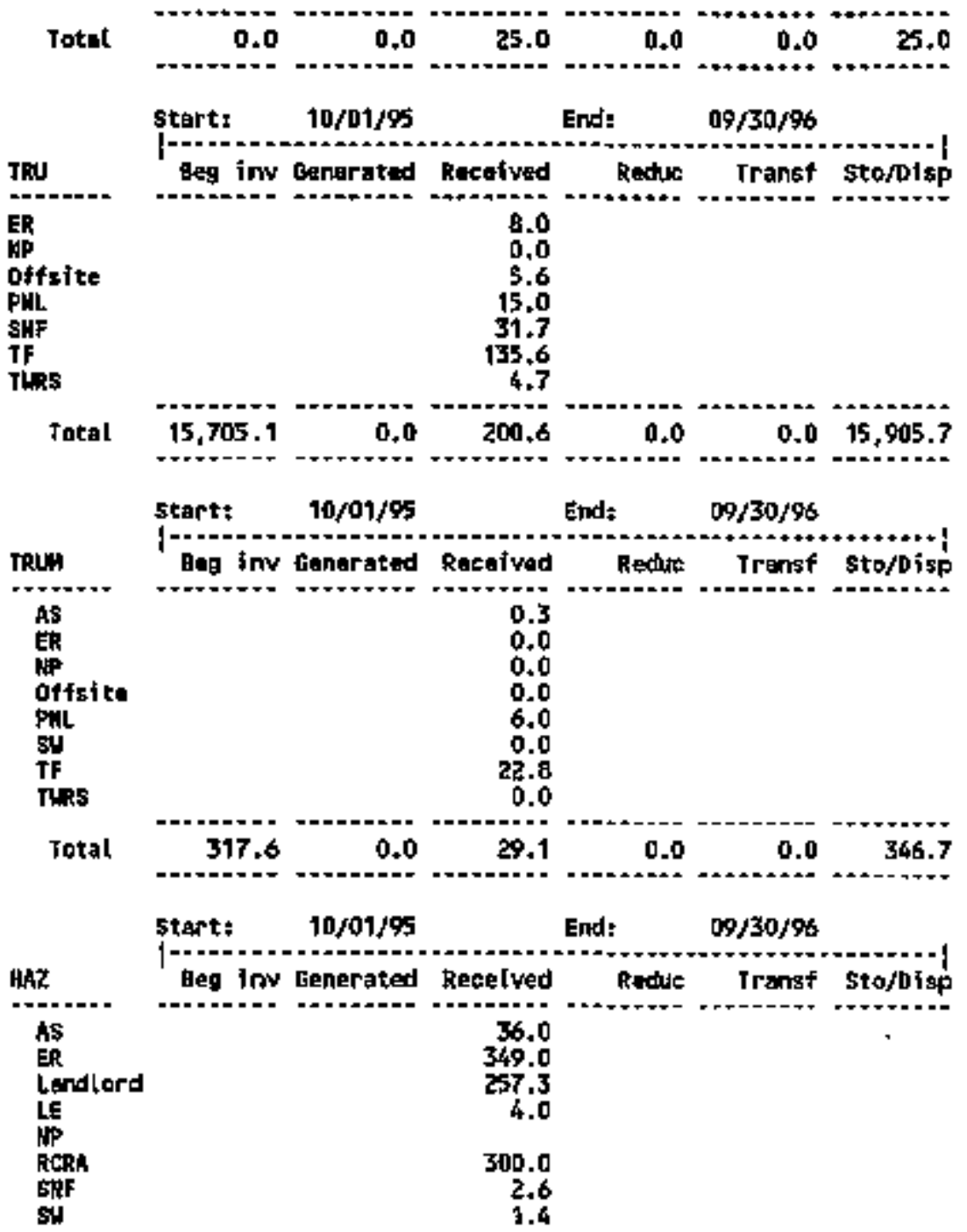

\section{A.6-3}


WHC-SP-1114, Rev. 1

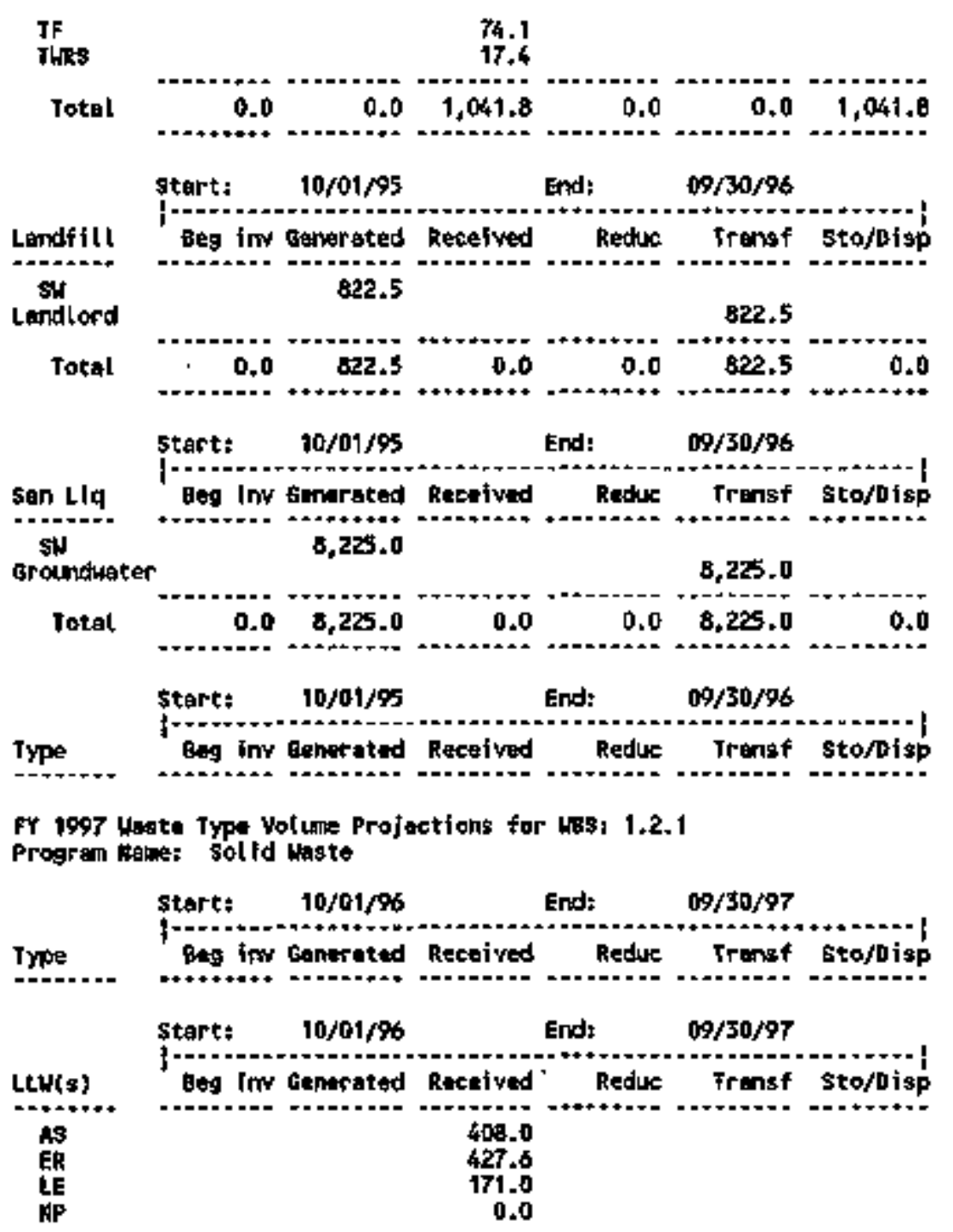

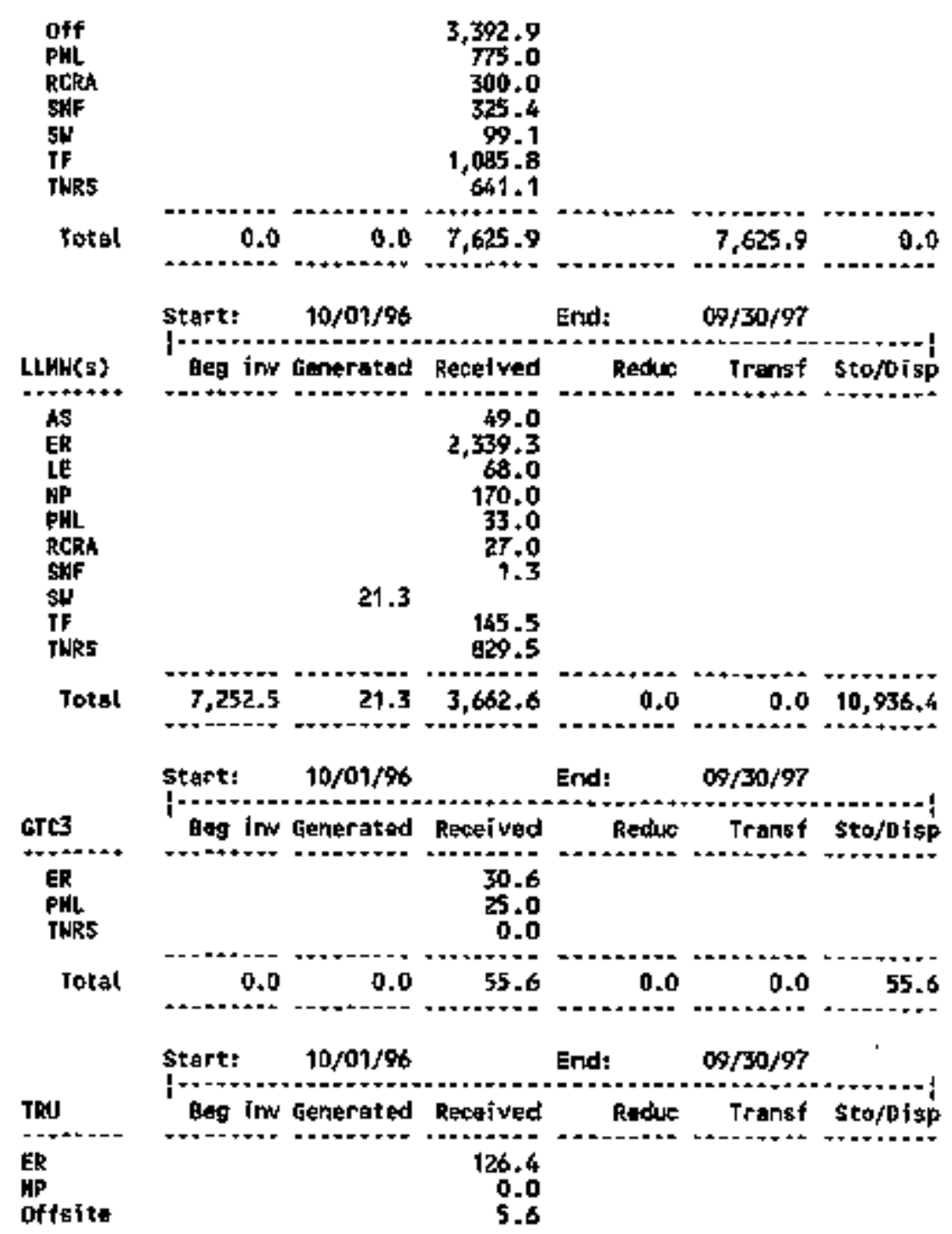

\section{A. 6-4}


WHC-SP-1114, Rev. 1
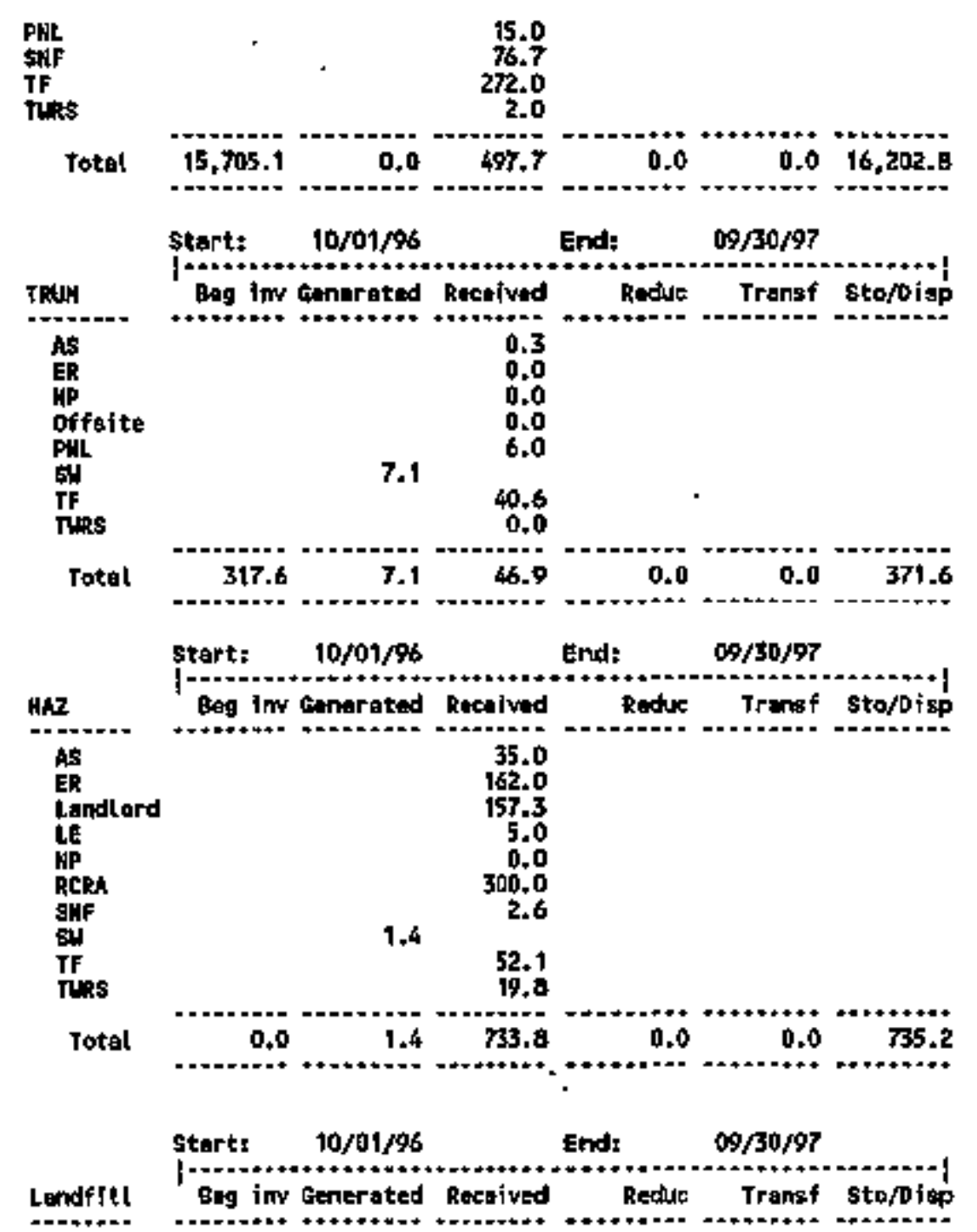

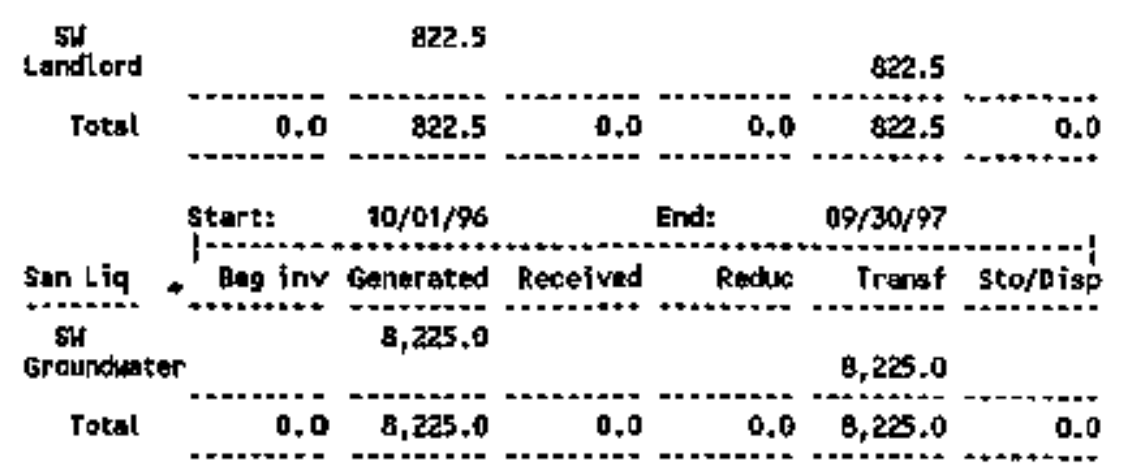

FY $19 \% 8$ Waste Typo Volume Projections for WBS: 1.2 .1

Progra Mane: sol id Waste

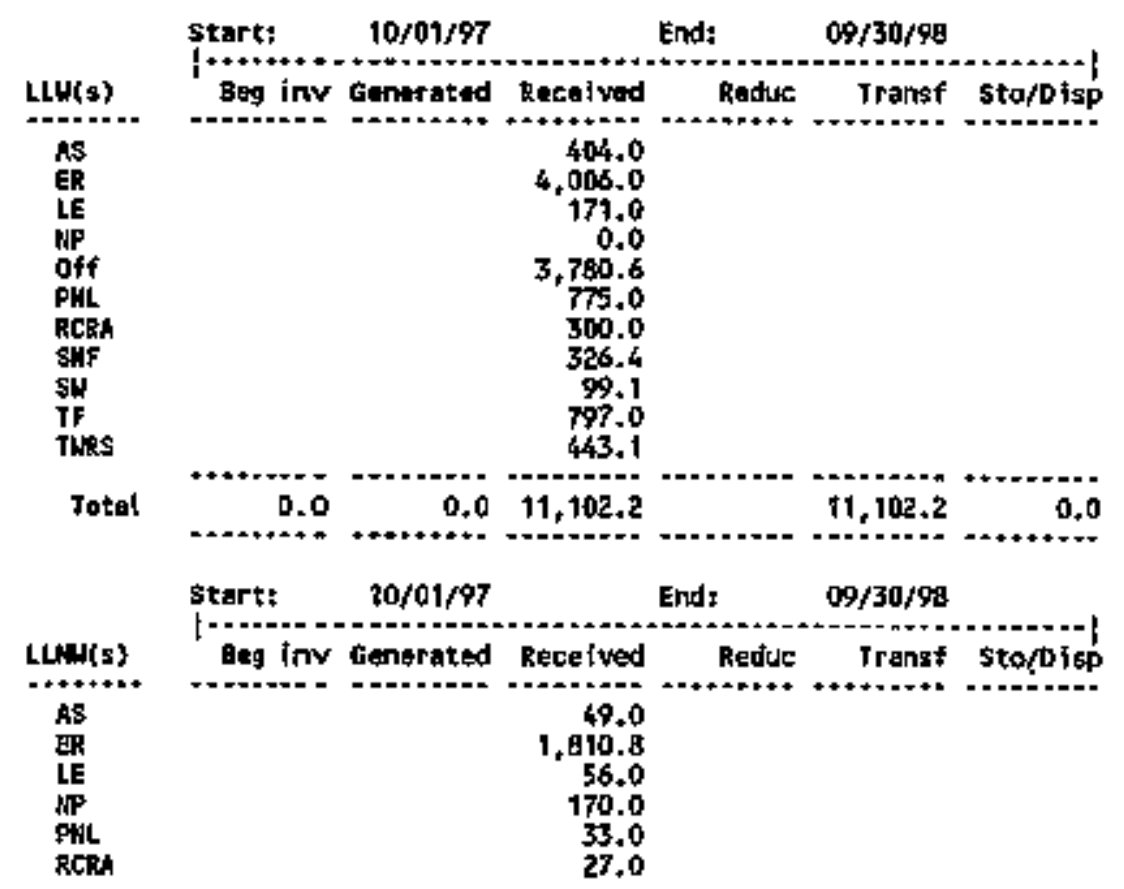

\section{A.6-5}


WHC-SP-1114, Rev. 1

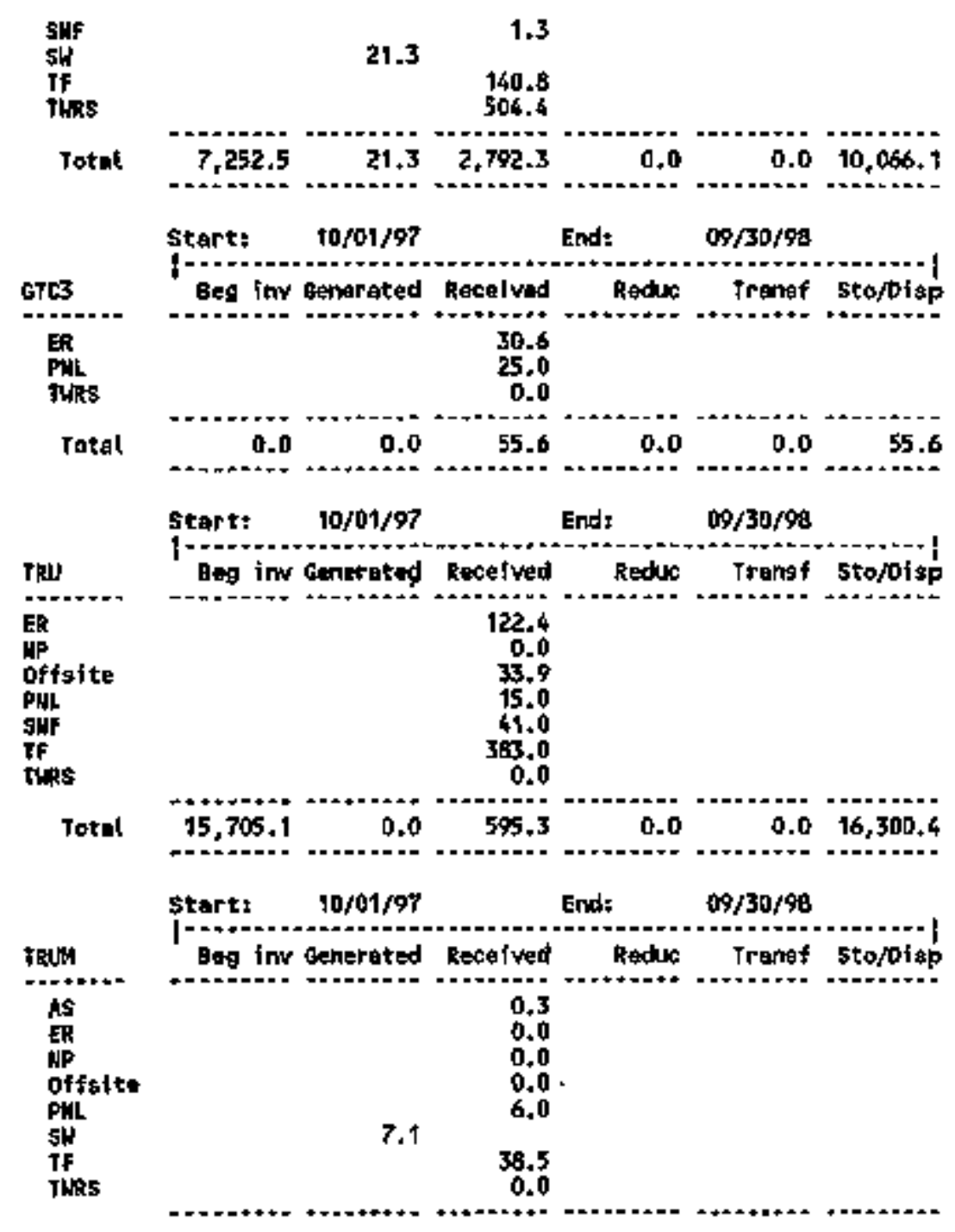

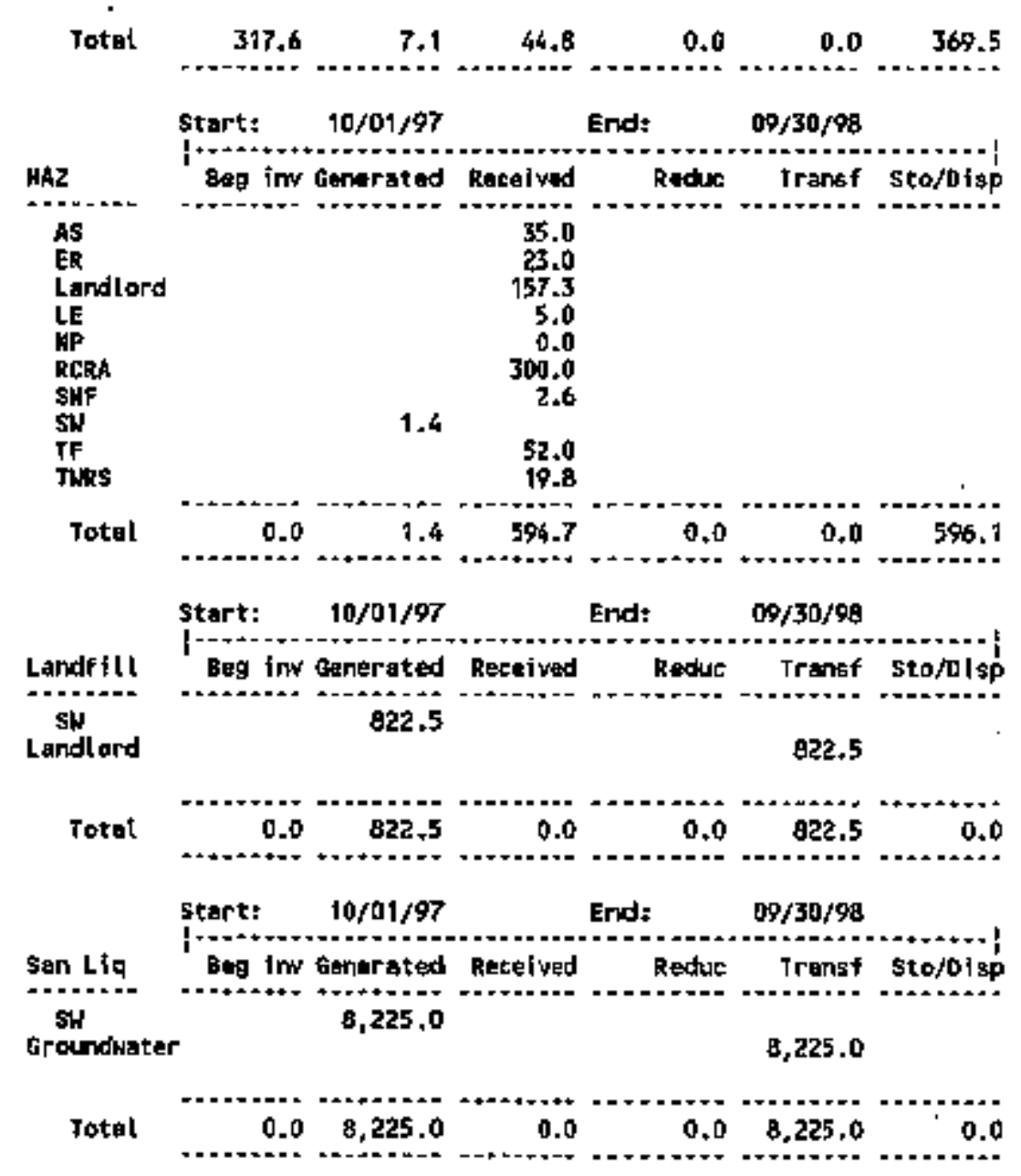

FY 1909 Wasto Type Yolune Projections for hBS: 1.2 .1 Progran Harne: Sol id haste
start:
$10 / 01 / 98$
End: $\quad 09 / 30 / 99$ 
WHC-SP-1114, Rev. 1
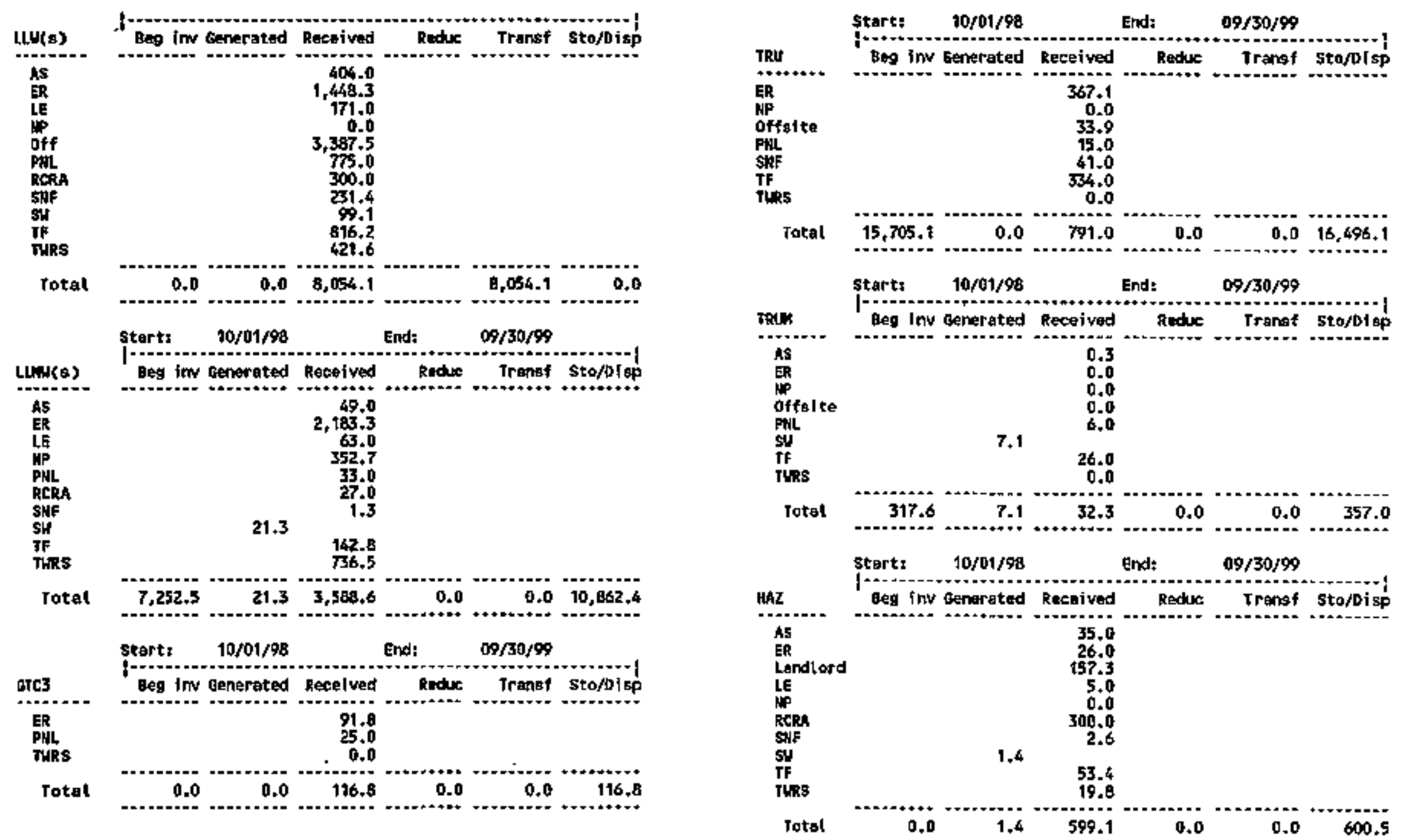

\section{A. 6-7}


WiC-SP-1114, Rev. 1

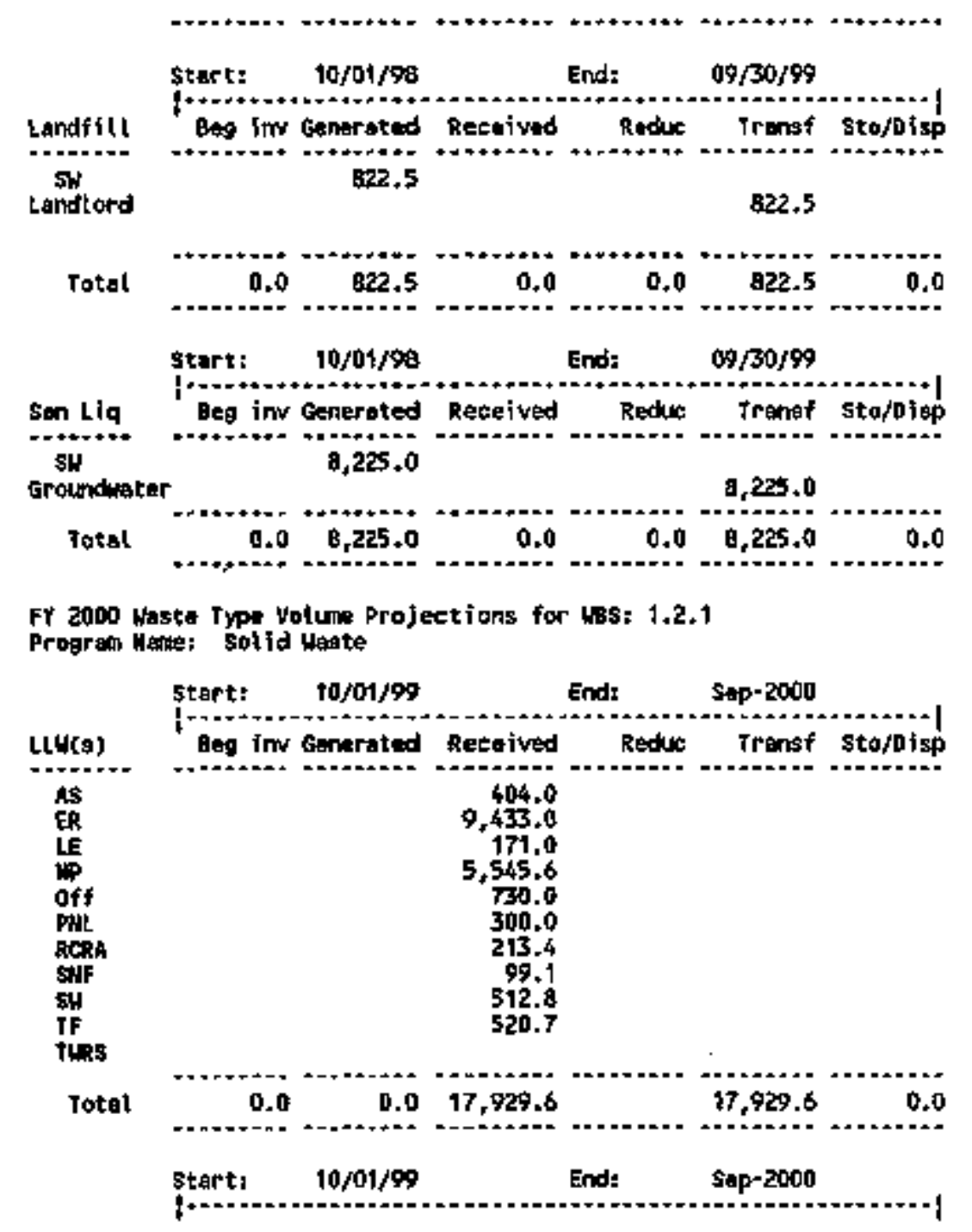

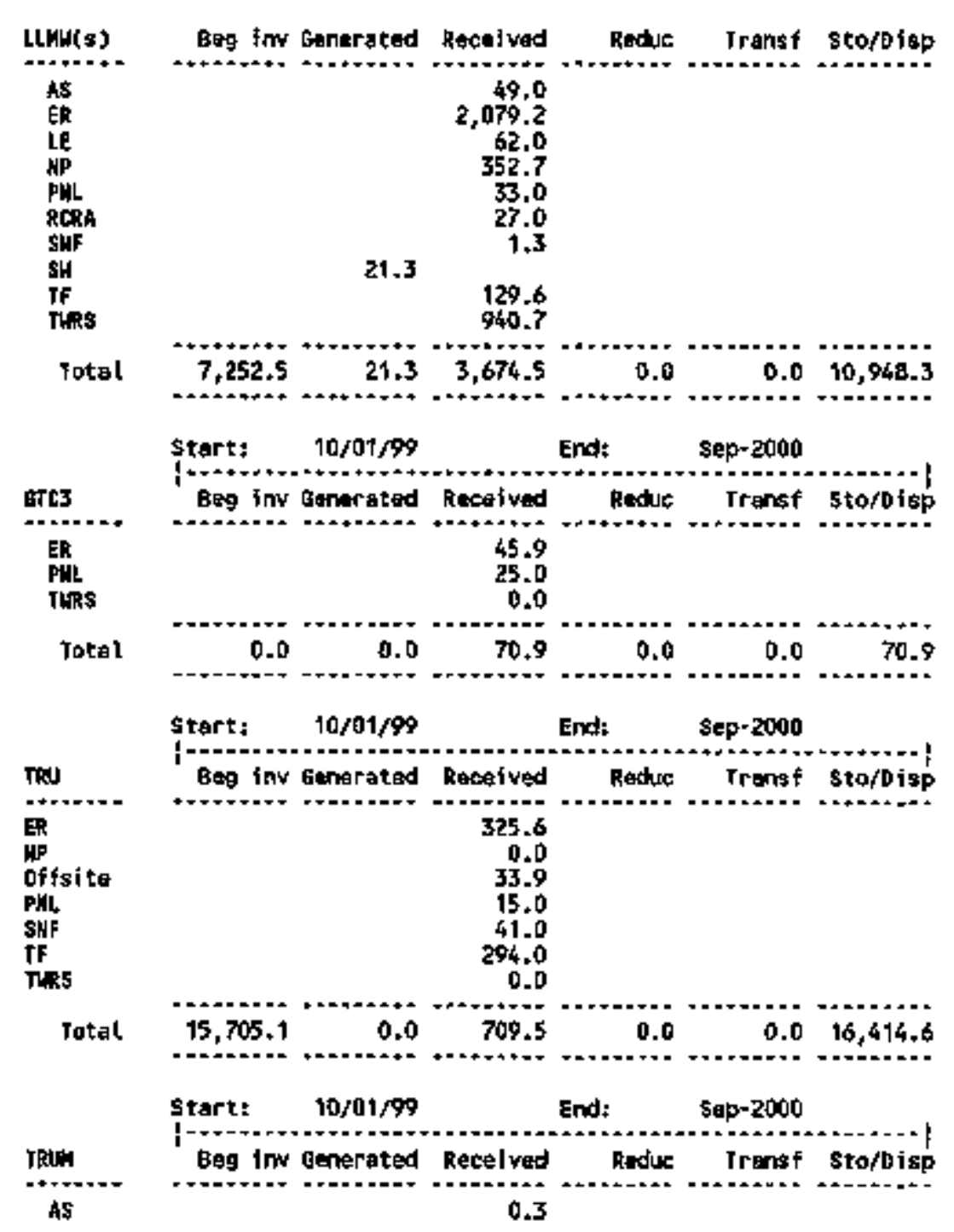

\section{A. 6-8}


MHC-SP-1114, Rev. 1

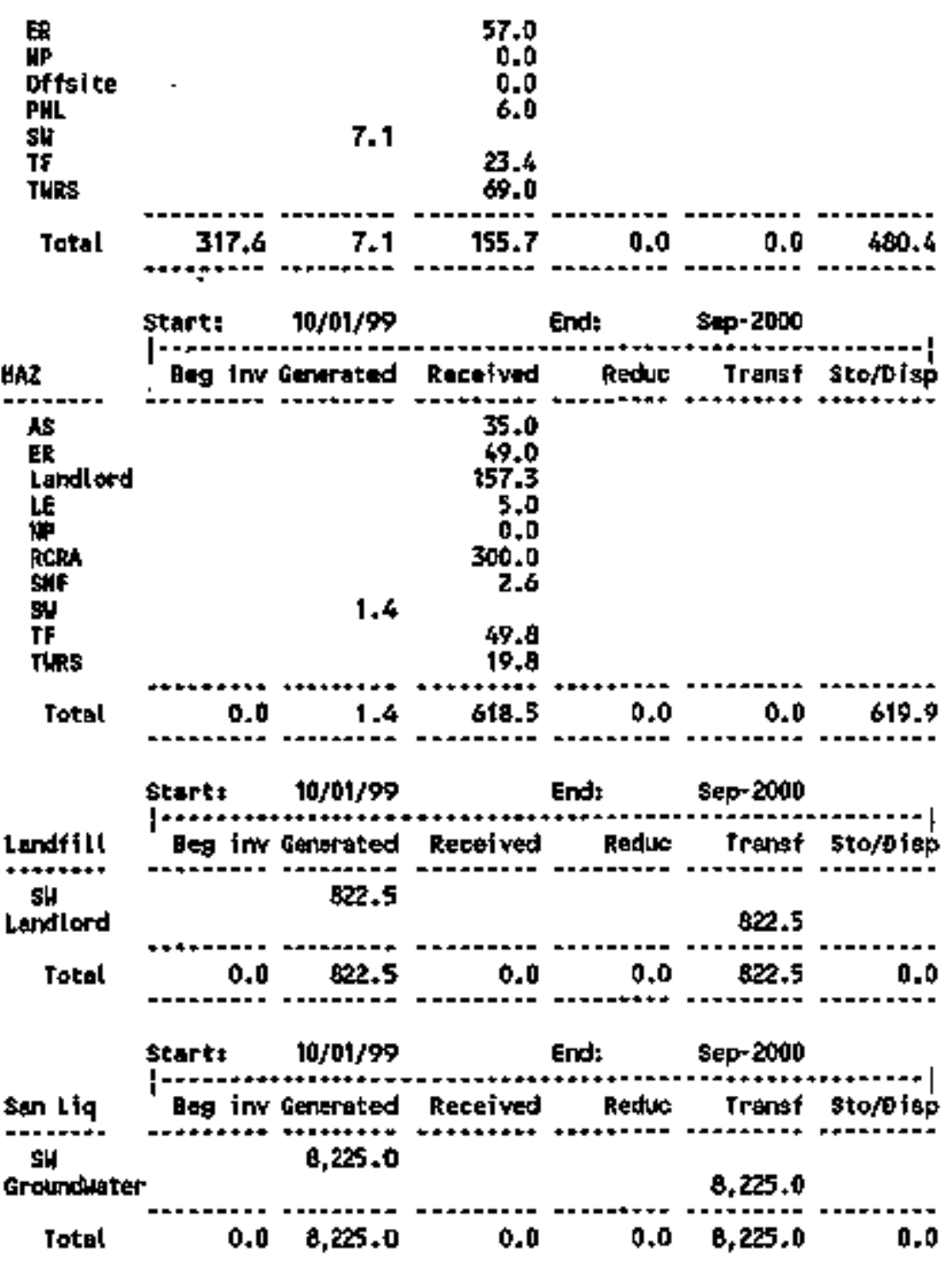

FY 200† to FY 2024 Waste Type Volume Projections for U95: 1.2.1

Progran Hand solial Haste
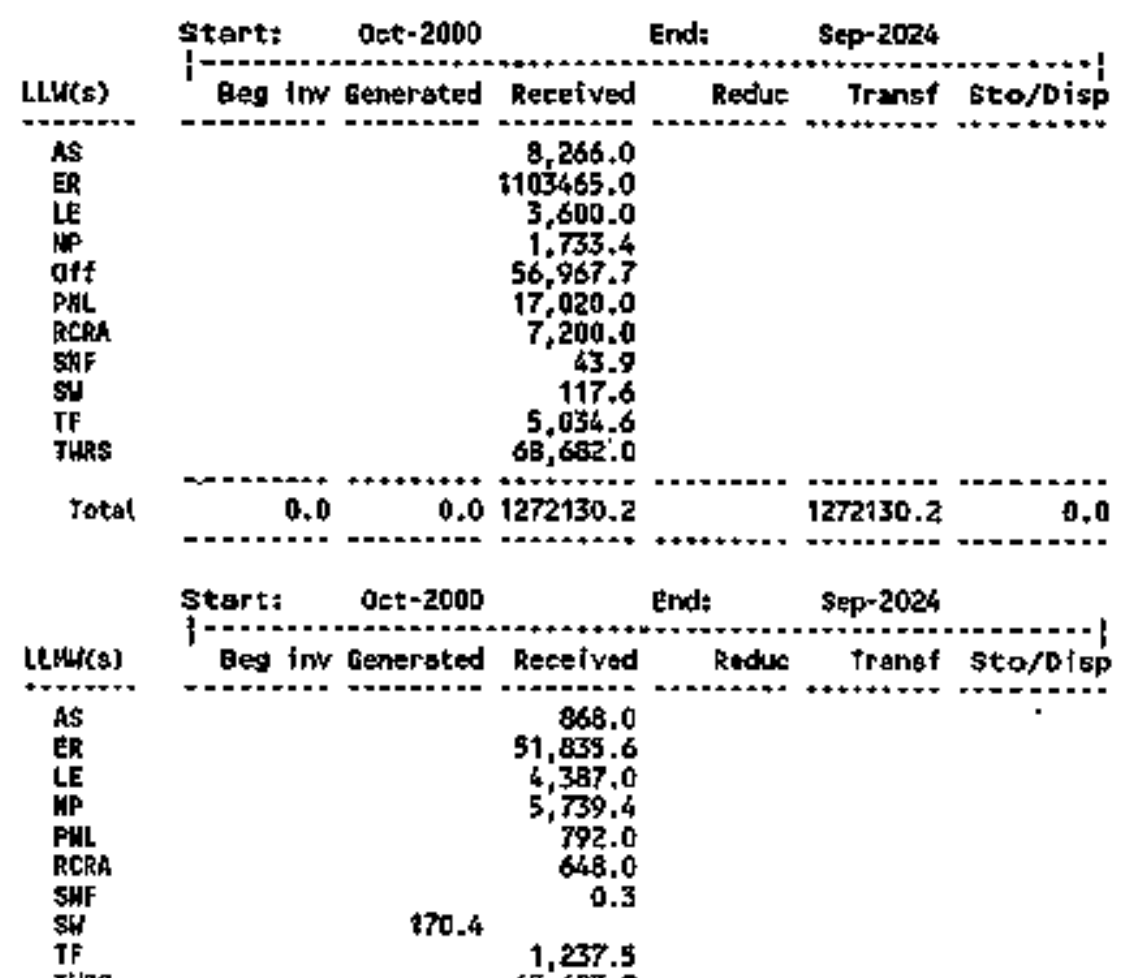

TWRS $45,683.9$

Total $7,252.5 \quad 170.4111,191.7 \quad \cdots \cdots$

$0,0 \quad 0.0118,614.6$

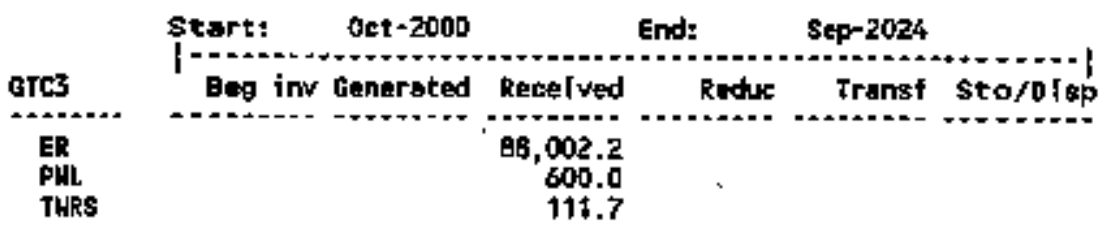




\section{WHC-5P-1114, Rev. 1}

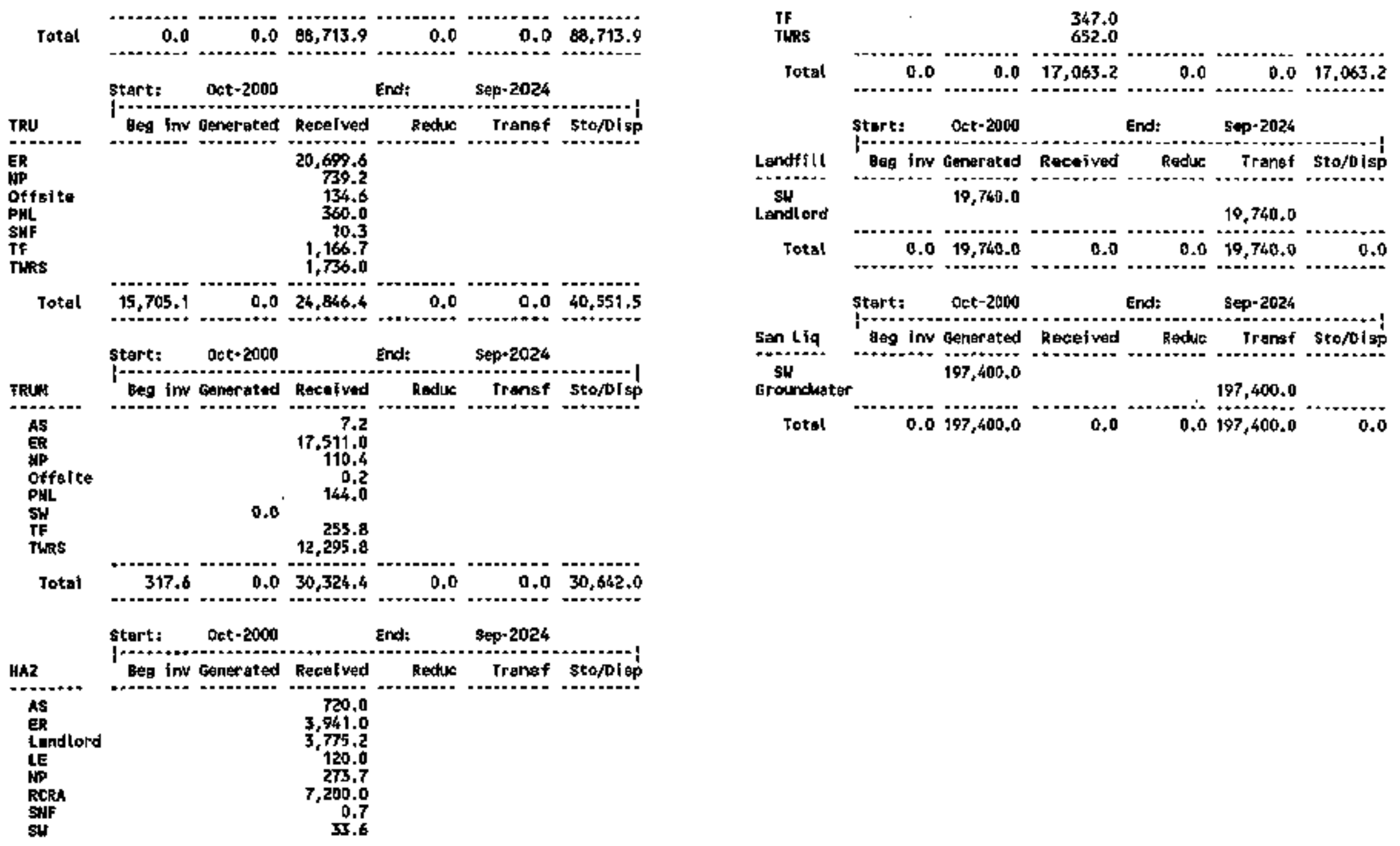




\section{B Schedule Basel ine}

This section of the Fiscal Year 1996 Solid Waste Program l4ult:-Year Program Plan contajns the following elements:

- 2.B.1 - Progran Logic Oiagram

- 2.B.2 - Program Haster Baseline Schedule

- 2.B.3 - Milestone List

- 2.B.4 - Mtlestone Description Sheets 
$F$

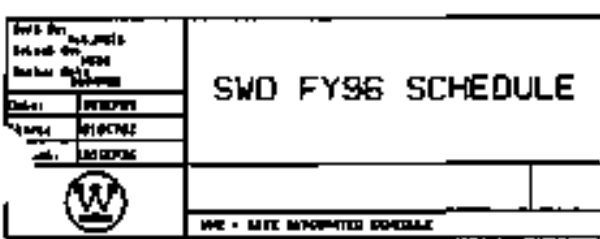

WHC - PROQRMM MASTER BASEUHE BCHEOULE

L2.t - SOLD WASTE DNTSKN FMGQ日O - FT200

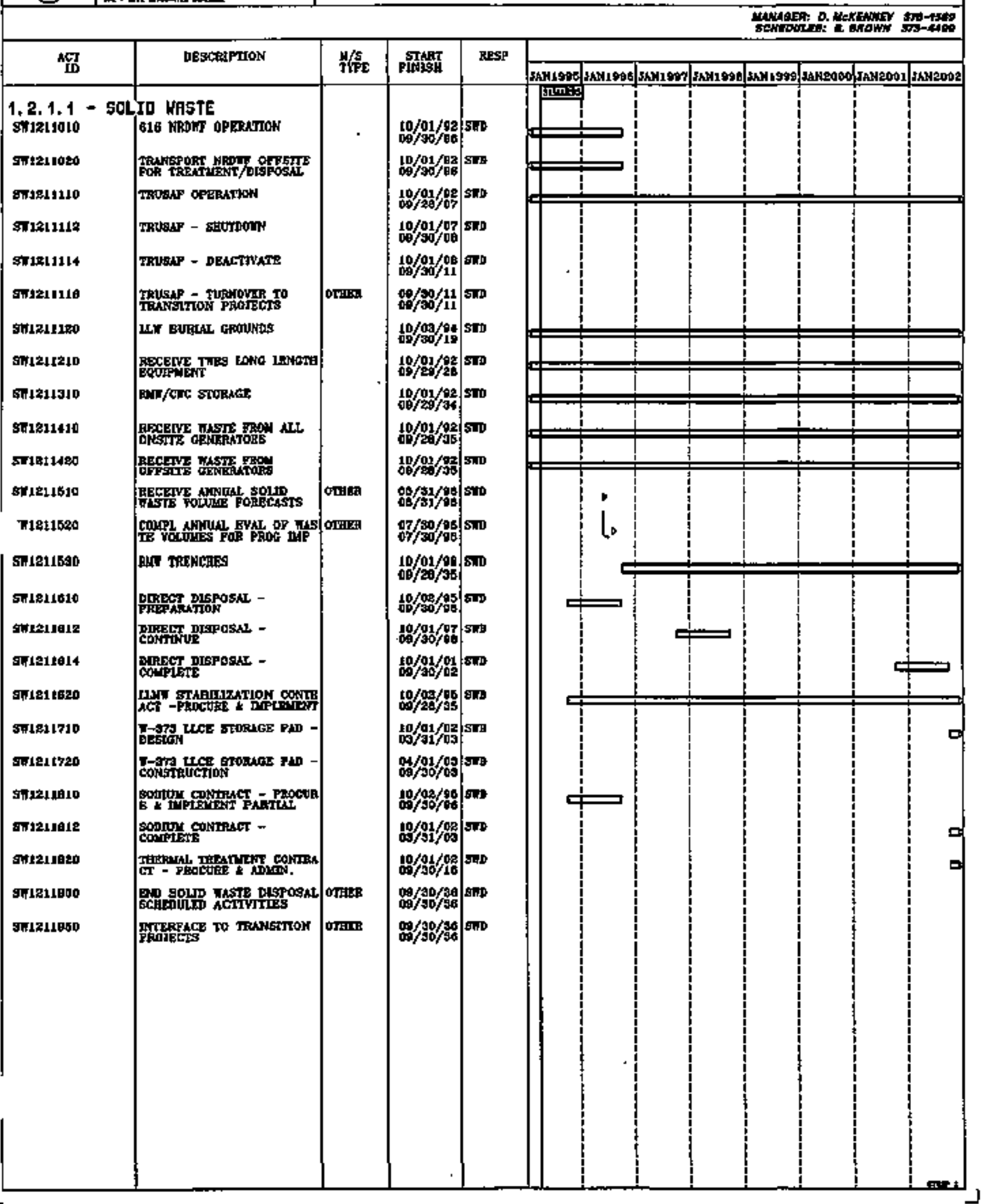




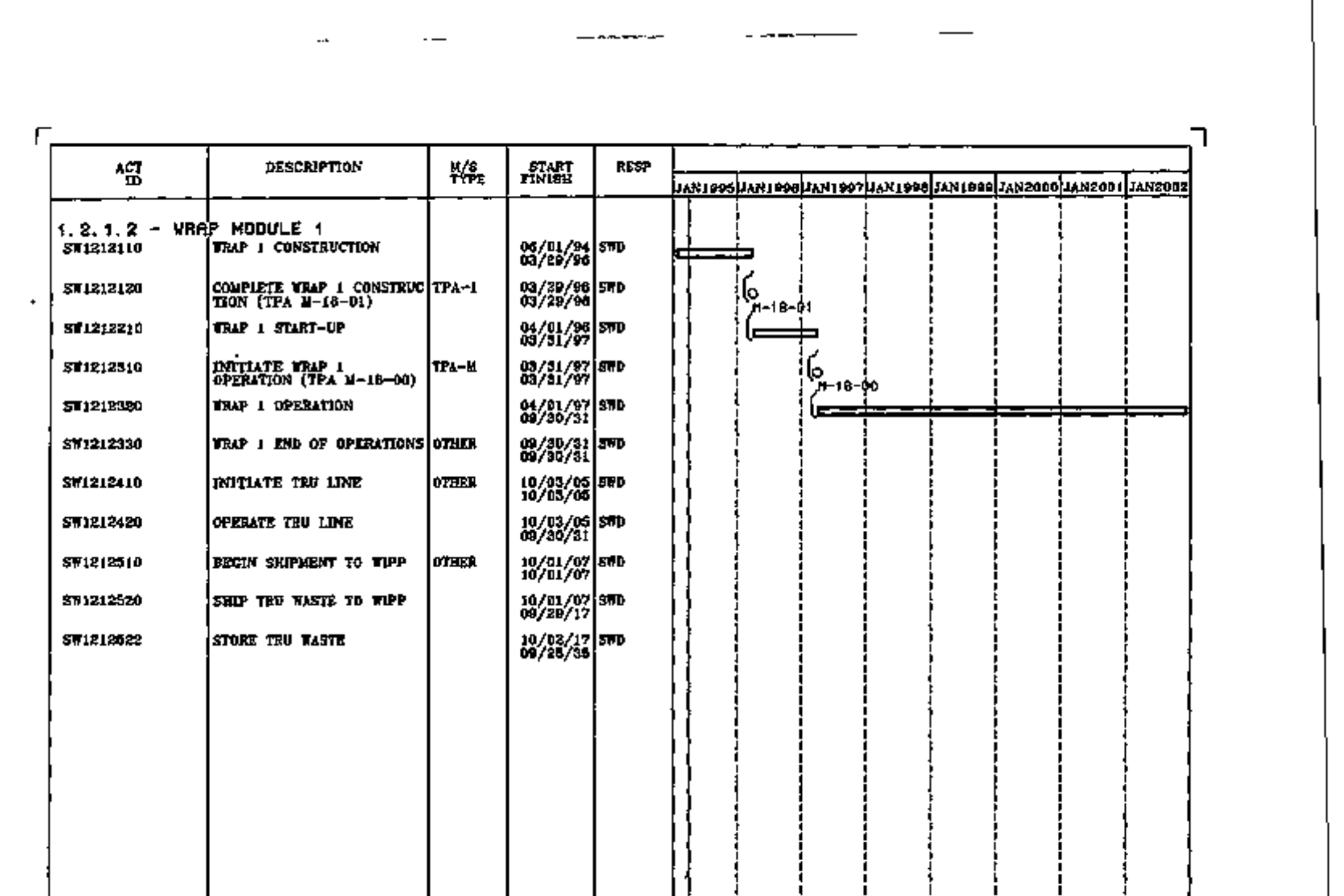




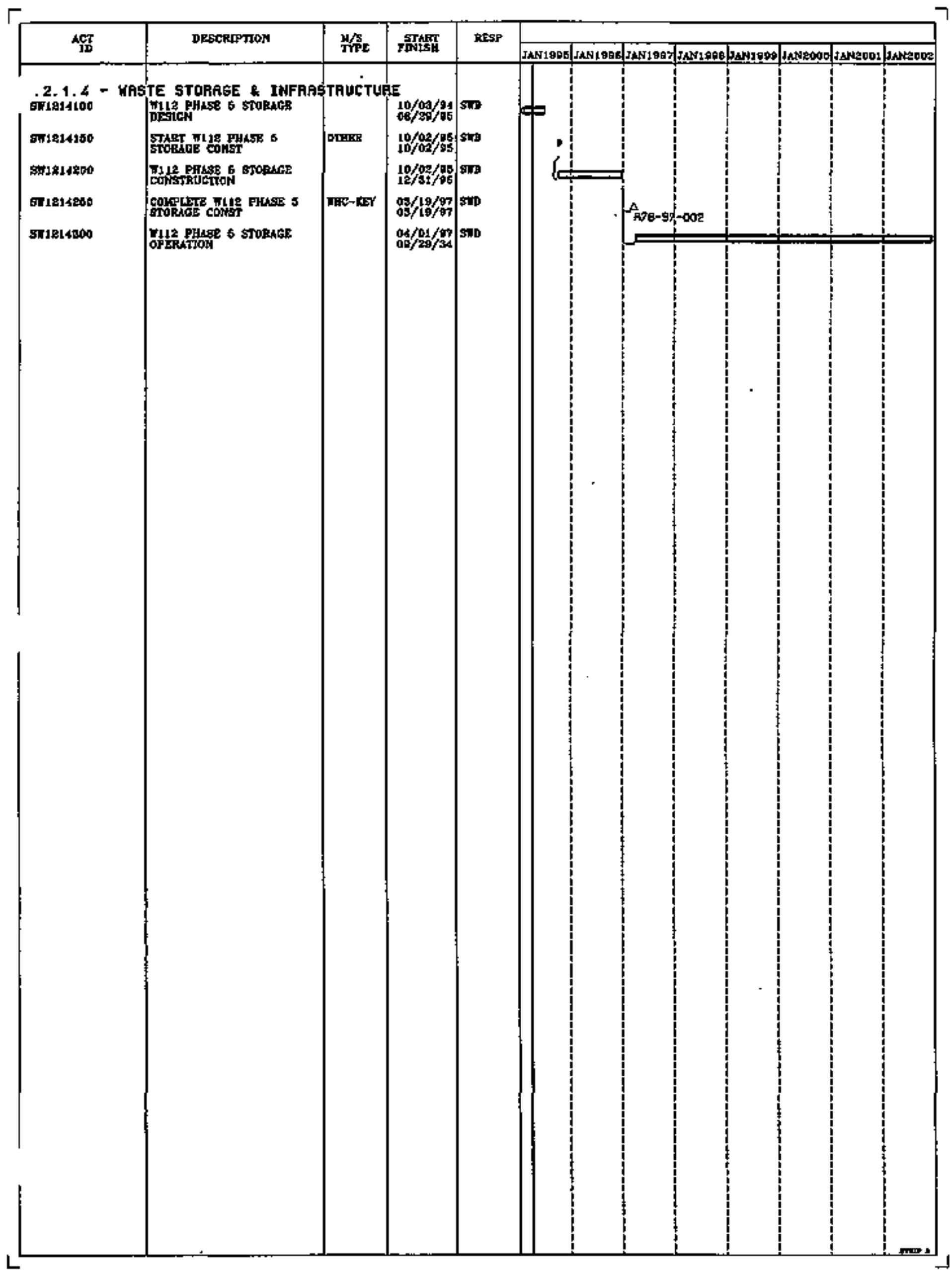




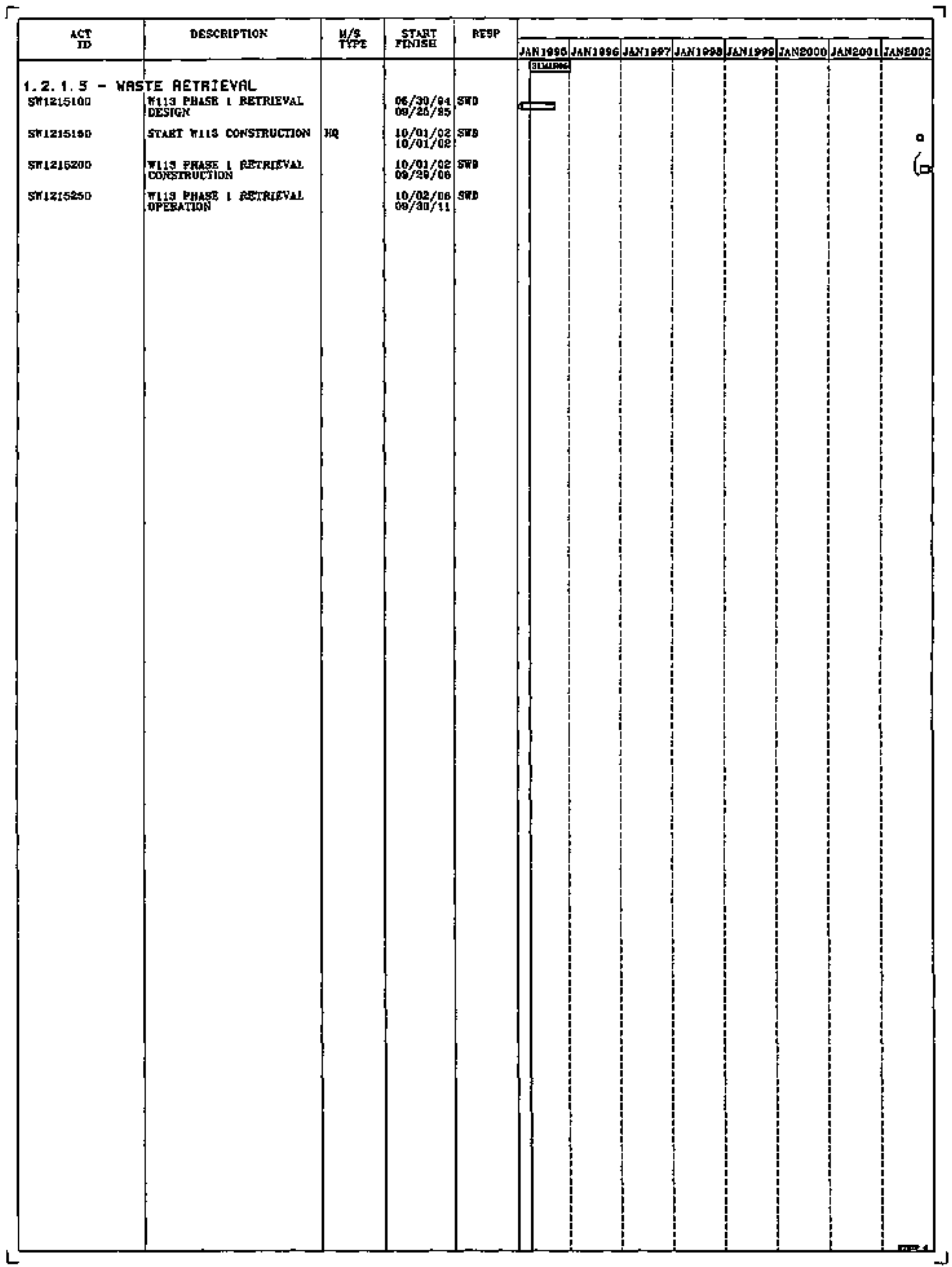




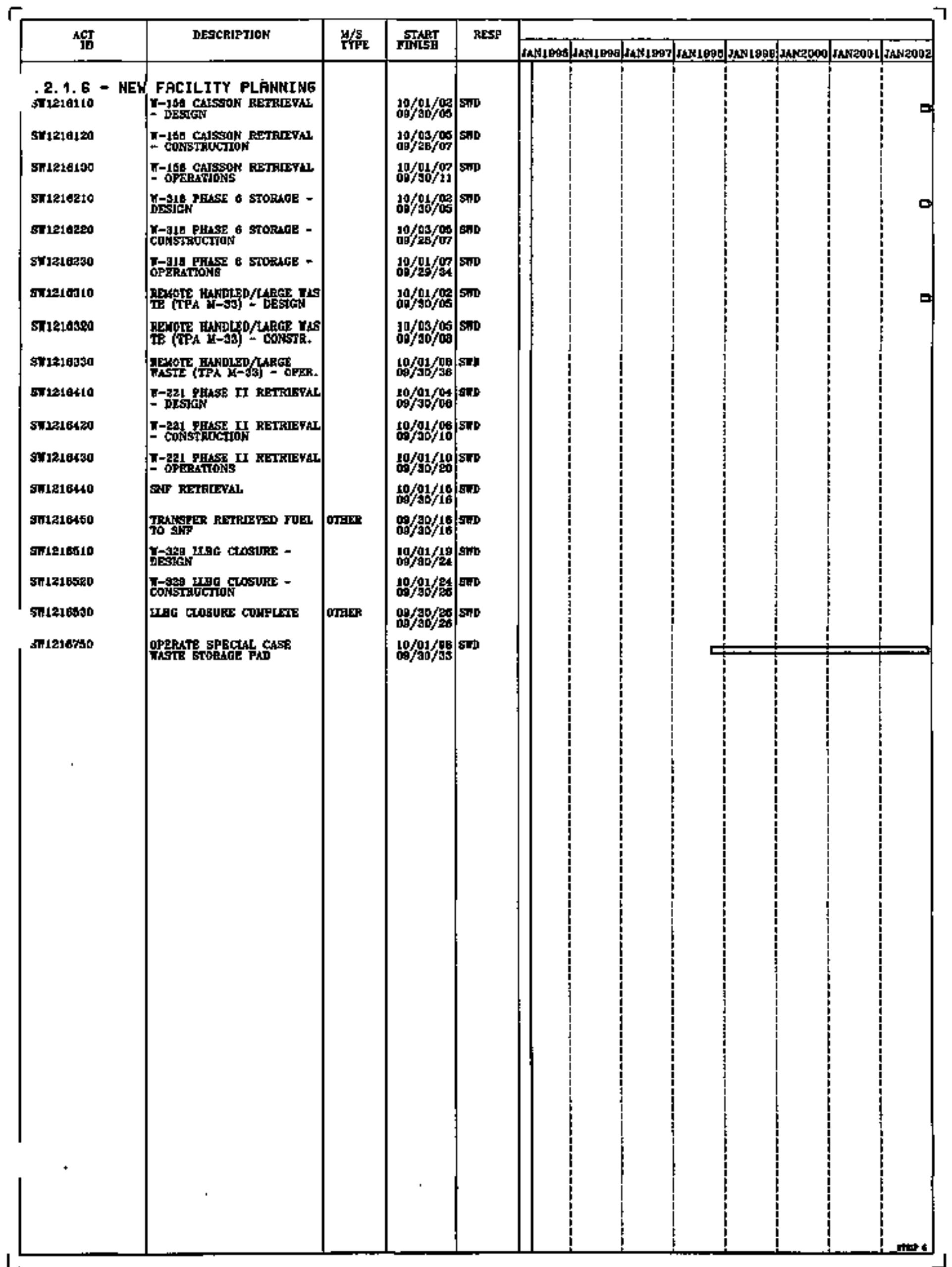




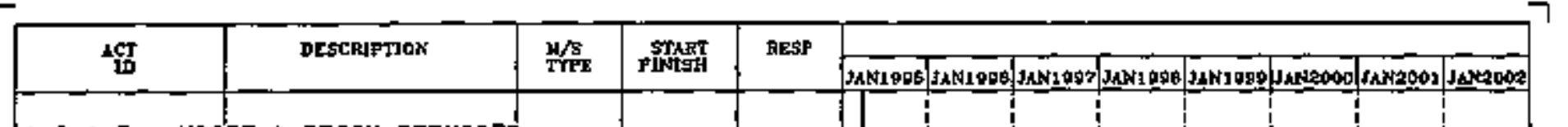

1.2.1.7 - WASTE DECDN SERYICES SW1217100 |

ST1217165 HGH DOSE EQUTPIXM/ TASTE DECOASAMTITHTION

STIP17P00

sinloritso

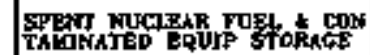
5n1217ses

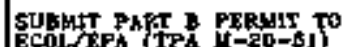

$\mathbf{T r} k-1$

simeryast

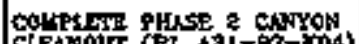
RL

ST1217600 TRunstak sPENT FUEl FPOH 10/00/as ST 0./20/16)

$10 / 03 / 9 s$, sits 09/2日/16 $10 / 0) / 90 \mid 50$ $12 / 31 / 95$

11/06/96/st. iifotio $10 / 01 / 03 / 510$ Dof $\mathrm{Aa} / \mathrm{b}$ $10 / 01 / 18]^{\sin }$ T-PLWNT - S80700179

Sn1217650 T-PLATT - DEACTTATE

sin1217to

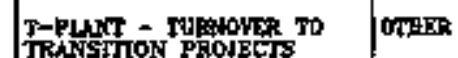
$10 / 01 / 19 / 50$ $00 / 29 / 2)$ sto

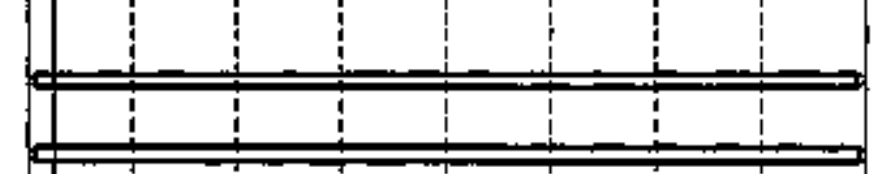


$\Gamma$

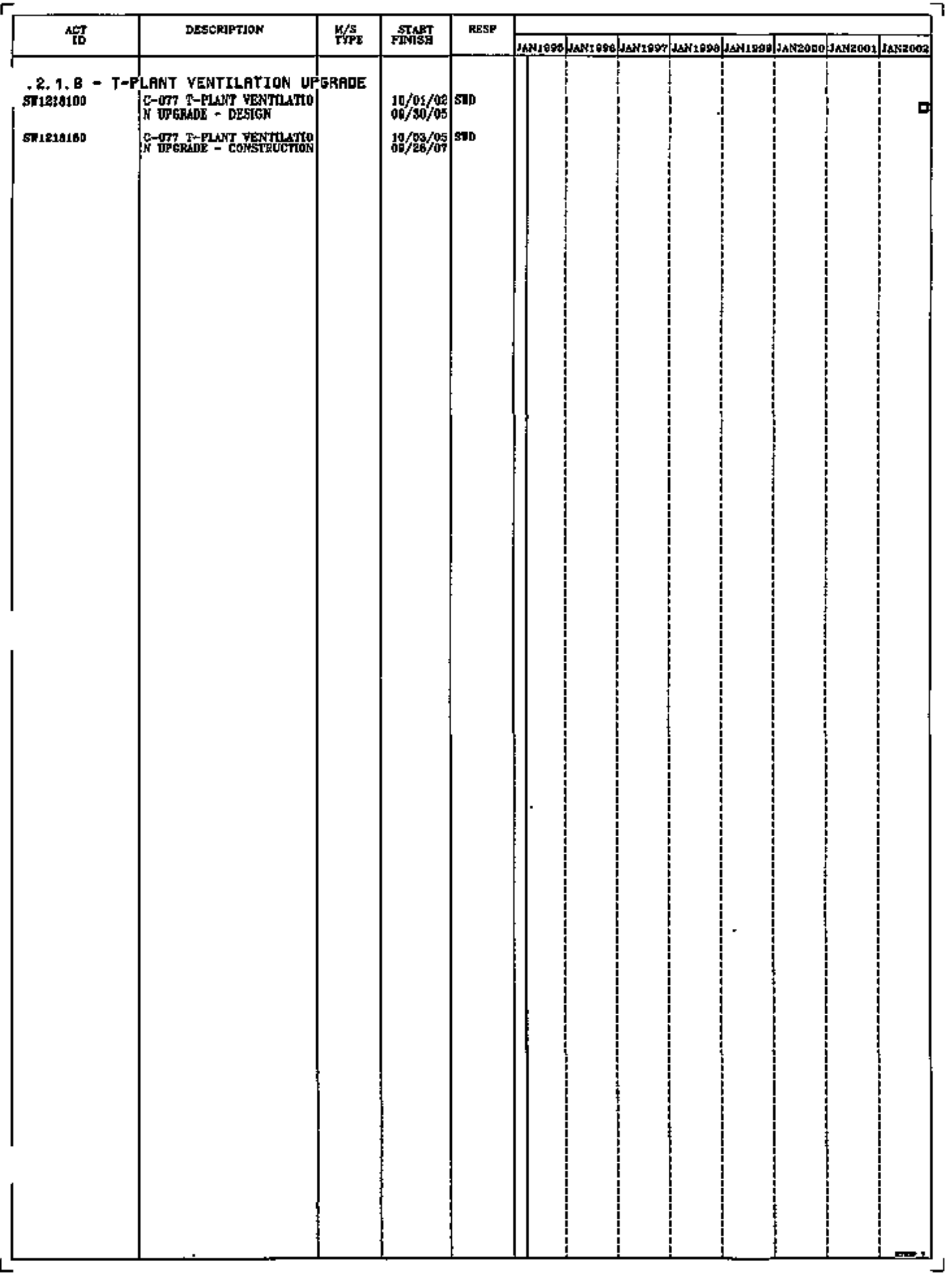




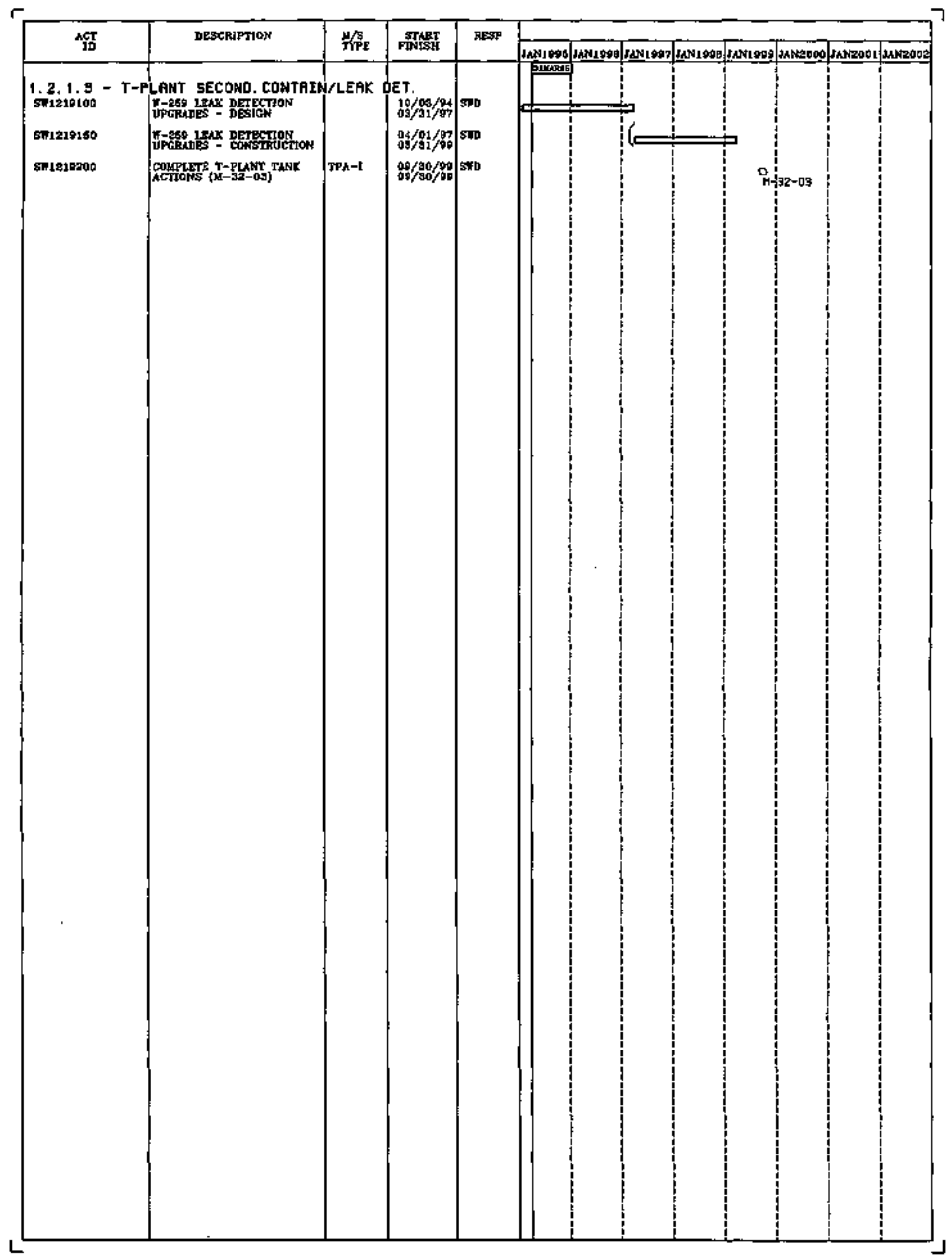




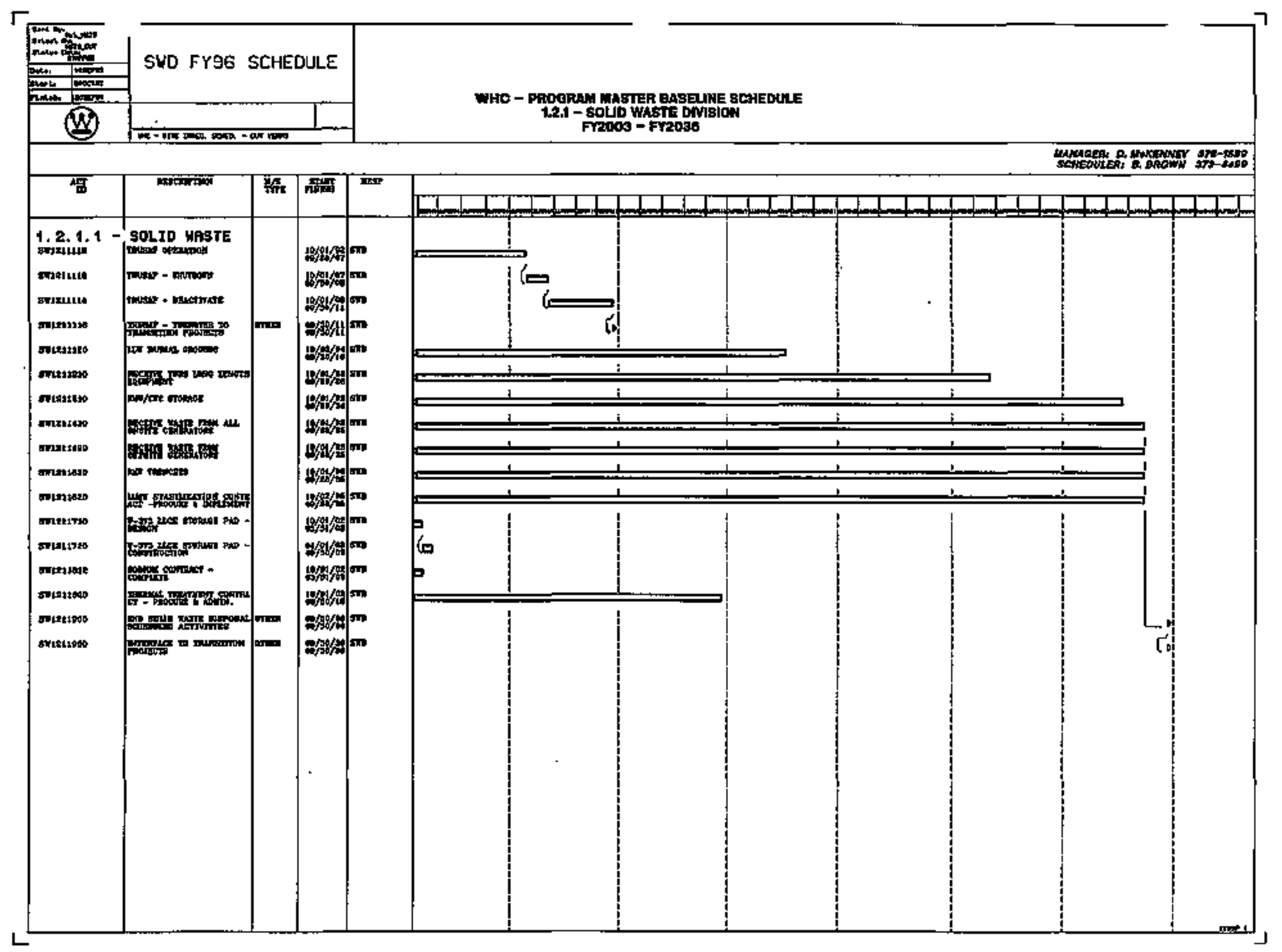




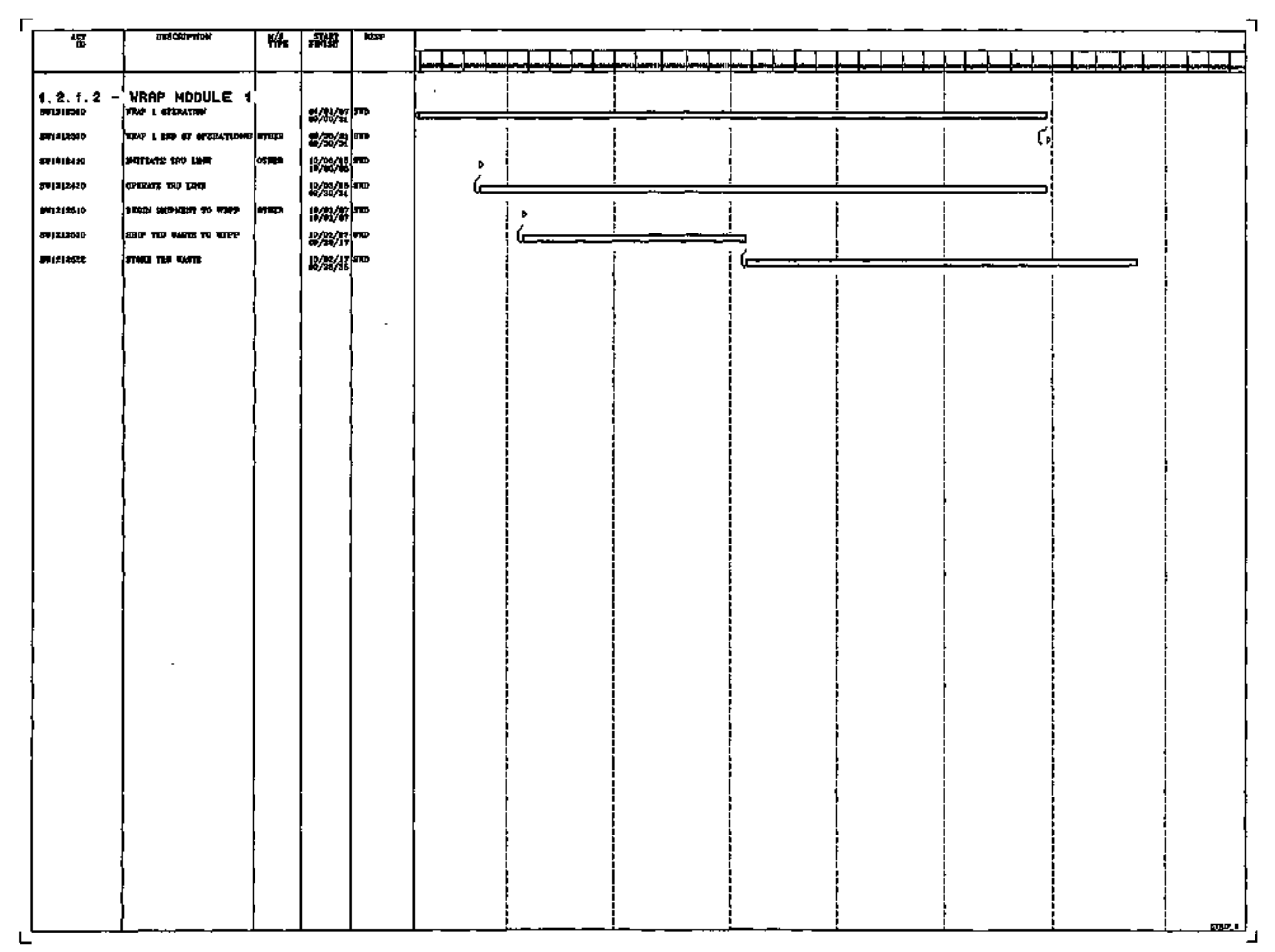





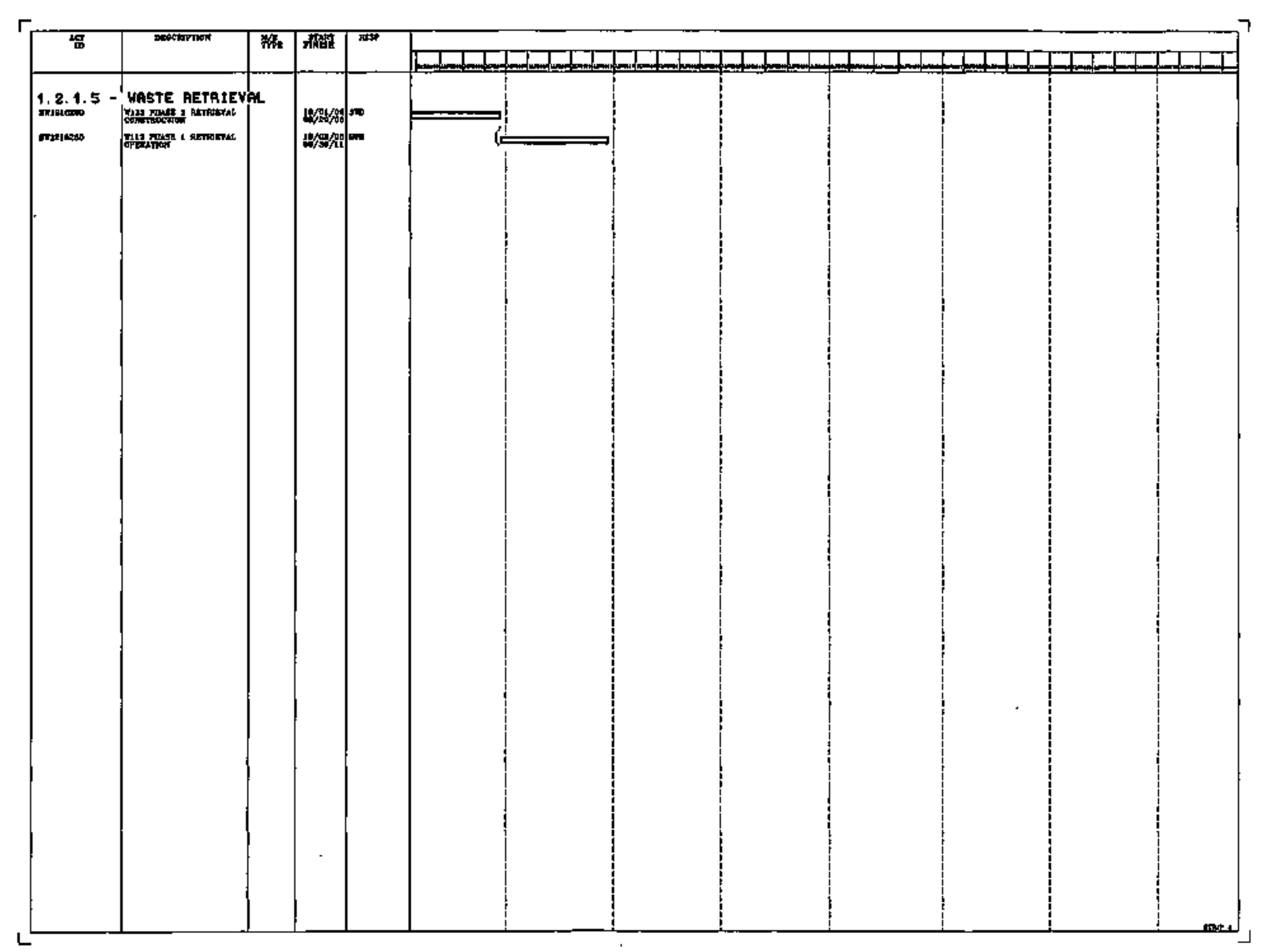




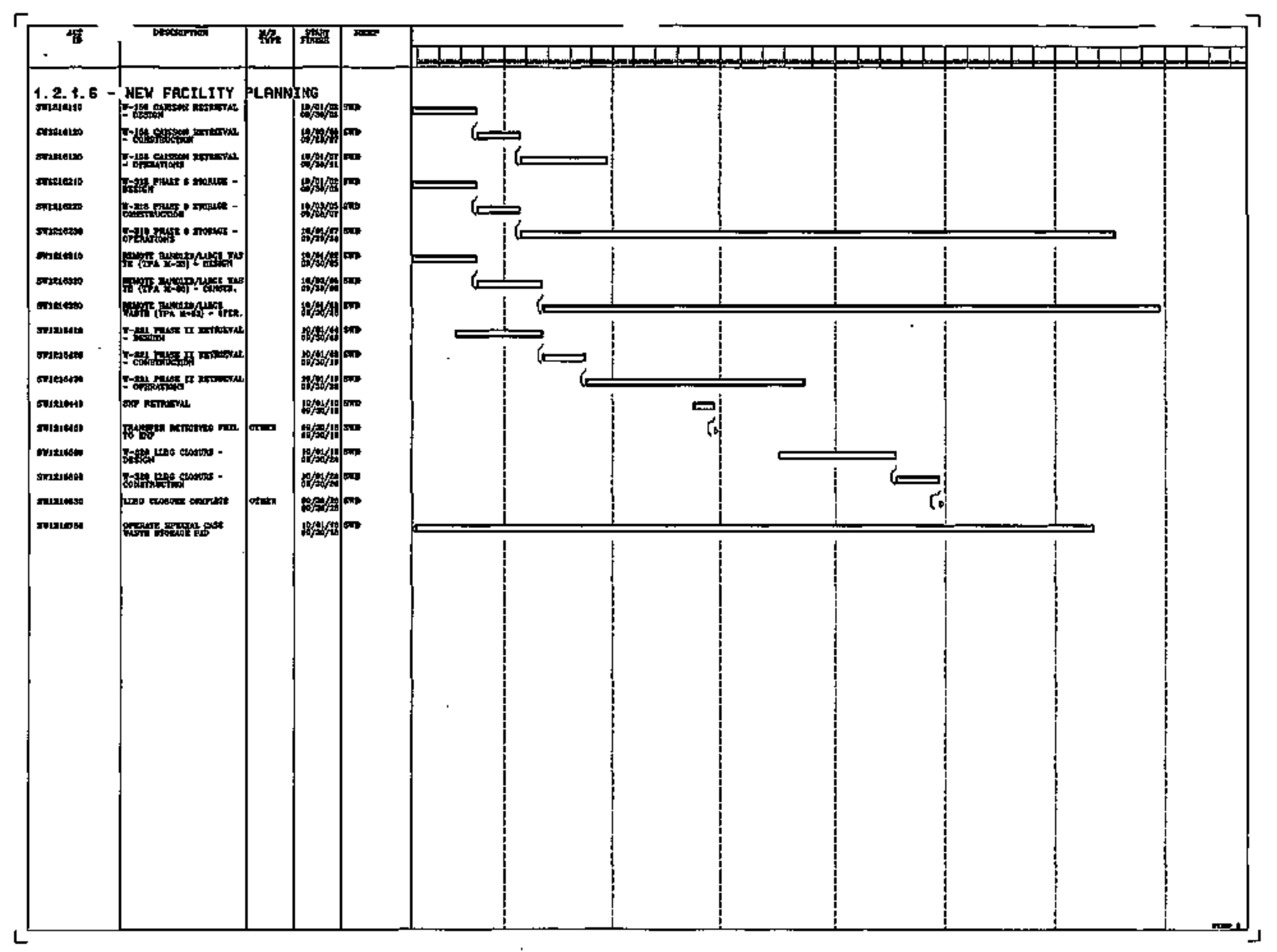




$=$




\begin{tabular}{|c|c|c|c|}
\hline \multicolumn{4}{|c|}{ MILESTONE LIST } \\
\hline $\begin{array}{c}\text { Mi Jestone } \\
\text { Type }\end{array}$ & $\begin{array}{l}\text { Contro1 } \\
\text { Nuluber }\end{array}$ & Mi]estane Description & $\begin{array}{c}\text { Milestone } \\
\text { Completion } \\
\text { Date }\end{array}$ \\
\hline $\mathbf{R L}$ & Al2-96-078 & 183-H BASIH FORMIC ACID VARIANCE PETITIOA & $02 / 29 / 96$ \\
\hline RL. & Al2-96-016 & ANARD LLHL STABILIZATION CONTRACT & $06 / 14 / 96$ \\
\hline RL & A12-96-090 & CMC OPERATIONS PLAN & $07 / 30 / 96$ \\
\hline $\mathbf{R L}$ & Al2-97-005 & CONPLETE TBP SHIPNENT FROM B PLANT & $06 / 30 / 97$ \\
\hline $\mathbf{R L}$ & A12-97-010 & COWPLETE YENDOR EA PREPARATIOA & $09 / 30 / 97$ \\
\hline RL & A $13-96-101$ & OPERATE TRUSAF IN ACCORDANCE WITH APPLICABLE REG. & $09 / 30 / 96$ \\
\hline $\mathbf{R L}$ & Al3-97-101 & OPERATE TRUSAF IN ACCORDANCE WITH APPLICABLE REG. & $09 / 30 / 97$ \\
\hline RL & Al3-98-101 & OPERATE JRUSAF IN ACCORDANCE WITH APPLICABLE REG. & $09 / 30 / 98$ \\
\hline RL & A13-99-101 & OPERATE TRUSAF IN ACCORDANCE WITH APPLICABLE REG. & $09 / 30 / 99$ \\
\hline RL & $\mathrm{A} 13-00-101$ & OPERATE TRUSAF IN ACCORDANCE YITH APPLICABLE REG. & $09 / 29 / 00$ \\
\hline RL & A13-01-101 & OPERATE TRUSAF IN ACCOROANCE WITH APPLICABLE REG. & $09 / 28 / 01$ \\
\hline RL & A13-02-101 & OPERATE TRUSAF IN ACCORDANCE WITH APPLICABLE REG. & $09 / 30 / 02$ \\
\hline RL & Al4-96-101 & STORE RHW \& OTHER WASTE REQ'RG LONG-TERM STORAGE & $09 / 30 / 96$ \\
\hline RL. & A14-97-101 & STORE RMW \& OTHER WASTE REQ'RG LONG-TERM STORAGE & $09 / 30 / 97$ \\
\hline$\underline{R L}$ & Al4-98-101 & STORE RMW \& OTHER WASTE REQ'RG LONG-TERH STORAGE & $09 / 30 / 98$ \\
\hline RL. & A14-99-101 & STORE RMW OTHER WASTE REQ'RG LONG-TERM STORAGE & $09 / 30 / 99$ \\
\hline RL & A14-00-101 & STORE RMW OTHER WASTE REQ'RG LONG-TERM STORAGE & $09 / 29 / 00$ \\
\hline RL & Al4-01-101 & STORE RHW \& OTHER WASTE REQ'RG LONG-TERM STORAGE & $09 / 28 / 01$ \\
\hline RL & A14-02-101 & STORE RAH' \& OTHER WASTE REQ'RG LONG-TERM STORAGE & $09 / 30 / 02$ \\
\hline RL & A15-96-501 & OPERATE 616 BLDG IN COMPL W/ APPL. REGULATIONS & $09 / 30 / 96$ \\
\hline RL & A16-96-701 & MAIMTAIN THE LOH-LEVEL ACTIVE BURIAL GROUNDS & $09 / 30 / 96$ \\
\hline RL & Al6-96-702 & DISPOSAL OF ALL CAT III WASTE IN INVENTORY & $09 / 30 / 96$ \\
\hline
\end{tabular}




\begin{tabular}{|c|c|c|c|}
\hline Rl. & A16-96-703 & MOVE 250 TRU DRUMS FROM BURIAL GROUND & $09 / 30 / 96$ \\
\hline RL & A16-97-701 & MAINTAIN THE LOH-LEVEL BURIAL GROLHDS & $09 / 30 / 97$ \\
\hline RL & A16-98-70I & MAINTAIN THE LOW-LEVEL BURIAL GROUNDS & $09 / 30 / 98$ \\
\hline $\mathbf{R L}$ & A16-99-701 & MAINTAIN THE LOW-LEVEL BURIAL GROUHOS & $09 / 30 / 99$ \\
\hline RL & Al6-00-701 & MAINTAIA THE LOH-LEVEL BURIAL GROUMDS & $09 / 29 / 00$ \\
\hline $\mathbf{R L}$ & Al6-01-701 & MAINTAIN THE LOW-LEVEL BURIAL GROUNDS & $09 / 28 / 01$ \\
\hline RL & A16-02-701 & MAINTAIN THE LOW-LEVEL BURIAL GROUHOS & $09 / 30 / 02$ \\
\hline RL & A17-96-101 & MNTN RMN DSPSL TRCHES IP ACCORDNCE W/ APPLC REGS & $09 / 30 / 96$ \\
\hline $\mathbf{R L}$ & A17-00-101 & MAINTAIN PWW DISPOSAL TRENCHES PER RCRA & $09 / 29 / 00$ \\
\hline $\mathbf{R L}$ & $A 17+01-101$ & MAINTAIN RH DISPOSAL TRENCHES PER RCRA & $09 / 28 / 01$ \\
\hline RL & A17-02-101 & MAINTAIN RWN DISPOSAL TRENCHES PER RCRA & $09 / 30 / 02$ \\
\hline $\mathbf{R L}$ & A19-96-KO1 & ISSUE REV 2 OF THE SOLID WASTE TECH BASELINE DOC & $09 / 30 / 96$ \\
\hline $\mathbf{R L}$ & A19-96-K02 & UPDATE AND PREPARE 3 INTERFACE CONTROL DOCUMENTS & $09 / 30 / 96$ \\
\hline $\mathbf{R L}$ & A19-97-K01 & ISSUE REV 3 OF THE SOLID WASTE TECH BASELINE DOC & $09 / 30 / 97$ \\
\hline $\mathbf{R L}$ & Al9-97-KO2 & UPDATE AND PREPARE 3 INTEREACE CONTROL DOCUMENTS & $09 / 30 / 97$ \\
\hline RL & A1A-95-510 & SUBMIT MYPP DOCUMENT TO DOE-RL & $08 / 31 / 96$ \\
\hline$\underline{\mathrm{RL}}$ & A1A-97-610 & SUBMIT MYPP DOCUMENT TO DOE-RL & $08 / 31 / 97$ \\
\hline RL & AlA-98-610 & SUBHIT MYPP DOCUNENT TO DOE-RL & $08 / 31 / 98$ \\
\hline$\underline{\mathbf{R L}}$ & A1A-99-610 & SUBMIT MYPP DOCUMENT TO DOE-RL & $08 / 31 / 99$ \\
\hline $\mathbf{R L}$ & $A 1 A-00-610$ & SUBMIT MYPP DOCUMENT TO OOE-RL & $08 / 31 / 00$ \\
\hline RL & AIA-01-610 & SUBHIT MYPP DOCUMENT TO DOE-RL & $08 / 31 / 01$ \\
\hline $\mathbf{R L}$ & AlA-02-610 & SUBHIT MYPP DOCUMENT TO DOE-RL. & $08 / 31 / 02$ \\
\hline RL. & $A 31-96-K 03$ & $\begin{array}{l}\text { PROCESS } 2000 \mathrm{FT}^{3} \text { IN } 221-T \text { CANYON FOR } \\
\text { REUSE/STORAGE/OISPOSAL }\end{array}$ & $09 / 30 / 96$ \\
\hline $\mathbf{R L}$ & $\mathrm{A3} 1-96-\mathrm{K} 01$ & ISSUE WHC APPR FY97 TREATMENT FORCAST FOR 221-T & $09 / 30 / 96$ \\
\hline RL & $\mathrm{A} 31-97-\mathrm{KO}_{0}$ & ISSUE WAC APPR FYgB TREATMENT FORCAST FOR 221-T & $09 / 30 / 97$ \\
\hline
\end{tabular}




\begin{tabular}{|c|c|c|c|}
\hline $\mathrm{RL}$ & $\mathrm{A} 32-96-\mathrm{KO} 03$ & PROCESS MINIMUM OF 23,000 FT3 OF CONT WASTE/EQUIP & $09 / 30 / 96$ \\
\hline RL & $\mathrm{A} 32-96-\mathrm{KOl}$ & ISSUE WHC APPROUED FY1997 TREATMENT FORECAST & $09 / 30 / 96$ \\
\hline RL & $\mathrm{A} 32-97-\mathrm{K}_{03}$ & PROCESS MIN OF 11,500 FT3 OF CONT WASTE/EQUIP & $09 / 30 / 97$ \\
\hline RL. & $\mathrm{A} 32-97-\mathrm{KOl}$ & ISSUE WHC APPROVED FY1998 TREATMEHT FORECAST & $09 / 30 / 97$ \\
\hline RL & $\mathrm{A} 33-96-\mathrm{K} 02$ & SUUEMIT CONT CERT DRAFT TO DOE PART "B" PERHIT & $11 / 30 / 95$ \\
\hline TPA & A33-96-TO1 & (M-20-51) SUPT SUBMIT PART B PERUIT TO ECOL/EPA & $12 / 31 / 95$ \\
\hline$\underline{\mathrm{RL}}$ & A33-96-K03 & STORE COMTAMINATED EQUIPAEENT & $09 / 30 / 96$ \\
\hline RL & A33-96-KO1 & STORE 72 FUEL ELEMEHTS & $09 / 30 / 96$ \\
\hline RL & A33-96-K05 & SUEMIT CONTRACTOR APPROVED PUR FUEL REMOVAL PLAN & $09 / 30 / 96$ \\
\hline$\underline{R L}$ & $\mathrm{~A} 333-97-\mathrm{KO}$ & STORE COMTAMINATED EQUIPAEENT & $09 / 30 / 97$ \\
\hline RL & $\mathrm{A} 33-97-\mathrm{K} 01$ & STORE 72 FUEL ELEMENTS & $09 / 30 / 97$ \\
\hline RL & A33-98-K03 & STORE COMTAMINATED EQUIPWENT & $09 / 30 / 98$ \\
\hline RL & A33-98-K0I & STORE 72 FUEL ELEMENTS & $09 / 30 / 98$ \\
\hline RL: & A33-99-K03 & STORE CONTAMIINATED EQUUIPWENT & $09 / 30 / 99$ \\
\hline RL. & $\mathrm{A} 33-99-\mathrm{KOI}$ & STORE 72 FUEL ELEMENTS & $09 / 30 / 99$ \\
\hline RL. & A33-00-K03 & STORE COMTAMINATED EQUIPPENT & $09 / 29 / 00$ \\
\hline RL & $\mathrm{A} 33-00-\mathrm{K} 01$ & STORE 72 FUEL ELEMENTS & $09 / 29 / 00$ \\
\hline RL & $\mathrm{A} 33-01-\mathrm{KO3}$ & STORE COMTAMINATED EQUIPAENT & $09 / 28 / 01$ \\
\hline RL, & $\mathrm{A} 33-01-\mathrm{KO} 01$ & STORE 72 FUEL ELEMEATS & $09 / 28 / 0 \mathrm{I}$ \\
\hline RL & $\mathrm{A} 33-02-\mathrm{KOO}$ & STORE COMTAMIINATED EQUUIPWENT & $09 / 30 / 02$ \\
\hline RL & $\mathrm{A} 33-02-\mathrm{K} 01$ & STORE 72 FUEL ELEMENTS & $09 / 30 / 02$ \\
\hline$\underline{R L}$ & $\mathrm{~A} 34-96-\mathrm{KO} 1$ & MAINT CERT FOR ONE RAILCAR & $09 / 30 / 96$ \\
\hline RL & A34-98-K01 & CMPT WORK NECESS. TO MAIHT CERT FOR 1 LWTC & $09 / 30 / 98$ \\
\hline RL & A34-99-KO1 & CMPT NORK NECESS. TO MAINT CERT FOR 1 LWTC & $09 / 30 / 99$ \\
\hline RL & A34-00-K01 & CMPT WORK NECESS. TO MAINT CERT FOR 1 LWTC & $09 / 29 / 00$ \\
\hline
\end{tabular}




\begin{tabular}{|c|c|c|c|}
\hline RL & A34-0I-K01 & CMPT WORK NECESS. TO MAINT CERT FOR 1 LWTC & $09 / 28 / 01$ \\
\hline RL. & $\mathrm{A} 34-02-\mathrm{KO}$ & CAPT WORK NECESS. TO MAINT CERT FOR 1 LNTC & $09 / 30 / 02$ \\
\hline TPA & 3A4-96-T01 & COAPLETE CONST, OF WRAP MODULE I (TPA :M-18-01) & $03 / 31 / 96$ \\
\hline Rl & A47-96-158 & ACCEPT RESPONSIBILITY FOR BLDG $2336-\not W$ & $04 / 10 / 96$ \\
\hline RL & A41-96-144 & COMP CONST IN ACCORD W/ APPR DESIGN MEOIA \& FDC & $04 / 30 / 96$ \\
\hline RLL & A47-96-239 & COWHENCE COLD RUNS OF SIM HST CONT THRU FACILITY & $06 / 28 / 96$ \\
\hline Rt. & $\mathrm{A} 46-96-001$ & ISSUE HHC APPROVED WRAPI FSAR & $09 / 30 / 96$ \\
\hline RL & A46-97-001 & ISSUE DOE APPROVEO WRAPI FSAR & $03 / 31 / 97$ \\
\hline TPA, RL & A47-97-001 & IHITIATE KRAP1 OPERATIONS(TPA AM-18-00) & $03 / 31 / 97$ \\
\hline RL & A47-97-100 & PROCESS LL/RMW WASTE ORUMS AS NEGOTIATED MITH RL. & $09 / 30 / 97$ \\
\hline $\mathrm{RL}$ & $\mathrm{A} 47-98-100$ & PROCESS LL/RTWW WASTE DRUMS AS NEGOTIATED WITH RL, & $09 / 30 / 98$ \\
\hline RL & $\mathrm{A} 47-99-100$ & PROCESS LL/RMH WASTE DRUMS AS NEGOTIATED WITH RL & $09 / 30 / 99$ \\
\hline RL & A47 $-00-100$ & PROCESS LL/RHA WASTE DRUMS AS NEGOTIATED WITH RL. & $09 / 29 / 00$ \\
\hline RL & $\mathrm{A} 47-01-100$ & PROCESS LL/RHW WASTE DRUMS AS NEGOTIATED WITH RL. & $09 / 28 / 01$ \\
\hline RL & A47-02-100 & PROCESS LL/RMW' WASTE DRLANS AS NEGOTIATEO WITH RL & $09 / 30 / 02$ \\
\hline RL. & A7B-96-139 & COMPLETE COHSTRUCTION OF OPS SUPPORT FACILITY & $08 / 31 / 96$ \\
\hline RL & $A B 1-96-K 01$ & 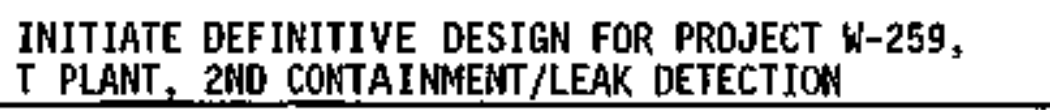 & $02 / 01 / 96$ \\
\hline RL & $A B 1-97-K 01$ & $\begin{array}{l}\text { COMPLETE DEFINITIVE DESIGN FOR PROJECT } W-259 \\
\text { I PLANT ZNO COHTAINMENT/LEAK OEIECTION }\end{array}$ & $04 / 01 / 97$ \\
\hline$T P A, R L$ & $A B 1-99^{\circ}-T 01$ & COMPLETE T-PLANT TANK ACTIONS (TPA:HM-32-03) & $09 / 30 / 99$ \\
\hline $\mathbf{R L}$ & MDD-96-001 & ISSUE SILYER RECLAMATION CONTRACT & $12 / 30 / 95$ \\
\hline RL & $M 00-97-001$ & ISSUE SILVER RECLAMATIOH CONFRACT & $12 / 30 / 96$ \\
\hline RLL & MDD-98-001 & ISSUE SILVER RECLAMATIOH CONTRACT & $12 / 30 / 97$ \\
\hline RL & MDD-99-001 & ISSUE SILVER RECLAMATION CONTRACT & $12 / 30 / 98$ \\
\hline RL & M0D-00-001 & ISSUE SILVER RECLAMATION CONTRACT & $12 / 30 / 99$ \\
\hline
\end{tabular}




\begin{tabular}{|c|c|c|c|}
\hline$\underline{\mathbf{R} L}$ & MOD-01-001 & ISSUE SILVER RECLAMAIION CONTRACT & $12 / 29 / 00$ \\
\hline RL & MDD-02-001 & ISSUE SILVER RECLAMATION CONTRACT & $12 / 31 / 01$ \\
\hline Rll & ML4-97-701 & RECEIVE/OISPOSE UP TO $200,000 \mathrm{CU}$ FT OF LLW & $09 / 30 / 97$ \\
\hline RL & ML4-98-701 & RECEIVE/DISPOSE UP TO 200,000 CU FT OF LLW & $09 / 30 / 98$ \\
\hline RL & ML4-99-701 & RECEIVE/OISPOSE UP TO 200,000 CU FT OF LLW & $09 / 30 / 99$ \\
\hline RL & HL4- $00-701$ & RECEIVE/DISPOSE UP TO 200,000 CU FT OF LLW & $09 / 29 / 00$ \\
\hline RL & MLL4-01-701 & RECEIVE/OISPOSE UP TO 200,000 CU FT Of LLH & $09 / 28 / 01$ \\
\hline RL. & ML4-02-701 & RECEIVE/OISPOSE UP TO 200,000 CU FT OF LLW & $09 / 30 / 02$ \\
\hline RL & MLL5-96-10I & REC' V UP TO 3500 C.F. OF TRU WASTE FOR STORAGE & $09 / 30 / 96$ \\
\hline RL & ML.5-97-101 & REC' $Y$ UP TO 3500 C.F. OF TRU WASTE FOR STORAGE & $09 / 30 / 97$ \\
\hline $\mathbf{R L}$ & ML5-98-101 & REC' $V$ UP TO 3500 C.F. OF TRU GIASTE FOR STORAGE & $09 / 30 / 98$ \\
\hline RL & MLL-5-99-101 & REC'Y UP TO 3500 C.F. OF TRU WASTE FOR STORAGE & $09 / 30 / 99$ \\
\hline RL. & ML5-00-101 & REC'V UP TO 3500 C.F. OF TRU WASTE FOR STORAGE & $09 / 29 / 00$ \\
\hline RL. & ML5-01-101 & REC' $V$ UP TO 3500 C.F. OF TRU WASTE FOR STORAGE & $09 / 28 / 01$ \\
\hline RL & ML5-02-101 & REC'V UP TO 3500 C.F. OF TRU WASTE FOR STORAGE & $09 / 30 / 02$ \\
\hline RL & HL6-96-10] & REC' $V$ UP TO 12,000 C.F. OF DRUMS \& BOXES OF RMH & $09 / 30 / 96$ \\
\hline $\mathbf{R L}$ & PHL6-97-101 & REC' $\cup$ UP TO 12,000 C.F. OF DRUMS \& BOXES OF RMN & $09 / 30 / 97$ \\
\hline $\mathbf{R L}$ & ML6-98-101 & REC'V UP TO 12,000 C.F. OF DRUMS \& BOXES OF RMW & $09 / 30 / 98$ \\
\hline RL & PL6-99-101 & REC'V UP TO 12,000 C.F. OF DRUMS \& BOXES OF RMW & $09 / 30 / 99$ \\
\hline RL. & ML6-00-101 & REC' $V$ UP TO 12,000 C.F. OF ORUMS \& BOXES OF RUP & $09 / 29 / 00$ \\
\hline $\mathbf{R L}$ & ML6-01-10I & REC' $V$ UP TO 12,000 C.F. OF DRUMS \& BOXES OF RMA & $09 / 28 / 0 \mathrm{I}$ \\
\hline $\mathbf{R L}$ & ML6-02-101 & REC' $Y$ UP TO 12,000 C.F. OF DRUMS \& BOXES OF RMW & $09 / 30 / 02$ \\
\hline RL & MLB-96-100 & RECEIPT OF 1200 CONTAIMERS-HAZ WASTE & $09 / 30 / 96$ \\
\hline
\end{tabular}




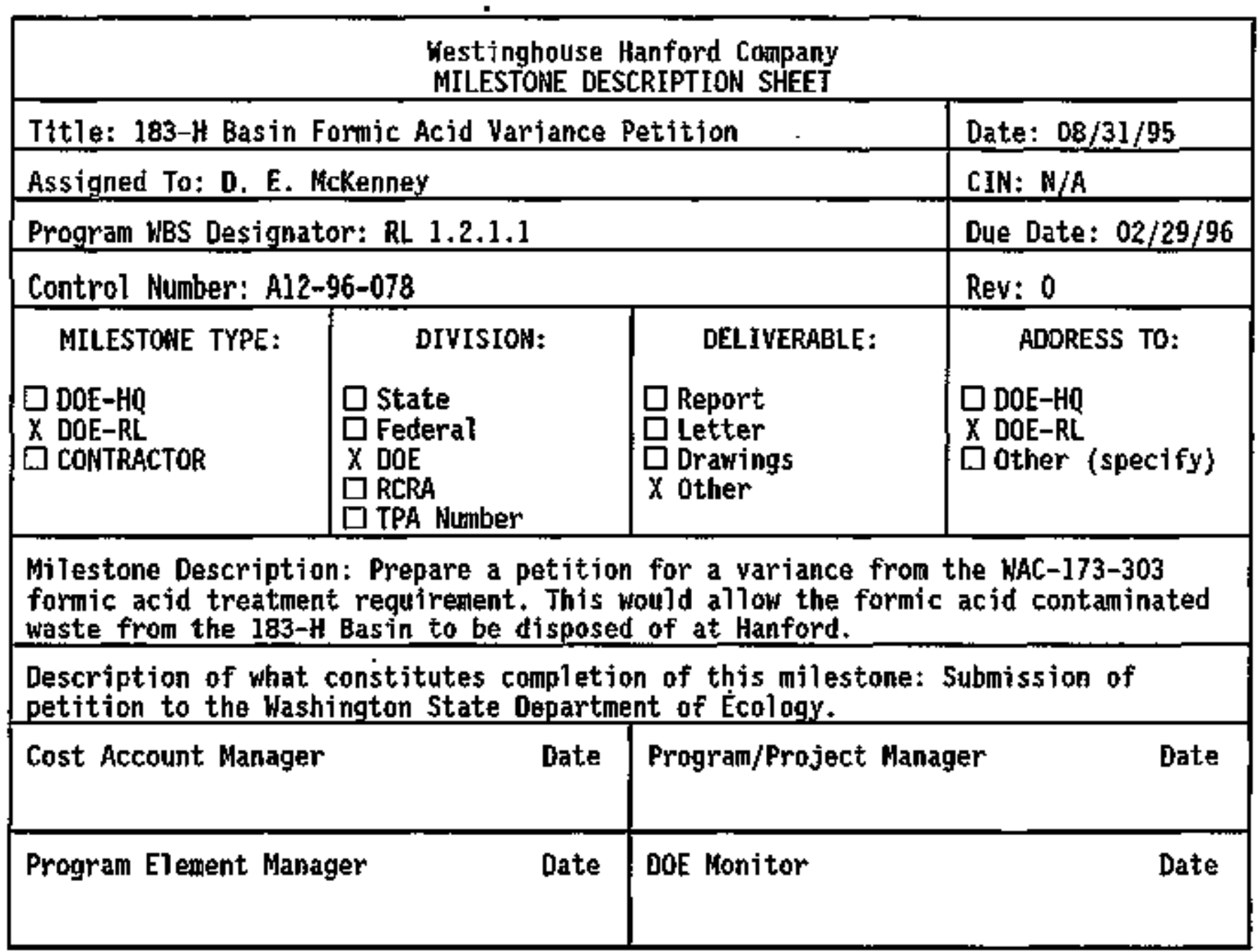




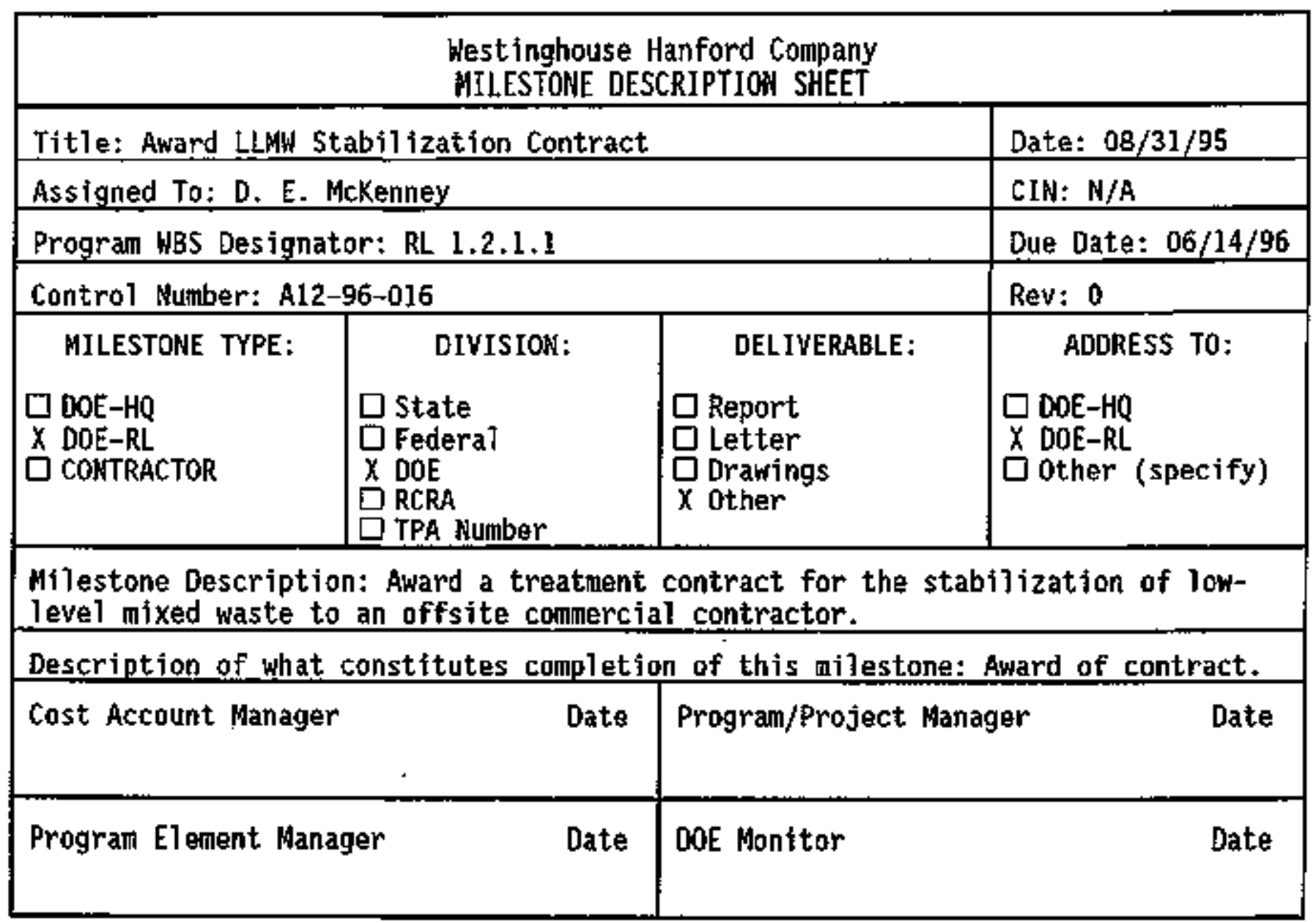




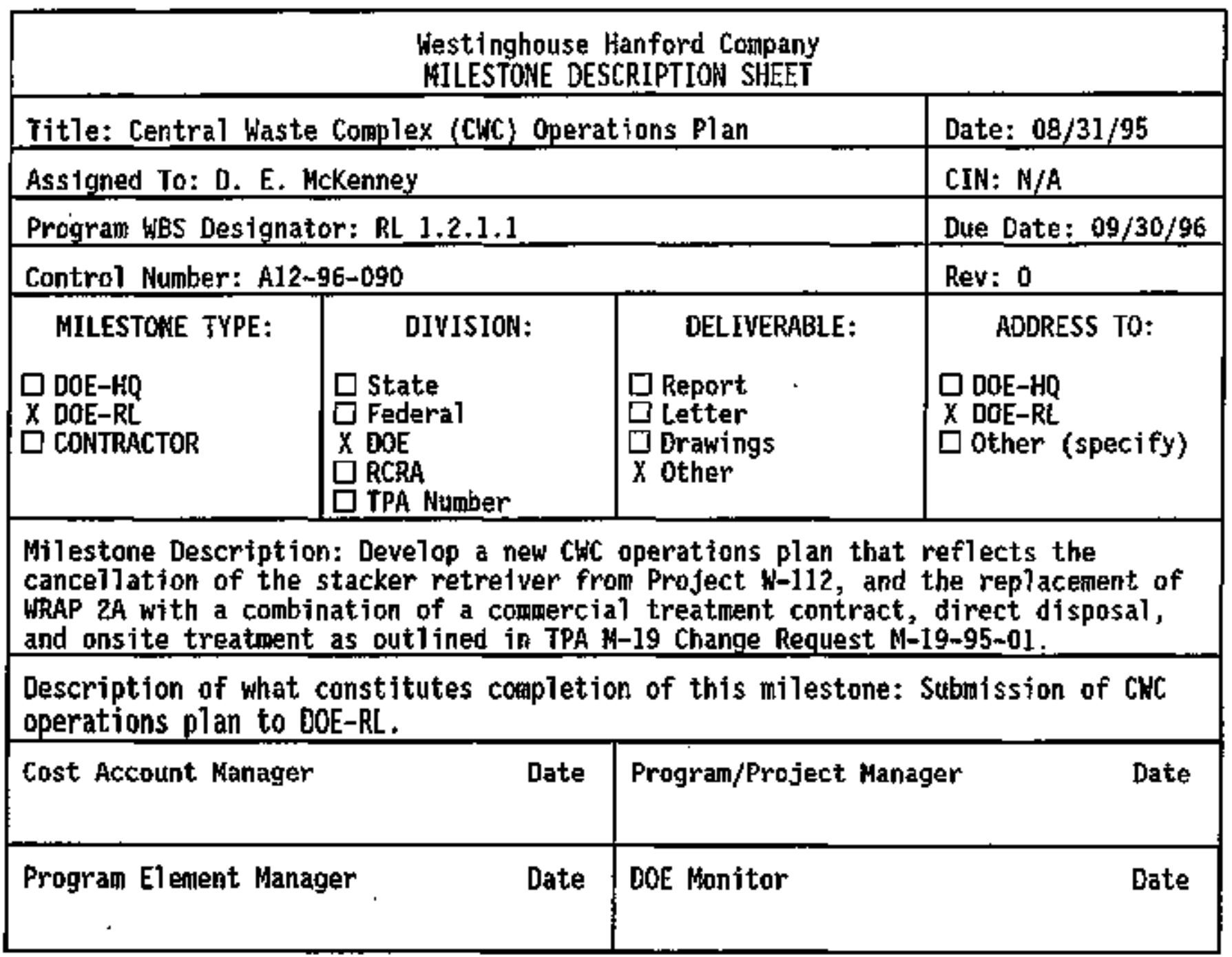




\section{SOLID MASTE PROGRAM}

WBS 1.2.1

FY 1996 Program P? an

WHC-SP-1114, ReV. 1

\begin{tabular}{|c|c|c|c|}
\hline \multicolumn{4}{|c|}{$\begin{array}{l}\text { Westinghouse Hanford Comparny } \\
\text { HILESTONE DESCRIPTION SHEET }\end{array}$} \\
\hline \multicolumn{3}{|c|}{ Title: Ship Tri-butyl Phosphate (TBP) from B Plant } & Date: $08 / 31 / 95$ \\
\hline \multicolumn{3}{|c|}{ Asstgned To: D. E. McKenney } & CIN: N/A \\
\hline \multicolumn{3}{|c|}{ Program WBS Designator: RL 1.2.1.I } & Duse Date: $06 / 30 / 97$ \\
\hline \multicolumn{3}{|c|}{ Control Number: A12-97-005 } & Rev: 0 \\
\hline $\begin{array}{l}\text { MILESTONE TYPE: } \\
\square \text { DOE-HQ } \\
X \text { DOE-RL } \\
\square \text { CONTRACTOR }\end{array}$ & $\begin{array}{l}\quad \text { DIVISION: } \\
\text { Q State } \\
\text { Q Federal } \\
x \text { DOE } \\
\text { D RCRA } \\
\square \text { TPA Number } \\
\end{array}$ & $\begin{array}{l}\text { DELIVERABLE: } \\
\square \text { Report } \\
\square \text { Letter } \\
\square \text { Drawings } \\
X \text { Dther }\end{array}$ & $\begin{array}{l}\text { ADDRESS TO: } \\
\square \text { DOE-HQ } \\
X \text { DOE-RL } \\
\square \text { other (specify) }\end{array}$ \\
\hline \multicolumn{4}{|c|}{$\begin{array}{l}\text { Milestone Description: Ship slightly radioactive TBP stored at B P1ant to an } \\
\text { offsite contractor for treatment. }\end{array}$} \\
\hline \multicolumn{4}{|c|}{$\begin{array}{l}\text { Description of what constitutes completion of this milestone: Shipment of all TBP } \\
\text { at B Plant to an offsite contractor. }\end{array}$} \\
\hline \multicolumn{2}{|c|}{ Cost Account Manager } & \multicolumn{2}{|c|}{ Program/Project Manager Date } \\
\hline \multicolumn{2}{|c|}{ Program E]ement Manager Date } & \multicolumn{2}{|c|}{ DOE Monitor Date } \\
\hline
\end{tabular}




\begin{tabular}{|c|c|c|c|}
\hline \multicolumn{4}{|c|}{$\begin{array}{l}\text { Westinghouse Hanford Company } \\
\text { MILESTONE DESCRIPTION SHEET }\end{array}$} \\
\hline \multicolumn{3}{|c|}{ Title: Vendor Environmental Assessment (EA) Preparation } & Date: $08 / 31 / 95$ \\
\hline \multicolumn{3}{|c|}{ Assigned To: D. E. Mckenney } & CIN: $N / A$ \\
\hline \multicolumn{3}{|c|}{ Program WBS Designator: RL 1.2.1.1 } & Due Date: $05 / 30 / 97$ \\
\hline \multicolumn{3}{|c|}{ Control Number: A12-97-010 } & Rev: 0 \\
\hline $\begin{array}{l}\text { MILESTONE TYPE: } \\
\text { Ф DOE-HQ } \\
X \text { DOE-RL } \\
\square \text { CONTRACTOR }\end{array}$ & $\begin{array}{l}\text { DIVISIOH: } \\
\square \text { State } \\
\square \text { Federal } \\
X \text { DOE } \\
\square \text { RCRA } \\
\square \text { TPA Number }\end{array}$ & $\begin{array}{l}\text { DELIVERABLE: } \\
\square \text { Report } \\
\square \text { Letter } \\
\square \text { Drawings } \\
x \text { Other }\end{array}$ & $\begin{array}{l}\text { ADDRESS TO: } \\
\square \text { DOE-HQ } \\
X \text { DOE-RL } \\
\square \text { Other (specify) }\end{array}$ \\
\hline \multicolumn{4}{|c|}{$\begin{array}{l}\text { Nilestone Description: The LUMN stabjlization contractor will be required to } \\
\text { complete the NEPA EA document and submit the document to RL by September } 30 \text {, } \\
1997 \text {. WHC wi11 support the contractor and track progress toward meeting the } \\
\text { required date. }\end{array}$} \\
\hline \multicolumn{4}{|c|}{$\begin{array}{l}\text { Description of what constitutes completion of this milestone: Notification of } \\
\text { DOE-RL via the SMS/PTS reporting system and delivery of the NEPA EA doctimentation } \\
\text { to DOE-RL by September } 30,1997 \text {. }\end{array}$} \\
\hline \multicolumn{2}{|c|}{ Cost Account Manager } & \multicolumn{2}{|c|}{ Program/Project Manager } \\
\hline Program Element $\mathrm{Ma}$ & Date & DOE Monitor & Date \\
\hline
\end{tabular}


WHC-SP-1114, Rev. I

\begin{tabular}{|c|c|c|}
\hline \multicolumn{3}{|c|}{$\begin{array}{l}\text { Westinghouse Hanford Company } \\
\text { MILESTONE DESCRIPTION SHEET }\end{array}$} \\
\hline \multicolumn{2}{|c|}{$\begin{array}{l}\text { Title: Operate TRUSAF in Accordance with Applicabie } \\
\text { Regulations }\end{array}$} & Date: $08 / 31 / 95$ \\
\hline \multicolumn{2}{|l|}{ Assigned To: P. L. Hapke } & CIN: $N / A$ \\
\hline \multicolumn{2}{|l|}{ Program HiBS Designator: RL 1.2.1.1 } & Due 0ate: $09 / 30 / 96$ \\
\hline \multicolumn{2}{|l|}{ Control Number: A13-96-101 } & Rev: 0 \\
\hline \begin{tabular}{l|l}
\multicolumn{1}{c|}{ MILESTONE TYPE: } & \multicolumn{1}{|c}{ DIVISION: } \\
$\begin{array}{l}\text { D DOE-HQ } \\
\text { X DOE-RL }\end{array}$ & $\square$ State \\
$\square$ CONTRACTOR & Federal \\
& $\chi$ DOE \\
& $\square$ RCRA \\
\end{tabular} & $\begin{array}{l}\text { DELIVERABLE: } \\
\text { Report } \\
\square \text { Letter } \\
\square \text { Drawings } \\
\text { X SAS/PTS Report }\end{array}$ & $\begin{array}{l}\text { ADDRESS TO: } \\
\square \text { OOE-HQ } \\
\times \text { DOE-RL } \\
\square \text { other (specify) }\end{array}$ \\
\hline \multicolumn{3}{|c|}{$\begin{array}{l}\text { Milestone Description: Operate TRUSAF on a monthiy basis in accordance with WHC- } \\
\text { EP-0063-4, Hanford Site Sol id Waste Acceptance Criteria; oOE/RL 91-51, 224-T } \\
\text { Transuranic Waste Storage and Assay Facility Dangerous Waste Permit Application, } \\
\text { Rev. 0. June 1992; WHC-50-WM-ISB-004, TRUSAF Interim Safety Basis from October } 1 \text {, } \\
1995 \text { through September 30, 1995. Washington State Department of Ecology issued } \\
\text { compliance orders with penalties will be used to determine compliance. }\end{array}$} \\
\hline \multicolumn{3}{|c|}{$\begin{array}{l}\text { Description of what constitutes completion of this milestone: This milestone is } \\
\text { complete when notification has been provided to RL via the Site Management System } \\
\text { report. }\end{array}$} \\
\hline Cost Account Manager Date & \multicolumn{2}{|c|}{ Program/Project Manager Date } \\
\hline Program Element Manager & DOE Nonitor & Oate \\
\hline
\end{tabular}


Title: Operate TRUSAF in Accordance with Applicable Regulations

Assigned To: P. L. Hapke CIN: N/A

Program WBS Designator: RL 1.2.1.1 Due Date: $09 / 30 / 97$

Control Number: A13-97-101

MILESTONE TYPE :

\section{DIVISION:}

口 DOE-HQ

$X$ DOE-RL

DIVISION:
$\square$ State
$\square$ Federal
$X$ DOE
$\square$ RCRA
$\square$ TPA Aumber

Date: $08 / 31 / 95$

$\square$ CONTRACTOR

Rev: 0

\begin{tabular}{|c|c|}
\hline DELIVERABLE: & ACDRESS TO: \\
\hline $\begin{array}{l}\square \text { Report } \\
\square \text { Letter } \\
\square \text { Drawings } \\
X \text { SMS/PTS Report }\end{array}$ & $\begin{array}{l}\square \text { DOE-HQ } \\
X \text { DOE-RL } \\
\square \text { other (specify) }\end{array}$ \\
\hline
\end{tabular}

MiTestone Description: Operate TRUSAF on a monthly basis in accordance with WHCEP-0063-4, Hanford Site Solid waste Acceptance Criteria; DOE/RL 91-51, 224-T

Transuranic Waste Storage and Assay Facility Dangerous Waste Permit Application, Rev. 0. June 1992; WHC-SD-4M-ISB-004, TRUSAF Interim Safety Basis from October 1, 1996 through September 30, 1997. Washington State Department of Ecology issued compliance orders with penalties will be used to determine compliance.

Description of what constitutes completion of this milestone: This milestone is complete when notification has been provided to RL via the Site Management System report.

\begin{tabular}{|ll|ll|}
\hline Cost Account Manager & Date & Program/Project Manager & Date \\
\hline Program Element Manager & Date & boE Monttor & Date \\
\hline
\end{tabular}




\section{SOLID WASTE PROGRAN \\ WBS 1.2.1}

Fy 1996 Program Plan

HHC-5P-1114, Rey, 1

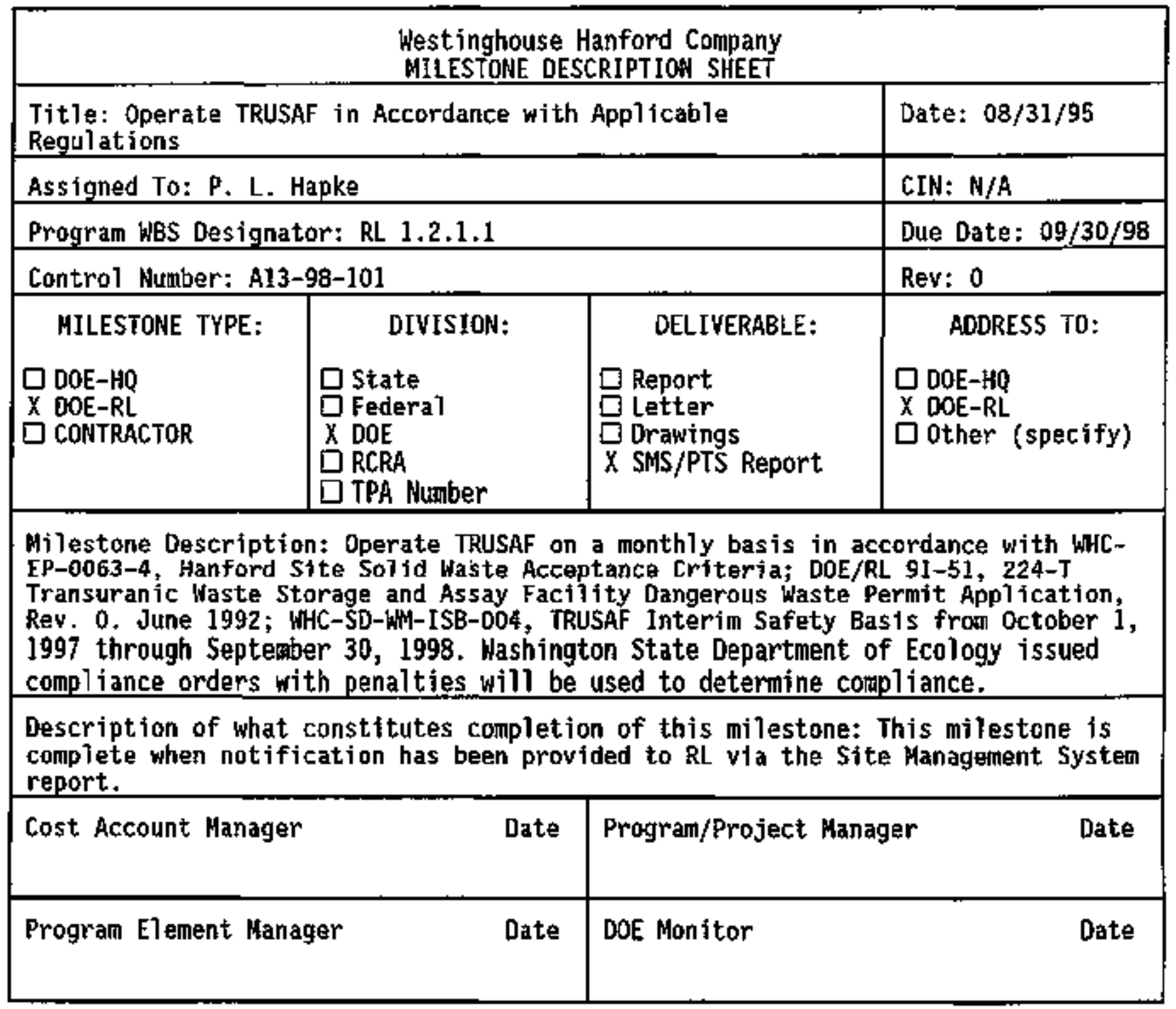


Westinghouse Hanford Company

MILESTONE DESCRIPTION SHEET

Title: Store RMW and Other Waste Requiring Long Term Storage Date: 08/31/95 in Compliance with Applicable Regulations

Assigned To: P. L. Hapke

Program WBS Designator: RL 1.2.1.1 CIN: N/A

Control Number: A14-96-101

MILESTONE TYPE:

DIVISION:

$\square$ DOE-HQ

$X$ DOE-RL

D CONTRACTOR

DIVISION:
$\square$ State
$\square$ Federa]
$X$ DOE
RCRA
$\square$ TPA Number

\section{DELIVERABLE:}

$\square$ Report

$\square$ Letter

$\square$ Drawings

$X$ SMS/PTS

Report

Milestone Description: Store radioactive mixed waste and other waste requiring long-term storage in the CWC on a monthly basis in accordance with the "Hanford Site Sol1d Waste Acceptance Criteria," WHC-EP-0063-4; D0E/RL 91/17, "Hanford CentraT Waste Complex Radtoact ive Mixed Waste Storage Facility Dangerous Waste Permit Application, "Revision 0, October 1991; and the "Central Waste Complex Interim Safety Basis, " WHC-SD-Nit-007 (Braft). Nashington State Department of Ecology issued compliance orders with penalties will be used to determine complifance.

Description of what constitutes completion of this milestone:

Storage in the Hanford Central Waste complex $100 \%$ of the radioactive mixed waste (drums and boxes) and other waste requiring long-tern storage offered by the Generators in compliance with the above regulations will constitute completion of this milestone.

\begin{tabular}{|ll|lc|}
\hline Cost Account Manager & Date & Program/Project Manager & Date \\
\hline Program Element Manager & Date & DOE Monttor & Date \\
& & & \\
\hline
\end{tabular}




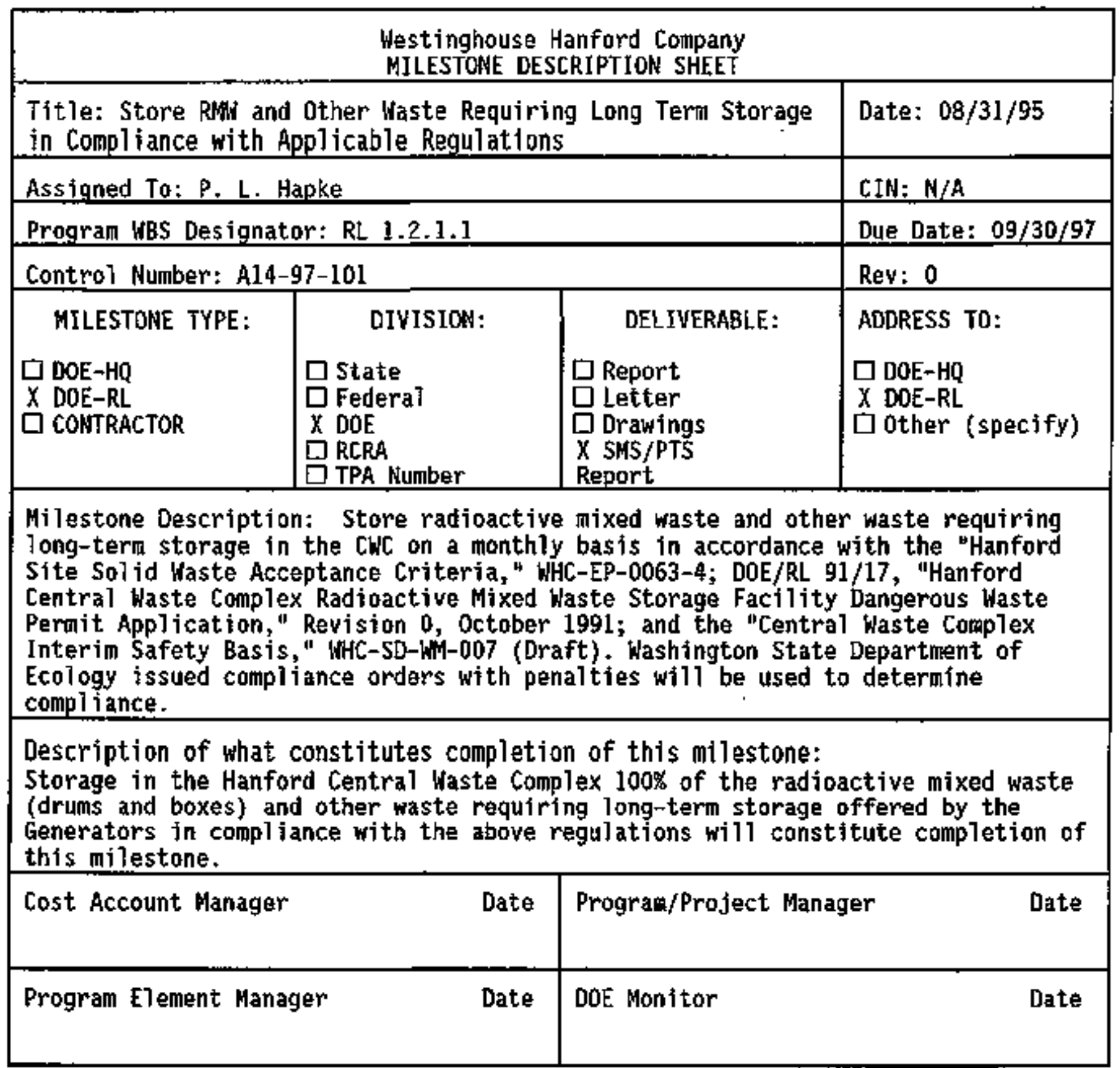


\begin{tabular}{ll|l} 
Title: Store RNW and Other Waste Requiring Long Term Storage & Date: 08/31/95
\end{tabular} in Compliance with Applicable Regulations

Assigned To: P. L. Hapke

CIN: N/A

Program UBS Designator: RL 1.2.1.1

Due Date: $09 / 30 / 98$

Control Number: A14-98-101

MILESTONE TYPE:

DIVISION:
State
Federal
$\times$ DOE
$\square$ RCRA
$\square$ TPA Mumber

DOE-HQ

$X$ DOE-RL

$\square$ CONTRACTOR

TPA Number

Rev: 0

ADBRESS TO:

I DOE-HO

$X$ DOE-RL

$\square$ other (spectfy)

Drawings

$X$ SMS/PTS

Report

Milestone Description: Store radioactive mixed waste and other waste requiring long-term storage in the CHC on a monthly basis in accordance with the "Hanford Sjte Solid Waste Acceptance Criteria," WHCC-EP-0063-4; DDE/RL 91/17, "Hanford Centra] Waste Complex Radioactive Míxed Waste Storage Facjlity Dangerous Waste Permit Application, "Revision 0, Octoger 1991; and the "Central haste Complex Interim Safety Basis," WHC-SD-Win-007 (Oraft). Washington State Department of Ecology issued compliance orders with penalties will be used to determine complitance.

Description of what constitutes completion of this milestone:

Storage in the Hanford Central Waste Complex $100 \%$ of the radioactive mixed waste (drums and boxes) and other waste requiring long-terce storage offered by the Generators in compliance with the above regulations will constitute completion of this milestone.

\begin{tabular}{|lc|lc|}
\hline Cost Account Manager & Date & Program/Project Manager & Date \\
\hline Program Element Manager & Date & DOE Monitor & Date \\
& & &. \\
\hline
\end{tabular}


Westinghouse Hanford Company

MILESTONE BESCRIPTION SHEET

\begin{tabular}{|c|c|c|c|}
\hline \multicolumn{3}{|c|}{$\begin{array}{l}\text { Title: Operate } 616 \text { Building in Compliance Hith Applicable } \\
\text { Regulations }\end{array}$} & Date: $08 / 31 / 95$ \\
\hline \multicolumn{3}{|c|}{ Asstgned To: R. 0. Pierce } & CIN: $N / A$ \\
\hline \multicolumn{3}{|c|}{ Progran WBS Designator: RL 1.2.1.1 } & Due Date: 09/30/96. \\
\hline \multicolumn{3}{|c|}{ Control Number: A15-96-501 } & Rev: 0 \\
\hline MILESTONE TYPE: & DIVISION: & DELIVERABLE: & ADDRESS TO: \\
\hline $\begin{array}{l}\square \text { DOE-HQ } \\
X \text { DOE-RL } \\
\square \text { COATRACTOR }\end{array}$ & $\begin{array}{l}\square \text { State } \\
\square \text { Federal } \\
x \text { DOE } \\
\square \text { RCRA } \\
\square \text { TPA Number }\end{array}$ & $\begin{array}{l}\square \text { Report } \\
\square \text { Letter } \\
\square \text { Drawings } \\
X \text { Dther (specify) } \\
\text { SMS/PTS Report }\end{array}$ & $\begin{array}{l}\square \text { DOE-HQ } \\
x \text { DOE-RL } \\
\square \text { Other (specify) }\end{array}$ \\
\hline
\end{tabular}

Milestone Description: Operate the 616 Building on a monthiy basis in coupliance with the RCRA Part B Perait from October 1, 1995 through September 30, 1996. Washington State Department of Ecology issued compliance orders with penalties will be used to determine compliance.

Description of what constitutes completion of this milestone: Th1s milestone is complete when notification has been provided to RL via the SHS/PTS reporting system.

\begin{tabular}{|ll|ll|}
\hline Cost Account Manager & Date & Progran/Project Manager & Date \\
\hline Program Element Manager & Date & DOE Monitor & Date \\
\hline
\end{tabular}




\begin{tabular}{|c|c|c|c|}
\hline \multicolumn{4}{|c|}{$\begin{array}{l}\text { Westinghouse Hanford Company } \\
\text { MILESTONE DESCRIPTION SHEET }\end{array}$} \\
\hline \multicolumn{3}{|c|}{ Title: Maintain the Low-Level Active Burial Grounds } & Date: $08 / 31 / 95$ \\
\hline \multicolumn{3}{|c|}{ Asstgned To: P. L. Hapke } & CIN: $N / A$ \\
\hline \multicolumn{3}{|c|}{ Program WBS Designator: RL 1.2.1.1 } & Due Date: $09 / 30 / 96$ \\
\hline \multicolumn{3}{|c|}{ Control Mumber: Al6-96-701 } & Rev: 0 \\
\hline $\begin{array}{l}\text { MILESTONE TYPE: } \\
\text { Q DOE-HQ } \\
X \text { DOE-RL } \\
\square \text { CONTRACTOR }\end{array}$ & $\begin{array}{l}\quad \text { OIVISION: } \\
\text { State } \\
\text { Stadera } \\
\text { feder } \\
\text { DoE } \\
\square \text { RCRA } \\
\square \text { TPA Number }\end{array}$ & $\begin{array}{l}\text { DELIVERABLE: } \\
\text { Report } \\
\square \text { Retter } \\
\square \text { Drawings } \\
X \text { Dther (specify) } \\
\text { SMS/PTS reporting }\end{array}$ & $\begin{array}{c}\text { ADDRESS TO: } \\
\square \text { DOE-HQ } \\
\chi \text { DOE-RL } \\
\square \text { Other (specify) }\end{array}$ \\
\hline \multicolumn{4}{|c|}{ 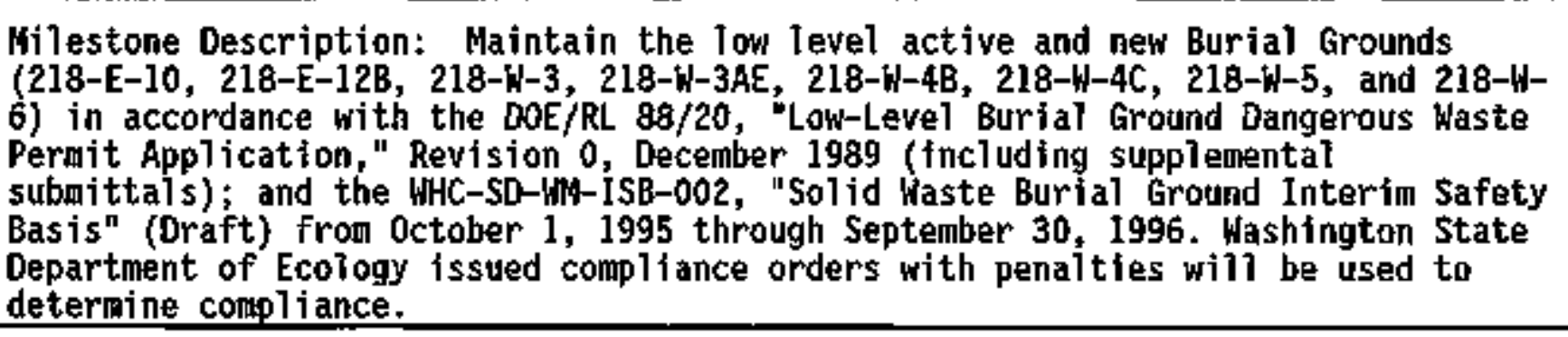 } \\
\hline \multicolumn{4}{|c|}{$\begin{array}{l}\text { Qescription of what constitutes completion of this milestone: } \\
\text { Watntenance of the low level active and new Buriai Grounds through September } 30 \text {, } \\
1996 \text { constitutes completion of this milestone. }\end{array}$} \\
\hline \multicolumn{2}{|c|}{ Cost Account Planager Date } & \multicolumn{2}{|c|}{ Program/Project Manager Date } \\
\hline \multicolumn{2}{|c|}{ Program El ement Manager Bate } & \multicolumn{2}{|l|}{ DOE Monitor } \\
\hline
\end{tabular}




\begin{tabular}{|c|c|c|c|}
\hline \multicolumn{4}{|c|}{$\begin{array}{l}\text { West inghouse Hanford Company } \\
\text { MILESTONE DESCRIPTION SHEET }\end{array}$} \\
\hline \multicolumn{3}{|c|}{$\begin{array}{l}\text { Title: Dispose of A11 Category III Waste in Inventory as of } \\
9 / 30 / 95\end{array}$} & Date: $08 / 31 / 95$ \\
\hline \multicolumn{3}{|c|}{ Asșigned To: P. L. Hapke } & CIN: $N / A$ \\
\hline \multicolumn{3}{|c|}{ Program WBS Designator: RL 1.2.1.1 } & Due Date: $09 / 30 / 96$ \\
\hline \multicolumn{3}{|c|}{ Control Number: A16-96-702 } & Rev: 0 \\
\hline $\begin{array}{l}\text { MILESTONE TYPE: } \\
\square \text { DOE-HQ } \\
\square \text { DOE-RL } \\
\square \text { CONTRACTOR }\end{array}$ & $\begin{array}{l}\text { DIVISIOH: } \\
\square \text { State } \\
\square \text { Federai } \\
X \text { DOE } \\
\square \text { RCRA } \\
\square \text { TPA Number }\end{array}$ & $\begin{array}{l}\text { DELIVERABLE: } \\
\square \text { Report } \\
\square \text { Letter } \\
\square \text { Drawings } \\
X \text { other (spec } \$ \text { fy) } \\
\text { SMS/PTS report ing }\end{array}$ & $\begin{array}{l}\text { ADDRESS TO: } \\
\square \text { DOE-HQ } \\
X \text { DOE-RL } \\
\square \text { Other (specify) }\end{array}$ \\
\hline \multicolumn{4}{|c|}{$\begin{array}{l}\text { Milestone Description: Dispose of a11 Category } 3 \text { waste in inventory as of } \\
9 / 30 / 95 \text { into high-integrity containers (HIC) by } 9 / 30 / 95 \text {. }\end{array}$} \\
\hline \multicolumn{4}{|c|}{$\begin{array}{l}\text { Description of what constitutes completion of this milestone: Report of } \\
\text { completion in the SMS/PTS monthly report to DOE-RL. }\end{array}$} \\
\hline Cost Account Manag & Date & \multicolumn{2}{|c|}{ Program/Project Manager } \\
\hline Program Element $\mathrm{Ha}$ & Date & \multicolumn{2}{|c|}{ DOE Monjtor Date } \\
\hline
\end{tabular}


Tit]e: Move 250 TRU Drums from Burial Grounds

Assigned To: P. L. Hapke

Date: $08 / 31 / 95$

Progran WBS Designator: RL 1.2.1.1

CIN: N/A

Control Number: A16-96-703

MILESTONE TYPE:

$\square$ DOE-HQ

$X$ DOE-RL

DIVISION:
$\square$ State
Federal
$X$ DOE
RCRA
$\square$ TPA Number

Due Date: $09 / 30 / 96$

$\square$ CONTRACTOR

DELIVERABLE:
$\square$ Report
Letter
$\square$ Orawings
$X$ other (spec ify)
SWS/PTS reporting

Rev: 0

ADORESS T0:

$\square$ DOE-HQ

$X$ DOE-RL

$\square$ other (specify)

Milestone Description: Move 250 TRU druns from the burial grounds into storage or dispose as LLW.

Description of what constitutes completion of this milestone: Report of completion in the SMS/PTS report to DDE-RL.

\begin{tabular}{|ll|lc}
\hline Cost Account Manager & Date & Program/Project Manager & Date \\
\hline Program ETement Manager & Date & DOE Monitor & Date \\
\hline
\end{tabular}




\section{SOLID MASTE PROGRAM}

WBS 1.2.1

FY 1996 Program P] an

HHC-SP-1114, Rev. 1

\begin{tabular}{|c|c|c|c|}
\hline \multicolumn{4}{|c|}{$\begin{array}{l}\text { Westinghouse Hanford Company } \\
\text { MILESTONE DESCRIPTION SHEET }\end{array}$} \\
\hline \multicolumn{3}{|c|}{ Title: Maintain the Low-Level Active Burial Grounds } & Date: $08 / 31 / 95$ \\
\hline \multicolumn{3}{|c|}{ Assigned To: P. L. Hapke } & CIN: N/A \\
\hline \multicolumn{3}{|c|}{ Program WBS Designator: RL 1.2 .1 .1} & Due Date: $09 / 30 / 97$ \\
\hline \multicolumn{3}{|c|}{ Control Number: A16-97-701 } & Rev: 0 \\
\hline $\begin{array}{l}\text { MILESTONE TYPE: } \\
\square \text { DOE-HQ } \\
X \text { DOE-RL } \\
\square \text { CONTRACTOR }\end{array}$ & $\begin{array}{l}\quad \text { DIVISION: } \\
\text { S State } \\
\square \text { Federal } \\
X \text { DOE } \\
\square \text { RCRA } \\
\square \text { TPA Number }\end{array}$ & $\begin{array}{l}\text { DELIVERABLE: } \\
\square \text { Report } \\
\square \text { Letter } \\
\square \text { Drawings } \\
\text { X Other (specify) } \\
\text { SMS/PTS reporting }\end{array}$ & $\begin{array}{l}\text { ADDRESS TO: } \\
\square \text { OOE-HQ } \\
X \text { DOE-RL } \\
\square \text { other (specify) }\end{array}$ \\
\hline \multicolumn{4}{|c|}{ 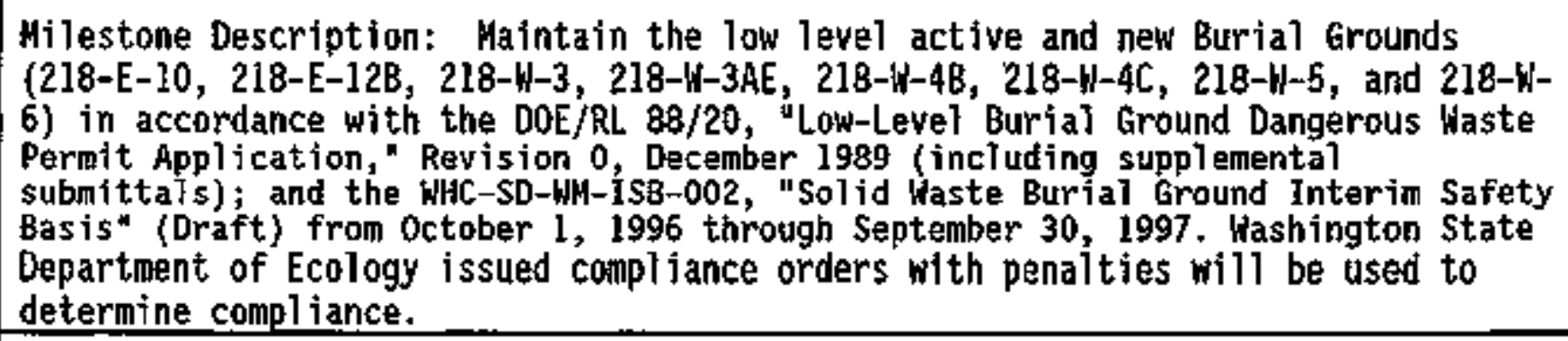 } \\
\hline \multicolumn{4}{|c|}{$\begin{array}{l}\text { Description of what constitutes completion of this milestone: } \\
\text { Maintenance of the low level active and new Burial Grounds through septenber } 30 \text {, } \\
1997 \text { constitutes completion of this milestone. }\end{array}$} \\
\hline \multicolumn{2}{|c|}{ Cost Account Manager } & \multicolumn{2}{|c|}{ Program/Project Manager Date } \\
\hline \multicolumn{2}{|c|}{ Program Elenent Manager } & \multicolumn{2}{|l|}{ DOE Monitor } \\
\hline
\end{tabular}




\begin{tabular}{|l|l|}
\hline \multicolumn{3}{|c|}{ Mest Inghouse Hanford Company } \\
MILESTOHE DESCRIPTION SHEET
\end{tabular}




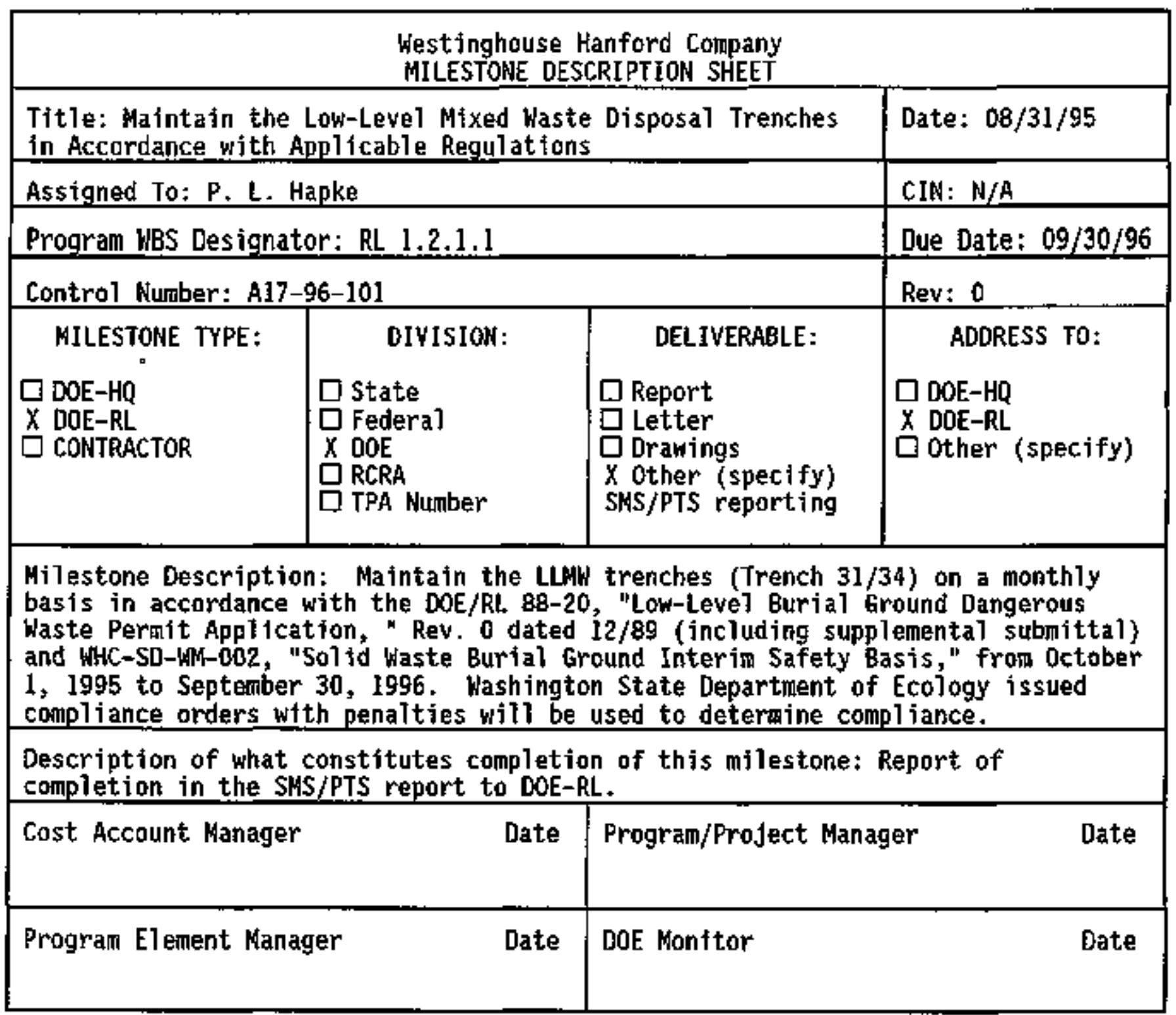


Westinghouse Hanford Company MILESTOME DESCRIPTION SHEET

Title: Issue Revision 2 of the "Soljd Waste Technical Baseline Document."

Assigned To: D. E. Mckenney

Program WBS Designator: RL 1.2.1.1 CIN: N/A

Control Number: A19-96-K01

MILESTOHE TYPE:

DIVISION:

D DOE-HQ

$X$ DOE-RL

$\square$ State

$\square$ Federal

$X$ DOE

$\square$ RCRA

$\square$ TPA Number

Date: $08 / 31 / 95$

$\square$ CONTRACTOR

Due Date: 09/30/96 Rev: 0

\begin{tabular}{|c|c|}
\hline DELLIVERABLE: & ADDRESS TO: \\
\hline $\begin{array}{l}\square \text { Report } \\
\text { Letter } \\
\square \text { Drawings } \\
X \text { Other (specify) }\end{array}$ & $\begin{array}{l}\square \text { DOE-HQ } \\
X \text { DOE-RL } \\
\square \text { other (specify) }\end{array}$ \\
\hline
\end{tabular}

Milestone Description: Issue Revision 2 of the "Solid Waste Technical Baseline Document."

Description of what constitutes completion of this milestone:

Issue Revision 2 of the "Solid Waste Technical Basel tne Docunent" by September $30,1996$.

\begin{tabular}{|ll|ll|}
\hline Cost Account Manager & Date & Progran/Project Manager & Date \\
\hline Program Element Hanager & Date & OOE Monitor & Date \\
\hline
\end{tabular}




\section{SOLID WASTE PROGRAM}

WBS 1.2 .1

FY 1996 Program Plan

WHC-SP-1114, Rev, 1

Hest $\uparrow$ nghouse Hanford Company

MILESTONE DESCRIPTION SHEET

Title: Update and Prepare Three Interface Control Documents Date: 08/31/95 (ICD).

Assigned To: D. E. McKenney

CIN: $N / A$

Program WBS Designator: RL 1.2.1.1

Due Date: 09/30/96

Control Number: A19-96-K02

HILESTONE TYPE:

DIYISION:

$\square \mathrm{DOE}-\mathrm{HO}$

$X$ DOE-RL

L] CONTRACTOR

DIVISION:
$\square$ State
$\square$ Federal
$X$ DOE
$\square$ RCRA
$\square$ TPA Number

Rev: 0

\begin{tabular}{|c|c|}
\hline DEL IVERABLE: & AODRESS TO: \\
\hline $\begin{array}{l}\square \text { Report } \\
\square \text { Letter } \\
\square \text { Drawings } \\
x \text { Other (specify) }\end{array}$ & $\begin{array}{l}\square \text { DOE-HQ } \\
X \text { DOE-RL } \\
\square \text { other (specify) }\end{array}$ \\
\hline
\end{tabular}

Milestone Description: Support sitewide systems analysis by preparing and/or updating three Interface Controt Documents by September 30, 1996.

Description of what constitutes coapletion of this milestone: Submission of three Interface Control Documents to DOE-RL by September 30, 1996.

\begin{tabular}{|ll|ll|}
\hline Cost Account Manager & Date & Program/Project. Panager & Date \\
\hline Program Element Manager & Date & DOE Monitor & Date \\
\hline
\end{tabular}




\begin{tabular}{|c|c|c|c|}
\hline \multicolumn{4}{|c|}{$\begin{array}{l}\text { Westinghouse Hanford Company } \\
\text { MILESTONE DESCRIPTION SHEET }\end{array}$} \\
\hline \multicolumn{3}{|c|}{$\begin{array}{l}\text { Title: Issue Revision } 3 \text { of the "Sol1d Waste Technifal } \\
\text { Baseline Document." }\end{array}$} & Date: $08 / 31 / 95$ \\
\hline \multicolumn{3}{|c|}{ Assigned To: D. E. McKenney } & CIN: N/A \\
\hline \multicolumn{3}{|c|}{ Program WBS Designator: RL 1.2.1.1 } & Due Date: $09 / 30 / 97$ \\
\hline \multicolumn{3}{|c|}{ - Control Number: A19-97-K01 } & Rev: 0 \\
\hline $\begin{array}{l}\text { MILESTOHE TYPE: } \\
\square \text { DOE-HQ } \\
X \text { BOE-RI } \\
\square \text { CONTRACTOR }\end{array}$ & $\begin{array}{l}\quad \text { DIVISION: } \\
\text { State } \\
\text { Stateral } \\
\text { Federal } \\
\text { DOE } \\
\text { RCRA } \\
\square \text { TPA Number }\end{array}$ & $\begin{array}{l}\text { DELIVERABLE: } \\
\square \text { Report } \\
\square \text { Letter. } \\
\text { Orawings } \\
X \text { Other (spec } f f y \text { ) }\end{array}$ & $\begin{array}{l}\text { ADDRESS TO: } \\
\square \text { OOE-HQ } \\
X \text { DOE-RL } \\
\square \text { other (specify) }\end{array}$ \\
\hline \multicolumn{4}{|c|}{$\begin{array}{l}\text { Milestone Description: Issue Revision } 3 \text { of the "Solid Waste Technical Baseline } \\
\text { Document." }\end{array}$} \\
\hline \multicolumn{4}{|c|}{$\begin{array}{l}\text { Description of what constitutes compietion of this mifestone: } \\
\text { Issue Reviston } 3 \text { of the "solfd Waste Technical Baseline Doctement" by September } \\
\text { 30, } 1997 \text {. }\end{array}$} \\
\hline \multicolumn{2}{|c|}{ Cost Account Manager Date } & \multicolumn{2}{|c|}{ Program/Project. Manager Date } \\
\hline \multicolumn{2}{|c|}{ Program Element Manager Date } & \multicolumn{2}{|c|}{ DOE Monitor Date } \\
\hline
\end{tabular}




\begin{tabular}{|c|c|c|c|}
\hline \multicolumn{4}{|c|}{$\begin{array}{l}\text { Hest fnghouse Hanford Company } \\
\text { MILESTONE DESCREPTION SHEET }\end{array}$} \\
\hline \multicolumn{3}{|c|}{$\begin{array}{l}\text { Title: Update and Prepare Three Interface Control Documents } \\
\text { (ICDs). }\end{array}$} & Date: $08 / 31 / 95$ \\
\hline \multicolumn{3}{|c|}{ Assigned To: 0. E. Mckenney } & CIN: $N / A$ \\
\hline \multicolumn{3}{|c|}{ Program WBS Designator: RL 1.2.1.1 } & Due Date: $09 / 30 / 97$ \\
\hline \multicolumn{3}{|c|}{ Control Number: A19-97-K02 } & Rev: 0 \\
\hline $\begin{array}{l}\text { HILESTOHE TYPE: } \\
\square \text { DOE-HQ } \\
X \text { DOE-RL } \\
\square \text { CONTRACTOR }\end{array}$ & \begin{tabular}{l}
\multicolumn{1}{l}{ DIVISION: } \\
D State \\
D Federal \\
$X$ DOE \\
$\square$ RCRA \\
$\square$ TPA NLmber
\end{tabular} & $\begin{array}{l}\text { DELIVERABLE: } \\
\text { Deport } \\
\square \text { Letter } \\
\square \text { Dratings } \\
X \text { Other (specify) }\end{array}$ & 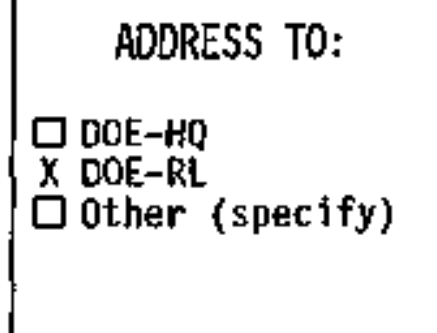 \\
\hline \multicolumn{4}{|c|}{$\begin{array}{l}\text { Milestone Description: Support sitewide systeas analysis by preparing and/or } \\
\text { updating three Interface Control Documents by September 30, } 1997 \text {. }\end{array}$} \\
\hline \multicolumn{4}{|c|}{$\begin{array}{l}\text { Description of what constitutes completion of this milestone: Submission of three } \\
\text { Interface Control Documents to DOE-RL by September } 30 \text {, I997. }\end{array}$} \\
\hline \multicolumn{2}{|c|}{ Cost Account Manager } & \multicolumn{2}{|c|}{ Program/Project Manager Date } \\
\hline \multicolumn{2}{|c|}{ Prograr Element Manager } & \multicolumn{2}{|c|}{ DOE Monitor Date } \\
\hline
\end{tabular}


Westinghouse Hanford Company MILESTONE OESCRIPTION SHEET

\begin{tabular}{|c|c|c|c|}
\hline \multicolumn{3}{|c|}{ Title: Subrit Solid Waste Multi-Year Pragram Plan } & Date: $08 / 31 / 95$ \\
\hline \multicolumn{3}{|c|}{ Assigned To: D. E. Mckenney } & CIN: $N / A$ \\
\hline \multicolumn{3}{|c|}{ Program HBSS Designator: RL 1.2.1.1 } & Due Date: $08 / 31 / 96$ \\
\hline \multicolumn{3}{|c|}{ Control Number: A1A-96-610 } & Rev: 0 \\
\hline MILESTONE TYPE: & DIVISION: & DELIVERABLE: & ADORESS TO: \\
\hline $\begin{array}{l}\text { DOE-HQ } \\
X \text { DOE-RL } \\
\square \text { CONTRACTOR }\end{array}$ & $\begin{array}{l}\square \text { State } \\
\square \text { Federal } \\
X \text { DOE } \\
\square \text { RCRA } \\
\square \text { TPA Number }\end{array}$ & $\begin{array}{l}x \text { Report } \\
\square \text { Letter } \\
\square \text { Drawings } \\
\square \text { other (specify) }\end{array}$ & $\begin{array}{l}\square \text { DOE-HQ } \\
X \text { DOE-RL } \\
\square \text { other (specify) }\end{array}$ \\
\hline
\end{tabular}

Milestone Description: This activity provides the Sol id Waste Multi-Year Program Plan for 1997 work scope. Prepare and deliver to DOE the Contractor's "FY 1997 Multi-Year Program PI an" by August 31, 1996.

Description of what constitutes completion of this milestone:

This milestone is complete when a WHC approved multi-year program plan has been transmitted to DOE-RL for review and concurrence.

\begin{tabular}{|ll|ll|}
\hline Cost Account Manager & Date & Program/Project Manager & Date \\
\hline Program Element Manager & Date & DOE Monitor & Date \\
& & & \\
\hline
\end{tabular}




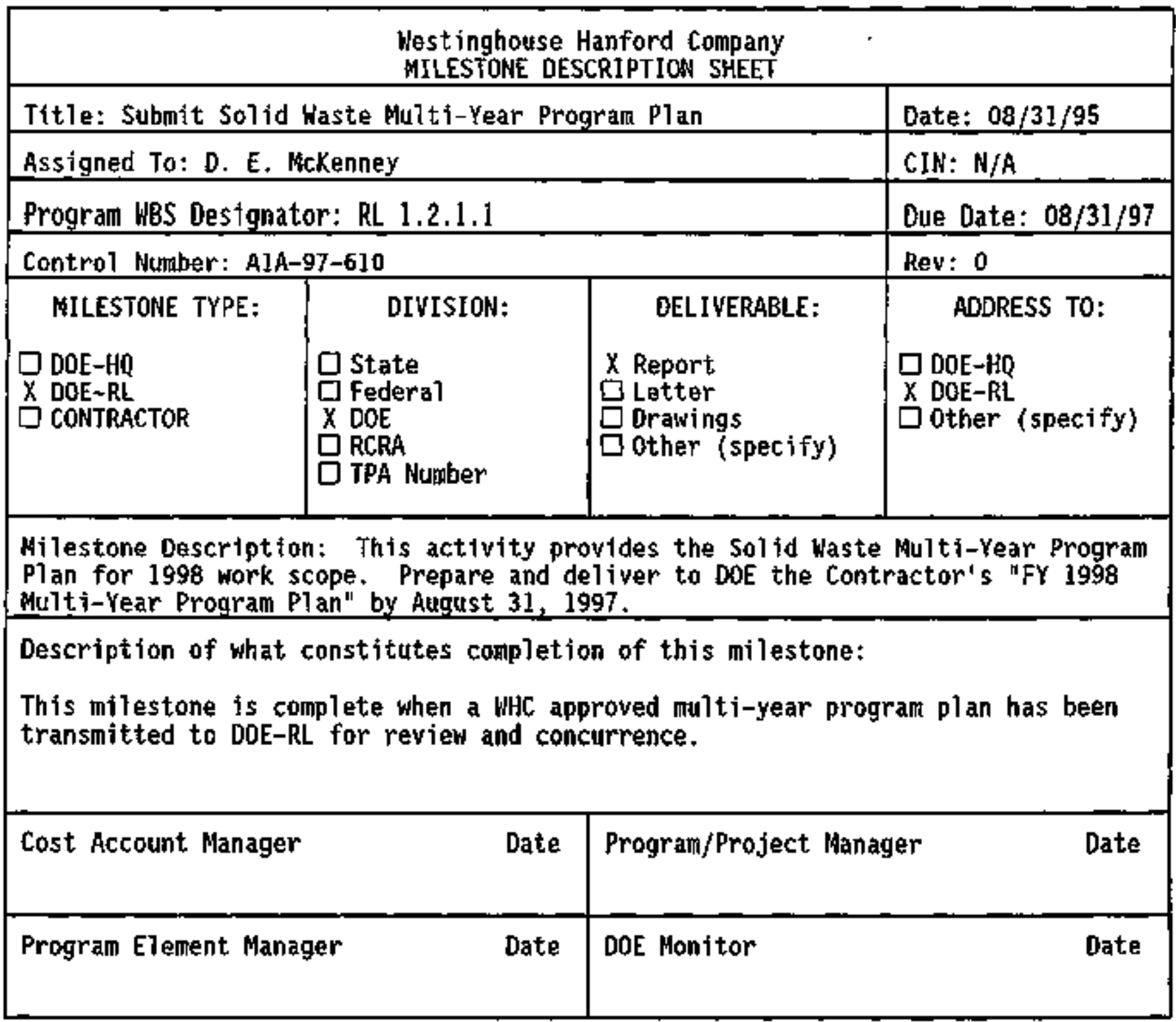




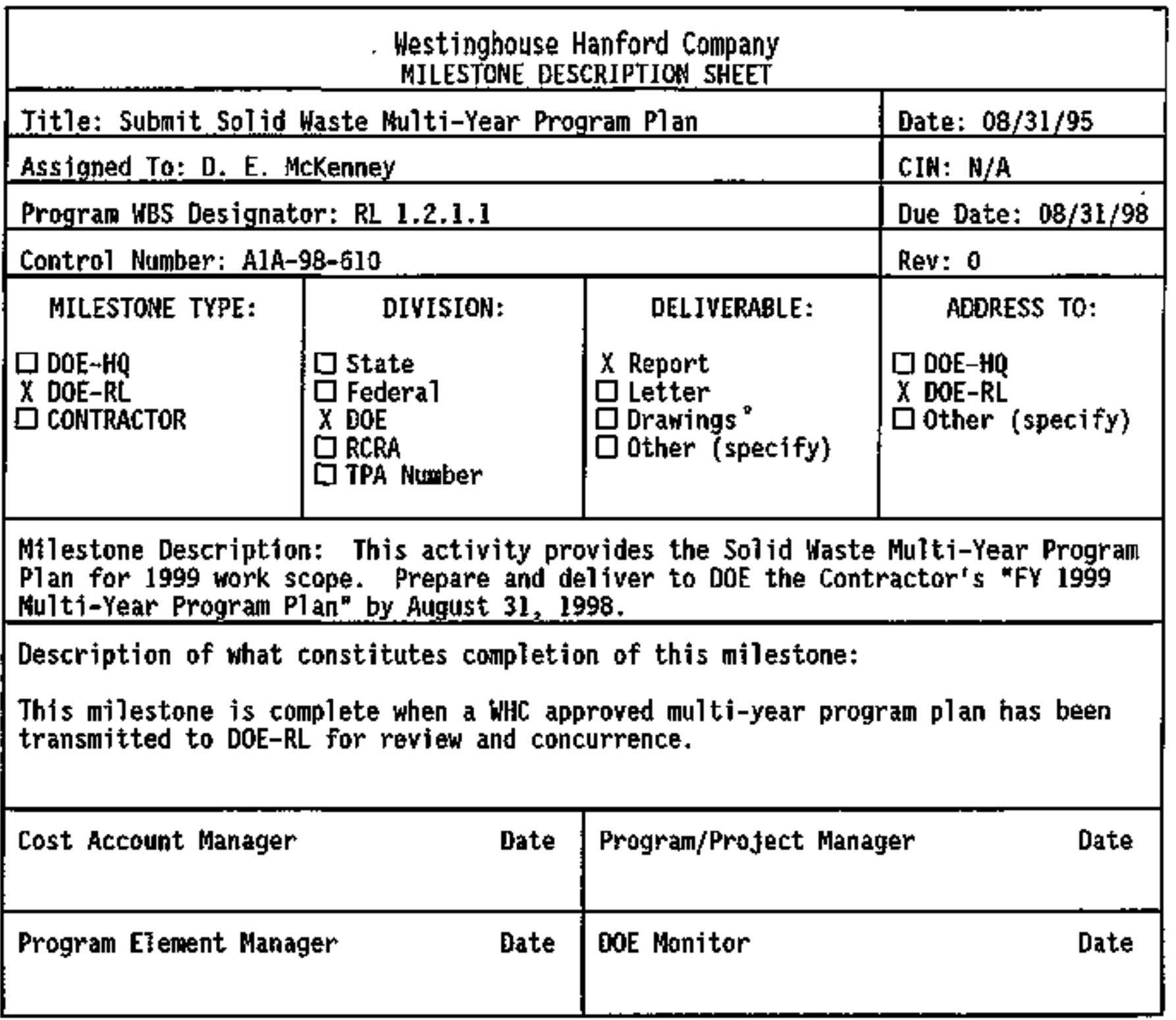




\section{SOLID WASTE PROGRAM}

WBS 1.2.1

Westinghouse Hanford Company MILESTOHE DESCRIPTION SHEET

Title: Process 2,000 $\mathrm{Ft}^{3}$ of Contaminated Equipment/Waste in $\quad$ Date: 08/31/95 the 221-T Canyon for Reuse/Storage/Disposal

Assigned To: S. D. ELLIOTT

Program WBS Designator: RL 1.2.1.7

CIN: N/A

Control Number: A31-96-k03

MILESTONE TYPE:

DIVISION:

$\square \mathrm{DOE}-\mathrm{HQ}$

$X$ DOE-RL

D CONTRACYOR

DIVISION:
$\square$ State
$\square$ Federal
DOE
$\square$ RCRA
$\square$ TPA Humber

T뭄

Milestone Description:

Process, package, and ship to the CHC, burial, or return to customer 2,000 cubic feet (based on exterior dimensions before processing) of waste/contaninated equipment for storage, disposal, and/or reuse in accordance with the "Hanford Stte Solid Waste Acceptance Criteria", WHC-EP-0063-4, (including page change effective as of 5eptember 30, 1994) from October 1, 1994 through September 30, 1995.

Description of what constitutes completion of this milestone:

Completion of this milestone will be documented in the site management system report.

\begin{tabular}{|ll|lc|}
\hline Cost Account Manager & Date & Progra甲/Project Manager & Date \\
\hline Program Element Manager & Date & DOE Monitor & Date \\
\hline
\end{tabular}




\section{Westinghouse Hanford Company MILESTONE DESCRIPTION SHEET}

\begin{tabular}{|c|c|c|c|}
\hline \multicolumn{3}{|c|}{$\begin{array}{l}\text { Title: Issue WHC Approved Fiscal Year } 1997 \text { Treatment Forecast } \\
\text { for 221-T Canyon Operations }\end{array}$} & Date: $08 / 31 / 95$ \\
\hline \multicolumn{3}{|c|}{ Assigned To: G. C. TRINER } & CIN: N/A \\
\hline \multicolumn{3}{|c|}{ Program WBS Designator: RL 1.2 .1 .7} & Due Date: $09 / 30 / 96$ \\
\hline \multicolumn{3}{|c|}{ Control Humber: A31-96-KO1 } & Rev: 0 \\
\hline $\begin{array}{l}\text { MILESTONE TYPE: } \\
\text { प DOE-HQ } \\
X \text { DOE-RL } \\
\square \text { CONTRACTOR }\end{array}$ & $\begin{array}{l}\text { DIVISION: } \\
\text { 口 State } \\
\square \text { Federal } \\
X \text { DOE } \\
\text { RCRA } \\
\square \text { TPA Humber }\end{array}$ & $\begin{array}{l}\text { DELIVERABLE: } \\
\text { X Report } \\
\text { L Letter } \\
\text { Drawings } \\
\square \text { Other (specify) }\end{array}$ & $\begin{array}{l}\text { ADORESS TO: } \\
\square \text { DOE-HQ } \\
X \text { DOE-RL } \\
\square \text { Other (specify) }\end{array}$ \\
\hline \multicolumn{4}{|c|}{$\begin{array}{l}\text { Milestone Description: } \\
\text { Report wi11 ident ify the type(s) and amount of equipment/waste to be processed in } \\
\text { the } 221-T \text { Canyon in FY } 1998 \text {. This information witi be utilized by SwD to assist } \\
\text { other Hanford sfte programs in forecasting, obtaining packaging and shipping } \\
\text { documentation, and assuring adequate capacity and processes exist at } T \text { Plant. }\end{array}$} \\
\hline \multicolumn{4}{|c|}{$\begin{array}{l}\text { Description of what constitutes completion of this milestone: } \\
\text { This milestone will be complete upon delivery of the WHC approved treatment } \\
\text { forecast to the U.S. Departiment of Energy, Richl and Operations office. }\end{array}$} \\
\hline \multicolumn{2}{|c|}{ Cost Account Manager } & \multicolumn{2}{|c|}{ Program/Project Manager Date } \\
\hline Program Element Ma1 & Date & DOE Monitor & Date \\
\hline
\end{tabular}




\section{Westinghouse Hanford Company}

MILESTOHE DESCRIPTION SHEET

\begin{tabular}{|l|l|l}
\hline T1tle: Isstue WHC Approved Fiscal Year 1998 Treatment Forecast & Date: 08/31/95
\end{tabular} for 221-T Canyon Operations

Assigned To: G. C. TRINER

Program HBS Designator: RL 1.2.1.7

CIN: $\mathrm{N} / \mathrm{A}$

Control Mumber: A31-97-K01

MILESTONE TYPE:

DIVISION:
$\square$ State
$\square$ FederaI
$X$ DOE
$\square$ RCRA
$\square$ TPA Humber

$\square$ DOE-HQ

$X$ DOE-RL

$\square$ CONTRACTOR

Due Date: 09/30/97

Rev: 0

DELIVERABLE:

$X$ Report

$\square$ Let.ter

$\square$ Drawings

other (specify)
ADORESS TO:

$\square$ DOE-HQ

$X$ DOE-RL

$\square$ Other (specify)

Milestone Description: Report witi identify the type(s) and amount of equipment/waste to be processed in the 221-T Canyon in FY 1999. This inforation will be utilized by SWO to assist other Hanford site programs in forecasting, obtaining packaging and shipping documentation, and assuring adequate capacity and processes exist at $\mathrm{T}$ Plant.

Description of what constitutes completion of this milestone:

Thts milestone will be complete upon delivery of the WHC approved treatment

forecast to the U.S. Department of Energy, Richland Dperations office.

\begin{tabular}{|ll|ll|}
\hline Cost Account Manager & Date & Program/Project Manager & Date \\
\hline Prograla Element Manager & Date & DDE Monitor & Date \\
\hline
\end{tabular}




\begin{tabular}{|c|c|c|c|}
\hline \multicolumn{4}{|c|}{$\begin{array}{l}\text { Westinghouse Hanford Company } \\
\text { MILESTONE DESCRIPTION SHEET }\end{array}$} \\
\hline \multicolumn{3}{|c|}{$\begin{array}{l}\text { Title: Process } 23,000 \mathrm{Ft}^{3} \text { of Contaminated Equipment/Waste for } \\
\text { Reuse/Storage/Disposal }\end{array}$} & Date: $08 / 31 / 95$ \\
\hline \multicolumn{3}{|c|}{ Assigned To: M. J. LABARGE } & $\mathrm{CIN}: \mathrm{N} / \mathrm{A}$ \\
\hline \multicolumn{3}{|c|}{ Program WBS Designator: RL 1.2.1.7 } & Due Date: $09 / 30 / 96$ \\
\hline \multicolumn{3}{|c|}{ Contro] Number: A32-96-K03 } & Rev: 0 \\
\hline $\begin{array}{l}\text { MILESTONE TYPE: } \\
\square \text { DOE-HO } \\
X \text { DOE-RL } \\
\square \text { CONTRACTOR }\end{array}$ & $\begin{array}{l}\quad \text { DIVISION: } \\
\text { State } \\
\text { Staderal } \\
\text { F } \\
\text { DOE } \\
\text { RCRA } \\
\text { RPA Number }\end{array}$ & $\begin{array}{l}\text { DELIVERABLE: } \\
\text { D Report } \\
\text { Letter } \\
\text { Drawings } \\
x \text { Other (specify) } \\
\text { SMS/PTS }\end{array}$ & $\begin{array}{l}\text { ADDRESS TO: } \\
\square \text { DOE-HQ } \\
X \text { DOE-RL } \\
\square \text { other (specify) }\end{array}$ \\
\hline \multicolumn{4}{|c|}{ 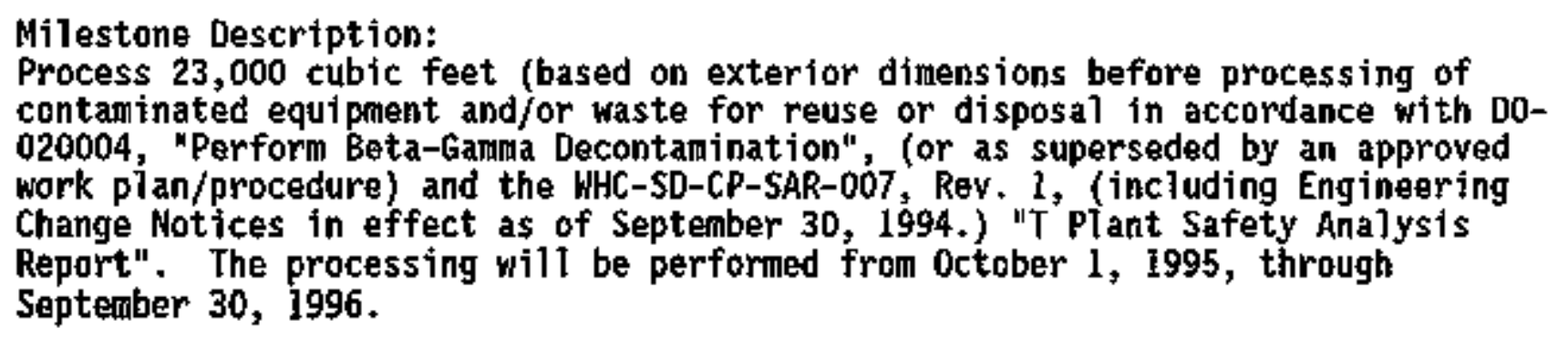 } \\
\hline \multicolumn{4}{|c|}{$\begin{array}{l}\text { Description of what constitutes completion of this milestone: } \\
\text { Completion of this milestone witl be documented in the site management system } \\
\text { report. }\end{array}$} \\
\hline \multicolumn{2}{|c|}{ Cost Account Manager } & \multicolumn{2}{|c|}{ Program/Project Hanager Date } \\
\hline \multicolumn{2}{|c|}{ Program Element. Manager } & DoE Honitor & Date \\
\hline
\end{tabular}




\begin{tabular}{|c|c|c|c|}
\hline \multicolumn{4}{|c|}{$\begin{array}{l}\text { West inghouse Hanford Company } \\
\text { MILESTONE DESCRIPTION SHEET }\end{array}$} \\
\hline \multicolumn{3}{|c|}{$\begin{array}{l}\text { Tit]e: Issue WHC Approved Fiscal Year } 1997 \text { Treatment Forecast } \\
\text { for } 2706-T \text { and Inspection/Repackaging Operations }\end{array}$} & Date: $08 / 31 / 95$ \\
\hline \multicolumn{3}{|c|}{ Asstgned To: G. C. TRINER } & CIN: N/A \\
\hline \multicolumn{3}{|c|}{ Program WBS Des ignator: RL 1.2.1.7 } & Due Date: $09 / 30 / 96$ \\
\hline \multicolumn{3}{|c|}{ Contral Number: A32-96-Kol } & Rev: 0 \\
\hline MILESTONE TYPE: & DIVISION: & DELIVERABLE: & ADDRESS T0: \\
\hline $\begin{array}{l}\text { D TPA } \\
\text { D DOE-HQ } \\
X \text { DOE-RL } \\
\square \text { CONTRACTOR }\end{array}$ & $\begin{array}{l}\square \text { State } \\
D \text { Federa1 } \\
X \text { DOE } \\
\square \text { RCRA } \\
\square \text { TPA Nuaber }\end{array}$ & $\begin{array}{l}x \text { Report } \\
D \text { Letter } \\
D \text { Drawings } \\
\square \text { other (specify) }\end{array}$ & $\begin{array}{l}\square \text { DOE-HQ } \\
X \text { DOE-RL } \\
\square \text { other (specify) }\end{array}$ \\
\hline \multicolumn{4}{|c|}{$\begin{array}{l}\text { Milestone Description: } \\
\text { Report will identify the type(s) and amount of equ1pment/waste to be processed in } \\
\text { the } 2706-T \text {. This information w111 be ut } 11 \text { ized by wos to assist other Hanford } \\
\text { site programs in forecasting, obtaining packaging and shipping documentation, and } \\
\text { assuring adequate capacjty and processes exist at } T \text { Plant. }\end{array}$} \\
\hline \multicolumn{4}{|c|}{$\begin{array}{l}\text { Description of what constitutes completion of this milestone: } \\
\text { This milestone will be complete upon delivery of the WHC approved treatment } \\
\text { forecast to the U.S. Department of Energy, Richland Operatlons office. }\end{array}$} \\
\hline \multicolumn{2}{|c|}{ Cost Account Manager Date } & \multicolumn{2}{|c|}{ Program/Project Manager $\quad$ Date } \\
\hline \multicolumn{2}{|c|}{ Program Element Manager Date } & \multicolumn{2}{|c|}{ DOE Monjtor } \\
\hline
\end{tabular}




\begin{tabular}{|c|c|c|c|}
\hline \multicolumn{4}{|c|}{$\begin{array}{l}\text { Westinghouse Hanford Company } \\
\text { MILESTOHE DESCRIPTION SHEET }\end{array}$} \\
\hline \multicolumn{3}{|c|}{$\begin{array}{l}\text { Tit1e: Process 11,500 } \mathrm{Ft}^{3} \text { of Contaminated Equipment/Haste for } \\
\text { Reuse/Storage/Disposal }\end{array}$} & Date: $08 / 31 / 95$ \\
\hline \multicolumn{3}{|c|}{ Assigned To: M. J. LABARGE } & CIN: $N / A$ \\
\hline \multicolumn{3}{|c|}{ Program UBS Designator: RL 1.2.1.7 } & Due Date: $09 / 30 / 97$ \\
\hline \multicolumn{3}{|c|}{ Contro] Number: A32-97-K03 } & Rev: 0 \\
\hline $\begin{array}{l}\text { MILESTONE TVPE: } \\
\square \text { DOE-HQ } \\
X \text { DOE-RL } \\
\square \text { CONTRACTOR }\end{array}$ & \begin{tabular}{l}
\multicolumn{1}{l}{ DIVISION: } \\
$\square$ State \\
$\square$ Federal \\
X DDE \\
RCRA \\
$\square$ TPA Number
\end{tabular} & $\begin{array}{l}\quad \text { OEL IVERABLE: } \\
\square \text { Report } \\
\square \text { Letter } \\
\square \text { Orawings } \\
\square \text { Other (specify) } \\
\text { SHS/PTS }\end{array}$ & $\begin{array}{c}\text { ADDRESS TO: } \\
\square \text { DOE-HQ } \\
X \text { DOE-RL } \\
\square \text { Other (specify) }\end{array}$ \\
\hline \multicolumn{4}{|c|}{ 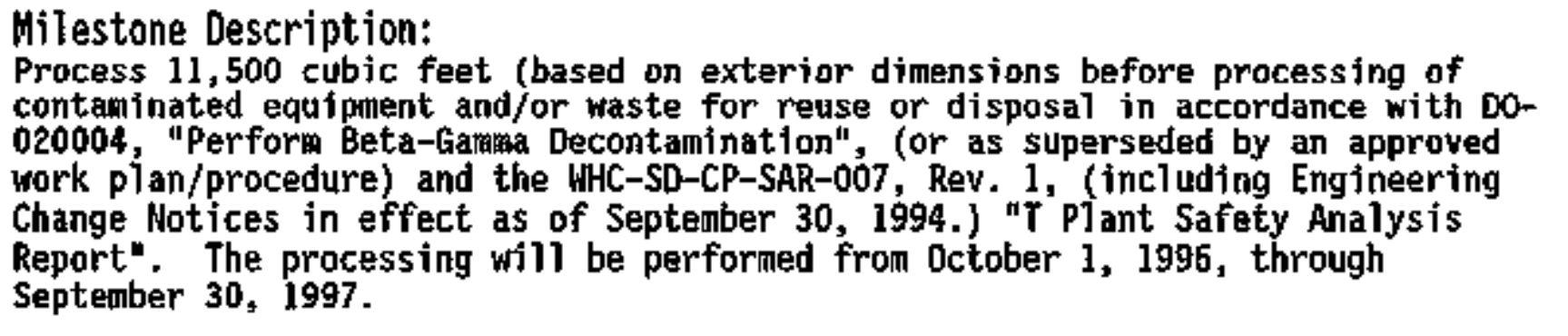 } \\
\hline \multicolumn{4}{|c|}{$\begin{array}{l}\text { Description of what constitutes completion of this milestone: } \\
\text { Completion of this milestone will be documented in the site management system } \\
\text { report. }\end{array}$} \\
\hline \multicolumn{2}{|l|}{ Cost Account Manager } & \multicolumn{2}{|c|}{ Program/Project Manager } \\
\hline Program Element Man & Date & DOE Monitor & Date \\
\hline
\end{tabular}




\begin{tabular}{|l|l|l|}
\hline \multicolumn{3}{|c|}{ Westinghouse Hanford Company } \\
MILESTOHE DESCRIPTION SHEET
\end{tabular}




\begin{tabular}{|c|c|c|c|}
\hline \multicolumn{4}{|c|}{$\begin{array}{l}\text { West1nghouse Hanford Company } \\
\text { MILESTONE DESCRIPTION SHEET }\end{array}$} \\
\hline \multicolumn{3}{|c|}{$\begin{array}{l}\text { Title: Submit a Contractor Certified Draft Part B Permit } \\
\text { Application to DoE-RL }\end{array}$} & Date: $08 / 31 / 95$ \\
\hline \multicolumn{3}{|c|}{ Asșigned To: G. C. TRINER } & CIN: N/A \\
\hline \multicolumn{3}{|c|}{ Program UBS Designator: RL 1.2.1.7 } & Oue Date: $11 / 30 / 95$ \\
\hline \multicolumn{3}{|c|}{ Control Number: $\mathrm{A} 33-96-\mathrm{K} 02$} & Rev: 0 \\
\hline \multirow{2}{*}{$\begin{array}{l}\text { MILESTONE TYPE: } \\
\square \text { DOE-HQ } \\
X \text { DOE-RL } \\
\square \text { CONTRACTOR }\end{array}$} & DIVISION: & DELIVERABLE: & ADDRESS TO: \\
\hline & $\begin{array}{l}\square \text { State } \\
\square \text { Federal } \\
x \text { DOE } \\
\square \text { RCRA } \\
\square \text { TPA Number }\end{array}$ & $\begin{array}{l}\square \text { Report } \\
\square \text { Letter } \\
\square \text { Drawings } \\
x \text { Other (specify) } \\
\text { APPLICATION }\end{array}$ & $\begin{array}{l}\square \text { DOE-HQ } \\
X \text { DOE-RL } \\
\square \text { Other (specify) }\end{array}$ \\
\hline \multicolumn{4}{|c|}{$\begin{array}{l}\text { Mijestone Description: } \\
\text { Submit T P Tant Complex Part B Permit Application to obtain RCRA final status } \\
\text { permit for T P ant to allow continuation of the waste treatment and waste } \\
\text { managenent mission, as required by Tri-Party Agreement milestane } M-20-51 \text {. }\end{array}$} \\
\hline \multicolumn{4}{|c|}{$\begin{array}{l}\text { Description of what constitutes completion of this milestone: } \\
\text { The WDS certified Part B Peraltit Appl fcation will be del ivered to the U.S. } \\
\text { Department of Energy, Richland Operations Office for subsequent submittal to } \\
\text { Ecology and EPA. }\end{array}$} \\
\hline \multicolumn{2}{|c|}{ Cost Account Manager Date } & \multicolumn{2}{|c|}{ Program/Project Manager $\quad$ Date } \\
\hline \multicolumn{2}{|c|}{ Program Element Manager } & \multicolumn{2}{|c|}{ DOE Monitor Date } \\
\hline
\end{tabular}




\begin{tabular}{|c|c|c|c|}
\hline \multicolumn{4}{|c|}{$\begin{array}{l}\text { Westinghouse Hanford Company } \\
\text { MILESTONE DESCRIPTION SHEET }\end{array}$} \\
\hline \multicolumn{3}{|c|}{$\begin{array}{l}\text { Title: Support Submittal of Part B Permit Application to } \\
\text { Ecology and EPA (M-20-5l) }\end{array}$} & Date: $08 / 31 / 95$ \\
\hline \multicolumn{3}{|c|}{ Assigned To: G. C. TRINER } & CIN: N/A \\
\hline \multicolumn{3}{|c|}{ Program WBS Designator: RL 1.2 .1 .7} & Due Date: $12 / 31 / 95$ \\
\hline \multicolumn{3}{|c|}{ Control Number: A33-96-J01 } & Rev: 0 \\
\hline $\begin{array}{l}\text { MILESTONE TYPE: } \\
\times \text { TPA } \\
\square \text { DOE-HQ } \\
\square \text { DOE-RL } \\
\square \text { CONTRACTOR }\end{array}$ & $\begin{array}{l}\text { DIVISION: } \\
\square \text { State } \\
\square \text { Federa1 } \\
\square \text { DOE } \\
\square \text { RCRA } \\
\times \text { JPA Number. } \\
\text { H-20-51 }\end{array}$ & 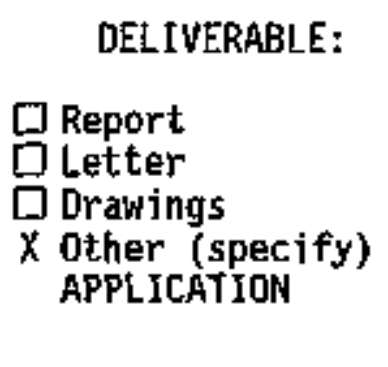 & $\begin{array}{l}\text { ADDRESS TO: } \\
\square \text { DOE-HQ } \\
\square \text { DOE-RL } \\
\square \text { Dther (specify) } \\
\text { TPA }\end{array}$ \\
\hline \multicolumn{4}{|c|}{$\begin{array}{l}\text { Mjlestone Description: } \\
\text { Subarit T Plant Complex Part B Permit Application to obtain RCRA final status } \\
\text { permit for T Plant to allow cont inuation of the waste treatruent and waste } \\
\text { management mission, as required by Tri-Party Agreement milestone M-20-51. }\end{array}$} \\
\hline \multicolumn{4}{|c|}{$\begin{array}{l}\text { Description of what constitutes completion of this milestone: } \\
\text { The WDS Part B Permit Application will be submitted by the U.S. Department of } \\
\text { Energy, Richland Operations Office to Ecology and EPA. }\end{array}$} \\
\hline \multicolumn{2}{|c|}{ Cost Account Manager } & \multicolumn{2}{|c|}{ Program/Project Manager Date } \\
\hline \multicolumn{2}{|c|}{ Program Element Manager } & DOE Monitor & Date \\
\hline
\end{tabular}




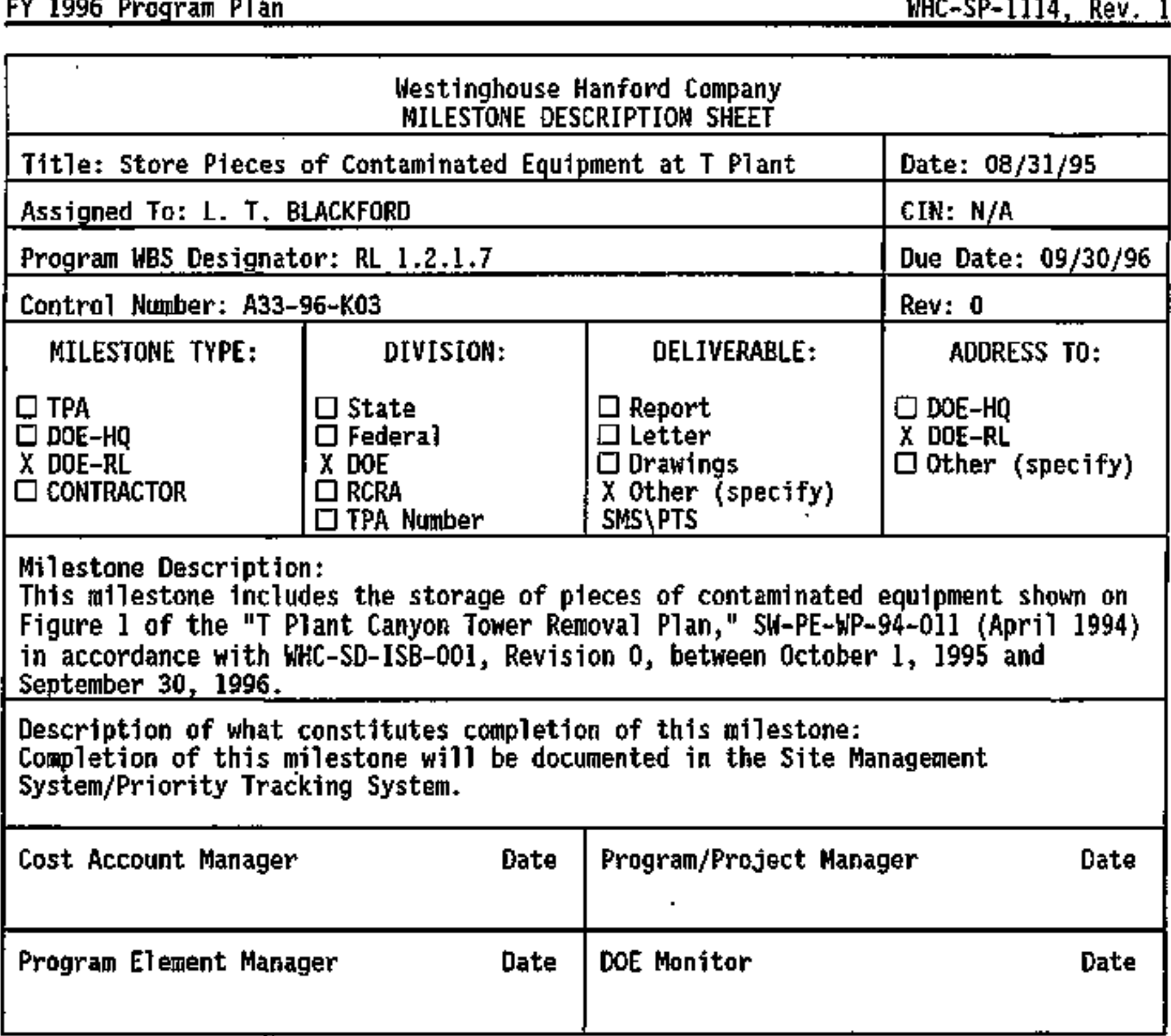




\begin{tabular}{|c|c|c|}
\hline \multicolumn{3}{|c|}{$\begin{array}{l}\text { Westinghouse Hanford Company } \\
\text { MILESTONE DESCRIPTION SHEET }\end{array}$} \\
\hline \multicolumn{2}{|c|}{ T1tle: Store 72 5pent Fuel Elements at T P1ant } & Date: $08 / 31 / 95$ \\
\hline \multicolumn{2}{|l|}{ Assigned To: S. D. ELLIOTT } & CIN: N/A \\
\hline \multicolumn{2}{|l|}{ Program WBS Designator: RL 1.2 .1 .7} & Due Date: $09 / 30 / 96$ \\
\hline \multicolumn{2}{|l|}{ Control Number: A33-96-k01 } & Rev: 0 \\
\hline 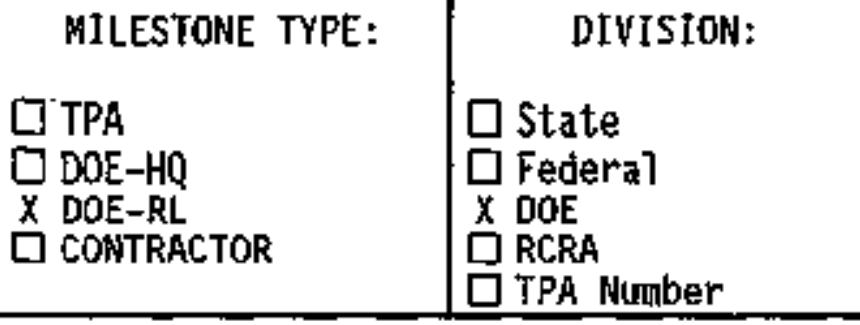 & $\begin{array}{l}\text { DELIVERABLE: } \\
\square \text { Report } \\
\square \text { Letter } \\
\square \text { Drawings } \\
\text { X Other (specify) } \\
\text { SMS/PTS REPORT }\end{array}$ & $\begin{array}{l}\text { ADDRESS TO: } \\
\square \text { DOE-HQ } \\
X \text { DOE-RL } \\
\square \text { other (spectfy) }\end{array}$ \\
\hline \multicolumn{3}{|c|}{$\begin{array}{l}\text { Milestone Description: } \\
\text { This milestone includes storing } 72 \text { Spent Fuel Elements at } T \text { Plant in accordance } \\
\text { with the "Solid Waste Division Interim Safety Besis for } 1 \text { plant FaciTity, " } \\
\text { between October } 1,1995 \text { and September } 30,1996 \text {. }\end{array}$} \\
\hline \multicolumn{3}{|c|}{$\begin{array}{l}\text { Description of what constitutes completion of this milestone: } \\
\text { A notification of continued storage of the } 72 \text { Spent Fuel Elements at } T \text { Plant will } \\
\text { appear in the Site Management System Report. }\end{array}$} \\
\hline Cost Account Manager & \multicolumn{2}{|c|}{ Program/Project Hanager Date } \\
\hline Program Element Manager & DOE Monitor & Date \\
\hline
\end{tabular}




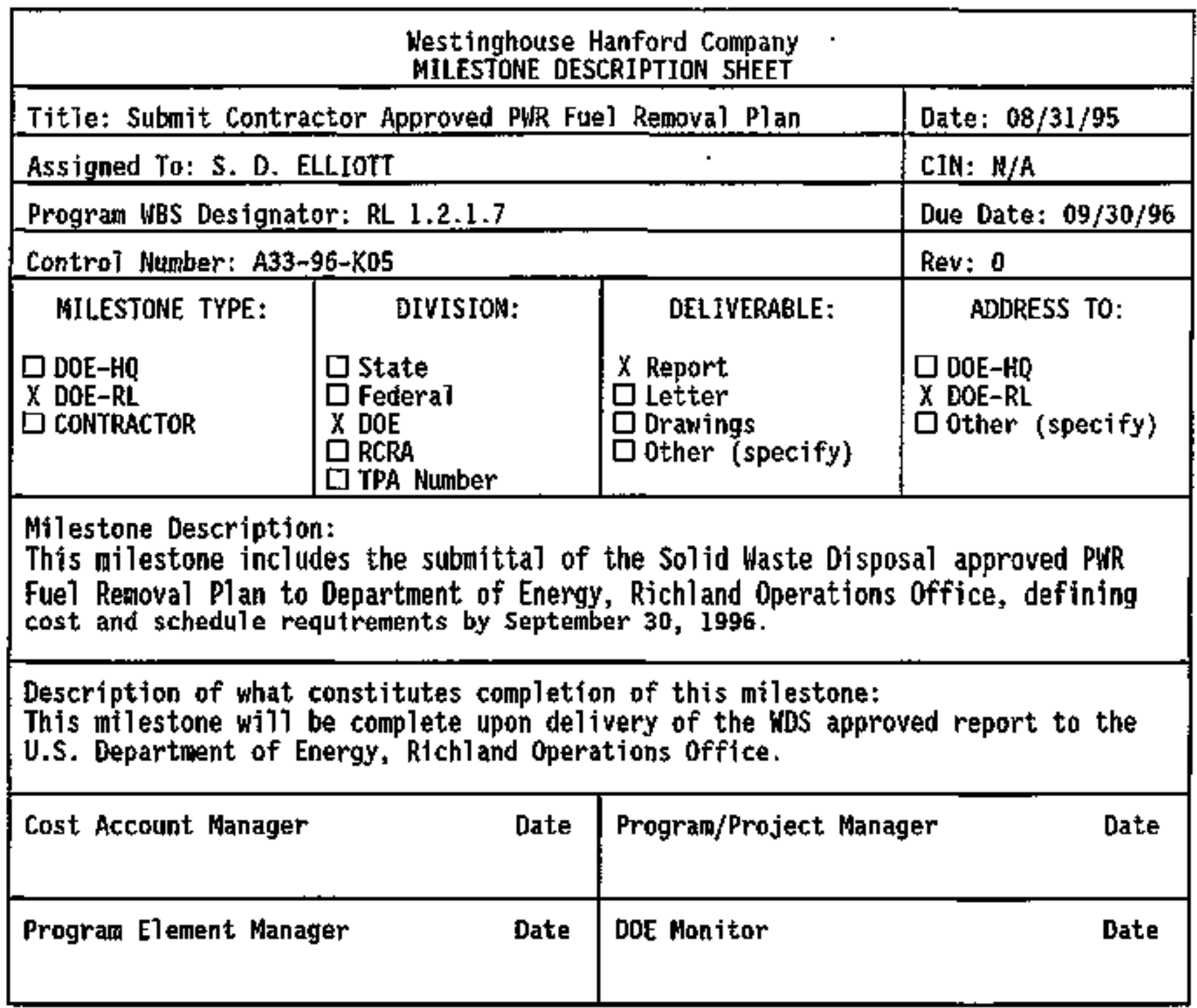




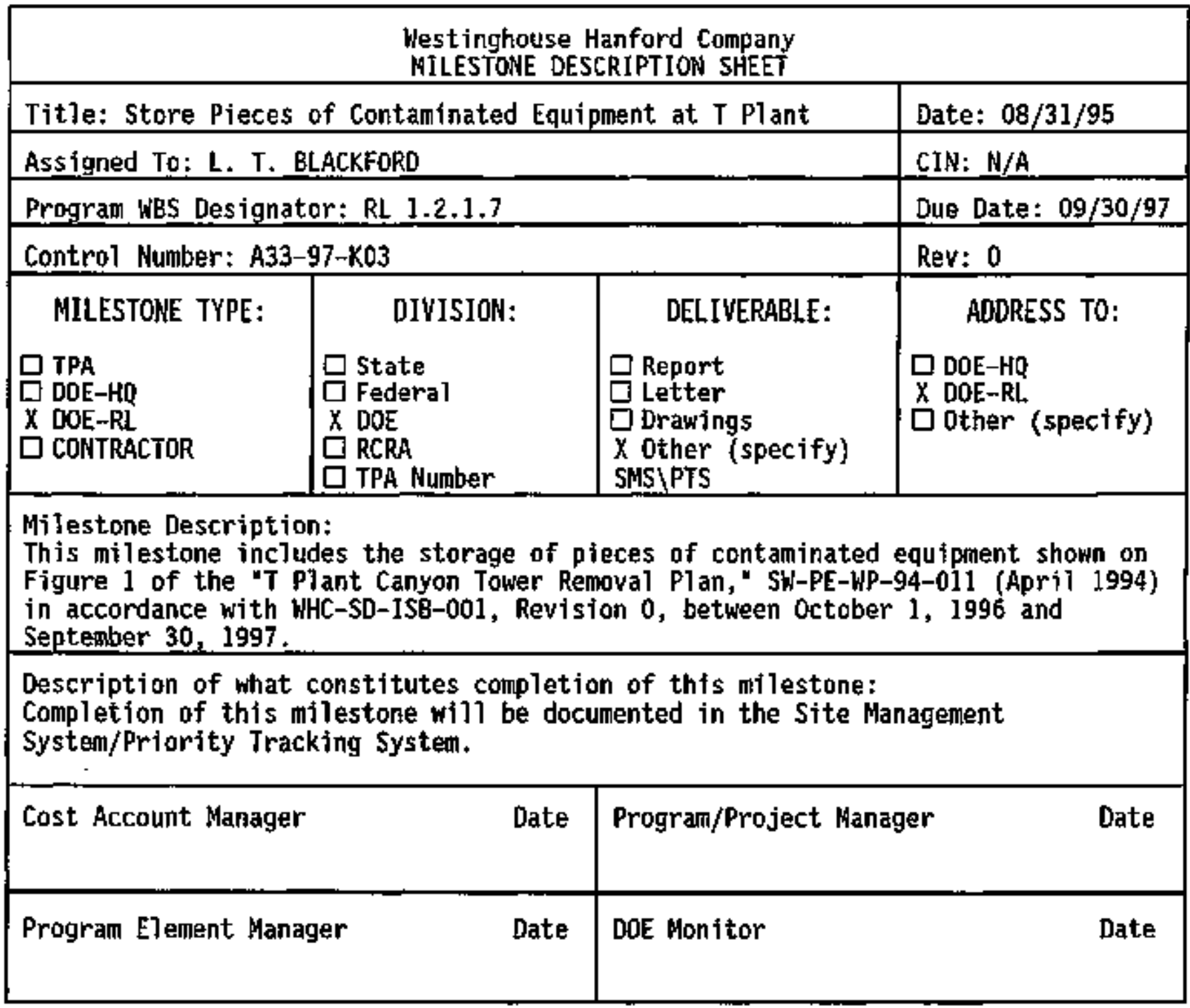




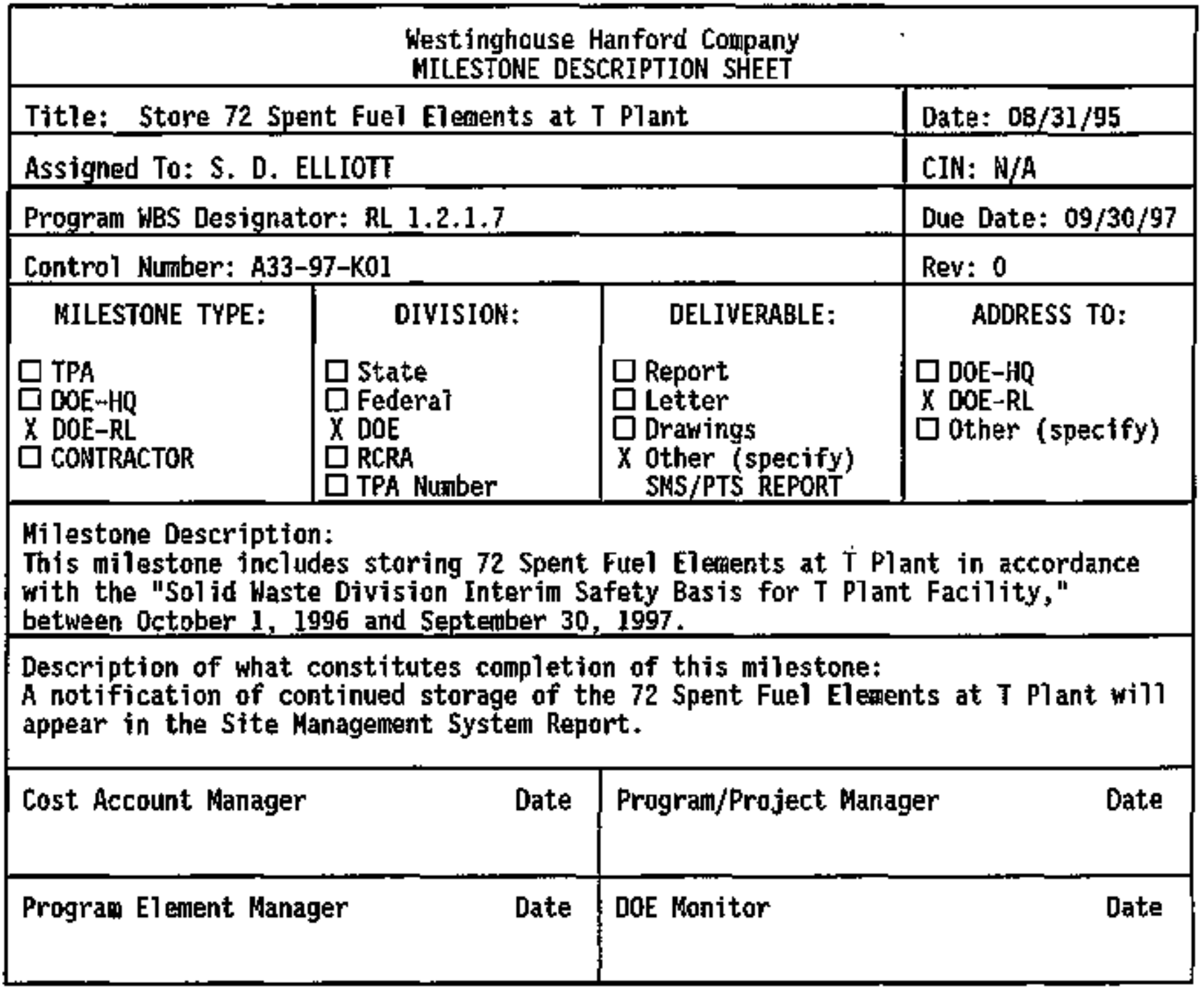




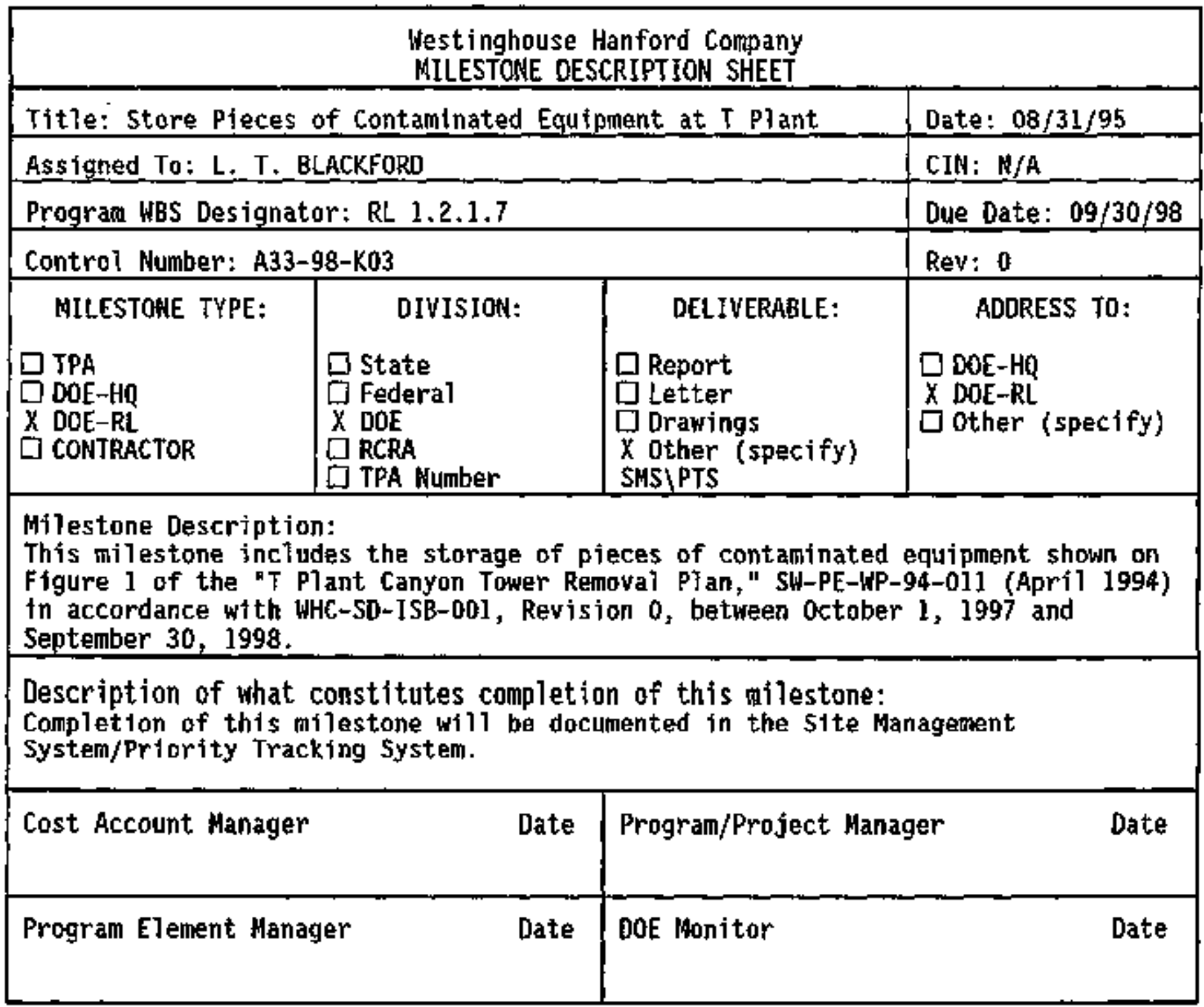




\begin{tabular}{|c|c|c|c|}
\hline \multicolumn{4}{|c|}{$\begin{array}{l}\text { Westinghouse Hanford Company } \\
\text { MILESTONE OESCRIPTION SHEET }\end{array}$} \\
\hline \multicolumn{3}{|c|}{ Title: Store 72 Spent Fuel Elements at T Plant } & Date: $08 / 31 / 95$ \\
\hline \multicolumn{3}{|c|}{ Assigned To: S. D. ELLIOTT } & CIN: $N / A$ \\
\hline \multicolumn{3}{|c|}{ Progran WBS Designator: RL 1.2.1.7 } & Due Date: $09 / 30 / 98$ \\
\hline \multicolumn{3}{|c|}{ Controi Number: A33-98-K01 } & Rev: 0 \\
\hline $\begin{array}{l}\text { MILESTONE TYPE: } \\
\text { TPA } \\
\square \text { DOE-HQ } \\
X \text { DOE-RL } \\
\square \text { CONTRACTOR }\end{array}$ & $\begin{array}{l}\text { DIVISION: } \\
\text { State } \\
\text { O Federal } \\
\times \text { DOE } \\
\text { RCRA } \\
\square \text { TPA Number }\end{array}$ & $\begin{array}{l}\text { DELIVERABLE: } \\
\square \text { Report } \\
\text { Retter } \\
\square \text { Drawings } \\
\text { X Other (specify) } \\
\text { SHS/PTS REPORT } \\
\end{array}$ & $\begin{array}{l}\text { ADDRESS TO: } \\
\square \text { OOE-HQ } \\
\text { X DOE-RL } \\
\square \text { Other (spectfy) }\end{array}$ \\
\hline \multicolumn{4}{|c|}{$\begin{array}{l}\text { Milestone Description: } \\
\text { This ailestone includes storing } 72 \text { Spent Fuel Elements at T Plant in accordance } \\
\text { with the "Solid Waste Division Interin Safety Basis for T Plant Facility," } \\
\text { between October 1, 1997 and September 30, I998. }\end{array}$} \\
\hline \multicolumn{4}{|c|}{$\begin{array}{l}\text { Description of what constjtutes completion of this mijestone: } \\
\text { A notification of continued storage of the } 72 \text { Spent Fue? Elements at } T \text { Plant will } \\
\text { appear in the Site Management System Report. }\end{array}$} \\
\hline \multicolumn{2}{|l|}{ Cost Account Nanager } & \multicolumn{2}{|c|}{ Program/Project Manager Date } \\
\hline \multicolumn{2}{|c|}{ Program E7ement Manager } & \multicolumn{2}{|c|}{ DOE Monitor Date } \\
\hline
\end{tabular}




\section{SOLID WASTE PROGRAFI}

HBS 1.2.1

FY 1996 Program P1an

WHC-SP-1114, ReV. 1

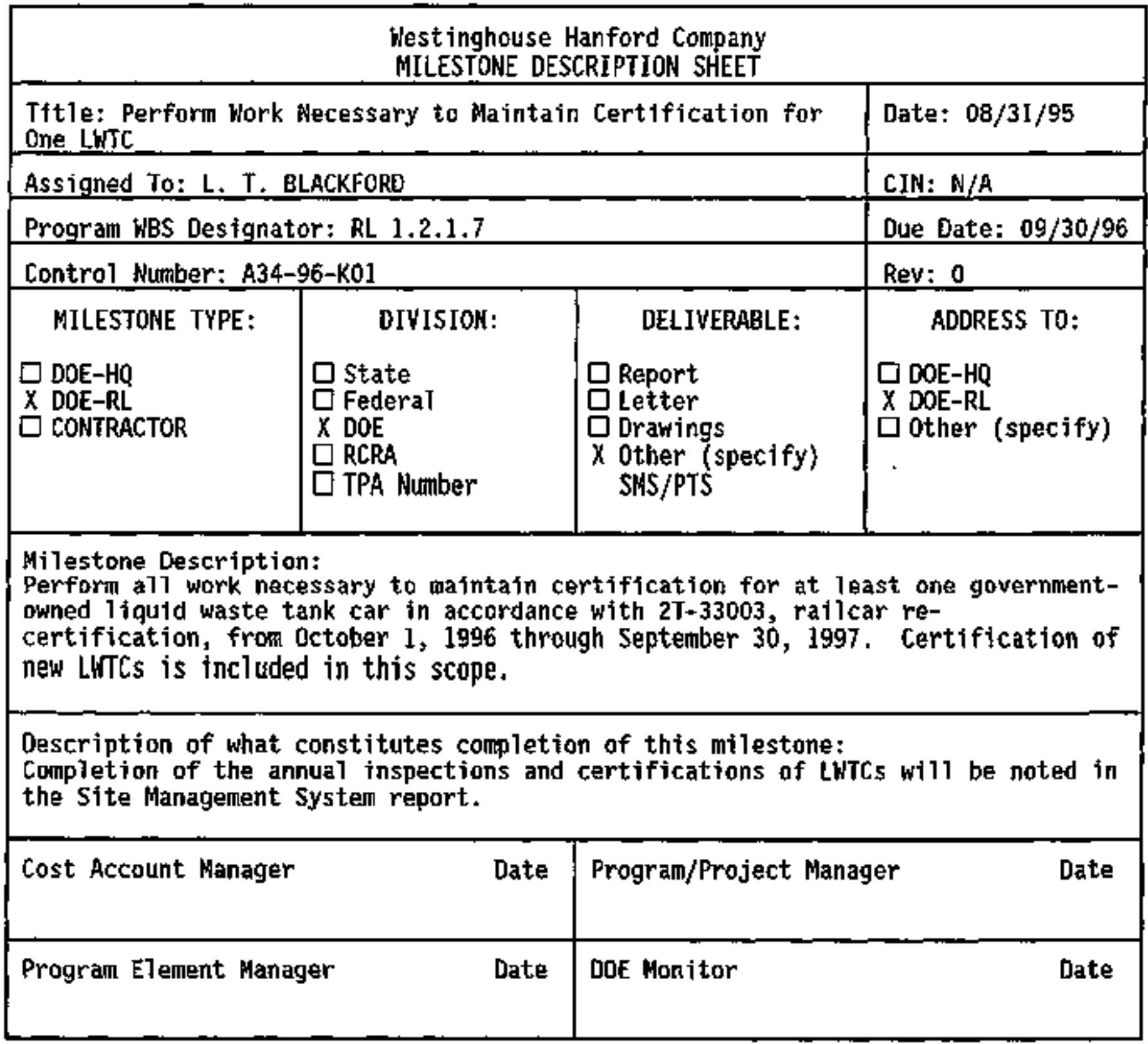




\begin{tabular}{|c|c|c|c|}
\hline \multicolumn{4}{|c|}{$\begin{array}{l}\text { Hestinghouse Kanford Company } \\
\text { MILESTONE DESCRIPTION SHEET }\end{array}$} \\
\hline \multicolumn{3}{|c|}{$\begin{array}{l}\text { Title: Perform Work Mecessary to Maintain Certification for } \\
\text { One LWTC }\end{array}$} & Date: $08 / 31 / 95$ \\
\hline \multicolumn{3}{|c|}{ Assigned To: L. T, BLACKFORD } & CIN: N/A \\
\hline \multicolumn{3}{|c|}{ Program WBS Designator: RL 1.2 .1 .7} & Due Date: 09/30/98 \\
\hline \multicolumn{3}{|c|}{ Control Number: A34-98-KO1 } & Rev: 0 \\
\hline $\begin{array}{l}\text { MILESTONE TYPE: } \\
\square \text { DOE-HQ } \\
X \text { DOE-RL } \\
\square \text { CONTRACTOR }\end{array}$ & $\begin{array}{l}\quad \text { DIVISION: } \\
\text { C State } \\
\square \text { Federal } \\
X \text { DOE } \\
\text { RCRA } \\
\square \text { TPA Number }\end{array}$ & $\begin{array}{l}\quad \text { DELIVERABLE: } \\
\square \text { Report } \\
\square \text { Letter } \\
\square \text { Lrawings } \\
\text { X Other (specify) } \\
\text { SMS/PTS }\end{array}$ & $\begin{array}{l}\text { ADDRESS TO: } \\
\square \text { DOE-HQ } \\
X \text { DOE-RL } \\
\square \text { other (specify) }\end{array}$ \\
\hline \multicolumn{4}{|c|}{$\begin{array}{l}\text { Milestone Description: } \\
\text { Perform all work necessary to maintain certification for at least one government } \\
\text { owned } 7 \text { iquid waste tank car in accordance with } 2 T-33003 \text {, rajlcar re- } \\
\text { certification, from october } 1 \text {, 1998 through September } 30,1999 \text {. Certification of } \\
\text { new LWTCs is included in this scope. }\end{array}$} \\
\hline \multicolumn{4}{|c|}{$\begin{array}{l}\text { Description of what constitutes completion of this milestone: } \\
\text { Completion of the annual inspections and certifications of LWTCs will be noted in } \\
\text { the Site Management System report. }\end{array}$} \\
\hline \multicolumn{2}{|l|}{ Cost Account Manager } & \multicolumn{2}{|c|}{ Program/Project Manager Date } \\
\hline \multicolumn{2}{|c|}{ Program Element Manager } & \multicolumn{2}{|c|}{ DOE Monitor } \\
\hline
\end{tabular}




\begin{tabular}{|c|c|c|c|}
\hline \multicolumn{4}{|c|}{$\begin{array}{l}\text { West inghouse Hanford Company } \\
\text { MILESTONE DESCRIPTION SHEET }\end{array}$} \\
\hline \multicolumn{3}{|c|}{ Title: Complete Construction of WRAP 1 Module 1} & Date: $08 / 31 / 95$ \\
\hline \multicolumn{3}{|c|}{ Assigned To: D. R. Lucas } & CIN: $N / A$ \\
\hline \multicolumn{3}{|c|}{ Program WBS Designator: RL 1.2.1.2 } & Due Date: $03 / 31 / 96$ \\
\hline \multicolumn{3}{|c|}{ Control Number: 3A4-96-T01 } & Rev: 0 \\
\hline $\begin{array}{l}\text { MILESTONE TYPE: } \\
\square \text { OOE-HQ } \\
X \text { DOE-RL } \\
\text { CONTRACTOR } \\
\square \text { TPA }\end{array}$ & \begin{tabular}{l}
\multicolumn{1}{c}{ DIVISION: } \\
$\square$ State \\
$\square$ FederaT \\
$X$ OOE \\
$\square$ RCRA \\
$X$ TPA Nutiber \\
M-I8-01 \\
\end{tabular} & $\begin{array}{l}\text { OELIVERABLE: } \\
\text { Report } \\
\text { Retter } \\
\text { Drawings } \\
\text { X Other (specify) }\end{array}$ & $\begin{array}{l}\text { AODRESS TO: } \\
\square \text { DOE-HQ } \\
X \text { DOE-RL } \\
\square \text { Other (specify) }\end{array}$ \\
\hline \multicolumn{4}{|c|}{$\begin{array}{l}\text { Milestone Description: Complete construction of the Waste Receiving and } \\
\text { Processing Module } 1 \text { Faciljty. }\end{array}$} \\
\hline \multicolumn{4}{|c|}{$\begin{array}{l}\text { Description of what constitutes completion of this milestone: Completion of the } \\
\text { intertm milestone wil1 be executed by the initiation of startup activities on } \\
\text { discreet systems within the WRAP Module I Facility that will accommodate } \\
\text { completion of TPA Milestone M-18-00, "Compiete WRAP Module } 1 \text { Construction and } \\
\text { Initiate Operations." }\end{array}$} \\
\hline \multicolumn{2}{|l|}{ Cost Account Manager } & \multicolumn{2}{|c|}{ Program/Project Manager $\quad$ Date } \\
\hline \multicolumn{2}{|c|}{ Progran Element Manager Date } & \multicolumn{2}{|c|}{ DOE Monitor Date } \\
\hline
\end{tabular}




\begin{tabular}{|c|c|c|c|}
\hline \multicolumn{4}{|c|}{$\begin{array}{l}\text { Hest inghouse Hanford Company } \\
\text { MILESTONE DESCRIPTION SHEET }\end{array}$} \\
\hline \multicolumn{3}{|c|}{ Title: Accept Responsibility of Building 2336-W } & Date: $08 / 31 / 95$ \\
\hline \multicolumn{3}{|c|}{ Assigned To: R. J. Bottenus } & CIN: N/A \\
\hline \multicolumn{3}{|c|}{ Program WBS Designator: RL 1.2.1.2 } & Due Date: $04 / 10 / 96$ \\
\hline \multicolumn{3}{|c|}{ Control Number: A47-96-158 } & Rev: 0 \\
\hline $\begin{array}{l}\text { MILESTONE TYPE: } \\
\square \text { DOE-HQ } \\
X \text { DOE-RL } \\
\square \text { CONTRACTOR }\end{array}$ & \begin{tabular}{ll} 
& \multicolumn{1}{c}{ DIVISION: } \\
& State \\
$\square$ & State \\
$\square$ & Federal \\
$X$ & DDE \\
$\square$ & RCRA \\
$\square$ & TPA Humber
\end{tabular} & \begin{tabular}{|ll} 
& DELLIVERABLE: \\
& Report \\
$\square$ & Letter \\
$\square$ & Drawings \\
$x$ & other (specify)
\end{tabular} & $\begin{array}{ll} & \text { ADORESS T0: } \\
\square & \text { DOE-HQ } \\
X & \text { DOE-RL } \\
\square & \text { Other (specify) }\end{array}$ \\
\hline \multicolumn{4}{|c|}{$\begin{array}{l}\text { Milestone Description: } \\
\text { Accept responstbility of Building 2336-H. }\end{array}$} \\
\hline \multicolumn{4}{|c|}{$\begin{array}{l}\text { Description of what constitutes completion of this milestone: } \\
\text { Completion of this ailestone is docunented by signing the official Acceptance of } \\
\text { Construction within } 30 \text { days of construction completion. }\end{array}$} \\
\hline \multicolumn{2}{|c|}{ Cost Account Manager: Date } & \multicolumn{2}{|c|}{ Program/Project. Manager Date } \\
\hline Prograft Element Ma & Date & DOE Honitor & Date \\
\hline
\end{tabular}




\section{SOLID HASTE PROGRAH}

WBS 1.2.1

Y 1996 Program Plan

WHE-SP-1114, Rev, 1

Westinghouse Hanford Company

MILESTOHE DESCRIPTION SHEET

Title: Complete Construction in Accordance with Approved

Design Media and FDC for Project W-026 WRAP I

Assigned To: D. R. Lucas

Program WBS Designator: RL 1.2.1.2

Date: $08 / 31 / 95$

Control Number: A41-96-144

MILESTONE TYPE:

DIVISION:

D DOE-HQ

$X$ DOE-RL

CONTRACTOR

\begin{tabular}{ll}
\multicolumn{1}{c}{ DIVISION: } \\
$\square$ & State \\
Federal \\
X & DOE \\
RCRA \\
TPA Number \\
\hline
\end{tabular}

OELIVERABLE:
$\square$ Report
Letter
$\stackrel{D}{\mathrm{D}}$ Orawings
Other (specify)

CIN: N/A

Due Date: $04 / 30 / 96$

Milestone Description:

Complete construction in accordance with approved design media and the

"Functional Design Criteria", lHHC-SD-W-026-FDC-001, Revision 2.

Description of what constitutes completion of this milestone:

Completion of this deliverable will be documented by submittal of the official Acceptance of Construction, (OAC) Section 1 to RL, within 30 days of compietion of construction.

\begin{tabular}{|lc|lc|}
\hline Cost. Account Manager: & Date & Program/Project Manager & Date \\
\hline Program Element Manager & Date & DoE Monitor & Date \\
\hline
\end{tabular}




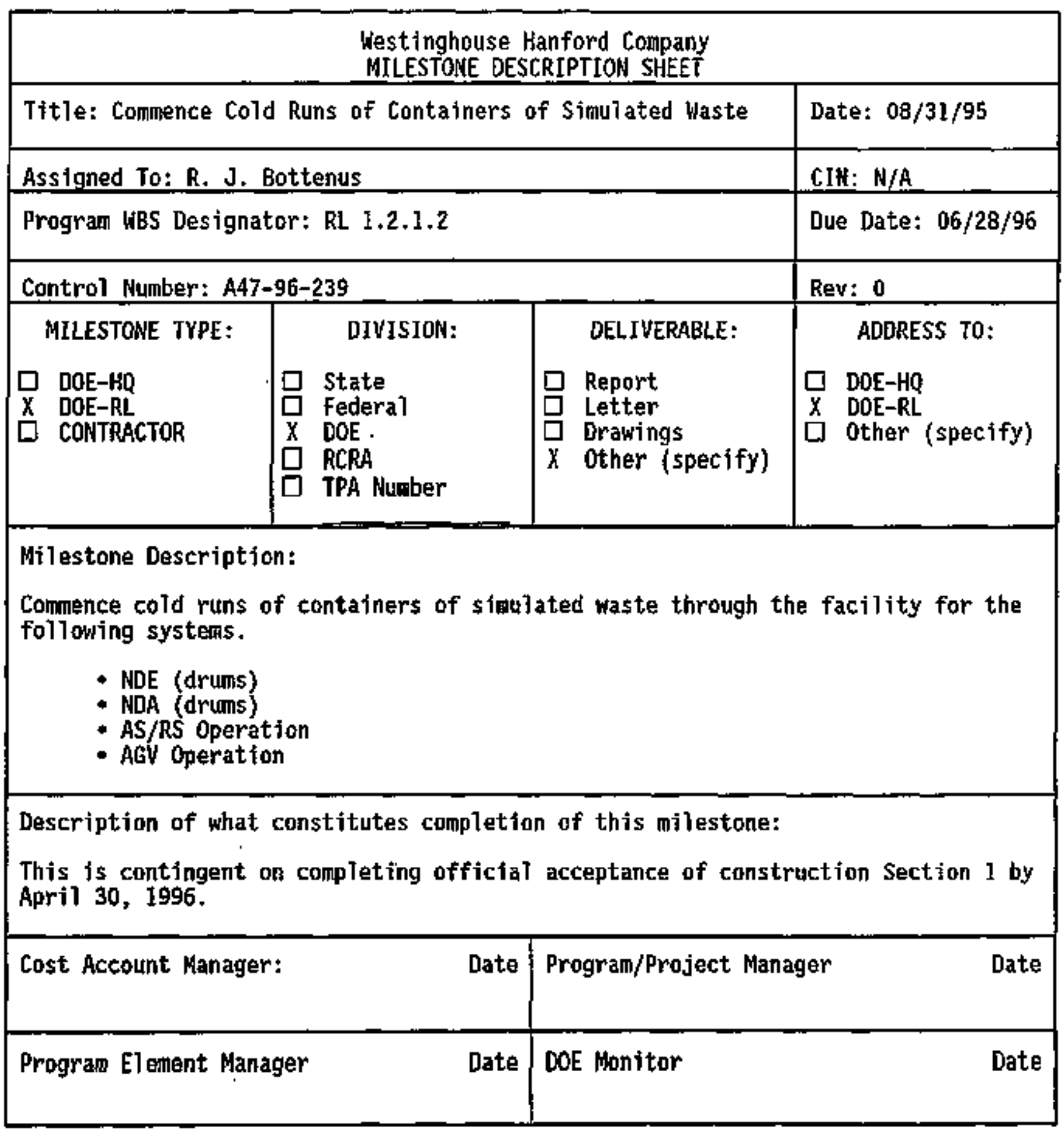




\begin{tabular}{|c|c|c|}
\hline \multicolumn{3}{|c|}{$\begin{array}{l}\text { West inghouse Hanford Company } \\
\text { MELESTONE OESCRIPTION SHEET }\end{array}$} \\
\hline \multicolumn{2}{|l|}{ Title: Issue WHC Approved WRAP I FSAR } & Date: $08 / 31 / 95$ \\
\hline \multicolumn{2}{|l|}{ Assigned To: R. J. Bottenus } & CIN:N/A \\
\hline \multicolumn{2}{|l|}{ Program WBS Designator: RL 1.2 .1 .2} & Due Date: $09 / 30 / 96$ \\
\hline \multicolumn{2}{|l|}{ Control Number: A46-96-001 } & Rev: 0 \\
\hline 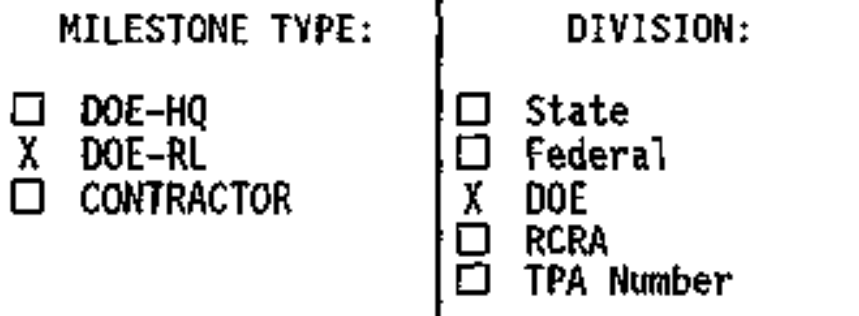 & $\begin{array}{ll} & \text { DELIVERABLE: } \\
\chi & \text { Report } \\
0 & \text { Letter } \\
0 & \text { Drawings } \\
\square & \text { Other (specify) }\end{array}$ & $\begin{array}{ll}\text { ADDRESS TO: } \\
\text { D DOE-HQ } \\
X \text { DOE-RL } \\
\square \text { Other (specify) }\end{array}$ \\
\hline \multicolumn{3}{|c|}{ Milestone Description: Issue WHC Approved FSAR } \\
\hline \multicolumn{3}{|c|}{$\begin{array}{l}\text { Description of what constitutes completion of this milestone: } \\
\text { Completion of this milestone is denoted by issuing the FSAR to DOE-RL for their } \\
\text { review. }\end{array}$} \\
\hline Cost Account Manager: & Progran/Project Man & Date \\
\hline Program Element Manager & DOE Monitor & Date \\
\hline
\end{tabular}




\begin{tabular}{|c|c|c|c|}
\hline \multicolumn{4}{|c|}{$\begin{array}{l}\text { Westinghouse Hanford Company } \\
\text { MILESTONE DESCRIPTION SHEET }\end{array}$} \\
\hline \multicolumn{3}{|c|}{ Title: Issue DOE Approved WRAP 1 FSAR } & Date: $08 / 31 / 95$ \\
\hline \multicolumn{3}{|c|}{ Assigned To: R. J. Bottenus } & CIN: N/A \\
\hline \multicolumn{3}{|c|}{ Progratr KBS Designator: RL 1.2.1.2 } & Due Date: $03 / 31 / 97$ \\
\hline \multicolumn{3}{|c|}{ Control Number: A46-97-00I } & Rev: 0 \\
\hline $\begin{array}{l}\text { MILESTONE TYPE: } \\
\square \text { DOE-HQ } \\
X \text { DOE-RL } \\
\square \text { CONTRACTOR }\end{array}$ & 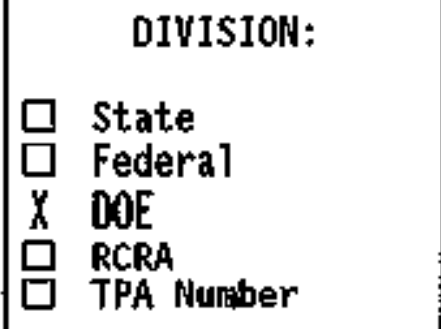 & \begin{tabular}{|ll} 
& OELIVERABLE: \\
$x$ & Report \\
0 & Letter \\
0 & Drawings \\
0 & Other (specify)
\end{tabular} & 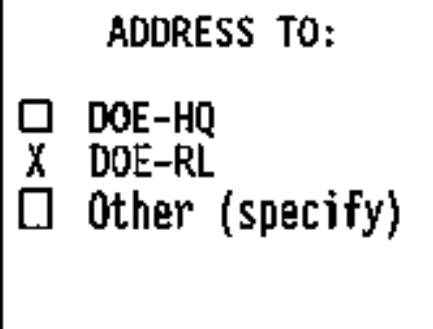 \\
\hline \multicolumn{4}{|c|}{ Milestone Description: Issue DOE Approved WRAP I FSAR } \\
\hline \multicolumn{4}{|c|}{$\begin{array}{l}\text { Description of what constitutes completion of this milestone: } \\
\text { Completion of this milestone is denoted by issuing the DOE approved FSAR to the } \\
\text { WHC document release station. }\end{array}$} \\
\hline \multicolumn{2}{|c|}{ Cost Account Manager: Date } & \multicolumn{2}{|c|}{ Program/Project Manager Date } \\
\hline \multicolumn{2}{|c|}{ Program Element Manager Date } & \multicolumn{2}{|c|}{ DOE Monitor Date } \\
\hline
\end{tabular}




\begin{tabular}{|c|c|c|c|}
\hline \multicolumn{4}{|c|}{$\begin{array}{l}\text { Westinghouse Hanford Company } \\
\text { MILESTONE OESCRIPTIOH SHEET }\end{array}$} \\
\hline \multicolumn{3}{|c|}{ Title: Complete WRAP I Construction and Initiate Operations } & Date: $08 / 31 / 95$ \\
\hline \multicolumn{3}{|c|}{ Assigned To: R. J. BOTTENUS } & CIN: N/A \\
\hline \multicolumn{3}{|c|}{ Program WBS Designator: RL 1.2.1.3 } & Due Date: $03 / 31 / 97$ \\
\hline \multicolumn{3}{|c|}{ Control Number: A47-97-001 } & Rev: 0 \\
\hline $\begin{array}{l}\text { MILESTONE TYPE: } \\
\text { D DOE-HQ } \\
X \text { DOE-RL } \\
\square \text { CONTRACTOR } \\
\square \text { TPA }\end{array}$ & $\begin{array}{l}\quad \text { DIVISION: } \\
\text { State } \\
\text { Sederai } \\
\text { X DOE } \\
\text { RCRA } \\
X \text { TPA Number } \\
\text { M- } 18-00 \\
\end{array}$ & $\begin{array}{l}\text { DELIVERABLE: } \\
\square \text { Report } \\
\square \text { Letter } \\
\square \text { Drawings } \\
\chi \text { Other (specify) }\end{array}$ & $\begin{array}{c}\text { ADDRESS TO: } \\
\square \text { DOE-HQ } \\
X \text { DOE-RL } \\
\square \text { Other (specify) }\end{array}$ \\
\hline \multicolumn{4}{|c|}{$\begin{array}{l}\text { Milestone Description: Initiate WRAP } 1 \text { operations. This a] so satifies TPA } \\
\text { Mifestone M-18-00. }\end{array}$} \\
\hline \multicolumn{4}{|c|}{$\begin{array}{l}\text { Description of what constitutes completion of this milestone: Initiation of } \\
\text { operations is signified by accepting the first container into the facility for } \\
\text { processing. }\end{array}$} \\
\hline \multicolumn{2}{|c|}{ Cost Account Manager } & \multicolumn{2}{|c|}{ Program/Project Manager $\quad$ Date } \\
\hline \multicolumn{2}{|c|}{ Progrant Element Manager Date } & \multicolumn{2}{|l|}{ DOE Monitor } \\
\hline
\end{tabular}




\begin{tabular}{|c|c|c|c|}
\hline \multicolumn{4}{|c|}{$\begin{array}{l}\text { West inghouse Hanford Company } \\
\text { MILESTONE DESCRIPTION SHEET }\end{array}$} \\
\hline \multicolumn{3}{|c|}{ Title: Process LL/RHM Naste Druts as Negotiated with RL. } & Date: $08 / 31 / 95$ \\
\hline \multicolumn{3}{|c|}{ Assigned To: R. J. Bottenus } & CIN: N/A \\
\hline \multicolumn{3}{|c|}{ Program MBS Designator: RL 1.2.1.2 } & Due Date: $09 / 30 / 97$ \\
\hline \multicolumn{3}{|c|}{ Control Number: A47-97-100 } & Rev: 0 \\
\hline $\begin{array}{l}\text { MILESTONE TYPE: } \\
\text { D DOE }-H Q \\
X \text { DOE-RL } \\
\square \text { CONTRACTOR }\end{array}$ & 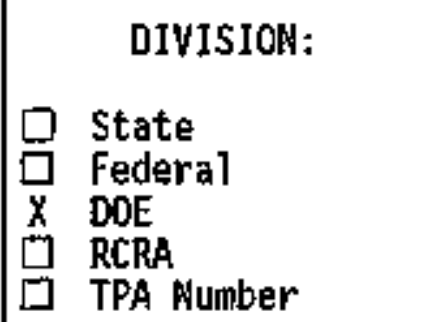 & \begin{tabular}{|ll} 
& DELIVERABLE: \\
& Report \\
& Letter \\
& Drawings \\
$X$ & Other (spec1fy)
\end{tabular} & \begin{tabular}{|ll} 
AOORESS TO: \\
DOE-HQ \\
$X$ DOE-RL \\
$\square$ & Other (specify)
\end{tabular} \\
\hline \multicolumn{4}{|c|}{$\begin{array}{l}\text { Milestone Description: } \\
\text { Process a negotiated amount (with RL) of LL/RHM Waste Drums through WRAP } 1 .\end{array}$} \\
\hline \multicolumn{4}{|c|}{$\begin{array}{l}\text { Description of what constitutes completion of this milestone: } \\
\text { This milestone is completed when the negotiated amount of waste drums (with RL) } \\
\text { are processed through WRAP } 1 \text {. }\end{array}$} \\
\hline \multicolumn{2}{|c|}{ Cost Account Manager: Date } & \multicolumn{2}{|c|}{ Program/Project Manager $\quad$ Date } \\
\hline Prọram E7ement Ma & Date & DOE Monitor & Date \\
\hline
\end{tabular}




\section{SOLID WASTE PROGRAM}

HBS 1.2 .1

Fy 1996 Program Plan

$\mathrm{NHC}-\mathrm{SP}-1114, \mathrm{ReV}, 1$

\begin{tabular}{|c|c|c|}
\hline \multicolumn{3}{|c|}{$\begin{array}{l}\text { Westinghouse Hanford Company } \\
\text { MILESTONE DESCRIPTION SHEET }\end{array}$} \\
\hline \multicolumn{2}{|c|}{ Title: Process LL/RGM Waste Orums as Negotiated with RL } & Date: $08 / 31 / 95$ \\
\hline \multicolumn{2}{|l|}{ Assigned To: R. J. Bottenus } & CIN: $N / A$ \\
\hline \multicolumn{2}{|l|}{ Program HBS Designator: RL 1.2 .1 .2} & Bue Date: $09 / 30 / 98$ \\
\hline \multicolumn{2}{|l|}{ Control Number: A47-98-100 } & Rev: 0 \\
\hline 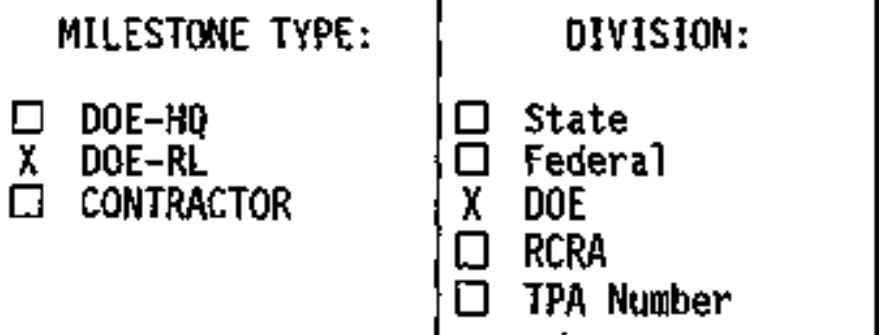 & \begin{tabular}{|ll} 
& OELIVERABLE: \\
& Report \\
$\square$ & Letter \\
0 & Orawings \\
$X$ & Other (specify)
\end{tabular} & $\begin{array}{ll}\text { ADDRESS TO: } \\
\square \text { DOE-HQ } \\
X \text { DOE-RL } \\
\square \text { other (specify) }\end{array}$ \\
\hline \multicolumn{3}{|c|}{$\begin{array}{l}\text { Milestone Description: } \\
\text { Process a negotiated amount (with RL) of LL/RWM Waste Drurus through WRAP } 1 .\end{array}$} \\
\hline \multicolumn{3}{|c|}{$\begin{array}{l}\text { Description of what constitutes completion of this milestone: } \\
\text { This milestone is completed when the regotiated amount of waste drums (with RL) } \\
\text { are processed through WRAP } 1 \text {. }\end{array}$} \\
\hline Cost Account Manager: & Program/Project Mani & Date \\
\hline Program Element Manager & DOE Monitor & Date \\
\hline
\end{tabular}


Westinghouse Hanford Company MILESTONE DESCRIPTION SHEET

Title: Complete Construction of the 0perations Support Facility

Assigned To: K. H. QUIGLEY

Program WBS Designator: RL 1.2.1.3

Control Nunber: A7B-95-139

MILESTOHE TYPE:

DIVISION:

$\square$ DOE-HO

$X$ DOE-RL

$\square$ CONTRACTOR

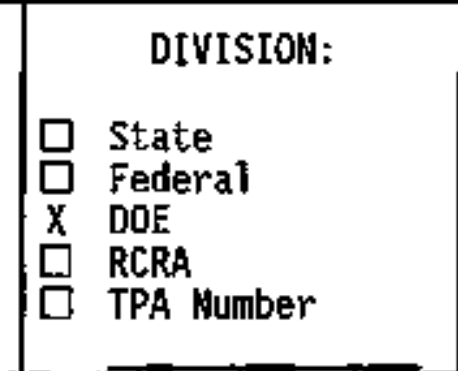

OELIVERABLE:

$\square$ Report.

Letter

$\square$ Drawings

$X$ other (specify)
CIN: $\mathrm{N} / \mathrm{A}$

Due Date: $08 / 31 / 96$

Rev: 0

Date: $08 / 31 / 95$

Rev: 0

AGDRESS TO:

$\square$ DOE-HQ

$X \quad$ DOE-RL

$\square$ Other (specify)

\section{Milestone Description:}

Complete construction of the Operations Support Facility in accordance wtth approved design media and the "Functional Design Criteria," $\mathrm{HC}-\mathrm{SD}-\mathrm{H}-\mathrm{I}$ 12-FDC-DO1, Revision 3.

Description of what const1tutes completion of this milestone:

Coapletion of this milestone is documented by signing the Official Acceptance of Construction, Part 1, within 30 days of completion of construction.

\begin{tabular}{|lc|lr|}
\hline Cost Account Manager: & Date & Program/Project Manager & Date \\
\hline Program Element Manager & Date & DOE Monitor & Date \\
& & & \\
\hline
\end{tabular}




\section{Westinghouse Hanford Company MILESTONE DESCRIPTION SHEET}

\begin{tabular}{|c|c|c|c|}
\hline \multicolumn{3}{|c|}{$\begin{array}{l}\text { Title: Initiate Definitive Design for Project } W-259, T \text { P lant } \\
\text { 2nd Containment/Leak Detection }\end{array}$} & Date: $08 / 31 / 95$ \\
\hline \multicolumn{3}{|c|}{ Assigned To: K. M. QUIGLEY } & CIN: $N / A$ \\
\hline \multicolumn{3}{|c|}{ Program WBS Designator: $\mathrm{RL} 1.2 .1 .9$} & Due Date: $02 / 01 / 96$ \\
\hline \multicolumn{3}{|c|}{ Contro\} Number: $A B 1-96-K O 1$} & Rev: 0 \\
\hline MILESTONE TYPE: & DIVISION: & DELIVERABLE : & ADDRESS TO: \\
\hline $\begin{array}{l}\text { प DOE-HQ } \\
X \text { BOE-RL } \\
\square \text { CONTRACTOR }\end{array}$ & $\begin{array}{l}\square \text { State } \\
\square \text { Federal } \\
X \text { DOE } \\
\square \text { RCRA } \\
\square \text { TPA Number }\end{array}$ & $\begin{array}{l}\square \text { Report } \\
\text { ketter } \\
\text { Drawings } \\
X \text { Other (specify) } \\
\text { SHS\PTS }\end{array}$ & $\begin{array}{l}\square \text { DOE-HQ } \\
X \text { DOE-RL } \\
\square \text { other (specify) }\end{array}$ \\
\hline
\end{tabular}

Milestone Description:

Project W-259, T Plant Secondary Containment and Leak Detection Upgrades, in support of Trí-Party Agreement mílestone $\mathbf{M - 3 2 - 0 3}$ "Complete T Plant Tank Actions, " will injtiate definitive design on February 1, 1996.

Description of what constitutes completion of this milestone:

The initiation of Defin1tive Design w1ll be in a Letter of Instruction to ICF Kaiser within 30 days of receipt of approved Project Authorization.

\begin{tabular}{|lc|lc|}
\hline Cost Account Manager & Date & Program/Project Manager & Date \\
\hline Program Element Manager & Date & DOE Monitor & Date \\
\hline
\end{tabular}




\begin{tabular}{|l|l|}
\hline \multicolumn{3}{|c|}{ Westinghouse Hanford Company } \\
MILESTONE DESCRIPTION SHEET
\end{tabular}




\begin{tabular}{|l|l|l|}
\hline \multicolumn{3}{|c|}{ Westinghouse Hanford Company } \\
MILESTONE DESCRIPTION SHEET
\end{tabular}




\begin{tabular}{|l|l|l|}
\hline \multicolumn{3}{|c|}{ Hestinghouse Hanford Company } \\
MILESTOME DESCRIPT ION SHEEI
\end{tabular}




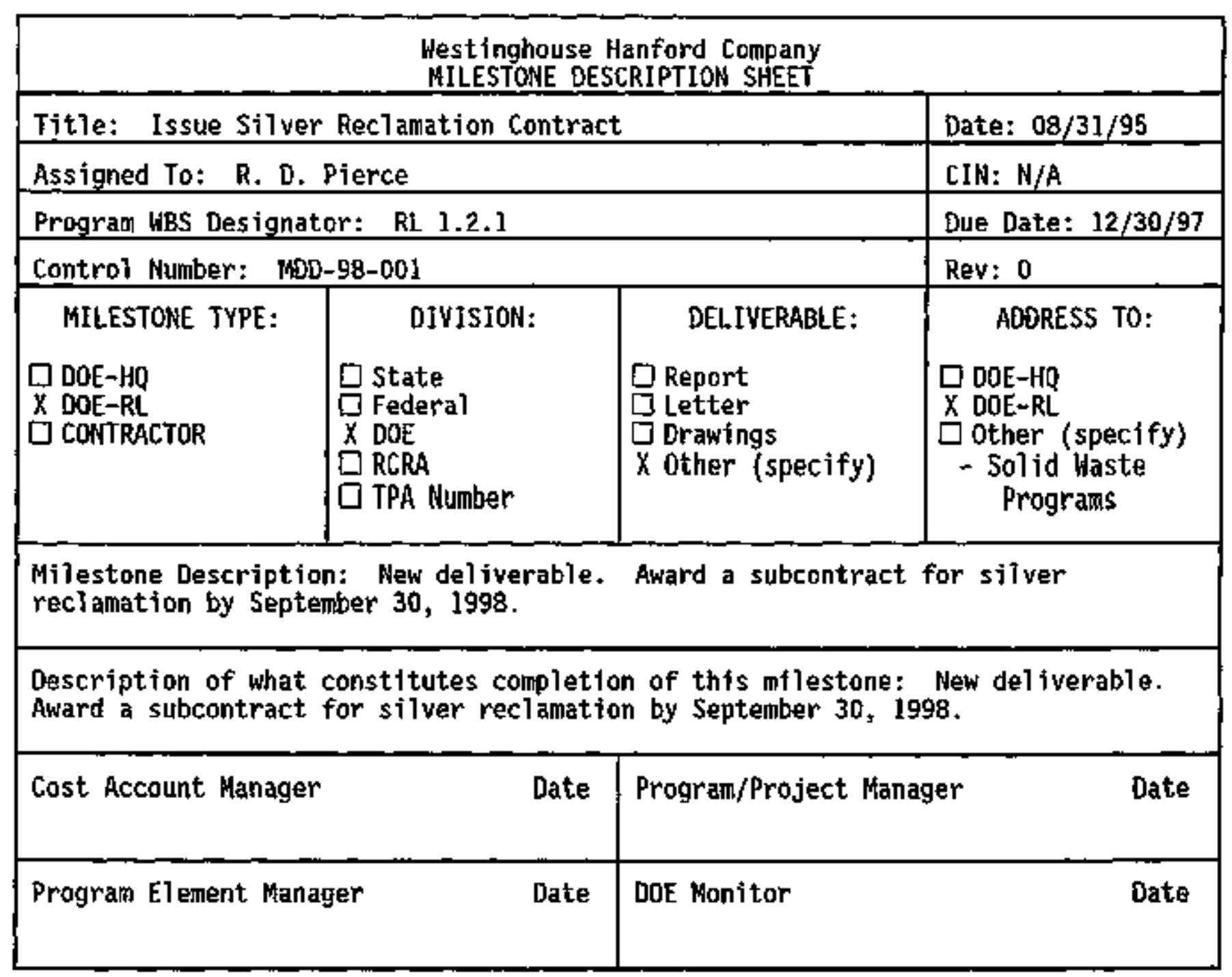


\begin{tabular}{|l|l|l}
\hline Title: Receive and Dispose up to 200,000 Cubic Feet of Low- & Date: 0B/31/95
\end{tabular} Level Waste

Assigned To: P. L. Hapke

Program WBS Designator: RL 1.2.1

Control Number: ML4-97-701

MILESTONE TYPE:

DOE-HQ

$X$ DOE-RL

$\square$ CONTRACTOR

DIVISION:
State
Gederal
$X$ DOE
RCRA
$\square$ TPA Humber

CIN: N/A

Due Date: $09 / 30 / 97$

Rev: 0

\begin{tabular}{|c|c|}
\hline DEL IVERABLE: & ADDRESS T0: \\
\hline $\begin{array}{l}\square \text { Report } \\
\square \text { Letter } \\
\square \text { Drawings } \\
X \text { Other (specify) }\end{array}$ & $\begin{array}{l}\square \text { DOE-HQ } \\
X \text { DOE-RL } \\
\square \text { other (specify) }\end{array}$ \\
\hline
\end{tabular}

Milestone Description: Receive and dispose $100 \%$ of the Low Level Waste (LLW) offered by the Generators. Normally, up to 200,000 cubjc feet of LLW is received. Receipt and disposal wit1 be in accordance with the DOE/RL 88/20, "Low-Level Burial Ground Dangerous Waste Permit Application," Revision 0 , December 1989 (including supplemental submitta1s); the "Hanford Site Sol1d Waste Acceptance Criteria," WHC-EP-0063-4, and WFC-5D-WM-ISB-002, "Solid Waste Burial Ground Interim Safety Basis," (Draft). Washington State Department of Ecology issued compliance orders with penalties wi11 be used to determine compliance.

Description of what constitutes completion of this railestone: Receipt and disposition of $100 \%$ of the Low Leve1 Waste (LLW) offered by the generators constitutes completion of this milestone.

\begin{tabular}{|lc|lc|}
\hline Cost Account Manager & Date & Program/Project Manager & Date \\
\hline Program Element Manager & Date & DDE Monjtor & Date \\
\hline
\end{tabular}


Title: Recefve and Dispose up to 200,000 Cubic Feet of LowLevel Waste

Assigned To: P. L. Hapke

Program WBS Designator: RL 1.2.1 CIN: N/A

Control Number: ML4-98-701

MILESTONE TYPE:

$\square$ DOE-HQ

$X$ DOE-RL

[] CONTRACTOR

\begin{tabular}{|c|c|}
\hline DIVISION: & DEL IVERABLE: \\
\hline $\begin{array}{l}\square \text { State } \\
\square \text { Federa] } \\
\text { X DOE } \\
\square \text { RCRA } \\
\square \text { TPA Number }\end{array}$ & $\begin{array}{l}\square \text { Report } \\
\square \text { Letter } \\
\square \text { Drawings } \\
x \text { other (specify) }\end{array}$ \\
\hline
\end{tabular}

Milestone Description: Receive and dispose $100 \%$ of the Low Level baste (LLW) offered by the Generators. Normaliy, up to 200,000 cubic feet of LLW is received. Receipt and djsposal will be in accordance with the DOE/RL 88/20, "Low-Leve1 Buria] Ground Dangerous Waste. Permit Appitcation," Revision 0, December 1989 (including supplemental submittals); the "Hanford Site Solid Waste Acceptance Criteria," WHC-EP-0063-4, and WHC-SD-WH-ISB-002, "Solid Waste Burial Ground Interim Safety Basis," (Draft). Washington State Department of EcoTogy issued compliance orders with penalties will be used to determine compliance.

Description of what constitutes completion of this milestone: Receipt and . disposition of $100 \%$ of the Low Level Waste (LIW) offered by the generators constitutes comp?etton of this milestone.

\begin{tabular}{|l|lll|}
\hline Cost Account Manager & Date & Progran/Project Manager & Date \\
\hline Program Element Manager & Date & DOE Monjtor & Date \\
\hline
\end{tabular}




\section{SOLID WASTE PROGRAM}

WBS 1.2 .1

FY 1996 Program PTan

HHC-SP-1114, ReV, I

\begin{tabular}{|c|c|c|c|}
\hline \multicolumn{4}{|c|}{$\begin{array}{l}\text { Westinghouse Hanford Company } \\
\text { MILESTOME DESCRIPTION SHEET }\end{array}$} \\
\hline \multicolumn{3}{|c|}{$\begin{array}{l}\text { Title: Receive Up to } 3,500 \text { Cubic Feet of TRU Waste Drums for } \\
\text { Storage }\end{array}$} & Date: $08 / 31 / 95$ \\
\hline \multicolumn{3}{|c|}{ Assigned To: P. L. Hapke } & CIN: N/A \\
\hline \multicolumn{3}{|c|}{ Program WBS Designator: RL 1.2.1 } & Due Date: 09/30/96 \\
\hline \multicolumn{3}{|c|}{ Control Number: ML5-96-101 } & Rev: 0 \\
\hline $\begin{array}{l}\text { MILESTDNE TYPE: } \\
\text { } \square \text { DOE-HQ } \\
X \text { DOE-RL } \\
\square \text { CONTRACTOR }\end{array}$ & $\begin{array}{l}\text { DIVISION: } \\
\text { State } \\
\text { Federal } \\
\text { X DOE } \\
\text { RCRA } \\
\square \text { TPA Number }\end{array}$ & $\begin{array}{l}\quad \text { DELIVERABLE: } \\
\square \text { Report } \\
\square \text { Letter } \\
\square \text { Orawings } \\
X \text { Other (specify) } \\
\text { SHS/PTS Report }\end{array}$ & $\begin{array}{l}\text { ADORESS T0: } \\
\square \text { DOE-HQ } \\
\text { X DOF-RL } \\
\square \text { Other (specify) }\end{array}$ \\
\hline \multicolumn{4}{|c|}{ 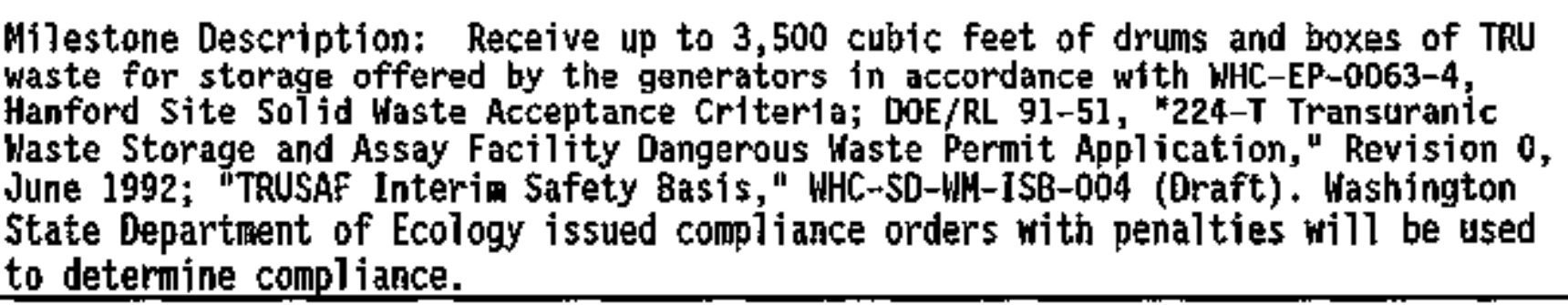 } \\
\hline \multicolumn{4}{|c|}{$\begin{array}{l}\text { Description of what constitutes completion of this milestone: Recejpt into the } \\
\text { Transuranic Storage and Assay Facility (TRUSAF) 100\% of the transuranjc drums } \\
\text { offered by the Generators. }\end{array}$} \\
\hline \multicolumn{2}{|c|}{ Cost Account Manager Date } & \multicolumn{2}{|c|}{ Program/Project Manager Date } \\
\hline \multicolumn{2}{|c|}{ Program Element Manager } & \multicolumn{2}{|c|}{ DOE Monitor } \\
\hline
\end{tabular}


WHC-SP-1114, Rev. I

\begin{tabular}{|c|c|c|c|}
\hline \multicolumn{4}{|c|}{$\begin{array}{l}\text { West inghouse Hanford Company } \\
\text { MILESTONE DESCRIPTION SHEET }\end{array}$} \\
\hline \multicolumn{3}{|c|}{$\begin{array}{l}\text { Title: Receive Up to } 3,500 \text { cubic Feet of TRU Waste Drums for } \\
\text { Storage }\end{array}$} & Date: $08 / 31 / 95$ \\
\hline \multicolumn{3}{|c|}{ Assigned To: P. L. Hapke } & CIN: N/A \\
\hline \multicolumn{3}{|c|}{ Program WES Designator: RL 1.2.1 } & Due Date: $09 / 30 / 97$ \\
\hline \multicolumn{3}{|c|}{ Control Number: HL5-97-101 } & Rev: 0 \\
\hline $\begin{array}{l}\text { MILESTONE TYPE: } \\
\square \text { DOE-HQ } \\
X \text { DOE-RL } \\
\square \text { CONTRACTOR }\end{array}$ & $\begin{array}{l}\quad \text { DIVISION: } \\
\text { State } \\
\text { SEdera] } \\
\times \text { DOE } \\
\text { RCRA } \\
\square \text { TPA Nupber }\end{array}$ & $\begin{array}{l}\text { DELIVERABLE: } \\
\square \text { Report } \\
\square \text { Letter } \\
\text { Drawings } \\
\text { X Other (specify) } \\
\text { SMS /PTS Report }\end{array}$ & $\begin{array}{l}\text { ADORESS TO: } \\
\square \text { DOE-HQ } \\
X \text { DOE-RL } \\
\square \text { other (specify) }\end{array}$ \\
\hline \multicolumn{4}{|c|}{ 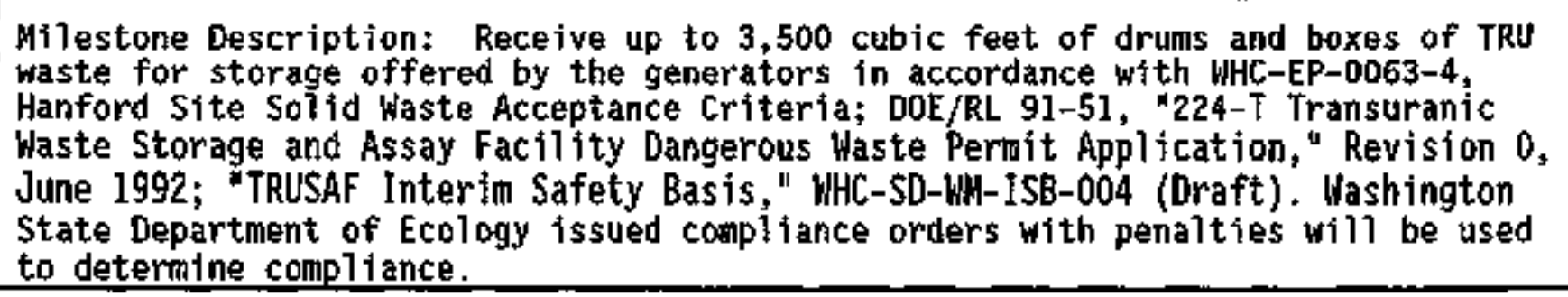 } \\
\hline \multicolumn{4}{|c|}{$\begin{array}{l}\text { Description of what constitutes completion of this milestone: Receipt finto the } \\
\text { Transuranic Storage and Assay Facility (TRUSAF) } 100 \% \text { of the transuranic drums } \\
\text { offered by the Generators. }\end{array}$} \\
\hline \multicolumn{2}{|c|}{ Cost Account Manager Date } & \multicolumn{2}{|c|}{ Program/Project Manager $\quad$ Date } \\
\hline \multicolumn{2}{|c|}{ Program Element Manager } & DOE Monitor & Datee \\
\hline
\end{tabular}


WHC-SP-1114, Rev. I

\begin{tabular}{|c|c|c|c|}
\hline \multicolumn{4}{|c|}{$\begin{array}{l}\text { Westinghouse Hanford Company } \\
\text { MILESTONE DESCRIPTION SHEET }\end{array}$} \\
\hline \multicolumn{3}{|c|}{$\begin{array}{l}\text { Tftle: Receive Up to } 3,500 \text { Cubic Feet of TRU Haste Drums for } \\
\text { Storage }\end{array}$} & Date: $08 / 31 / 95$ \\
\hline \multicolumn{3}{|c|}{ Assigned To: P. L, Hapke } & CIN: N/A \\
\hline \multicolumn{3}{|c|}{ Program WBS Designator: RL 1.2.1 } & Due Date: $09 / 30 / 98$ \\
\hline \multicolumn{3}{|c|}{ Contro] Number: ML5-98-101 } & Rev: 0 \\
\hline $\begin{array}{l}\text { MILESTONE TYPE: } \\
\square \text { DOE-HQ } \\
X \text { DOE-RL } \\
\square \text { CONTRACTOR }\end{array}$ & $\begin{array}{l}\quad \text { DIVISION: } \\
\text { State } \\
\text { Federal } \\
X \text { DOE } \\
\text { RCRA } \\
\text { TPA Number }\end{array}$ & $\begin{array}{l}\quad \text { DELIVERABLE: } \\
\square \text { Report } \\
\square \text { Letter } \\
\square \text { Drawings } \\
X \text { Other (specify) } \\
\text { SMS/PTS Report }\end{array}$ & $\begin{array}{l}\text { ADDRESS TO: } \\
\square \text { DOE-HQ } \\
X \text { DOE-RL } \\
\square \text { 0ther (specify) }\end{array}$ \\
\hline \multicolumn{4}{|c|}{ 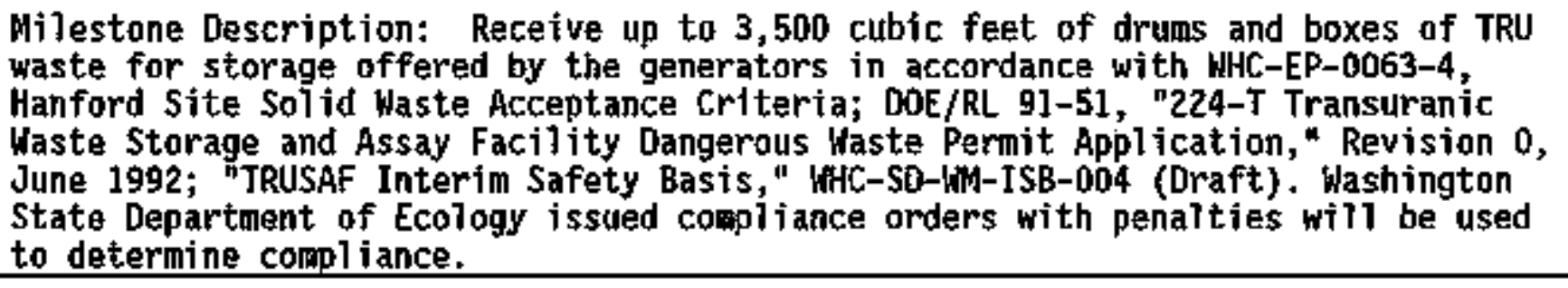 } \\
\hline \multicolumn{4}{|c|}{$\begin{array}{l}\text { Description of what constitutes completion of this milestone: Receipt into the } \\
\text { Transuranic Storage and Assay Facility (JRUSAF) } 100 \% \text { of the transuranic drums } \\
\text { offered by the Generators. }\end{array}$} \\
\hline \multicolumn{2}{|c|}{ Cost Account Manager Date } & \multicolumn{2}{|c|}{ Program/Project Manager Date } \\
\hline \multicolumn{2}{|c|}{ Program EJement Manager } & \multicolumn{2}{|l|}{ DOE Monitor } \\
\hline
\end{tabular}




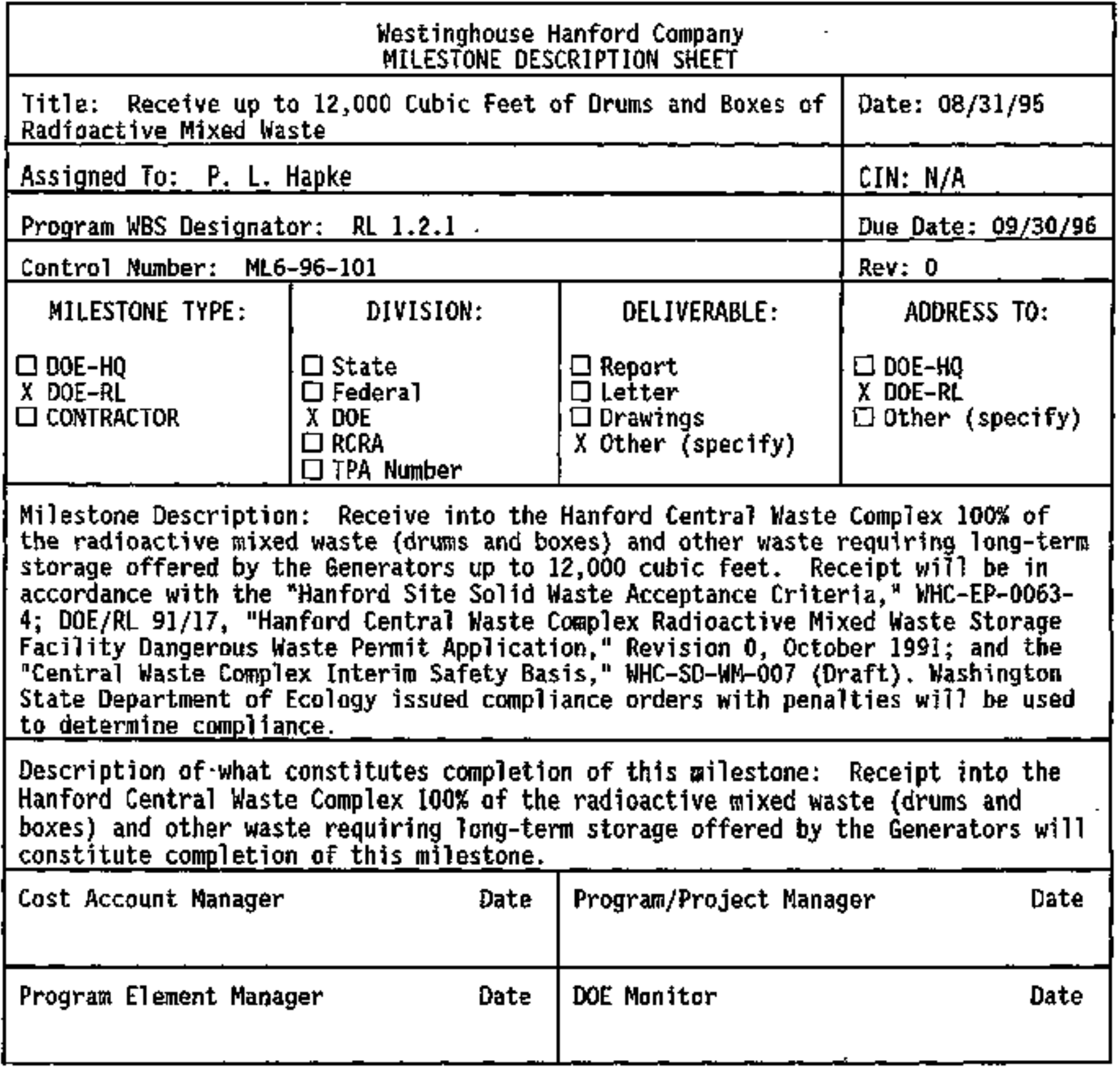




\begin{tabular}{|c|c|c|}
\hline \multicolumn{3}{|c|}{$\begin{array}{l}\text { Westinghouse Hanford Company } \\
\text { HILESTONE DESCRIPTION SHEET }\end{array}$} \\
\hline \multicolumn{2}{|c|}{$\begin{array}{l}\text { Title: Recefve up to } 12,000 \text { Cubic Feet of Drums and Boxes of } \\
\text { Radioactive Mixed Waste }\end{array}$} & Date: $08 / 31 / 95$ \\
\hline \multicolumn{2}{|l|}{ Assigned To: P. 1. Hapke } & CIN: $N / A$ \\
\hline \multicolumn{2}{|l|}{ Program WBS Designator: RL 1.2.1 } & Due Date: $09 / 30 / 97$ \\
\hline \multicolumn{2}{|l|}{ Control Humber: HL6-97-10] } & Rev: 0 \\
\hline 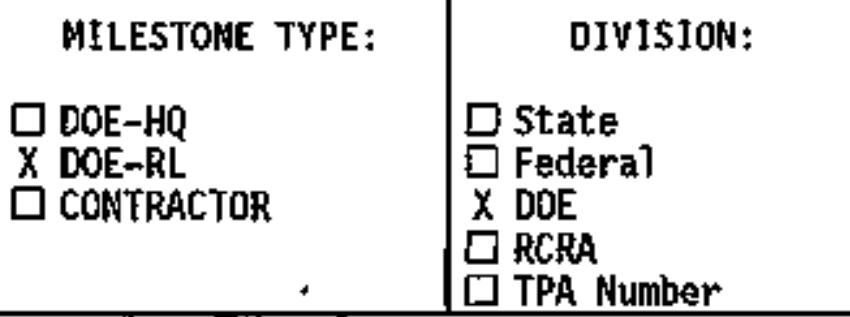 & $\begin{array}{l}\text { DEL IVERABLE: } \\
\square \text { Report } \\
\square \text { Letter } \\
\square \text { Orawings } \\
\square \text { other (specify) }\end{array}$ & $\begin{array}{l}\text { ADDRESS TO: } \\
\square \text { DOE-HQ } \\
\times \text { DOE-RL } \\
\square \text { Other (specify) }\end{array}$ \\
\hline \multicolumn{3}{|c|}{ 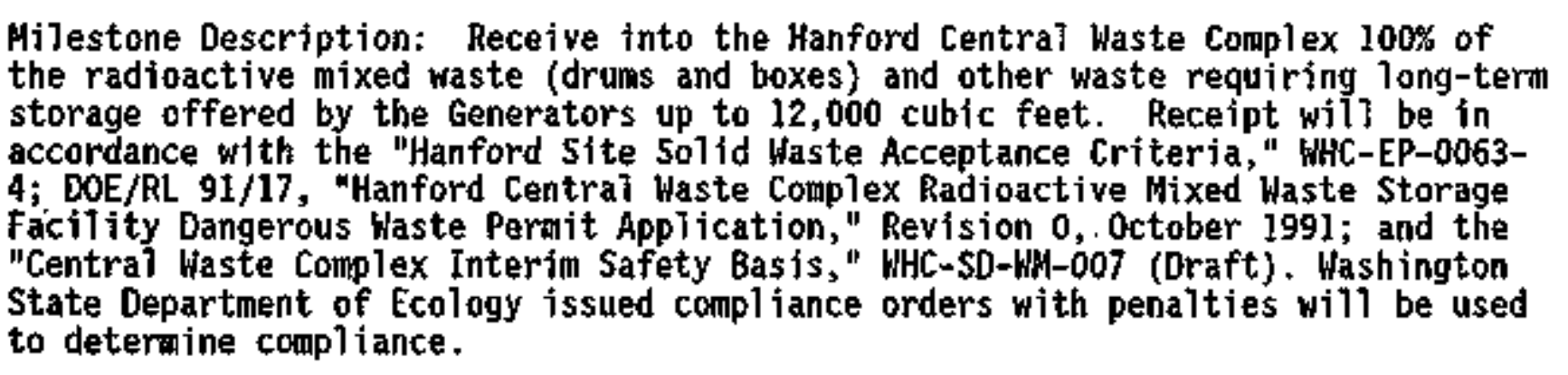 } \\
\hline \multicolumn{3}{|c|}{$\begin{array}{l}\text { Description of what constitutes completton of this mtlestone: Receipt into the } \\
\text { Hanford Centrat Waste Complex } 100 \% \text { of the radioactive mixed waste (drums and } \\
\text { boxes) and other waste requiring long-term storage offered by the Generators will } \\
\text { constitute completion of this milestone. }\end{array}$} \\
\hline Cost Account Manager & \multicolumn{2}{|c|}{ Program/Project. Manager . Date } \\
\hline Program Element Manager & DOE Monitor & Date \\
\hline
\end{tabular}




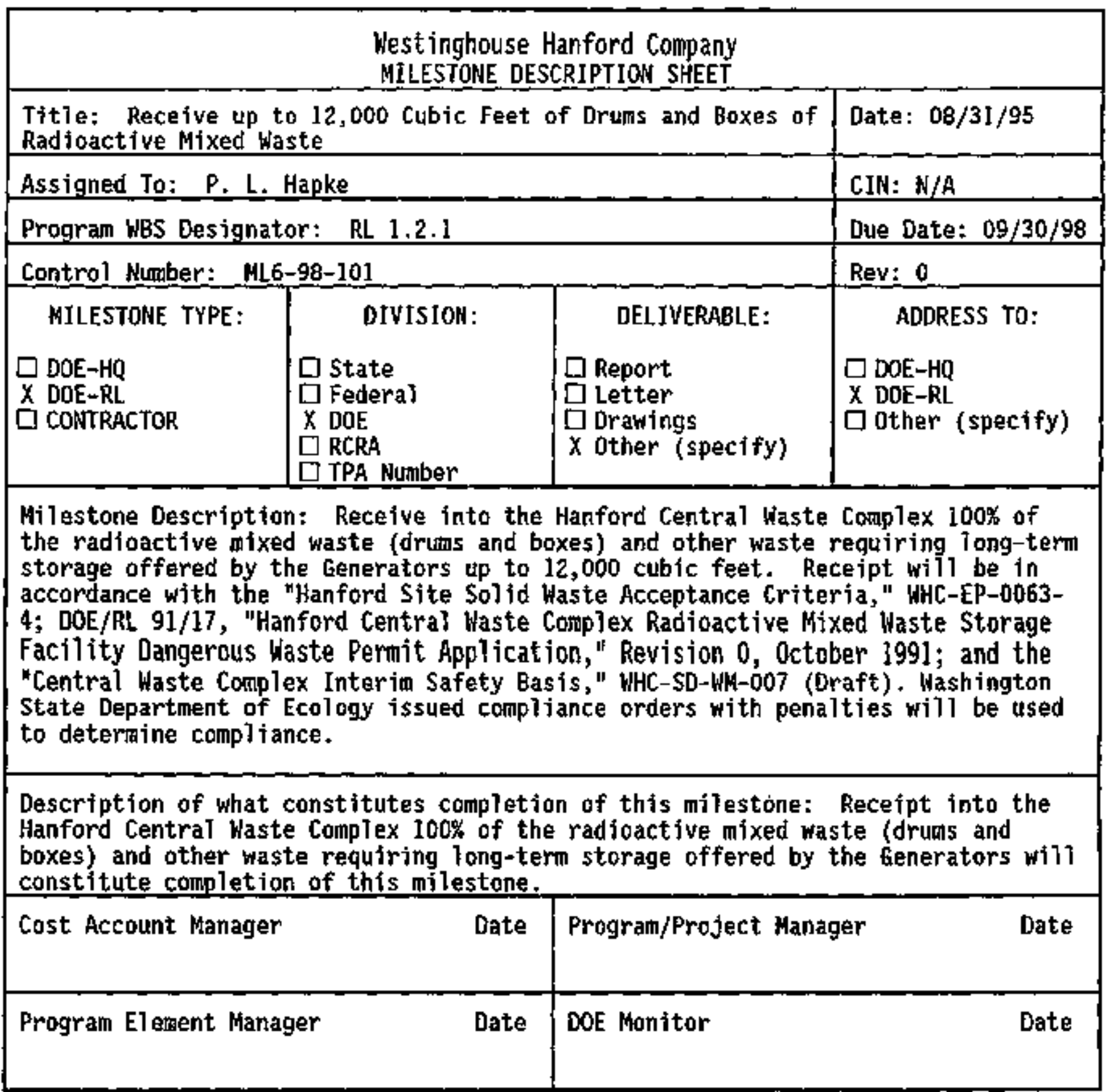




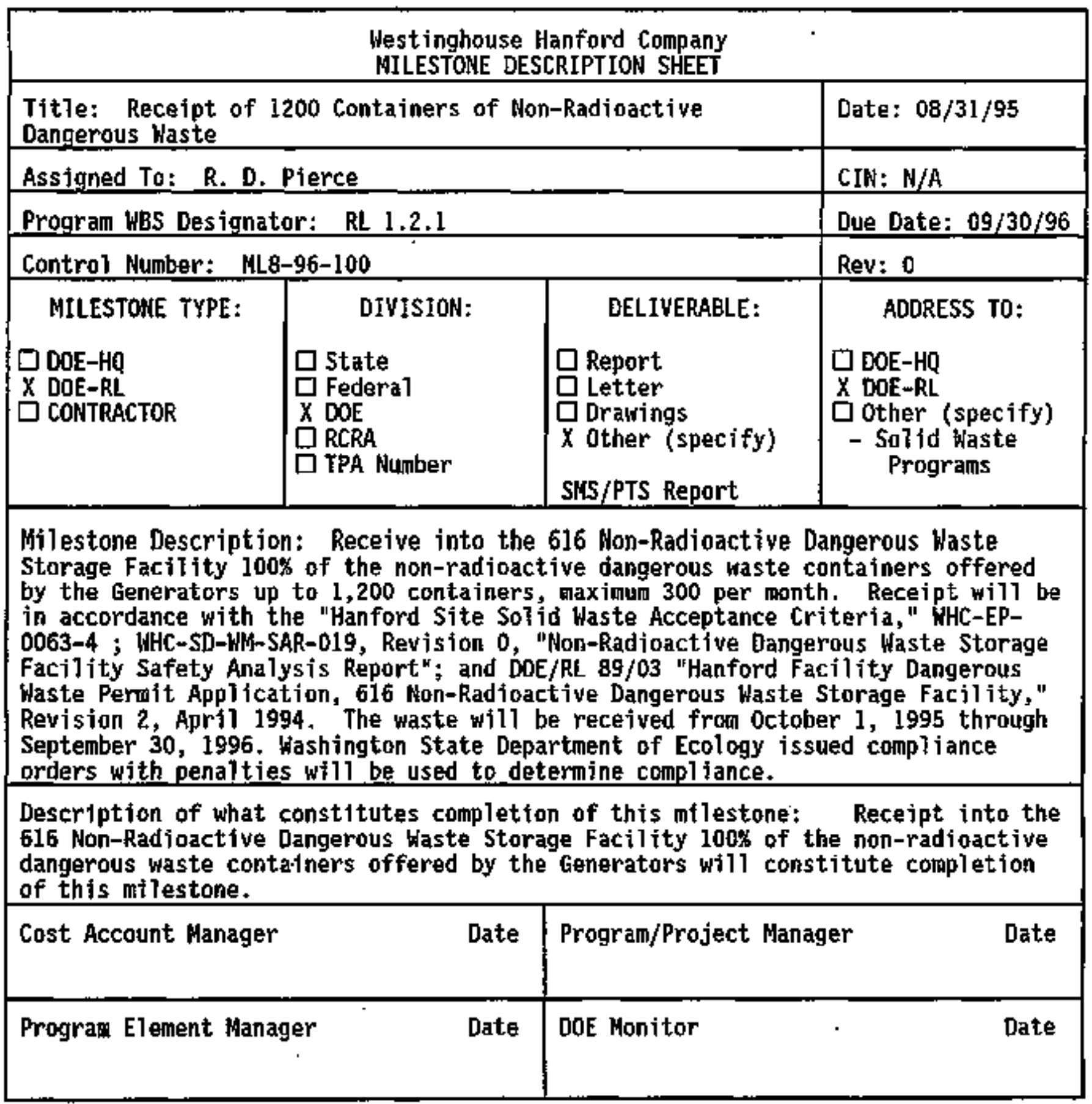




\section{C Cost Baselíne}

This section of the Fiscal Year 1996 Solid Haste Program Hulti-Year Program Plan contajns the following elements:

- 2.C.1 - Cost Baseline Summary By Year

- 2.C.2 - Basis of Estimate

- 2.C.3 - Pianned Staffíng Profíles

- 2.C.4 - Performance Measures 


\begin{tabular}{|c|c|c|c|c|c|c|c|c|}
\hline \multicolumn{9}{|c|}{ Cost Baseline summary ( $s$ in Thousands) } \\
\hline $\begin{array}{l}\text { Program } \\
\text { Element/ } \\
\text { ADS\# } \\
\end{array}$ & $\begin{array}{l}\text { Fund Type } \\
\text { (OP, CE, } \\
\text { GPP, LI) } \\
\end{array}$ & $\begin{array}{l}\text { FY95 SMS } \\
\text { Guidance }\end{array}$ & FY96 Plan & FY97 Ptan & FY98 Plan & Fy99 P1an & $\begin{array}{l}\text { FY2000 } \\
\text { Plan }\end{array}$ & $\begin{array}{l}\text { Fyzool } \\
\text { Plan }\end{array}$ \\
\hline $\begin{array}{l}\text { SOLIS } \\
\text { WASTE (A1) }\end{array}$ & OP & 25,280 & 33,575 & 23,462 & 20,623 & 20,433 & 23,663 & 29,253 \\
\hline \multirow[t]{3}{*}{$2200-0$} & CE & 1,581 & 754 & 615 & 500 & 590 & 360 & 160 \\
\hline & GPP & 351 & 300 & 350 & 200 & 300 & 300 & 210 \\
\hline & LI & & & & & & & \\
\hline $\begin{array}{l}\text { WASTE/ } \\
\text { DECON (A3) }\end{array}$ & $\mathrm{OP}$ & 25,524 & 21,384 & 18,676 & 15,850 & 15,850 & 15,850 & 15,850 \\
\hline \multirow[t]{3}{*}{$2320-0$} & CE & 363 & 150 & 1,224 & 150 & 150 & 150 & 150 \\
\hline & GPP & 836 & 1,000 & & & & & \\
\hline & LI & & & & & & & \\
\hline & & & & & & & & \\
\hline $\begin{array}{l}\text { WRAP } 1 \\
(A 4)\end{array}$ & $\mathrm{OP}$ & 4,866 & 11,275 & 9,400 & 10,377 & 10,377 & 10,377 & 10,377 \\
\hline \multirow[t]{3}{*}{$2220 \mathrm{~m} 1$} & $\mathrm{CE}$ & & & 500 & 500 & 500 & 500 & 500 \\
\hline & $\mathrm{GPP}$ & & & & & & & \\
\hline & LI & 3,603 & & & & & & \\
\hline $\begin{array}{l}\text { WRAP } 2 A \\
\text { (A5) }\end{array}$ & OP & 546 & & & & & & \\
\hline $2230-1$ & L.I & 200 & & & & & & \\
\hline
\end{tabular}




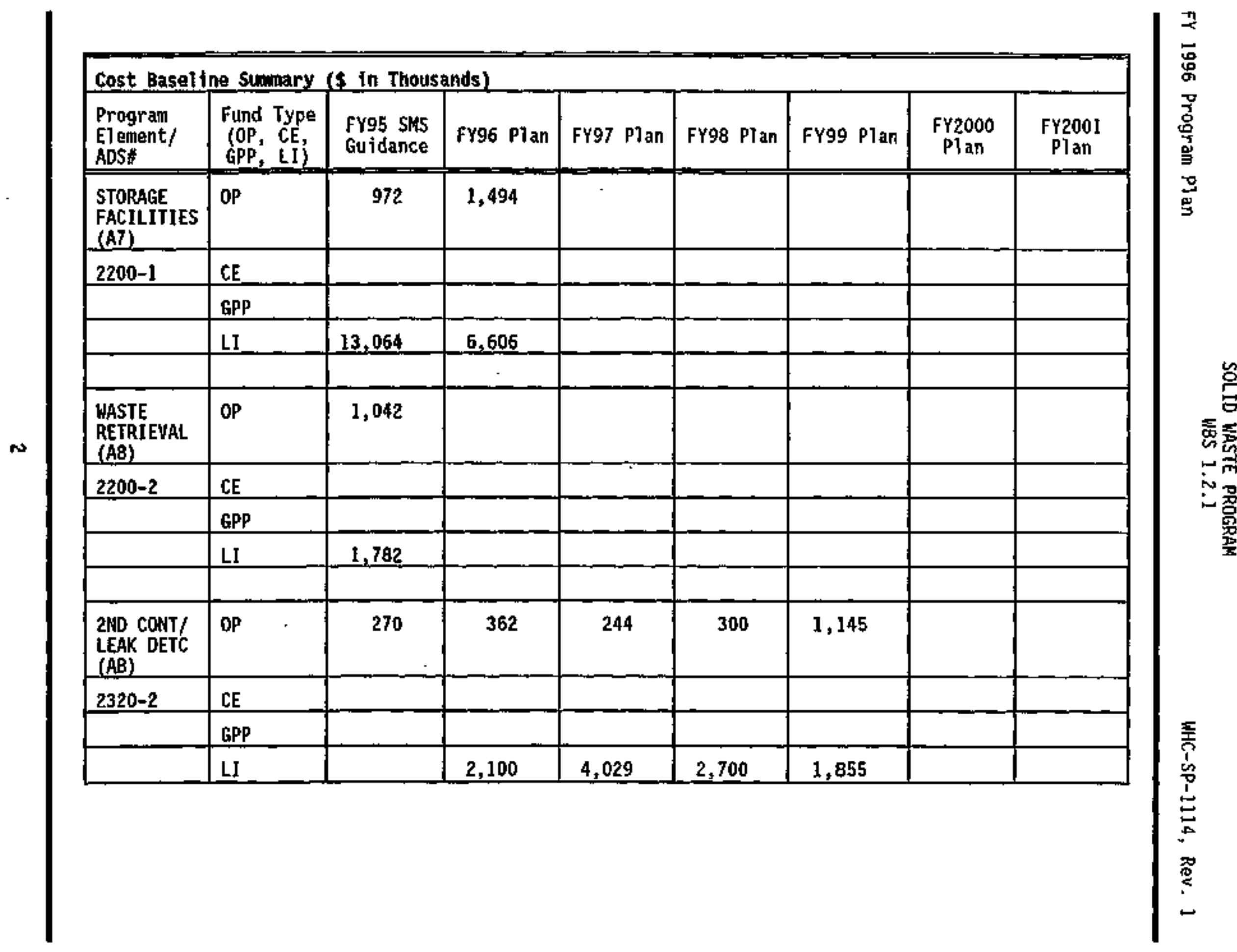




\begin{tabular}{|c|c|c|c|c|c|c|c|c|}
\hline $\begin{array}{l}\text { Progral } \\
\text { Element/ } \\
\text { ADS\# }\end{array}$ & $\begin{array}{l}\text { Fund Type } \\
\text { (OP, CE, } \\
\text { GPP, LI) }\end{array}$ & $\begin{array}{l}\text { Frg5 SMS } \\
\text { Guidance }\end{array}$ & FY96 Plan & FY97 Plan & FY98 Plan & Fy9g Pian & $\begin{array}{c}\text { Fy2000 } \\
\text { plan }\end{array}$ & $\begin{array}{c}\text { FY2001 } \\
\text { PTan }\end{array}$ \\
\hline \multirow[t]{4}{*}{ SUB-TOTAL, } & $\mathrm{OP}$ & 58,500 & 68,090 & 51,782 & 47,150 & 47,805 & 49,890 & 55,480 \\
\hline & CE & 1,944 & 904 & 2,339 & 1,150 & 1,240 & 1,010 & 810 \\
\hline & GPP & 1,187 & 1,300 & 350 & 200 & 300 & 300 & 210 \\
\hline & LI & 18,649 & 8,706 & 4,029 & 2,700 & 1,855 & & \\
\hline CARRYOVER & $O P$ & 2,912 & 6,333 & & & & & \\
\hline \multirow[t]{4}{*}{ TOTAL $(A)$} & $\mathrm{OP}$ & 61,412 & 74,423 & 51,782 & 47,150 & 47,805 & 49,890 & 55,480 \\
\hline & CE & 1,944 & 904 & 2,339 & 1,150 & 1,240 & 1,010 & 810 \\
\hline & GPP & 1,187 & 1,300 & 350 & 200 & 300 & 300 & 210 \\
\hline & LI & 18,649 & 8,706 & 4,029 & 2,700 & 1,855 & & \\
\hline MDDOP & $\mathrm{OP}$ & 475 & 286 & 295 & 303 & 302 & 311 & 320 \\
\hline ML. & $0 p$ & 12,541 & 8,996 & 8,323 & 8,558 & 9,307 & 9,534 & 9,820 \\
\hline
\end{tabular}

MOTE: The FY97-2002 cost information for MDOOP and ML is based upon Financial Data System rates as of September 12, 1995. 


\section{SOLID NASTE PROGRAM}

NBS 1.2.1

FY 1996 Program Plan

WHC-SP-1114, Rey, 1

\section{C.2 Basjs of Estimate}

The record copy of the Basis of Estimate for the Solid Waste Program resides with the appropriate Program Cognizant Engineer 7ocated in M0278/200 West Area. 


\begin{tabular}{|c|c|c|c|c|c|c|c|c|}
\hline \multicolumn{9}{|c|}{ Planned Staffing (Full Time Equivalent) } \\
\hline Job Family & Nutiber & & & & & & & \\
\hline Job Category & & 1996 & 1997 & 1998 & 1999 & 2000 & 2001 & 2002 \\
\hline \multicolumn{9}{|l|}{ MANAGERS } \\
\hline First Line & 1010 & 26.6 & 21.7 & 19.7 & 18.7 & 18.6 & 18.5 & 19.1 \\
\hline General/Execut jve & M020 & 18.2 & 16.8 & 15.4 & 15.4 & 15.6 & 15.4 & 15.9 \\
\hline Project/Progran & M030 & 2.7 & 2.2 & 1.6 & 1.5 & 1.0 & 1.0 & 1.0 \\
\hline $\begin{array}{l}\text { Project Program } \\
\text { Engineers }\end{array}$ & M040 & 2.2 & .3 & .3 & 1.0 & .2 & .2 & .2 \\
\hline Subtotal & & 49.8 & 41.0 & 37.0 & 36.6 & 35.4 & 35.1 & 36.2 \\
\hline \multicolumn{9}{|l|}{ ENGTNEERS } \\
\hline Chemical & $\mathrm{E} 010$ & 20.6 & 16.1 & 10.8 & 10.6 & 10.8 & 10.7 & 10.7 \\
\hline Civil & $\mathrm{E} 020$ & 4.0 & 2.4 & 1.2 & 1.3 & 1.3 & 1.3 & 1.3 \\
\hline Computer & E030 & 3.1 & .6 & & & & & \\
\hline Electrical & $E 040$ & 7.4 & 5.3 & 3.7 & 3.6 & 3.4 & 3.2 & -3.0 \\
\hline Environmental & $E 050$ & 19.1 & 13.6 & 11.8 & 12.3 & 12.9 & 14.9 & 17.1 \\
\hline Industria] & $E 060$ & .9 & 1.0 & 1.0 & .9 & 1.0 & 1.0 & 1.0 \\
\hline Mechantical & $\mathrm{E} 070$ & 23.2 & 14.5 & 13.7 & 13.9 & 13.9 & 13.8 & 13.7 \\
\hline Nuclear & $E 080$ & 3.2 & 1.2 & 1.3 & 1.3 & 1.5 & 1.5 & 1.5 \\
\hline Plant & $E 100$ & 40.1 & 33.9 & 28.7 & 28.3 & 29.3 & 29.0 & 28.8 \\
\hline Quality Control & E110 & 6.8 & 3.9 & 3.2 & 3.1 & 3.5 & 3.5 & 4.0 \\
\hline Safety & E120 & 11.9 & 9.6 & 8.7 & 8.9 & 9.1 & 9.0 & 11.7 \\
\hline Dther & $\mathrm{E} 130$ & 60.8 & 38.7 & 25.7 & 30.5 & 25.8 & 26.1 & 32.1 \\
\hline Subtotal & & 201.1 & 140.8 & 109.8 & 114.7 & 112.5 & 114.0 & 124.9 \\
\hline
\end{tabular}




\begin{tabular}{|c|c|c|c|c|c|c|c|c|}
\hline \multicolumn{9}{|c|}{ Planned Staffing (Full Time Equivalent) } \\
\hline Job $\tan 41 \mathrm{y}$ & Musber & & & & & & & \\
\hline Job Category & & 1996 & 1997 & 1998 & 1999 & 2000 & 2001 & 2002 \\
\hline \multicolumn{9}{|l|}{ SCIEMTISTS } \\
\hline Chemist & 5010 & 3.5 & 2.0 & 1.8 & 1.4 & 1.5 & 1.5 & 1.5 \\
\hline Environmental & S020 & 14.3 & 10.8 & 9.7 & 9.8 & 10.5 & 10.5 & 10.4 \\
\hline Geologist & so30 & 1.1 & 1.1 & .9 & .4 & .4 & .4 & .4 \\
\hline Life Sctentists & SO40 & .6 & & & & & & \\
\hline other & sogo & 2.7 & .4 & .4 & .4 & .5 & .5 & .5 \\
\hline Subtotal & & 22.3 & 14.3 & 12.8 & 12.0 & 12.9 & 12.9 & 12.8 \\
\hline \multicolumn{9}{|l|}{$\begin{array}{l}\text { ADNINISTRATOR/ } \\
\text { OTHER } \\
\text { PROFESSIONALS }\end{array}$} \\
\hline Accountant/Auditor & P010 & 8.7 & 6.2 & 6.0 & 5.8 & 5.6 & 5.6 & 5.7 \\
\hline Buyer/Procurement & P030 & 1.2 & 1.0 & 1.0 & 1.0 & 1.0 & 1.0 & 1.0 \\
\hline $\begin{array}{l}\text { Compliance } \\
\text { Inspectors }\end{array}$ & P050 & .3 & & & & & & \\
\hline $\begin{array}{l}\text { Computer Systems } \\
\text { Analyst }\end{array}$ & P060 & 1.4 & 1.3 & 1.5 & 1.5 & 1.5 & 1.5 & 1.5 \\
\hline $\begin{array}{l}\text { Cost Estimator/ } \\
\text { Planner/Scheduler }\end{array}$ & P070 & 25.2 & 20.8 & 20.8 & 21.0 & 21.1 & 20.8 & 22.1 \\
\hline Health Physicist & P080 & 9.3 & 8.3 & 8.9 & 8.7 & 8.7 & 8.9 & 10.1 \\
\hline Industrial Hygiene & $P 090$ & .7 & & .2 & .1 & .2 & 1.1 & 2.3 \\
\hline Attomey & P100 & .1 & .1 & .1 & .1 & .1 & .1 & .1 \\
\hline $\begin{array}{l}\text { Safeguard \& } \\
\text { Security Specialist }\end{array}$ & $\mathrm{P} 140$ & 3.2 & 3.0 & 3.2 & 3.2 & 3.2 & 3.2 & 3.2 \\
\hline Trainer & P150 & 9.1 & 7.3 & 7.4 & 8.2 & 8.2 & 8.1 & 11.8 \\
\hline
\end{tabular}




\begin{tabular}{|c|c|c|c|c|c|c|c|c|}
\hline \multicolumn{9}{|c|}{ Planned Staffing (Full Time Equivalent) } \\
\hline Job Fanjy & Humber. & ? & $\therefore$ & $\therefore$ & 管" & : & & \\
\hline Job Category & & 1996 & 1997 & 1998 & 1999 & 2000 . & 2001 & 2002 \\
\hline $\begin{array}{l}\text { Technical Hriter \& } \\
\text { Editor }\end{array}$ & P160 & .4 & .4 & .4 & .4 & .4 & .4 & .4 \\
\hline other & P170 & 6.5 & 4.3 & 5.2 & 5.2 & 5.4 & 5.3 & 5.5 \\
\hline Subtotal. & & 66.0 & 52.7 & 54.7 & 55.2 & 55.4 & 56.0 & 63.7 \\
\hline $\begin{array}{l}\text { GEHERAL } \\
\text { ADHIHISRATOR/ } \\
\text { SECRETARY/CLERK }\end{array}$ & sind & : & $\sqrt{x}$ & ming & क & & $\begin{aligned} & \ddots \ddots \\
& \therefore \therefore \\
&\end{aligned}$ & \\
\hline $\begin{array}{l}\text { Adninistrative } \\
\text { Assistant }\end{array}$ & 6010 & 1.2 & 1.1 & 1.1 & .9 & .9 & .9 & .9 \\
\hline $\begin{array}{l}\text { Office Clerk } \\
\text { (General) }\end{array}$ & 6020 & 10.7 & 9.8 & 10.6 & 10.6 & 10.5 & 10.2 & 13.6 \\
\hline $\begin{array}{l}\text { Office clerk } \\
\text { (Special ist) }\end{array}$ & 6030 & 2.6 & 2.3 & 2.2 & 2.0 & 2.1 & 2.1 & 2.1 \\
\hline Secretary & G040 & 20.4 & 17.0 & 15.0 & 14.9 & 14.9 & 14.7 & 15.6 \\
\hline Sybtotal & & 35.0 & 30.2 & 28.9 & 28.4 & 28.4 & 27.9 & 32.2 \\
\hline TECHNICIAMS & 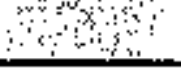 & & $:$ & & $\therefore$ & $\because$ & & \\
\hline $\begin{array}{l}\text { Computer } \\
\text { Operator/Coder }\end{array}$ & Tolo & 1.9 & .4 & 1.8 & 1.8 & 1.8 & 1.8 & 1.8 \\
\hline Drafter & T020 & 3.3 & 2.9 & 2.8 & 2.8 & 2.7 & 2.6 & 2.5 \\
\hline $\begin{array}{l}\text { Engineering } \\
\text { Technician }\end{array}$ & T030 & 5.0 & 4.7 & 4.3 & 4.2 & 4.3 & 4.3 & 4.3 \\
\hline $\begin{array}{l}\text { Hea] th Physics } \\
\text { Technician } \\
\end{array}$ & T050 & 35.9 & 33.4 & 32.8 & 31.4 & 31.4 & 33.1 & 38.8 \\
\hline $\begin{array}{l}\text { Instruments/Control } \\
\text { Technician }\end{array}$ & T070 & 5.4 & 5.8 & 5.1 & 3.7 & 3.6 & 3.5 & 3.4 \\
\hline
\end{tabular}




\begin{tabular}{|c|c|c|c|c|c|c|c|c|}
\hline \multicolumn{9}{|c|}{ Planned Staffing (Full Time Equivalent) } \\
\hline Job Famtly & Number & & & & & & & \\
\hline Job Category & & 1996 & 1997 & 1998 & 1999 & 2000 & 2001 & 2002 \\
\hline other & $\underline{T 110}$ & 2.4 & 1.9 & 1.5 & $\underline{1.3}$ & 1.3 & 1.3 & 1.3 \\
\hline Other Technictans & $T 120$ & .1 & & & & & & \\
\hline Sybtotal & & 53.9 & 49.1 & 48.3 & 45.2 & 45.1 & 46.6 & 52.1 \\
\hline \multicolumn{9}{|l|}{ CRAFTS } \\
\hline Carpenter & $\mathrm{CO10}$ & .5 & .5. & .5 & .5. & .5 & .5 & .5 \\
\hline Electrician & $\mathrm{CO20}$ & 7.4 & 7.3 & 6.9 & 5.1 & 5.0 & 4.9 & 4.8 \\
\hline Machinist & CO40 & .6 & 1.2 & 1.5 & 1.5 & 1.5 & 1.5 & 2.5 \\
\hline Millwright & $\mathrm{co6} 0$ & 3.1 & 2.9 & 2.7 & 2.4 & 2.4 & 2.3 & 2.3 \\
\hline Painter & $\mathrm{co70}$ & 2.7 & $\underline{2.6}$ & 2.5 & 2.5 & 2.6 & 2.6 & 2.6 \\
\hline Plumber/Pipefitter & $\cos 8$ & 4.6 & 4.3 & 3.7 & 3.2 & 3.1 & 3.0 & 2.9 \\
\hline $\begin{array}{l}\text { Structural/Metal } \\
\text { Uarker }\end{array}$ & $\operatorname{coso}$ & 3.2 & 2.8 & 2.5 & 2.4 & 2.3 & 2.3 & 2.3 \\
\hline $\begin{array}{l}\text { Vehicle/Moblle } \\
\text { Equipment Mechanic }\end{array}$ & $\mathrm{C} 100$ & 1.3 & 1.0 & .8 & .8 & 1.1 & 2.3 & 3.3 \\
\hline Helder & $\mathrm{C} 110$ & .5 & .3 & .3 & .3 & .3 & .3 & .3 \\
\hline other & $\mathrm{C120}$ & 8.8 & 8.1 & 9.9 & 9.0 & 8.9 & 9.3 & 11.1 \\
\hline Subtotal & & 33.1 & 31.0 & 31.3 & 27.7 & 27.7 & 29.0 & 32.6 \\
\hline \multicolumn{9}{|l|}{ OPERATORS } \\
\hline $\begin{array}{l}\text { Material Moving } \\
\text { Equipment. }\end{array}$ & $\mathrm{R} 030$ & 1.0 & .5 & .3 & .3 & .3 & .3 & .3 \\
\hline Nuclear Plant & $\mathrm{R} 040$ & 1.4 & 1.2 & .9 & 1.0 & 9 & .9 & .8 \\
\hline $\begin{array}{l}\text { Nuclear Waste } \\
\text { Process }\end{array}$ & R050 & 68.7 & 55.6 & 56.3 & 58.6 & 59.2 & 63.6 & 82.1 \\
\hline
\end{tabular}




\begin{tabular}{|c|c|c|c|c|c|c|c|c|}
\hline \multicolumn{9}{|c|}{ Planned Staffing_(Full Time Equivalent) } \\
\hline Job Fatally & Netaber & & & & & & & \\
\hline Job Category & & 1996 & 1997 & 1998 & 1999 & 2000 & 2001 & 2002 \\
\hline Utilities System & 8070 & .1 & .1 & .1 & .1 & .1 & .1 & .1 \\
\hline Other Operators & $\mathrm{R} 080$ & .4 & & & & & & \\
\hline Subtotal & & 71.7 & 57.4 & 57.6 & 60.0 & 60.5 & 64.9 & 83.3 \\
\hline \multicolumn{9}{|l|}{$\begin{array}{l}\text { LABORERS \& GENERAL } \\
\text { WORKERS }\end{array}$} \\
\hline Firofighter & Lo10 & .4 & .4 & .3 & .3 & .2 & .2 & .1 \\
\hline Janitor/Cleaner & Lozo & 5.2 & 6.2 & 6.6 & 6.7 & 6.6 & 6.6 & 6.8 \\
\hline General Laborer & Loso & 1 & .1 & .1 & .1 & .1 & & \\
\hline $\begin{array}{l}\text { Light Vehicle } \\
\text { Drivers } \\
\end{array}$ & Lo70 & 2.7 & 2.4 & 2.2 & 2.2 & 2.2 & 2.2 & 2.2 \\
\hline Subtotal & & 8.4 & 9.1 & 9.2 & 9.3 & 9.1 & 9.0 & 9.1 \\
\hline TOTAL_FTES & & 541.2 & 425.6 & 389.6 & 389.1 & 387.0 & 395.4 & 446.9 \\
\hline
\end{tabular}

NOTE: The information within this table may vary within one percent of the fiscal year total because of rounding and minor corrections to the data subsequent to the table being generated. 
2.C.4 Performance Measures

Please see Solid Waste Disposal Contract for contract-based performance based initiatives (PBI). 


\section{A. Technical Objectives}

General and specific technical objectives or activities must occur in the execution year to support the Solid Waste Program Mission. The Solid Waste Program is a mature Hanford Site program whose activities have been constant for a number of years and are expected to relatin so. The efficiency and effectiveness with which these activjties have been performed has steadily improved, but the activities themselves have not changed. The technjcal objectives, therefore, are similar to the functions listed in Section 1.B.1, Technical Functions/Requirements. The functions from Section 1.B.1. are repeated here for completeness.

- Maintain a safe and compliant solid waste operational environment by continually assessing and maintaining the solid waste operational environment In a safe and compltant condftion.

- Maintain safe and compliant solid waste systems, equtpment and structures. Continually assess and aintain the solid waste facility systems, equiprent, and structures, and their operations in a safe condition. Majntaín a qualified solid waste staff, and maintain required solid waste facility and operating documentation.

- Maintain safe and compljant solid waste documentation (SARs, OSRs, Waste Acceptance Criteria, etc.). Maintain required sol id waste facility and operating documentation related to safe and compliant operations.

- Provide program control including scheduling, financial analysis, change control preparation, organizational financial/business managenent, programmatic organizational financial/schedule analysis and program/performing organizational business reporting activities. Scheduling activities include fiscal year and outyear development, cost account monjtoring and change control tasks and reporting. Financial activities include fiscal year budget developnent and outyear requirements planning, cost account monitoring and change control tasks including varjance analysis reports and estimate at completion report preparation.

- Retrieve suspect TRU solid waste. This includes retrieval planning, solid waste access and removal, obtaining waste characterfzation samples, generating waste information, estabilishing retrieval capability requirements, and forecasting future retrieval operations. (Currently not funded.)

- Treat soljd waste. Provide for the treatment of solid waste to convert the waste to an acceptable form for final disposttion. (Some activities are restricted by funding Tevels.)

- Inspect container contents of newly generated waste to meet reguirements for waste verification and inspect contents of "unknown" or "backlog" wastes to provide segregation and compliant packaging of waste.

- Store solid waste in a safe and compliant manner prior to shipment to approved treatment and/or dtsposal stte. 


\section{SOLID WASTE PROGRAN \\ WBS 1.2 .1}

- Receive newly generated solid waste generated during Hanford cleanup mission operations and from other missions. Recejpt of new waste includes soljd waste receipt planning, characterization information verification for acceptance, estab]ishing receipt capability requirements, and forecasting future receipt operations.

- Ship solid waste. Prepare the necessary shipment requests and documentation. Coordinate the solid waste package shipment with shippers and receivers to verify that the waste transfer was accomplished. Document the solid waste shipment status.

- Dispose solid waste. Schedule solid waste package disposal and prepare waste transport package and disposal documentation. Provide finai inspection of the solid waste package load and place in disposal site.

- Through a systems engtneering approach develop and maintain baseline documentation including defining the technical baseline of the program and interfaces with other site programs, develop alternatives and trade studies for alaterfals requiring a path-definition for management and disposition (e.g., materials within the scope of TPA Milestone M-33). Provide records management and tracking of solid waste through the Solid Waste Information Tracking Systen (SWITS).

- Process high dose waste and contaniaated equipment to meet applicable standards for disposa], storage, re-use, or free release. The equipment to be processed will include Tank Farms augers and contaminated equipment stored in the $T$ Plant canyon. In addition, other high dose contaminated equipment and waste being stored at site facilities undergoing decommissioning and generated during the site cleanup wili be treated. Treatment of high dose waste and equipment will take place in the 221-T canyon.

- Receive, store, contajn, perforri decontarination and repackage incoming wastes for reduction in radiation fieids and recategorization of wastes from mixed to non-aixed and/or Greater Than Category 3 low-level to Category I or 3 lowlevel or if possible to levels making the waste a releasable (nonradioactive) material.

- Package solid waste. Ensure solid waste is in a container suttable for the waste type. Provide solid waste packaging documentation including packaging certifications and solid waste feed stock traceability. Maintain appropriate labeling and inspection of the containers of solid waste.

- Provide contaminated equipment storage and storage of spent fúc elements at T plant.

- Perform al1 work necessary to maintaín certification of at least one liquid waste tank car.

- Prepare solid waste disposition specifications. Generate speciftcations for solid waste operations including treatment, packaging, certification, storage, shipping, disposition, and archiving samples and records. 
- Disposition solid waste. Provide packaging and shipping, treatment/storage and /or imabitization for packaging and certifying the solid waste, store or ship it as needed, treat and/or tmmobiltze it, and accomplish final disposjtion.

- When necessary identify, inventory, and characterize the waste received from generators by reviewing the process operations (current and historical), by reviewing new waste information and by sampling and analyzing the waste inventory. Assess the current condjtion of the waste. Archive the results of these investigations. (5ome activities are restricted by funding ievels.)

- Assess solid waste characterization information. Evaluate the solid waste characterization information consisting of generator request inforation, process knowledge information, and waste sample characterization data, against the solid waste disposition requirements and provide validation of meeting the disposition requirements.

- Determtne solid waste disposition requirements by compiling and sorting inposed requirements, defining requirements that result from proposed solutions and required capabilities, and assessing constraints under which the function must operate.

- Certify and verify solid waste. Certify that solid waste is treated according to the disposition requirements and that the documentation is in order to al]ow the solid waste package to be disposftioned.

- Assess the solid waste treaturent, storage, and disposal (TSO) capabitity needs and resources based on requjred versus design throughput and capacities.

- Provide archival storage and retrieval operations for the analyzed solid waste process sample analysis data. 
WHC - SOLIO WASTE PROGRAM FY 1996 SCHEDULE 1A1A0I - DOE PROGRAM SUPPORT

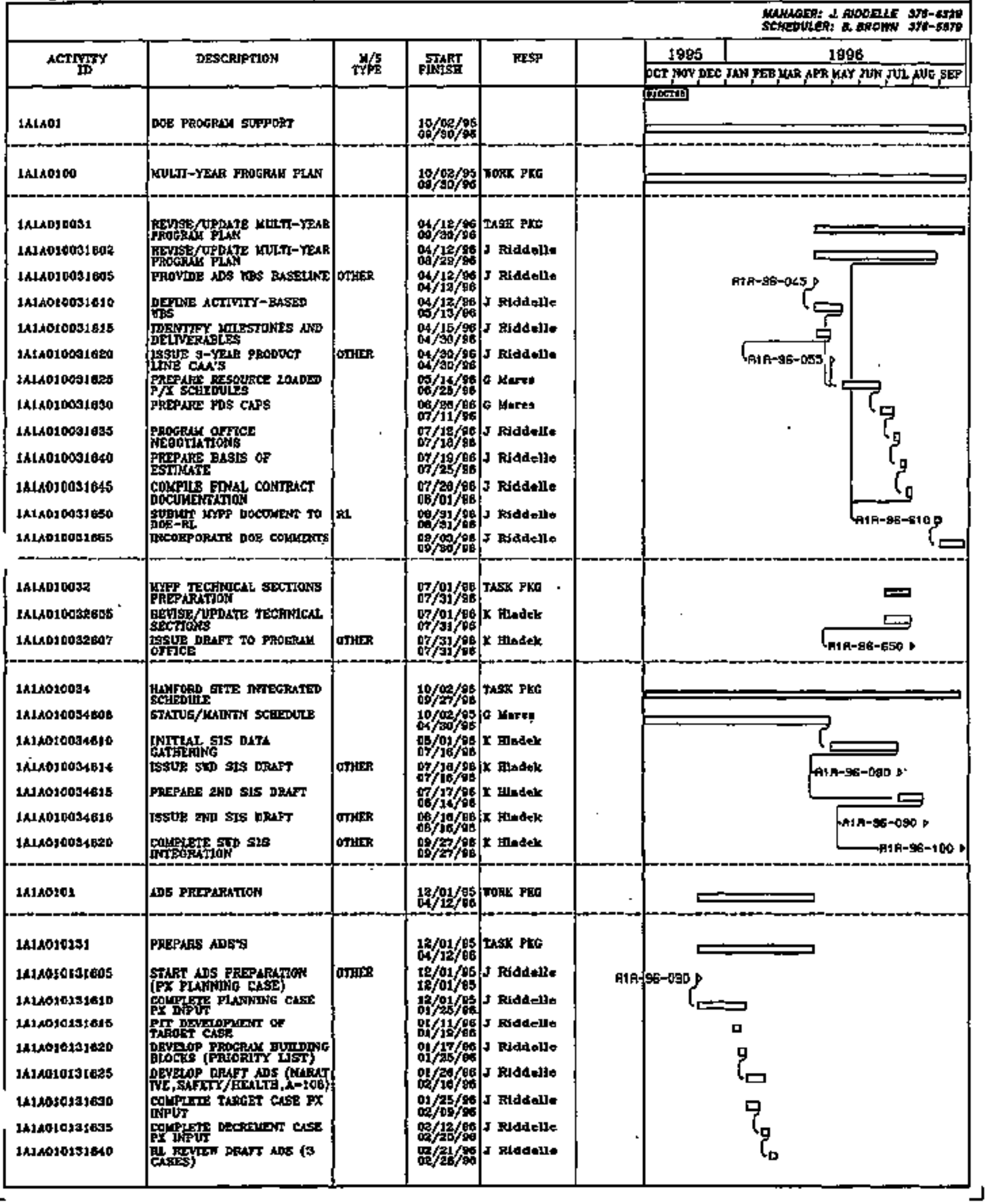




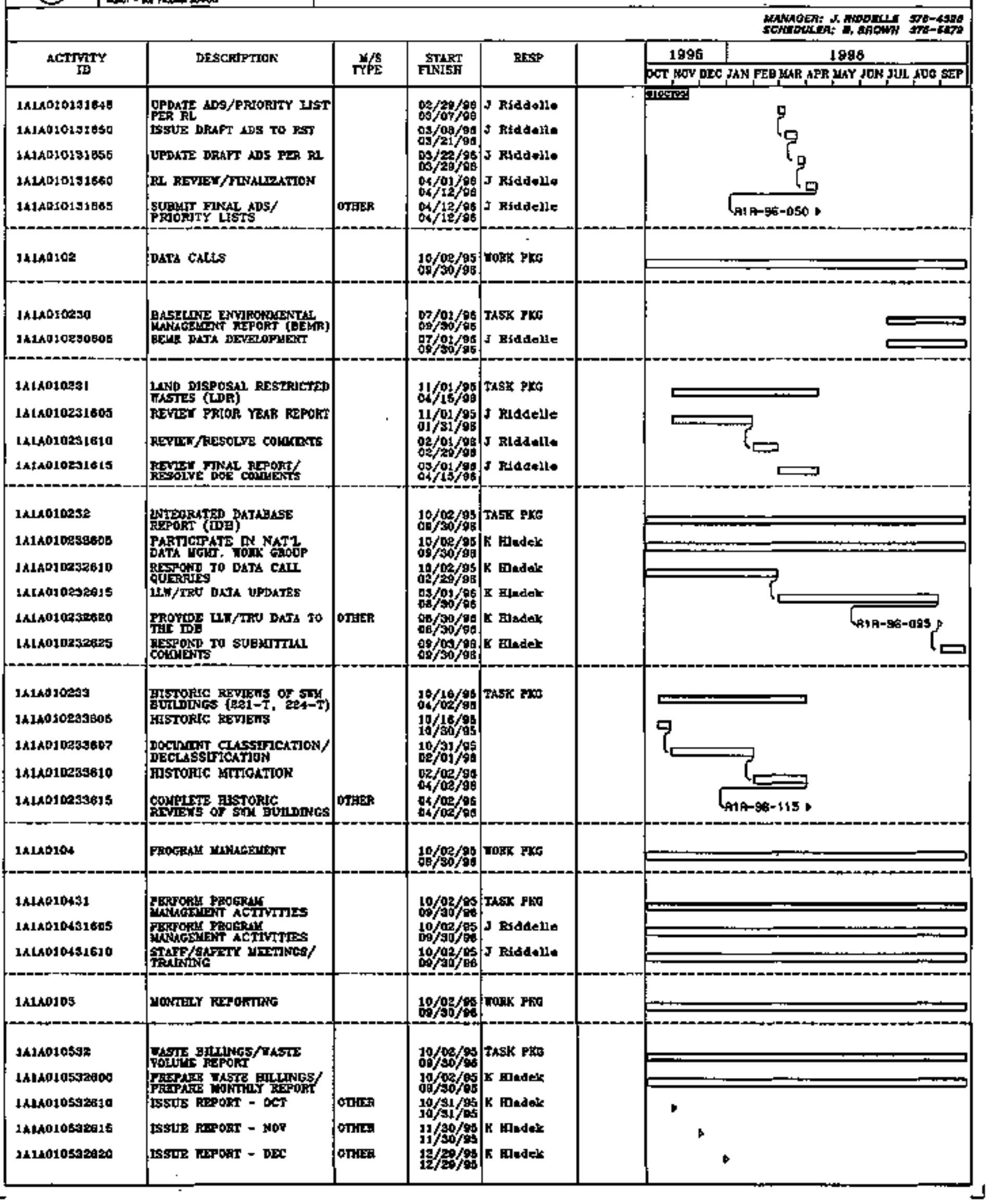


WHC - SOLID WASTE PROGRAM FY 1996 SCHEDULE 1A1A01 - DOE PROGRAM SUPPORT

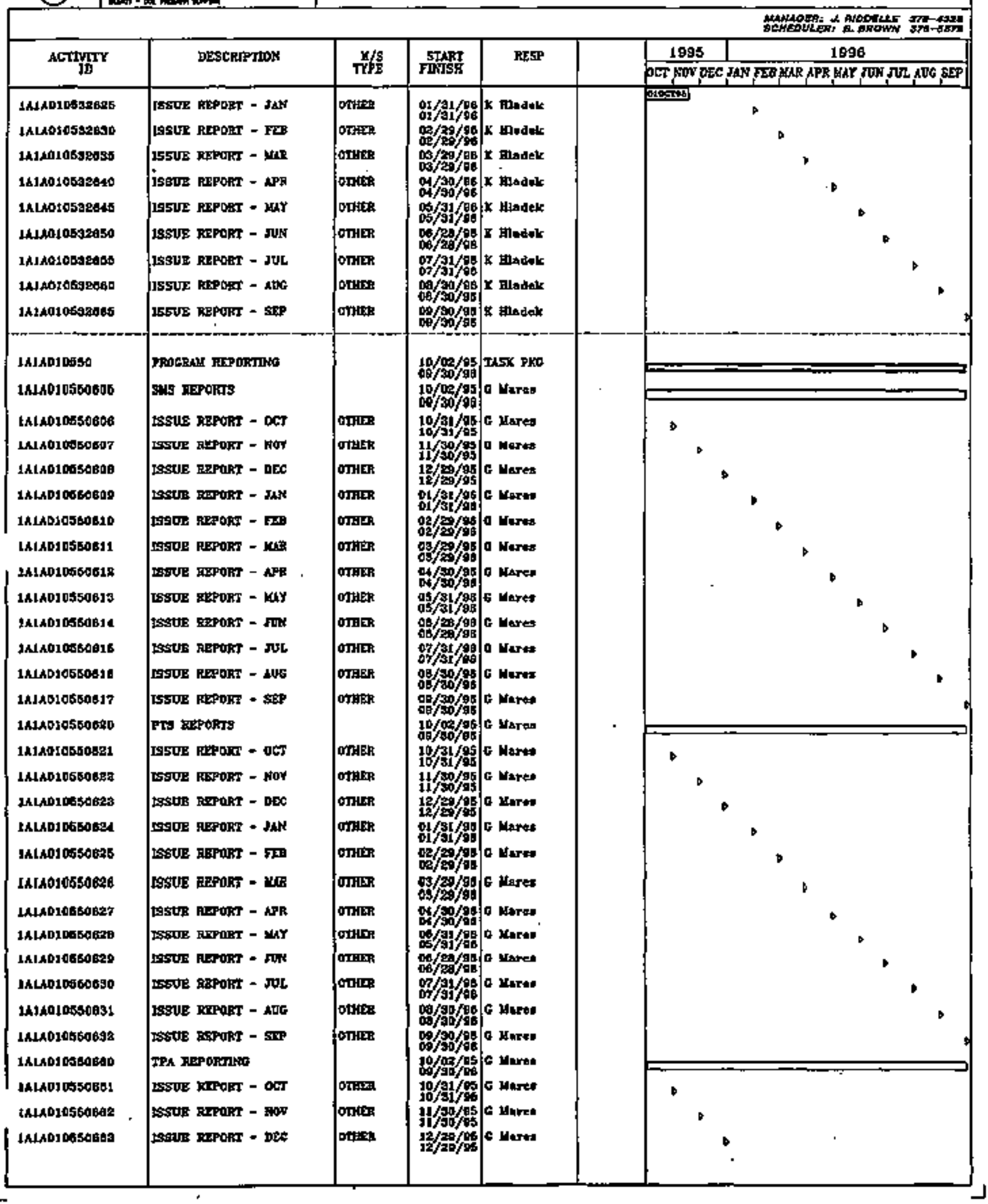




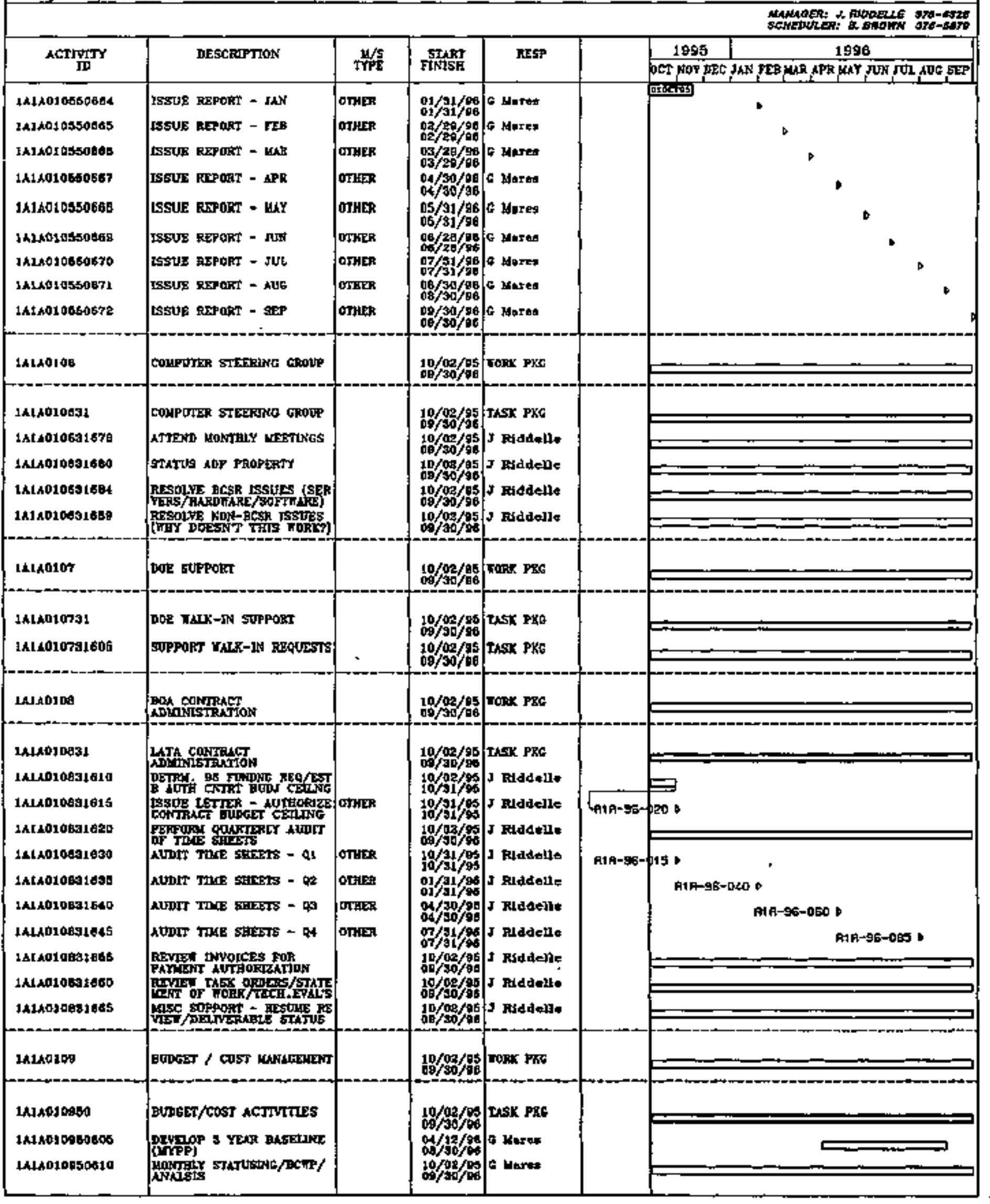




\section{WHC - SOLID WASTE PROGRAM FY 1996 SCHEDULE TA1AOA - DOE PROGRAM SUPPORT}

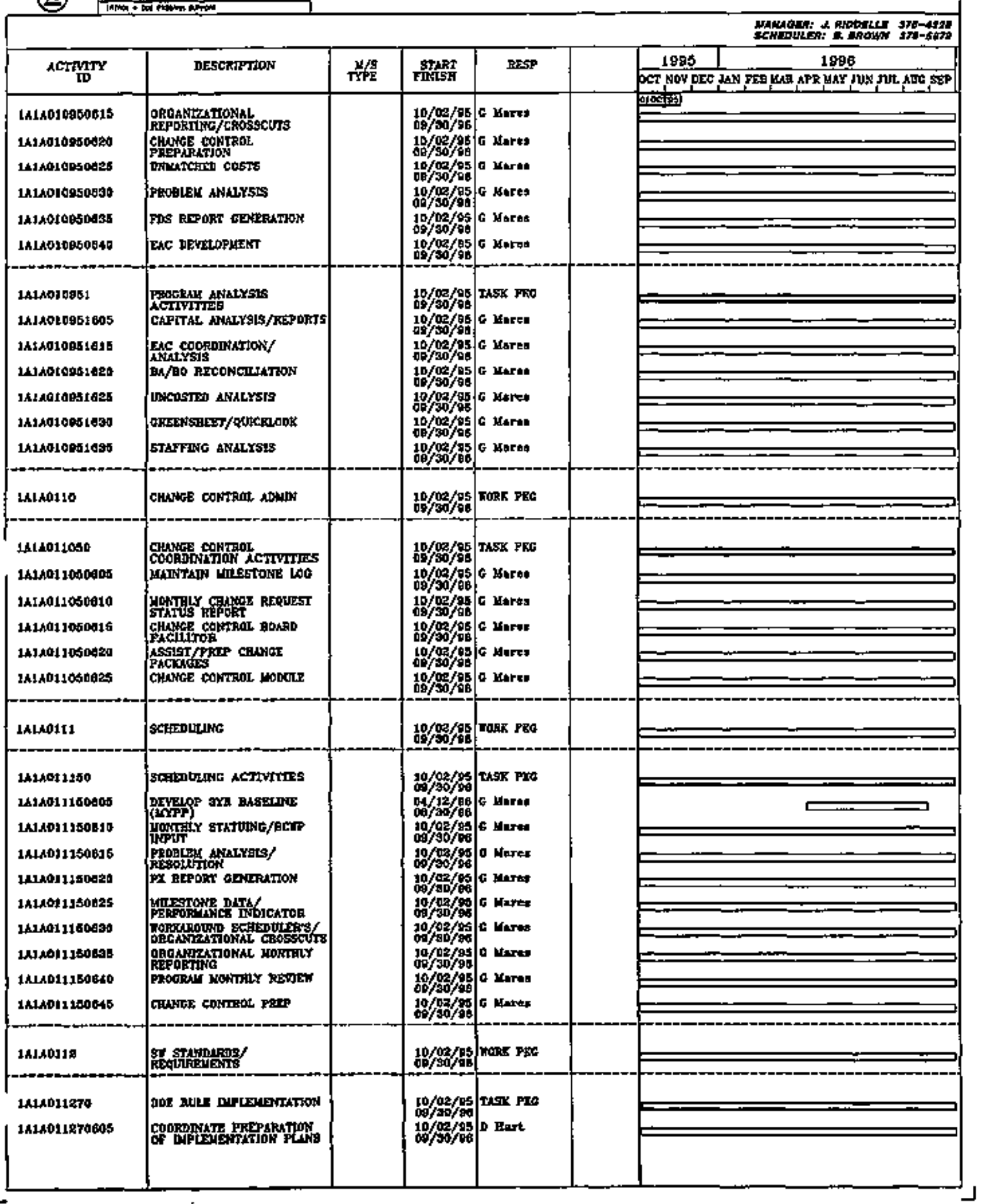


a

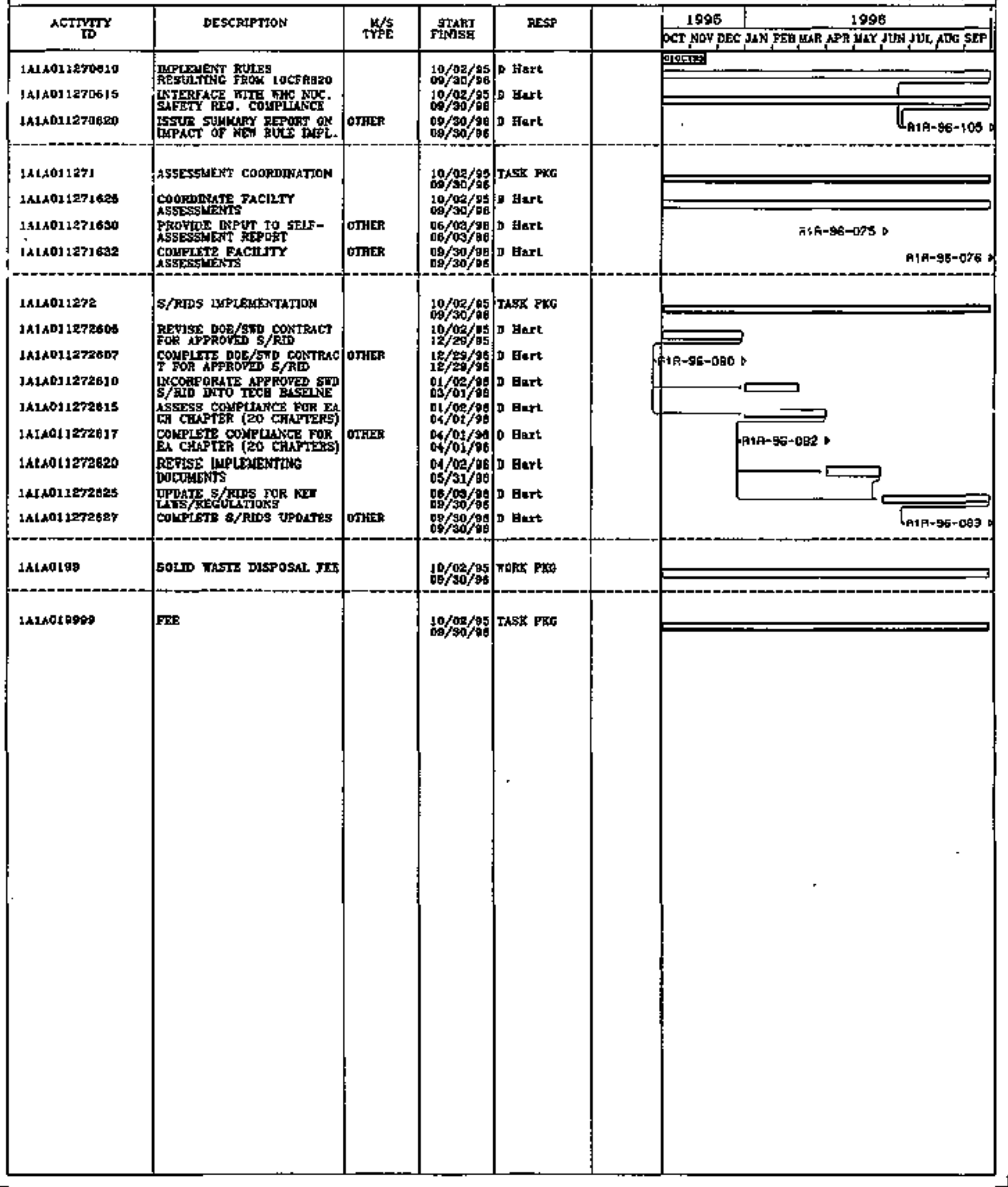




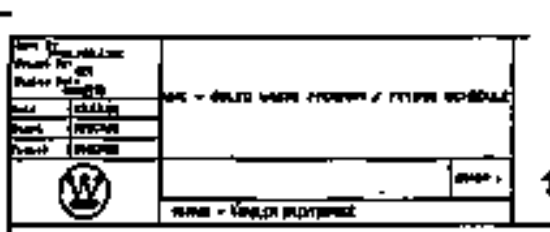

WHC - SOLID WASTE PAOCRAM

FY 1996 SCHEDULE

TA1AO2 - TAALER MAINTENANCE - AS OF 25NULOS

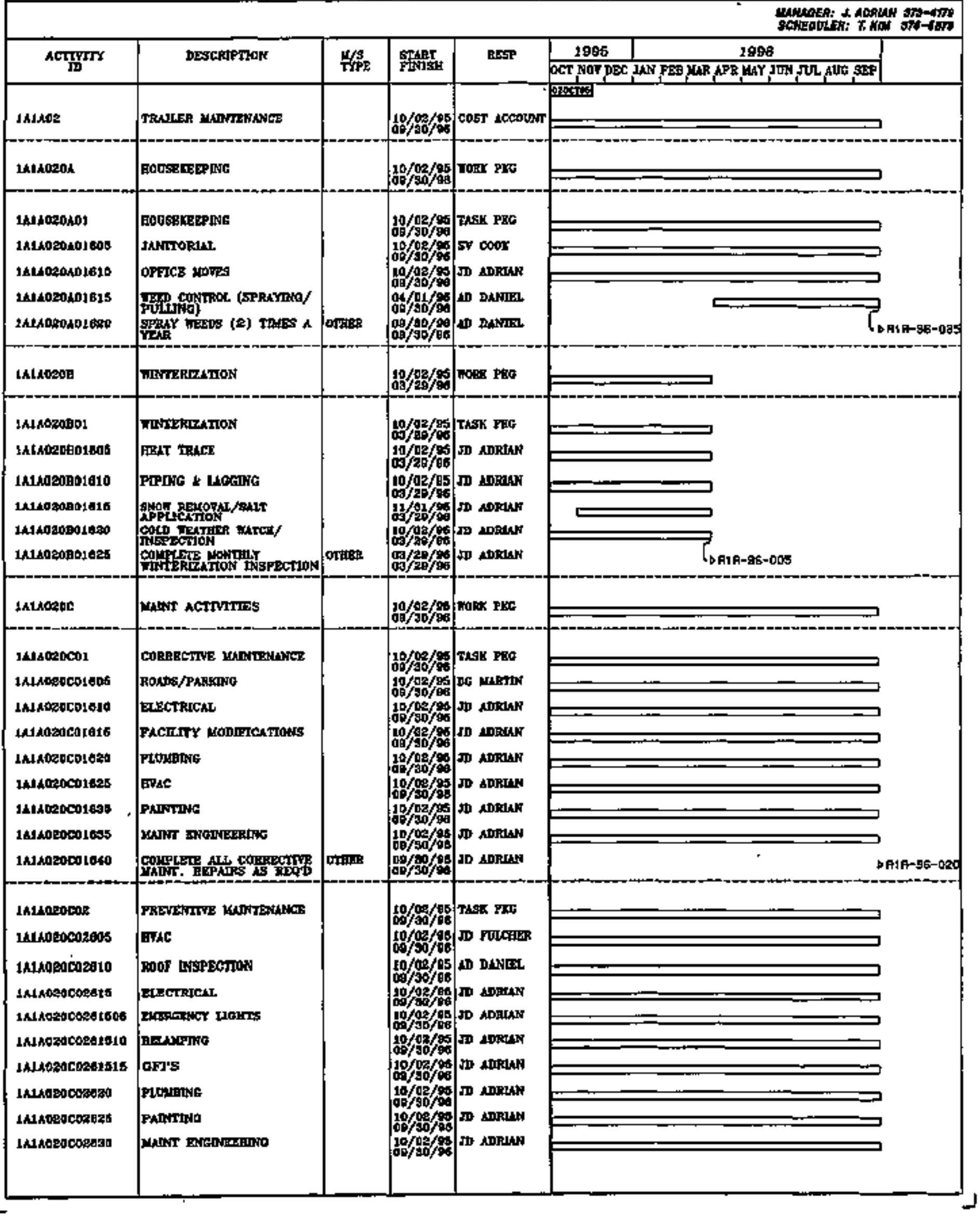




\section{WHC - SOLID WASTE PROERAM FY TOS日 \$CHEDULE \\ 1A1AO2 - TRAILER MAINTENANCE - AS OF 25 JULO5}

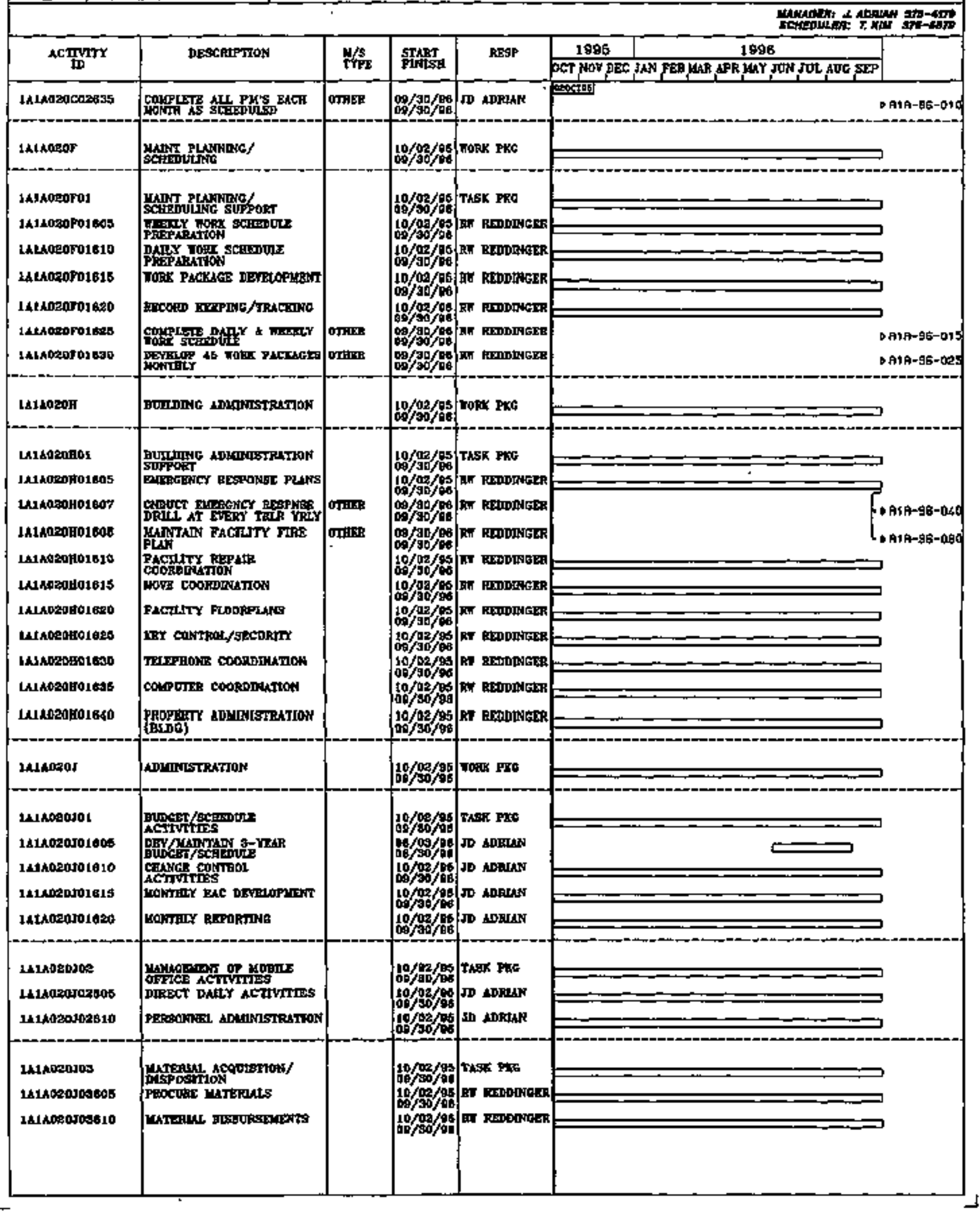




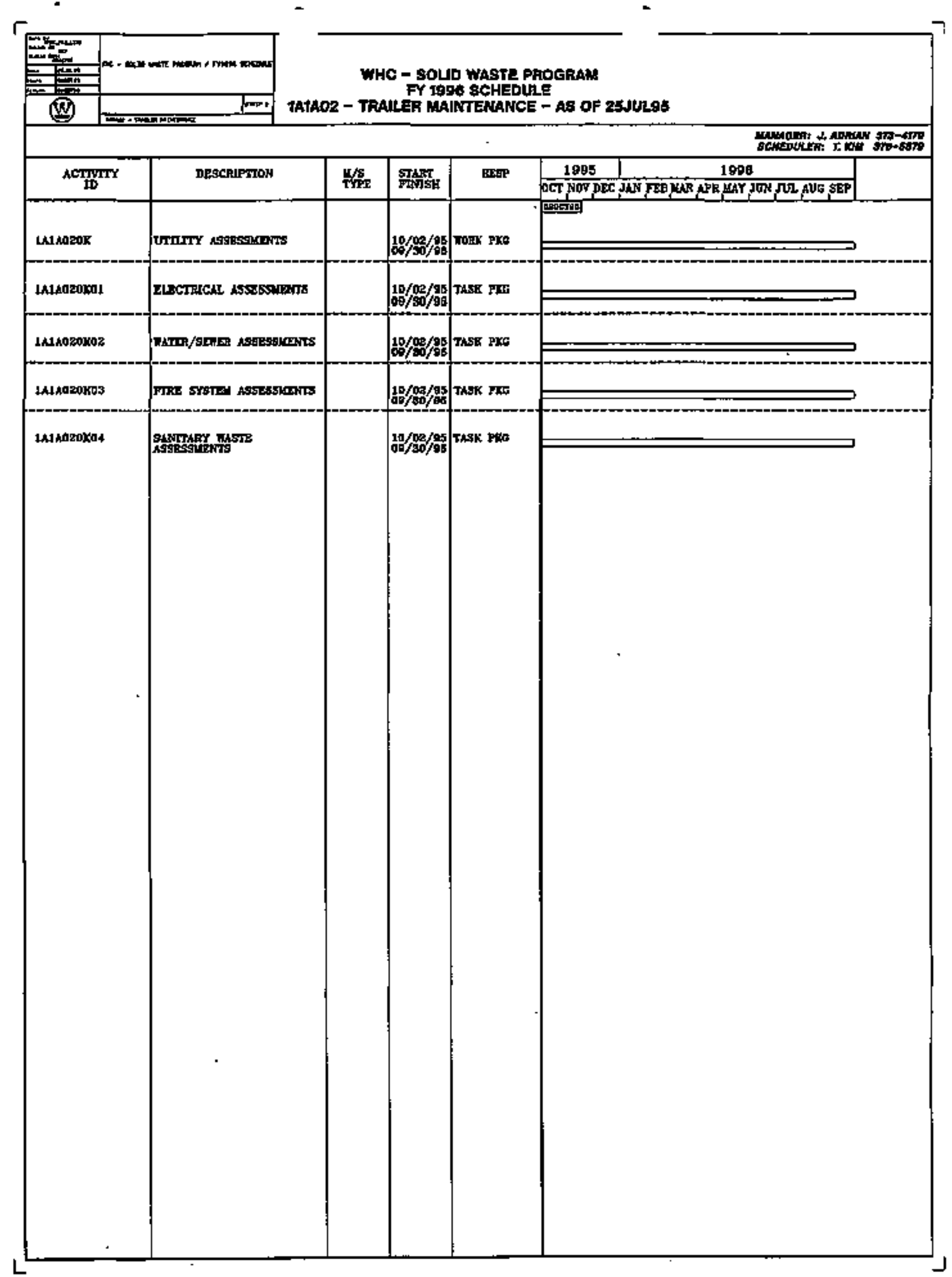




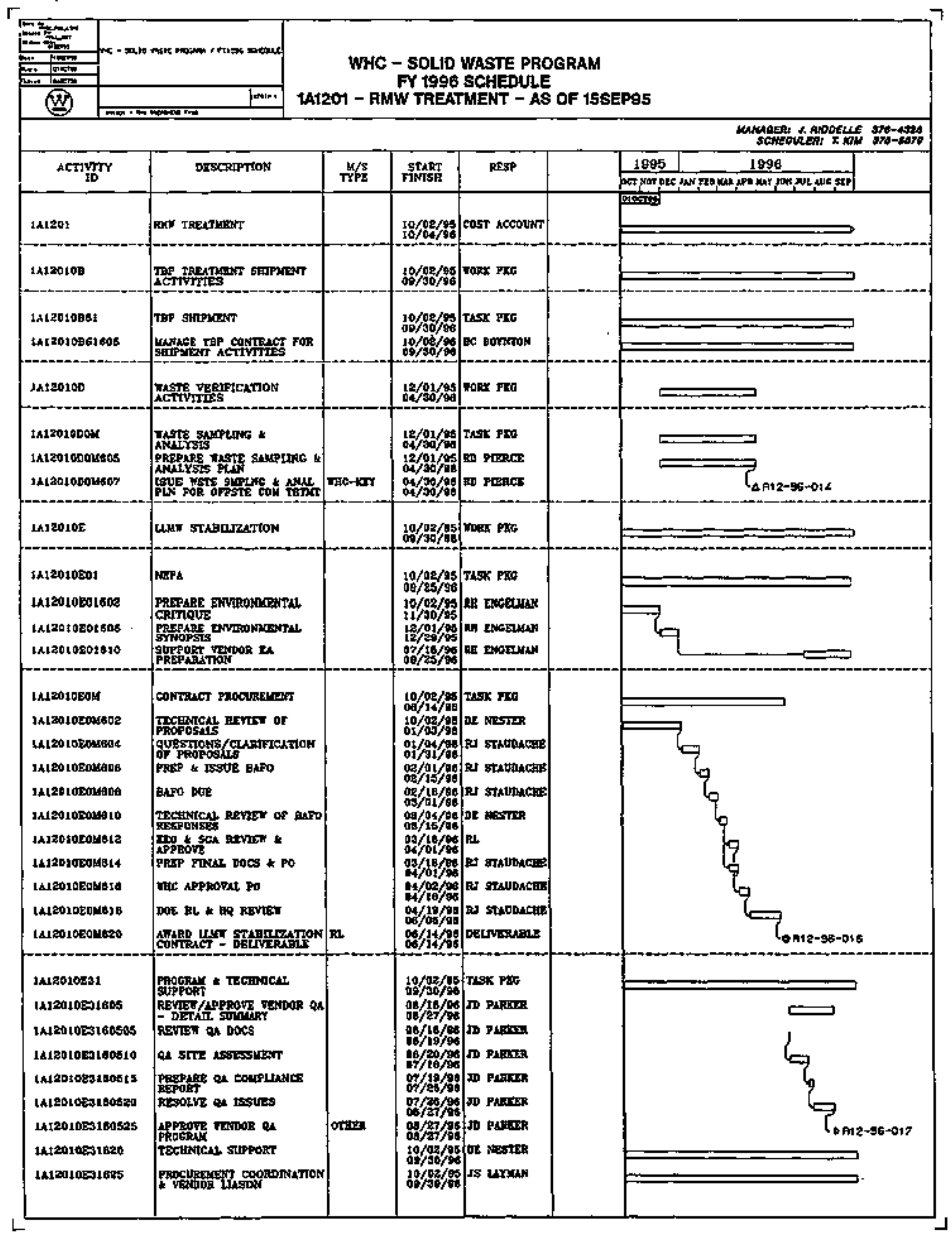




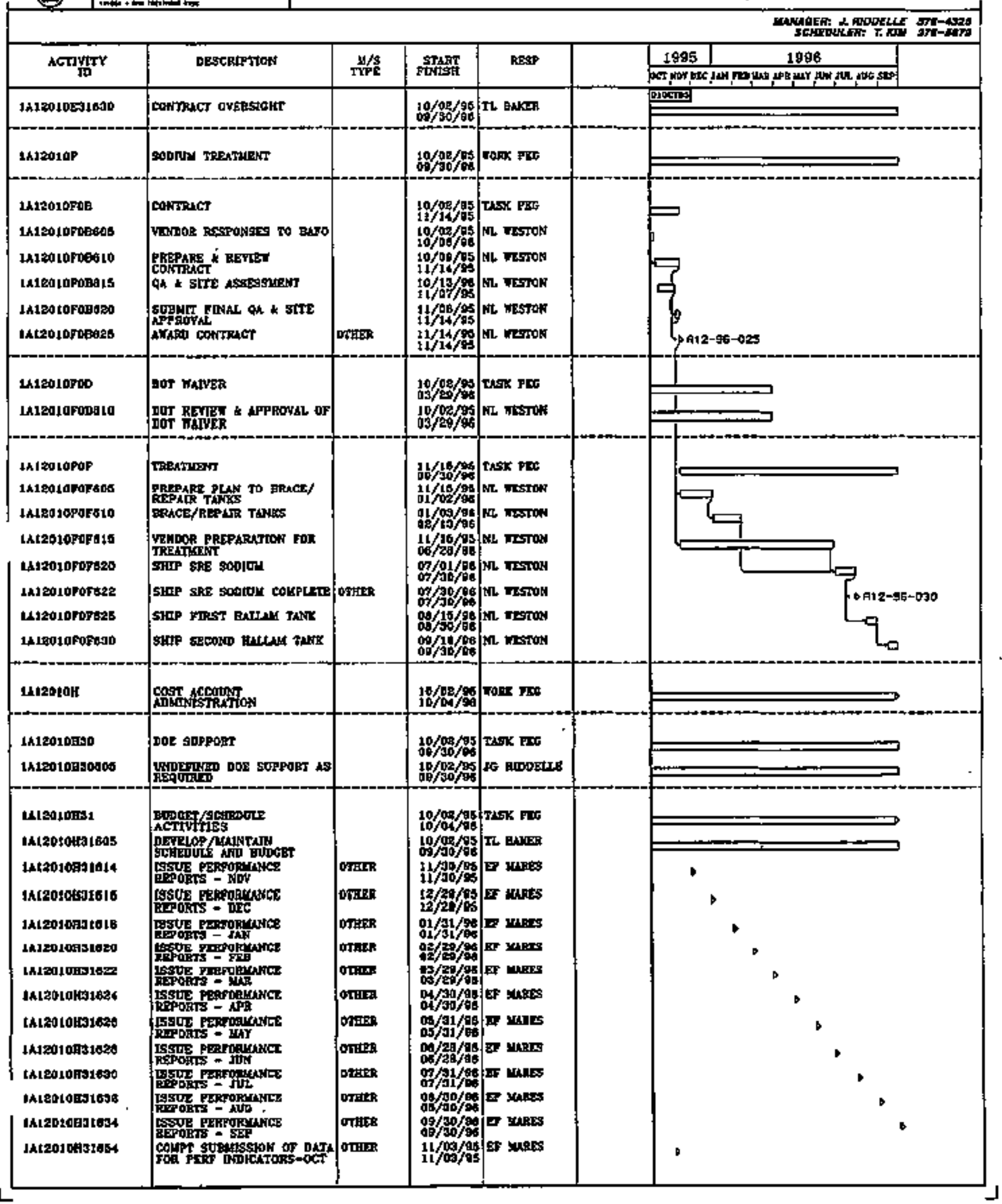




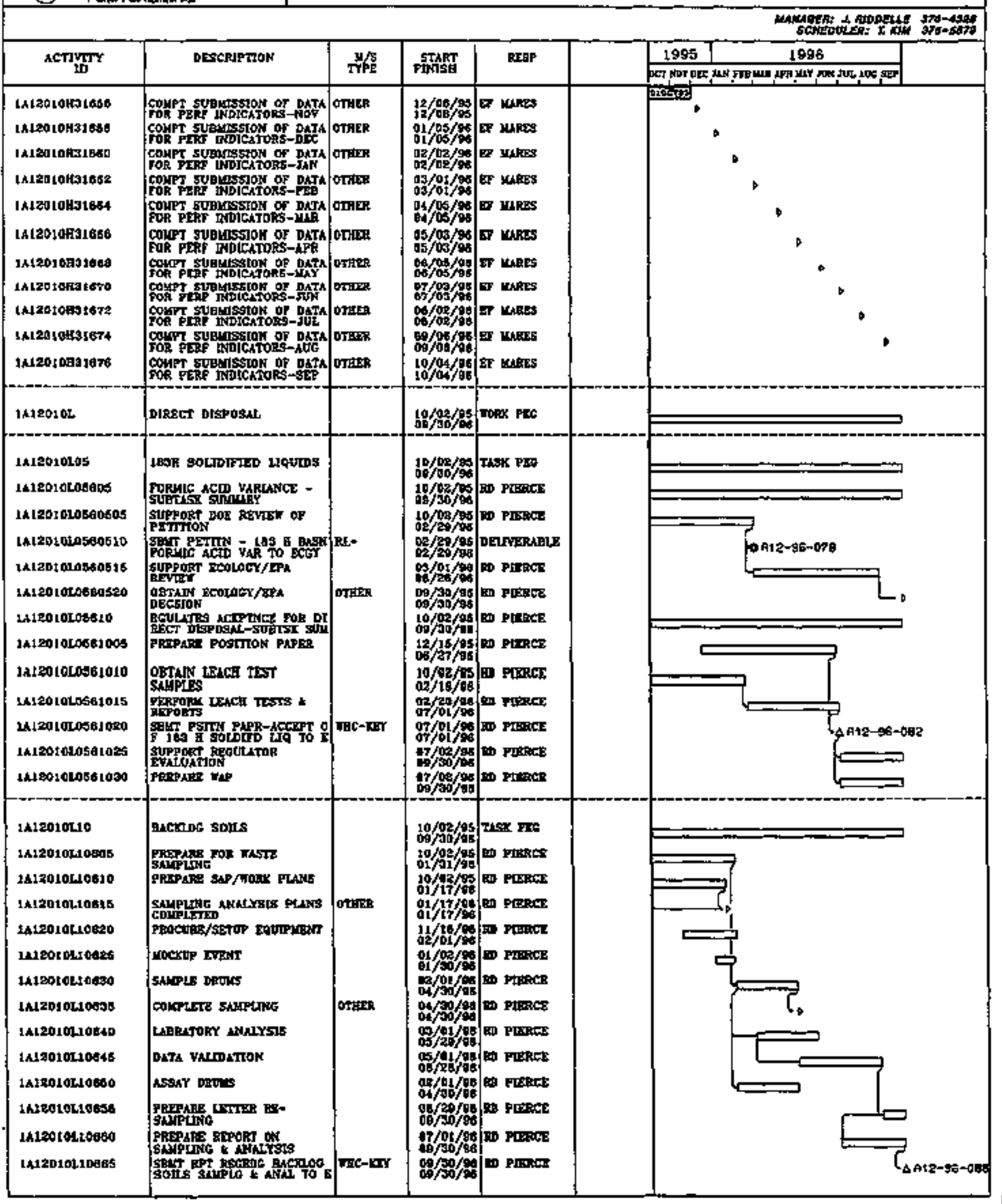


r

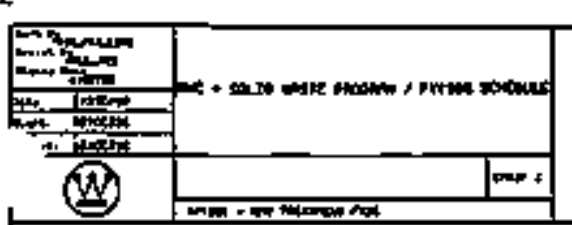

\section{WHC - SDLID WASTE PROGRAM \\ FY 1996 SCHEOULE \\ 1A1201 - RMW TREATMENT - AS OF 15SEP9S}

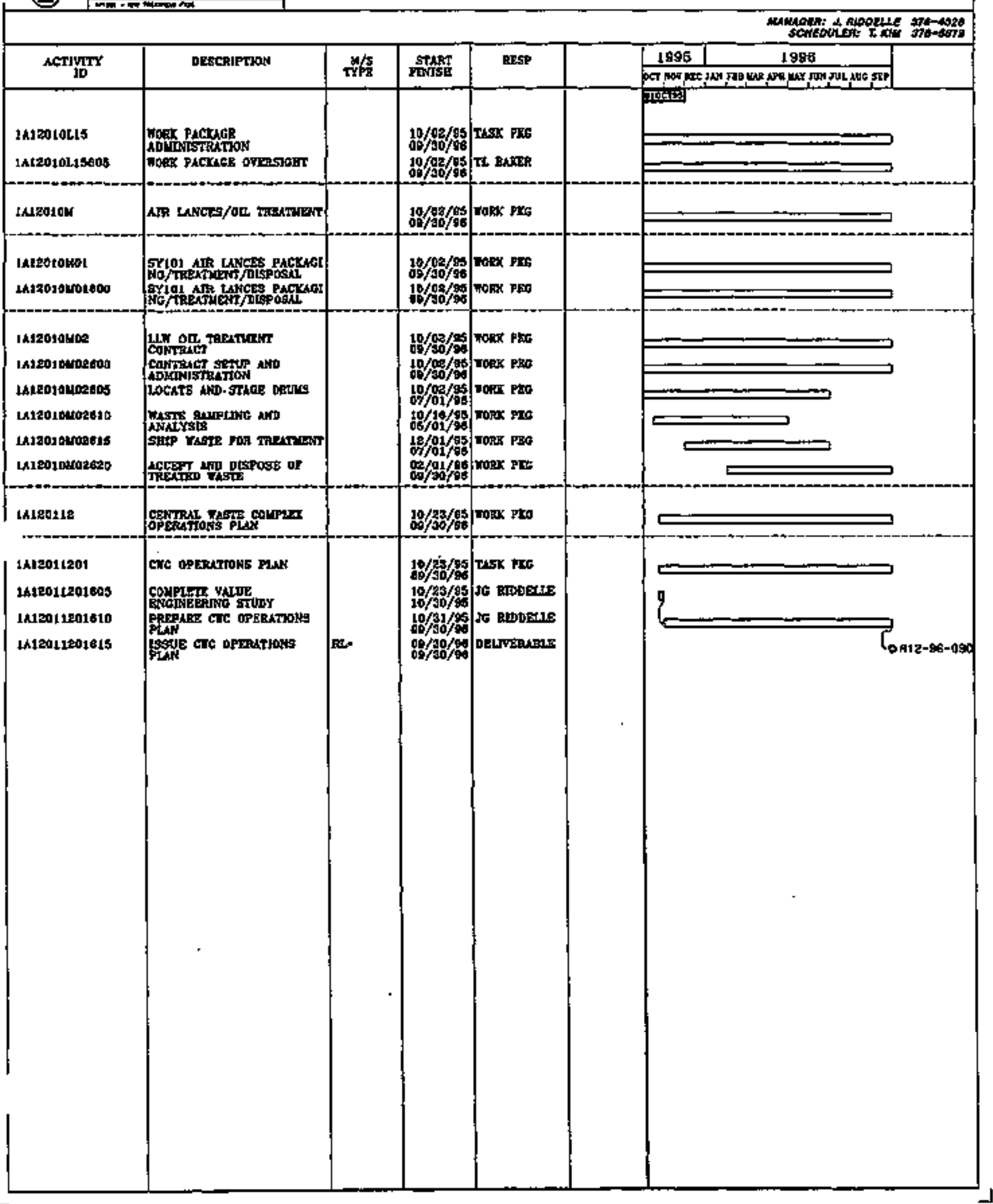


WHC - SOLID WASTE PROGEAM

FY 1 1996 SCHEDULA 1A1203- MACBOENEAPSULATION OF MIXED WASTE OEBFIS

(W)

二का

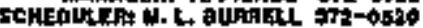

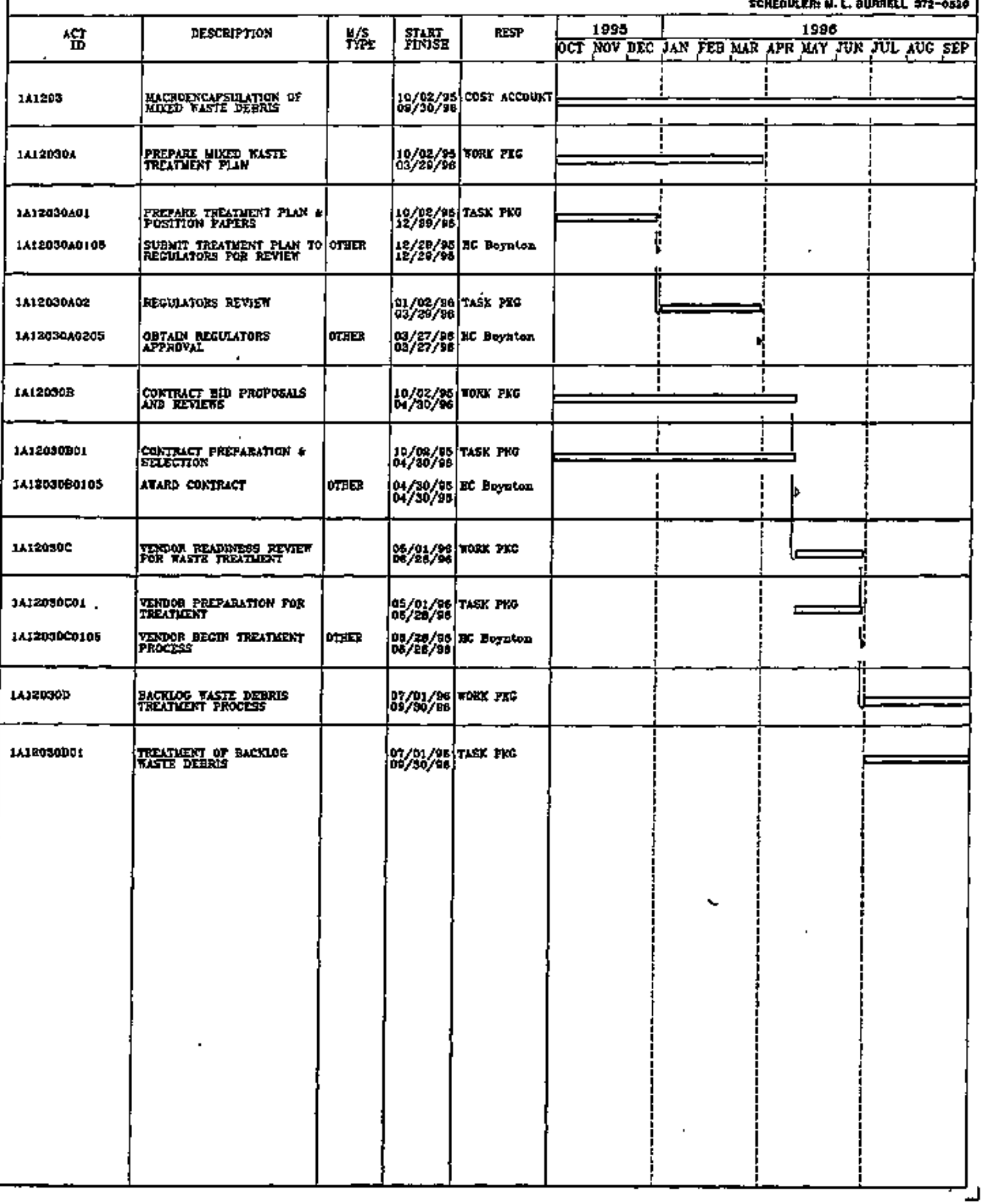


WHC - SOLID WASTE PROGRAM

FY 1996 SCHEDULE 1A1301 - TRU STORAGE

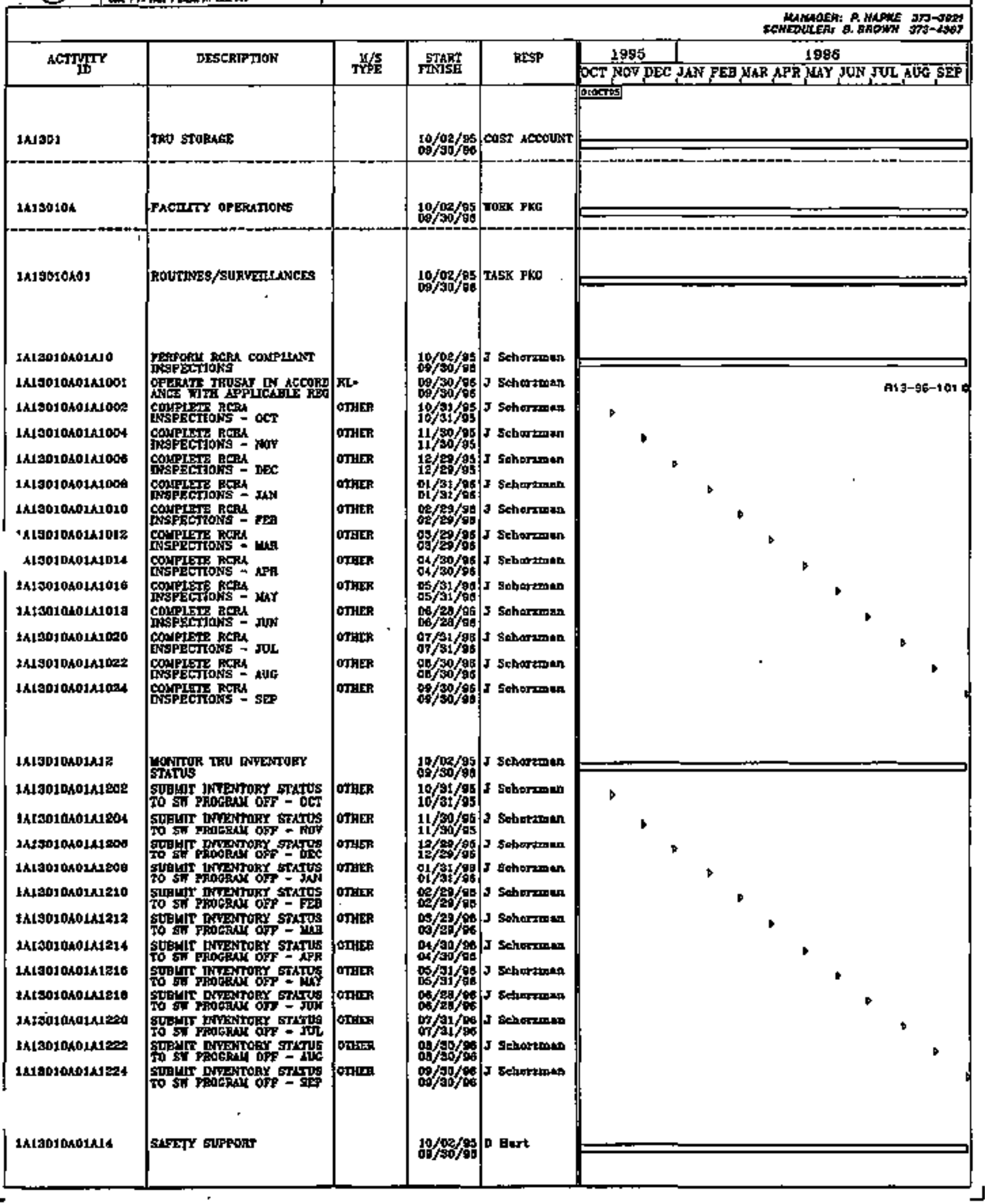


5 |

WHC - SOLID WASTE PROGAAM FY 1996 SCHEDULE TA1301 - TRU STORAGE

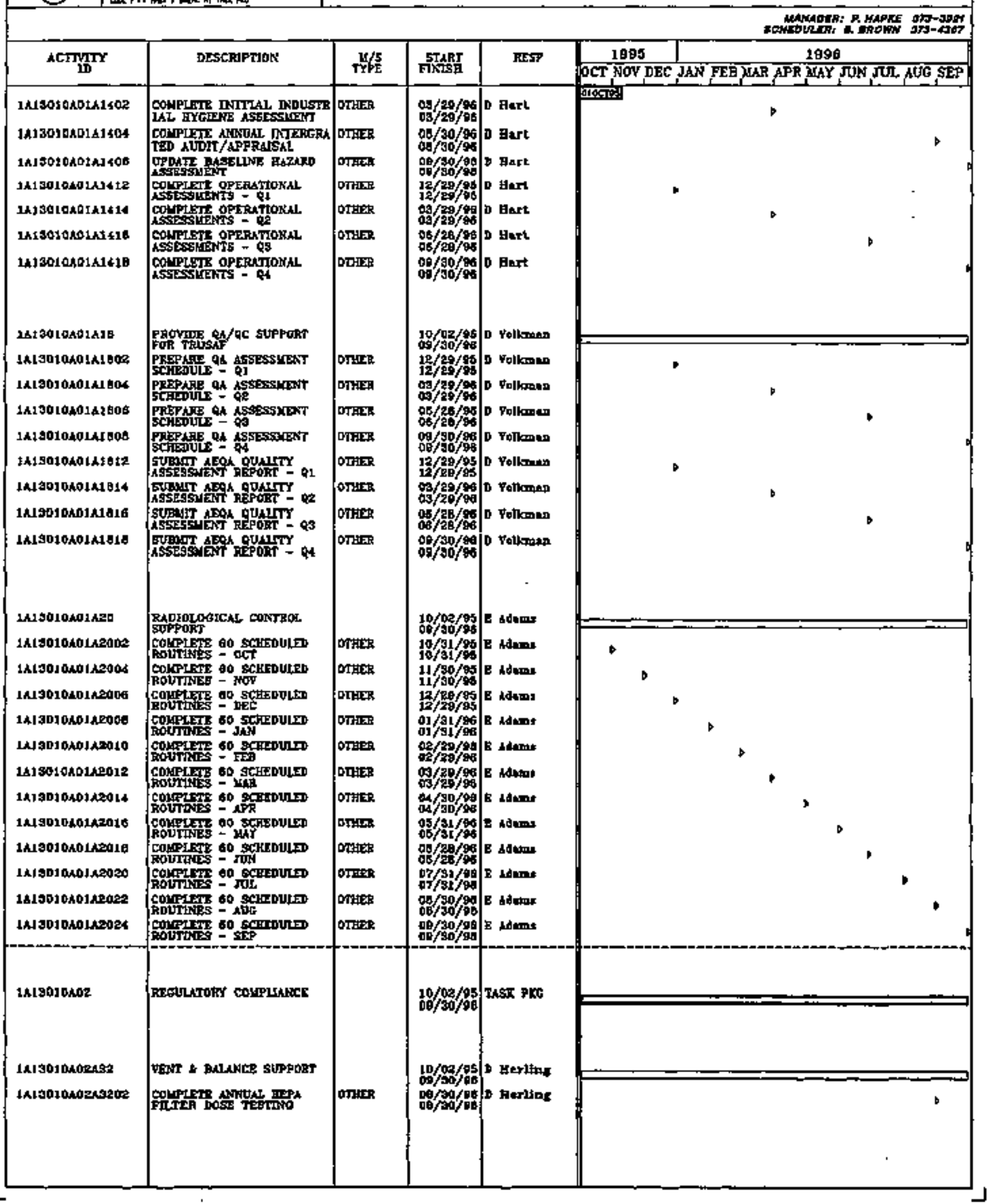




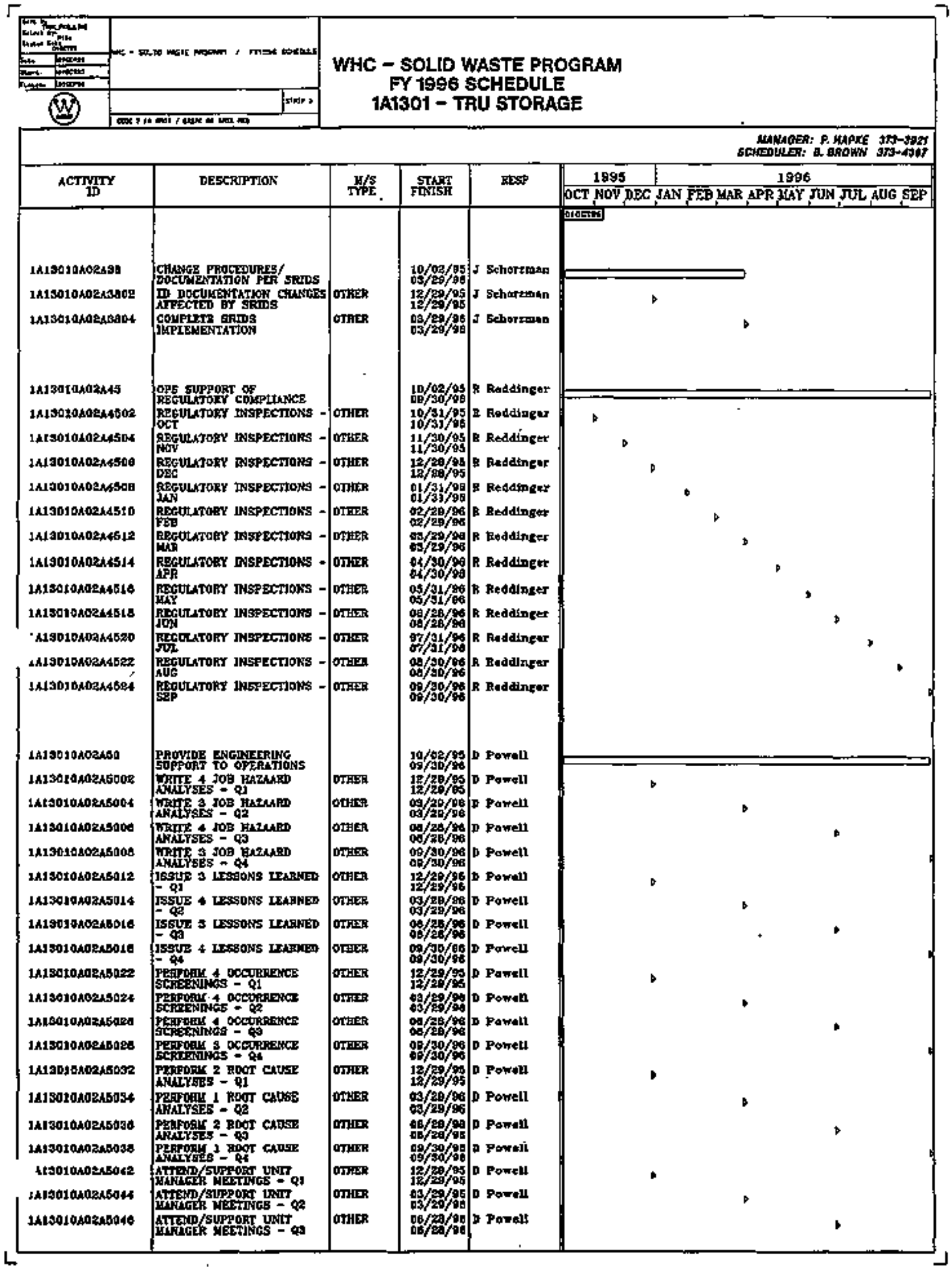




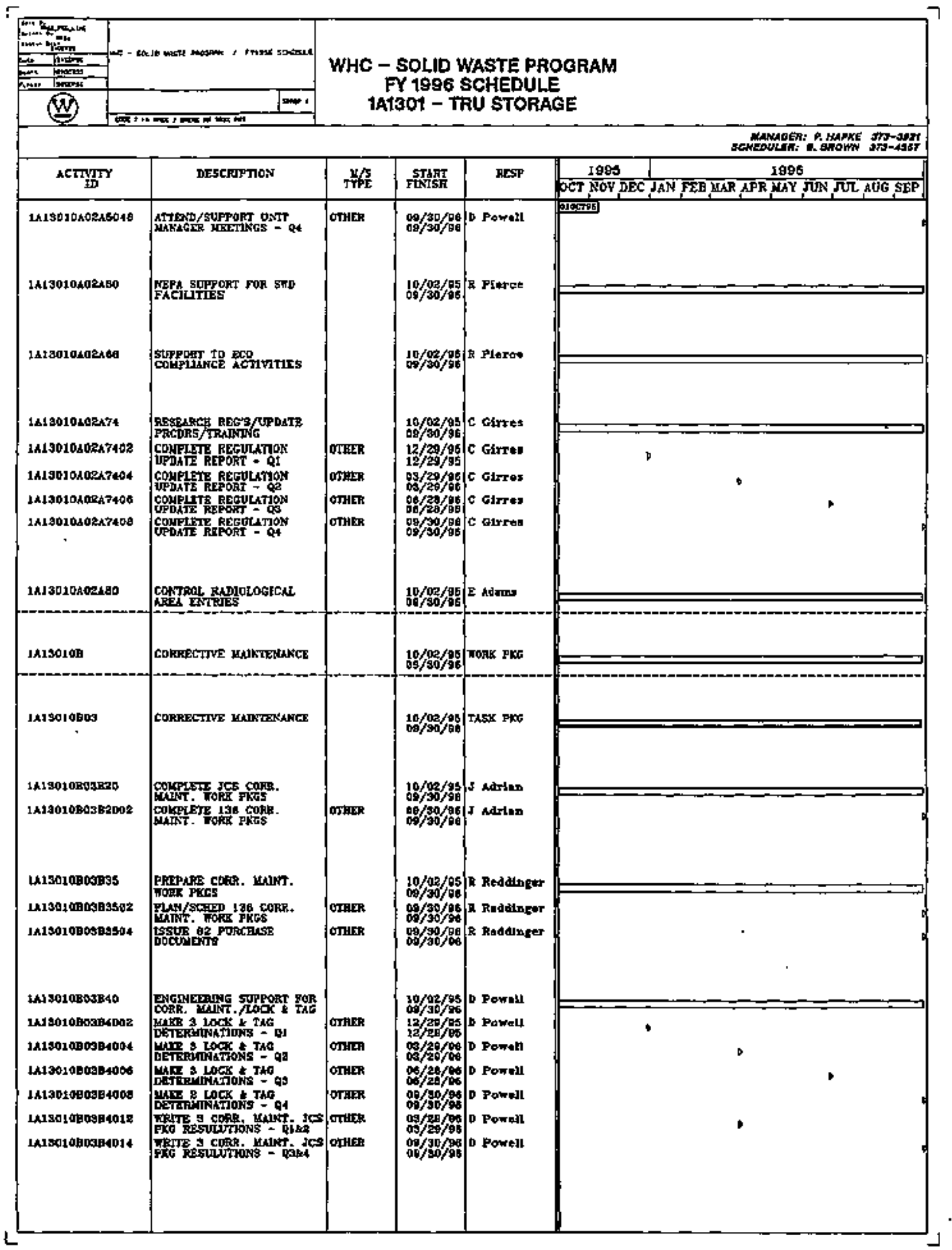



TA1SO1 - TRU STORAGE

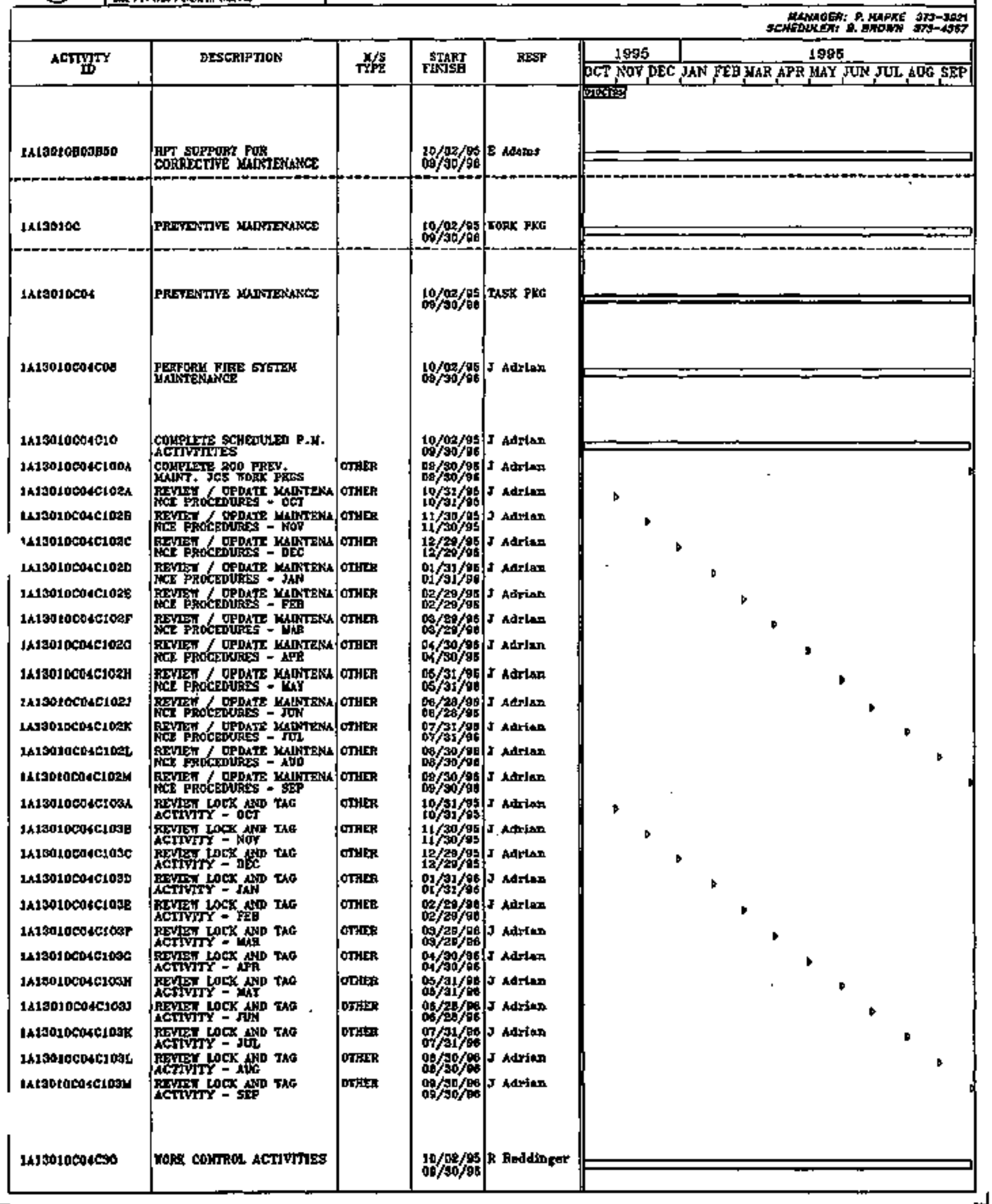


WHC - SOLID WASTE PAOGRAM

FY 1996 SCHEDULE

1A13O1 - TRU STORAGE

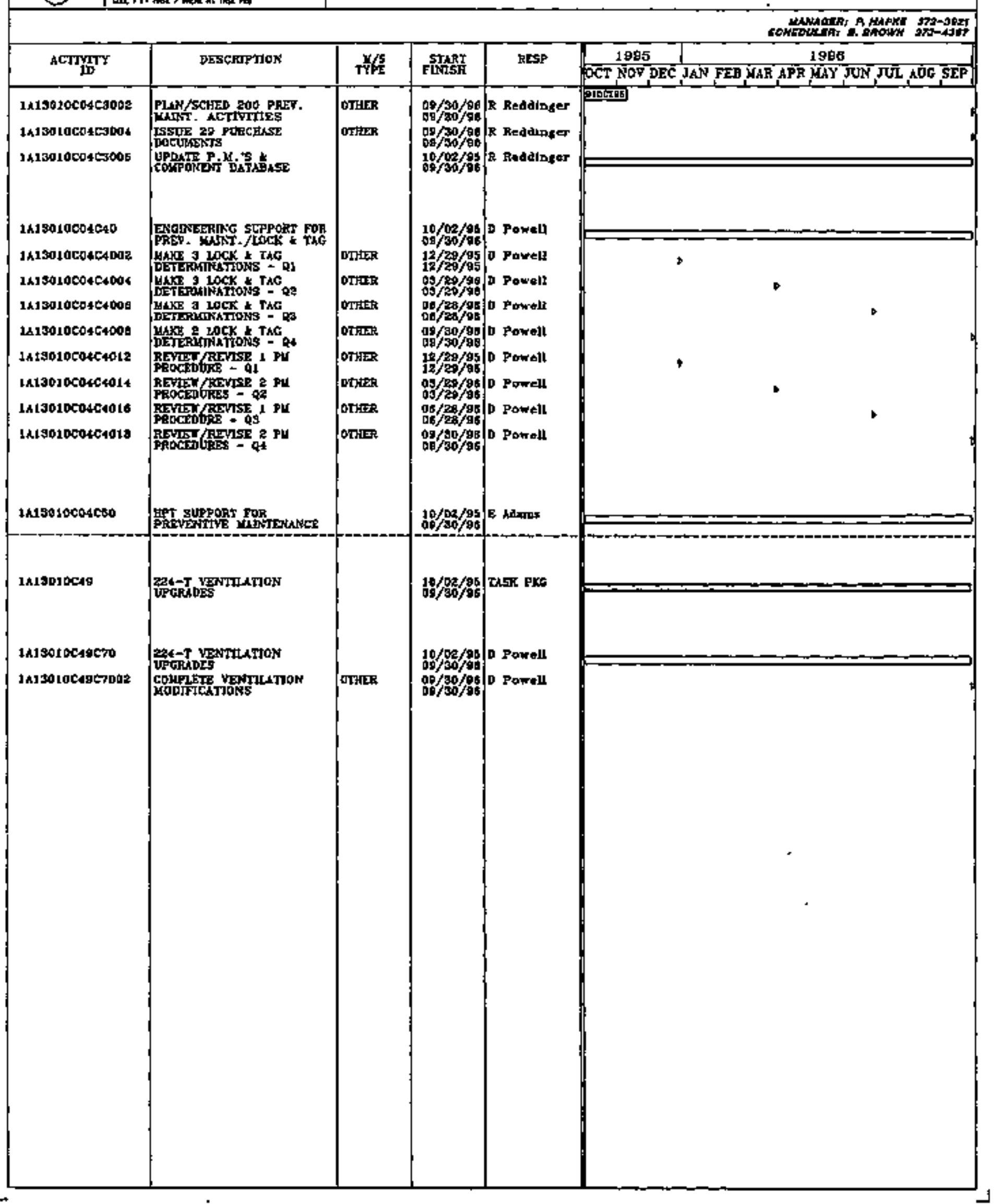




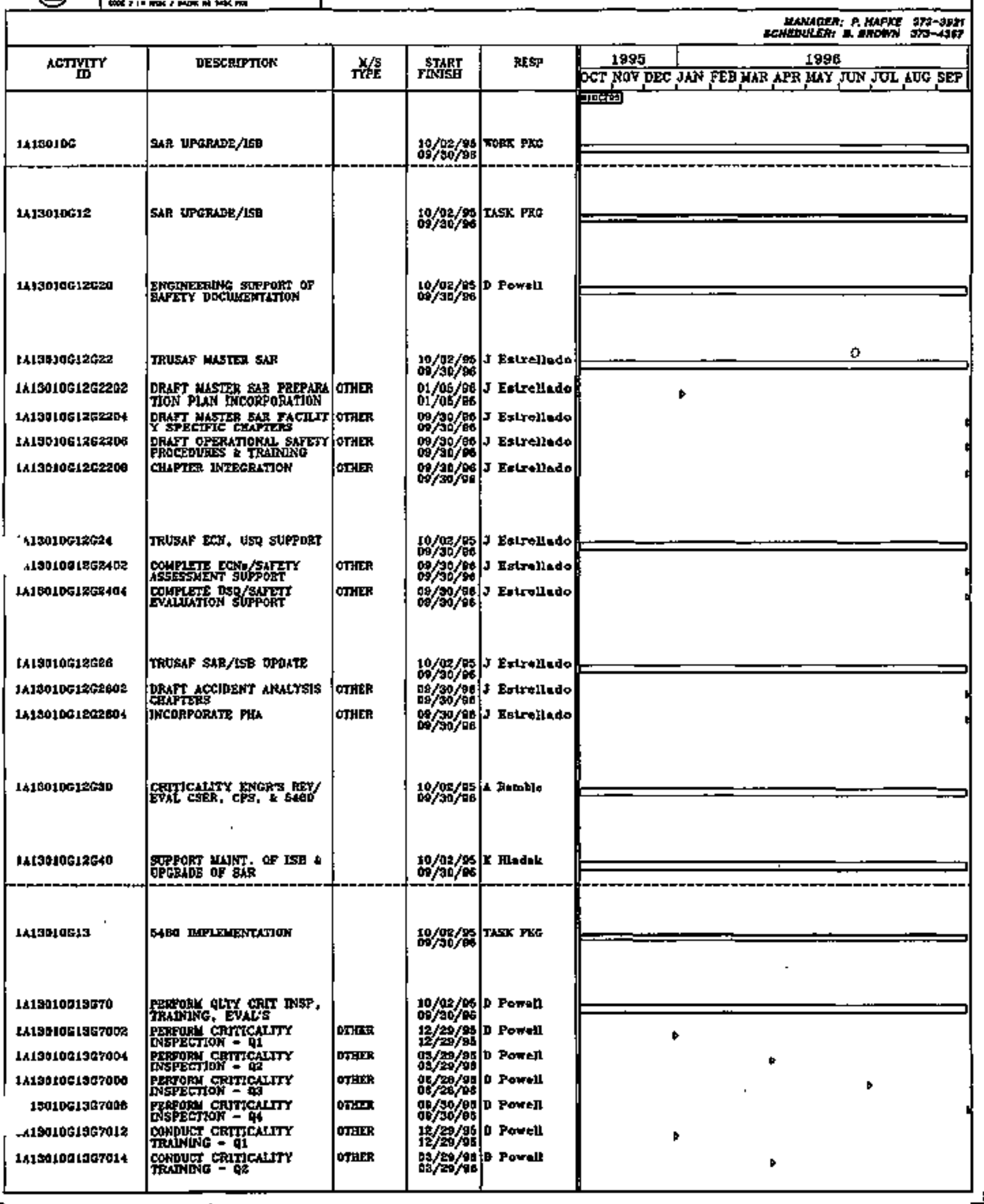




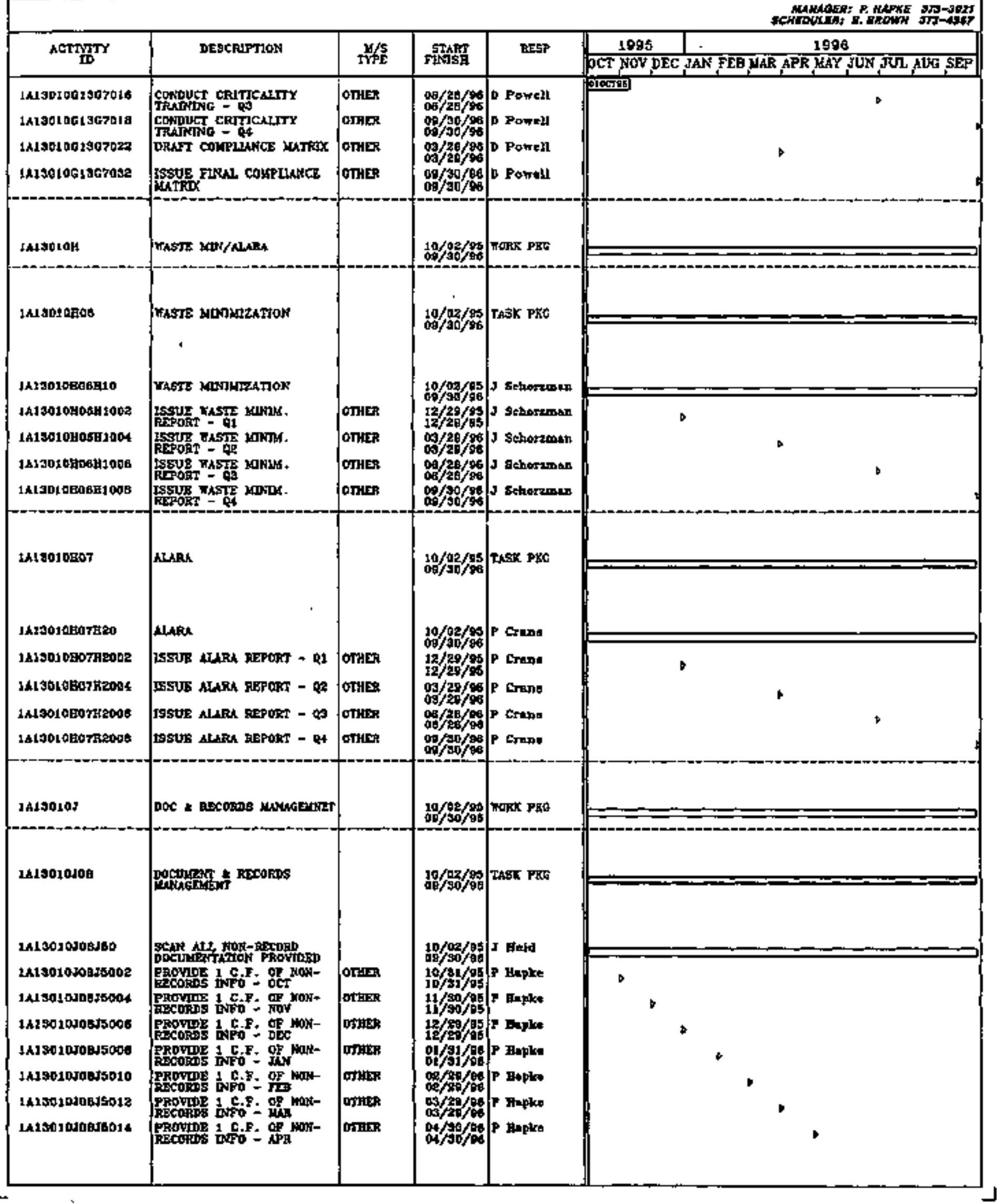


r

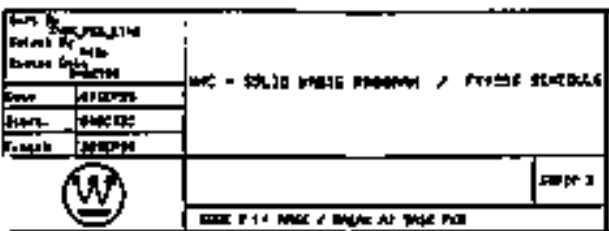

WHC - SOLID WASTE PROGRAM

FY 1996 SCHEDULE

1A13O1 - TRU STORAGE

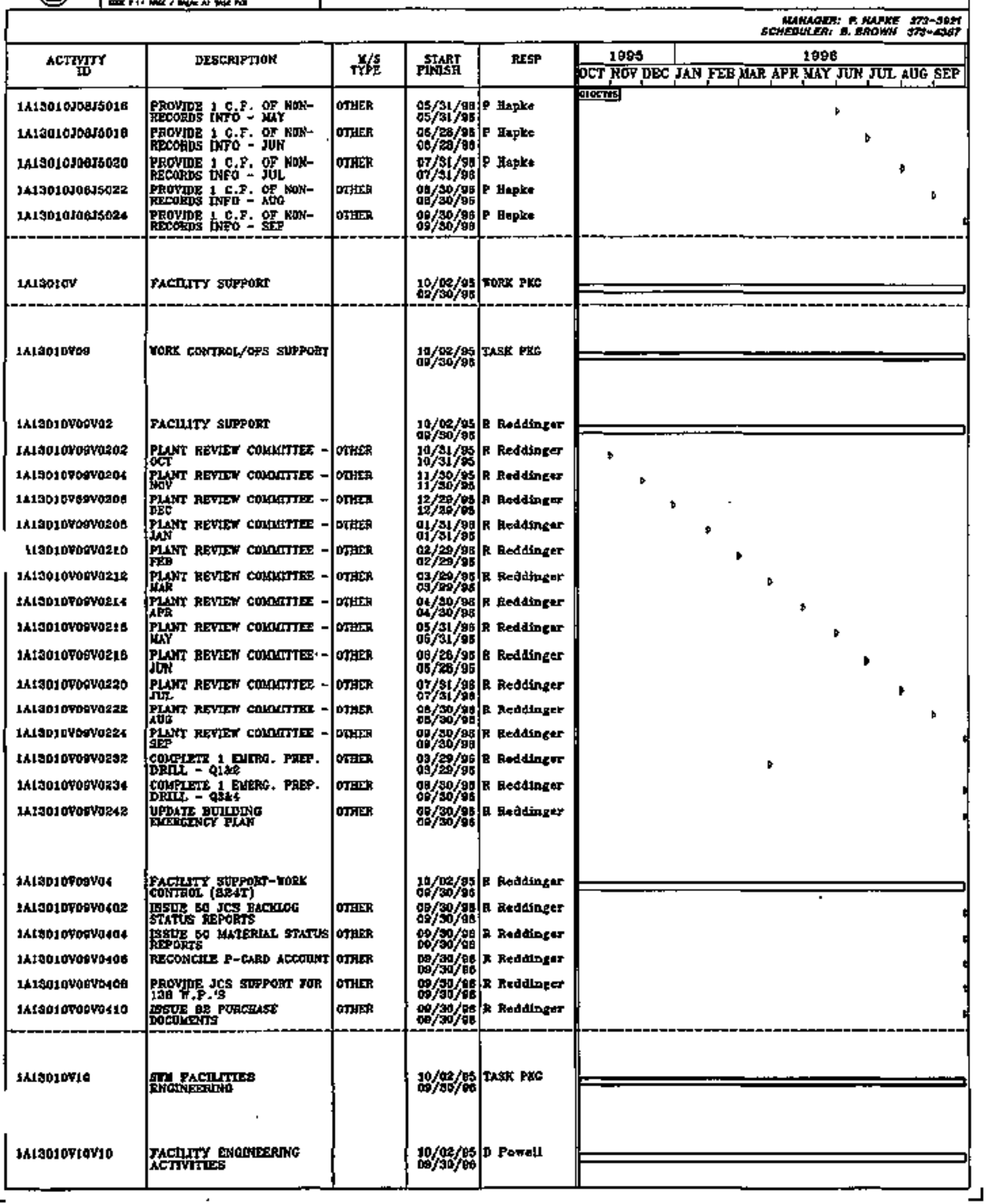



IA1301 - TRU STORAGE

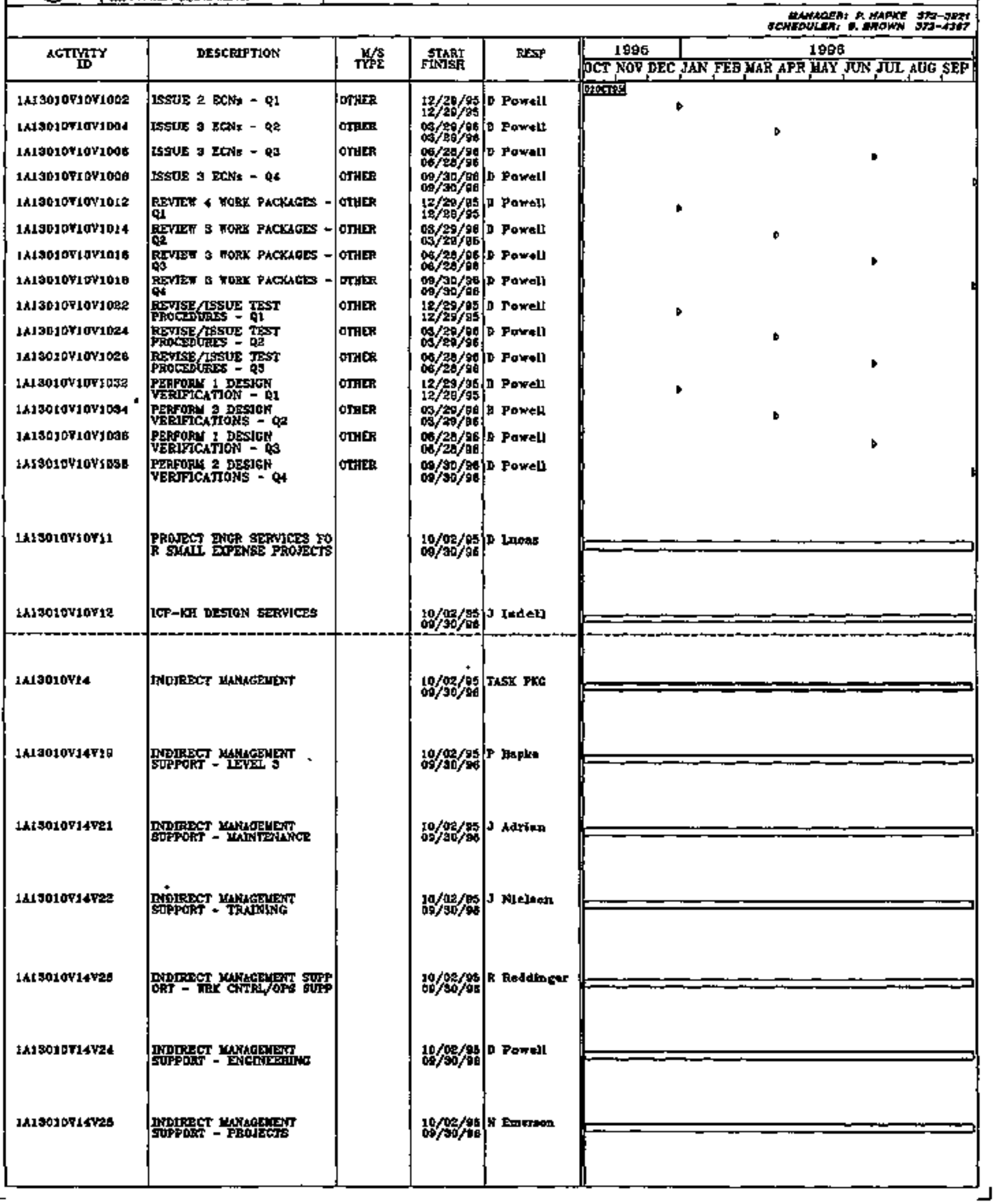


WHC - SOLID WASTE PROGRAM

FY 1996 SCHEDULE

1A1301 - TRU STORAGE

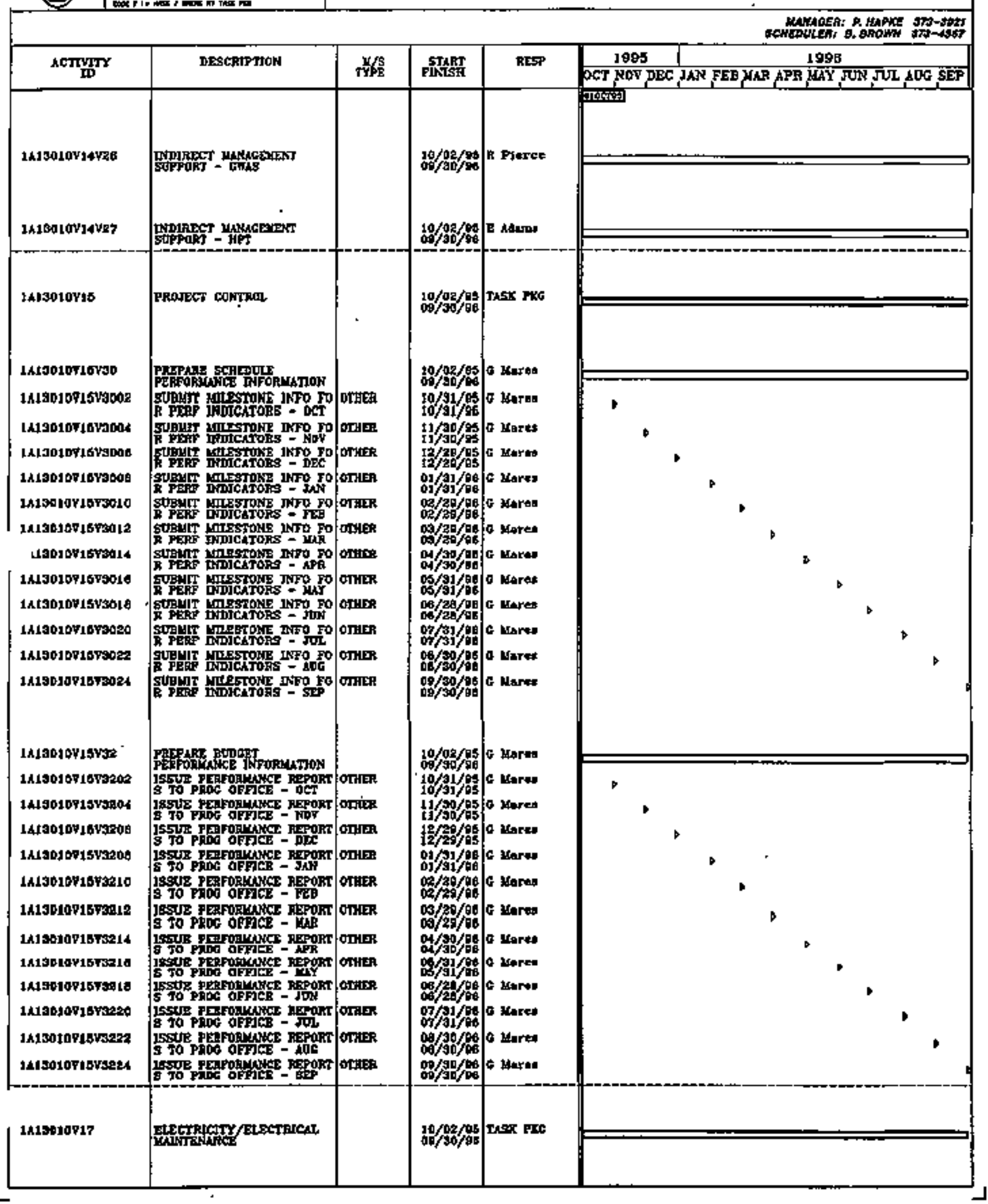




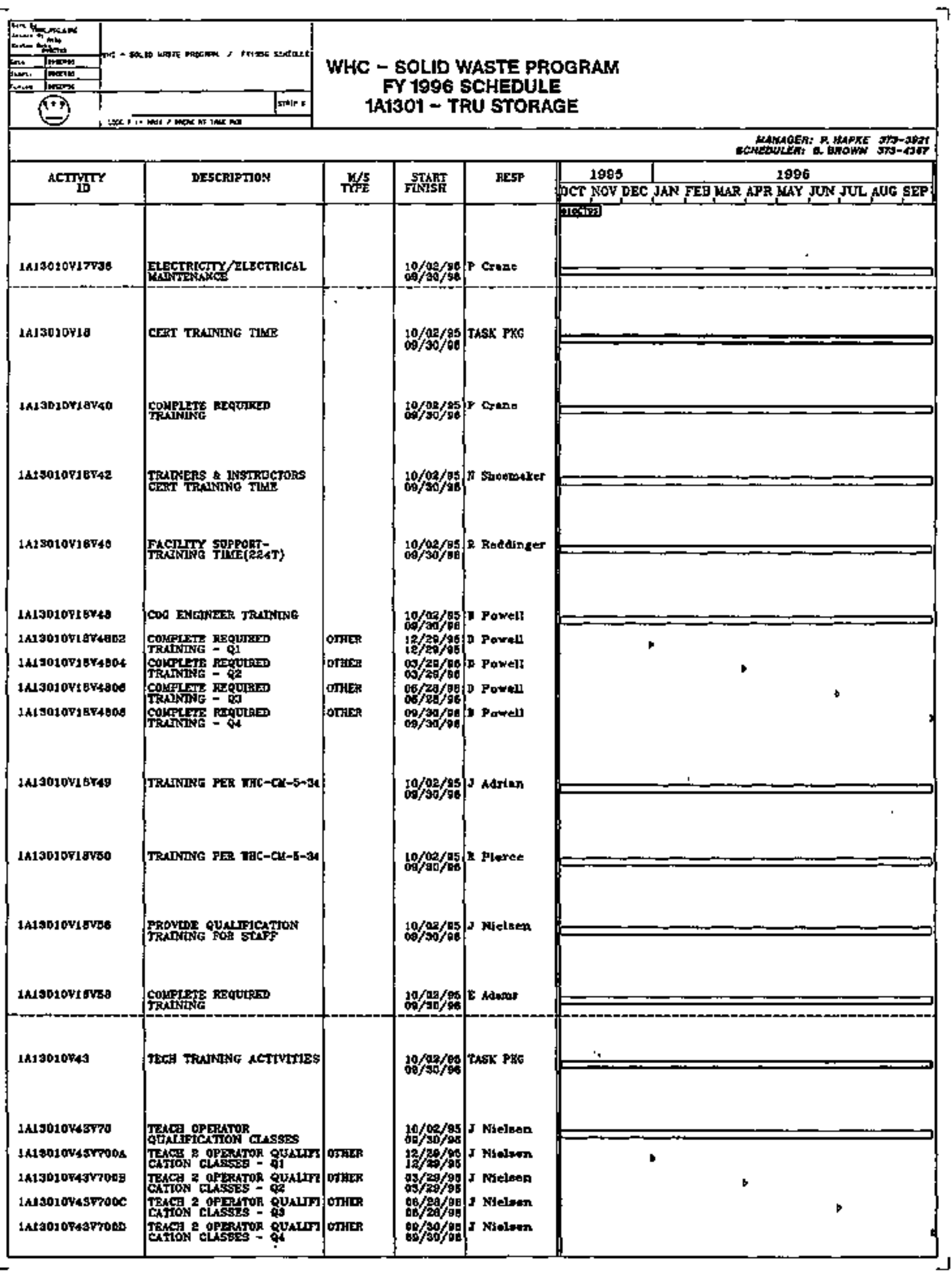


$\Gamma$

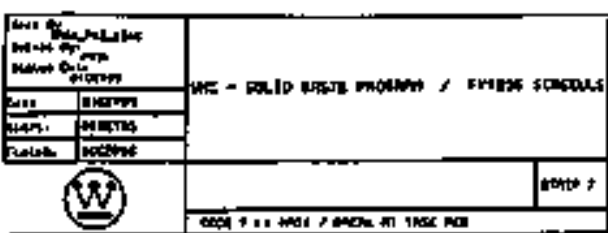

WHC - SOLID WASTE PROGRAM

FY 1996 SCHEDULE 1A1301 - TRU STORACE

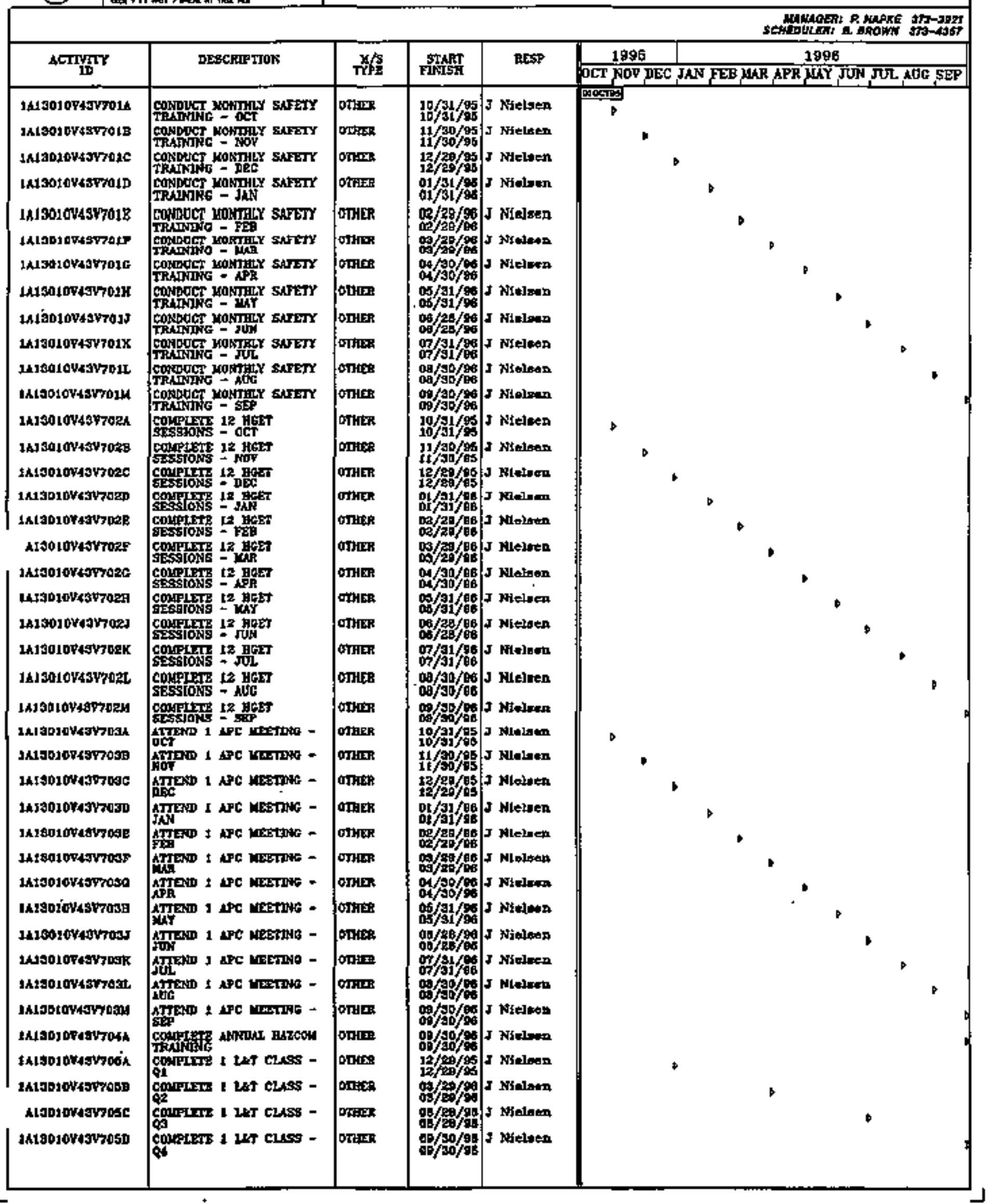


WHC - SOLID WASTE PROGRAM FY 1996 SCHEDULE IAIJOT TRU STORAGE

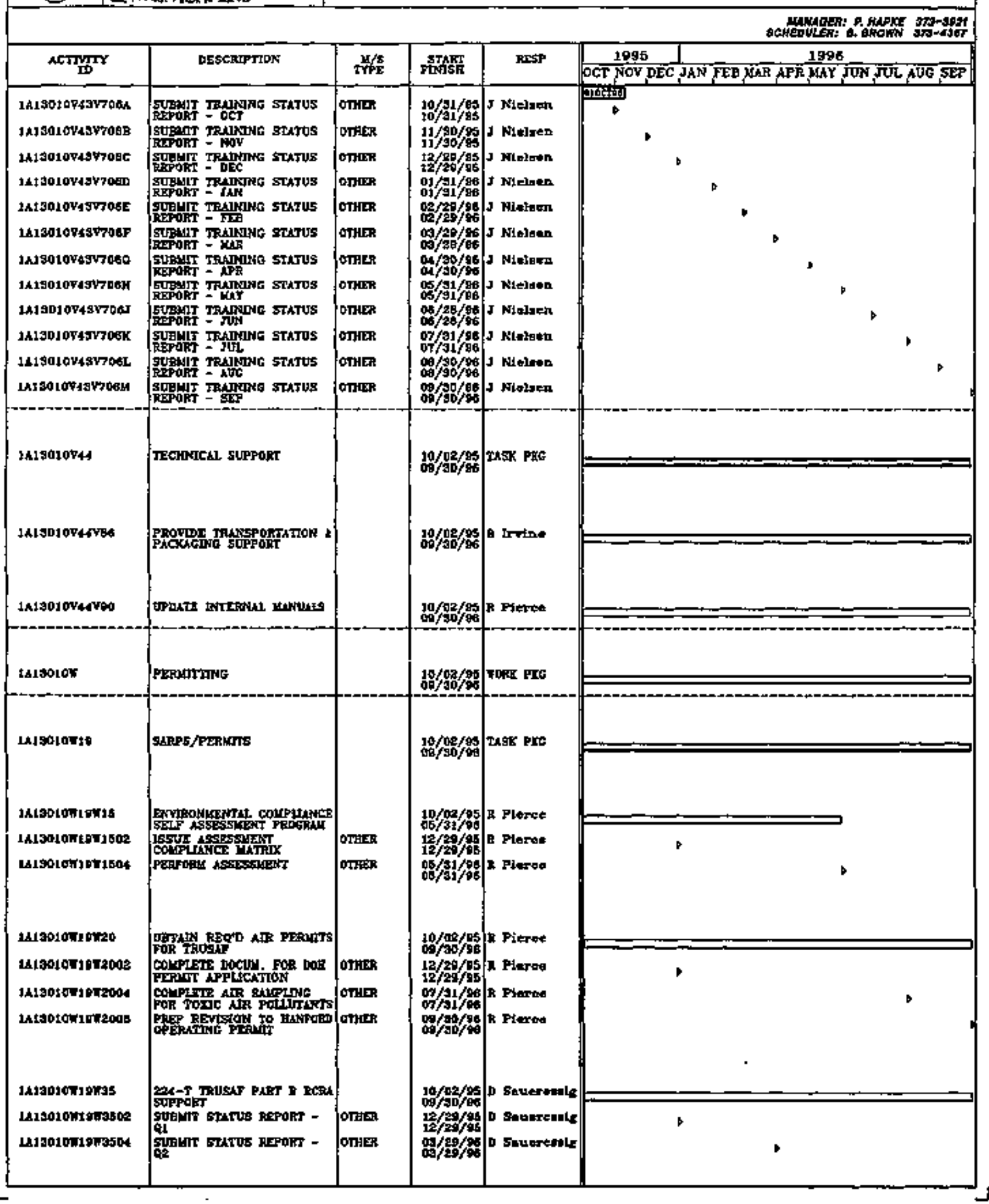



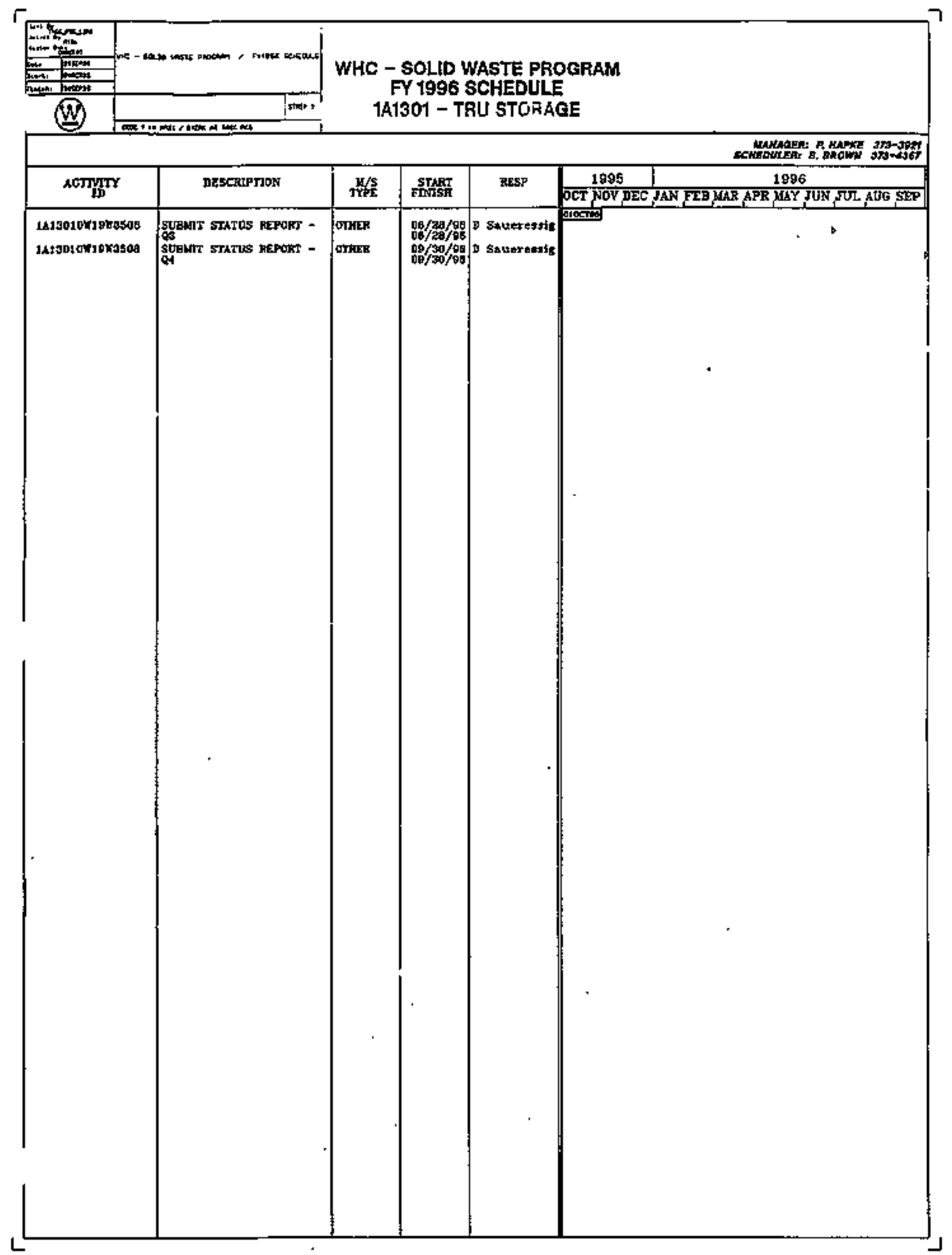
WHC - SOLID WASTE PROGRAM

FY 1996 SCHEDULE

1AF401 - RMW/CWC STORAGE

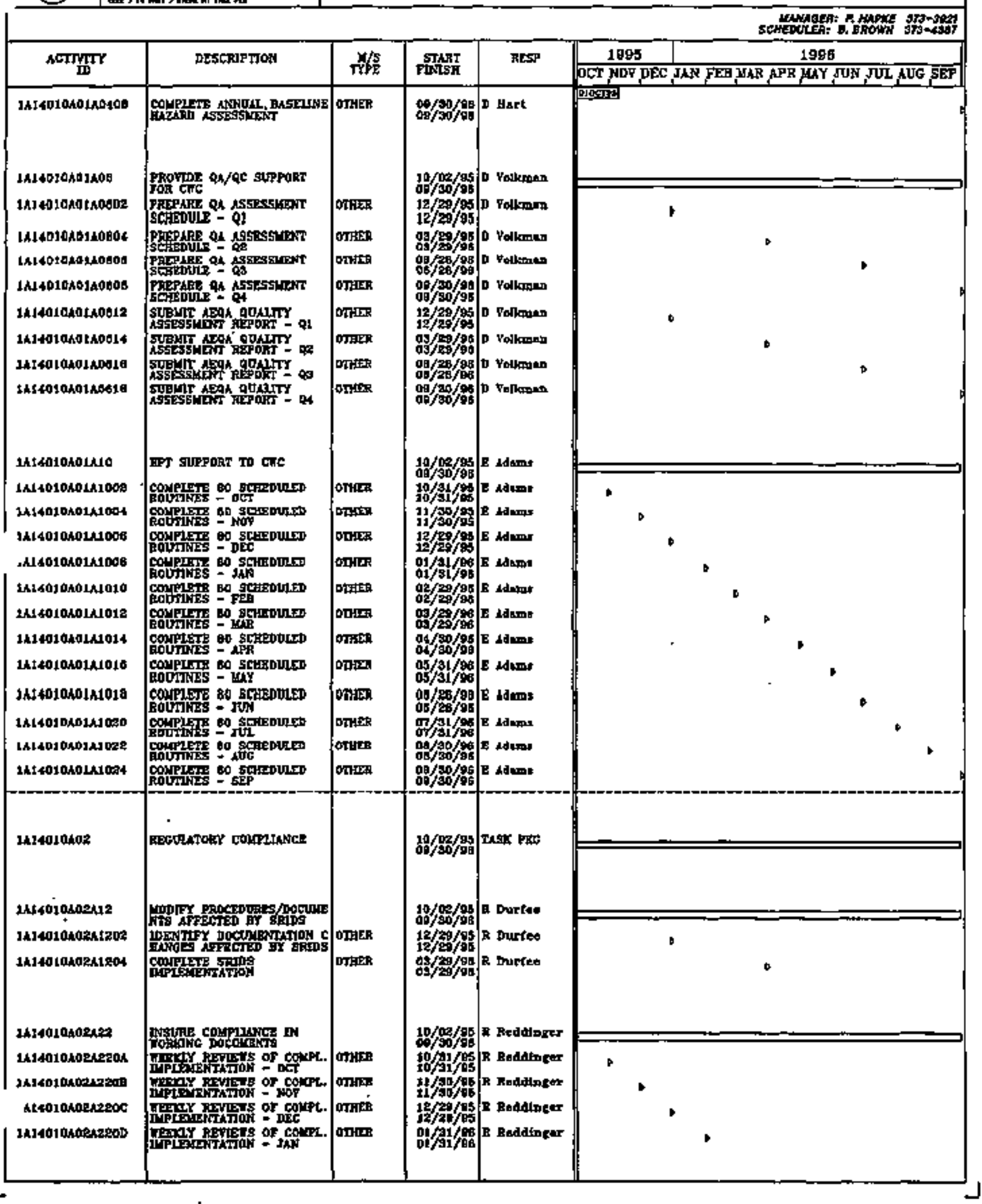




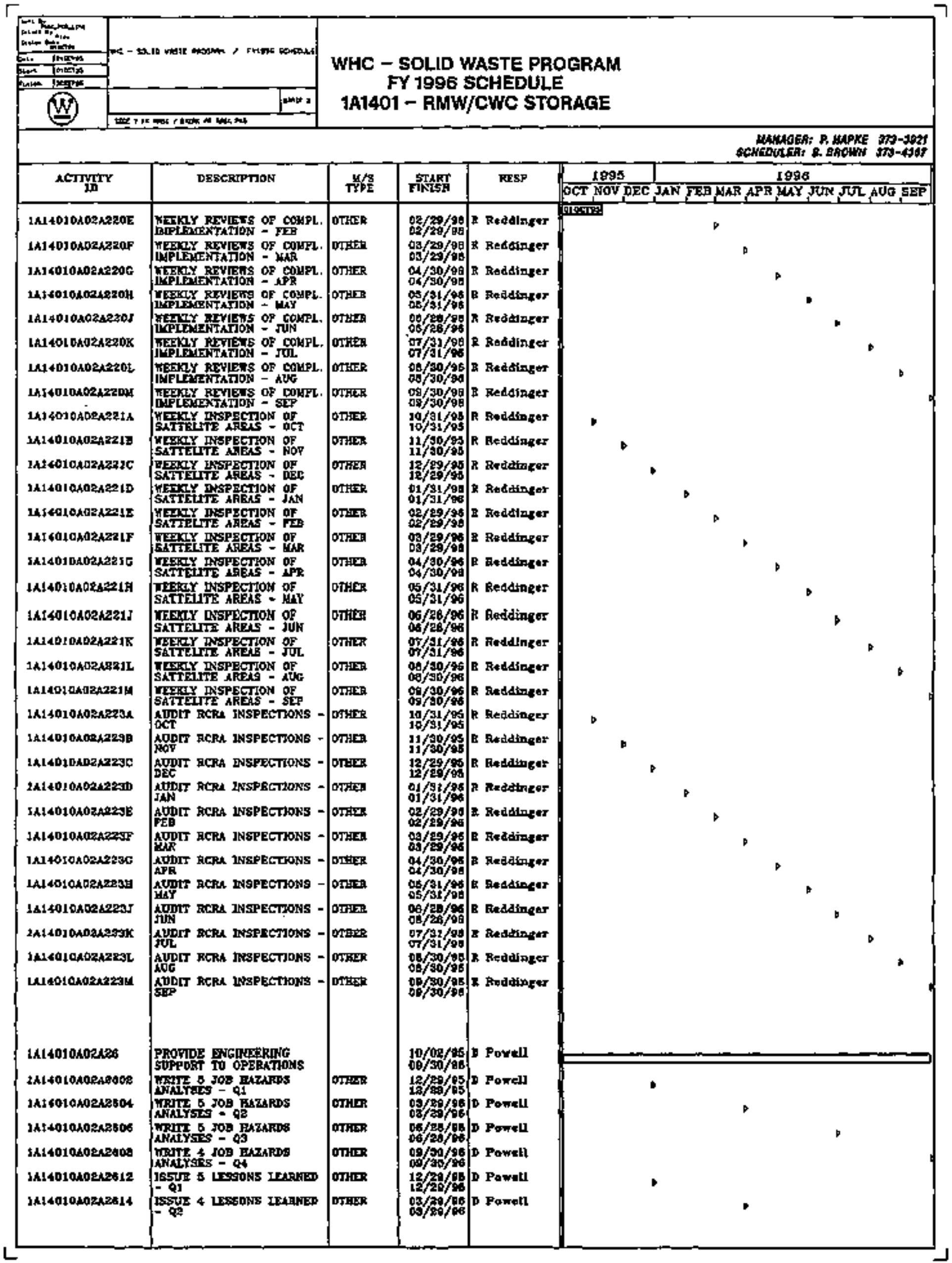


WHC - SOLID WASTE PROGRAM FY 1996 SCHEDULE TAJ401 - RMW/CWC STORACE

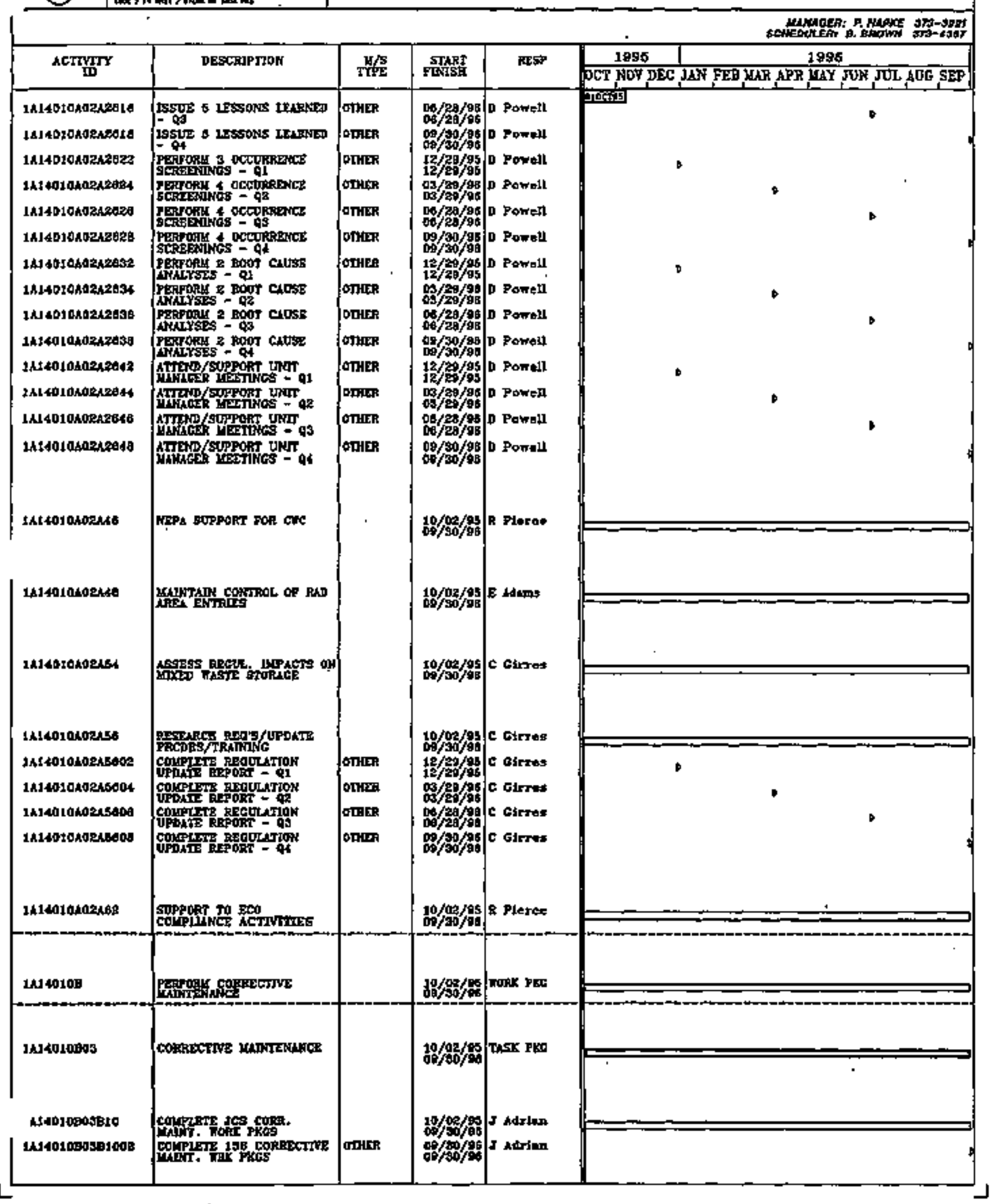




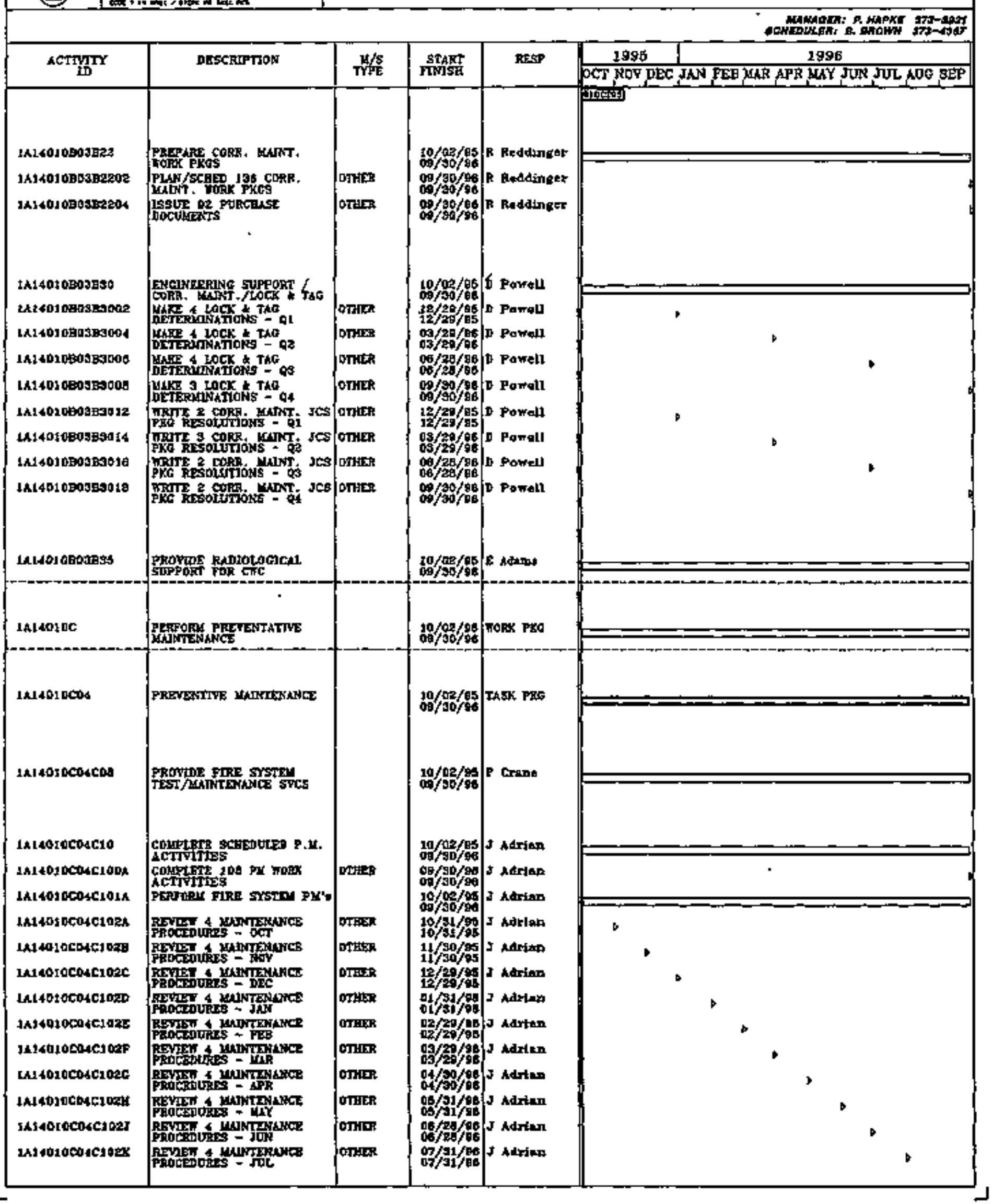




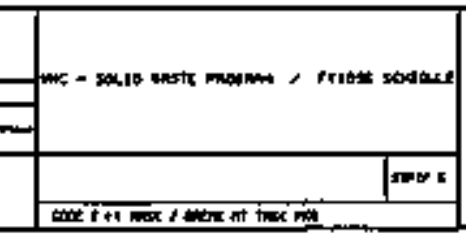

WHC - SOLJD WASTE PROGRAM

FY 1996 SCHEDULE 1At40T - RMW/CWC STORAGE

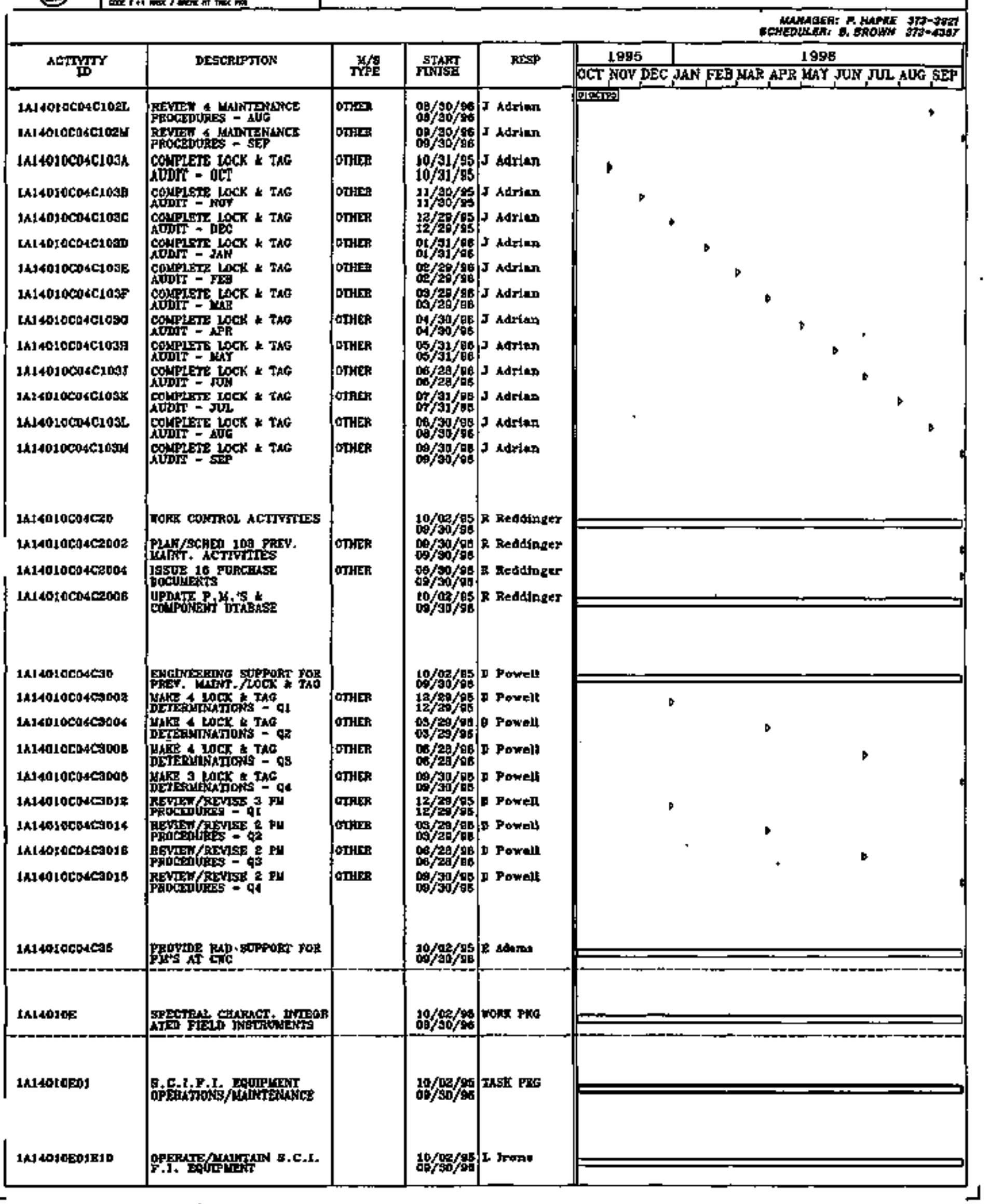


WHC - SOLID WASTE PROGRAM FY 1996 SCHEDULE 1A1401 - RMW/CWC STORAGE

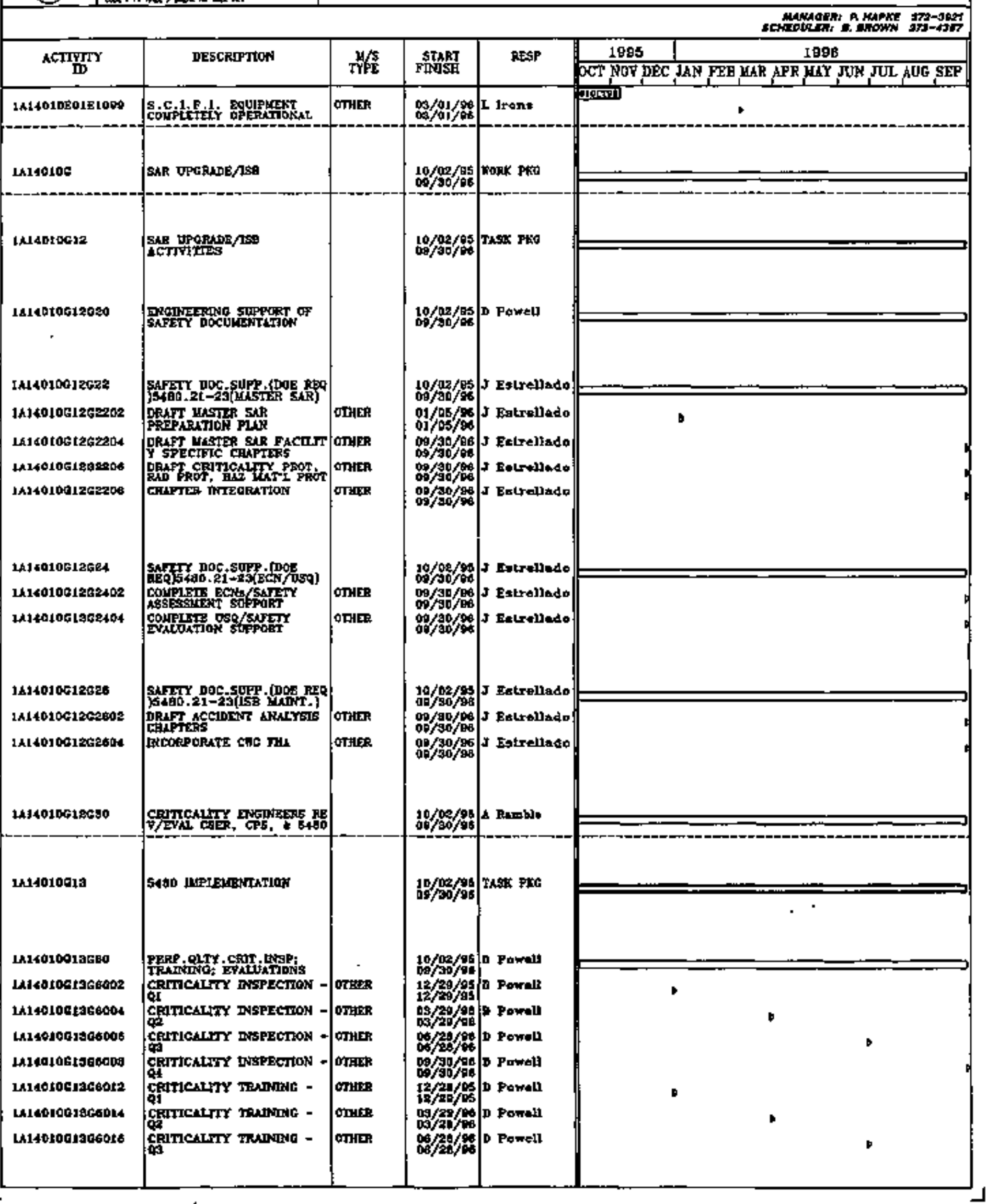


WHC - SOLID WASTE PROGRAM

FY 1996 8CHEDULE

1A1401 - AMW/CWC STORAGE

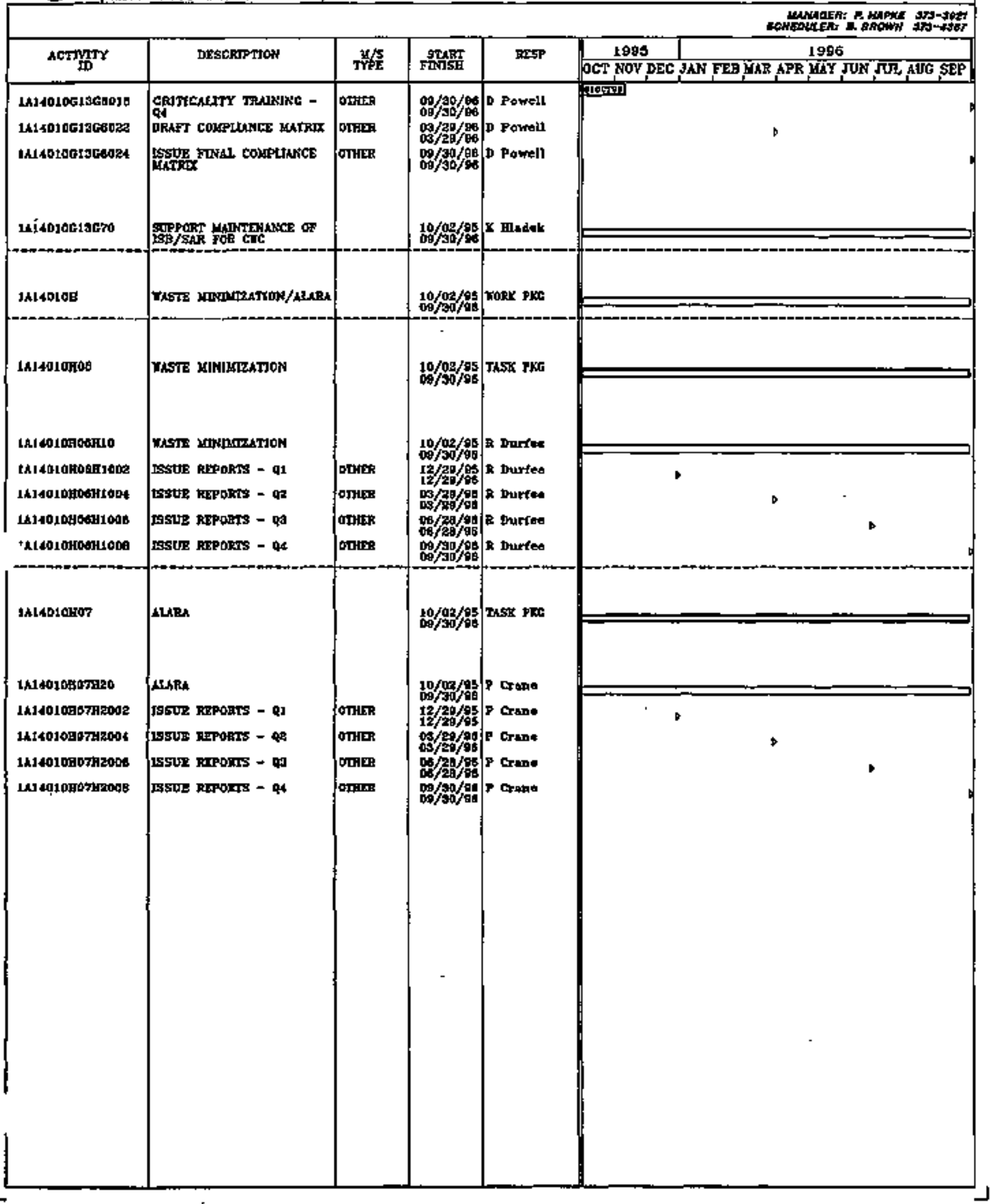




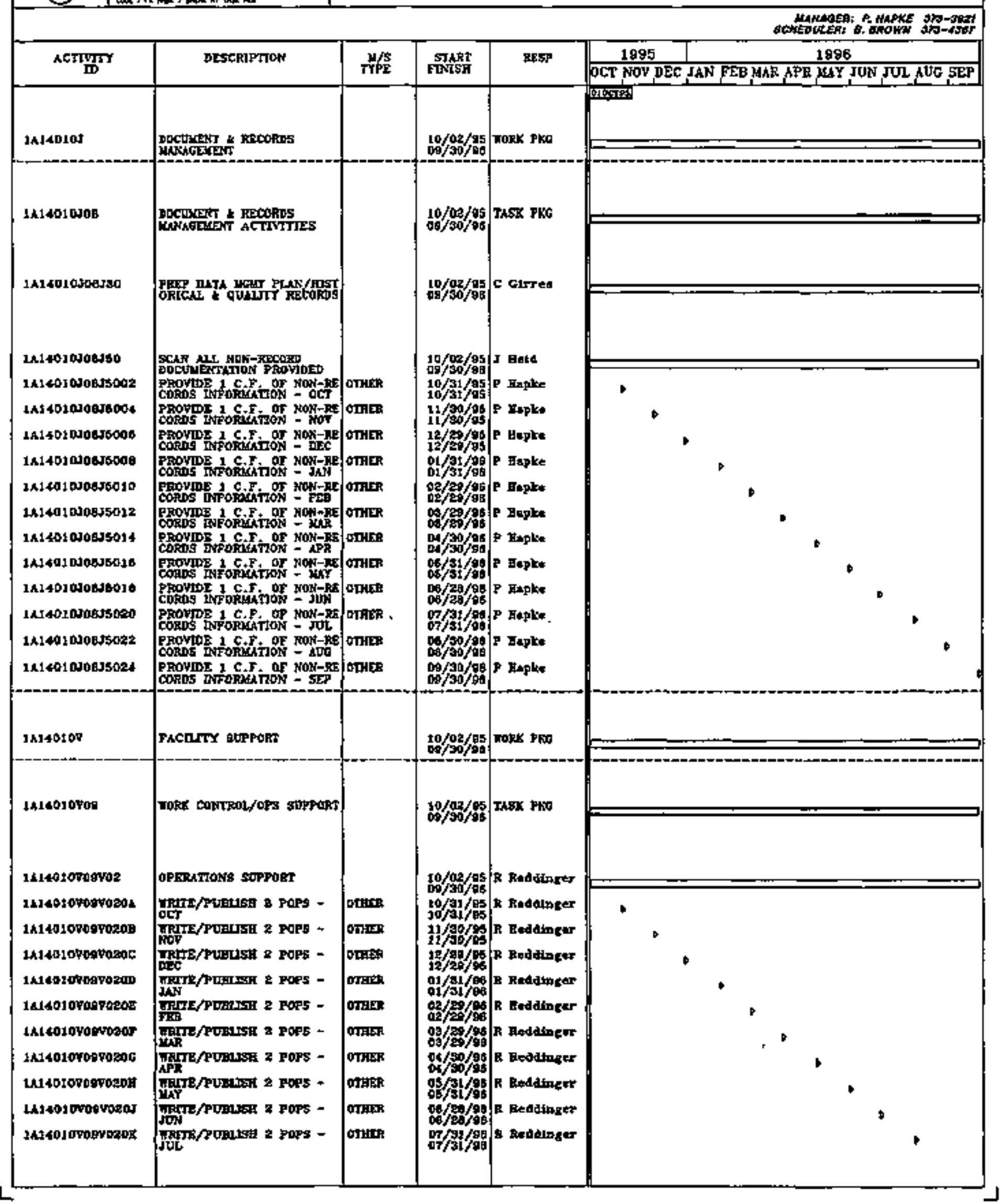




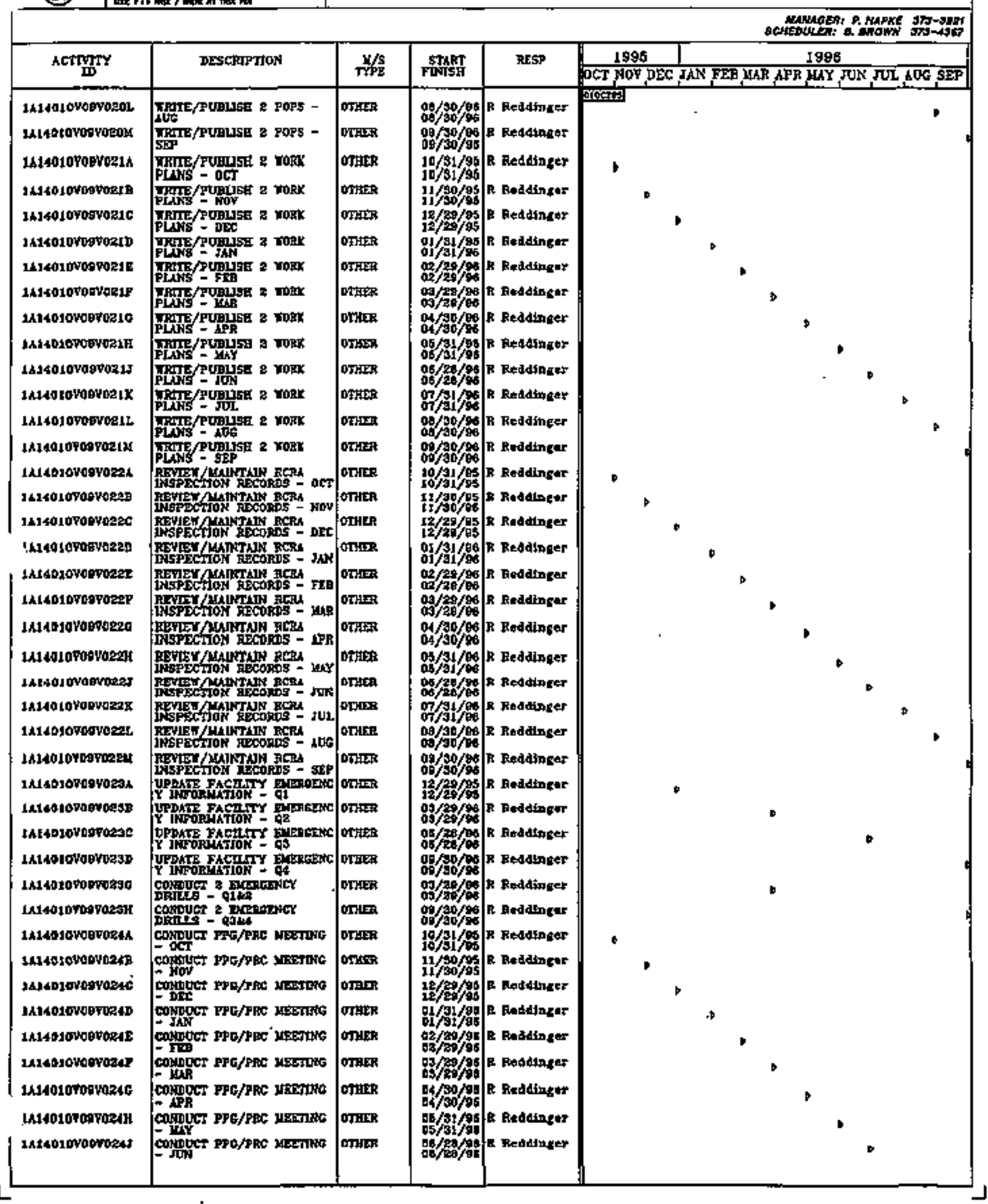


WHC - SOLID WASTE PROGRAM FY 1996 5CHEDULE 1A1401 - RMW/CWC STORAGE

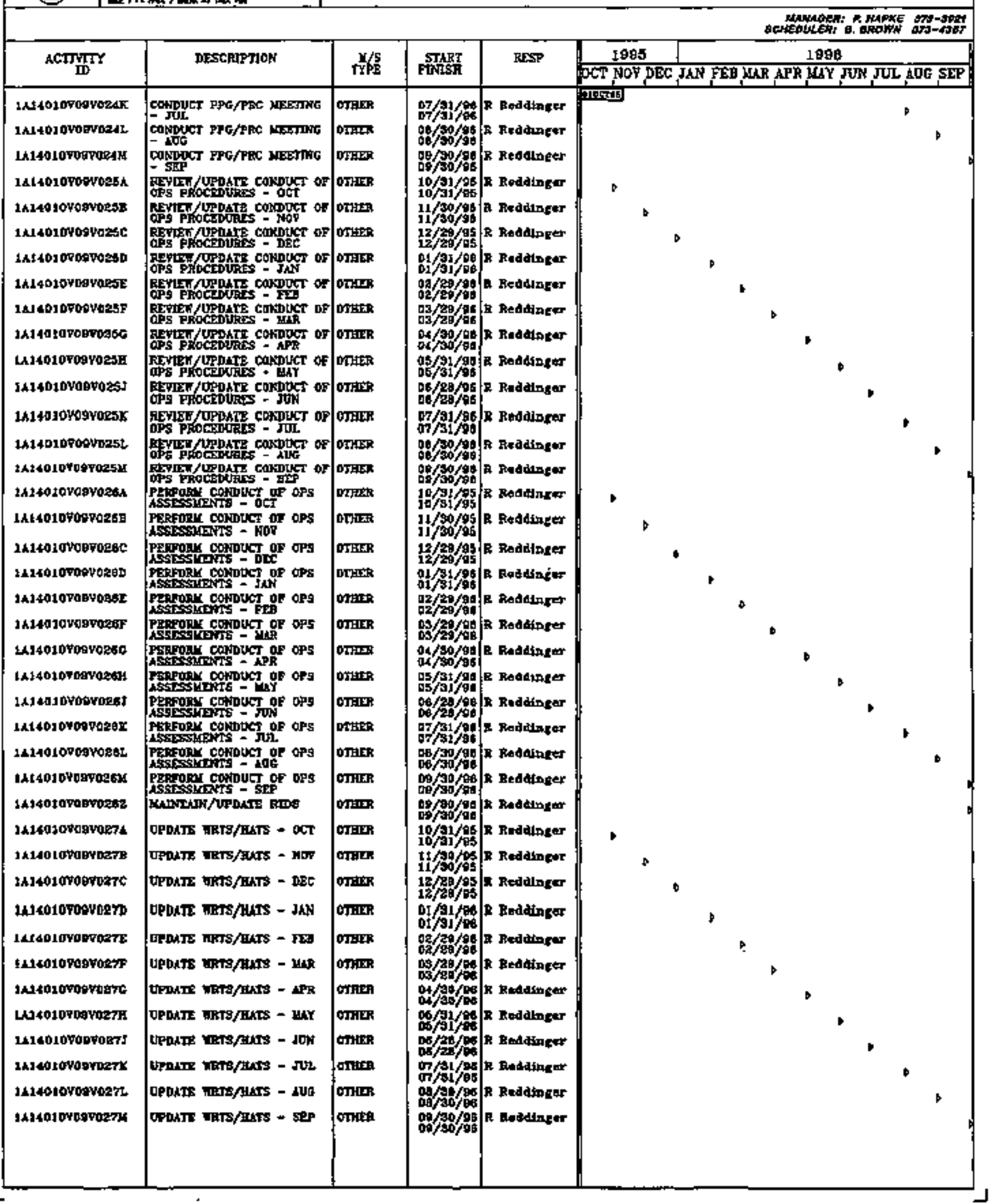


WHC - SOLID WASTE PROGRAM

FY 1996 SCHEDULE IA1401 - RMW/CWC STORAGE

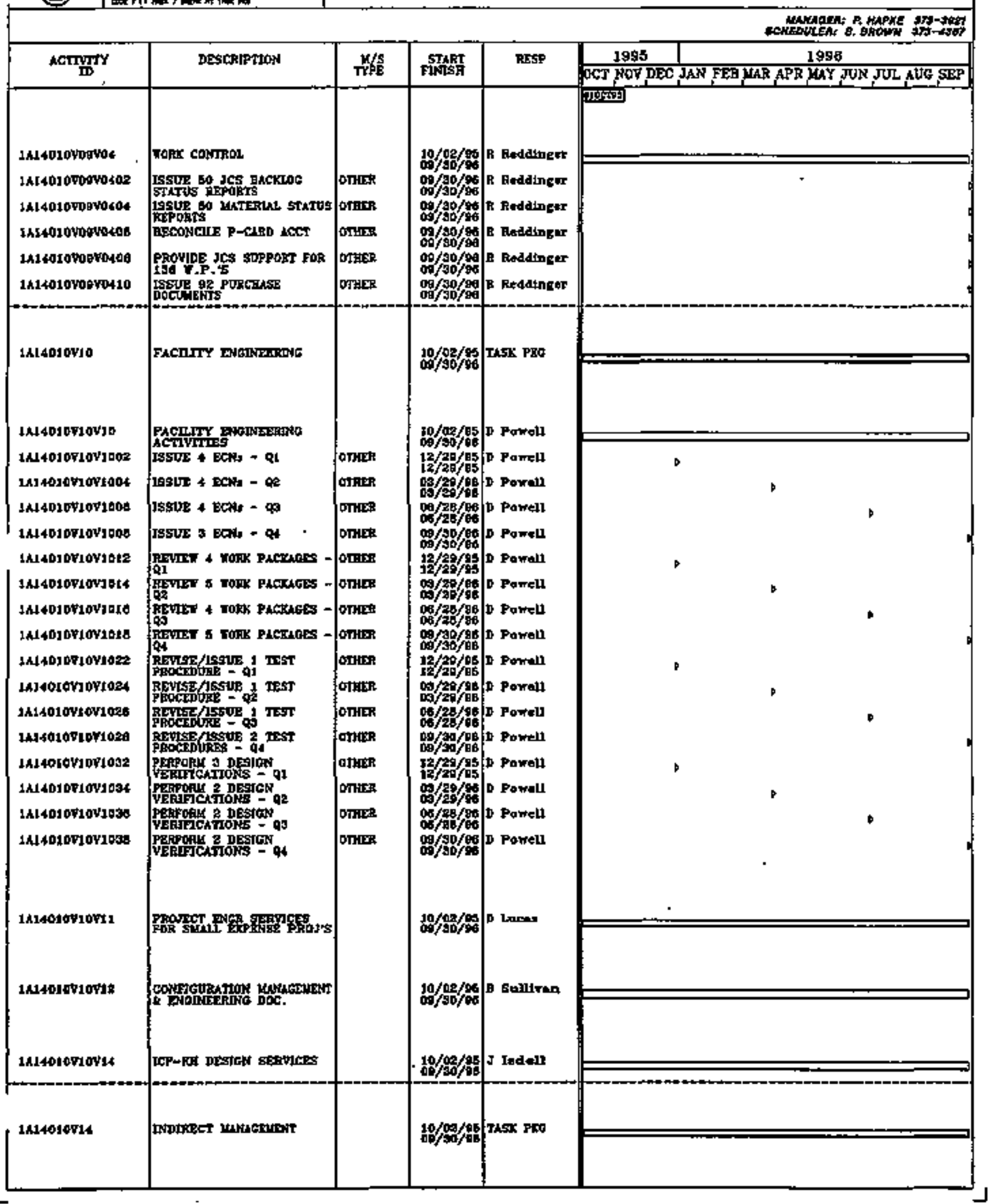


WHC - SOLID WASTE PROGRAM

FY 1996 SCHEDULE

IA1401 - RMW/CWC STORAGE

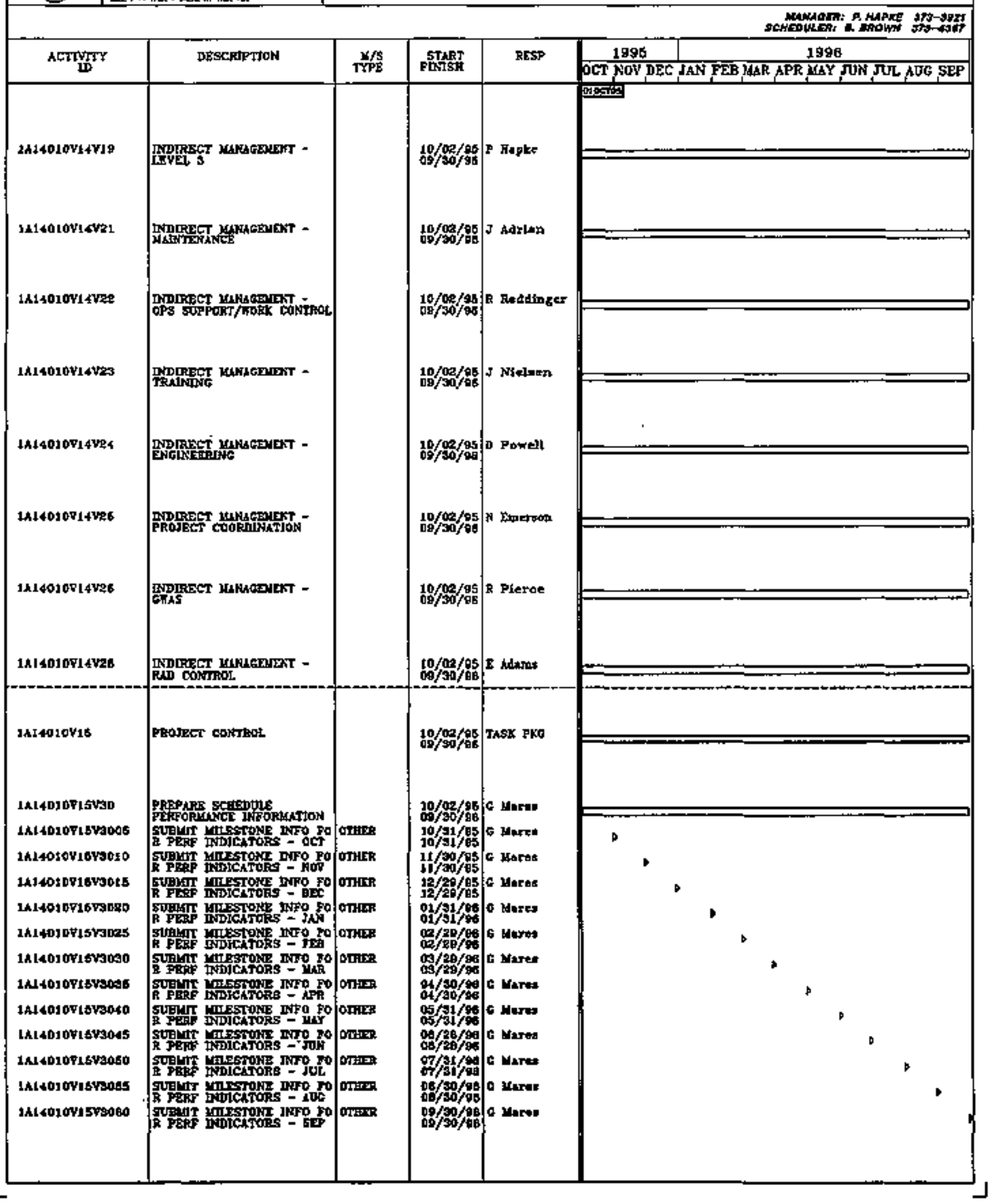


$\Gamma$

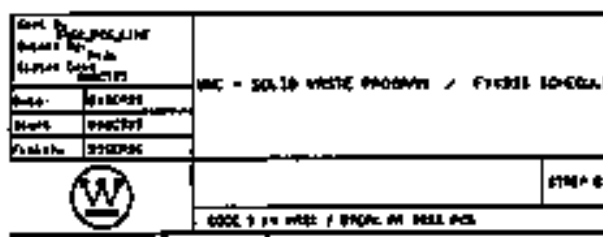

WHC - SOLID WASTE PROGRAM

FY 1996 SCHEDULE

IA1401 - RMW/CWC STORAGE

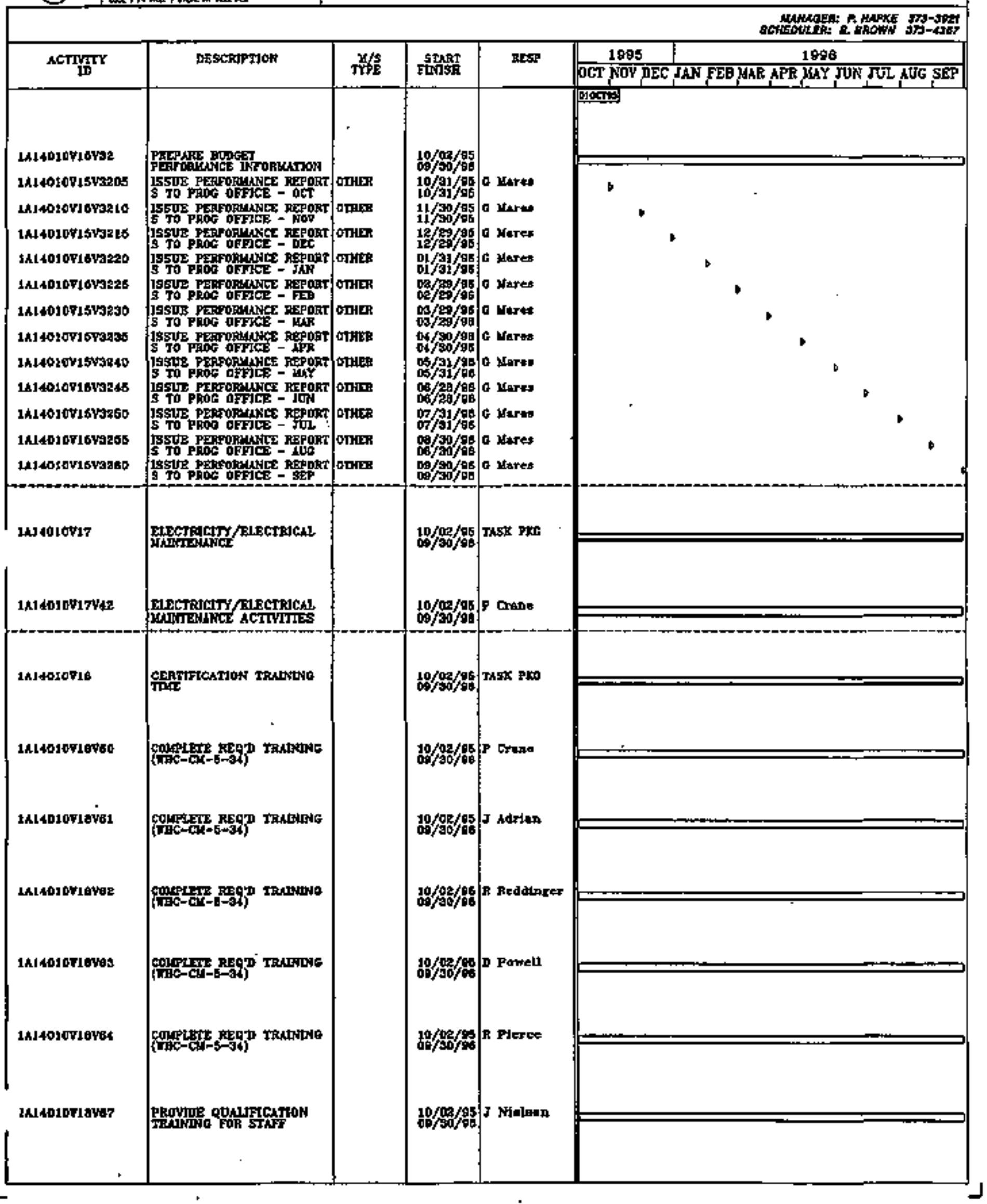




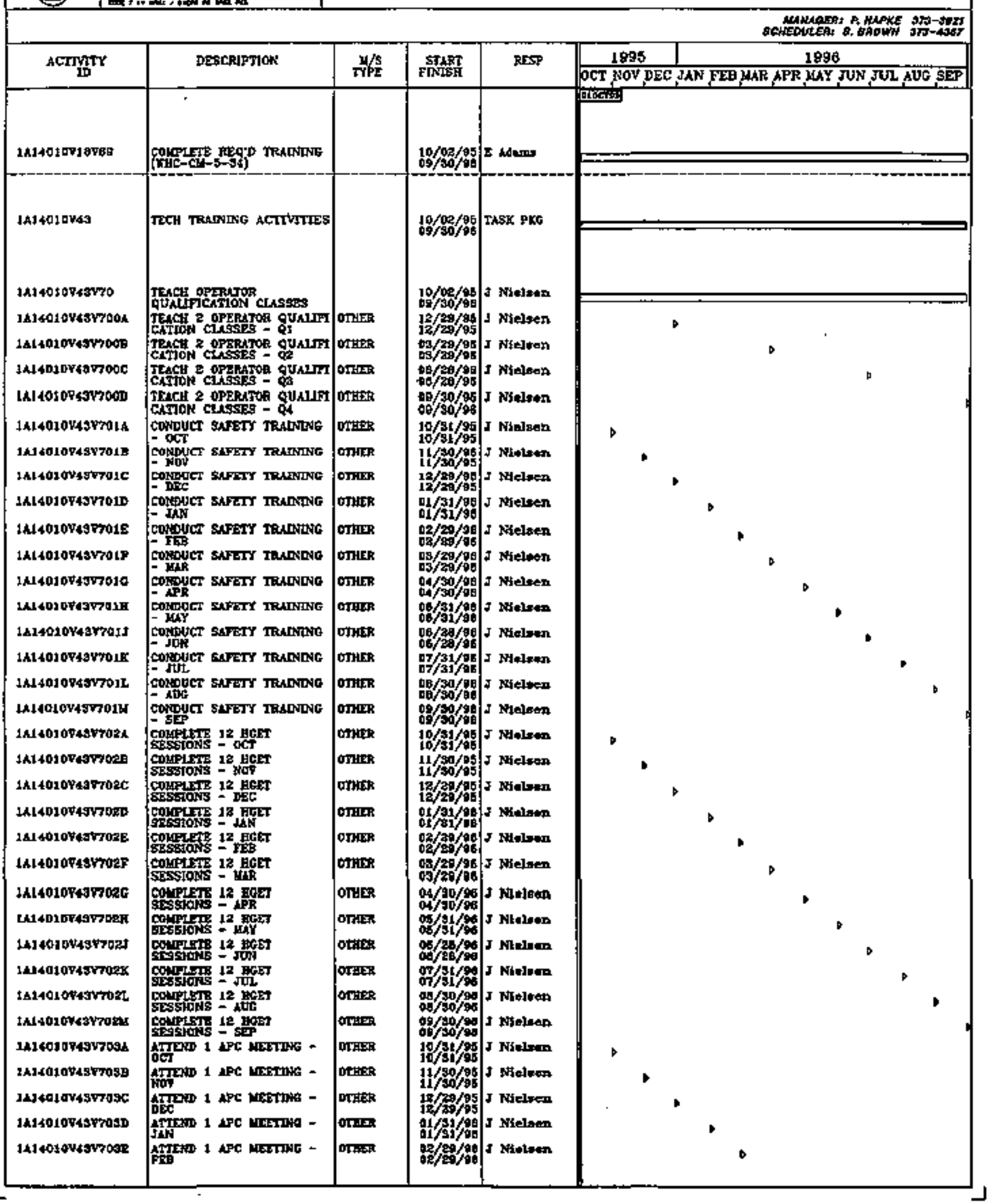


WHC - SOLID WASTE PROGRAM

FY 1996 SCHEDULE

1A1401 - RMW/CWC STORAGE

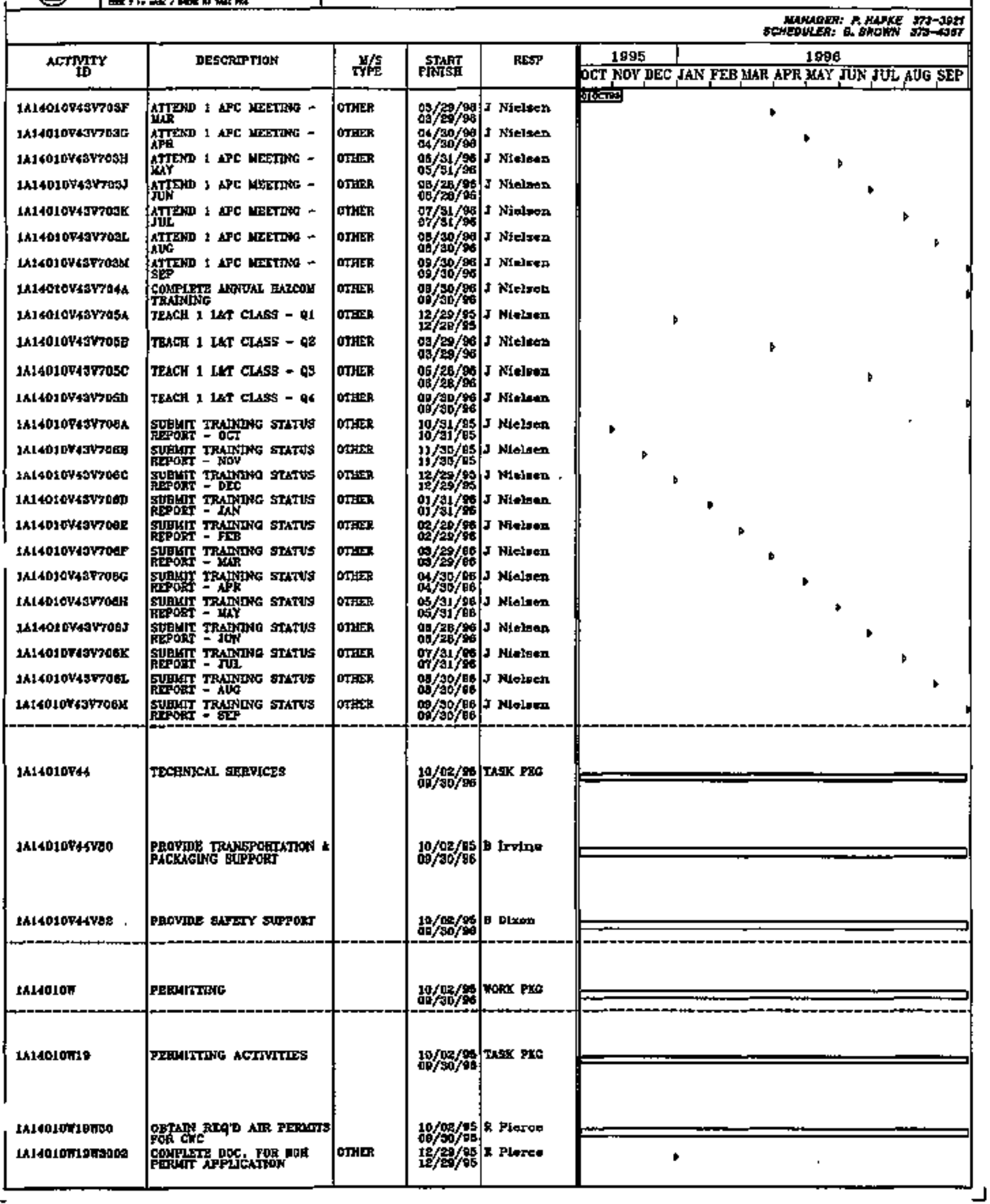




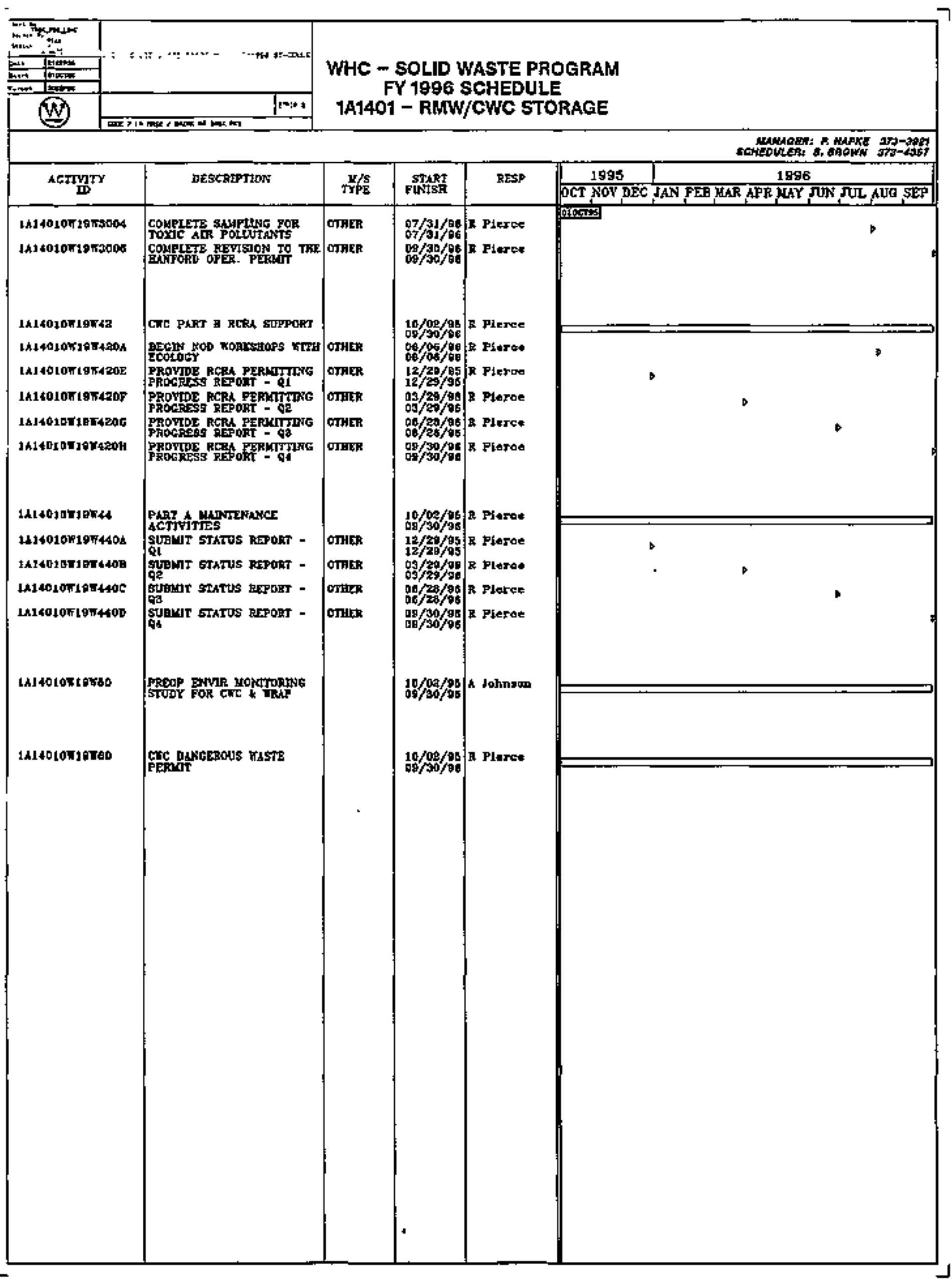




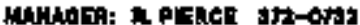

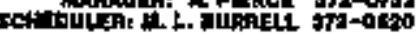

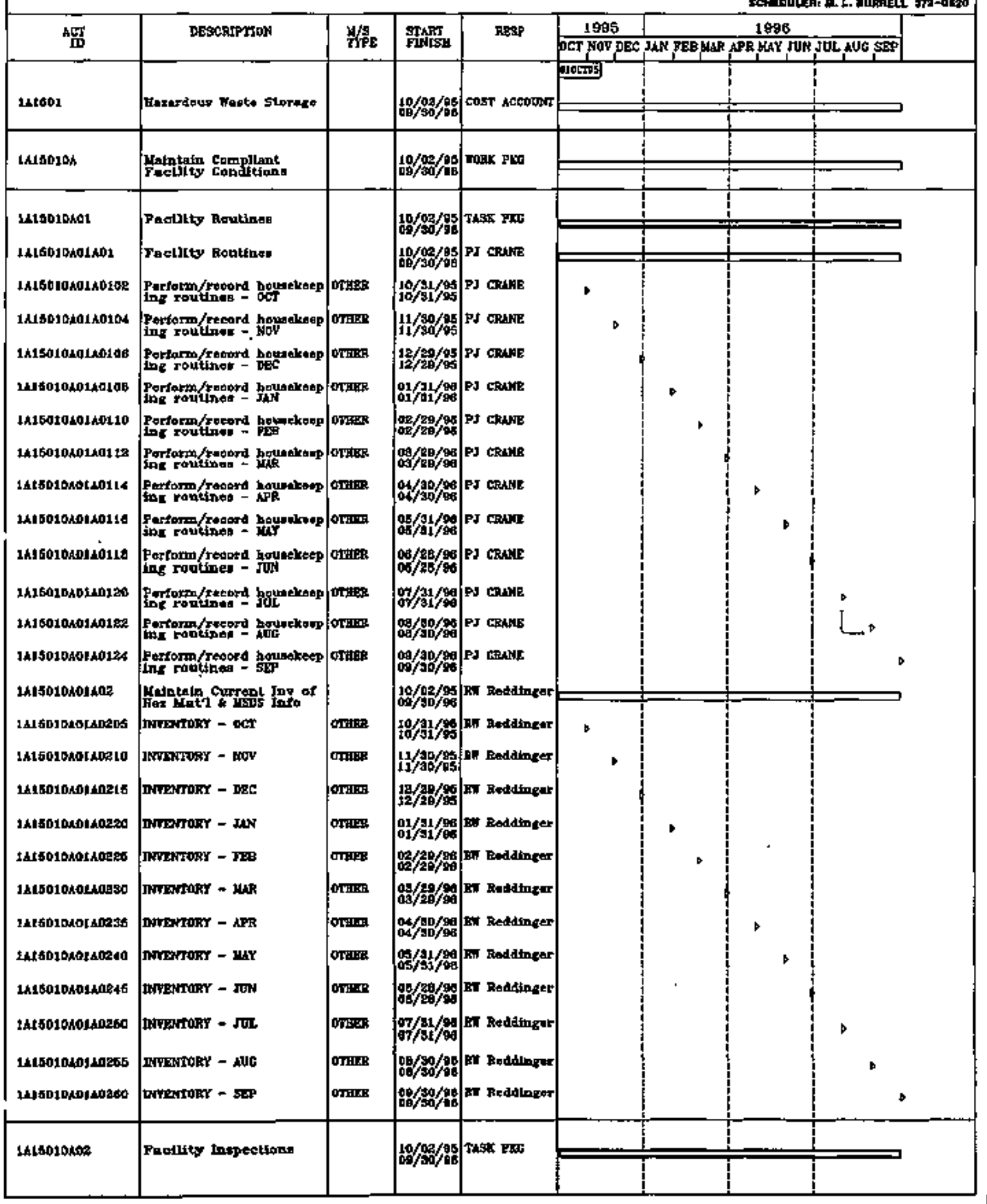




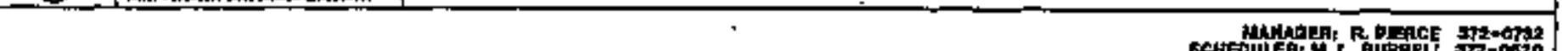

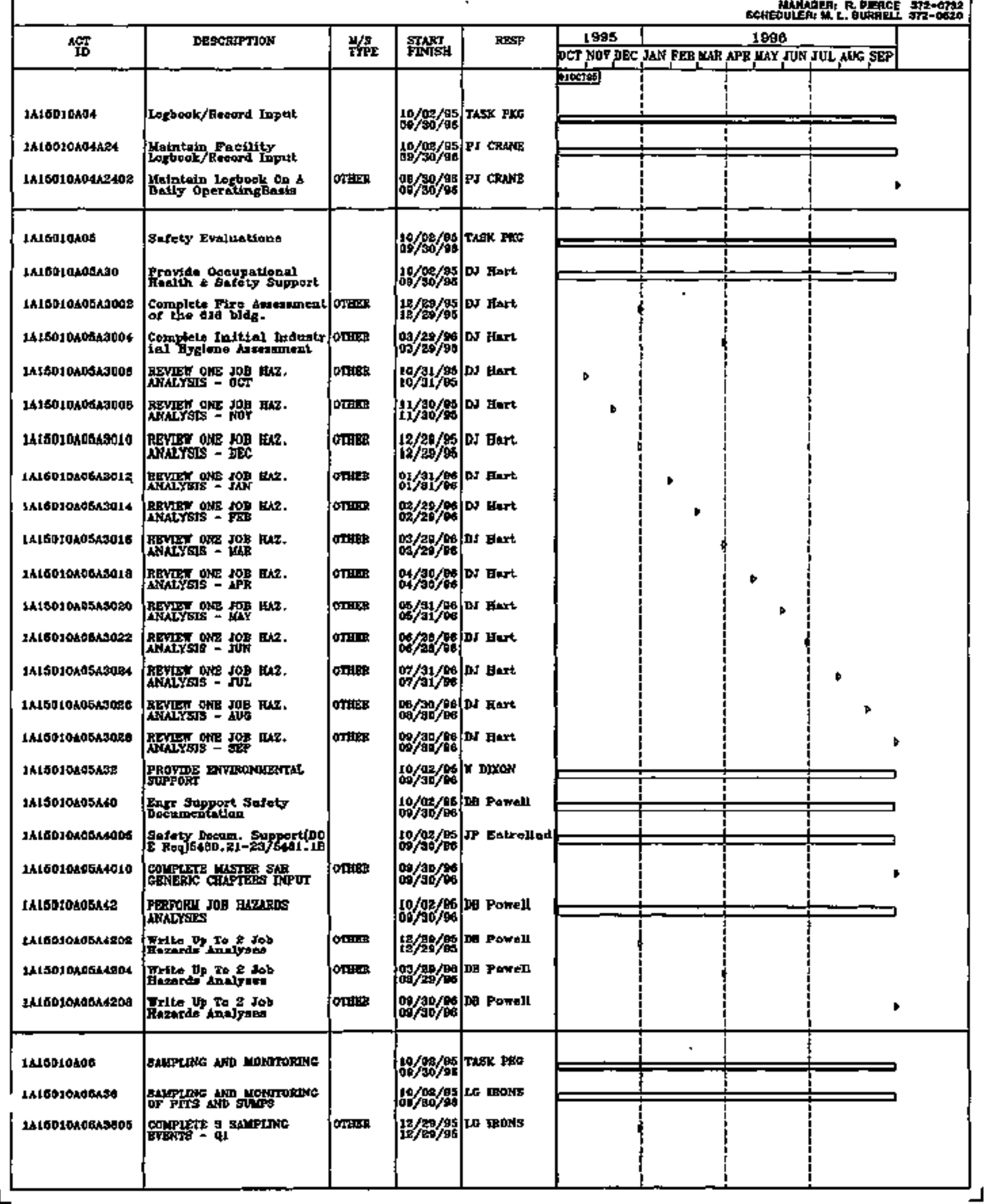




\section{WHC - SOLID WASTE PROCAAM \\ FY 1998 SCHEOUEE \\ 1A15OT - HAZAFDOUS WASTE GHAPKENT COOADINATION}

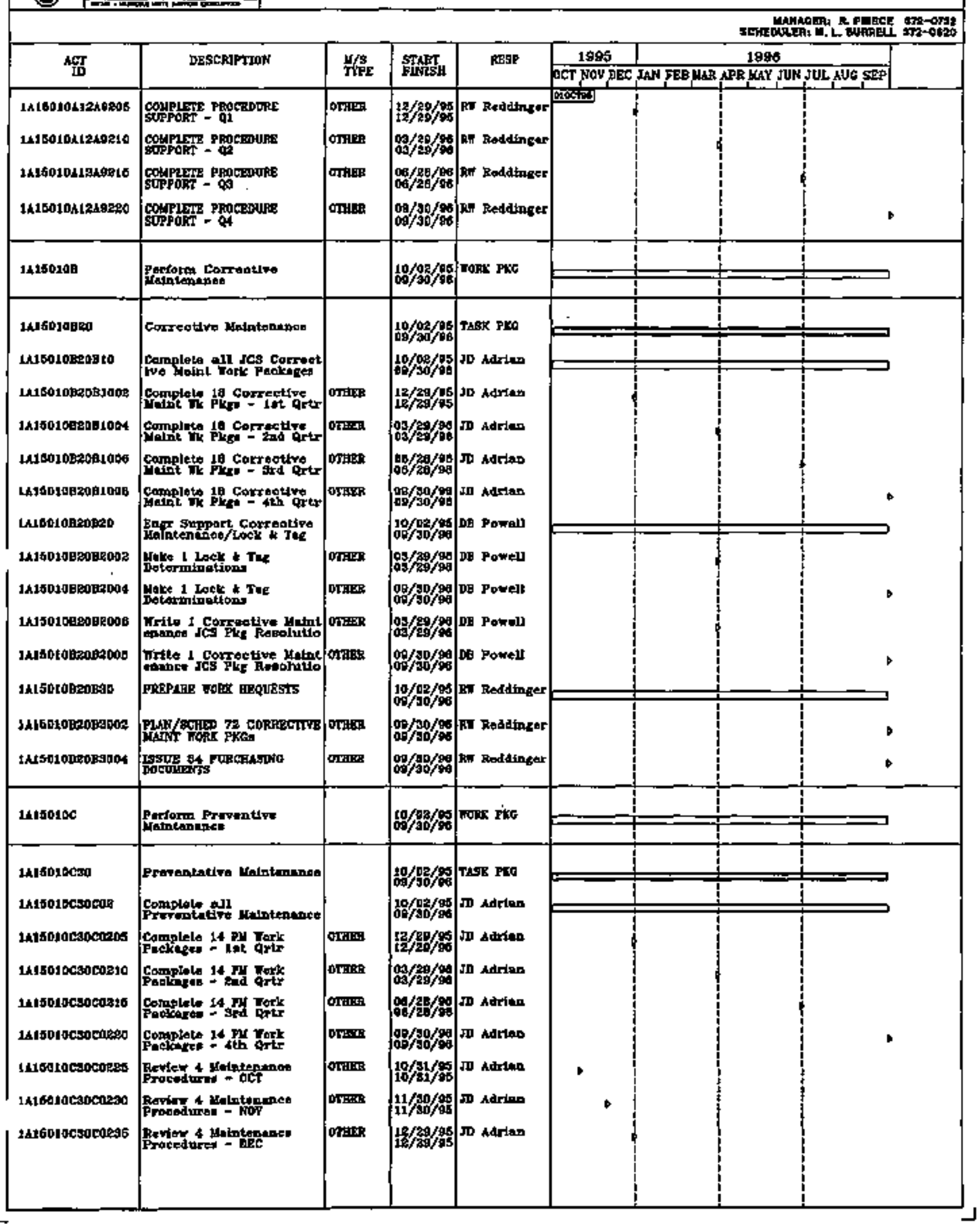




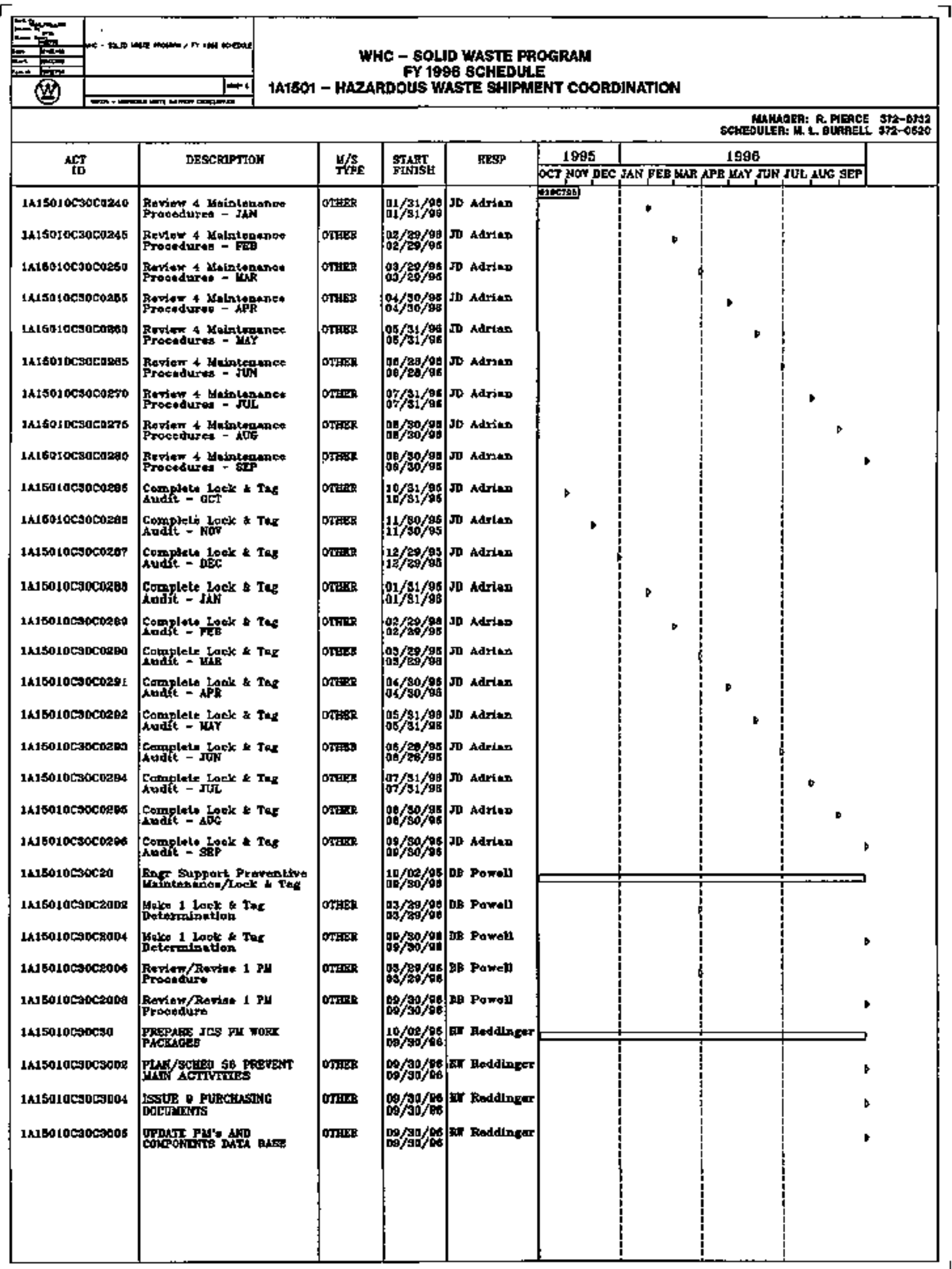




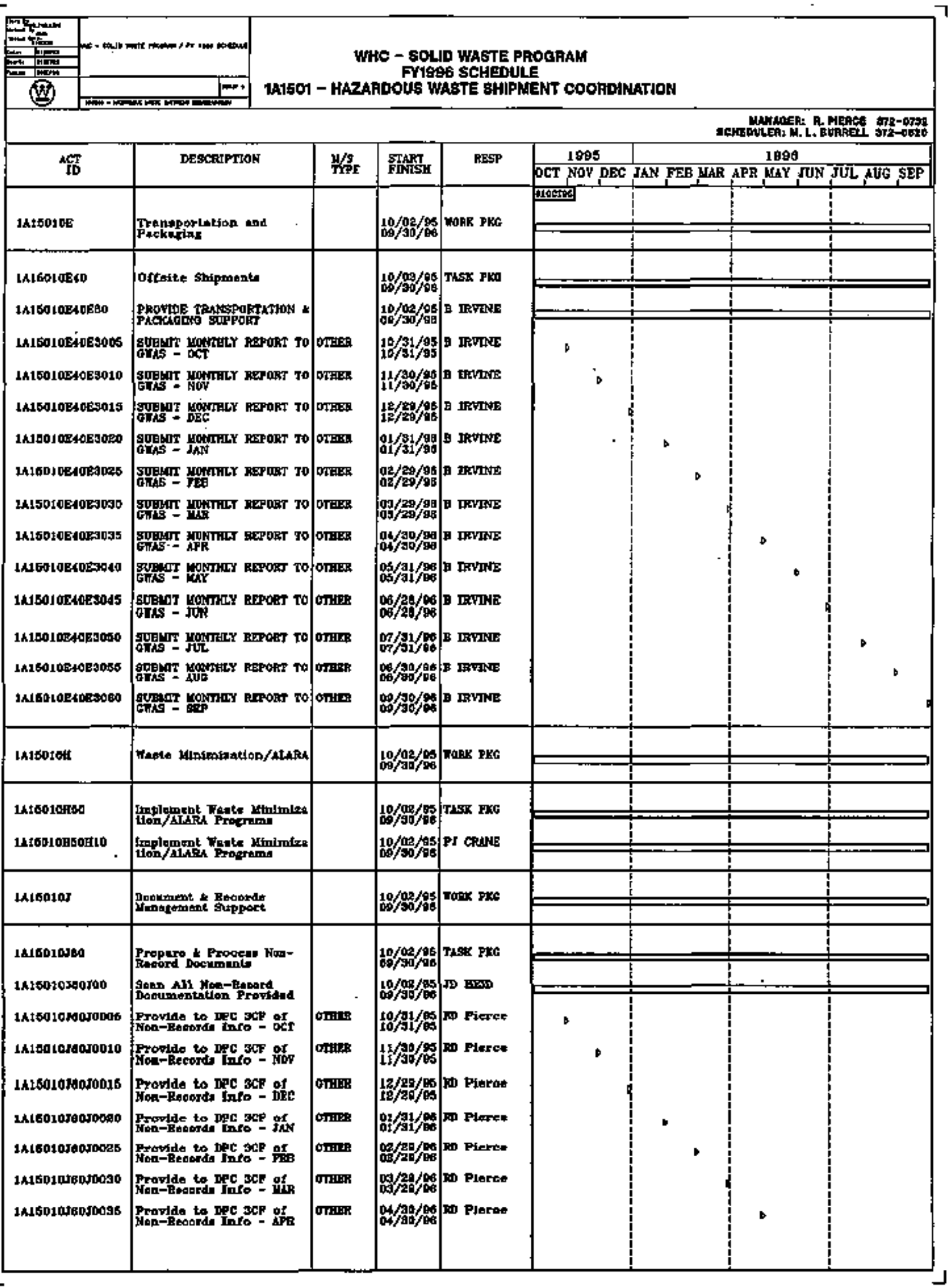




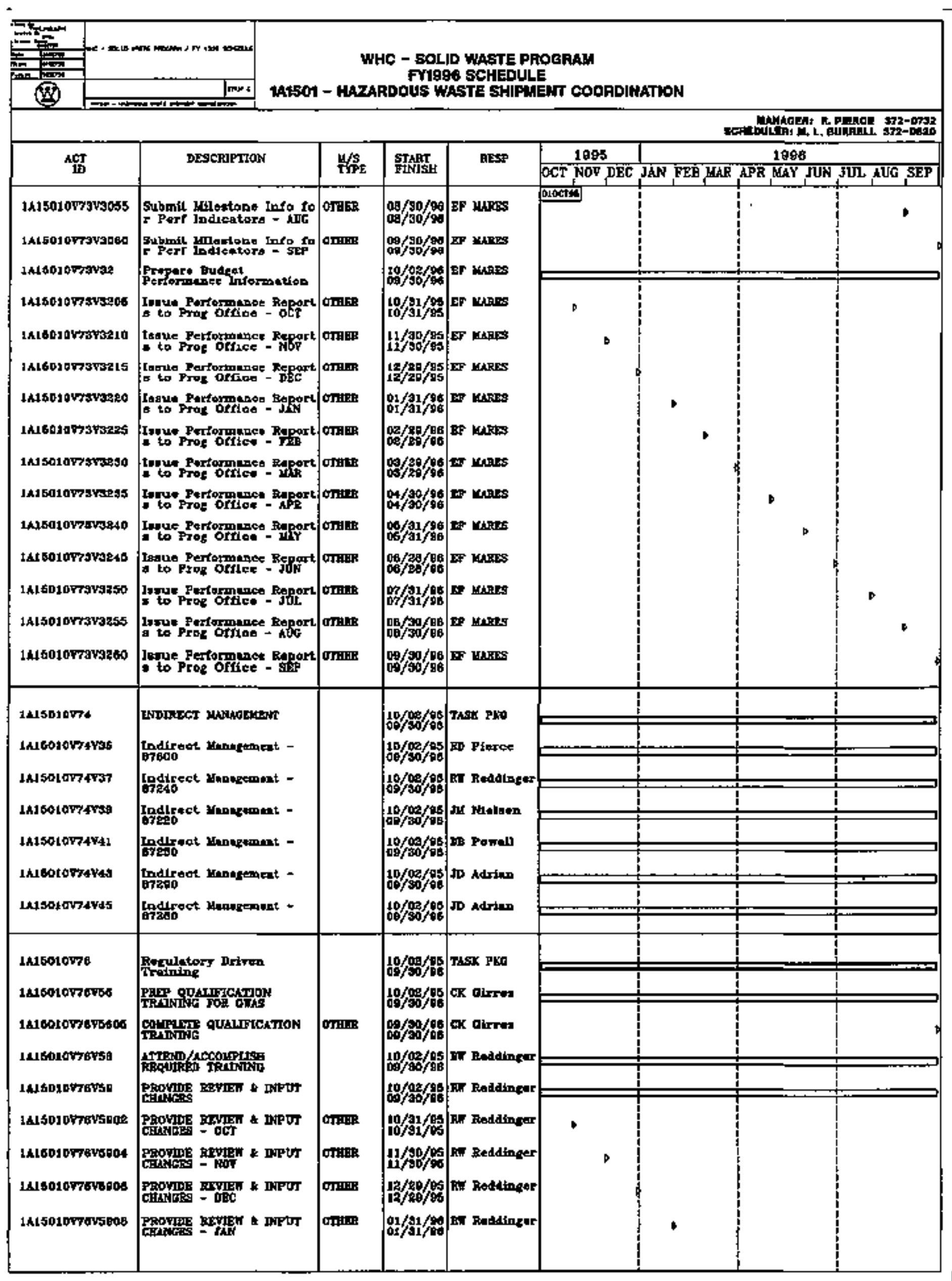




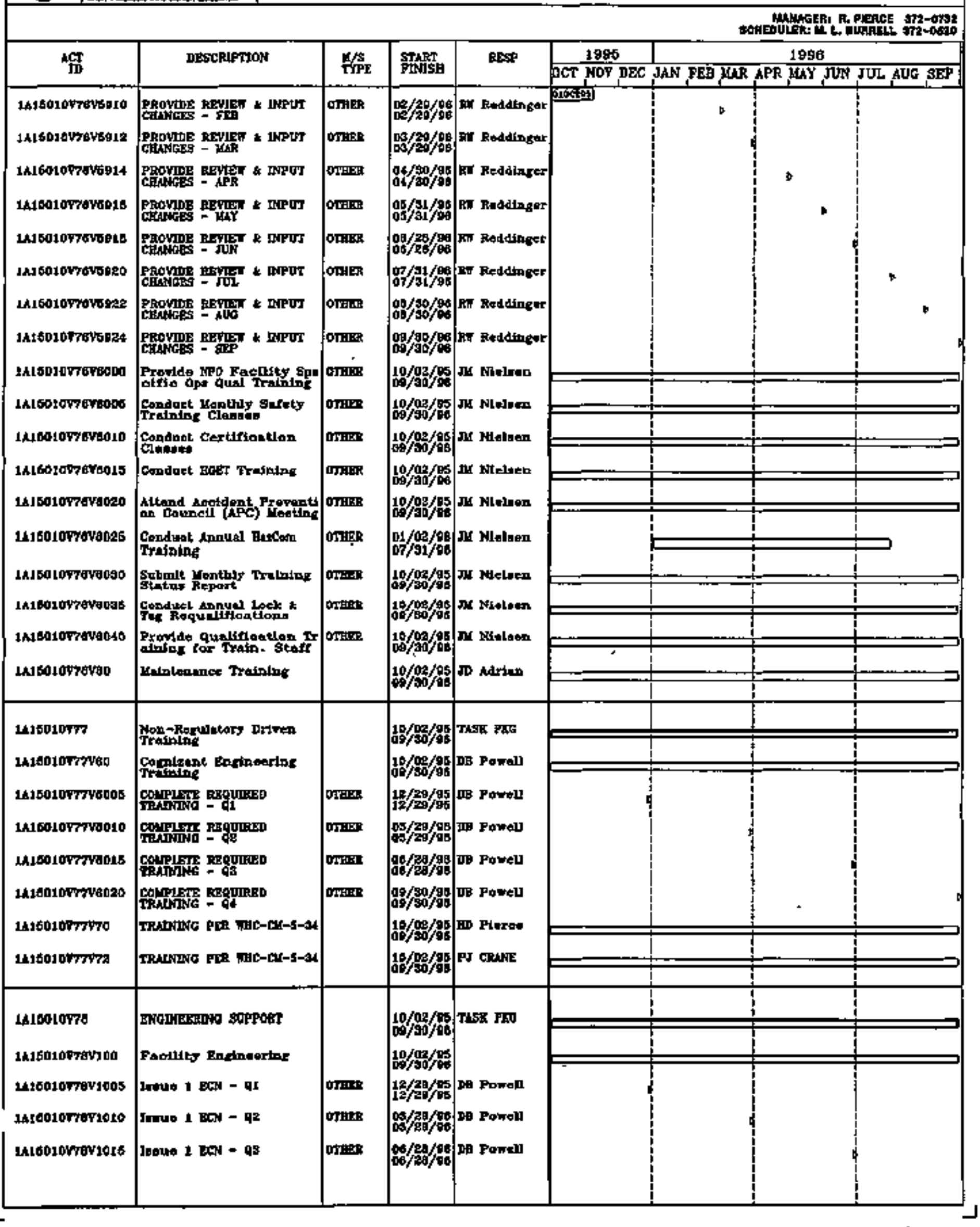




\section{$\Delta$}

IALEQSOVTEYIORO

1A16010V70Floes

1AJ6010978v1090

1Aj5010F7evjous

1AJ5010Y7ar1040

1k150layzoriass

14]501078ave000

L1501077arso00

14]5010r7ay40

341501078V4006

14J5010770V4010

AdSO10770740!5

A16010478YBR

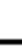

1A15BJOVO

LA15010\%7970

LAL5010ThTV1005

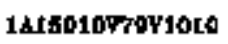

1M501079VJOL

1A1601079y toed

Idsotorrertors

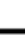

14sojapa

IAHSBJOVEIY4O

1455010\%8174005

1Asosopat74010

1450107a IFadG

LAEDIDHSYYOEO

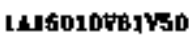

MIs0107b1 17ados

2A1S01DFAJvo10

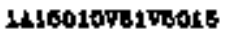

LAt601076178020

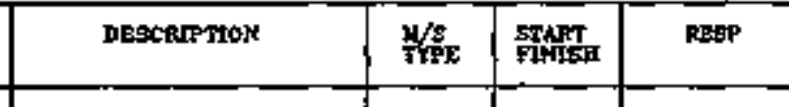

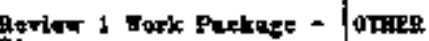

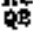

Roview 1 Hork Package =

Rerief /saute Teat rosedusto - ad

Perfoym 1 Derien

Pertorm 1 Detim Verifontion - oskt

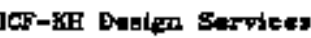

Configaration Kaagtematit 4 Enginearing Doo

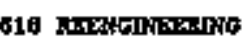

IDFNIIT 3 options $\mathrm{FOR}$ IIDG B18 USh

Divelop L 1SSTE COST ᄂ SCHATULs REPOETS

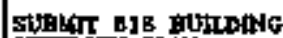
SHOTDOT PLA

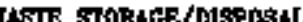
TICGEMGAL REYAL

WOFI CONTRSE

wodx comproc.

IFYO 30 JCS Hack LOC

ISSUE SO MTERIAL STATUS GTHER WEDRTS

BECONCILE P-CARE ACCOUST OTHER

FROYIDE JCS SOPPORT YOR OHHER 72 Work PKo's

ISSUE 34 Amerasula Dections

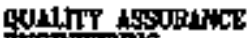

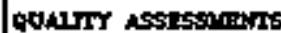

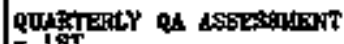

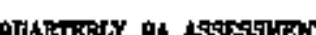

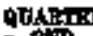

atiperaxay al ussessicant

Qthikat - $\mathbf{s i b}$

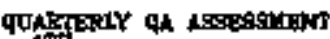

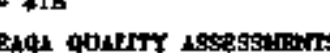

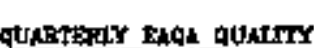

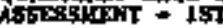

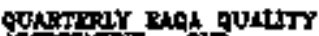

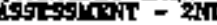

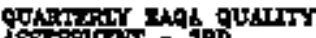
SSESTiknt - JP

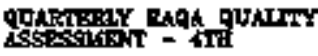

12/30/05 DO POFEll $12 / 25 / 95$

03/20/Bo Do Pomell $09 / \mathrm{BO} / 90$

06/20/96 Do Powell $\infty / 26 / \infty$

oo/ayes Da Powell $00 / 0 / 0$

OTHEB D3/20/06 De Powall $00 / 21 / 10$

OTHER $09 / 39 / 96$ De Powell $09 / 90 / 8 \mathrm{~B}$

10/02/95 De Powell $0.2 / 50 / 06$

10/0z/os to Powell $06 / 30 / 100$

oe/oj/go De Doptop $06 / 20 /$ at

OS/01/p6 He Hoyaton os/a1/0 ot

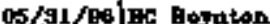
$05 / 91 / 106$

ac/89/06/EC Boyaton in $/ 20 / 06$

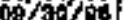

$10 / 02 / 05:$ TLST FYG

at/o/80.

$10 / 09 / 90$

$0 \% / 00$

$00 / 50 / 0$ $0 \% / 50 / 0$

og/9y/ge In Reddinger (3)

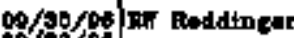
由/ $150 \%$ Do/ $19 / 06$

$00 / 50 / 06$

10/08/95: Do vellmm

18/28/05 to vallem

t. $/ 20 / 45$

os/ap/9s Do volkmm

$0 \mathrm{~s} / 2 \mathrm{D} / \mathrm{As}$

06/20/96 Do Fallman

$06 / 2=/ 106$

09/30/40 Do Volloman

$\mathrm{De} / \mathrm{aO} / \mathrm{O}$

to/0e/es Dn Vothen

$00 / 50 / 00$

12/2e/gs me Volkman $12 / 20 / 85$

03/29/09 to volknens $03 / 20 / 45$

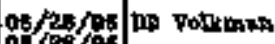
o.j/8

op/9p/96 bo votruat

$100 / 90 / 96$
10/008/05 tic Bogratom

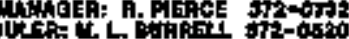
GehEOUU Th:

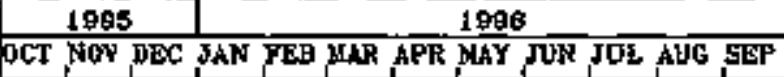

OLOCF

OTHER Do/ge/po Rit Rodalinger

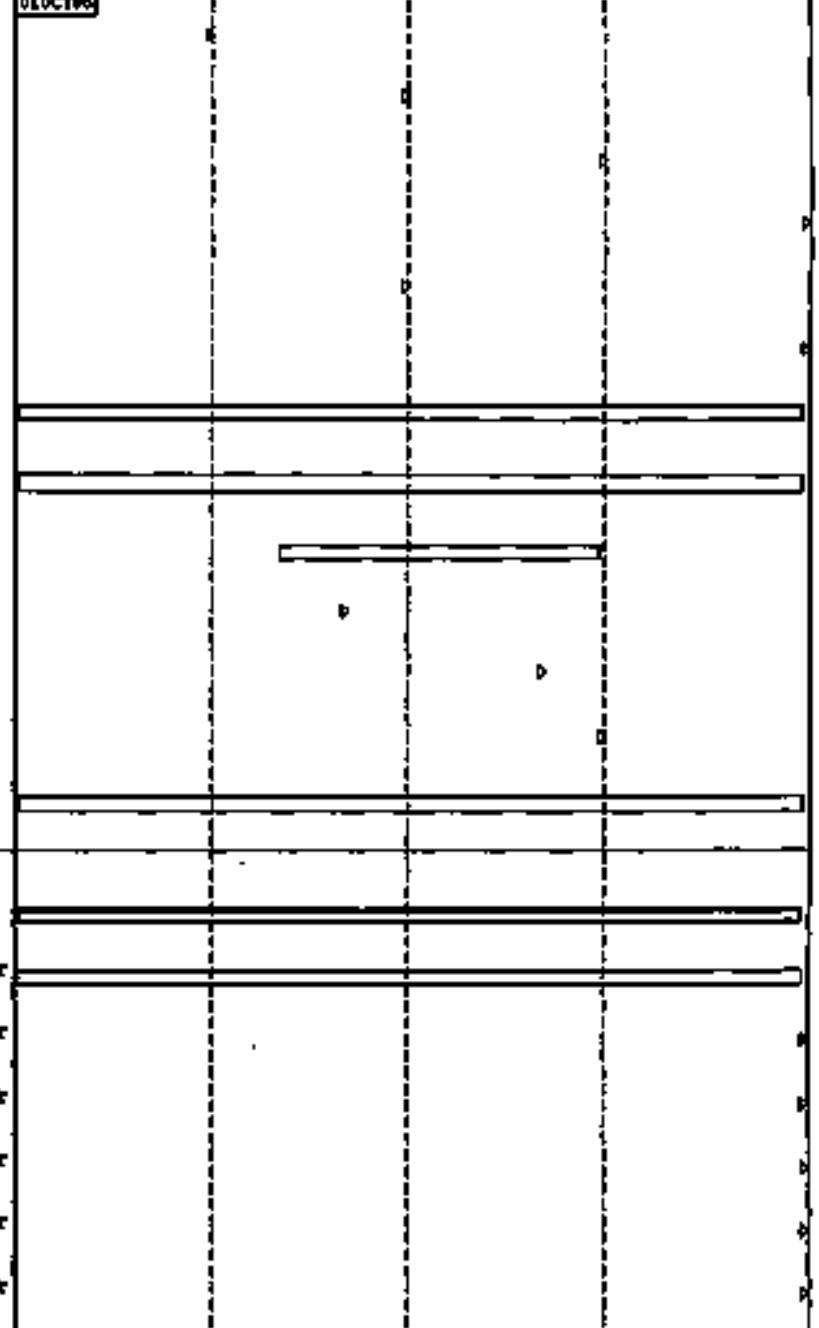

0/02/05 TLST PKG

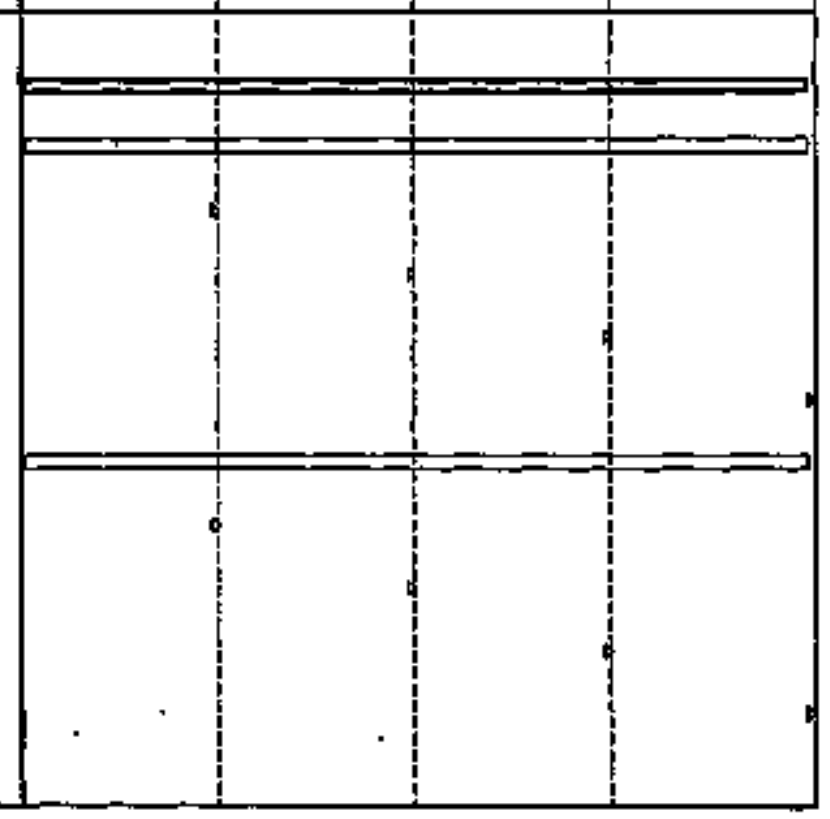




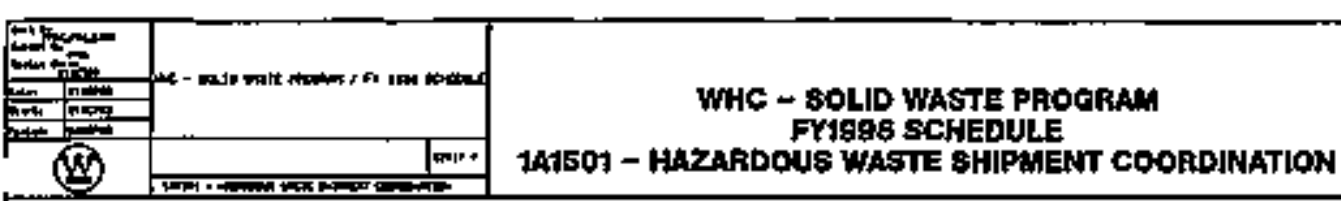

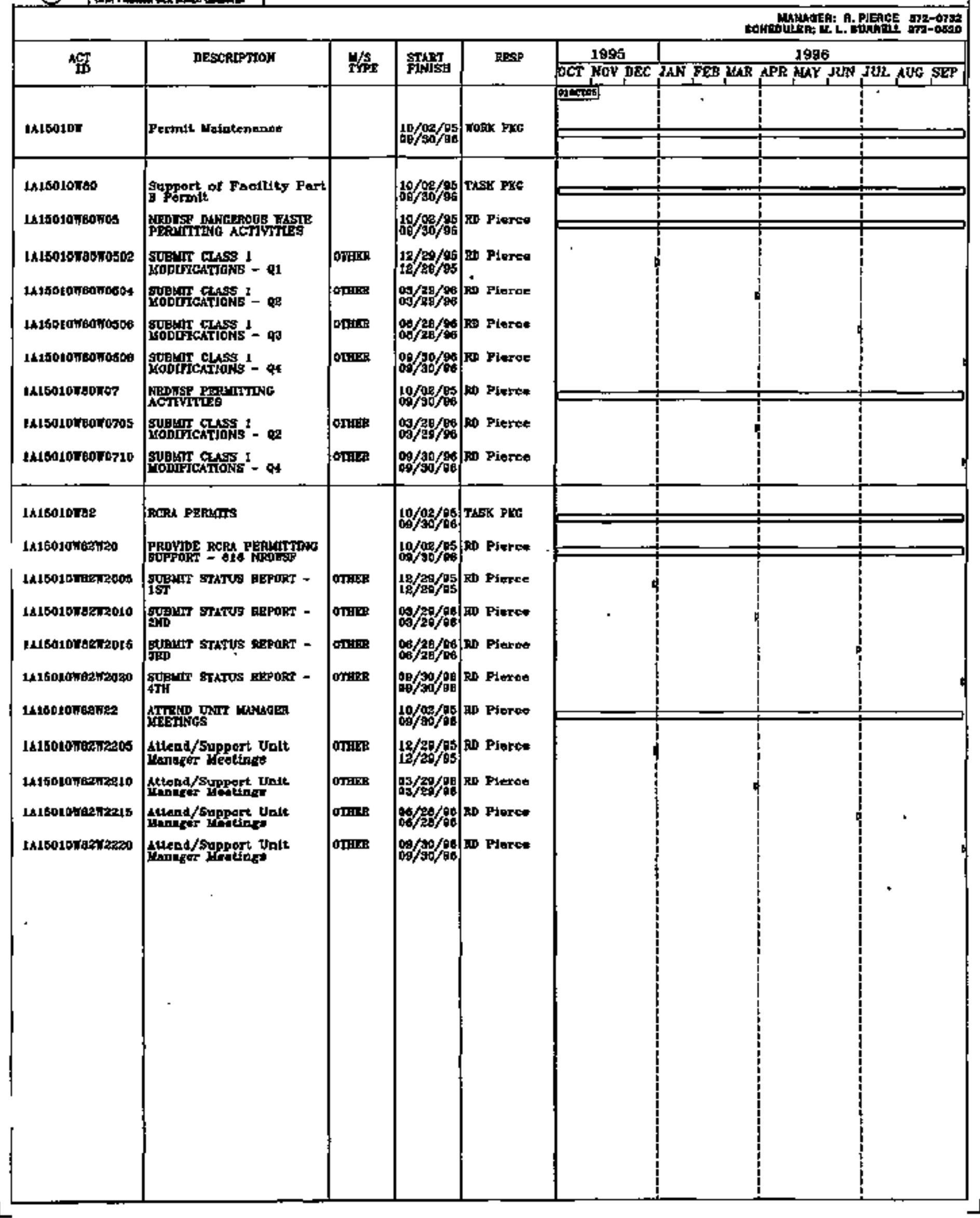




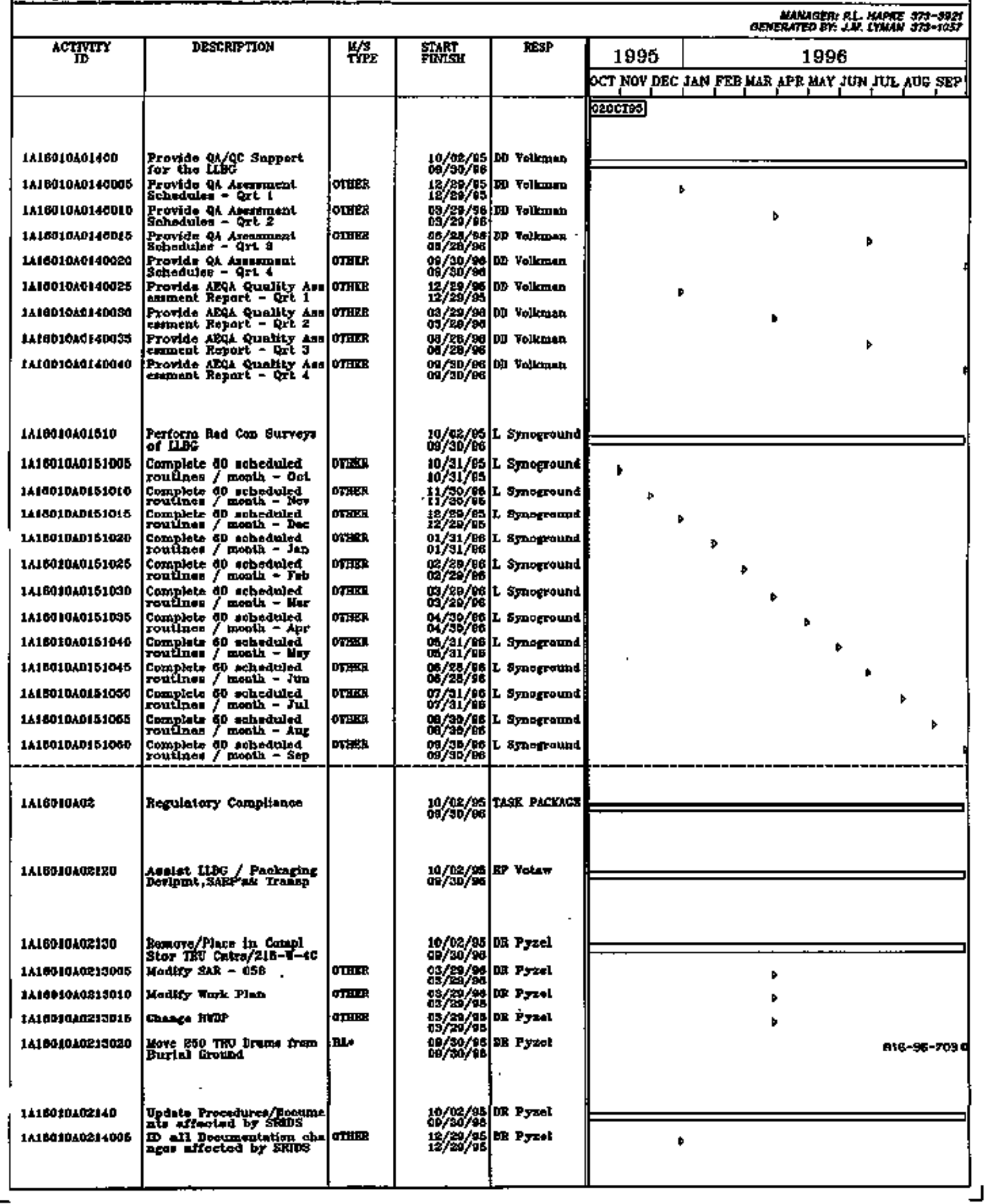


$\Gamma$

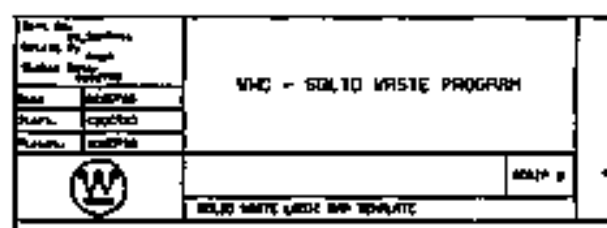

WHC - SOLID WASTE PRORAAM

FY 1996 SCHEDULE

1A1601 - LOW LEVEL BURIAL GROUNDS

Haxe s7s-90:

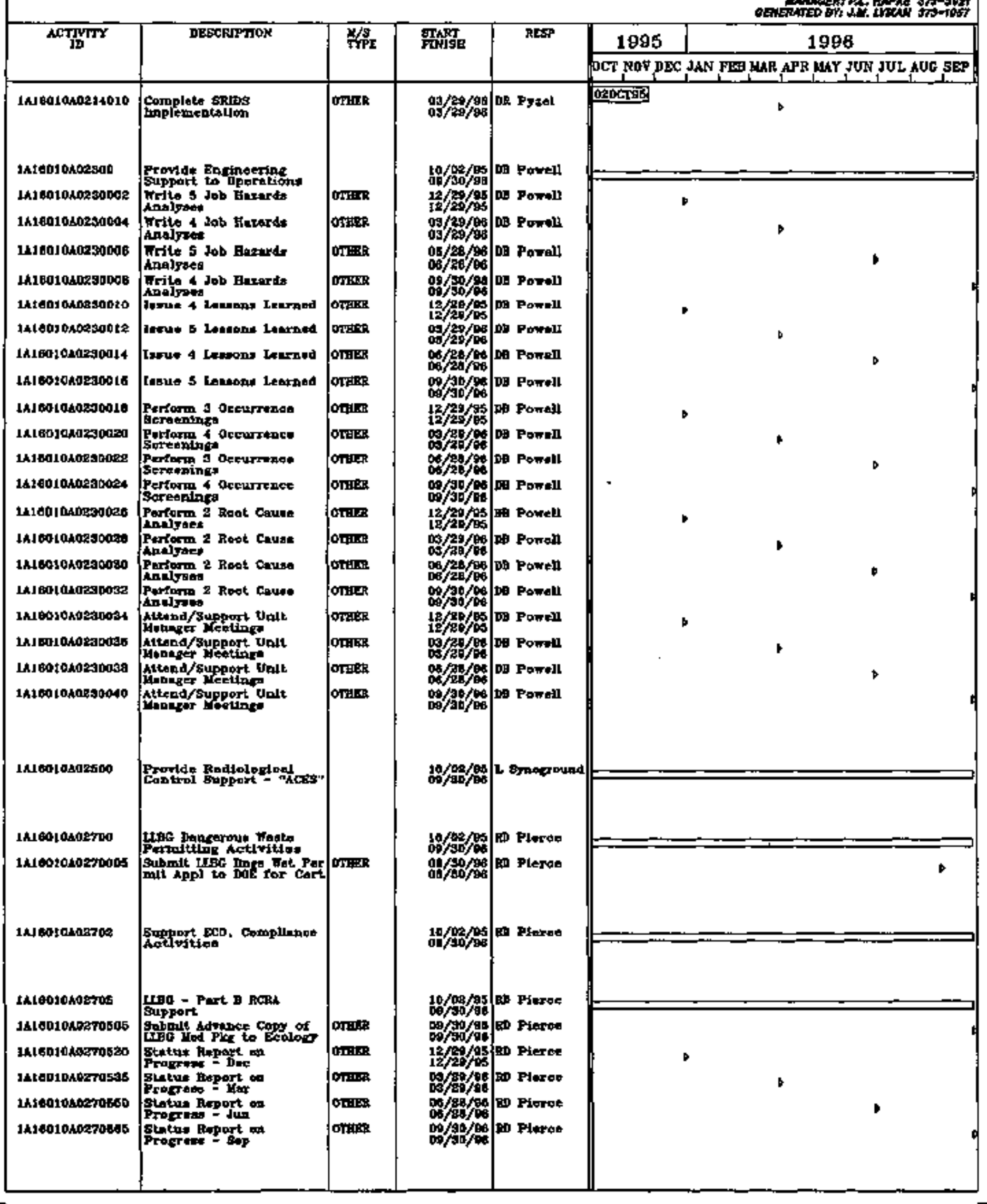




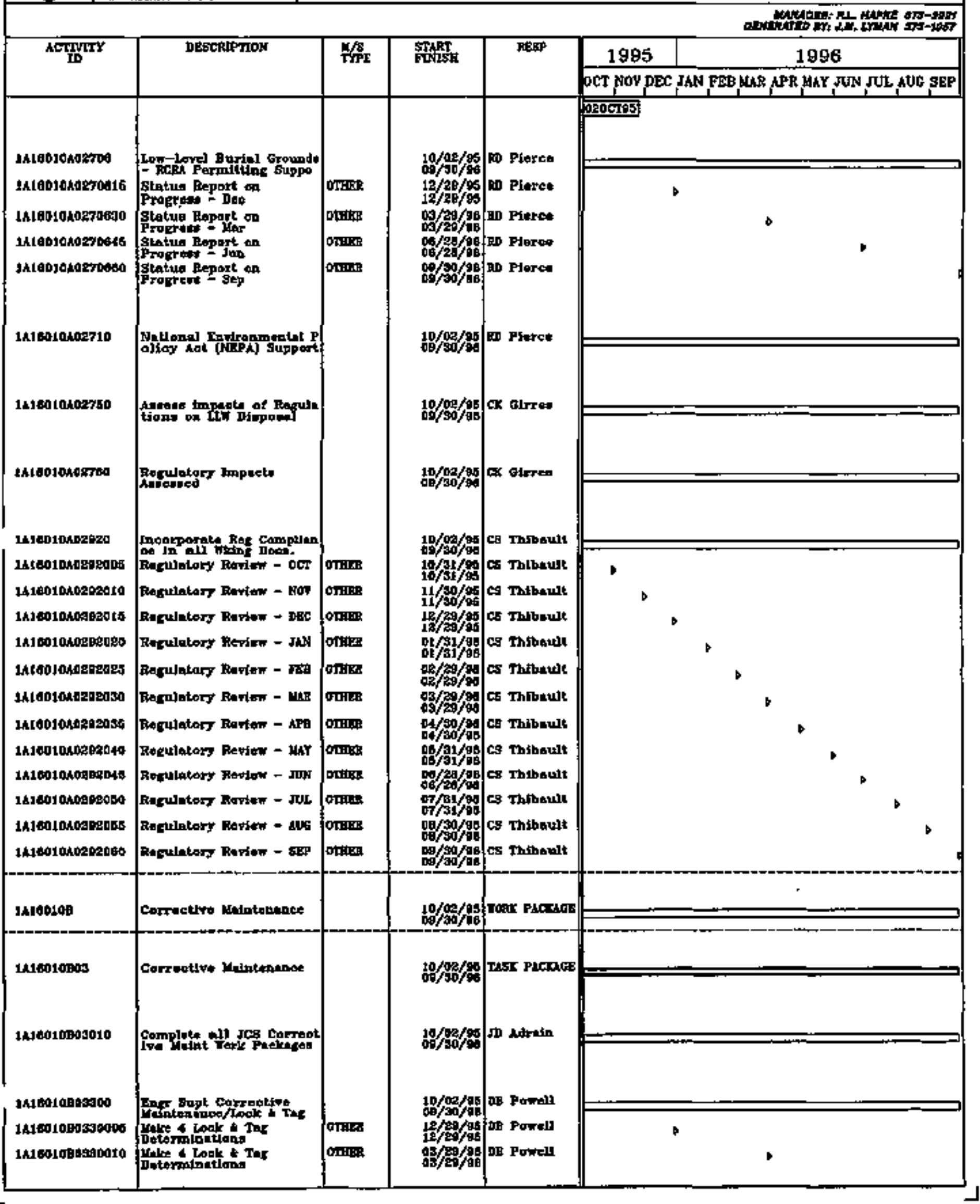




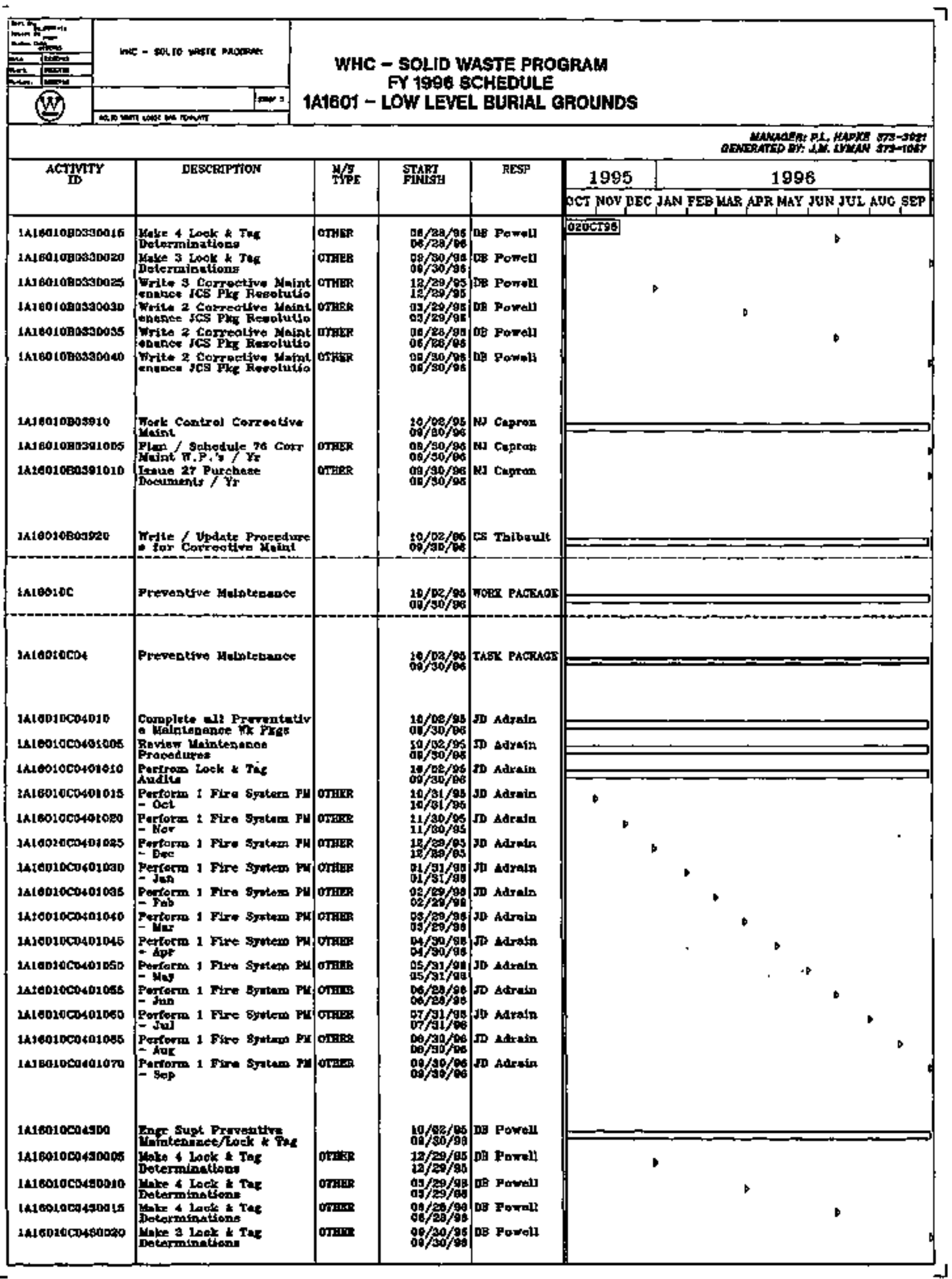



FY 1996 SCHEDULE 141601 - LOW LEVEL BURIAL, CROUNDS

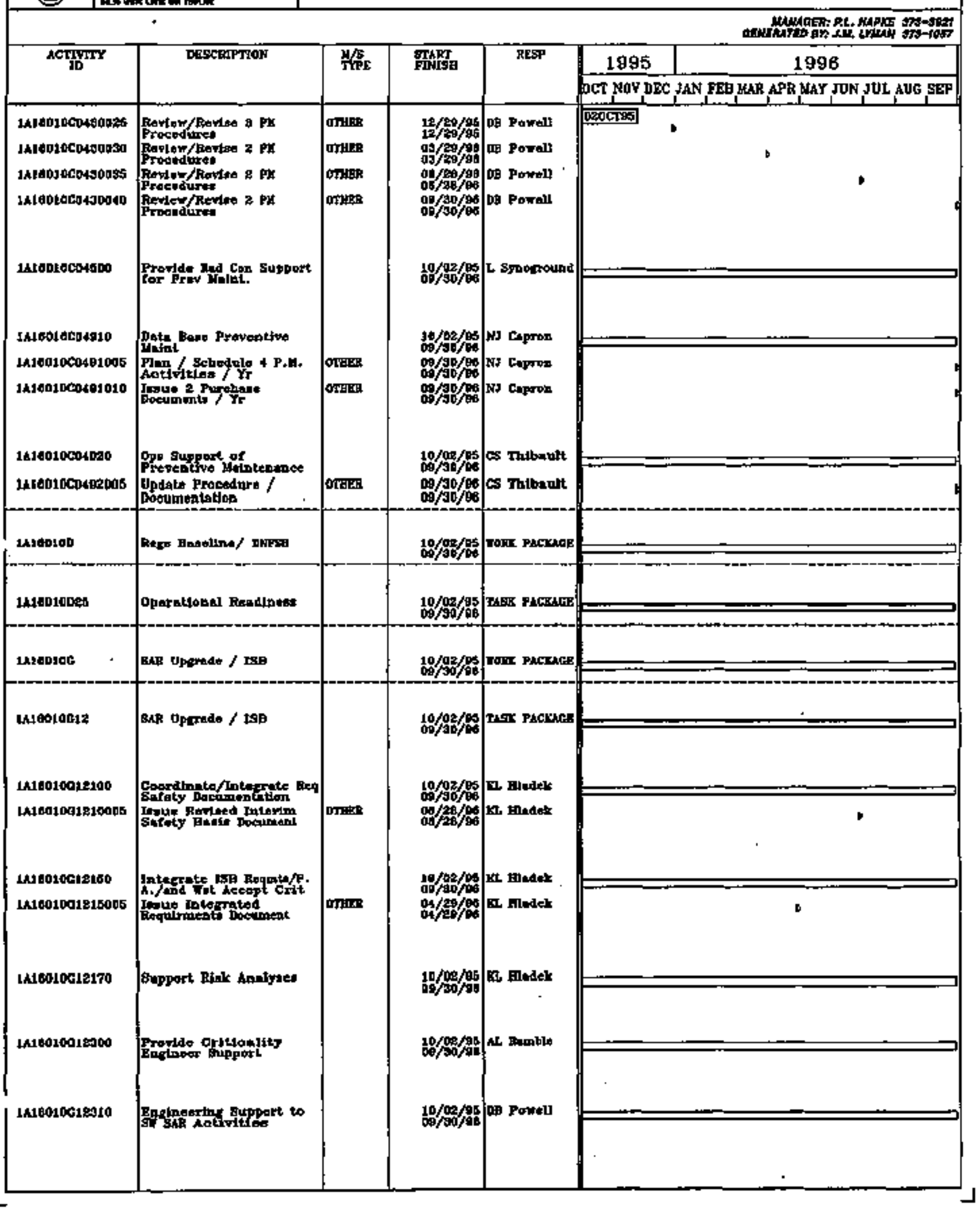




\section{WHC - SOLID WASTE PROGRAM} FY 1996 SCHEDULE 1A1601 - LOW LEVEL BUAIAL OROUNOS

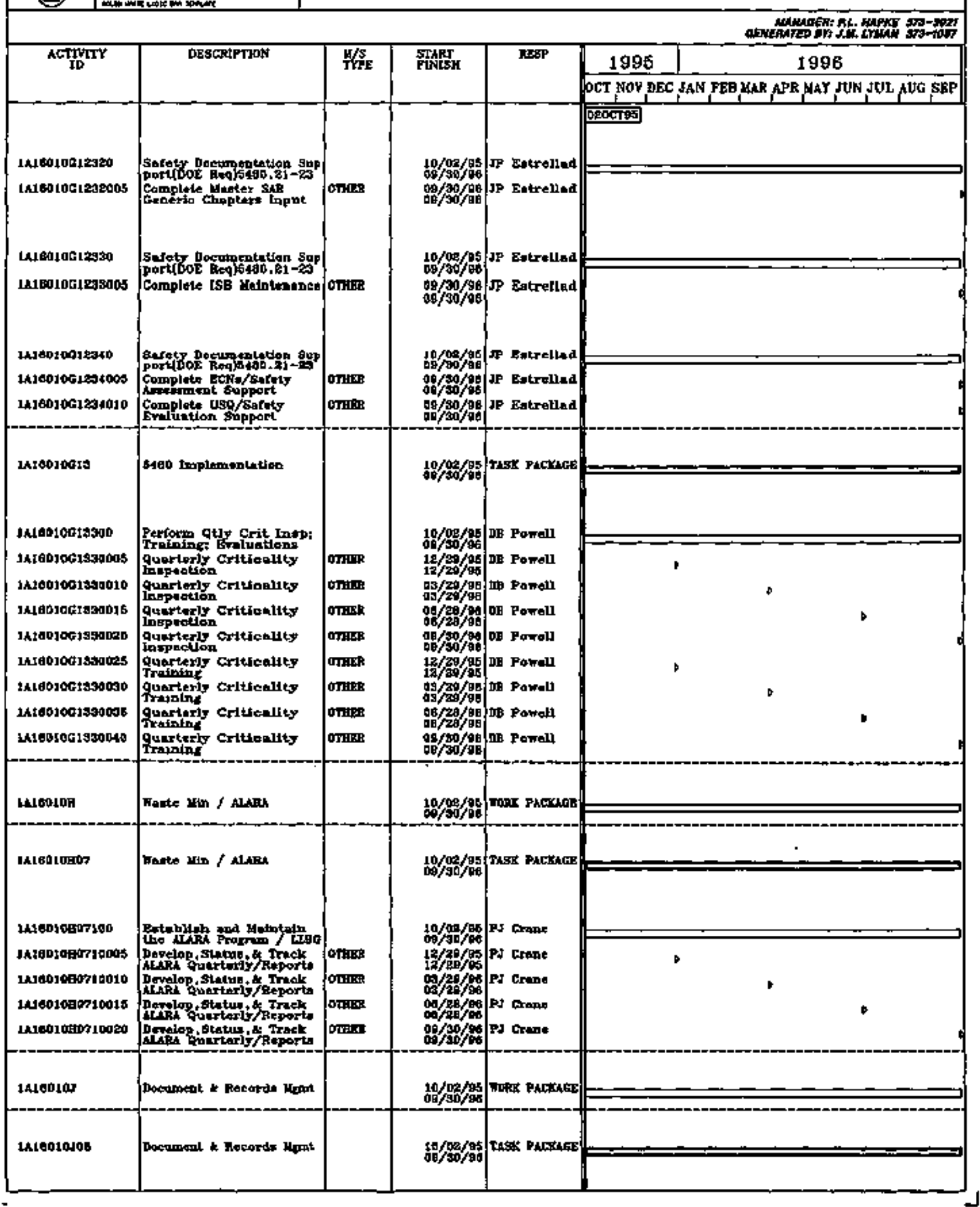


$\Gamma$

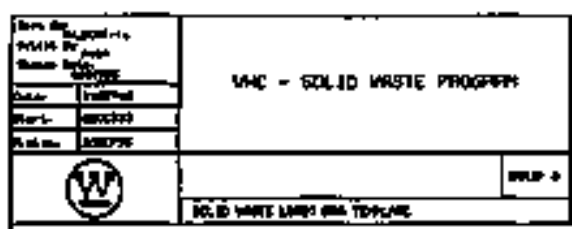

$$
\begin{gathered}
\text { WHC - SOLID WASTE PROGRAM } \\
\text { FY } 1996 \text { SCHEDULE } \\
\text { } \text { \$A1601 - LOW LEVEL EURIAL GROUNDS }
\end{gathered}
$$

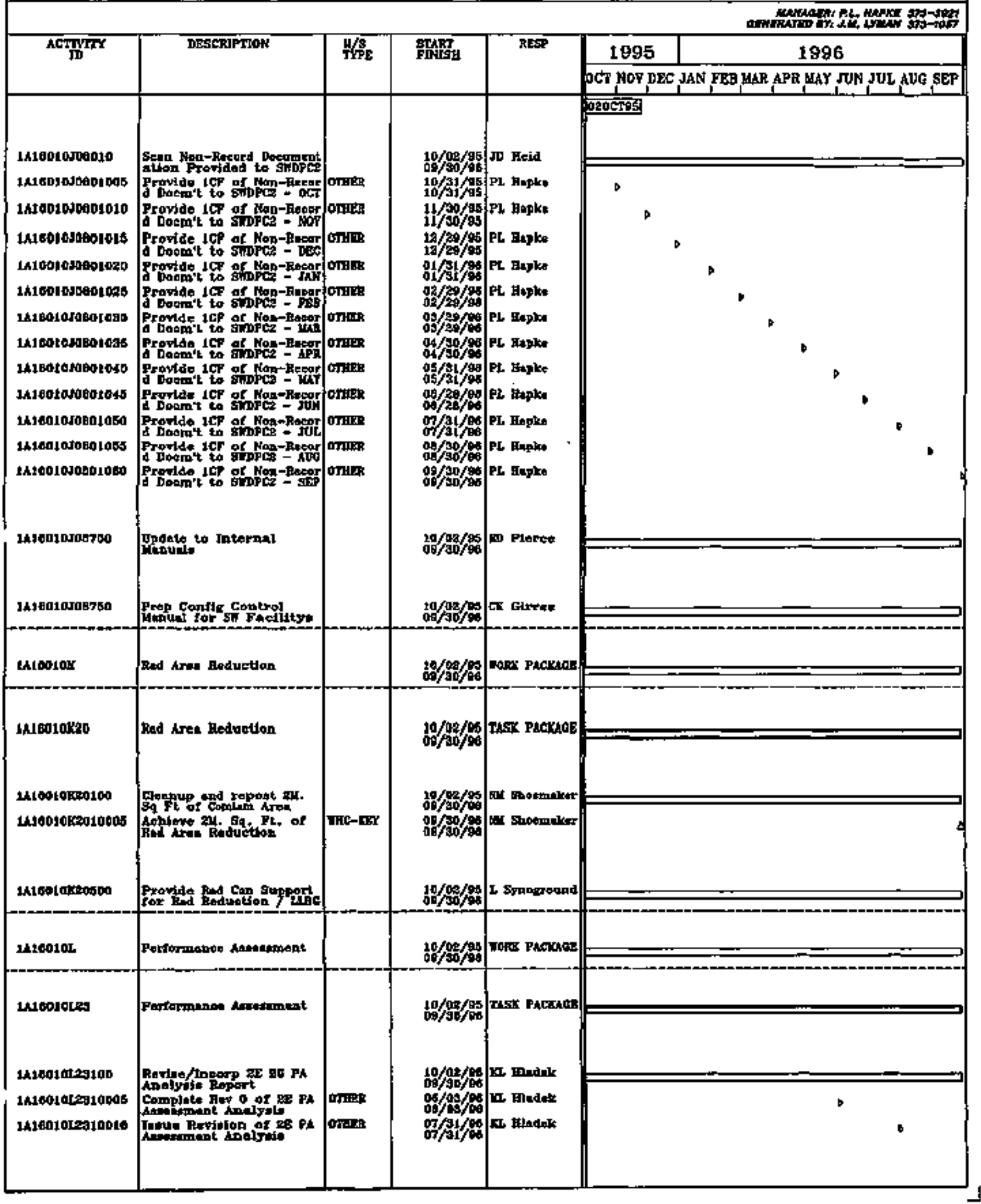




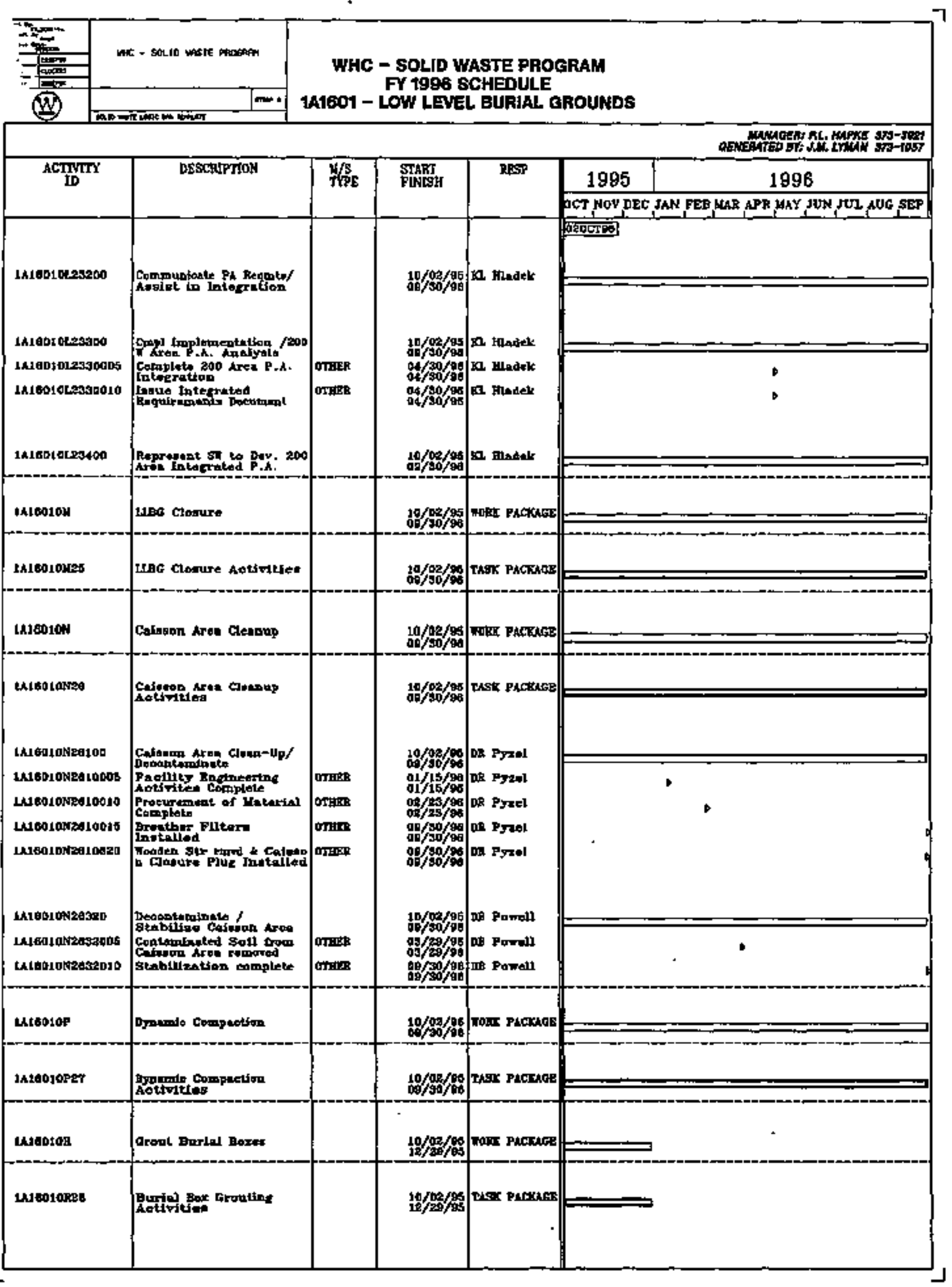



FY 1996 SCHEDULE IA1601 - LOW LEVEL BURIAL GROUNDS

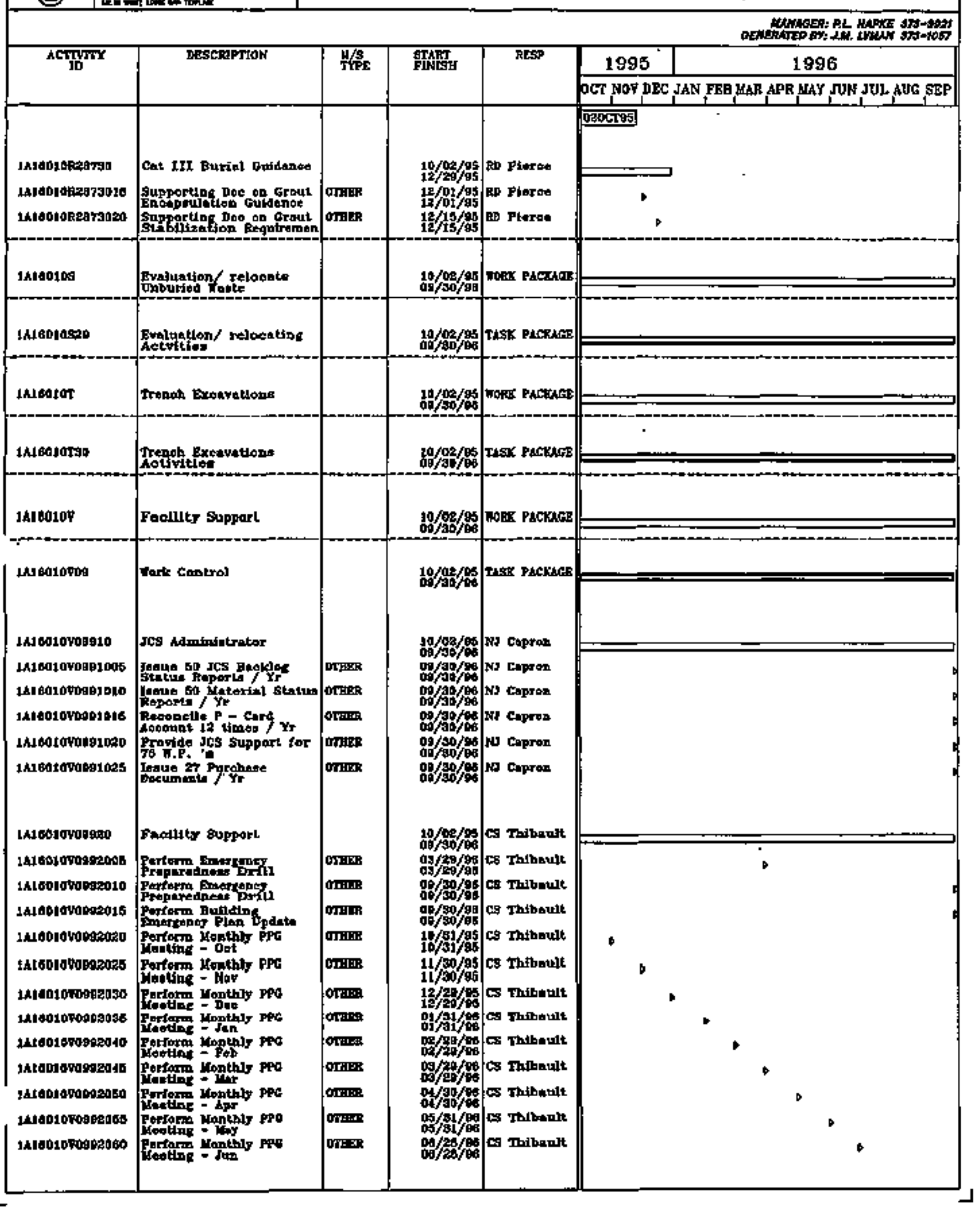




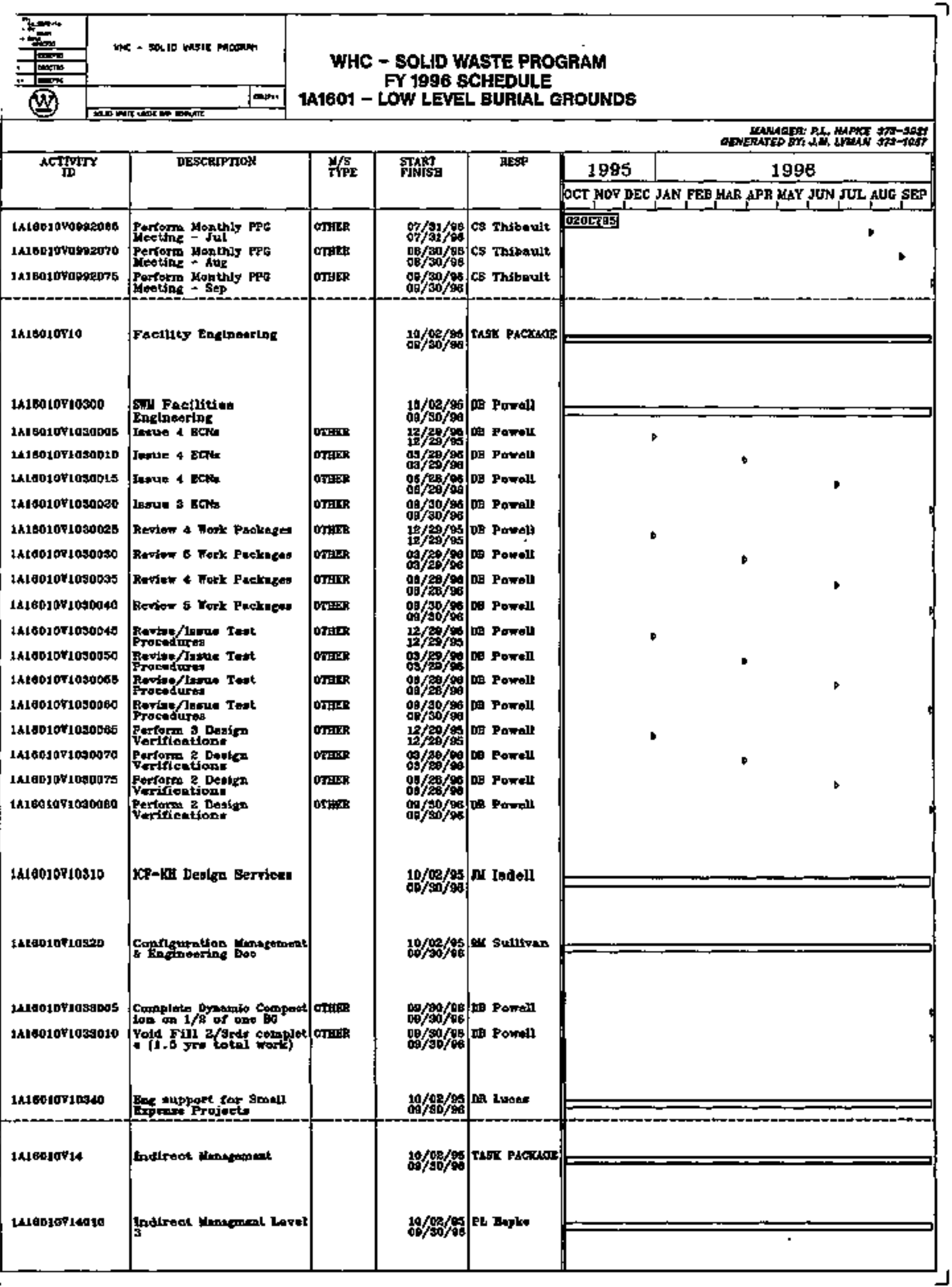


WHC - SOLID WASTE PROGRAR FY T996 SCHEDULE TAt601 - LOW LEVEL BUATAL QROUNDS

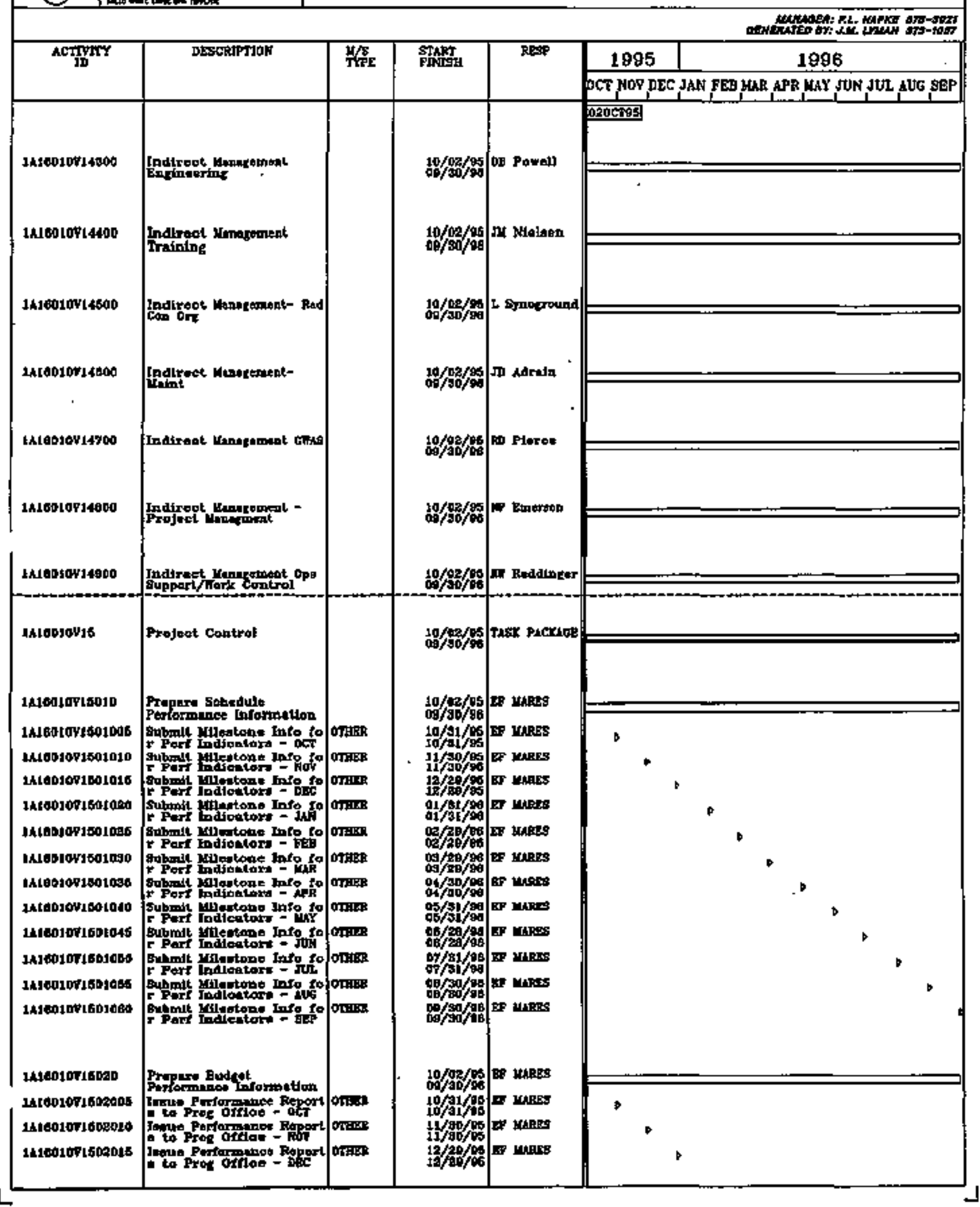


WHC - SOLID WASTE PROGRAM FY 1996 SCHEDULE 1A1601 - LOW LEVEL BUAIAL GROUNDS

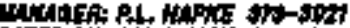

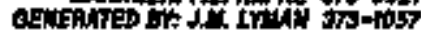

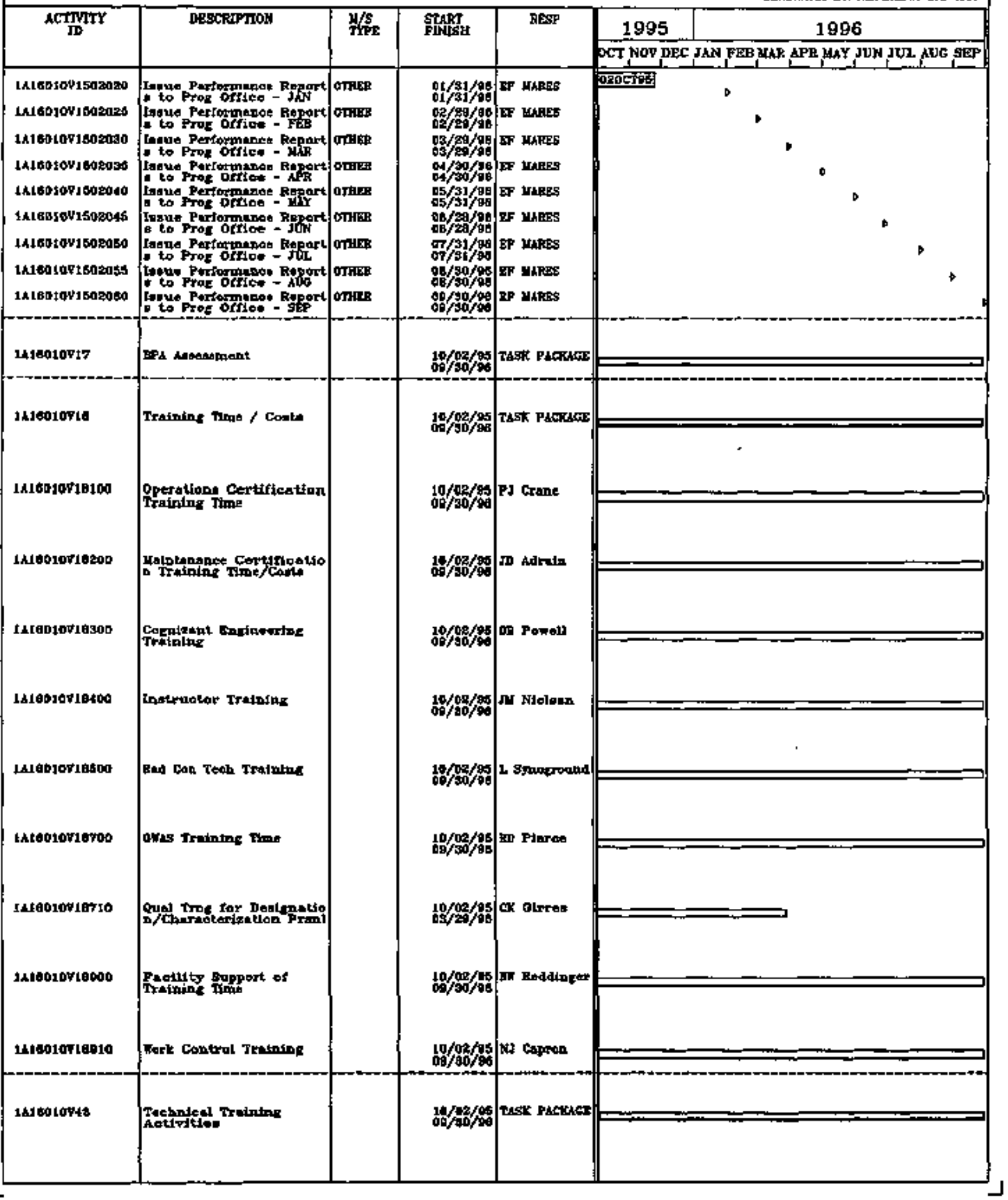




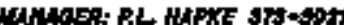

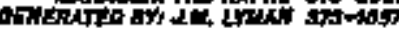

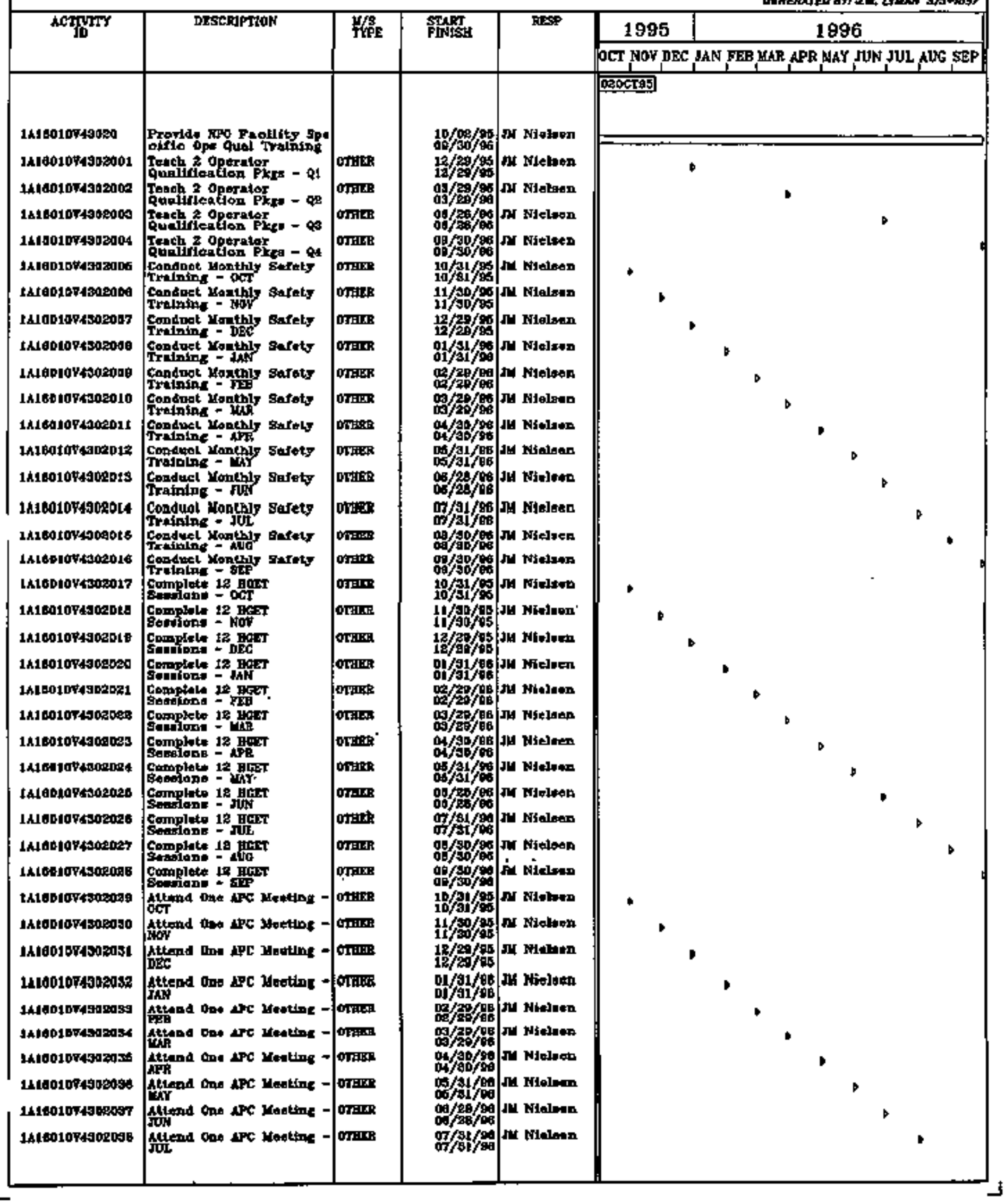


WHC - SOLID WASTE PROGRAN FY 1996 SCHEDULE 1A16D1 - LOW LEVEL BUAIAL GROUNDS

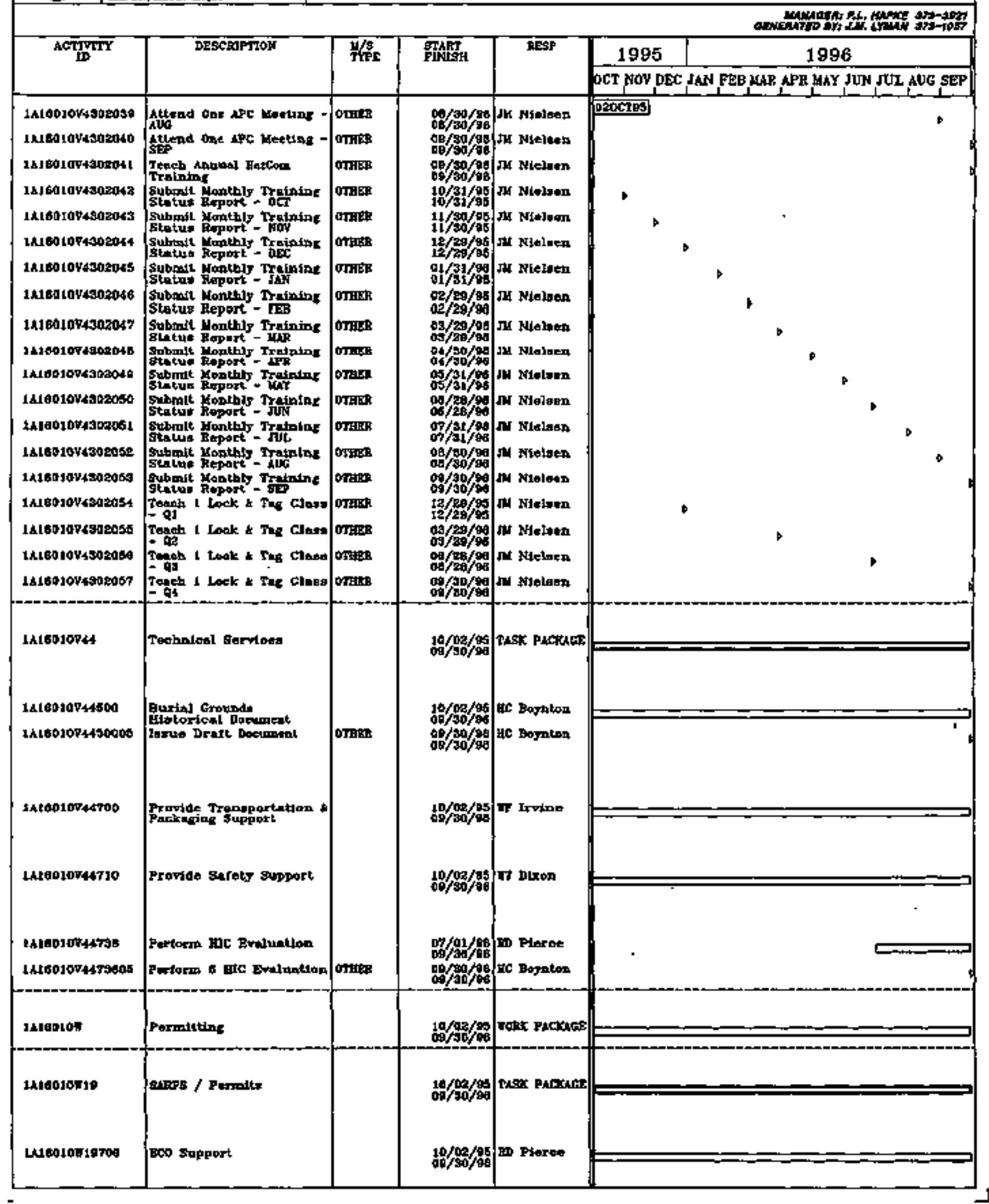


$\Gamma$

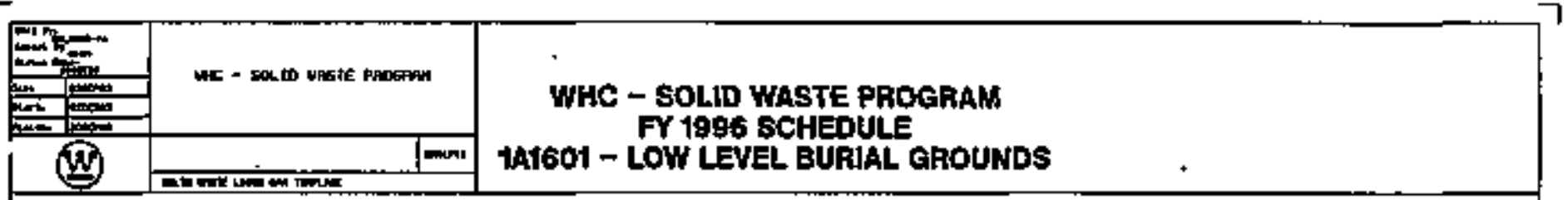

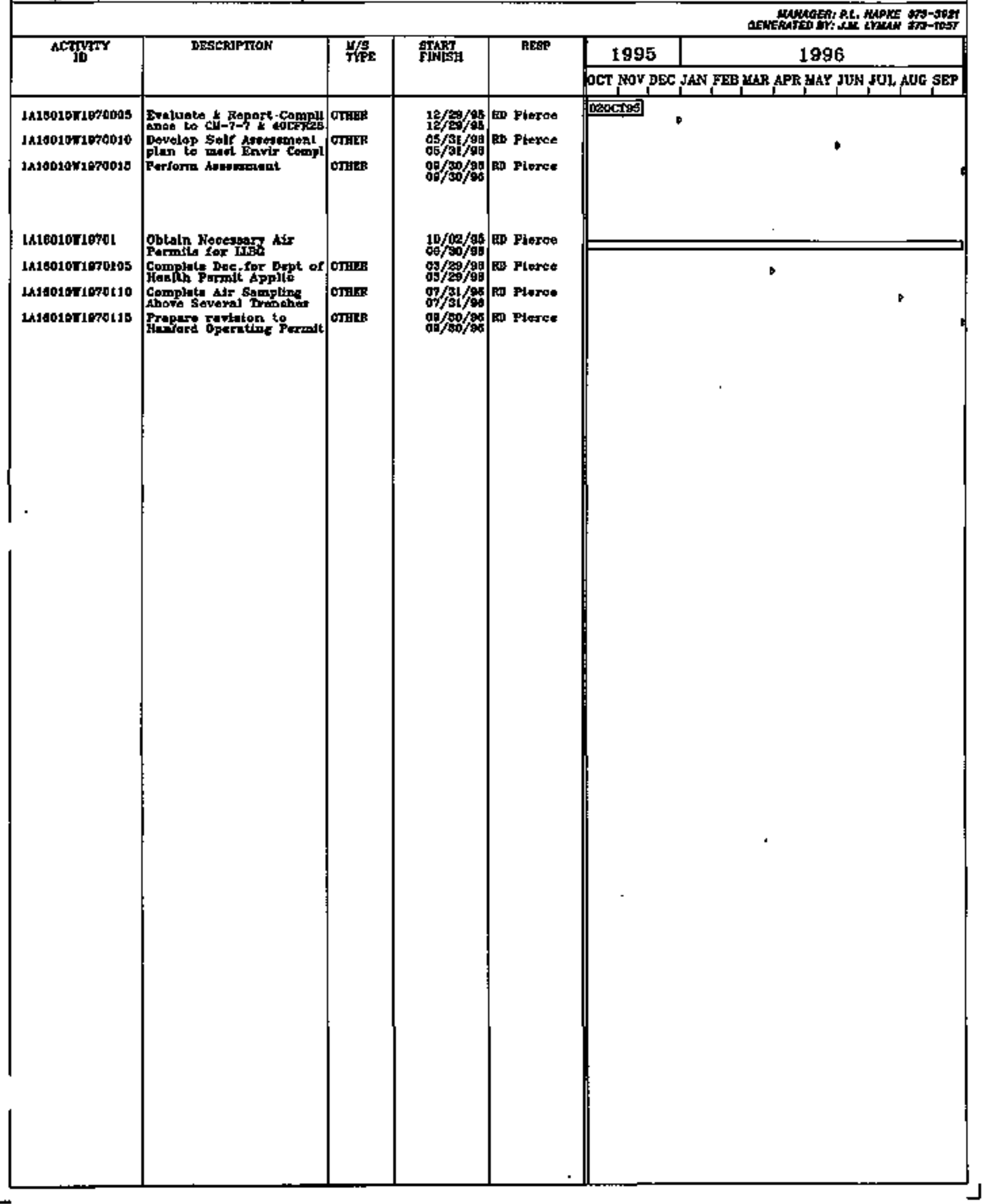




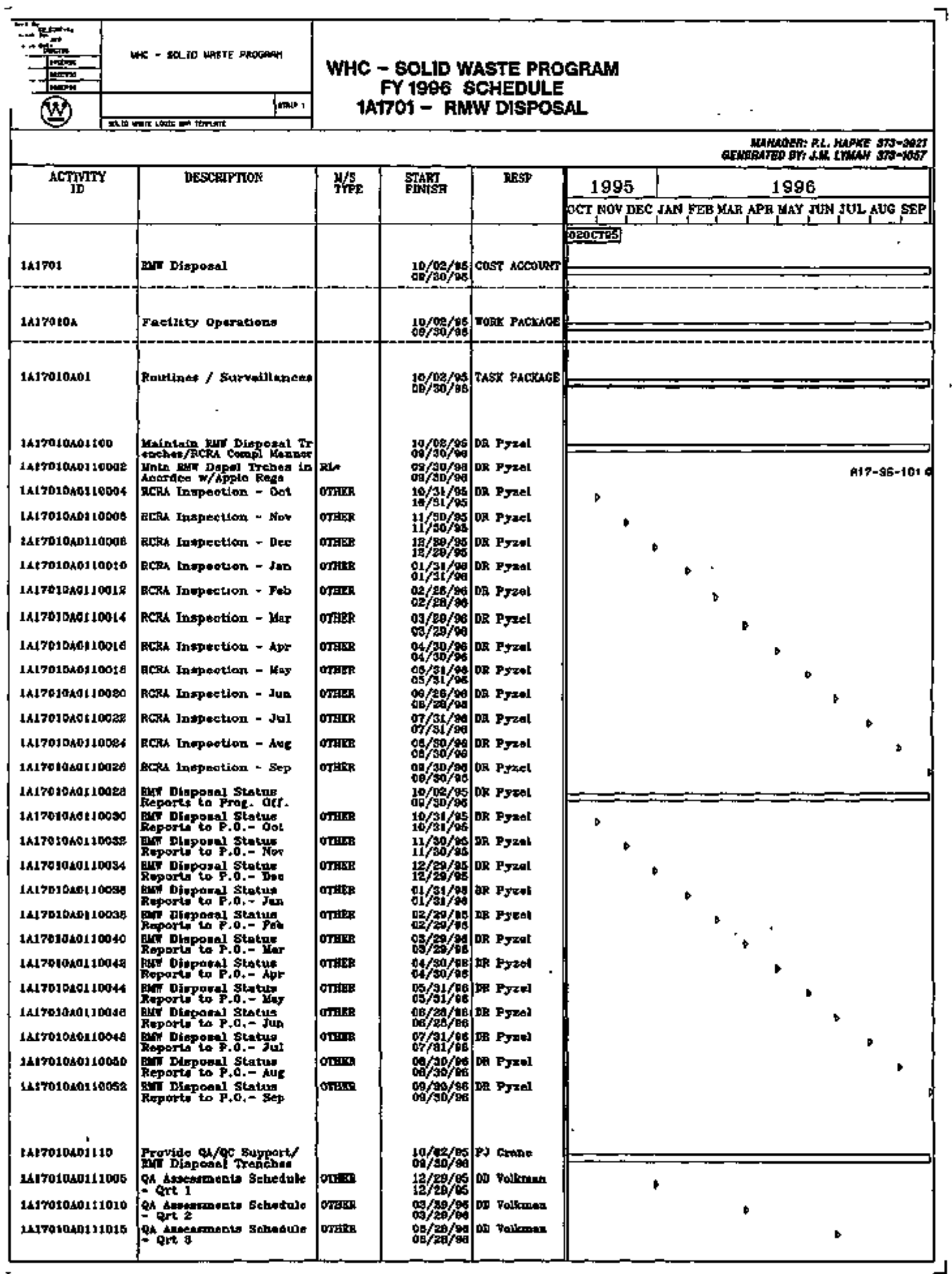




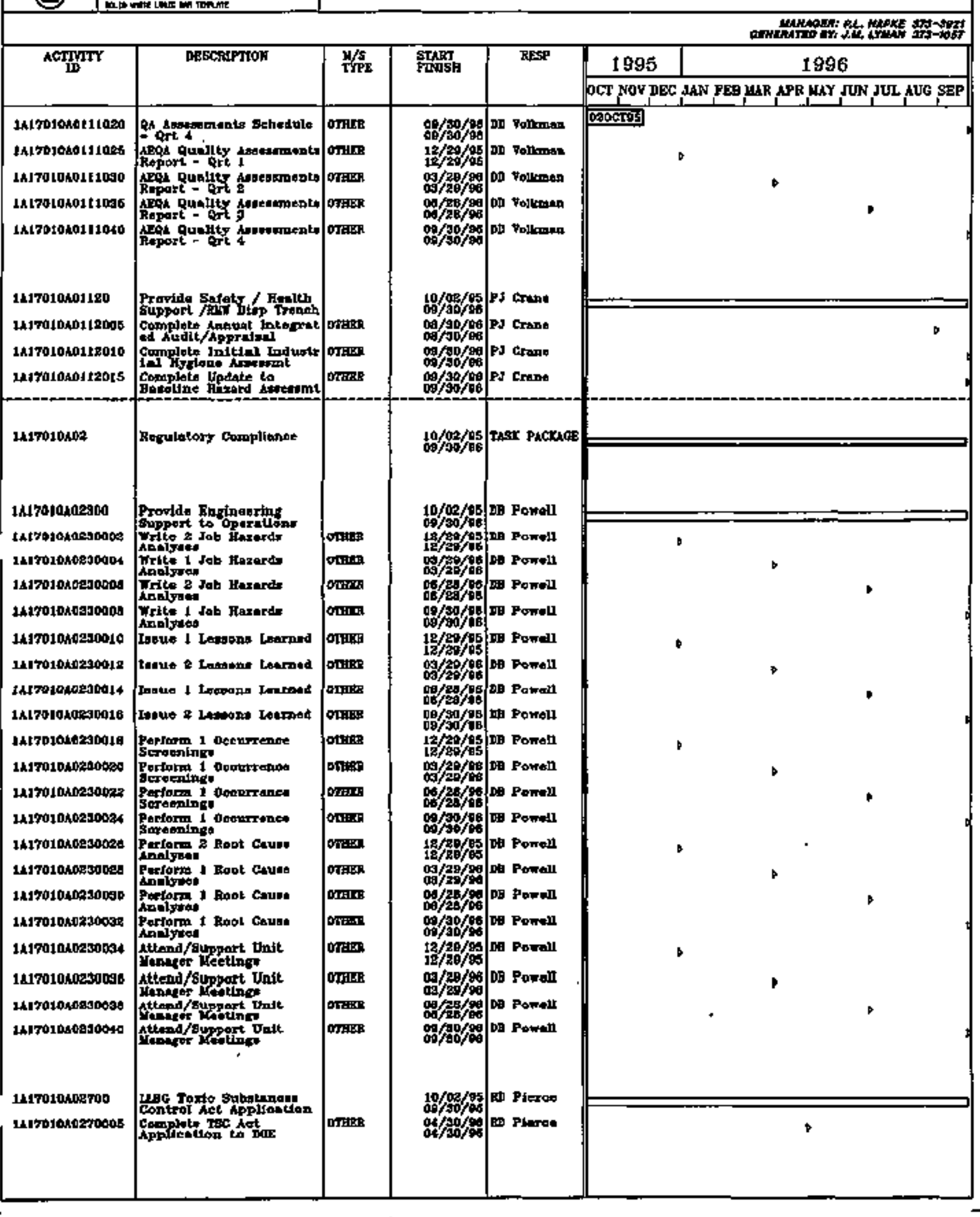




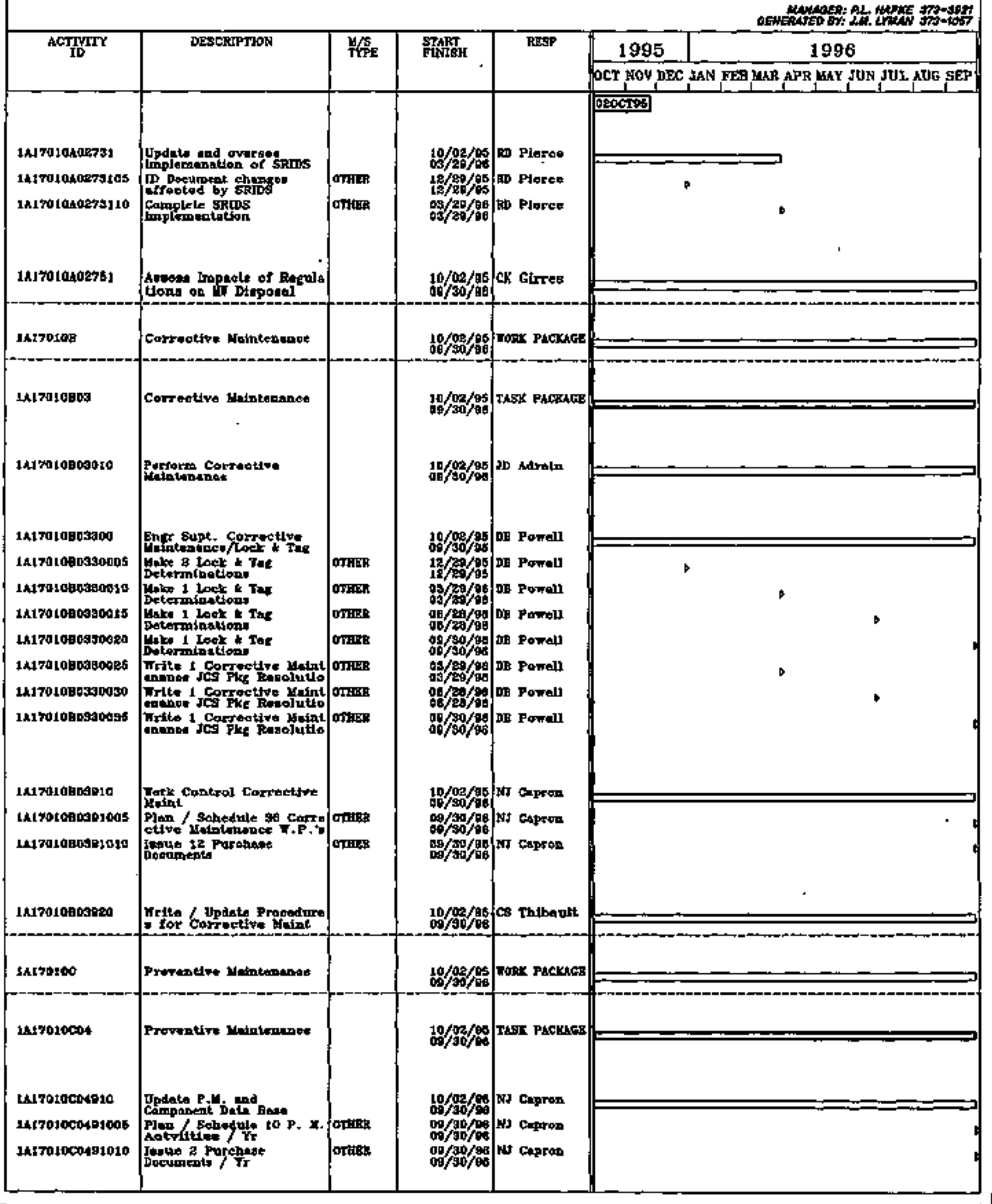


WHC - SOLID WASTE PROGRAM

FY 1996 SCHEDULE

1A7701 - RMW DISPOSAL

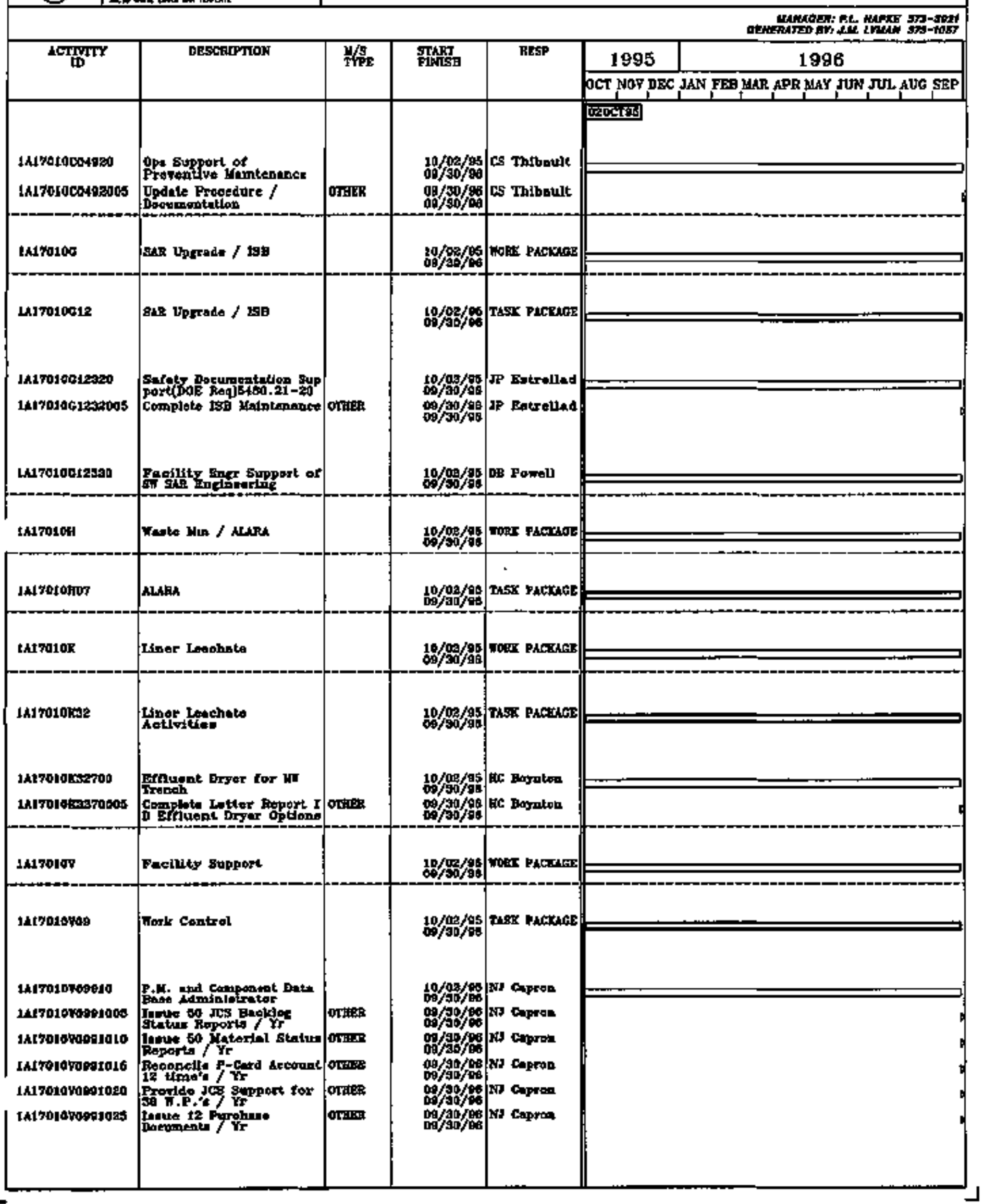




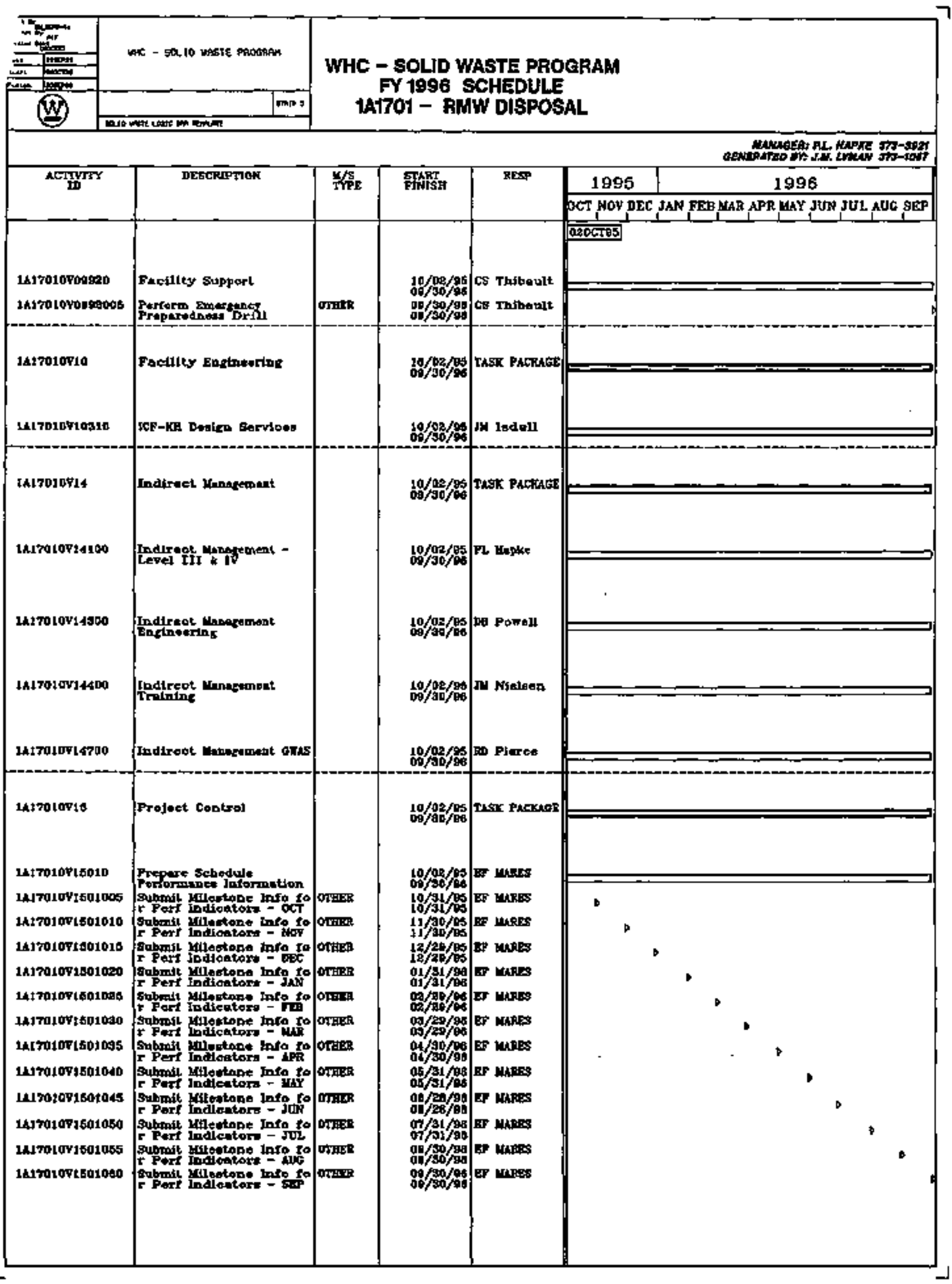


WHC - SOLID WASTE PROGRAM FY 1996 SCHEDULE 1At701 - FMW DISPOSAL

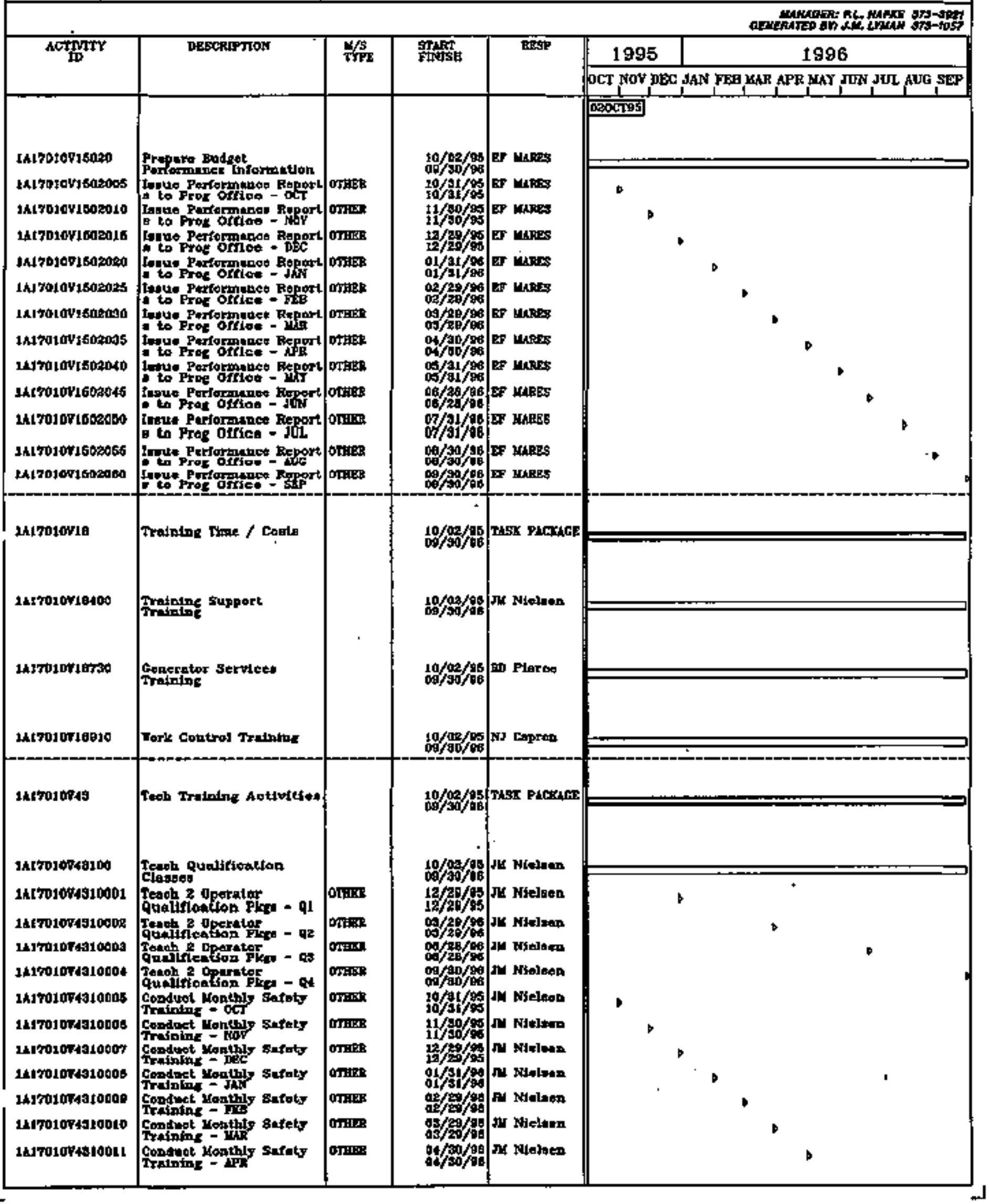


WHC - SOLD WASTE PROQRAM

FY 1996 SCHEDULE

IA1701 - RMW DISPOSAL

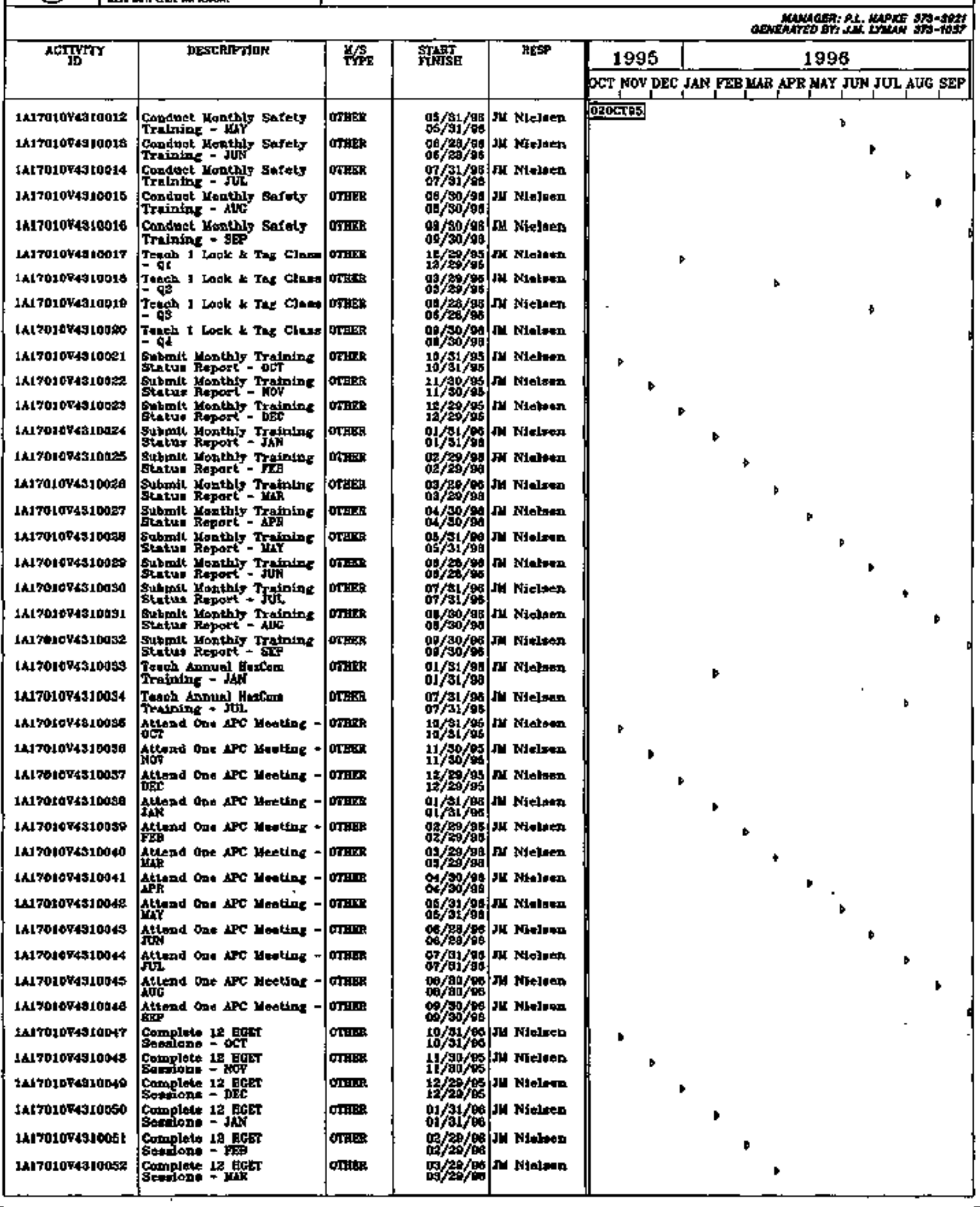


WHC - SOLID WASTE PROERAM

FY 1996 SCHEDULE

1A1701 - RMW DISPOSAL

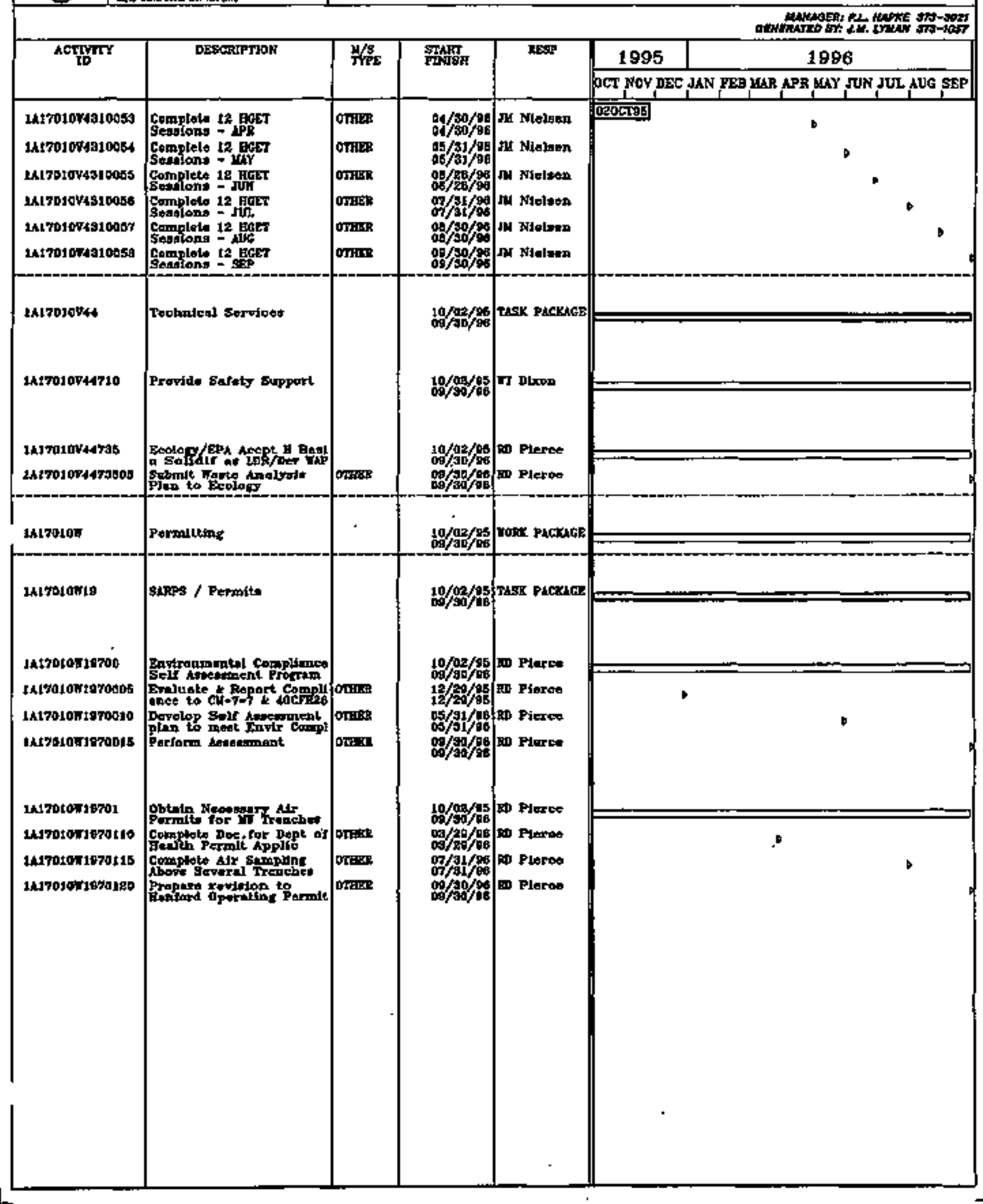




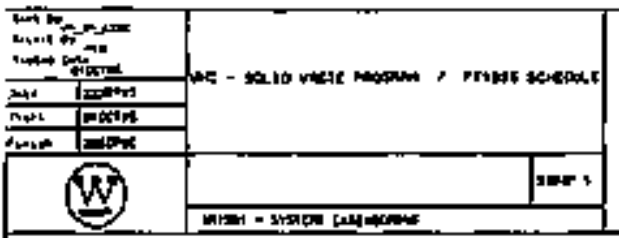

WHC - SOLDD WASTE PROCRAM FY 1996 SCHEDULE 1A1901 - SYSTEMS ENGINEERING

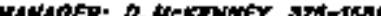

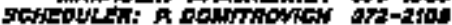

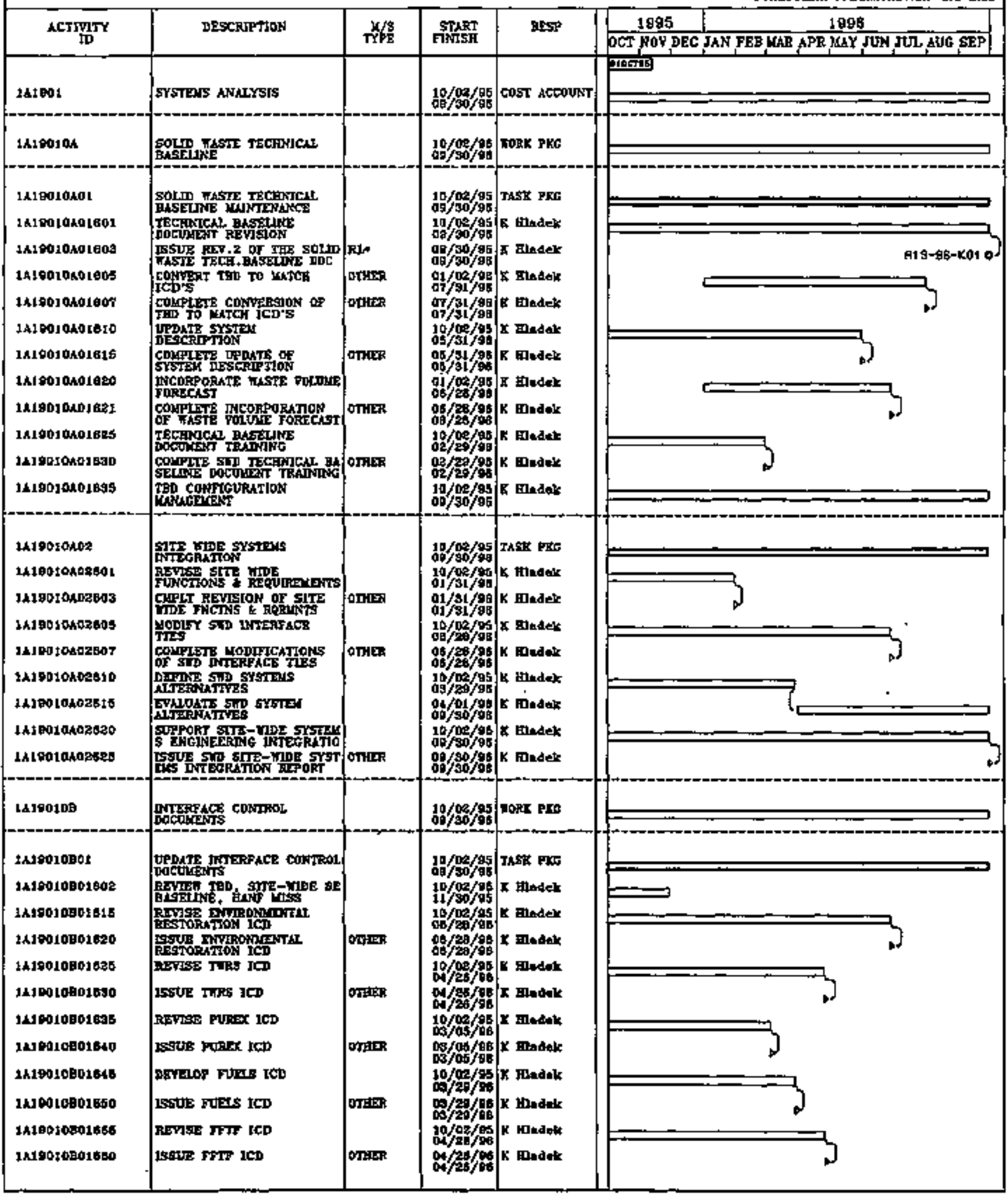


WHC - SOLID WASTE PROGRAM FY 1996 SCHEDULE 1A19OT - SYSTENS ENGINEERING

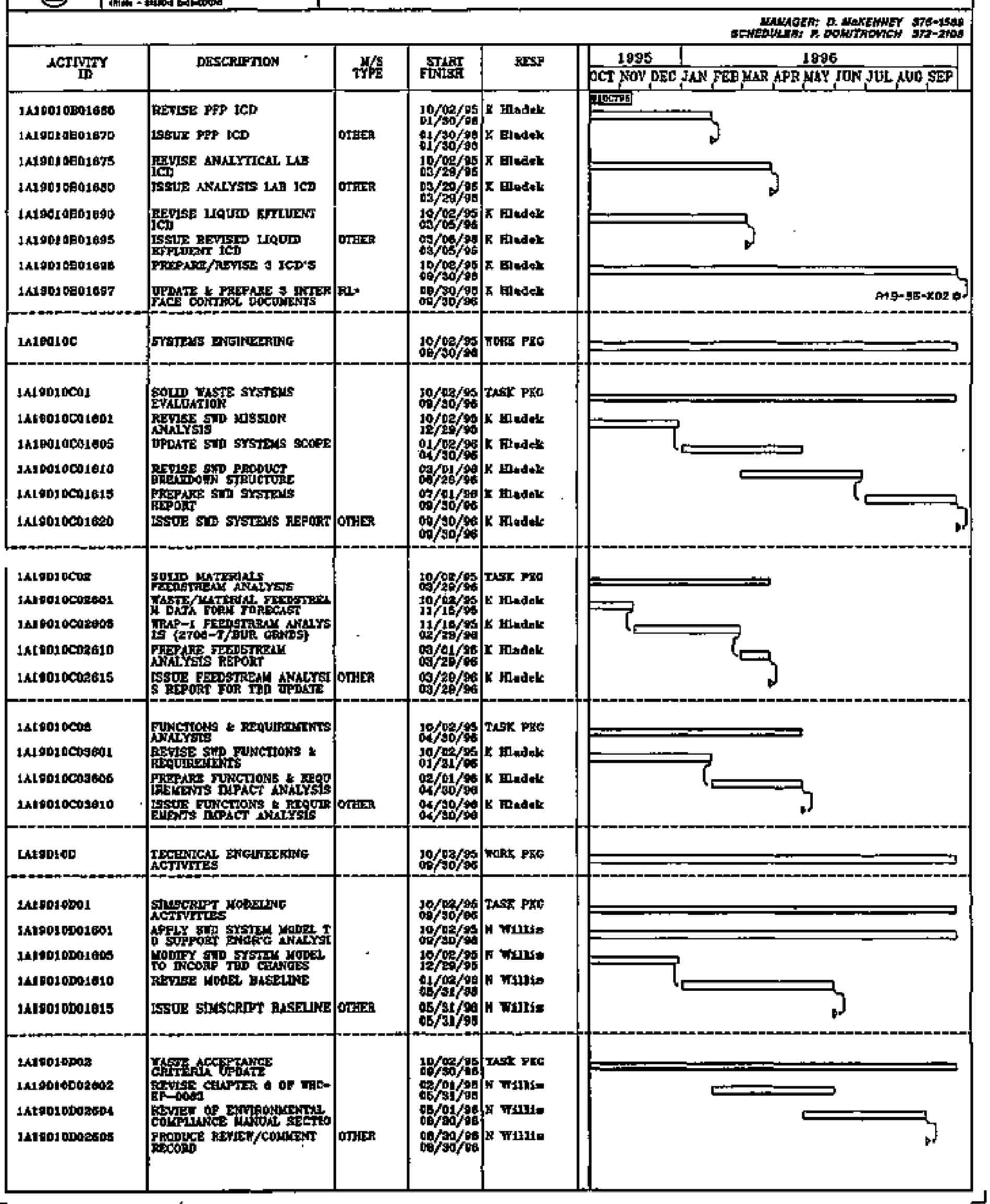


WHC - SOLED WASTE PROGRAM FY 1996 SCHEDULE IAT9O1 - SYSTEMS ENQINEERINO

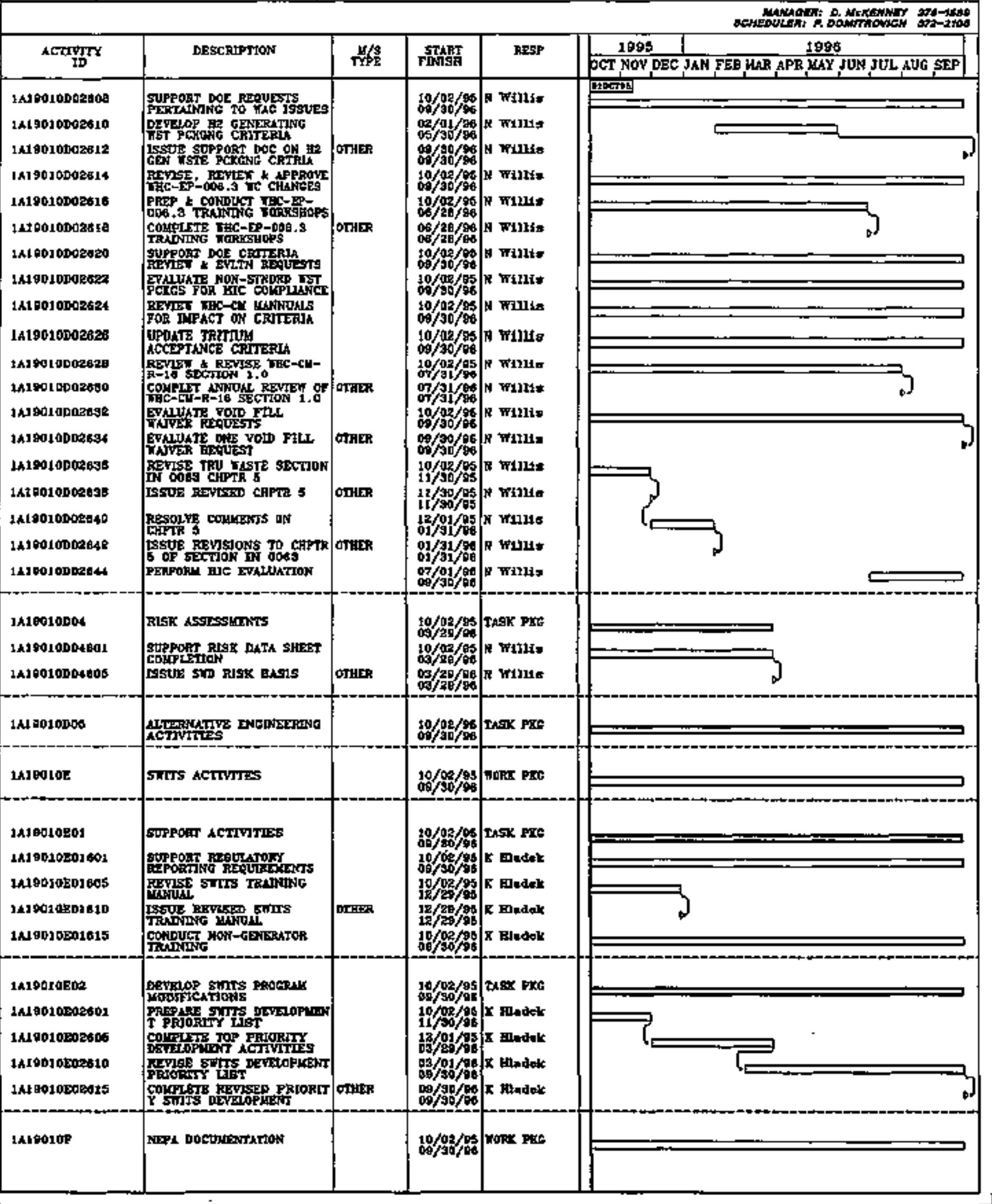


WHC - SOLID WASTE PROQRAM FY 1996 SCHEDULE TA1901 - SYSTEMS ENGINEERING

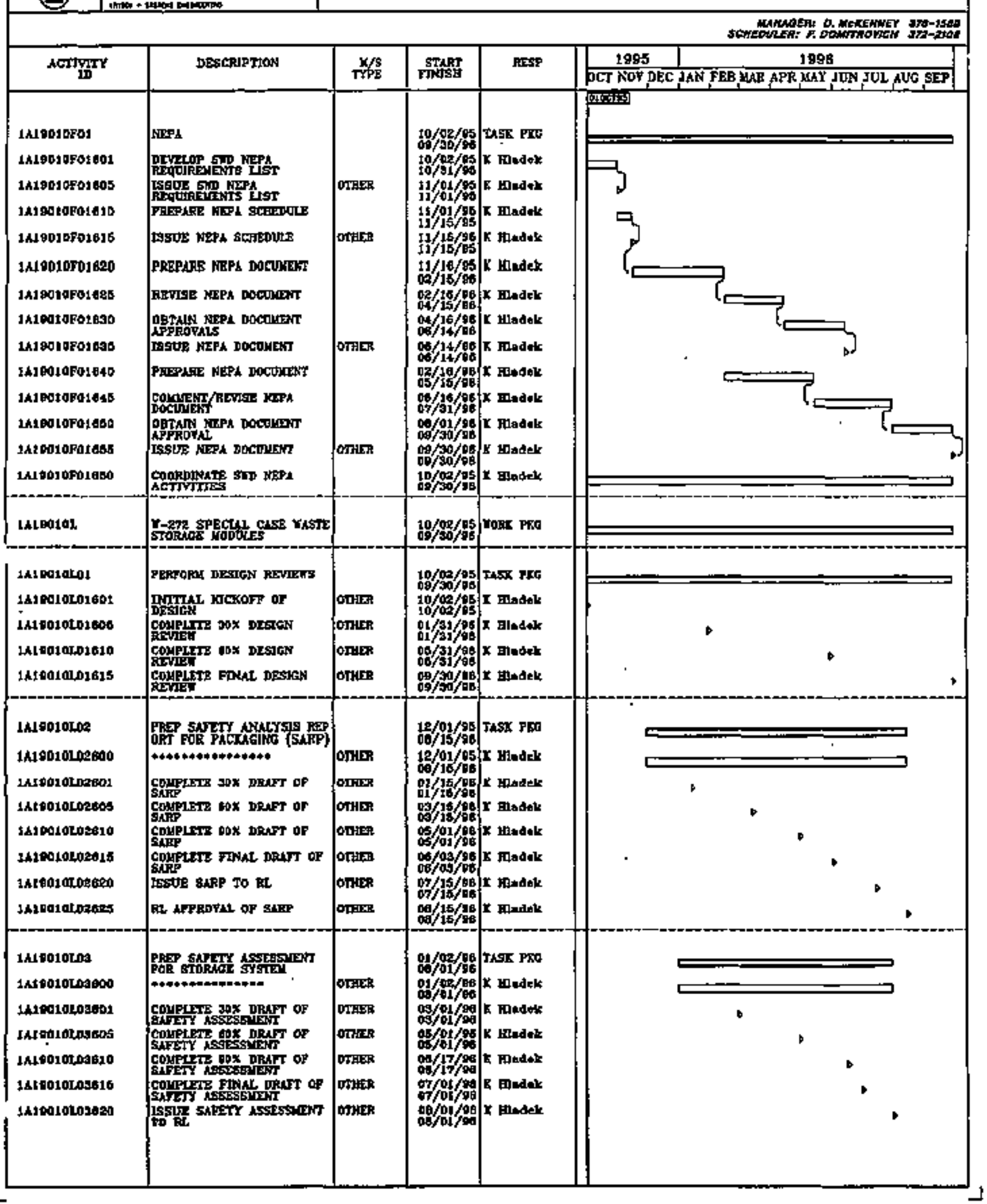


WHC - SOLID WASTE PROGFAM

FY 1996 SCHEDULE

1A1901 - SYSTEMS ENOINEERING

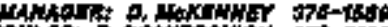

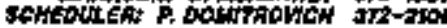

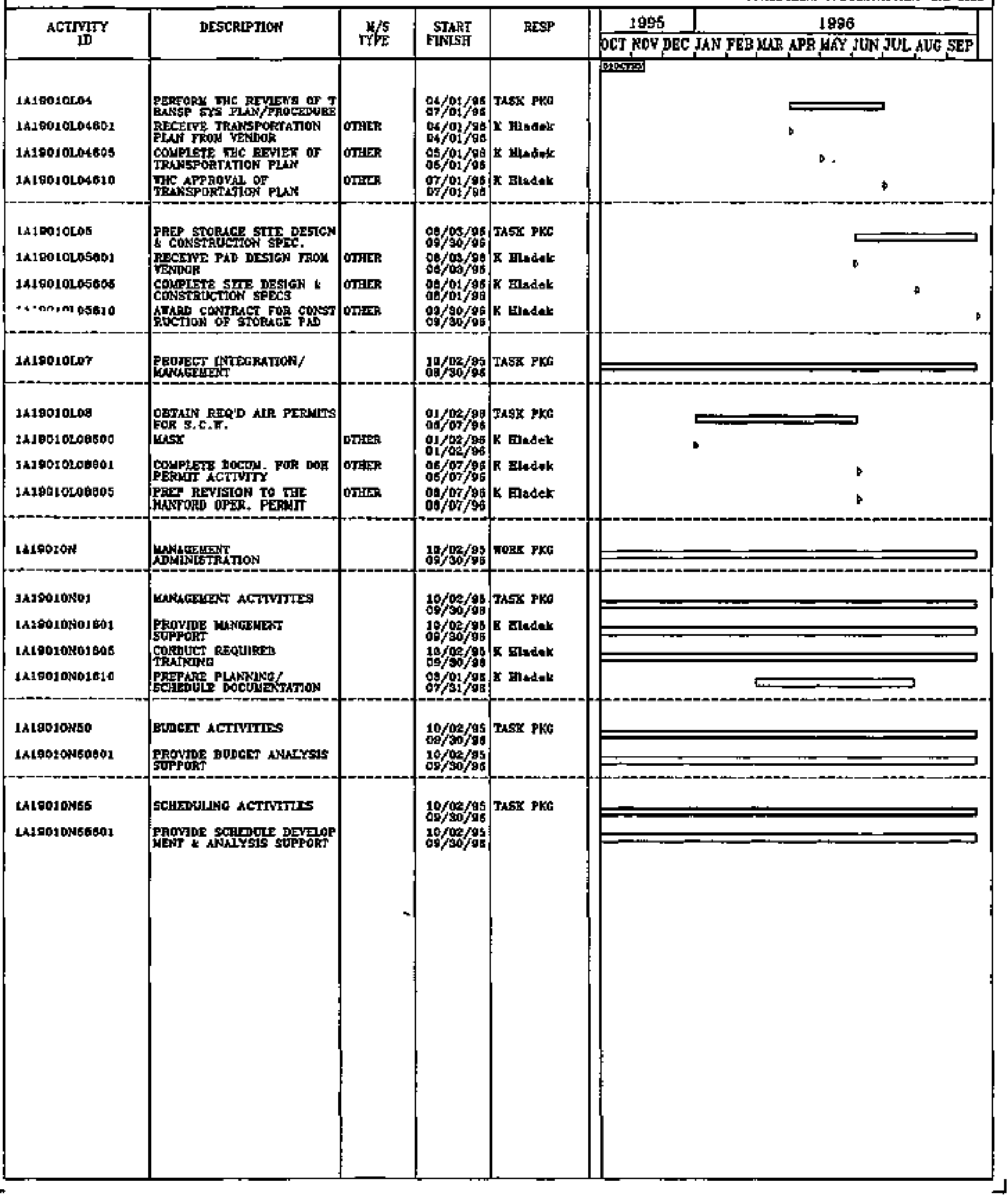




\section{WHC - SOLID WASTE PROGRAH 1A3104 FY 96 221-T CANYON OPEAATIONS}

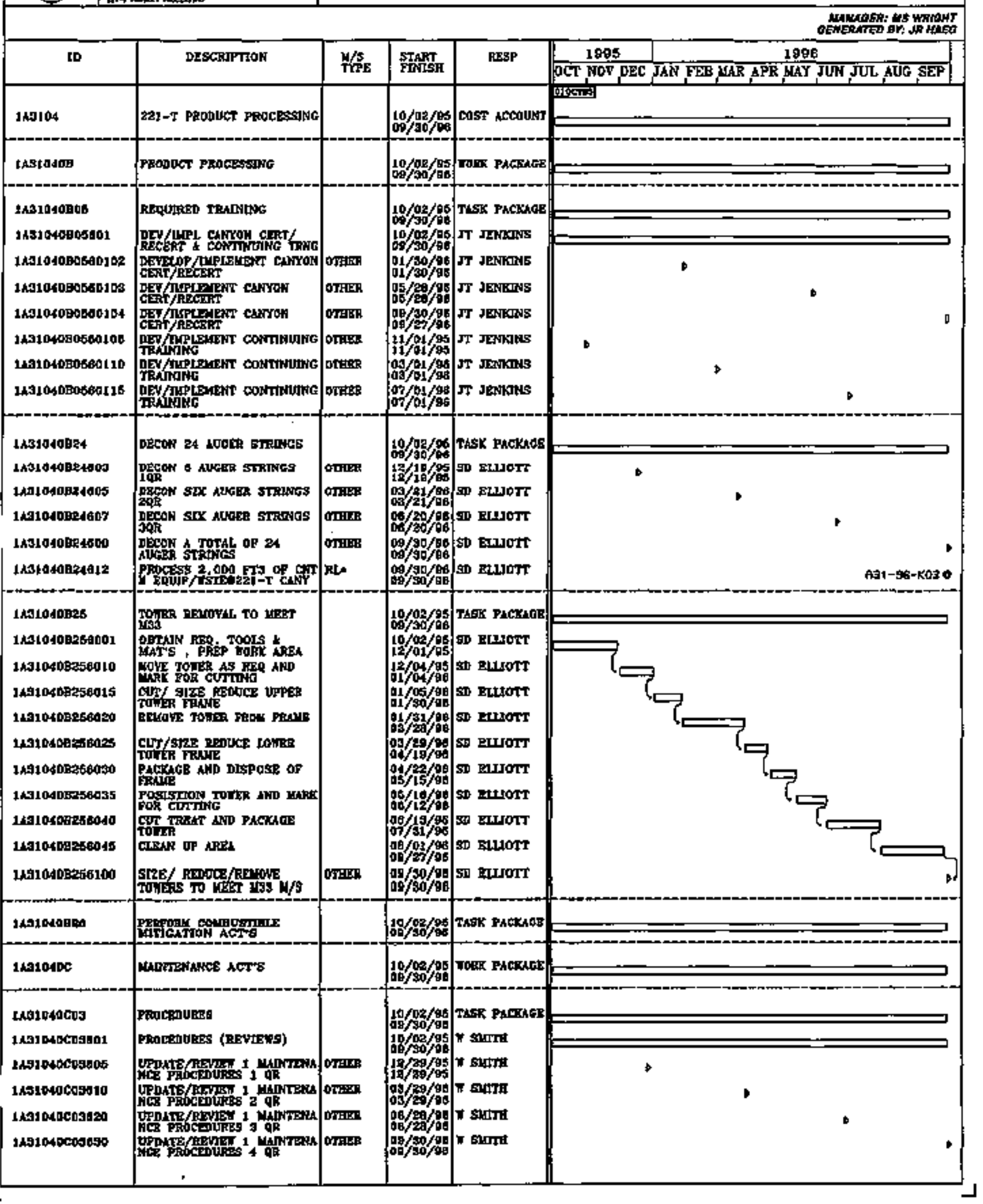




\section{WHC - SOLID WASTE PROGRAM $1 A 3202$ \\ 2706-T HOT \$TANDEY}

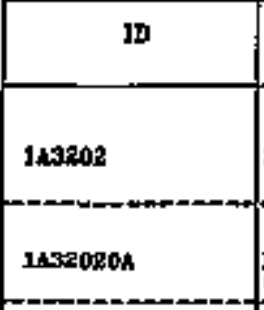

\section{usapaspyty}

LA320ROABROOOI

IA320001006005

IAA200204026010

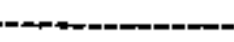

1hatearanod

Haragenomboot

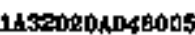

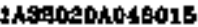

--C---

1hatenzars

1492020ht70001

IA320204176010

|---20--

1Asgarokal

1A020Z0A2LEOD

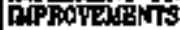

1 topocones

ld3a02012a:001

IAS908OHARA010

J16rozods8

1450020ksadool

H3roponagacos

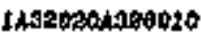

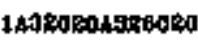

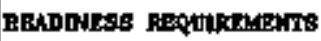

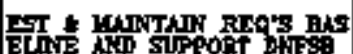

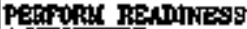

ACTIVIrIins

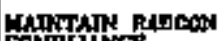

Dorithince

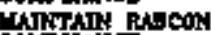

Cotphisc

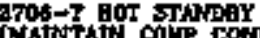

(COAD)

cupr zran-7 not stumbor

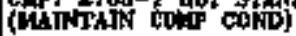

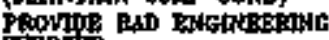

Fupqat

1haparoc

WNTEAAKCE ACTTVITIES

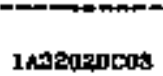

1At60204058001

\section{PROCEDOALS}

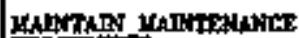

THOEEOUA

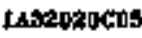

ld5goasco6eot

ingagocos

1herordenatorot

\section{Trintiva}

Whthrenthier TRAHilig

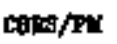

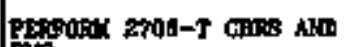
PIS

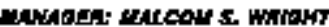

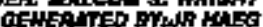

\begin{tabular}{|c|c|c|}
\hline$\frac{y}{\mathrm{~T}} / \mathrm{s}$ & START & RPSP \\
\hline & $10 / 02 / 95$ & CDST ACCOUST \\
\hline & $10 / 02 / 95$ & च0स木 FК: \\
\hline
\end{tabular}

\begin{tabular}{l|l}
1995 & 1996 \\
\hline
\end{tabular} OCT NOV DEC IAN FEB IAR APR UAY JTA JUL AUC SESP

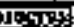

$$
+
$$

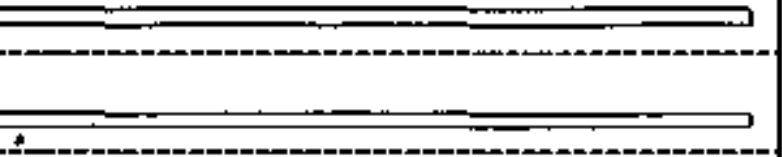

$10 / 02 / 96$ TLSK PUt

ad $/ 100 / 9$ a

$10 / 0 \% / 9$ )

ang $/ 90 / 96$

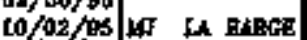

$09 / 30 / 0 \%$

$10 / 02 / 89$

IA BAREE

$\infty / 50 / 0$

$10 / 02 / 05$ THST PYC

$00 / 90 / 06$

0 $\$ / 01 / 06$. B sim

$04 / 30 / 06$.

06/05/80 क ज्ञाता

$09 / 30 / 60$

10/02/05 日 surit

$00 / 50 / 06$

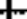

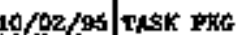

á $/ 90 / 96$

10/0e/tos as ancron

$10 / 02 / 96$ TI ERICXSON

$00700 / 9$

Thes rue

$08 / 09 / 86$

ar $/ 27 / 96$

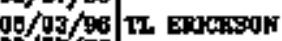

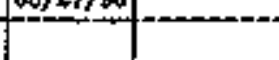

10/92/C5 TAKi PLO

$00 / 30 / 06$

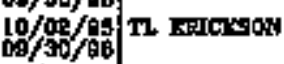

10/09/95 Is stets

$00 / 00 / 0$ B

10/00/06 T/45\% gxa

$10 / 02 / 60$

$10 / 02 / 05$ DE ज्ञाtroT

$00 / 50 / 5$

10/08/05 yt LA BARE

Q $/ 5 \%$ at

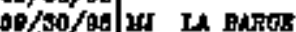

$0130 / 9$

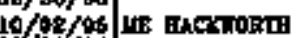
on $/ 00 / 0$

$00 / 700$
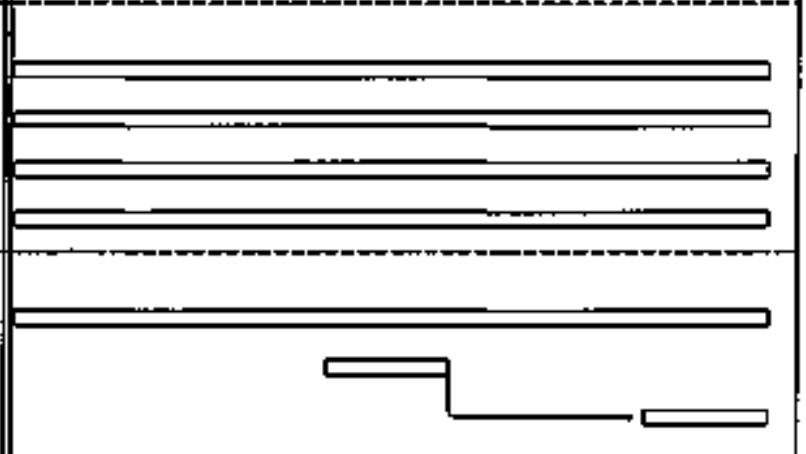

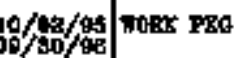


WHC - SOLID WASTE PROGRAM 143202 2706-T HOT STANDBY

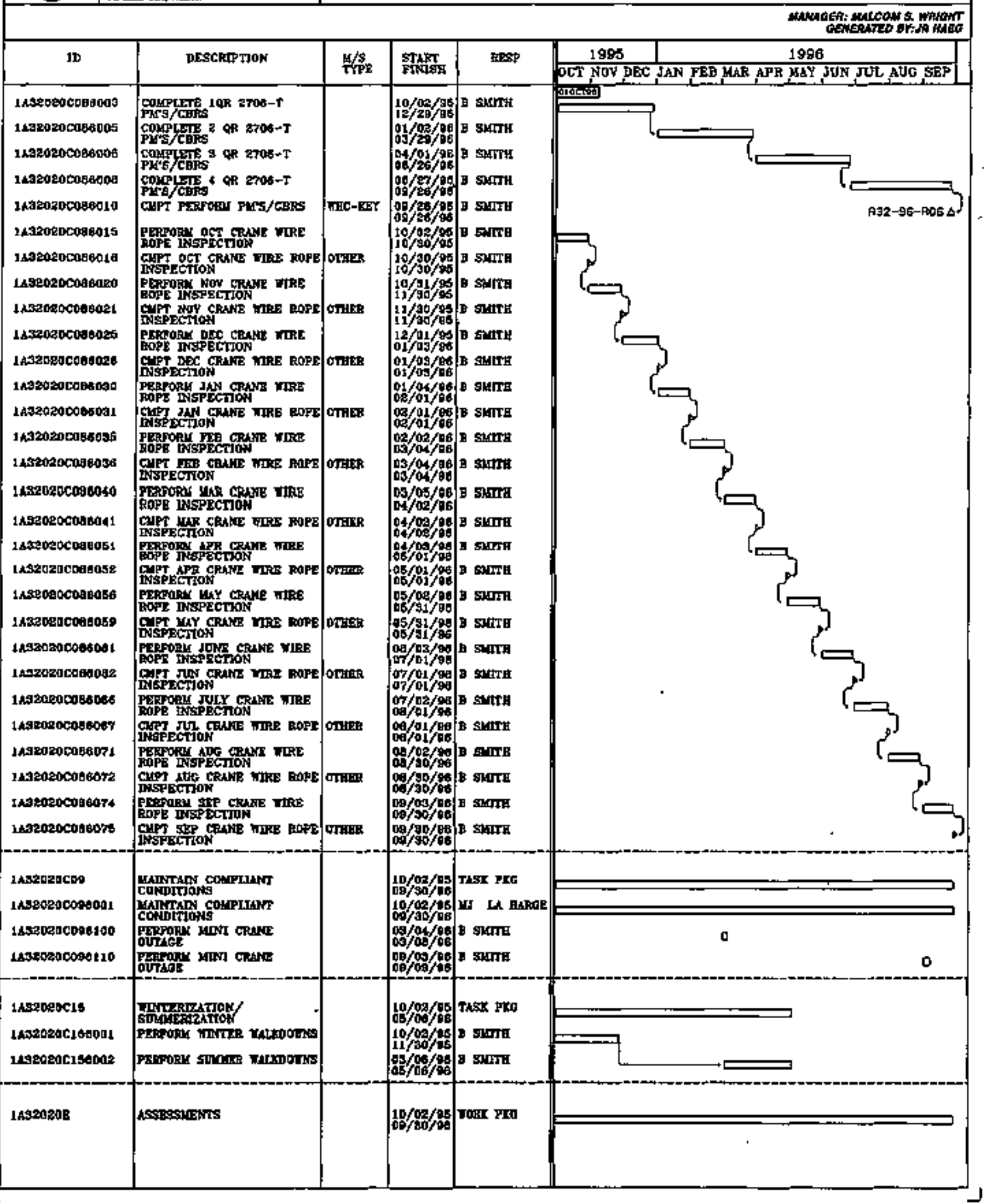




\section{WHC - SOLID WASTE PROGRAM $1 \mathrm{A3202}$ 2706-T HOT STANDEY}

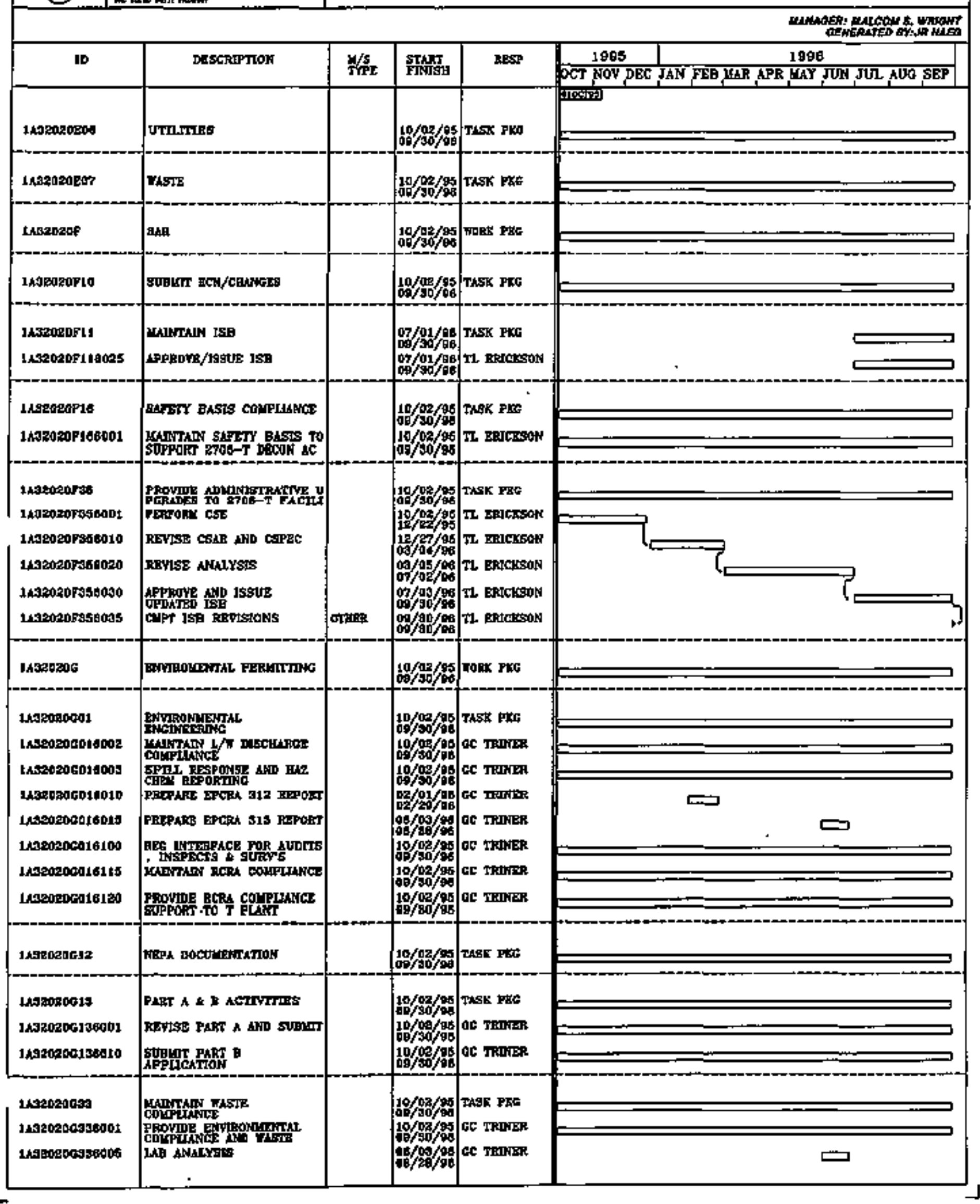




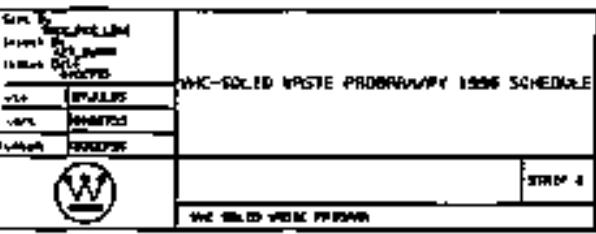

\section{WHC - SOLID WASTE PROCRAM 143202 2706-T HOT STANDEY}

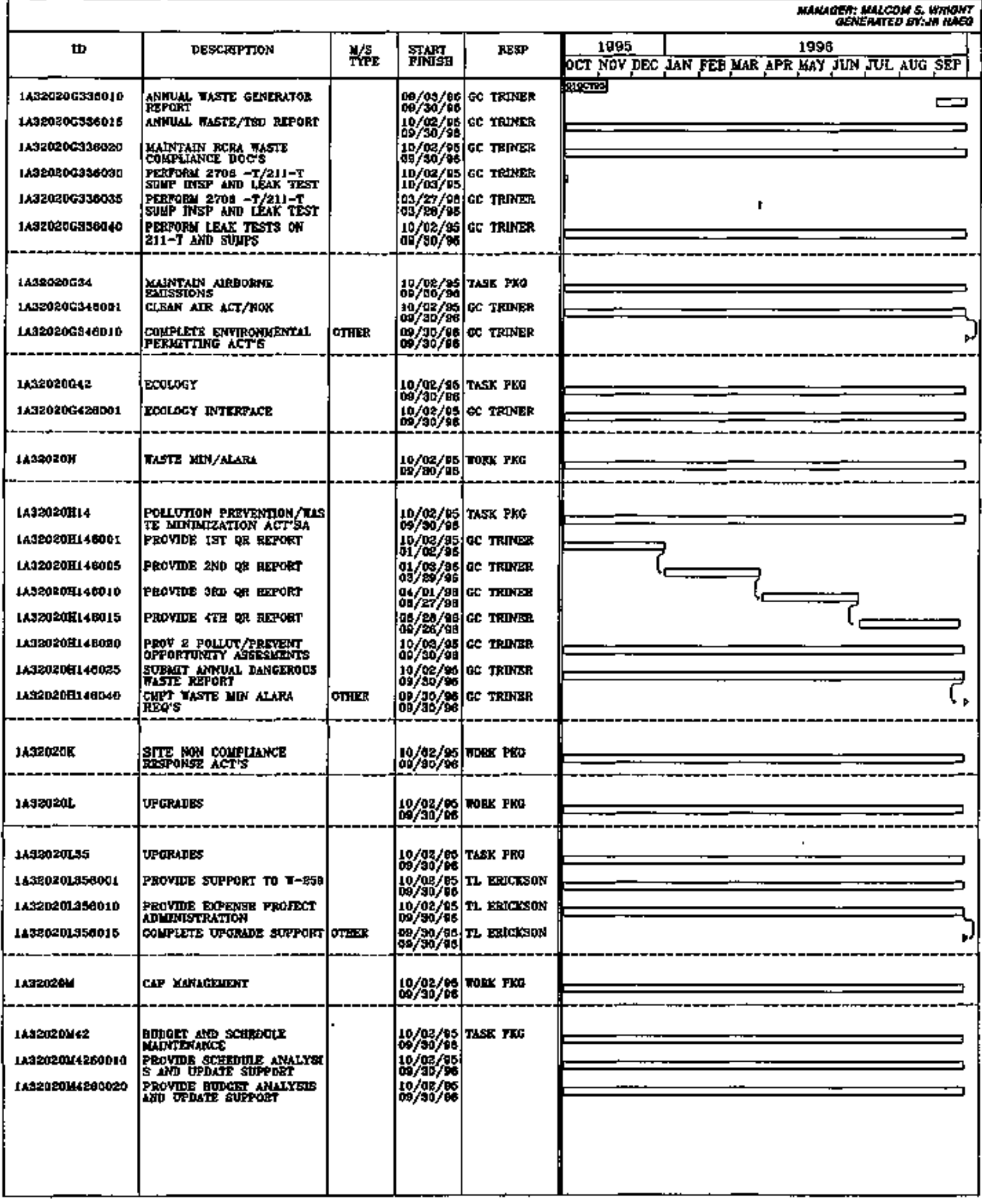


WHC - SOLD WASTE PROCRAM 143204

2706-T PRODUCT PROCESSINE

\begin{tabular}{|c|c|c|c|c|c|}
\hline \multirow[b]{2}{*}{ IP } & \multirow[b]{2}{*}{ pescantiox } & \multirow[b]{2}{*}{ 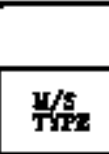 } & \multirow[b]{2}{*}{ STMET } & \multirow[b]{2}{*}{ FESP } & 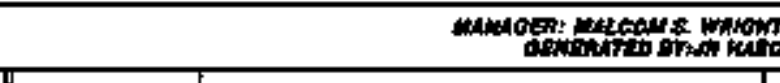 \\
\hline & & & & & 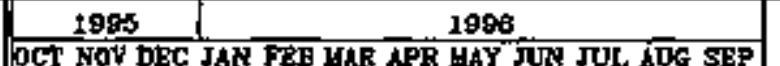 \\
\hline IAsabs & Prob-I PRopilat & & top/atos & cost account & 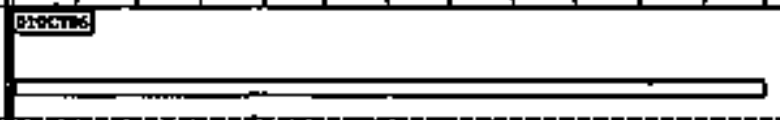 \\
\hline It:804ak & 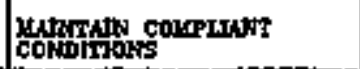 & & $01 / 00 / 90$ & rope ptat & \\
\hline 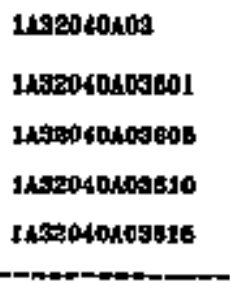 & 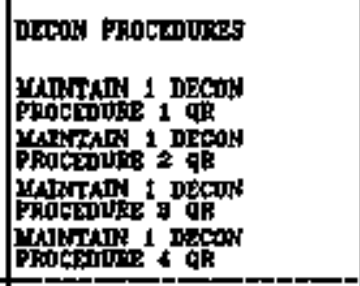 & & 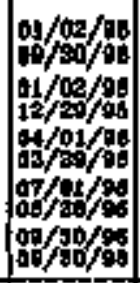 & 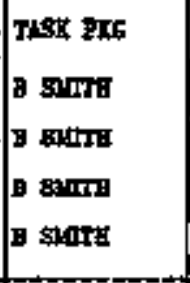 & : \\
\hline HOEO40B & 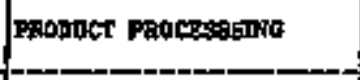 & & $10 / 92 / 90$ & morex pro & \\
\hline 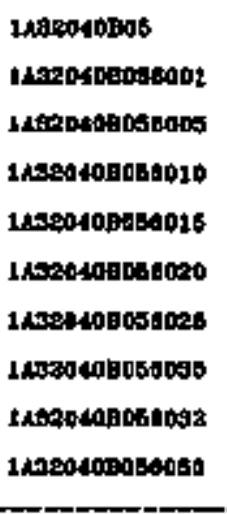 & 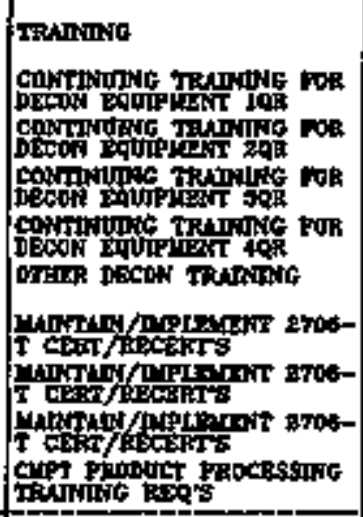 & ortitax & 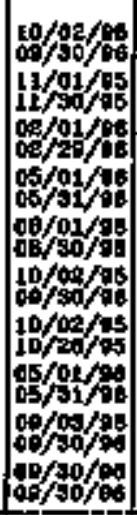 & 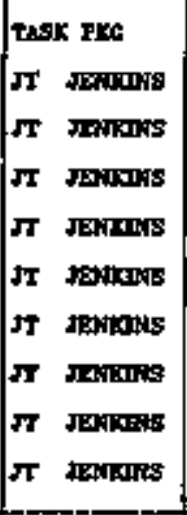 & للى \\
\hline 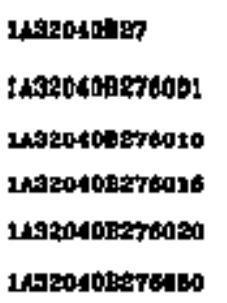 & 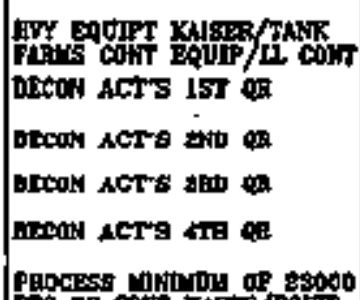 & & 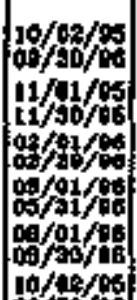 & 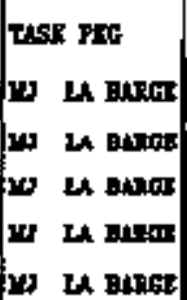 & \\
\hline 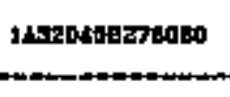 & 然 & 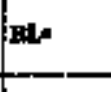 & & 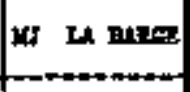 & 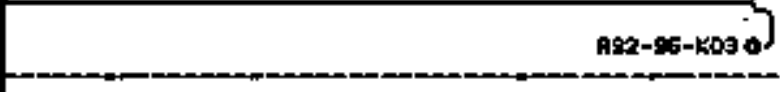 \\
\hline 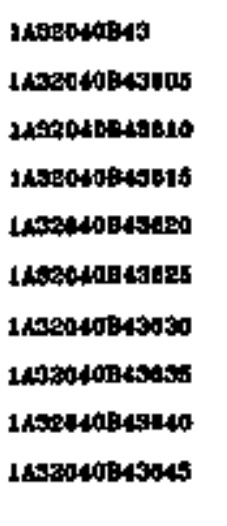 & 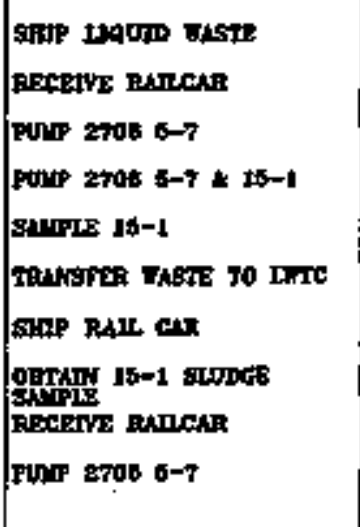 & & 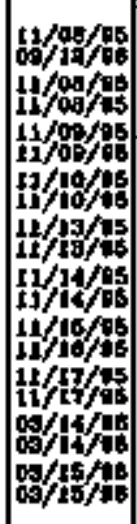 & 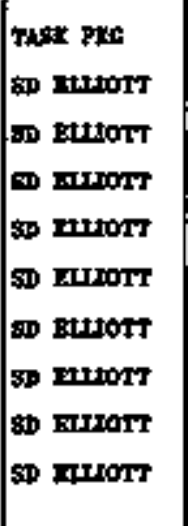 & b \\
\hline
\end{tabular}




\section{WHC - SOLID WASTE PROGRAM . 143204 2706-T PRODUCT PROCESSINO}

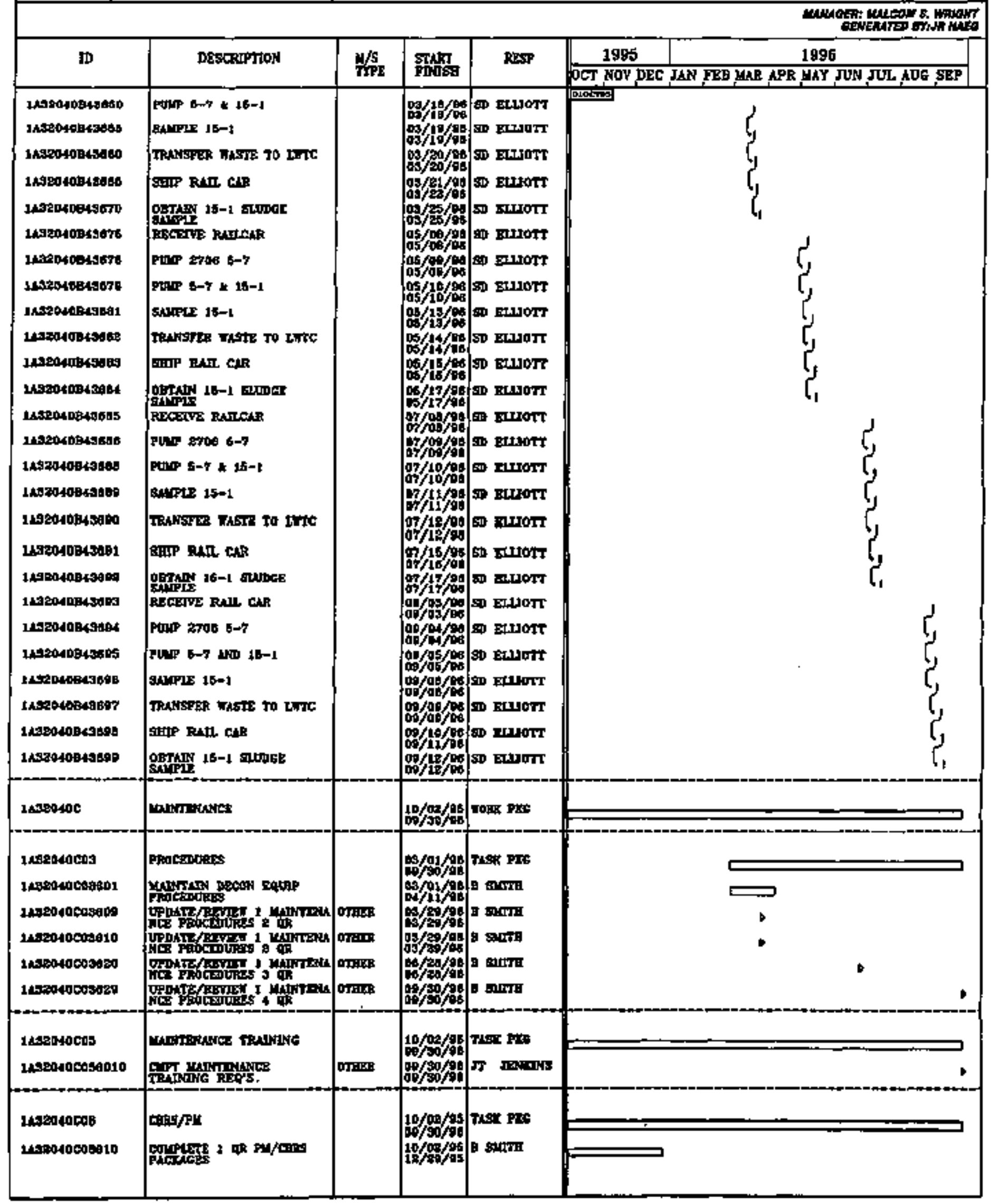


$B$ | WHC - SOLID WASTE PROGRAM
2706-T PRODUCT PROCESSING

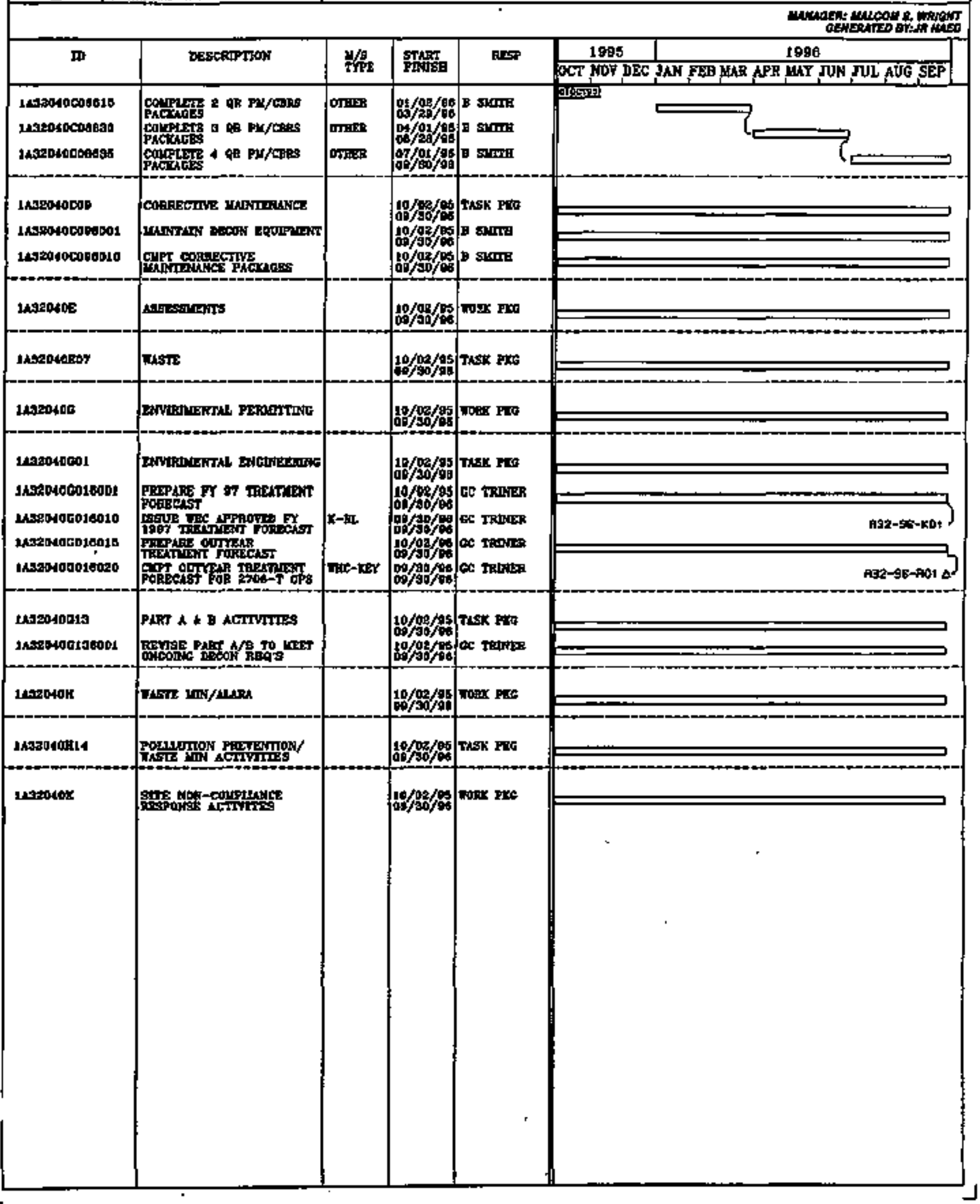




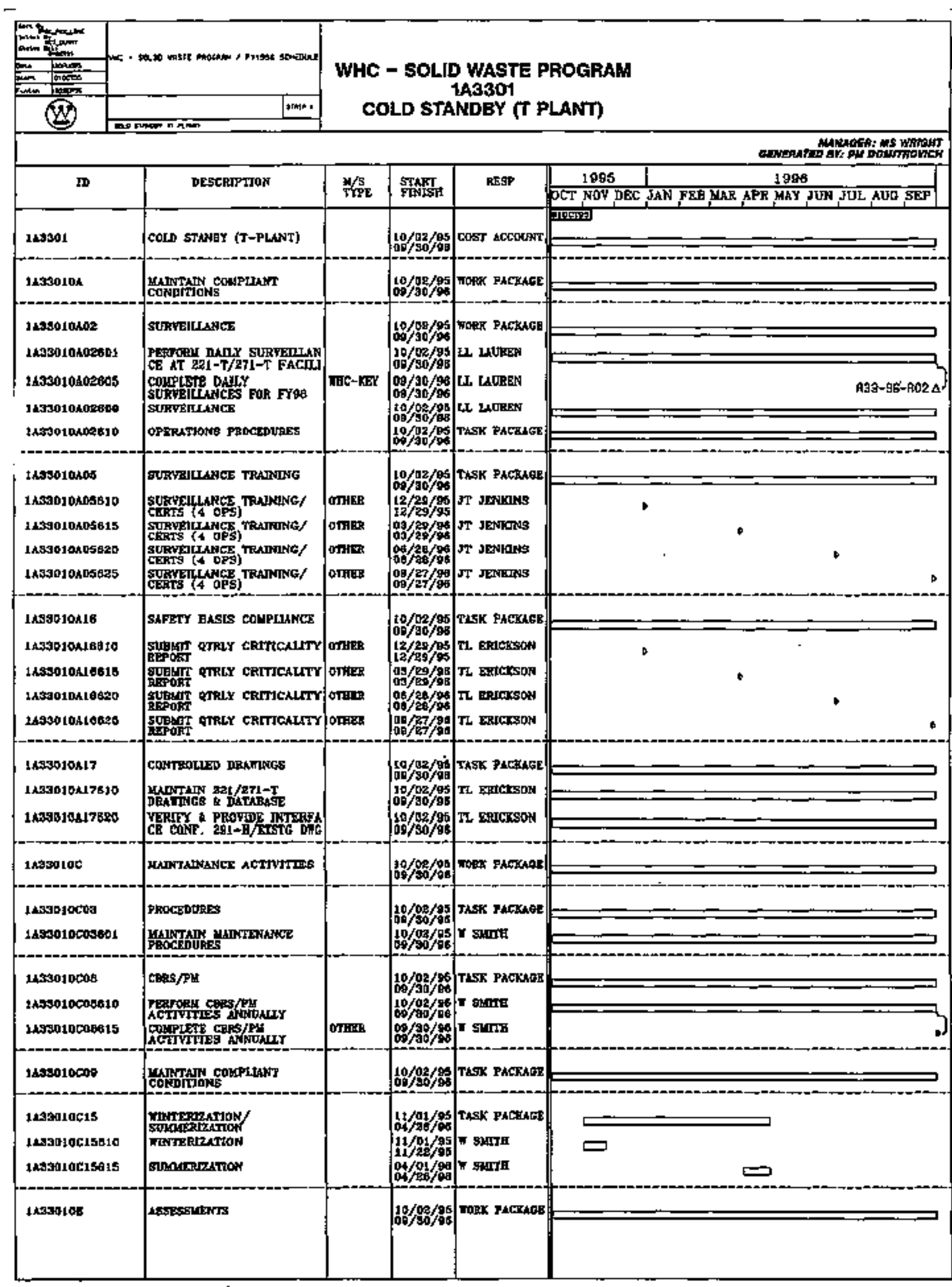




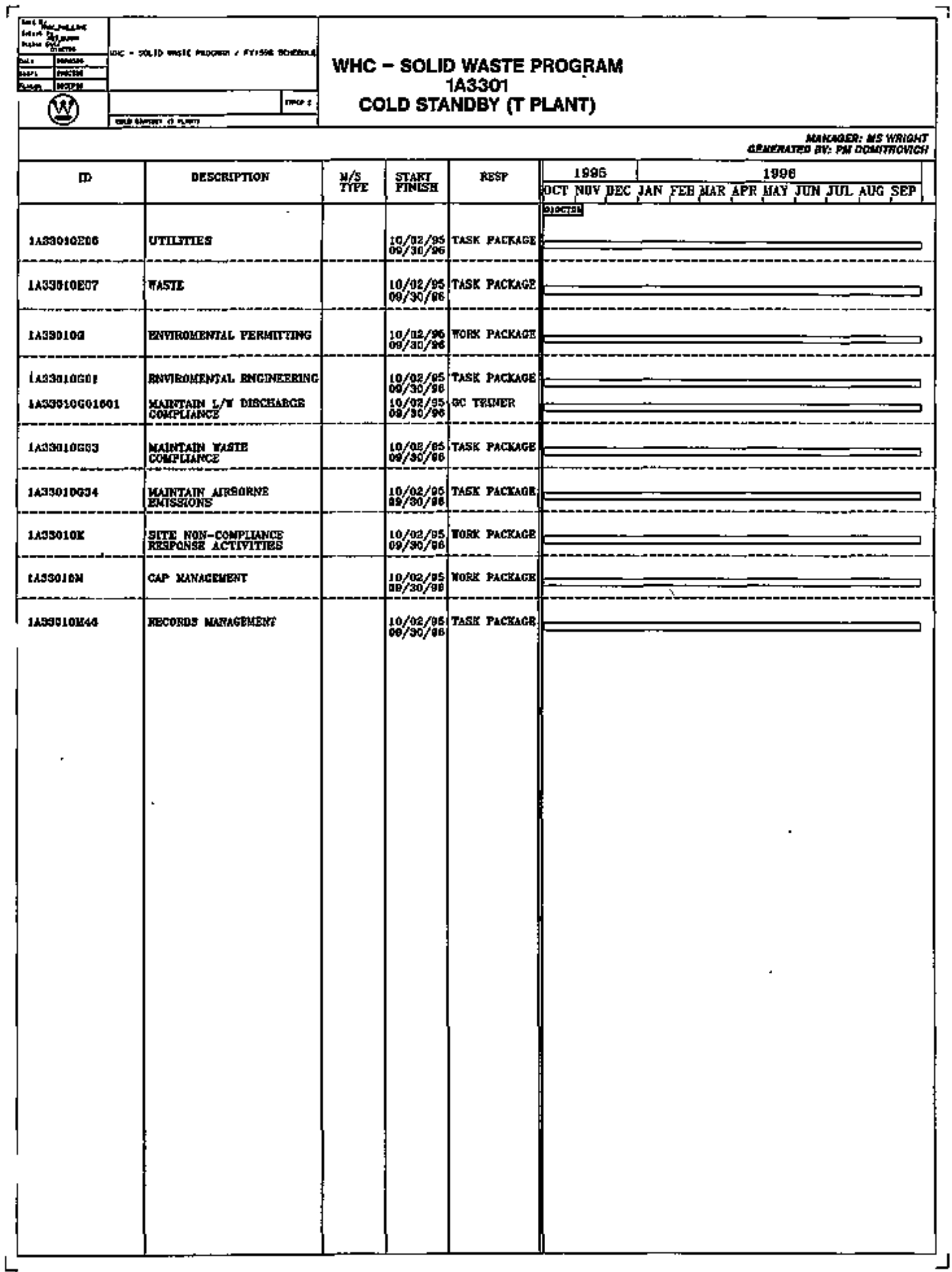




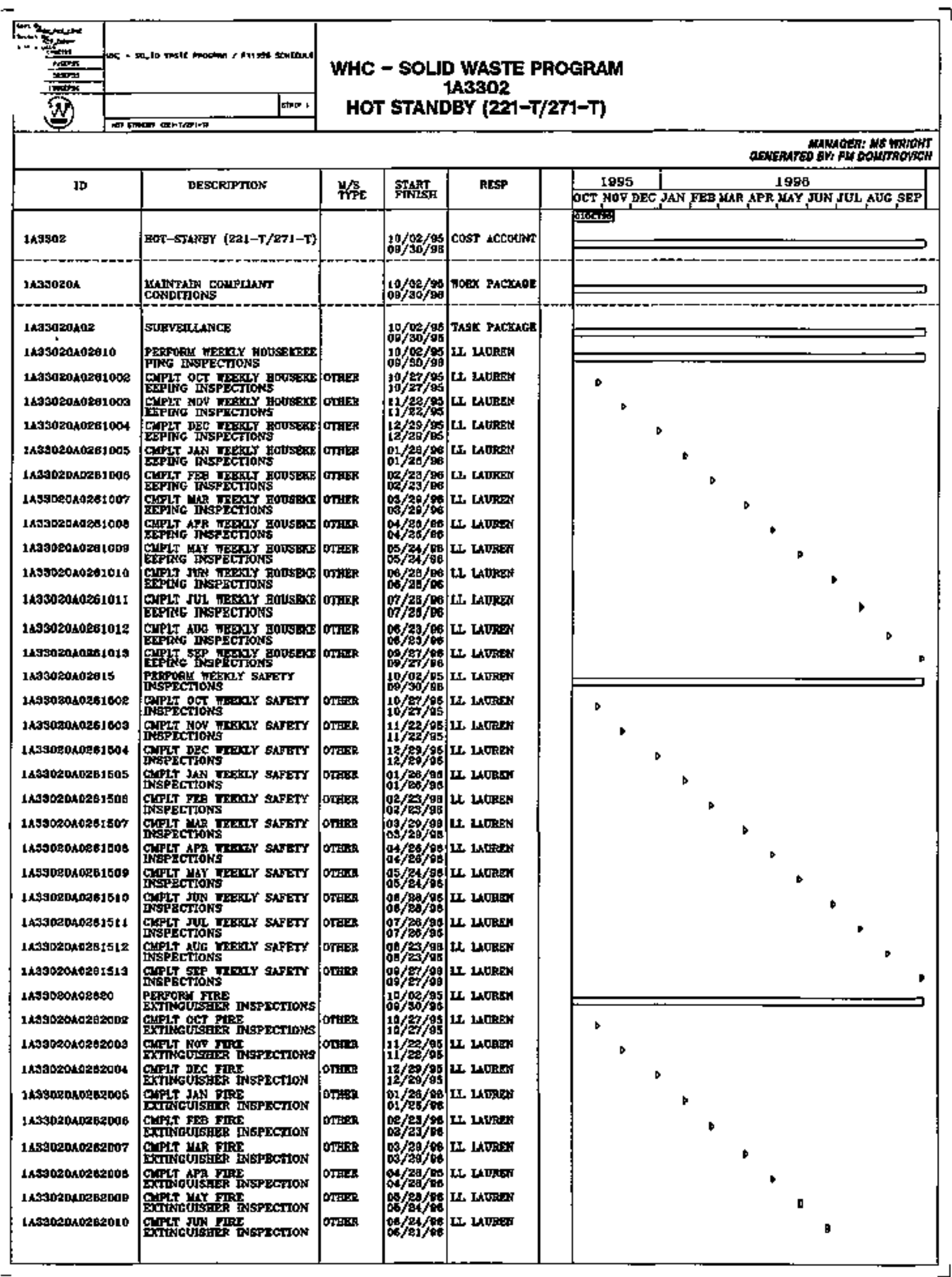


WHC - SOLID WASTE PROGRAM $1 \mathrm{A3302}$ HOT STANDBY (221-T/271-T)

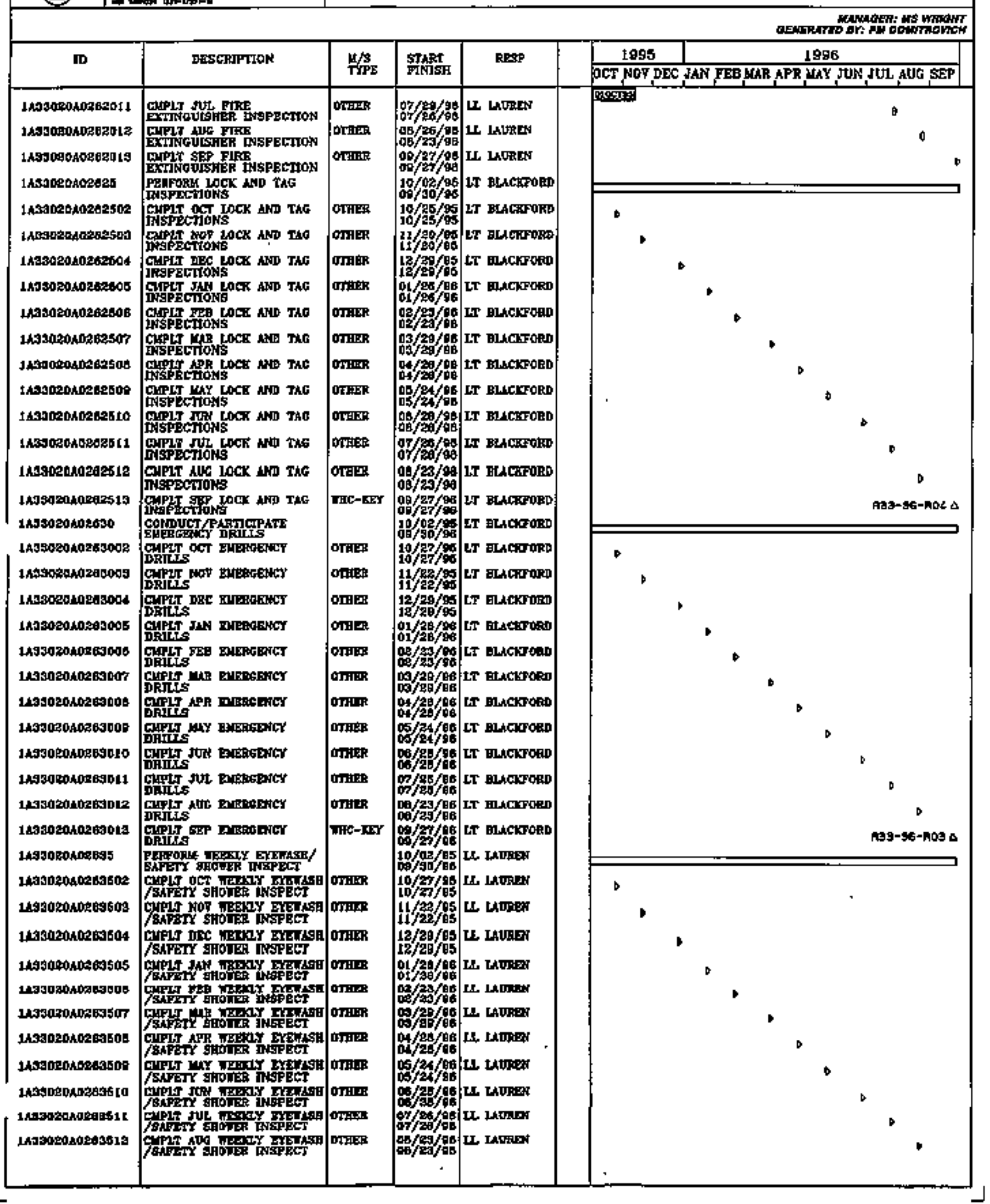




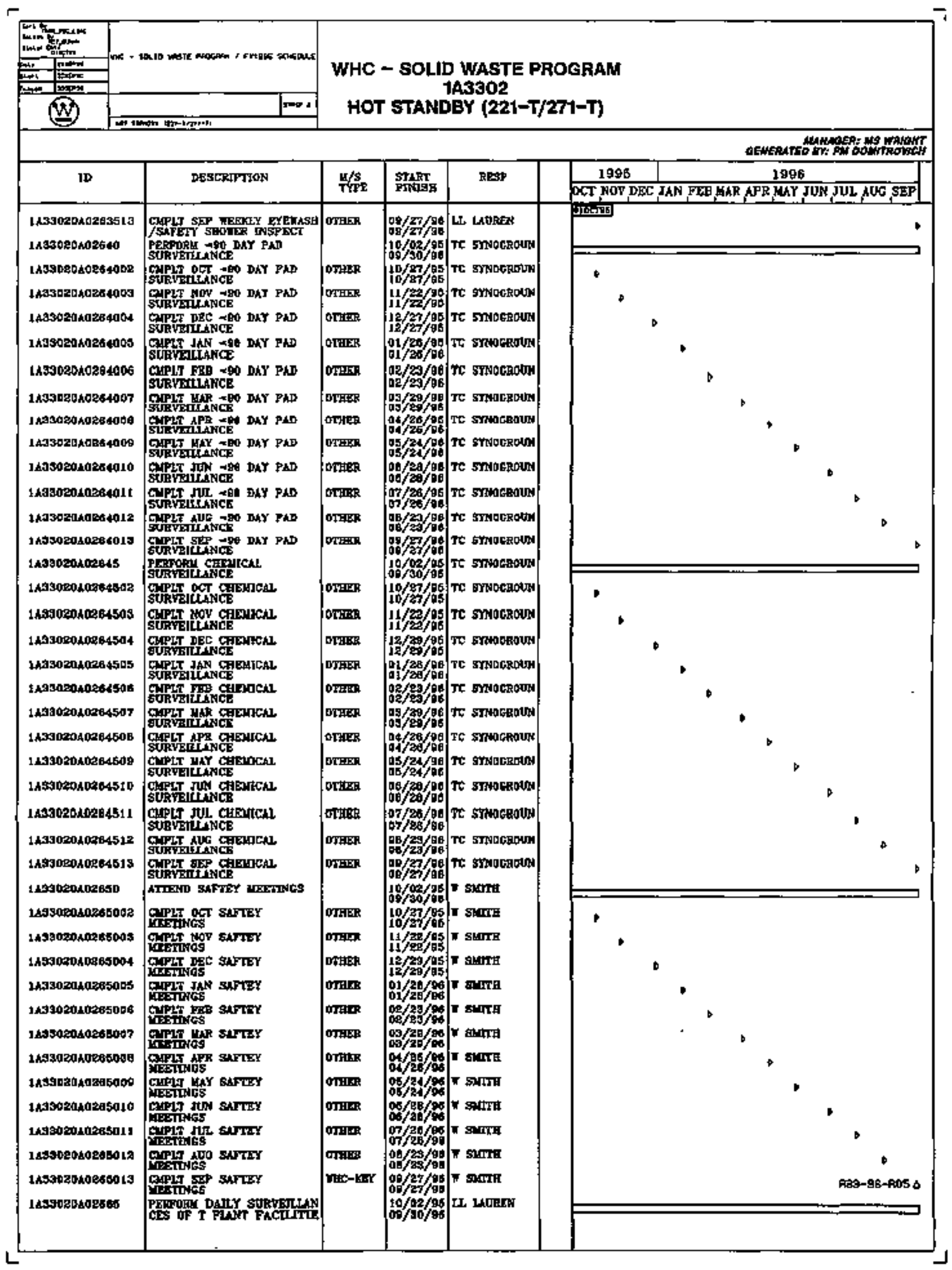


WHC - SOLID WASTE PROGRAM 1A3302 HOT STANDBY (221-T/271-T)

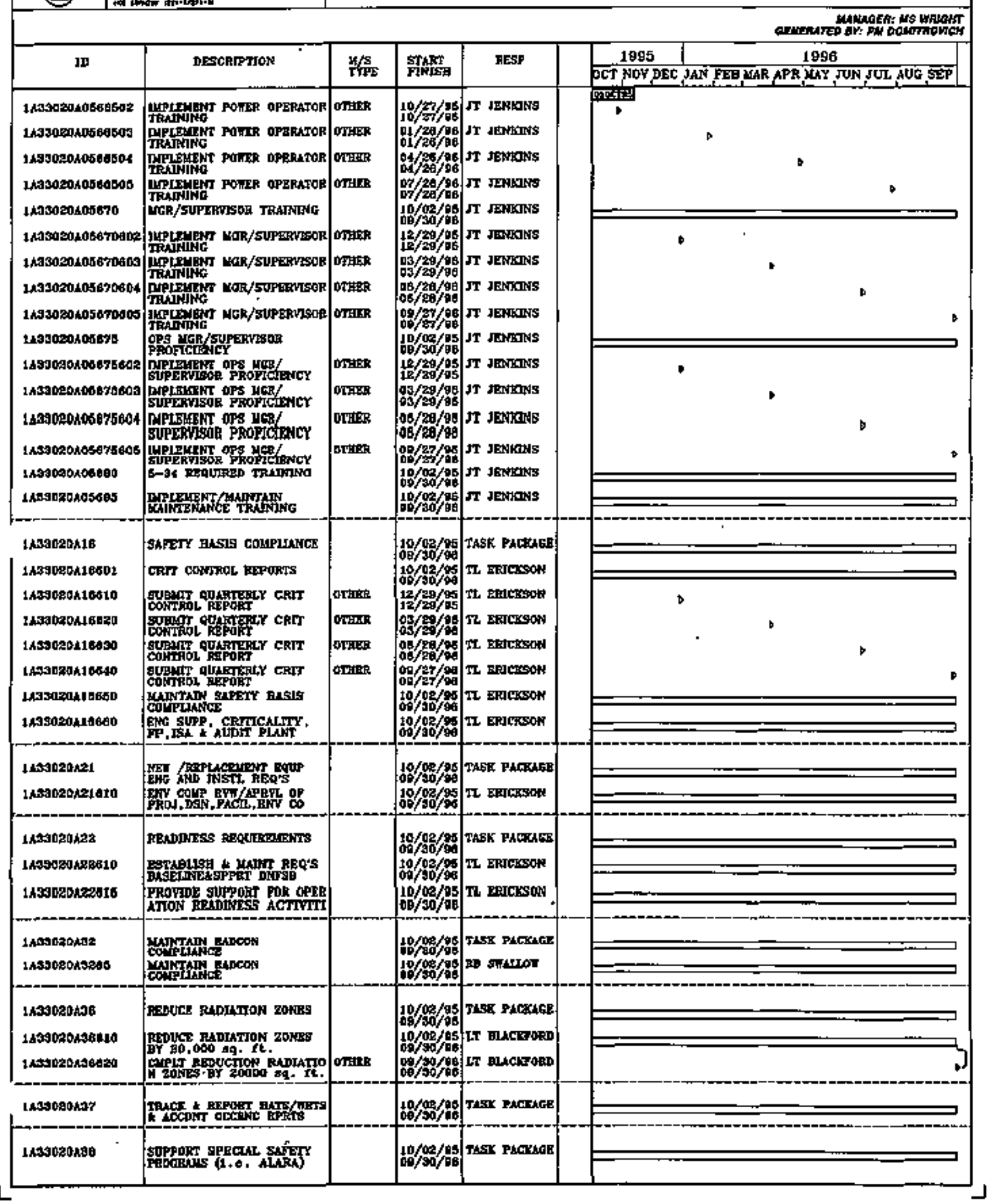




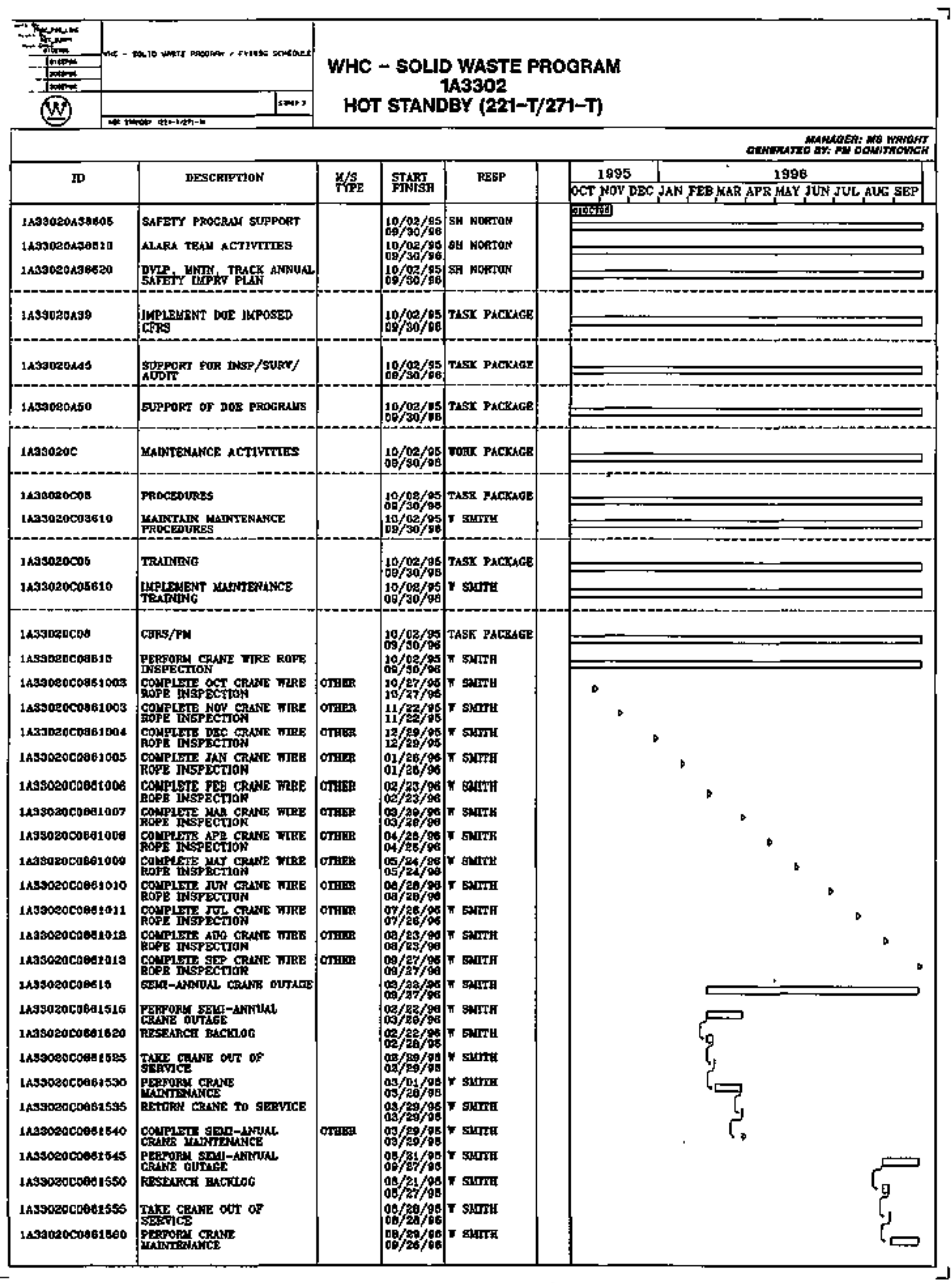


WHC - SOLID WASTE PROGRAM $1 A 3302$

HOT STANDBY (221-T/271-T)

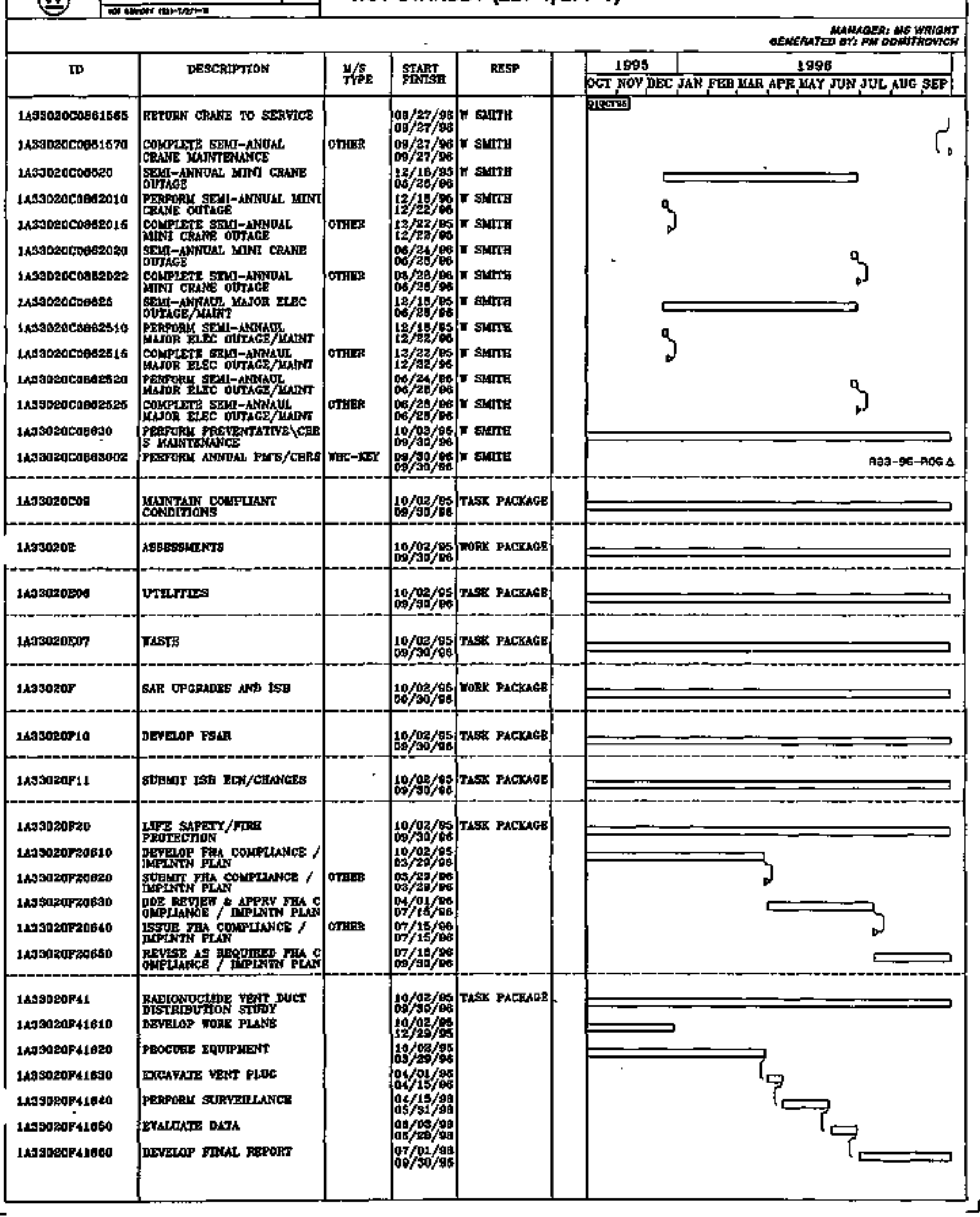




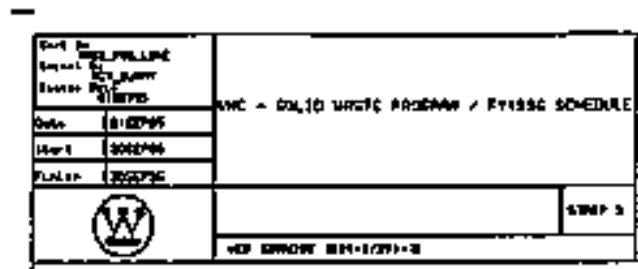

\section{WHC - SOLID WASTE PROGRAM $1 \mathrm{A3} 302$ \\ HOT STANDBY (221-T/271-T)}

Mucass: wo warins

oEH

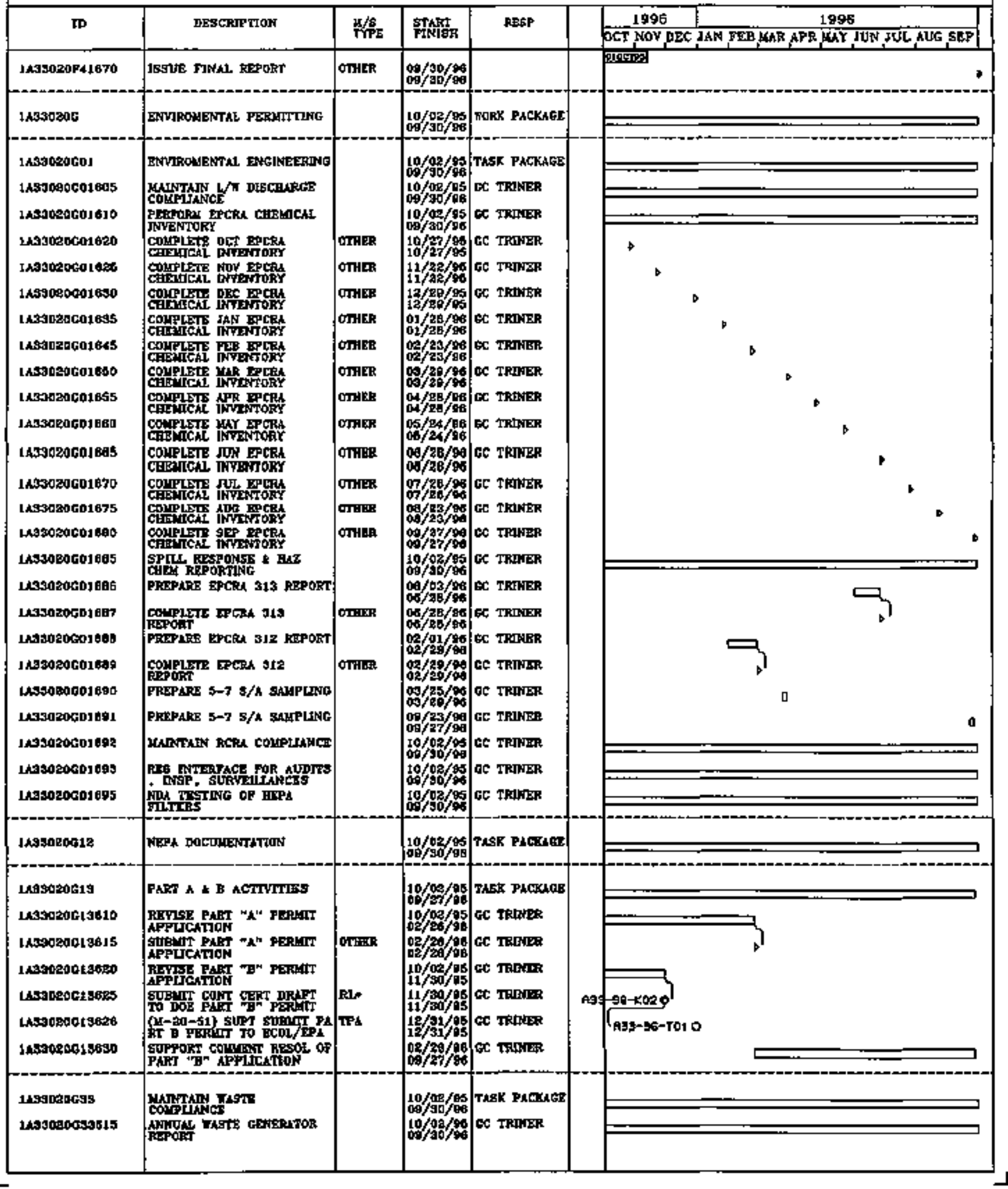


WHC - SOLID WASTE PROGRAM 143302

HOT STANDEY (221-T/271-T)

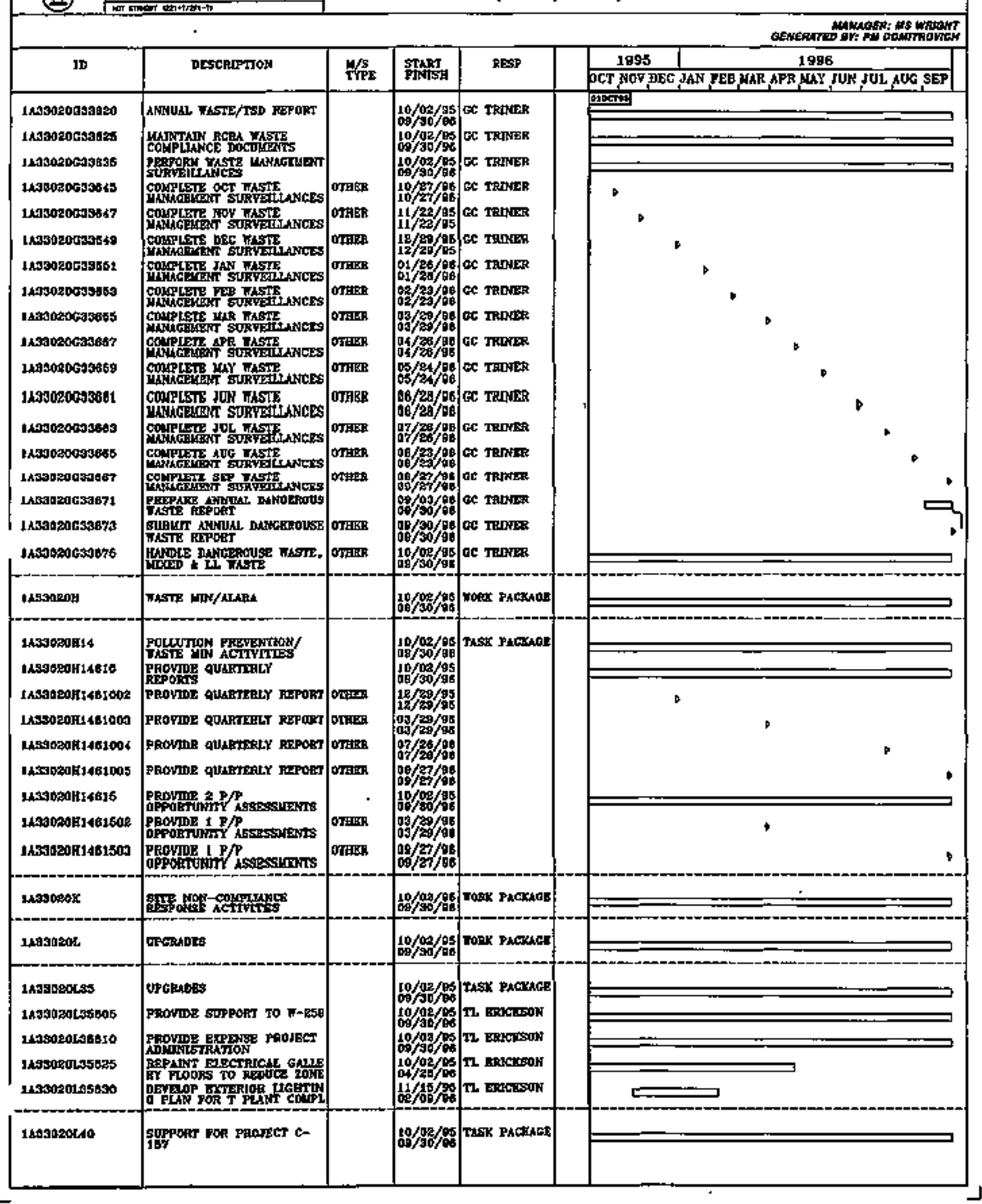




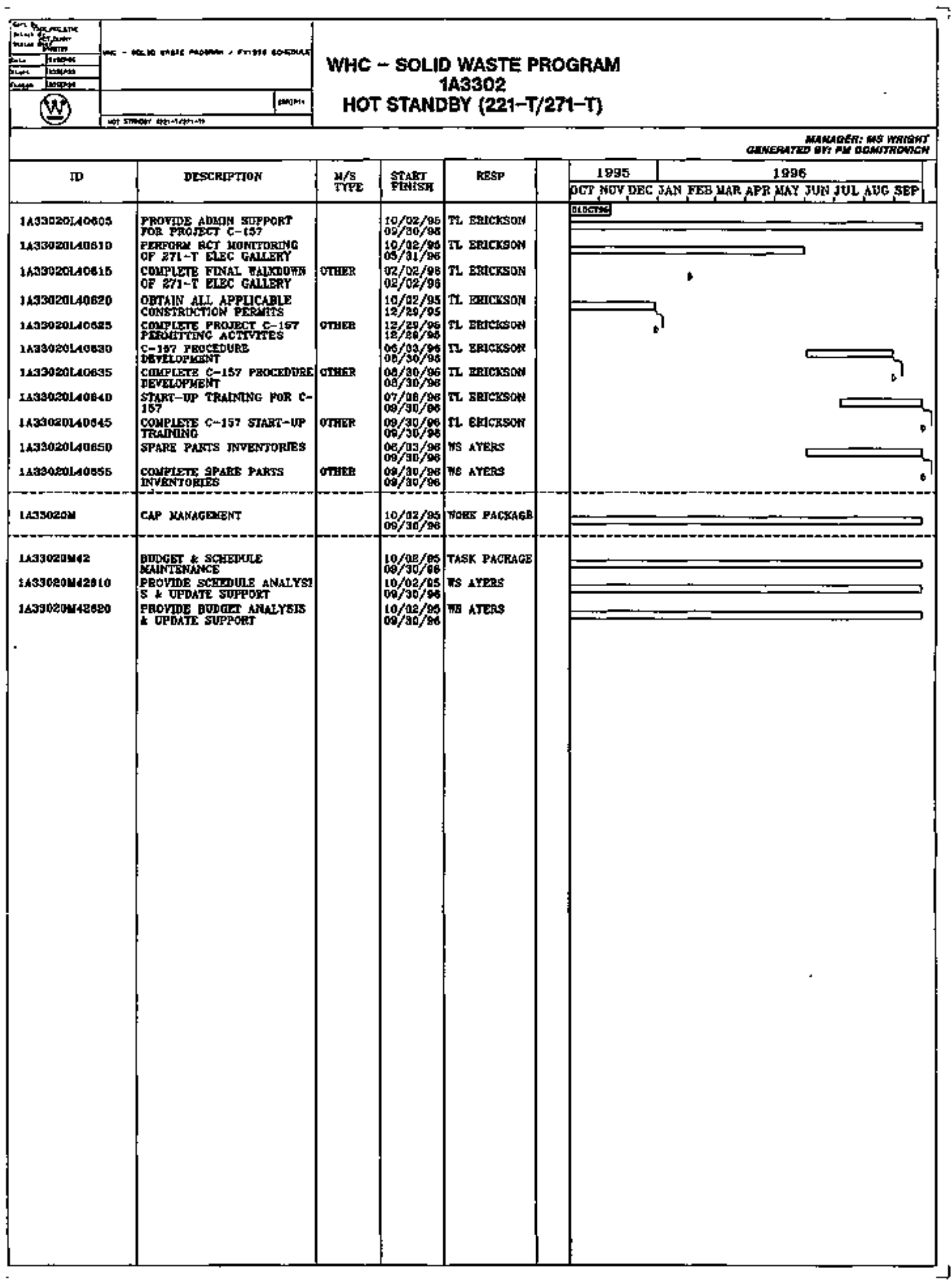




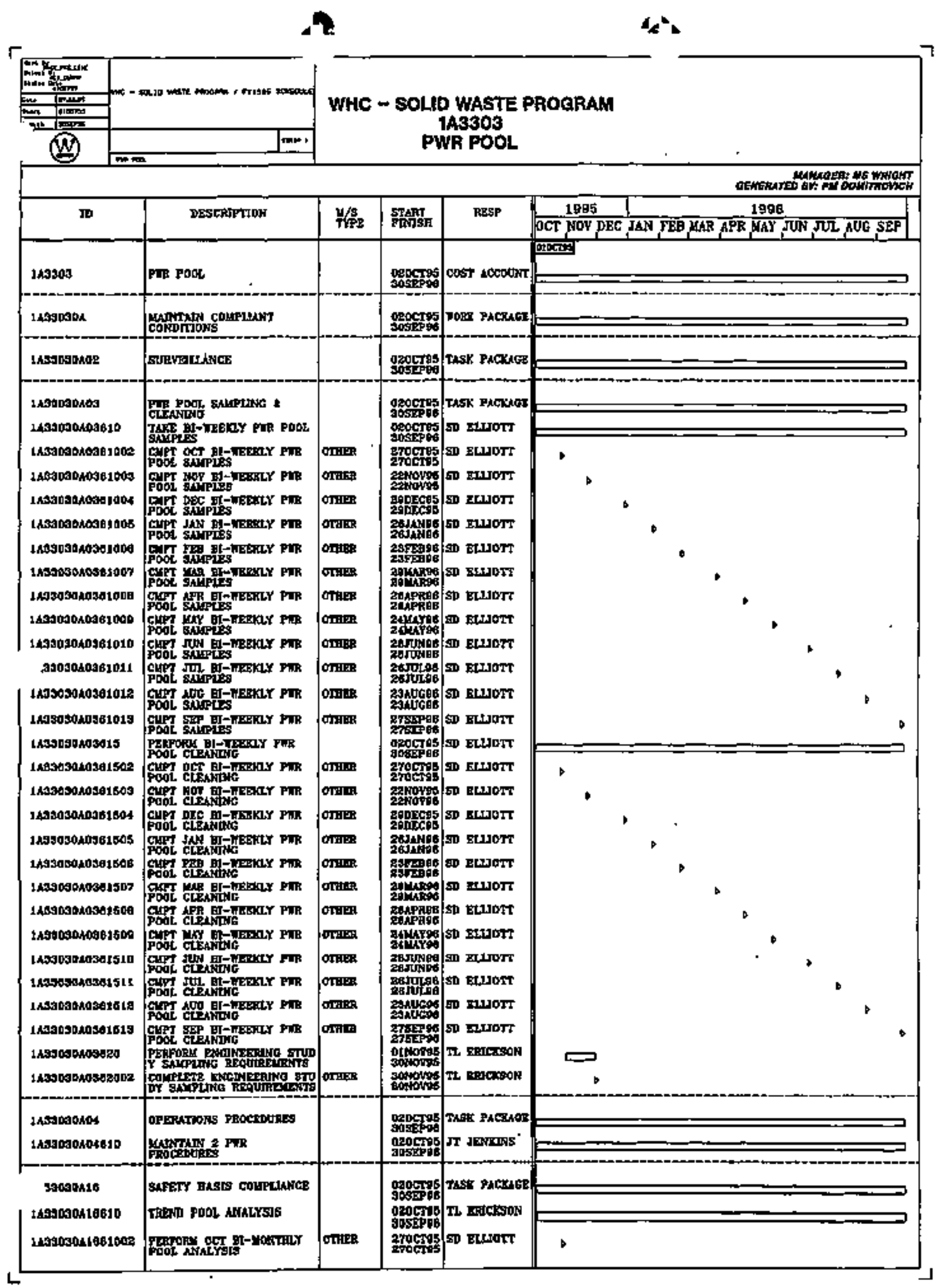




\section{WHC - SOLID WASTE PROGRAM $1 \mathrm{A3} 402$ RAILCAR CERTIFICATION}

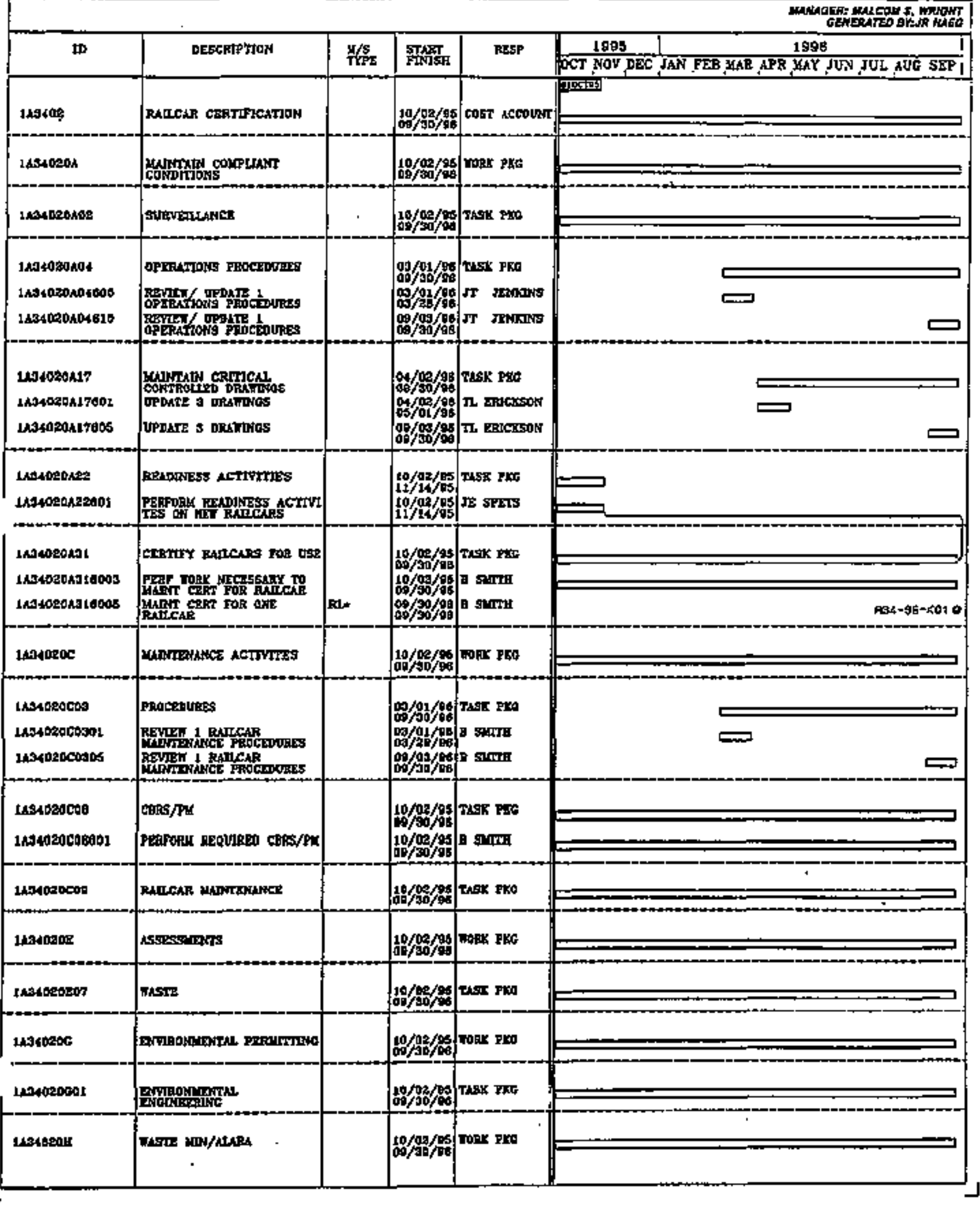





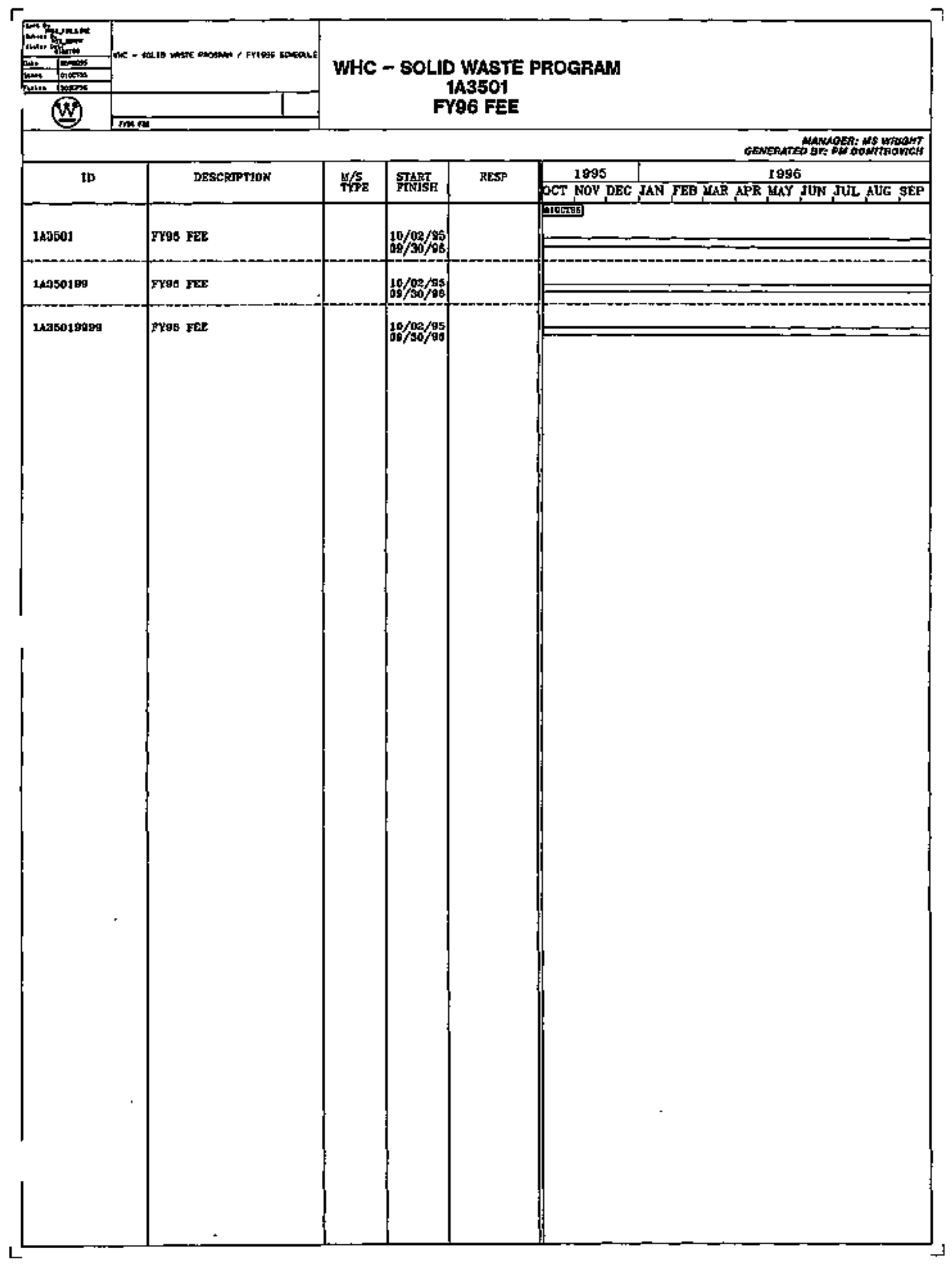




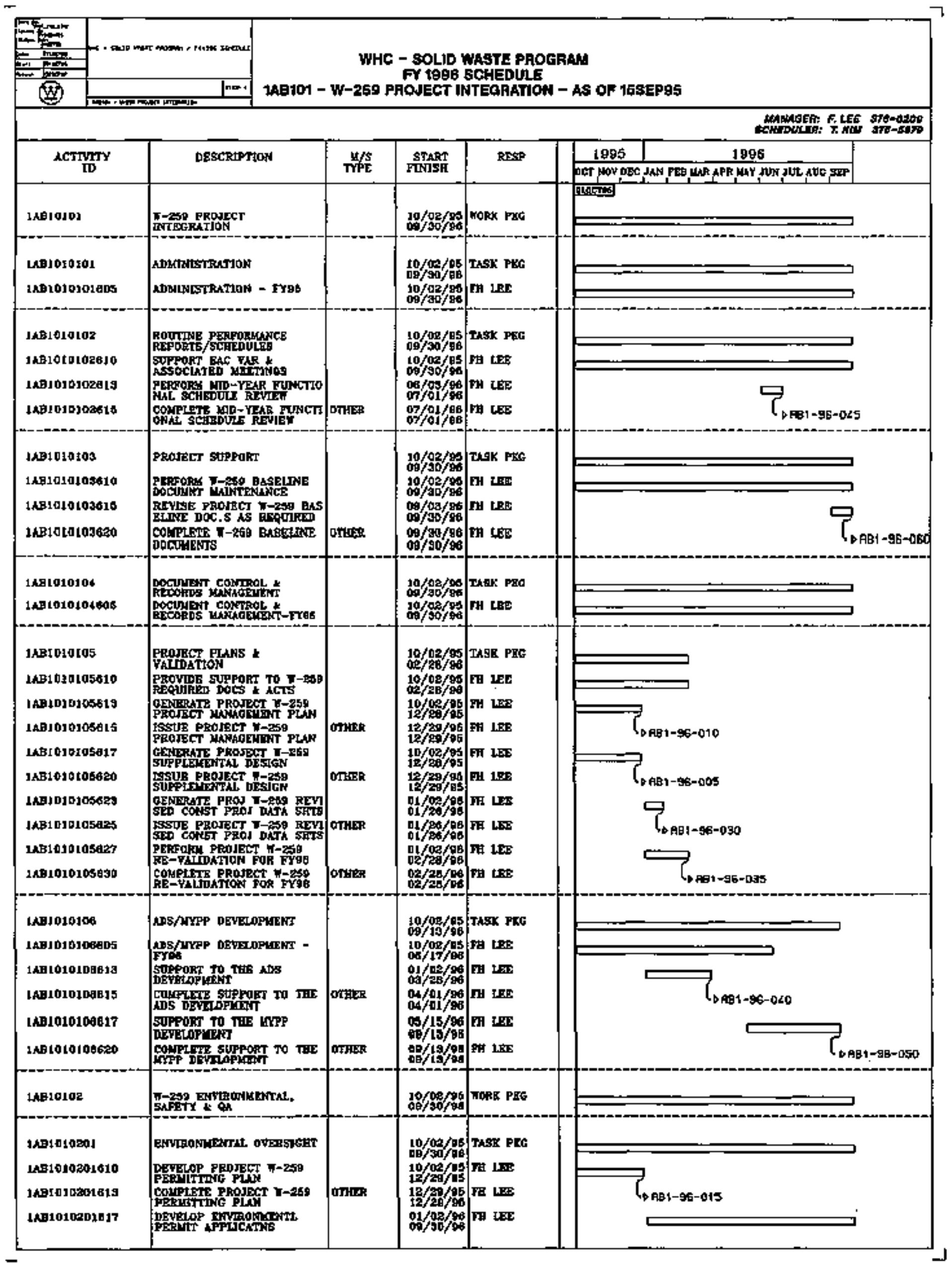




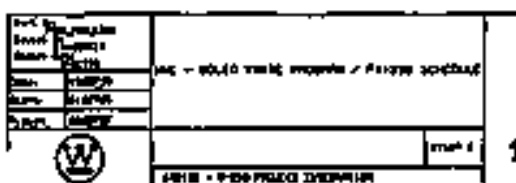

\section{WHC - SOLID WASTE PAORRAM FY 的6 SCHEDUNE 1ABFO1 - W-259 PRONECT INTEERATLON - AS OF 15SEPSS}

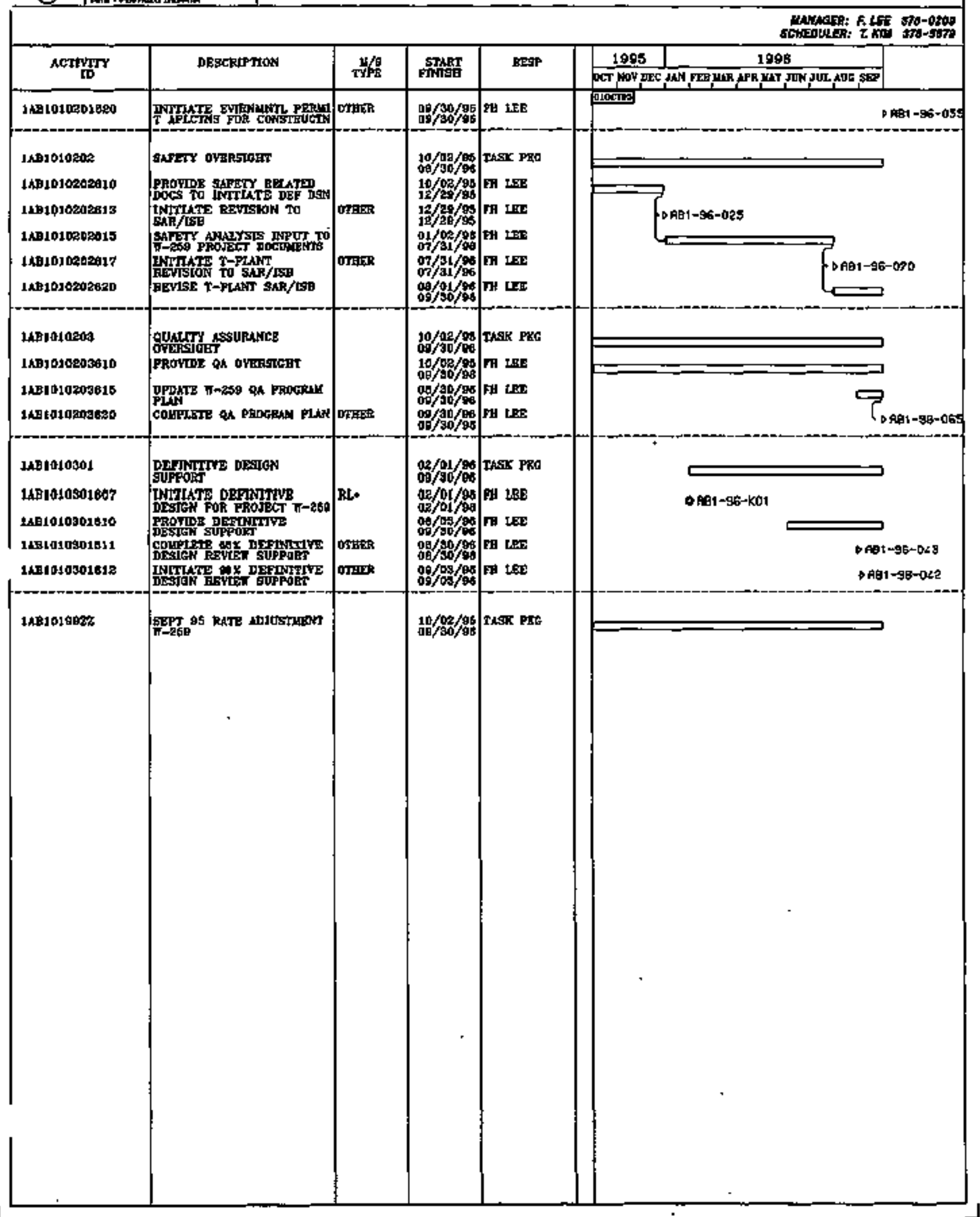




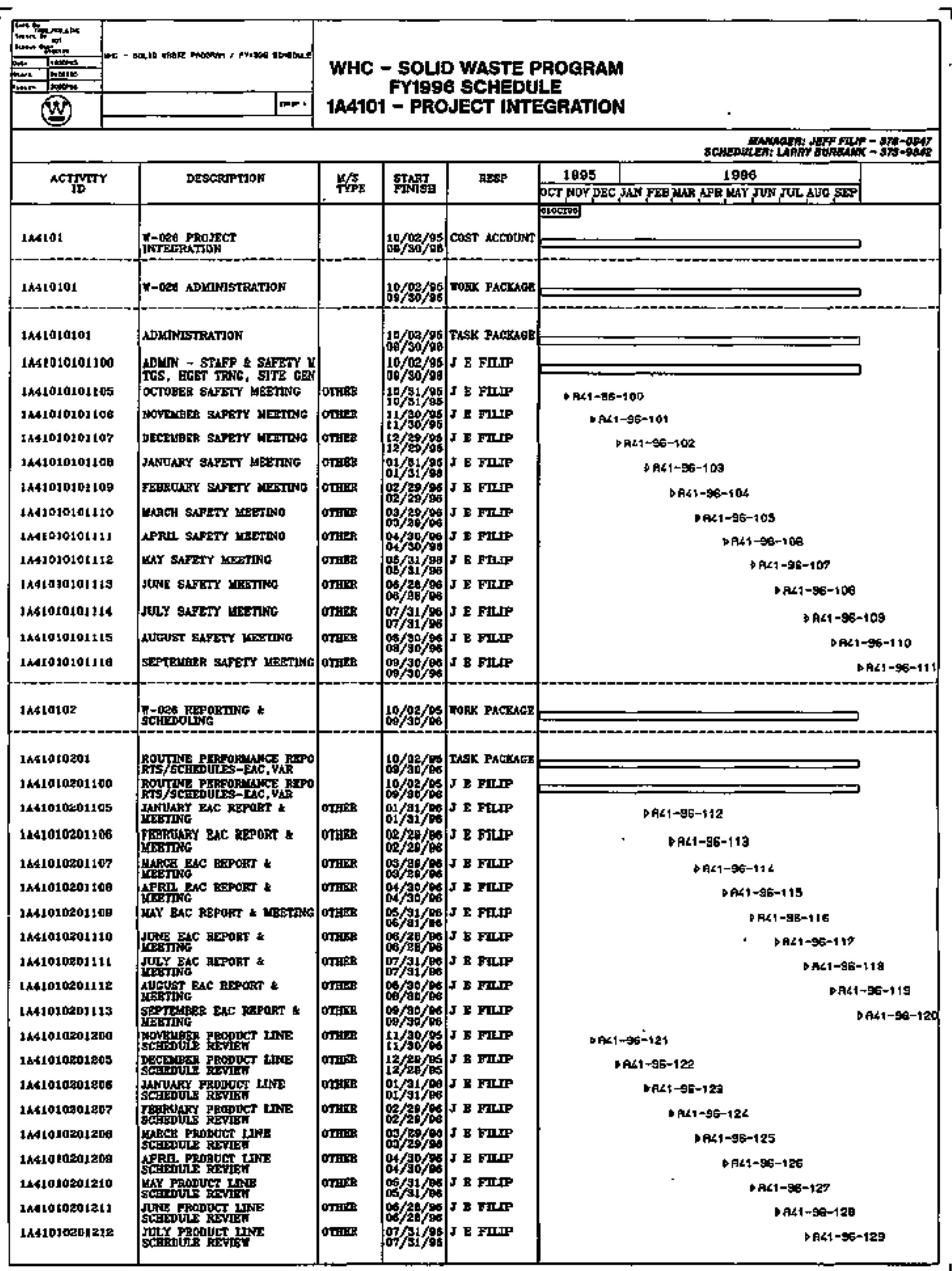




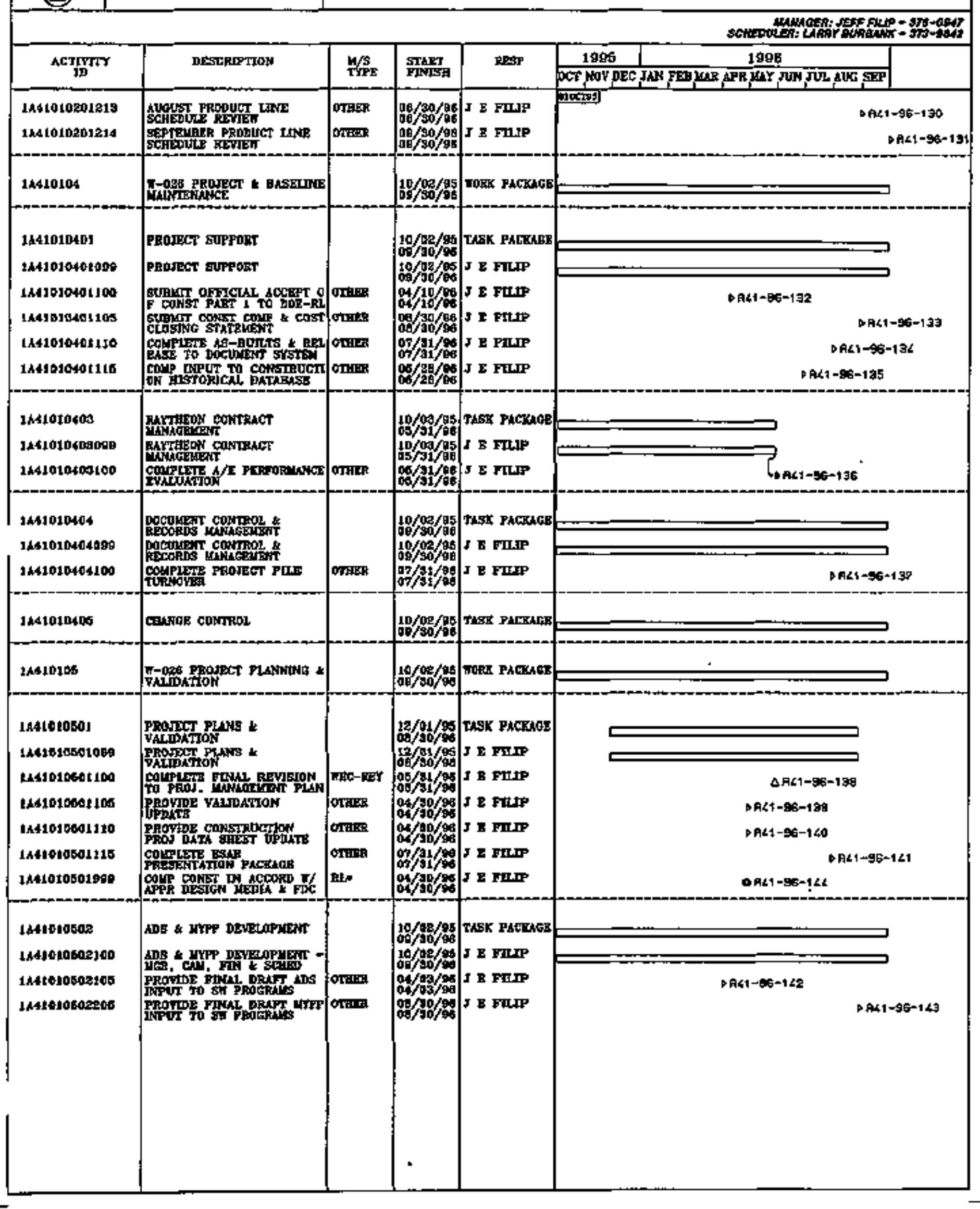




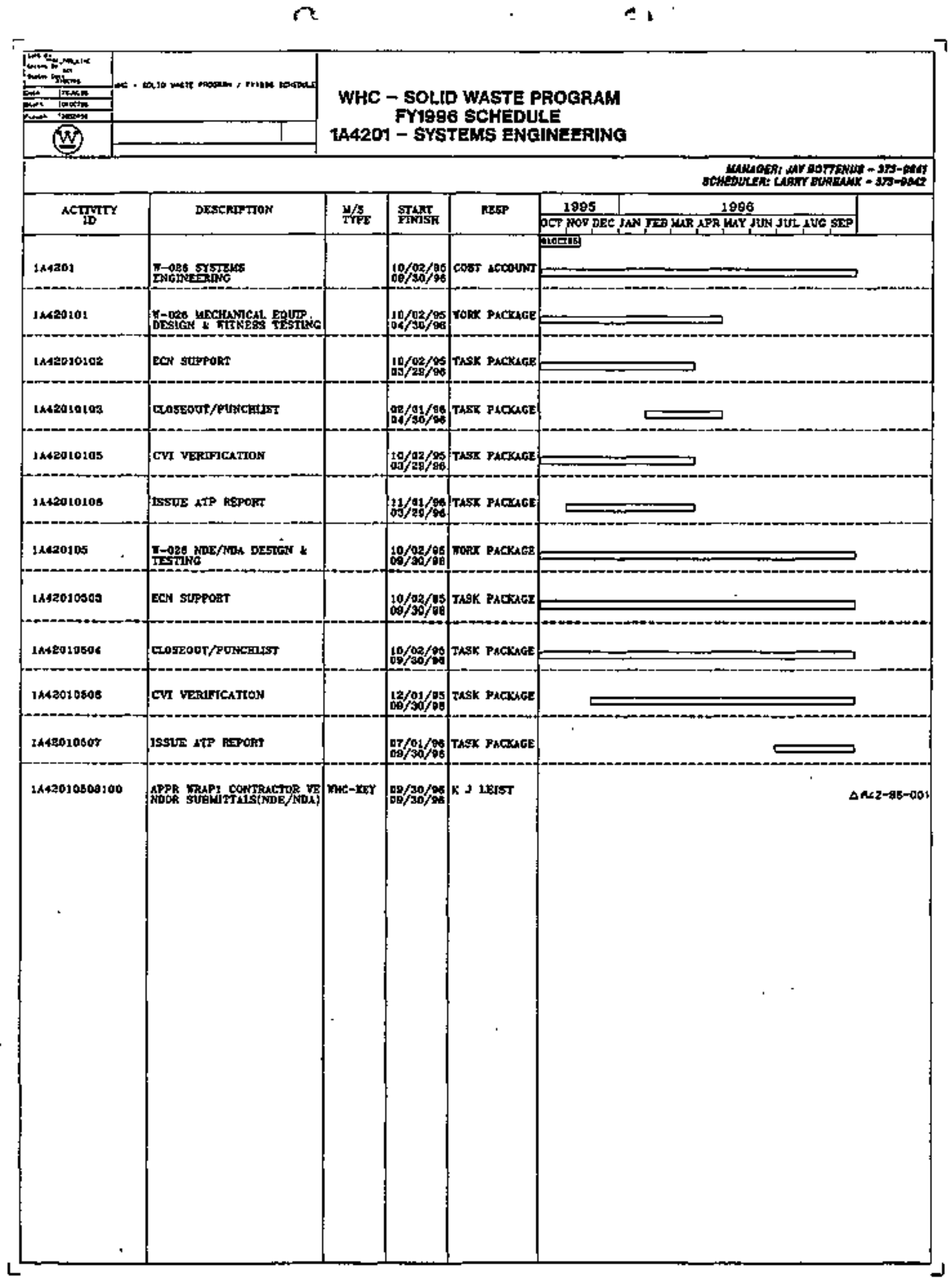


* 4 4.

WHC - SOLID WASTE PROERAM FY1996 SCHEDULE 1A4301 - PROJECT STARTUP

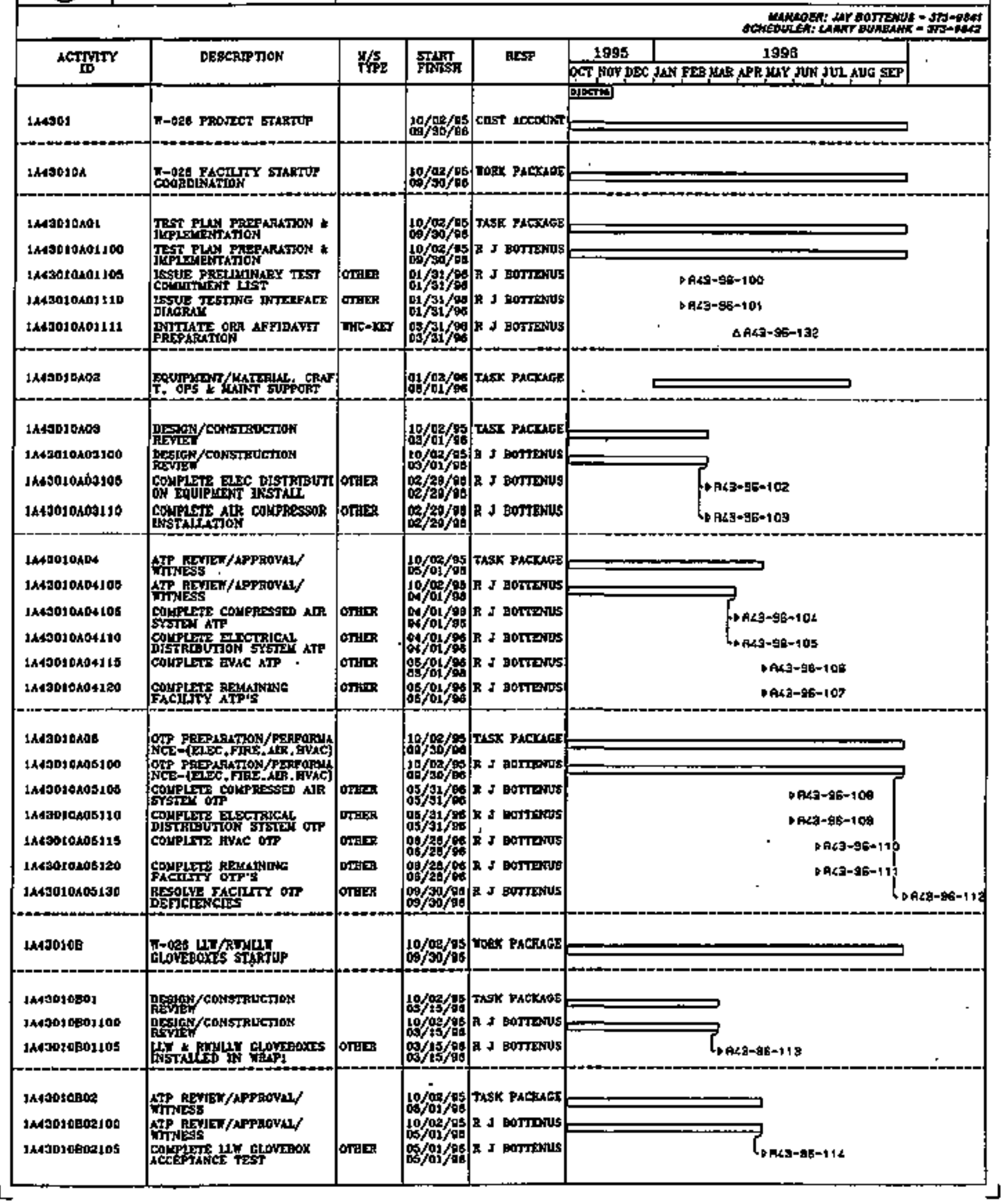


WHC - SOLID WASTE PROERAM FY1996 SCHEDULE $1 A 4301$ - PROJECT STARTUP

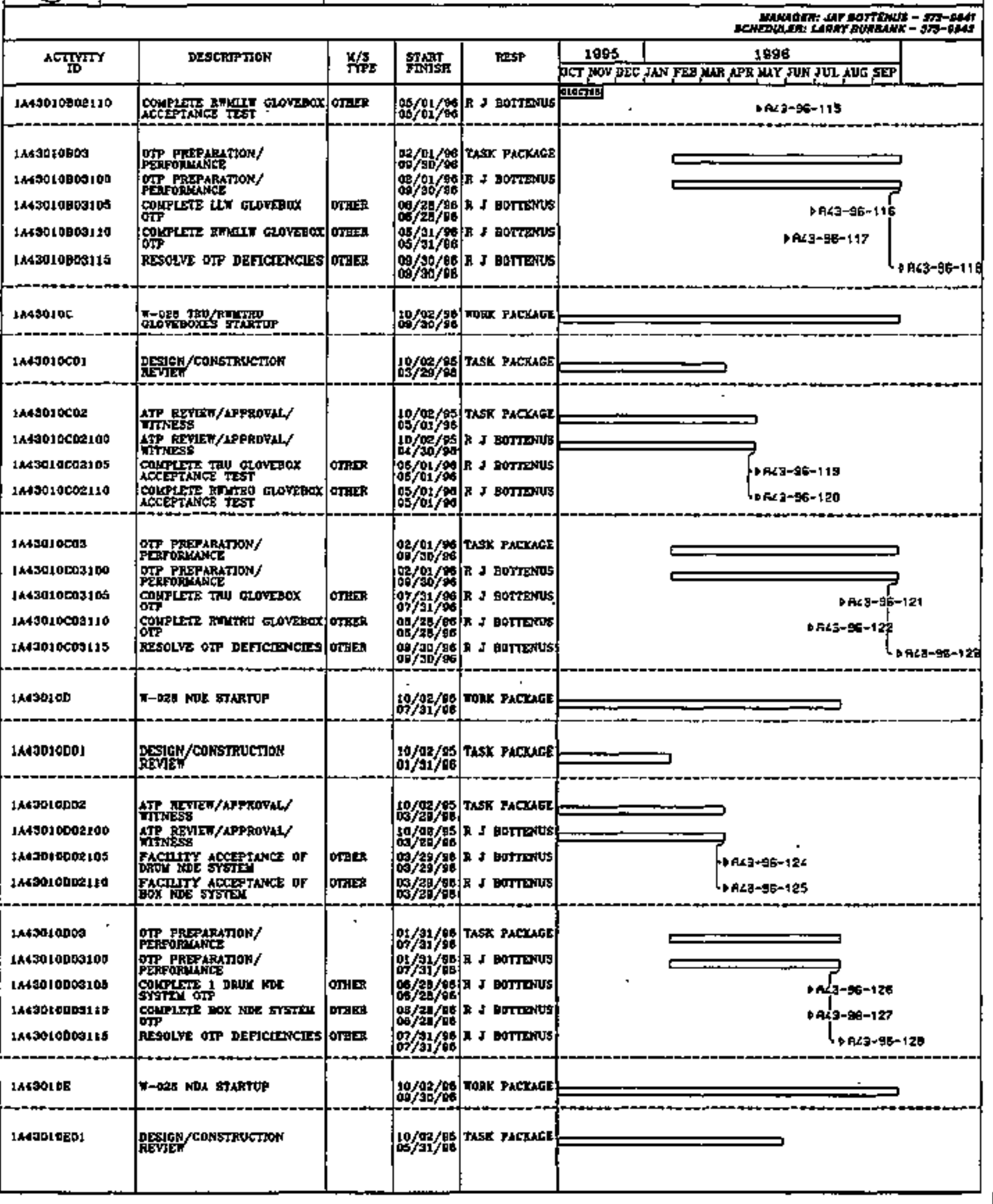




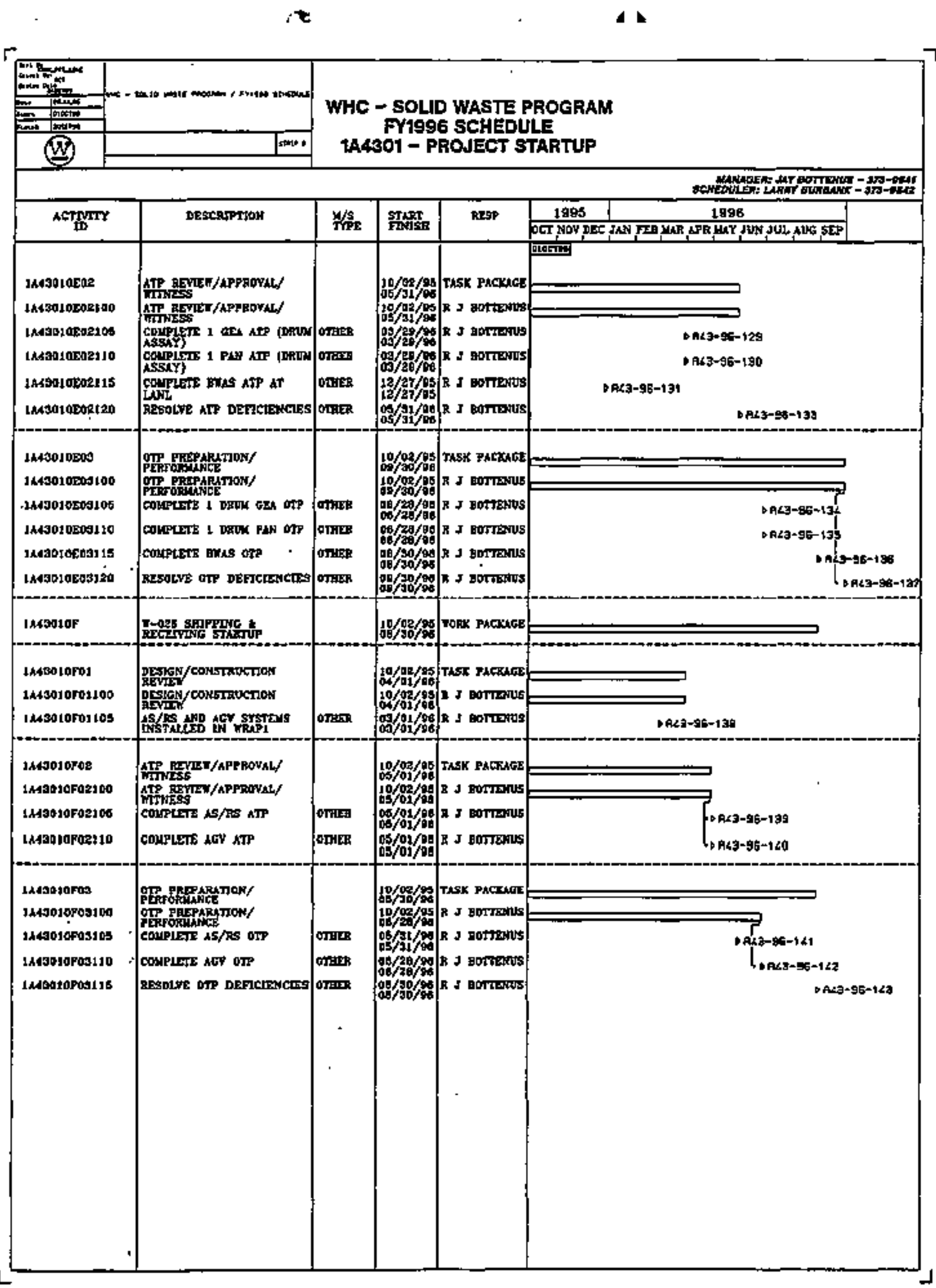


5

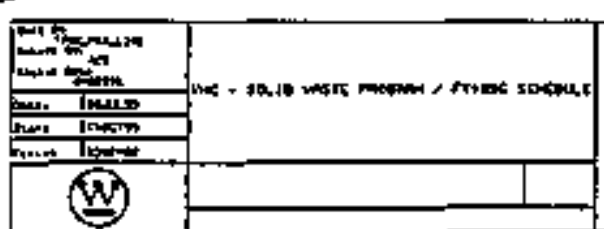

WHC - SOLID WASTE PROGRAM FY1996 SCHEDULE

1A4501 - DMS/PCS DEVEAOPNENT

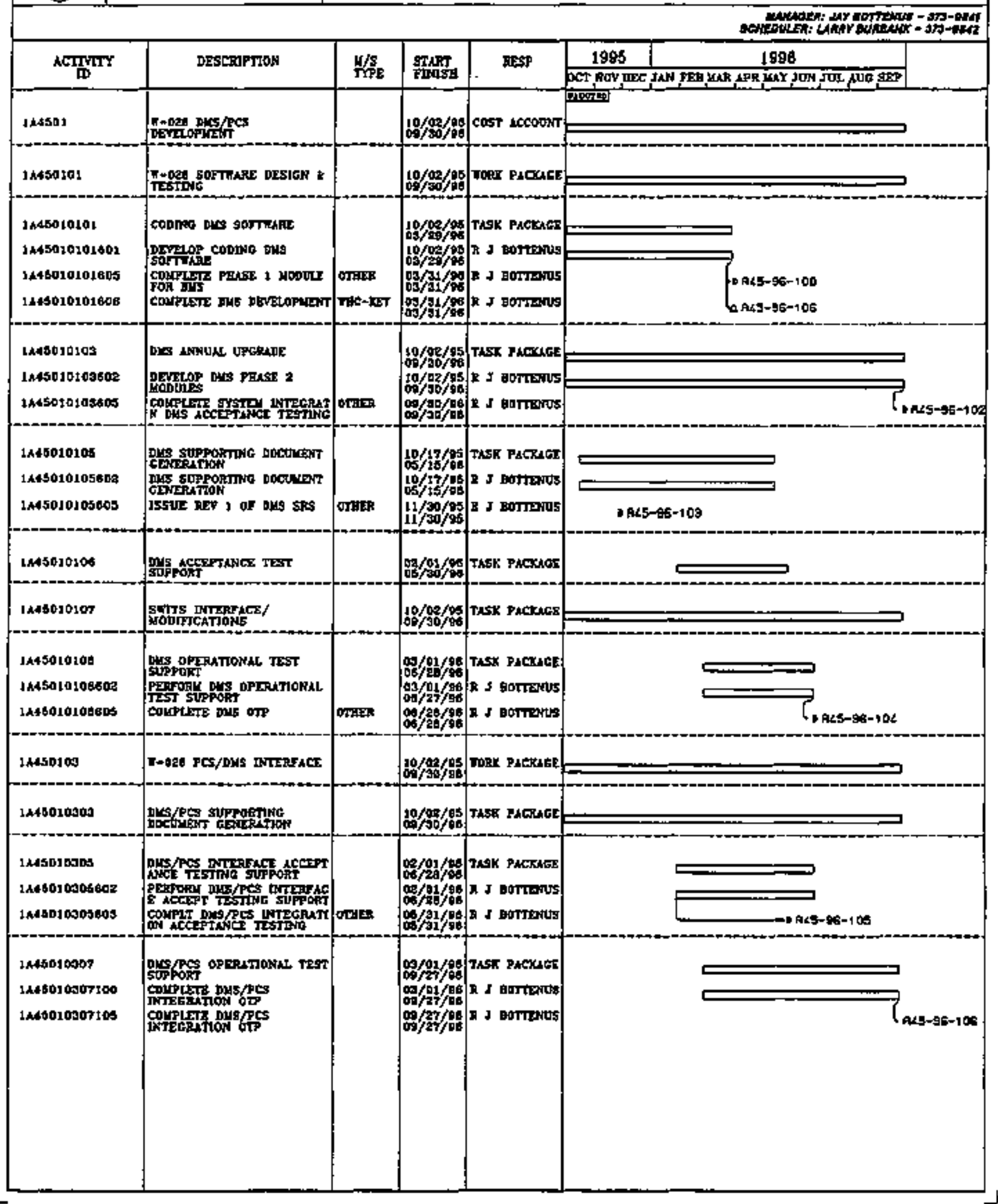


WHC - SOLID WASTE PROGRAM FY1996 SCHEDULE

$1 A 4601$ - ENVRON., SAFETY $\&$ OA

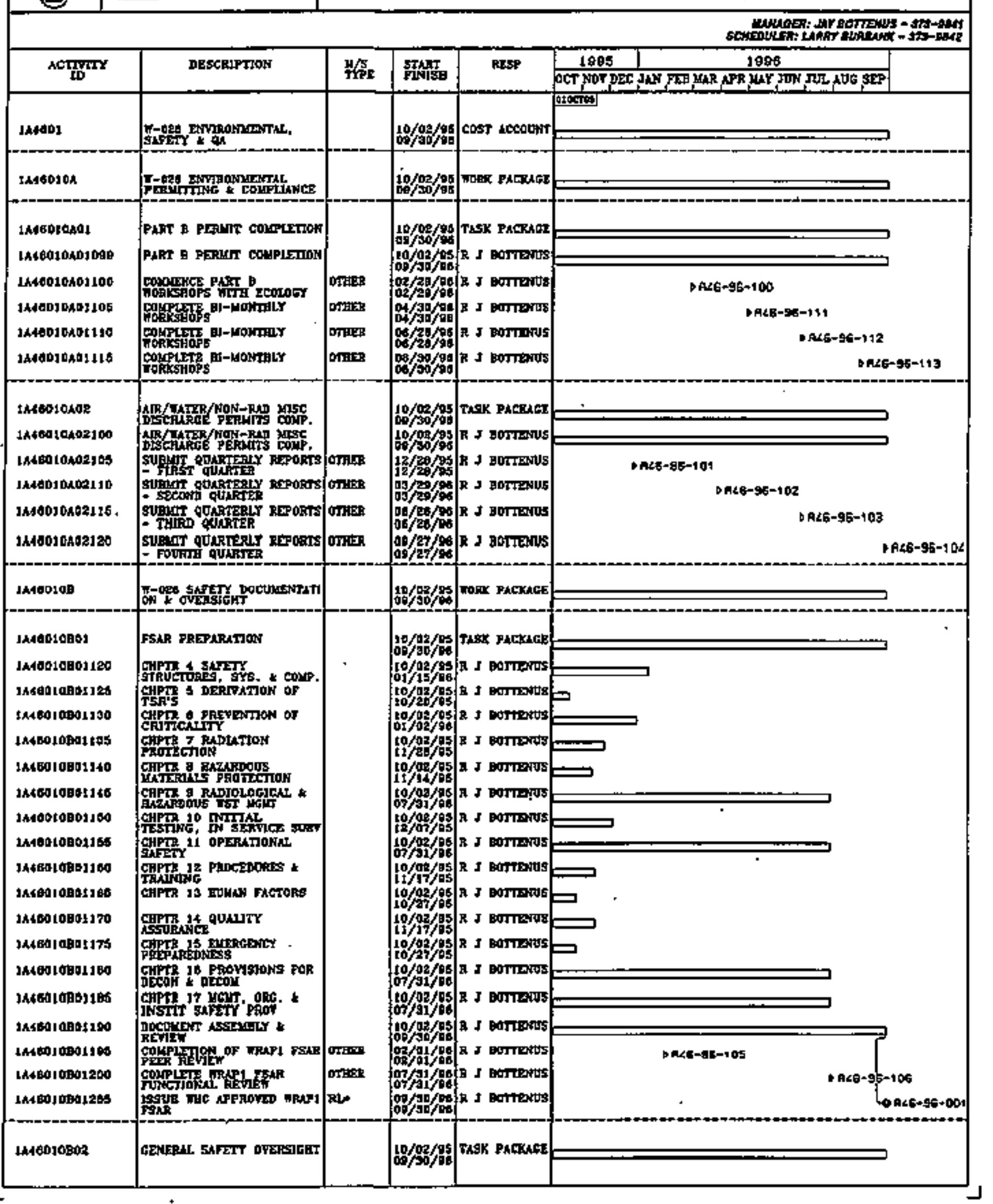




\section{WHC - SOLD WASTE PAOCRAM FY198B BCHILDULLE 1AMT20 - MANTANA WRAP CONOLANT FACILITY CONDITION8}

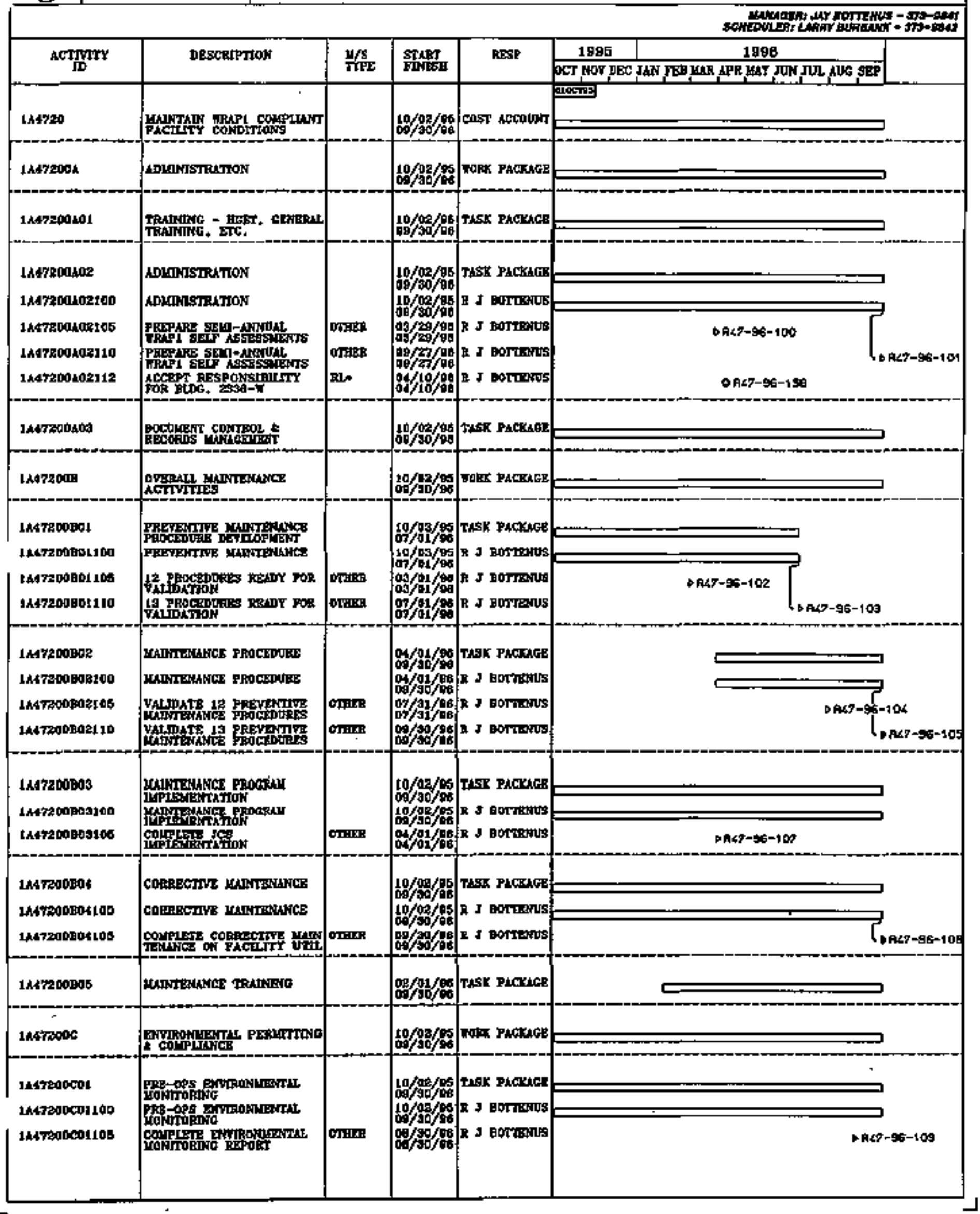




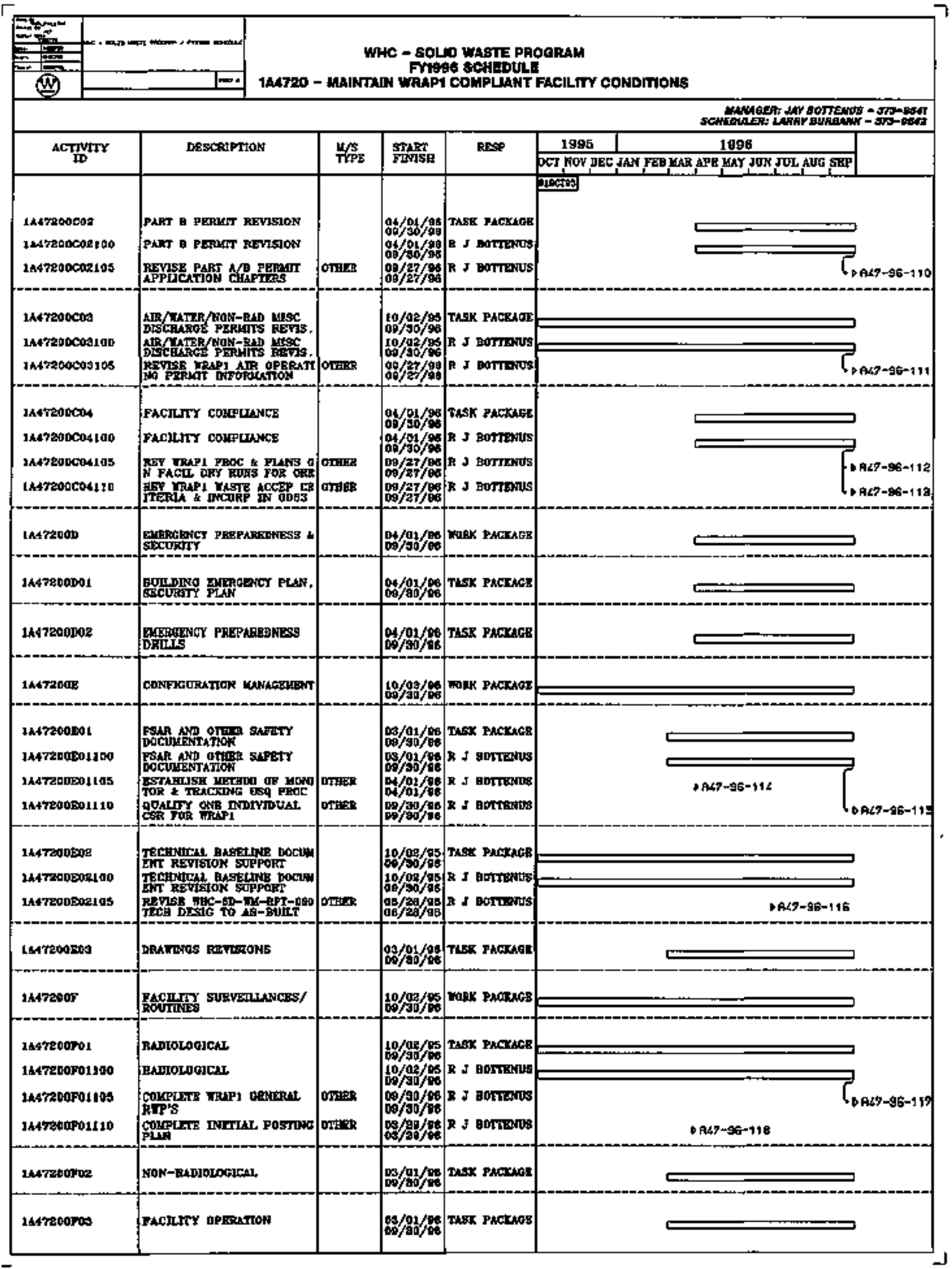


Fr1989 SOHFOULE 144720 - MADNTAN WRAPI COMPUANT FACILTY CONDMONS:

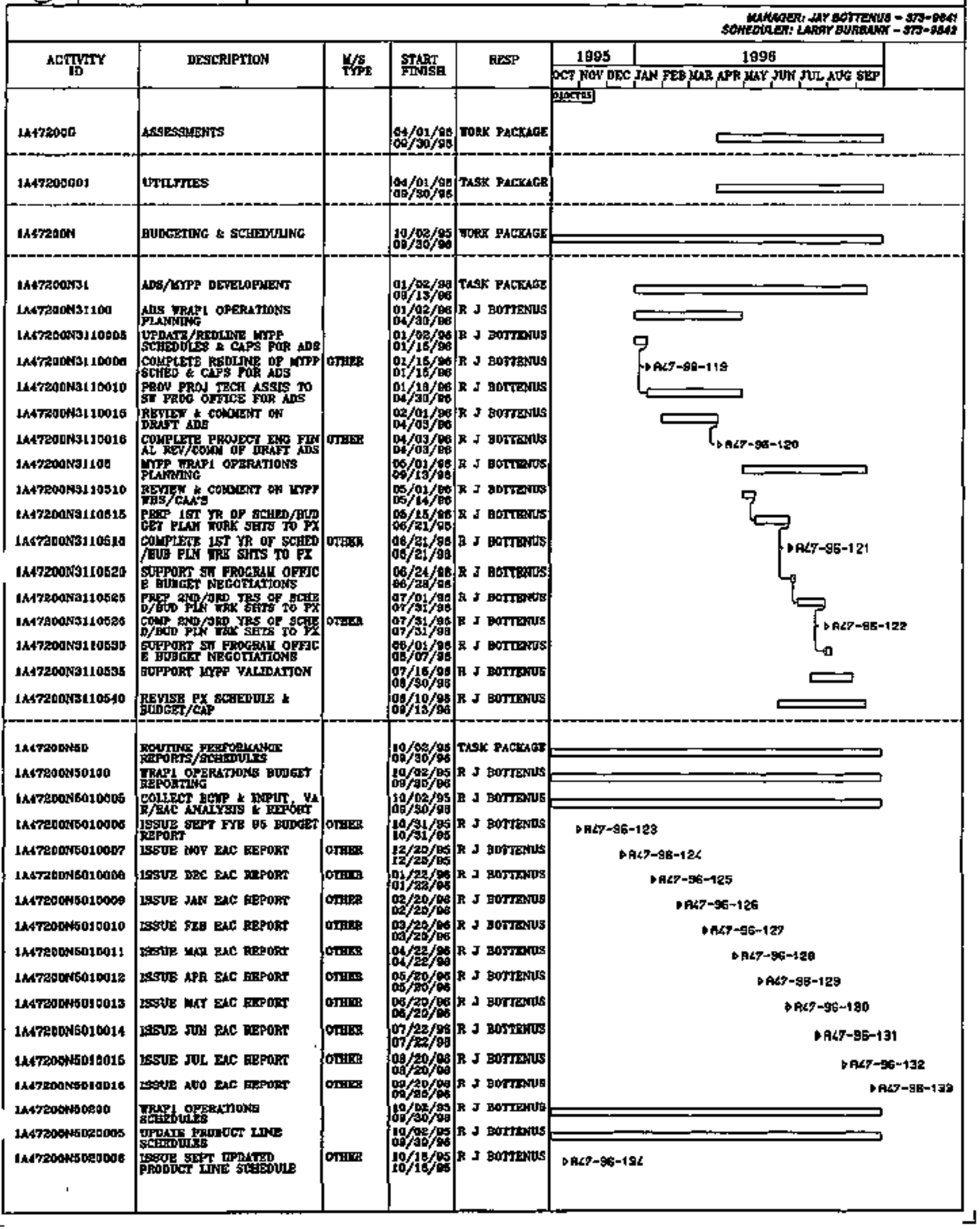




\begin{tabular}{|c|c|}
\hline ACTIVTY & DESCRIPJON \\
\hline IAd721 & Fin \\
\hline IAL72TOB & 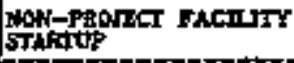 \\
\hline
\end{tabular}

InT2LOG01

L4425eFarjao

\section{4+3210日01206}

1447elattolsio

IN77zlahols15

FACIITY COKGWHAT

genrt rasts

CETTIFICATION SULPLAPRS

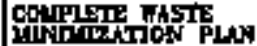

IA472107102.

IAsza10h0z100

* IMT73 minos105

Lx+72] 04102110

LA4721040115

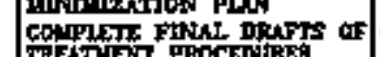

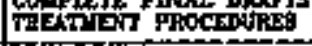

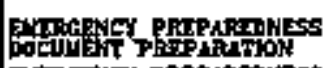

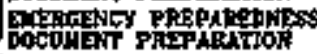

bocousit shipathiog

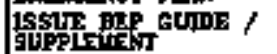

1Ss1te sectrity Ptin

(2)

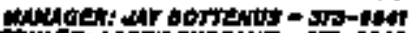
dentovis: LATr allis

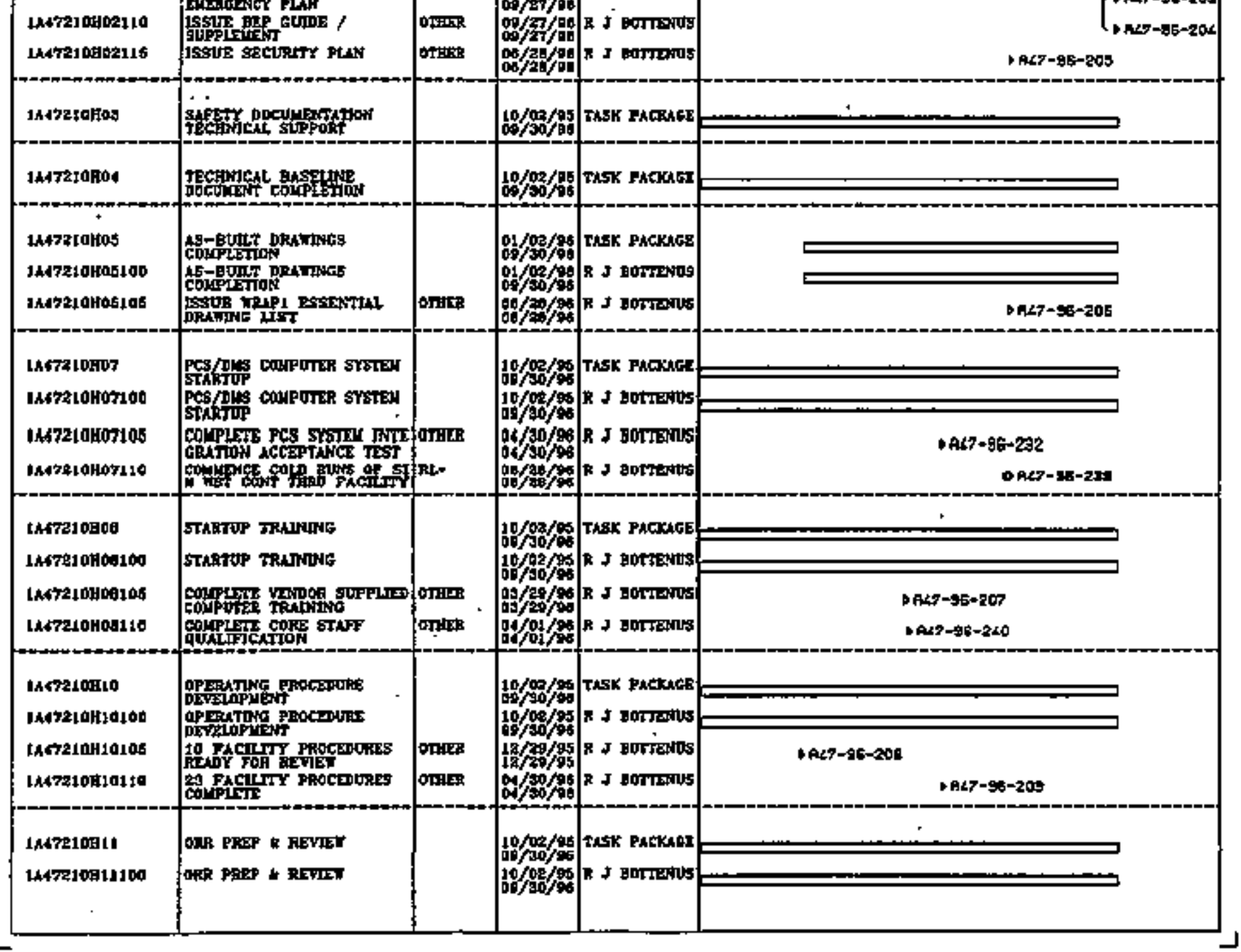


r $\quad$ a.

5

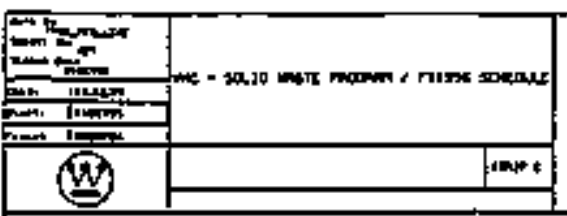
WHC - SOLD WASTE PROGRAM
FY199S SCHEDULE
1A4721 - WRAP1 NOH-PROJECT STARTUP

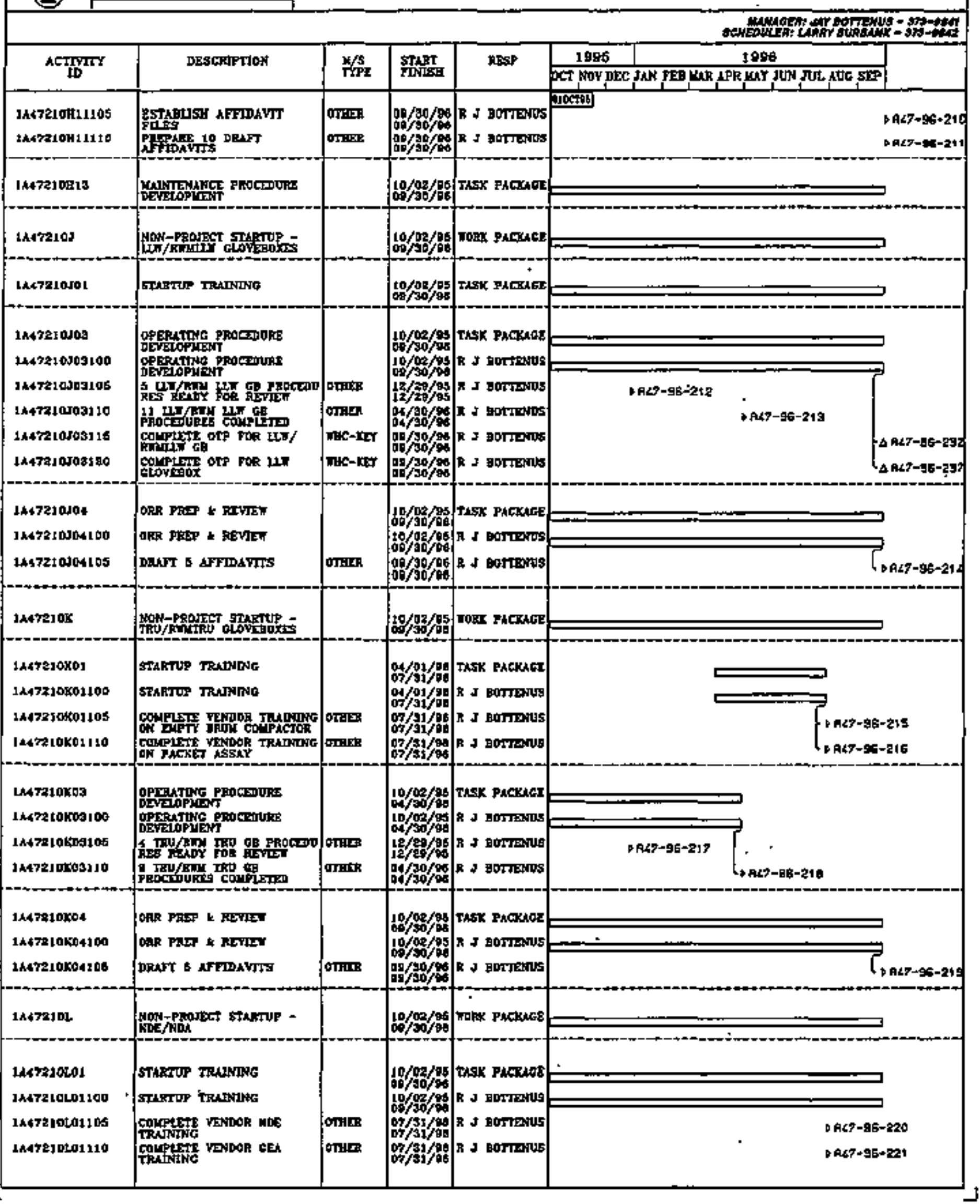




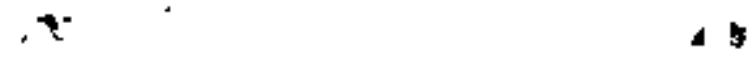

WHC - \$OLID WASTE PROGRAM FY1996 SCHEDULE \$A4721 - WRAPt NON-PROJECT STAFTUP

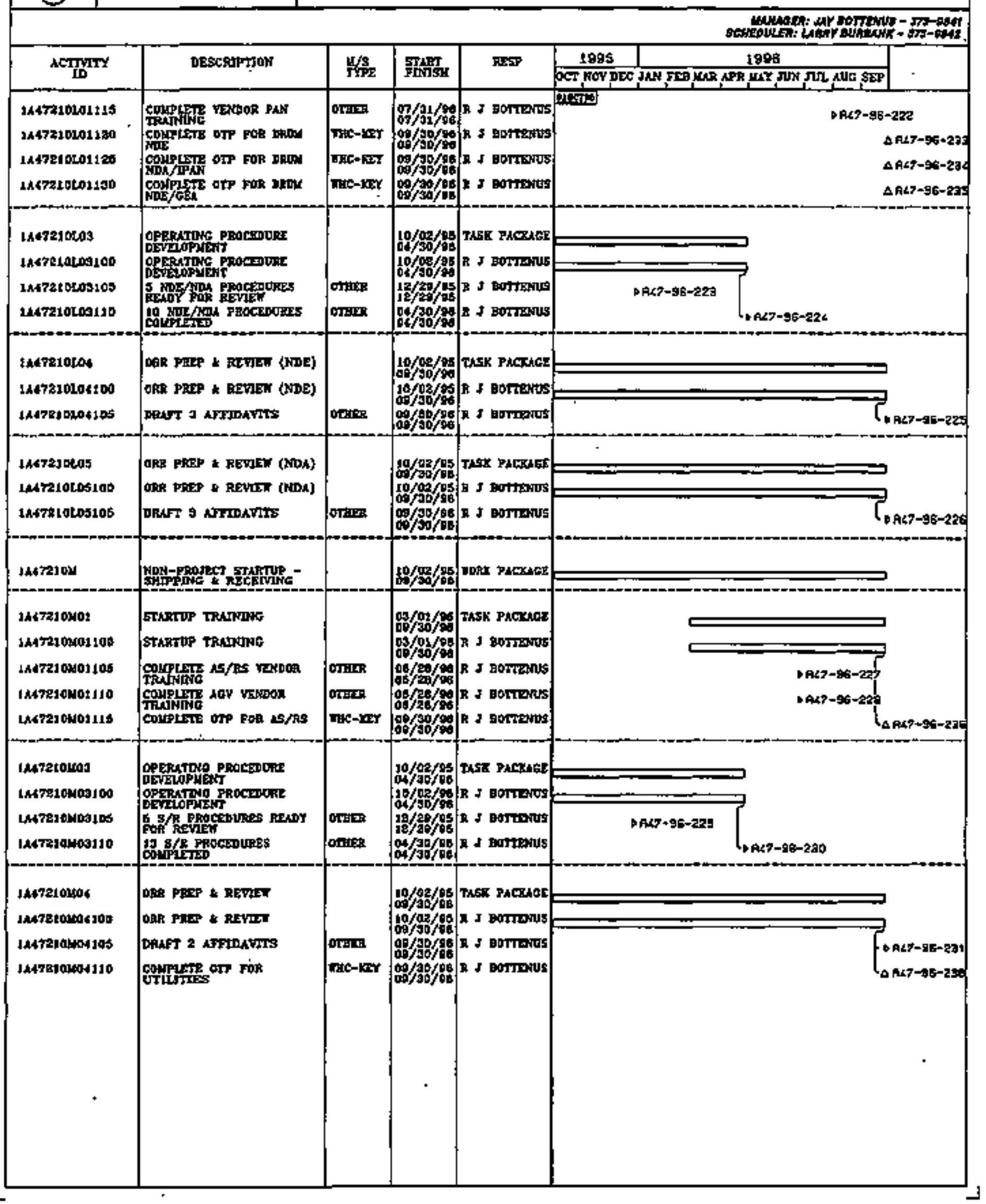


WHC - SOLID WASTE PROGRAM FY1996 SCHEDULE 144723 - WRAP1 NDE/NDA OPERATION

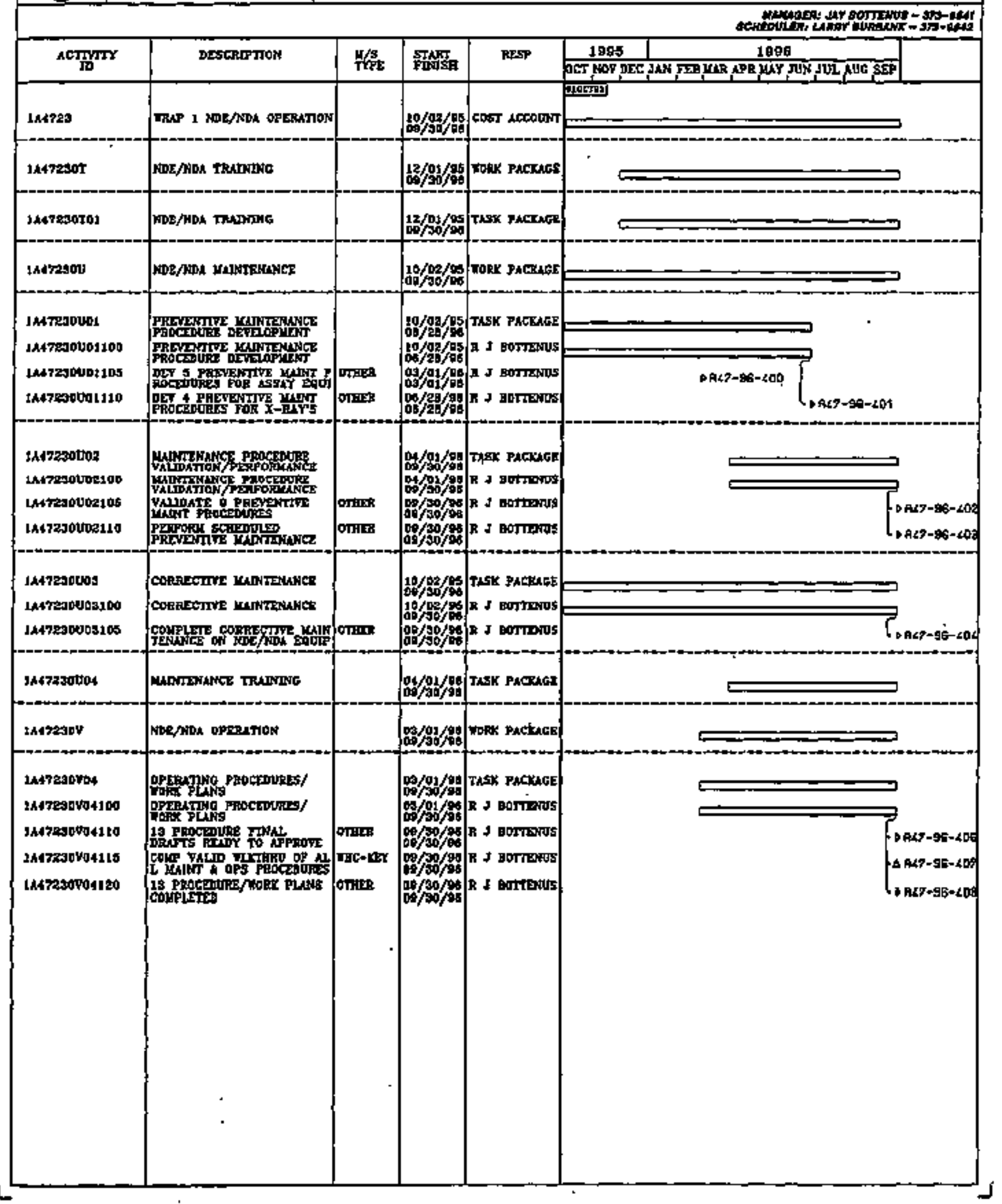




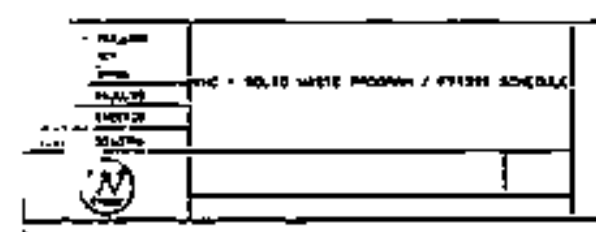

\section{WHC - SOLID WASTE PROGAAM FY1996 SCHEDULE $1 A 4724$ - WRAP1 SHIPPING \& RECENING}

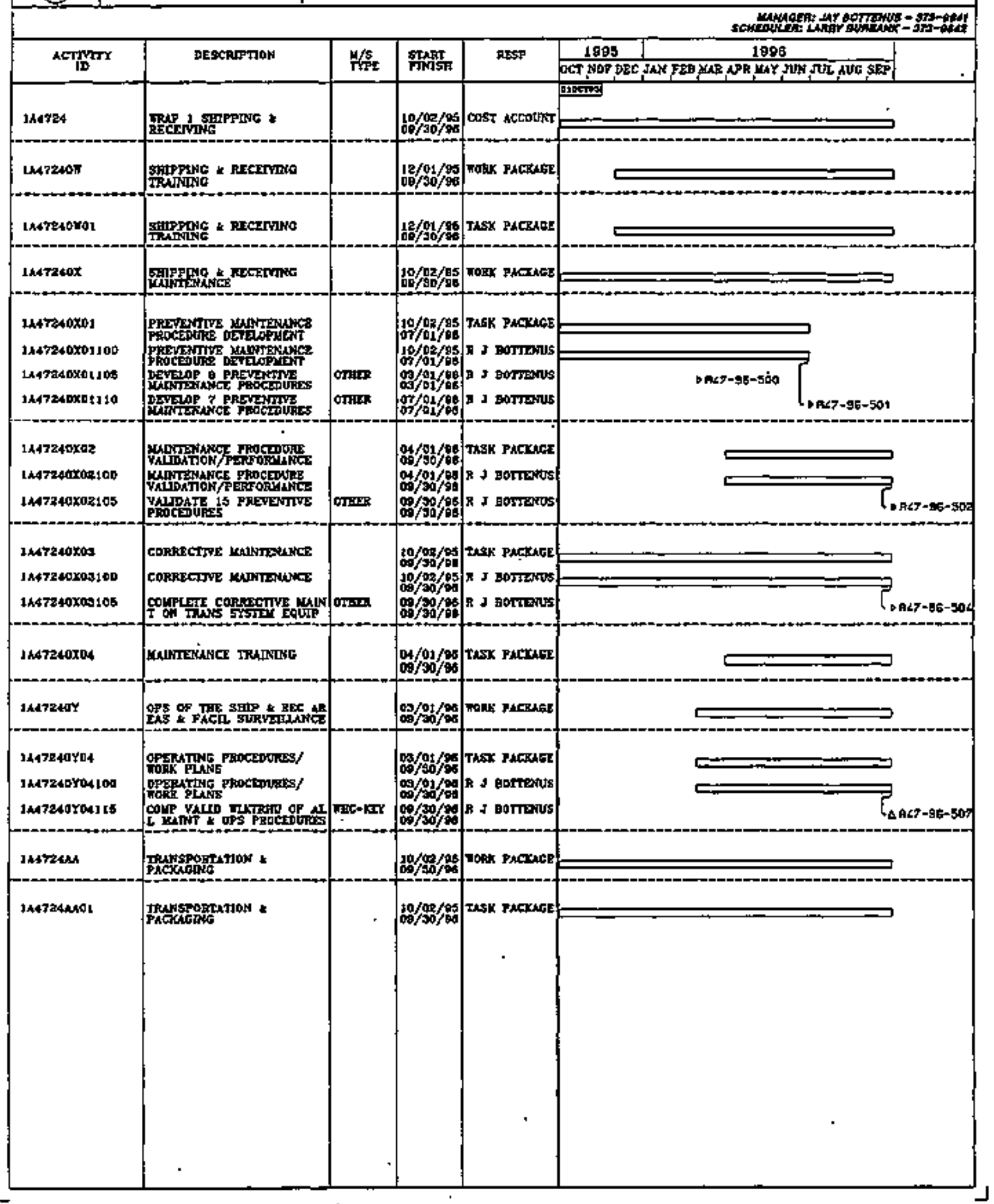




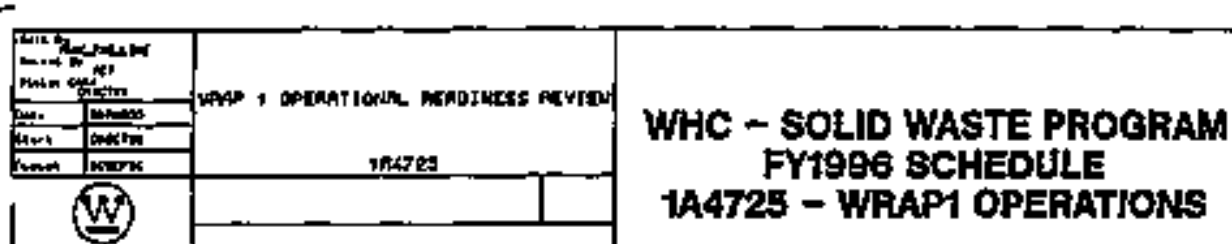

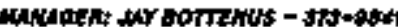

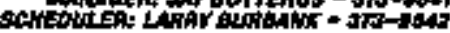

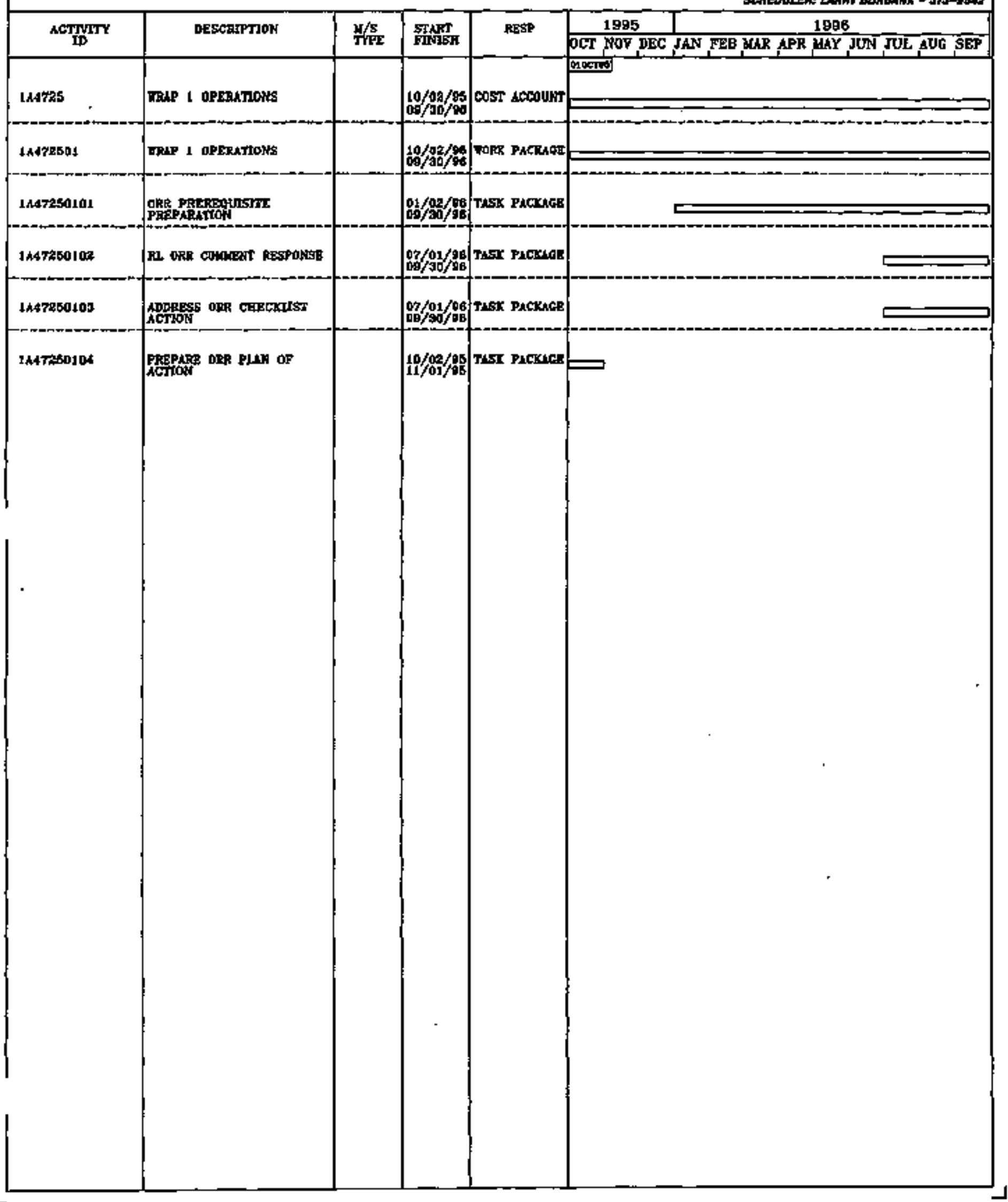




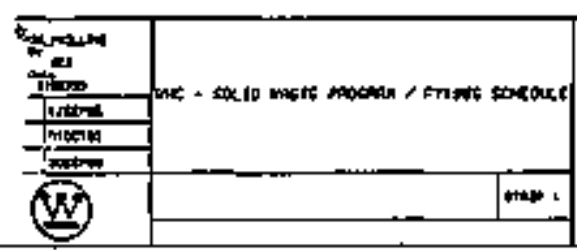

\section{WHC - SOLID WASTE PROGRAM FYI996 SCHEDULE 1AT101 - PRONECT INTEGRATION}

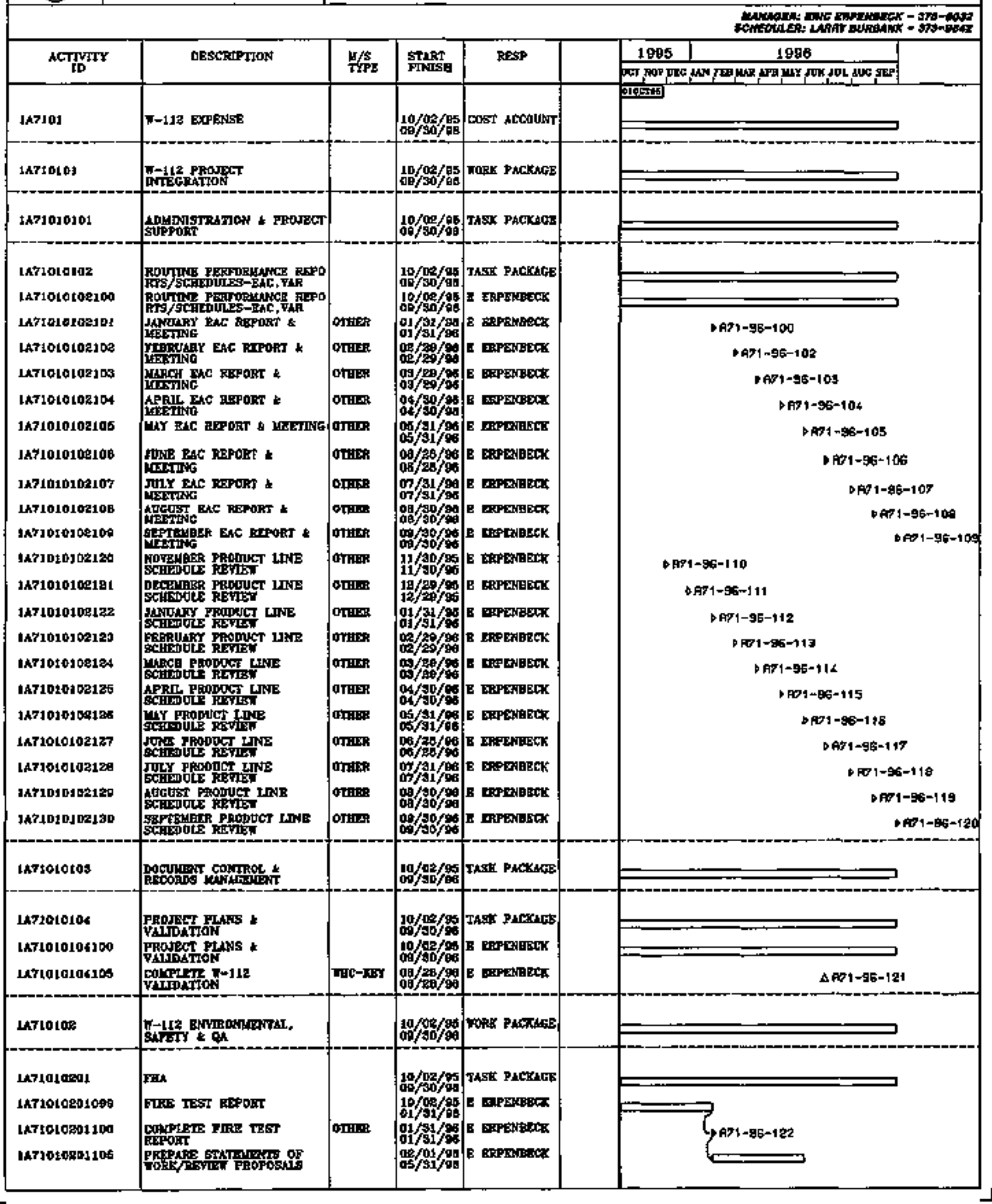




\section{WHC - SOLID WASTE PROQRAM FY1996 SCHEDULE 1A7101 - PROJECT INTECRATION}

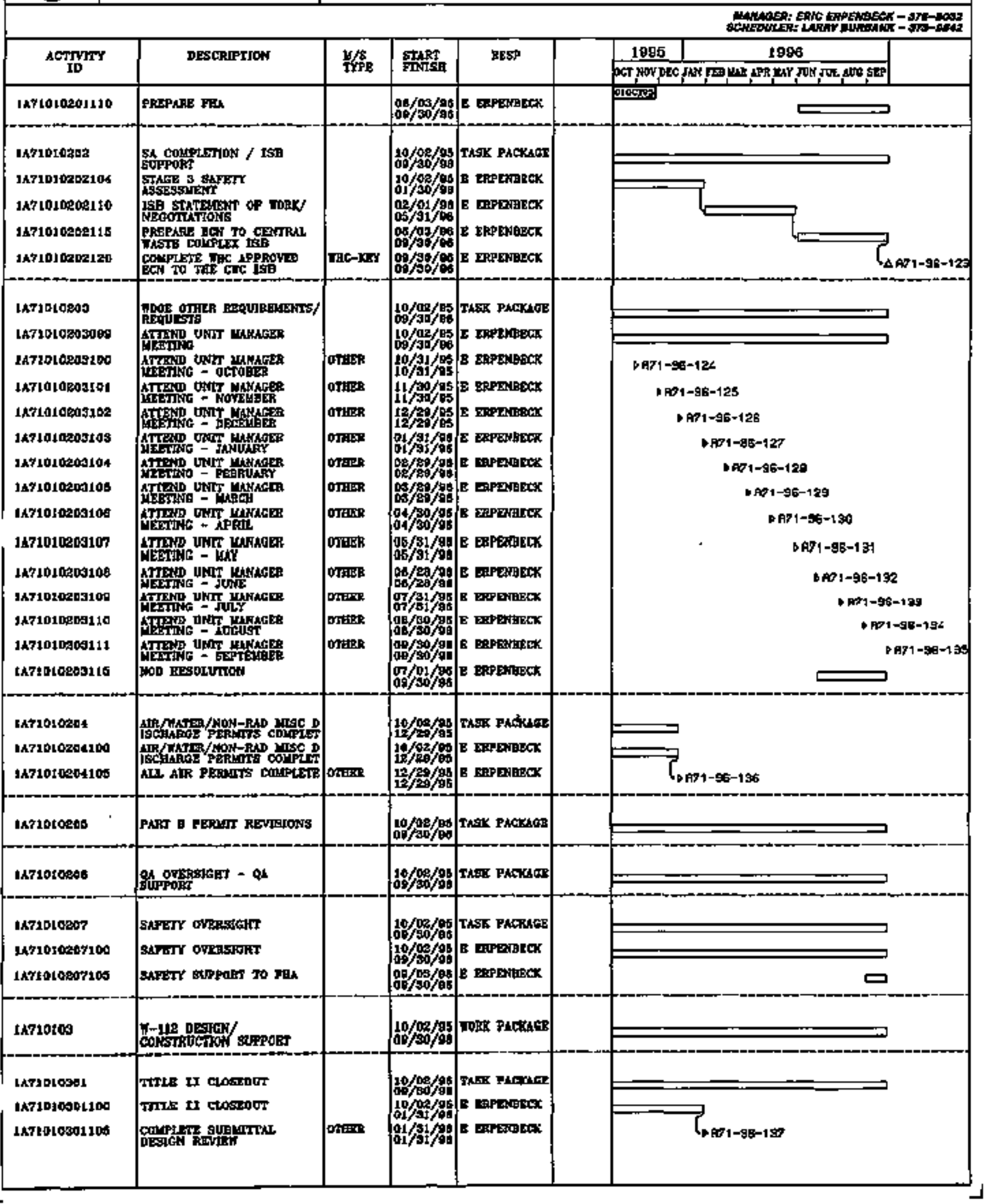




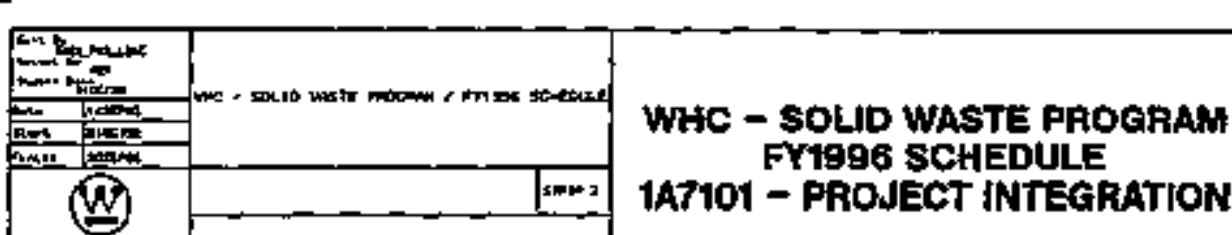

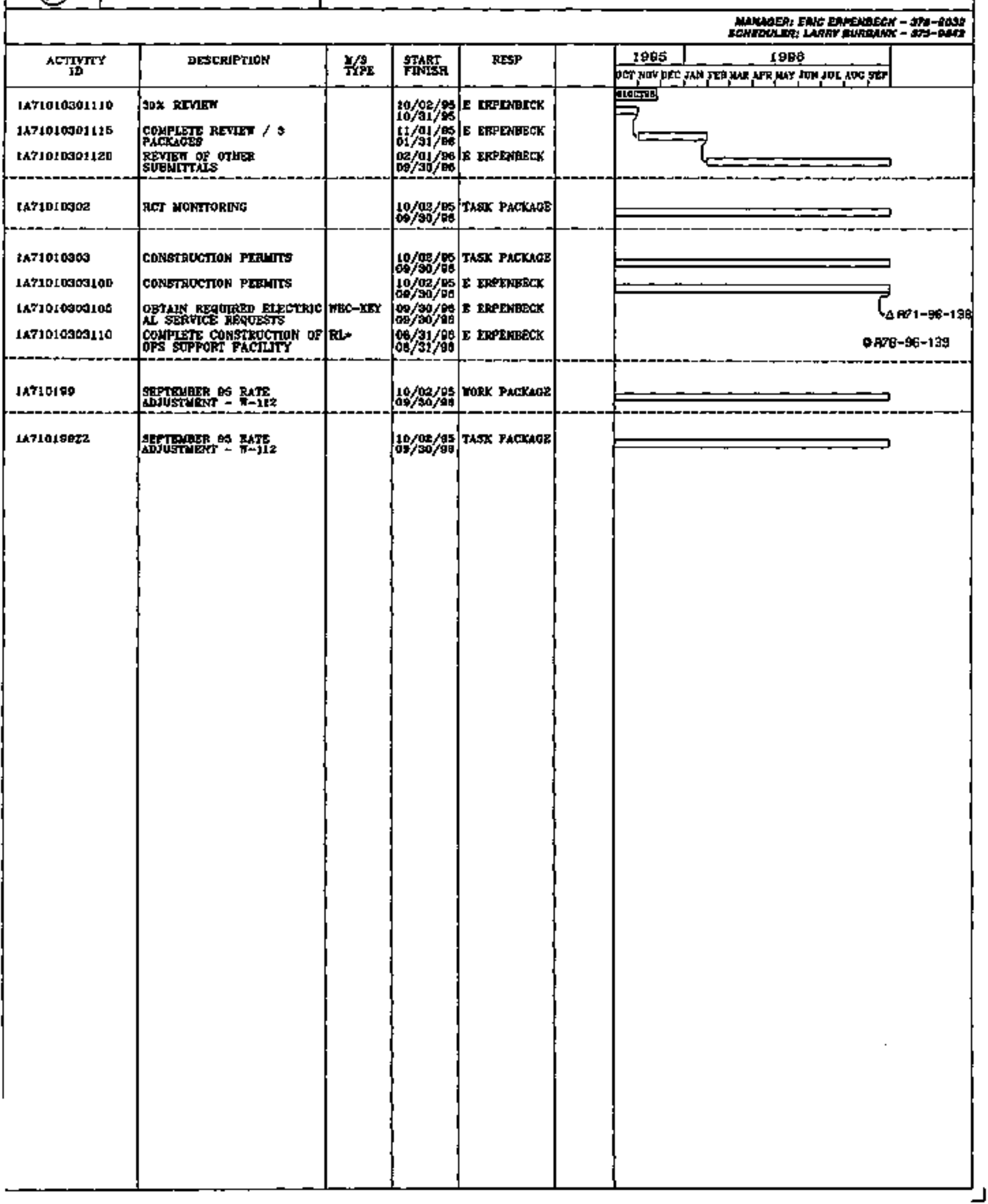


WHC - SOLID WASTE PROGRAM

FY1996 8CHEDULE

1A7301 - PROJECT STARTUP

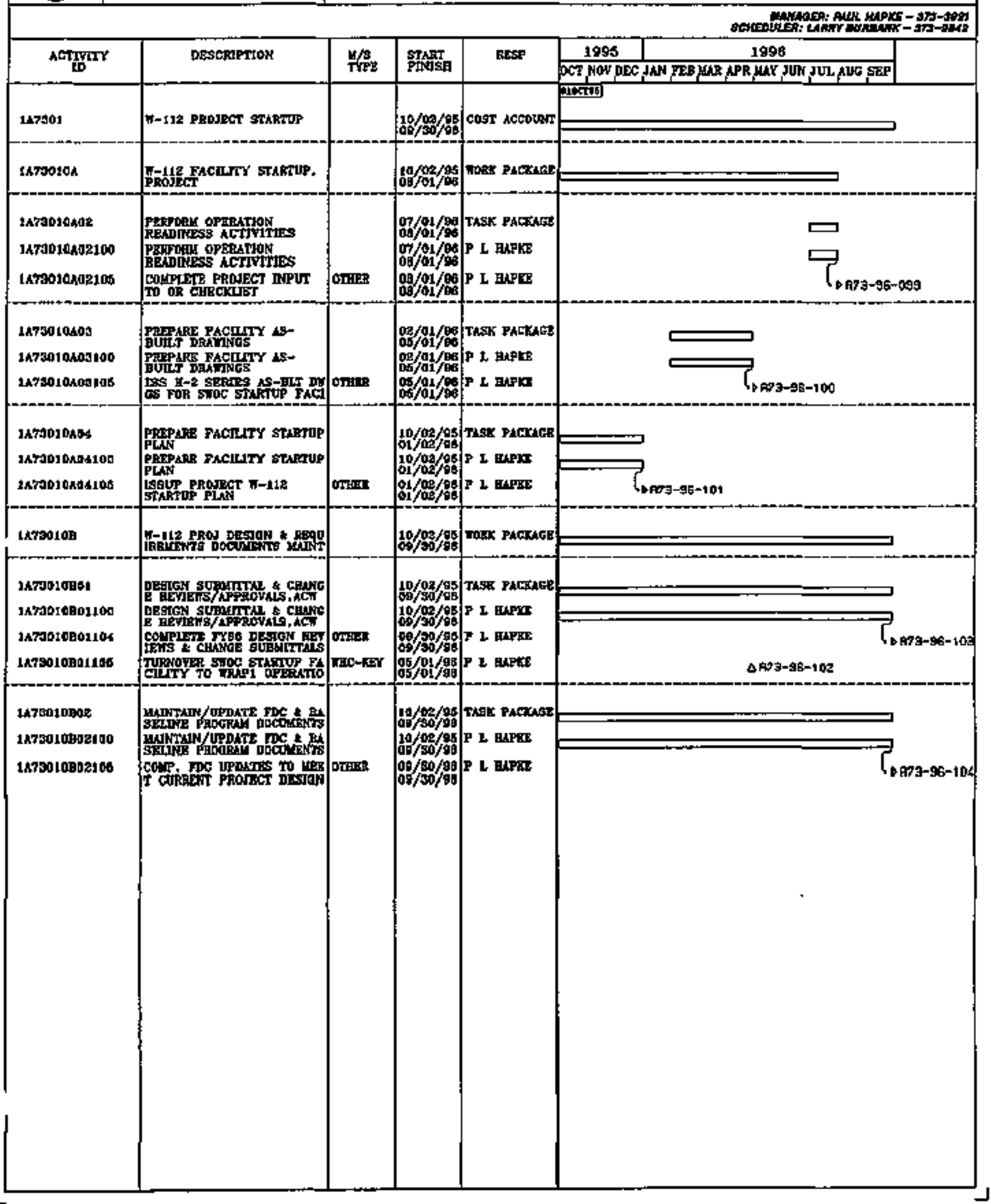




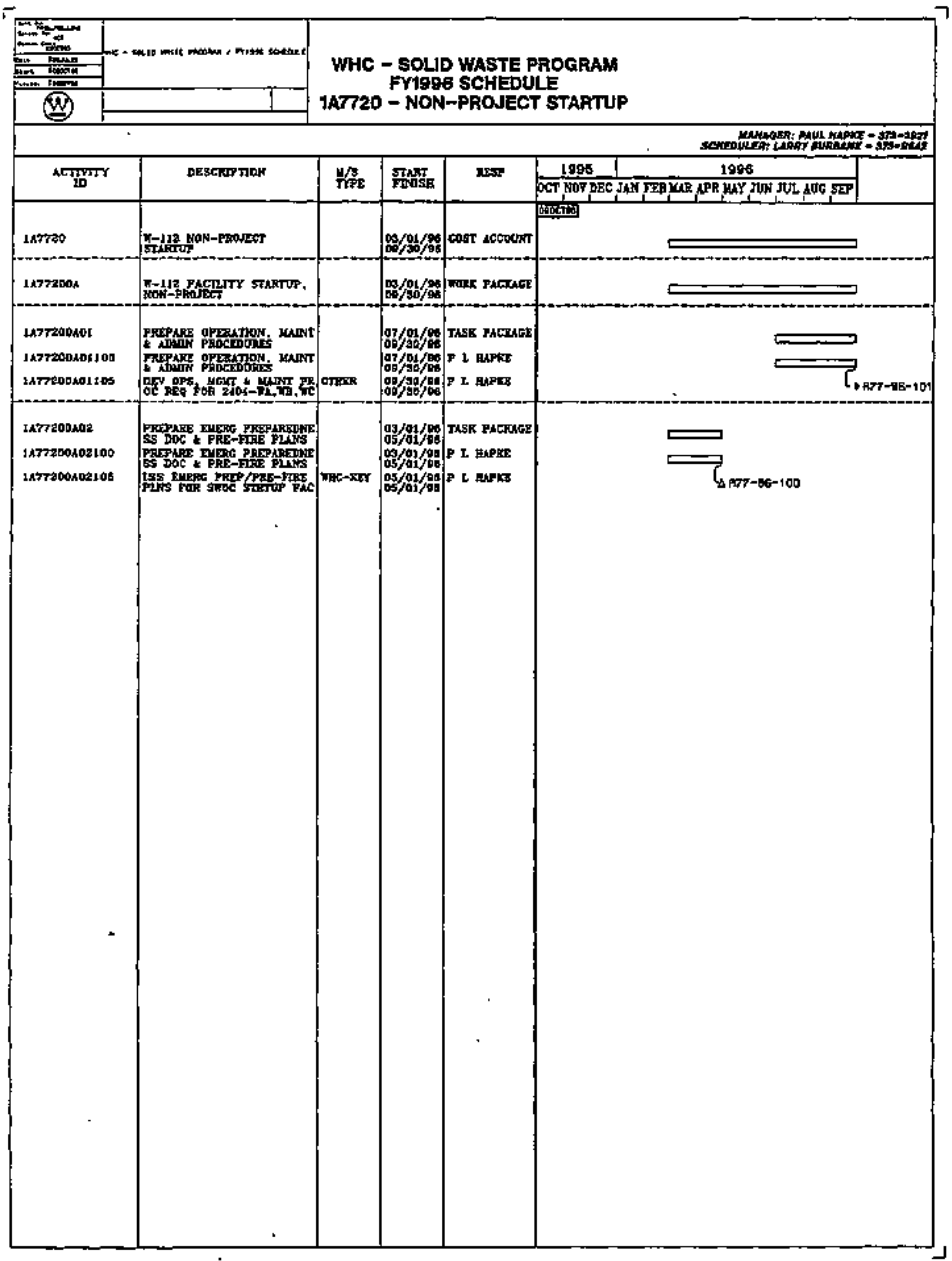




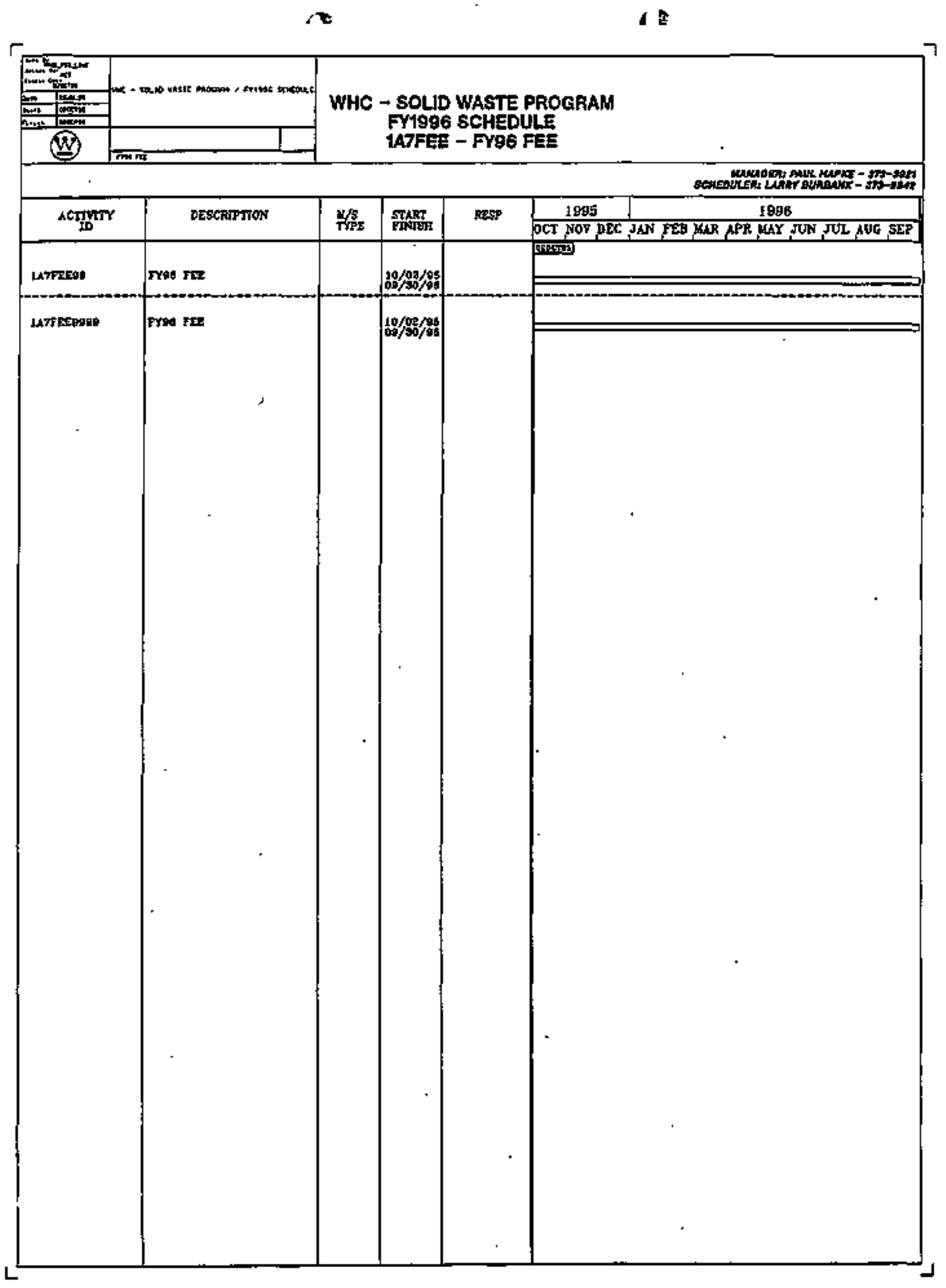




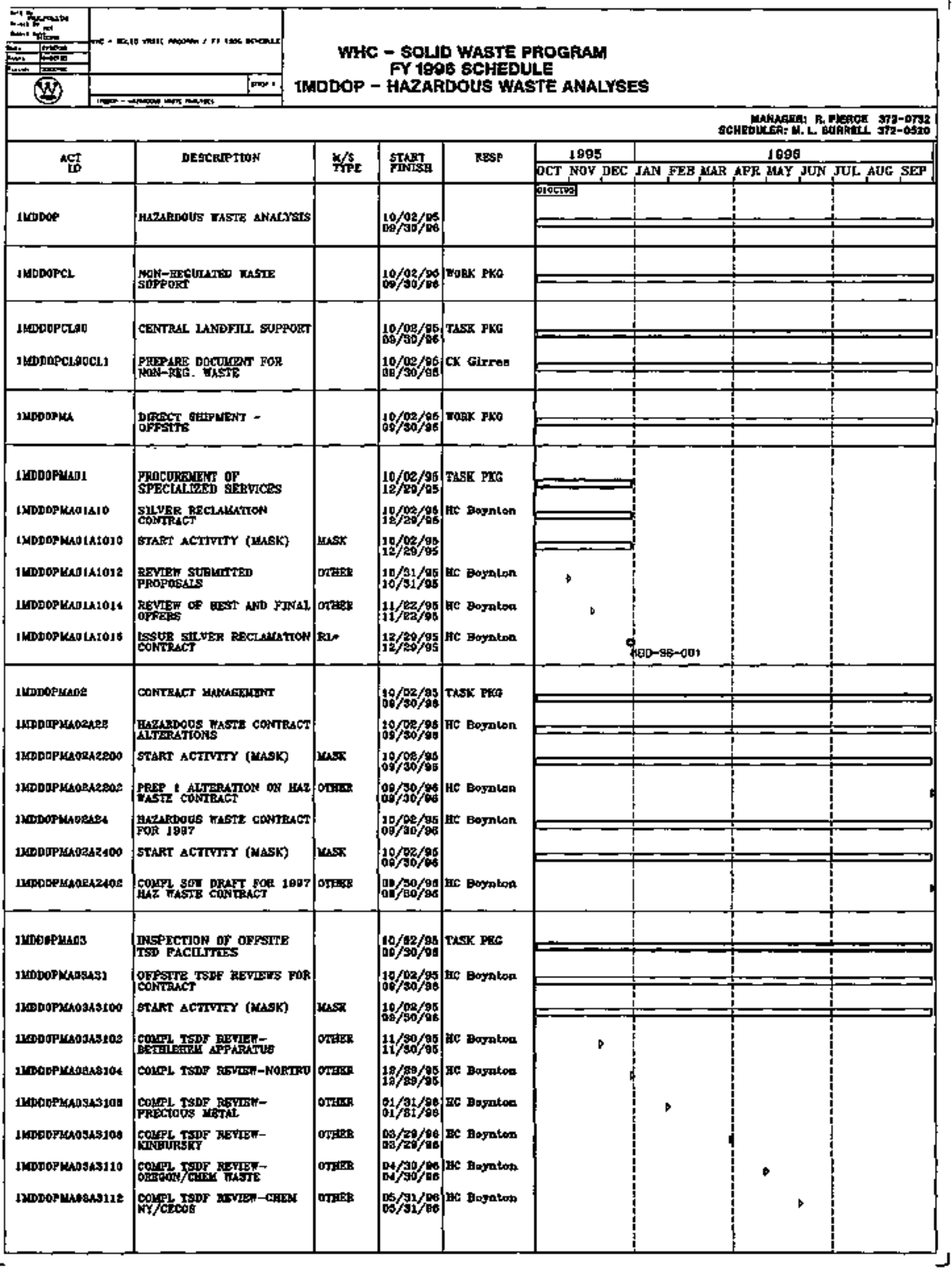


WHC - SOLID WASTE PROQRAM

FY 1998 SCHEDULE 1MDDOP - HAZAFDOUS WASTE ANALYSES

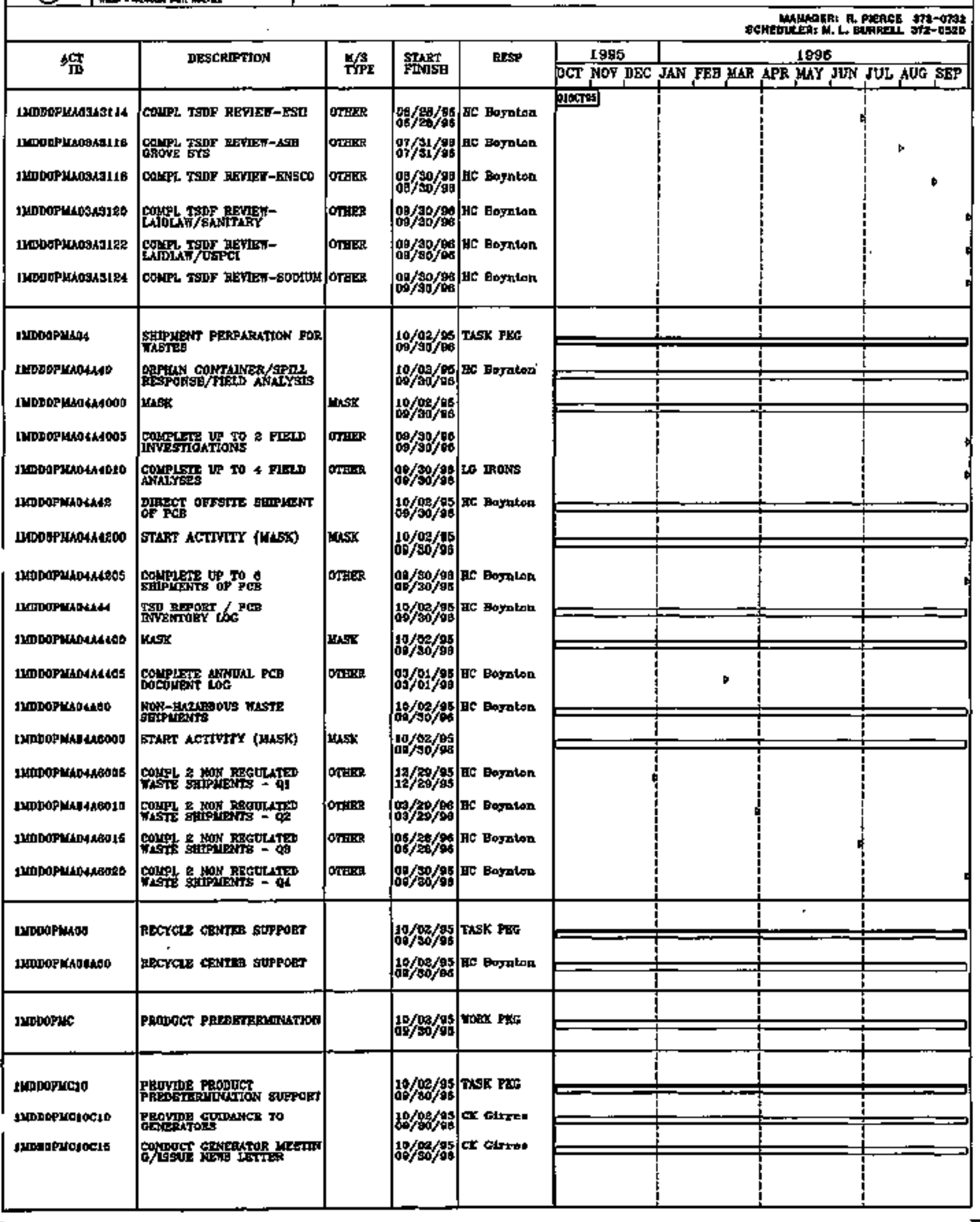




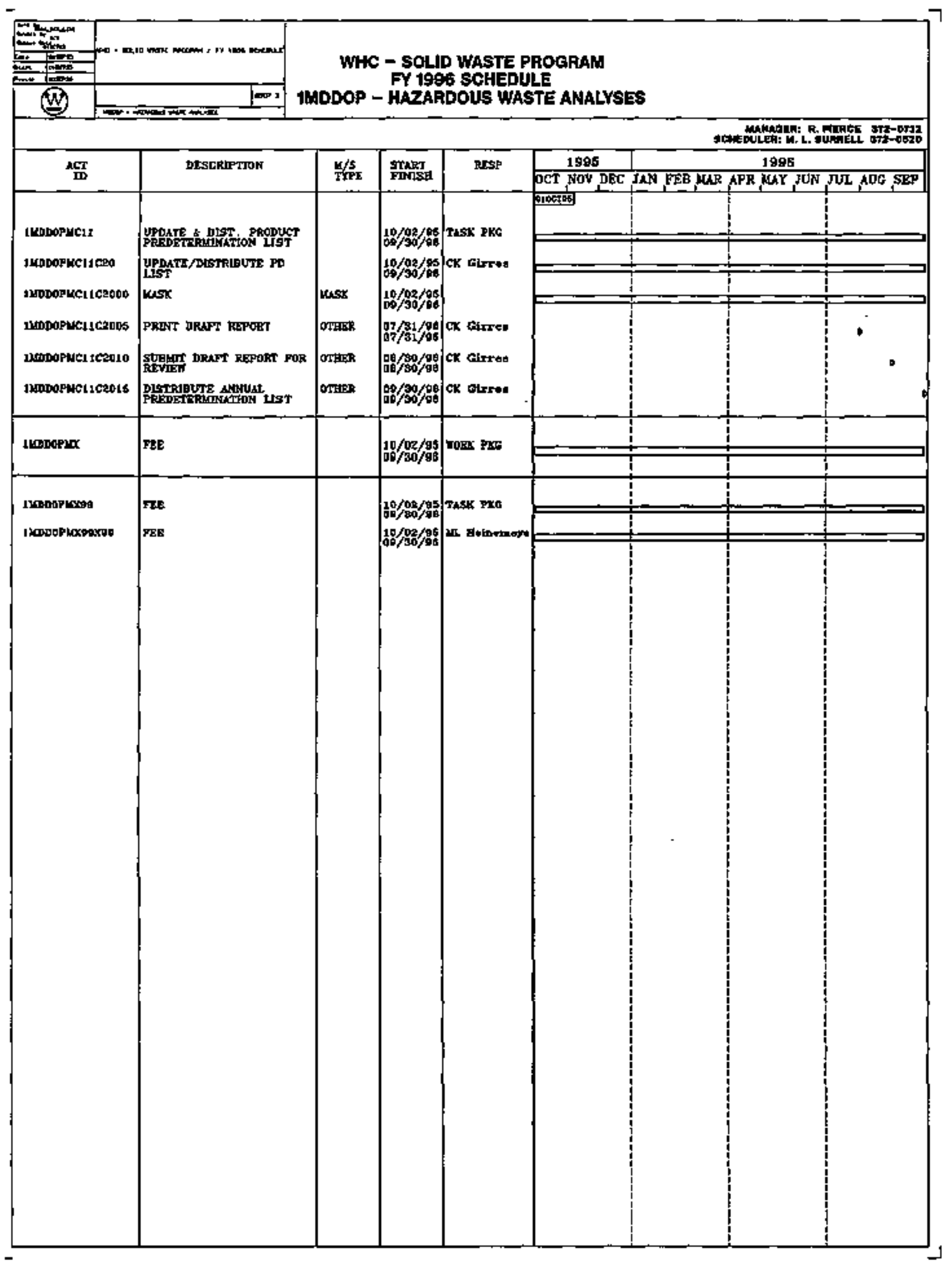




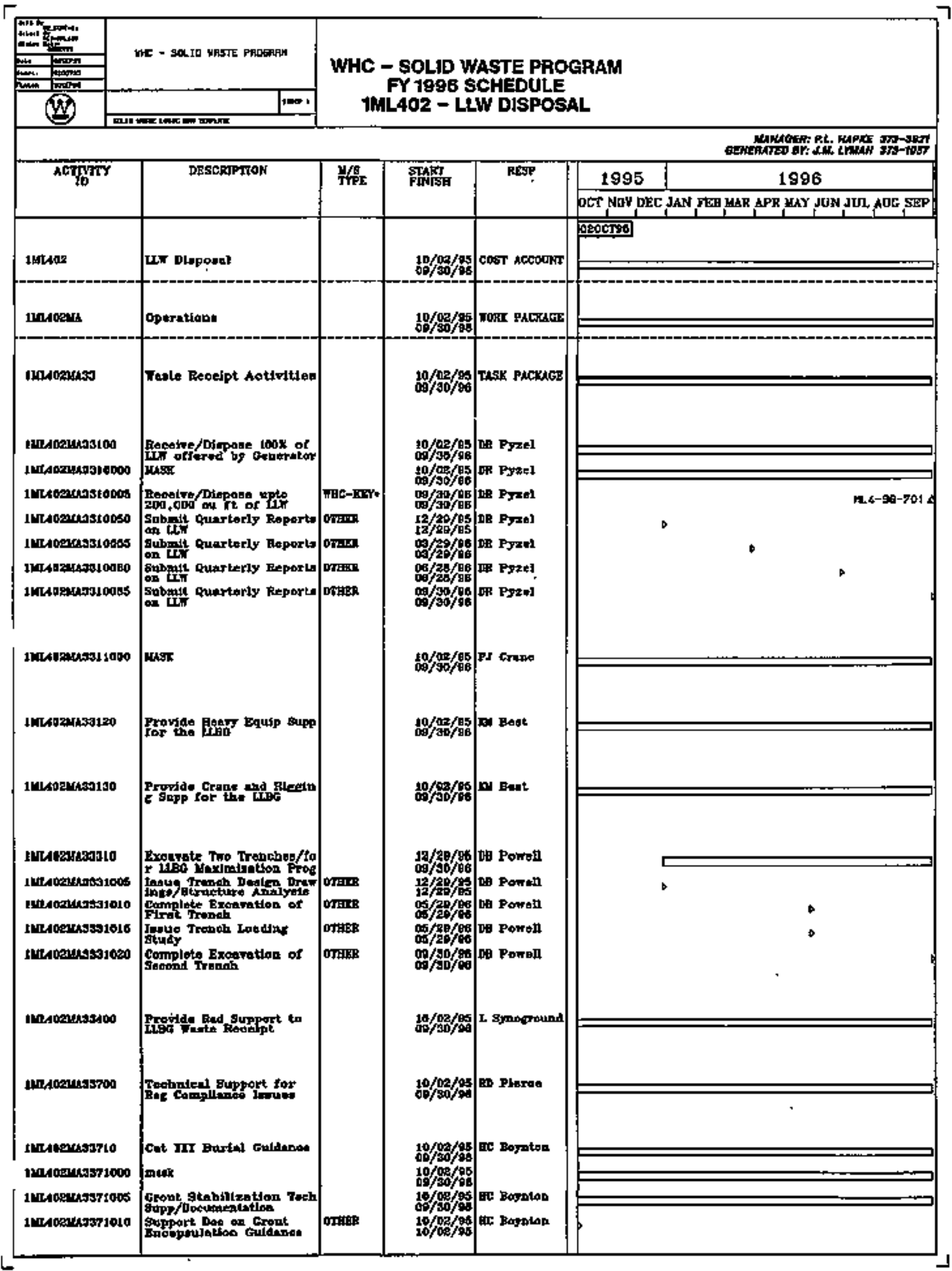




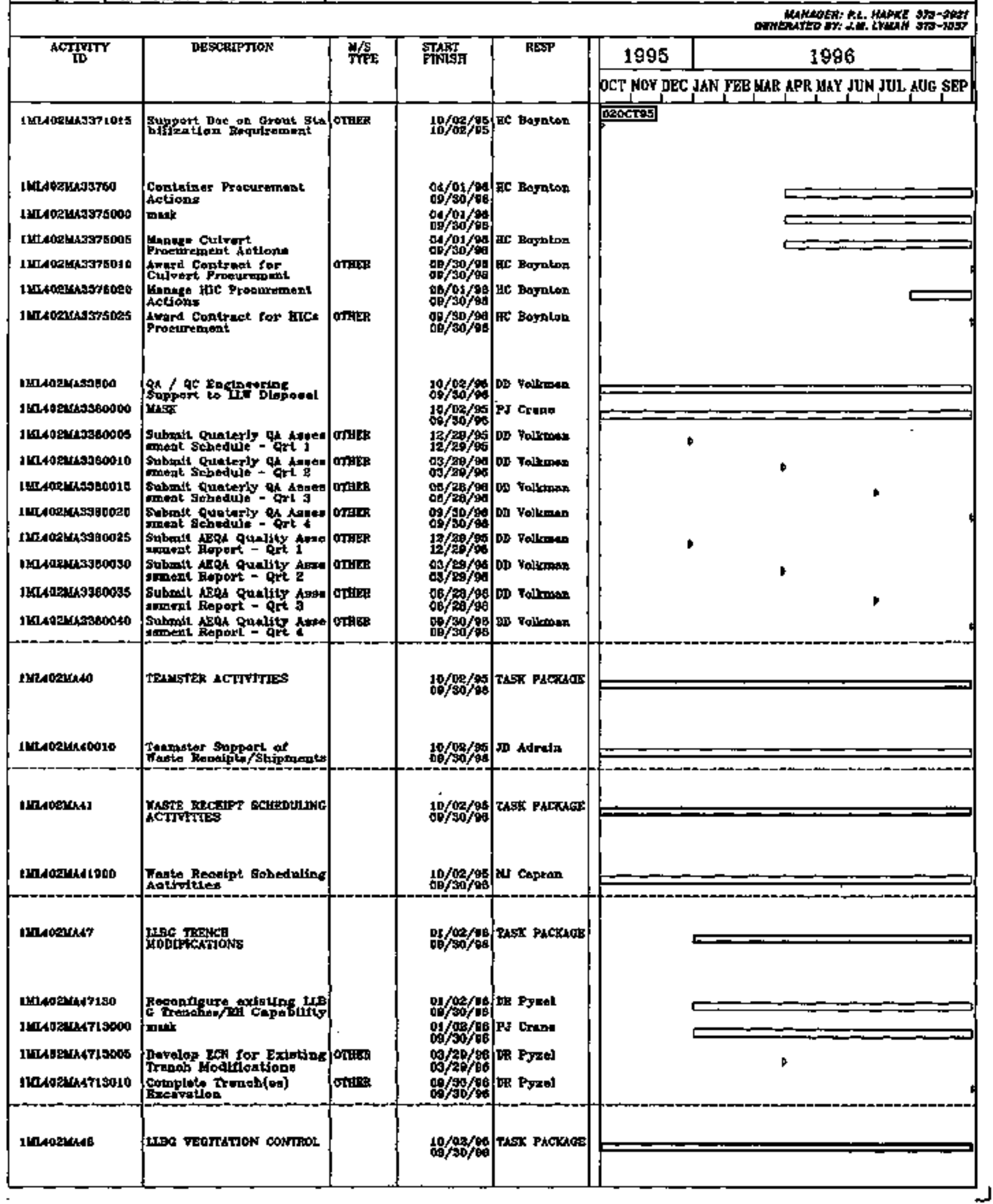


WHC - SOLID WASTE PROGRAM FY 1996 SCHEDULE 1ML402 - LLW DISPOSAL

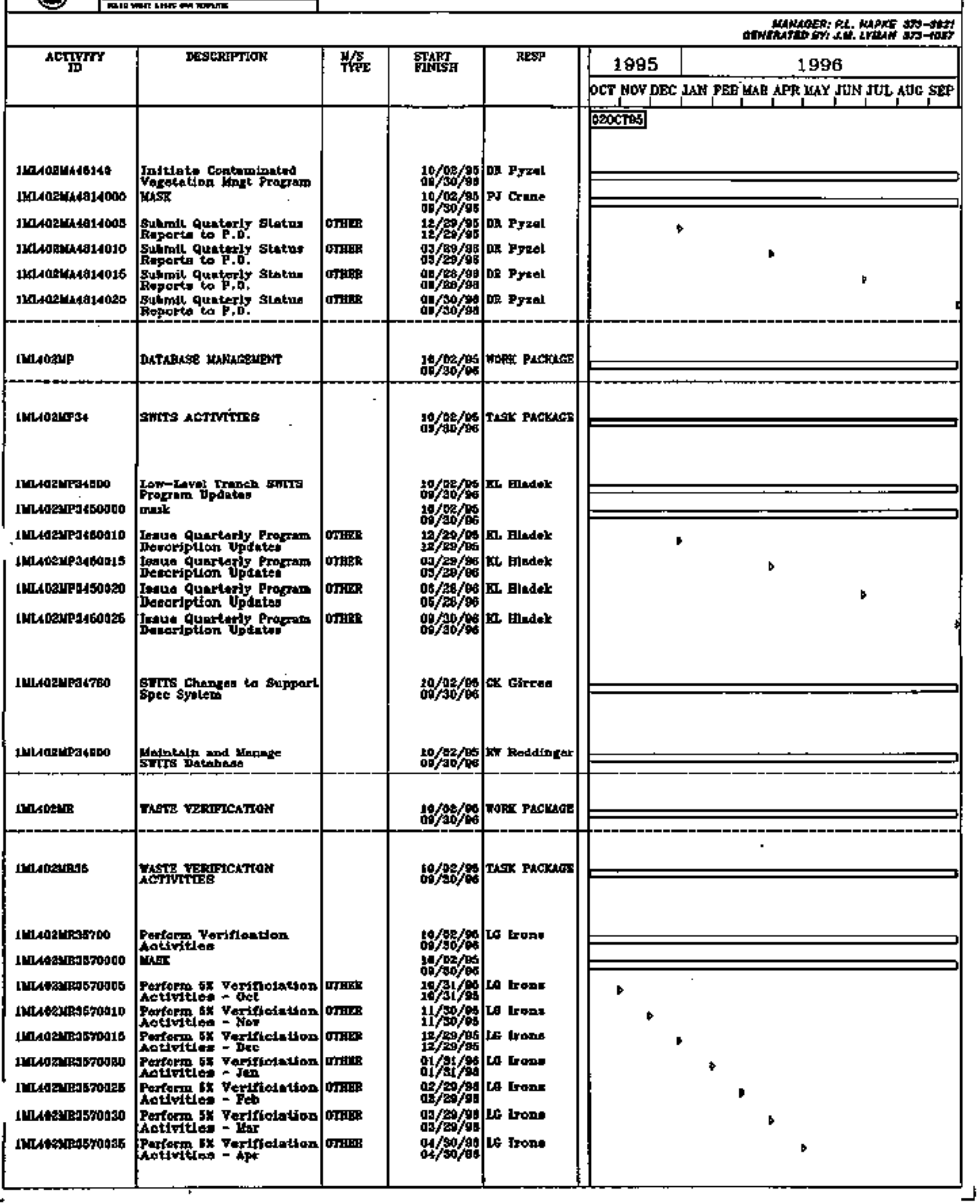




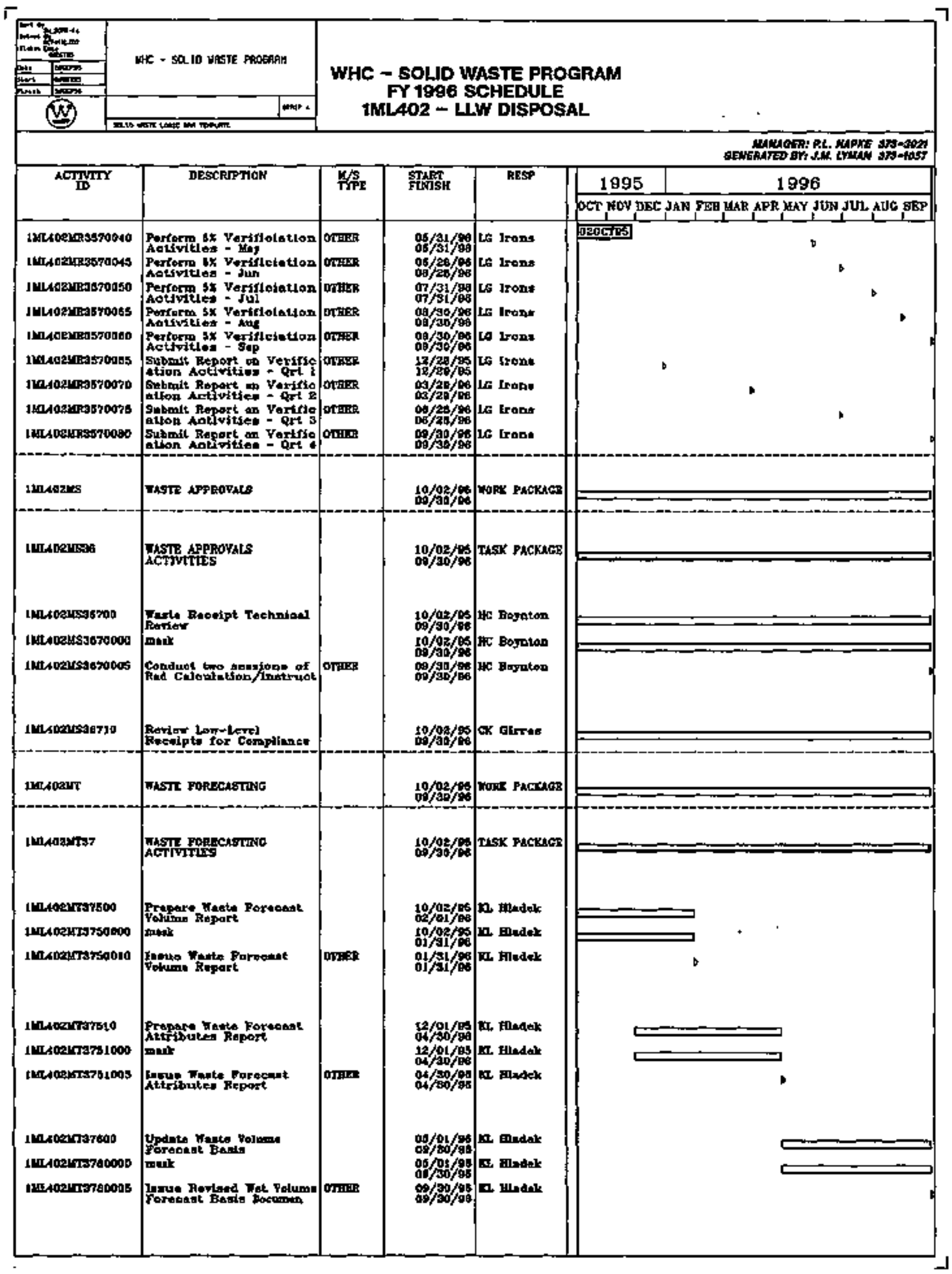


WHC - SOLID WASTE PROERAM

FY 1996 SCHEDULE TMLAD2 - LLW DISPOSAL

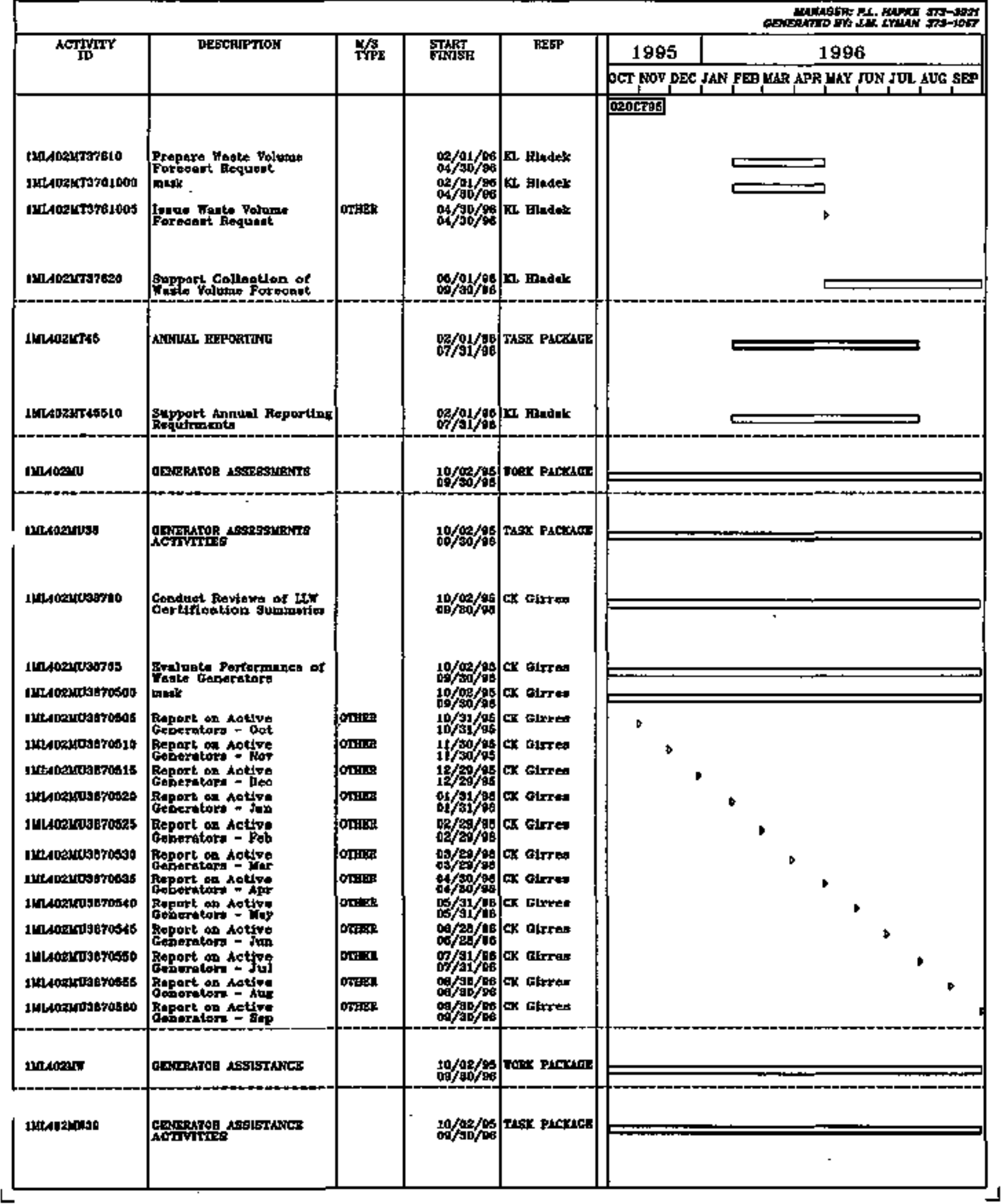


(i)

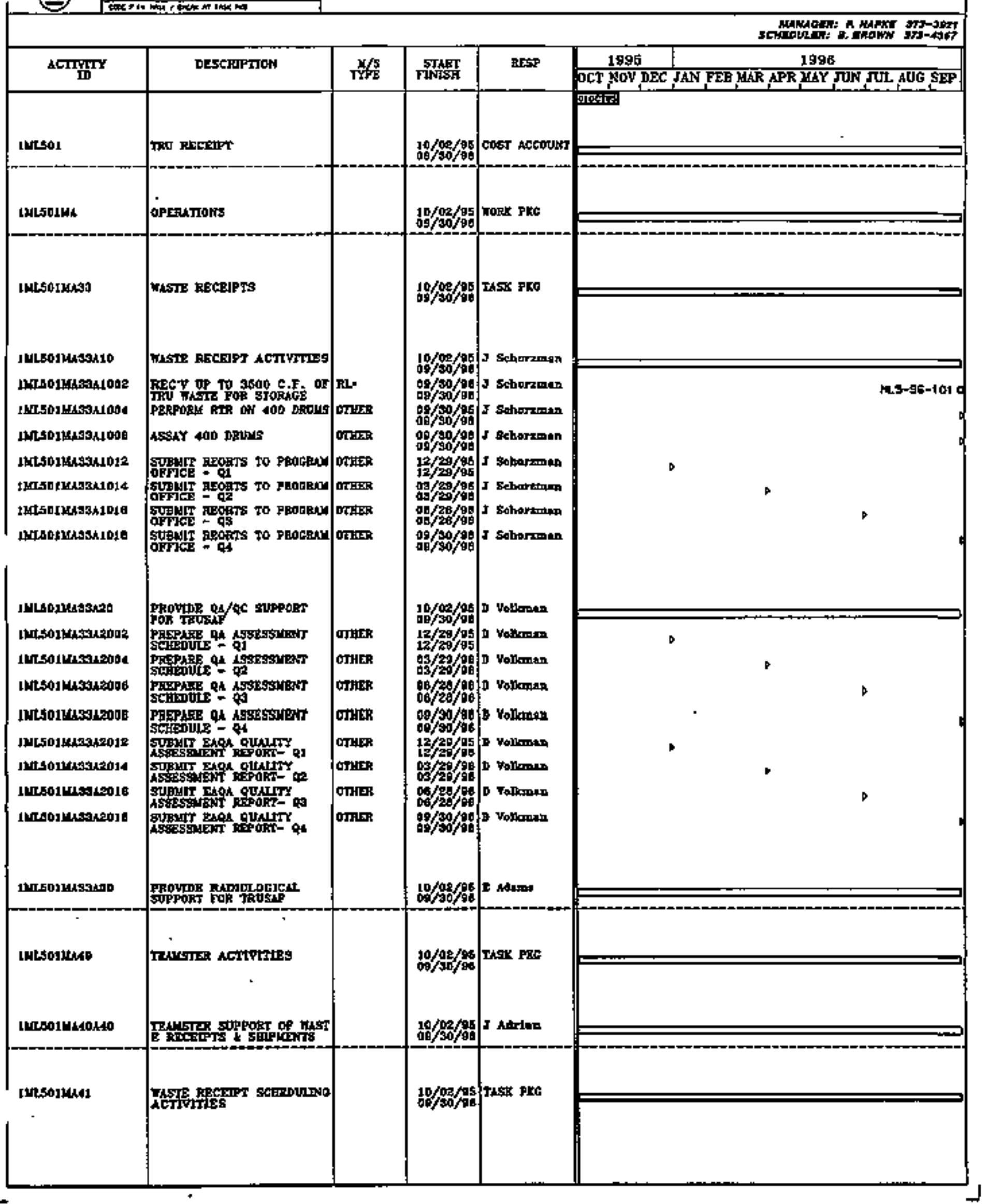




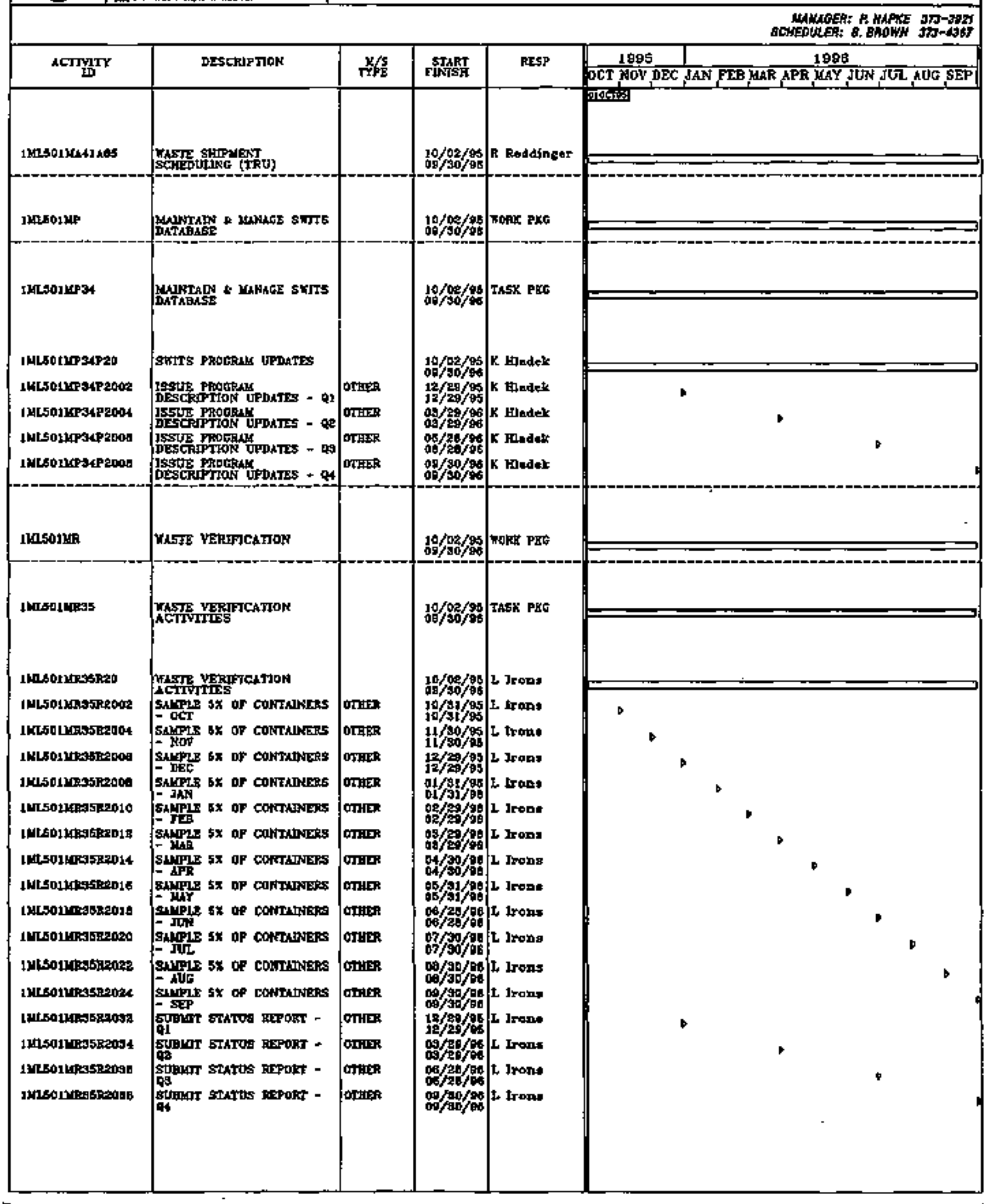


WHC - SOLID WASTE PROGRAM

FY 1996 SCHEDULE

1ML501 - TRU RECEIPTS

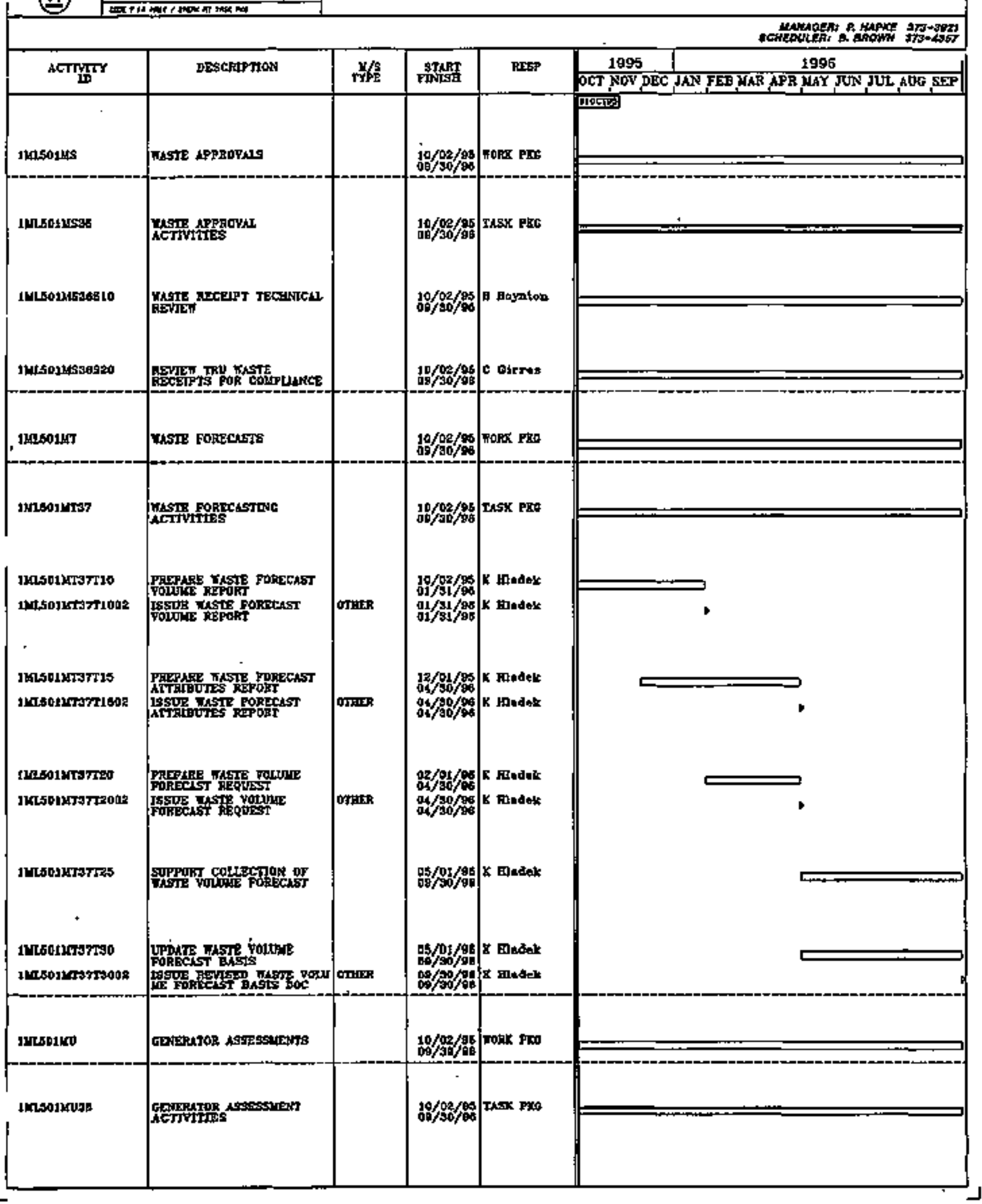




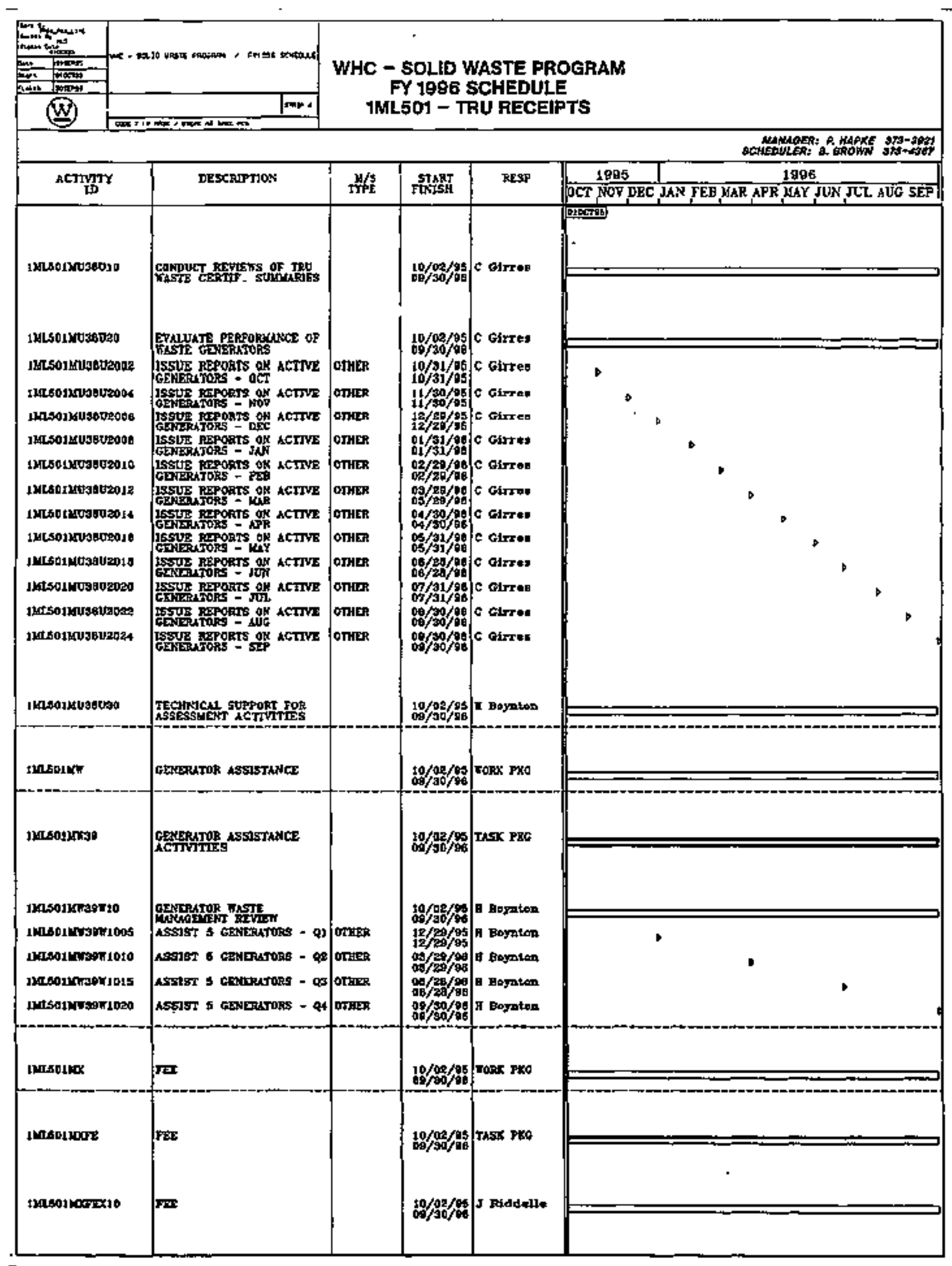


WHC - SOLID WASTE PROGRAM

FY 1996 SCHEDULE

1ML603 - RMW/CWC RECEIPTS

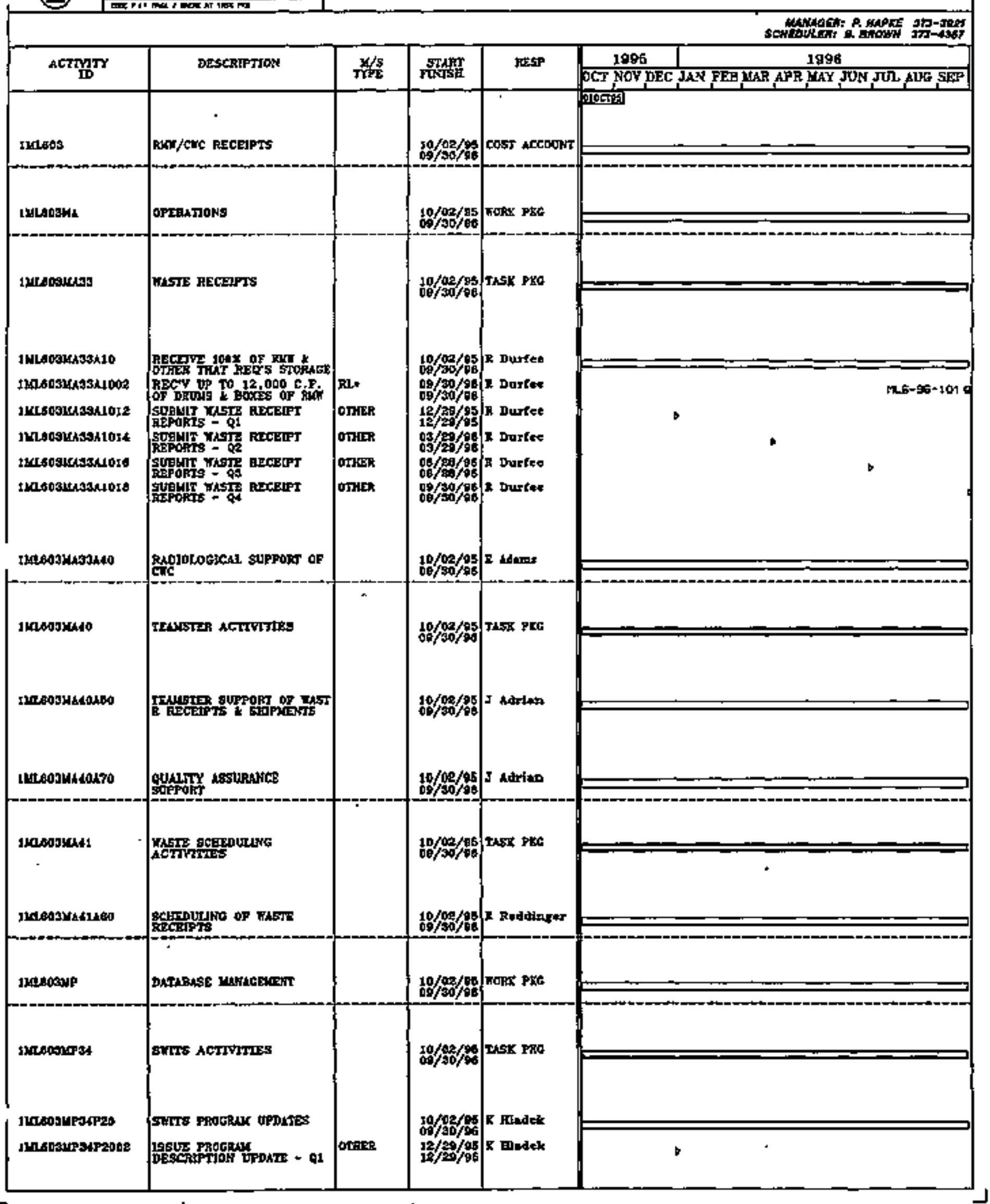




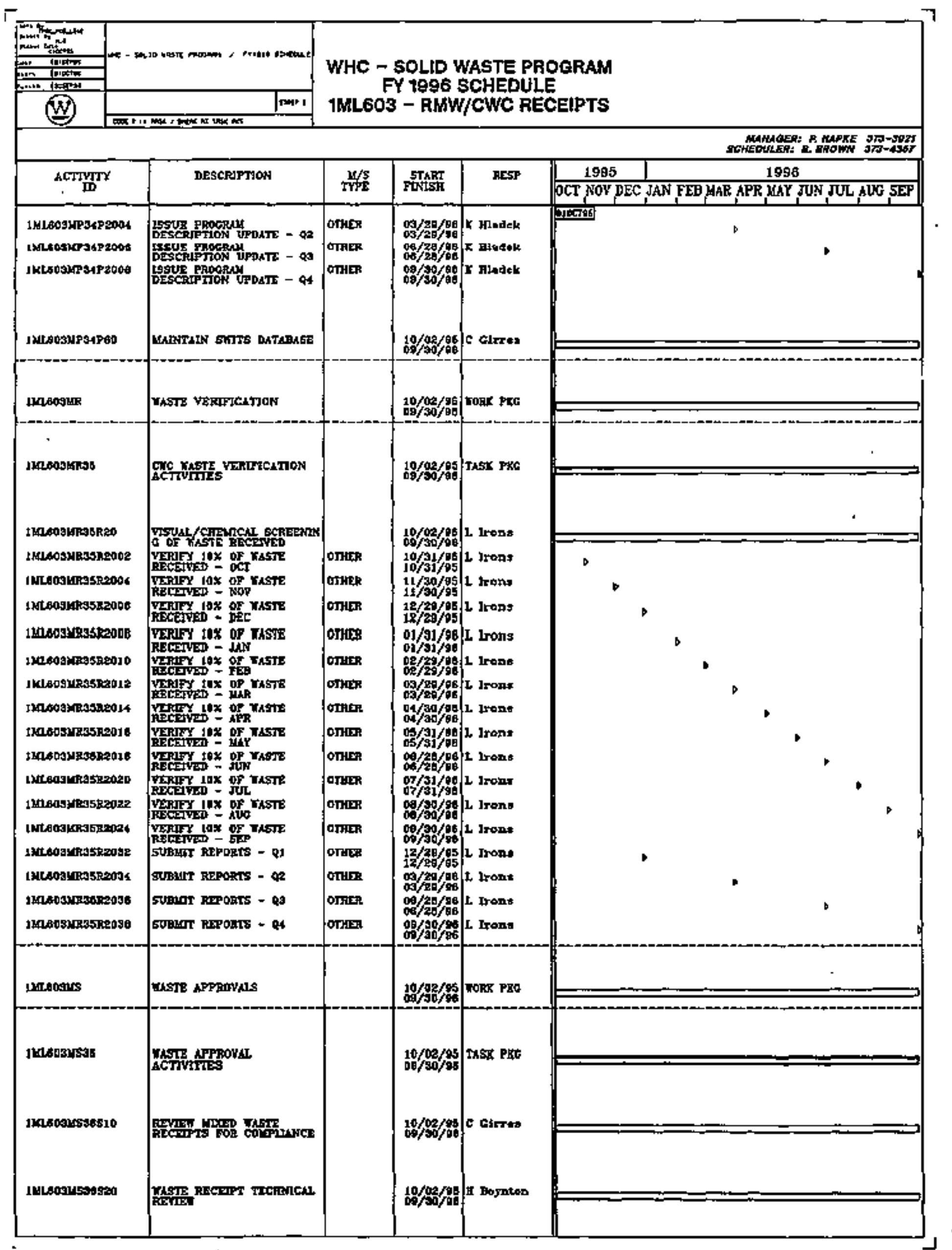


WHC - SOLID WASTE PROGRAM FY 1996 SCHEDULE IFíLÉOS - RMW/CWC RECEIPTS

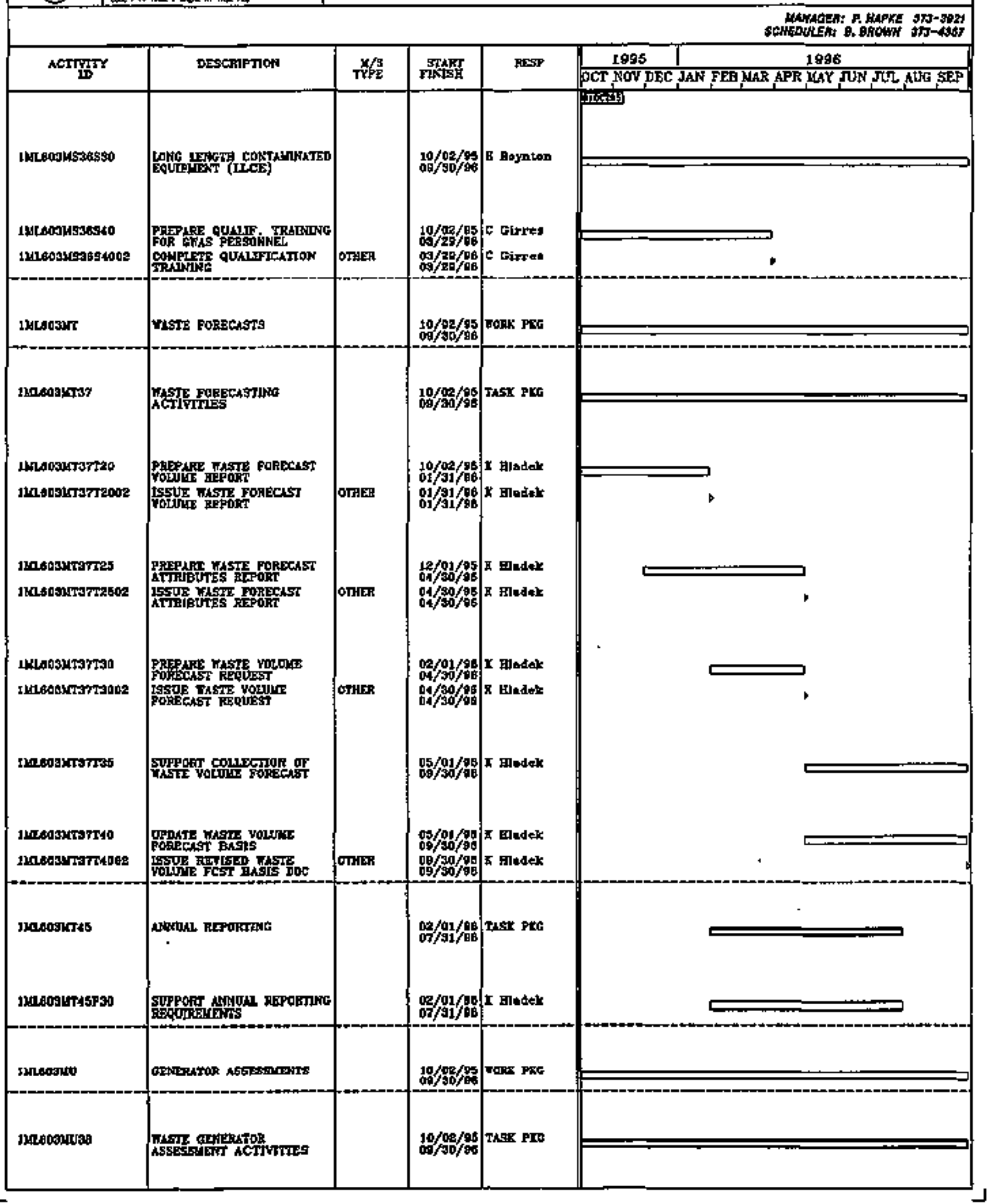


WHC - SOLID WASTE PROGRAM FY 19SS SCHEDULE IML603 - RWW/CWC RECEIPTS

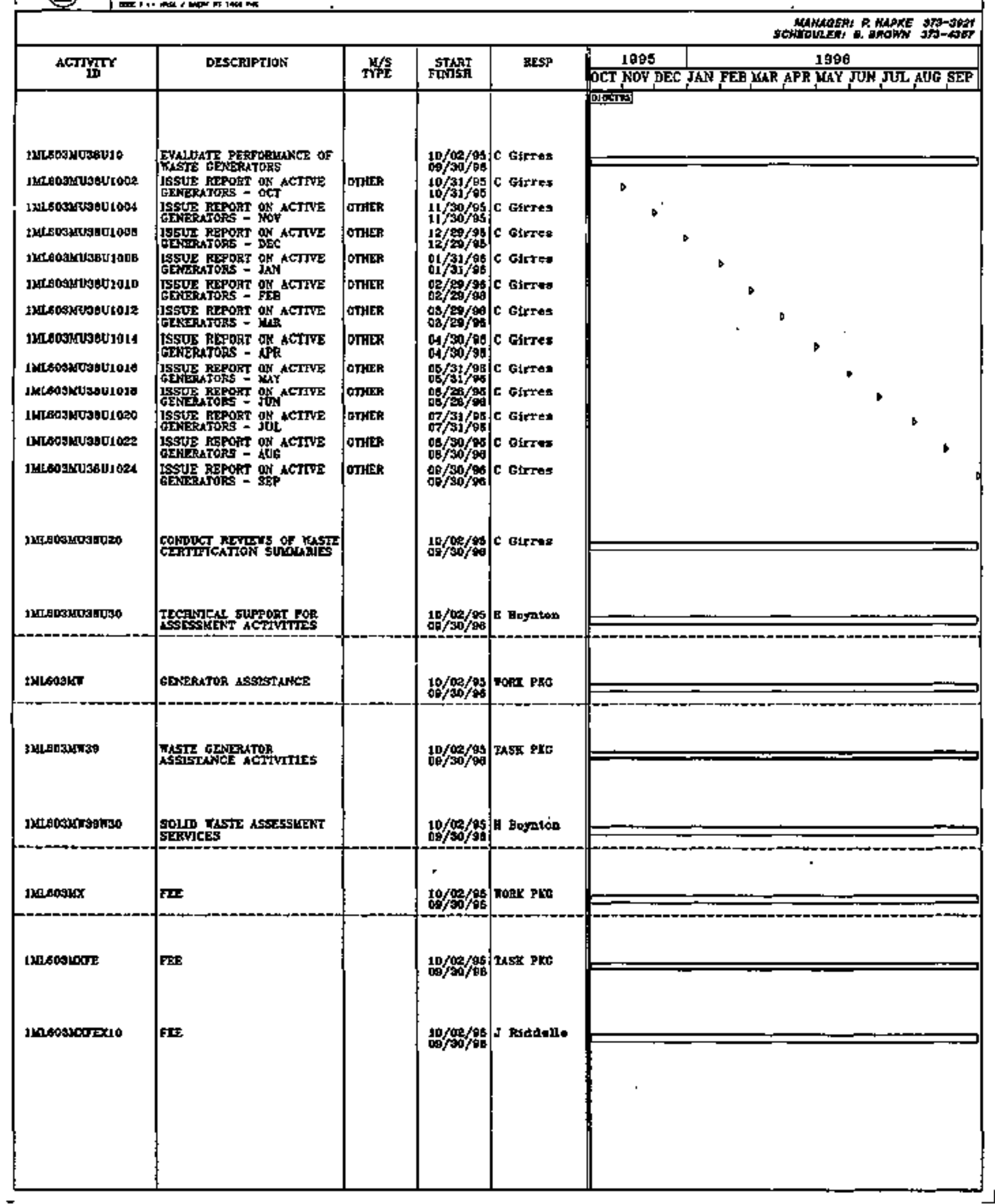




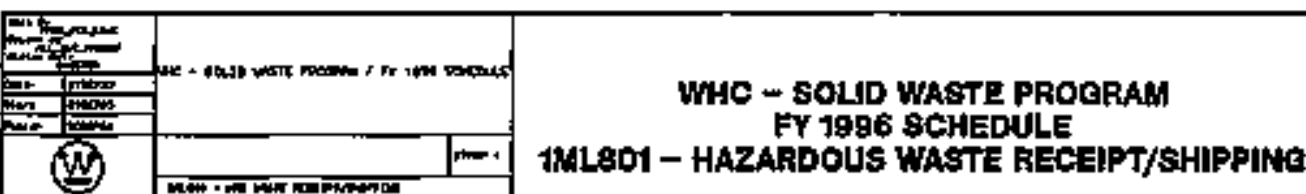

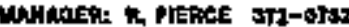

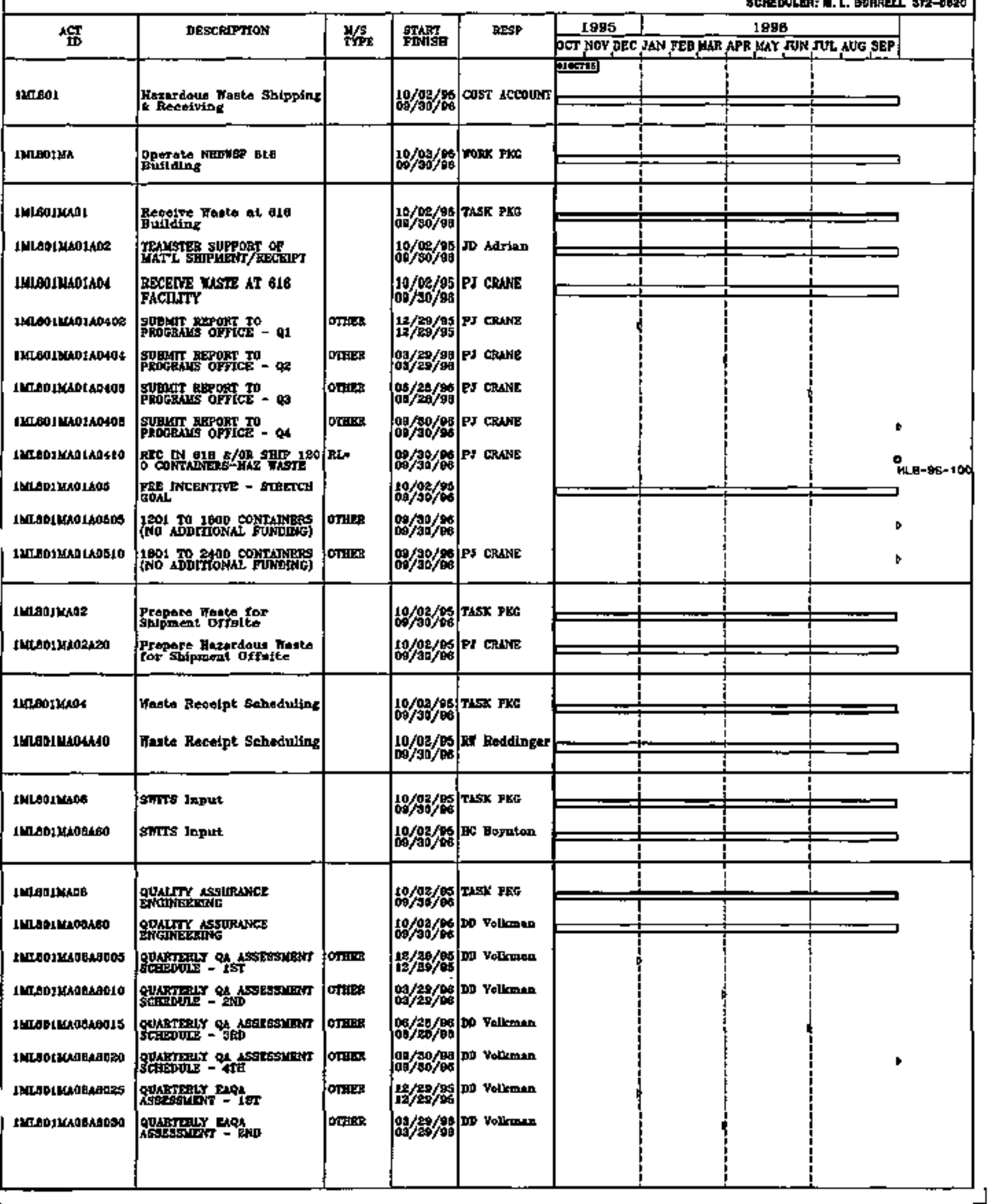




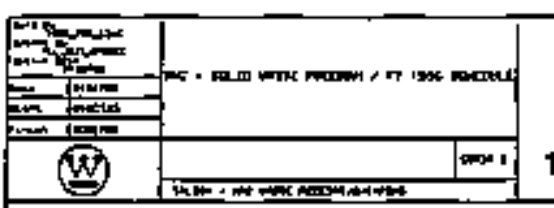

WHC - SOLID WASTE PFOGRAM
FY 1996 SCHEDULE
MLAO1 - HAZARDOUS WASTE RECEIPT/SHIPPING

\begin{tabular}{|c|c|c|c|c|c|c|c|c|c|}
\hline & & & & & & & \multicolumn{3}{|c|}{ 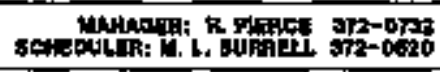 } \\
\hline \multirow{2}{*}{ ACt } & \multirow[t]{2}{*}{ DESCRIPTION } & \multirow{2}{*}{$\frac{\mathrm{H}}{\mathrm{T}} \mathrm{s}$} & \multirow{2}{*}{$\begin{array}{l}\text { START } \\
\text { FIMISE }\end{array}$} & \multirow[t]{2}{*}{ RESP } & 1095 & & 1996 & & \\
\hline & & & & & \multicolumn{4}{|c|}{ OCT HOY DEC JAN FEB MAR APR WAY JIN JUL DUG SEP } & \\
\hline 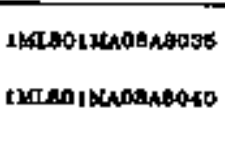 & 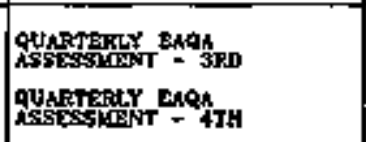 & OTFER & 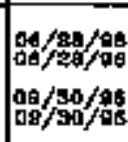 & De valtomen & 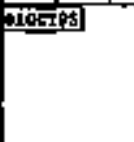 & & & $p$ & \\
\hline 1uchantax & thazerdou Therte Shipment & & jag/30/95 & vate PSE & & & & & \\
\hline IXeteliminad & Inopomt/Trangport Ho:/ & & 30/00/25 & TASK PXE & & & & & \\
\hline 13asolbosojocos & INSRET/TRANSPORT TASTL & POREa & $\frac{10 / 81 / 95}{30 / 31 / 25}$ & Hic Bojnten & - & & & & \\
\hline ILLedmanosóto & INSPEET/TRANSPORT TASTE, & orikes & $111 / 30 / 95$ & HC Boynlon & D & & & & \\
\hline IMLajkporogls & DNSPECI/TRANSPORT TASTE & OTHER & $212 / 29 / 95$ & He Baynton & & & & & \\
\hline 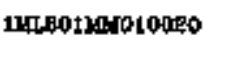 & NESPEET/TRANSPORT WASTE & ATHER & $01 / 91 / 95$ & HC Boyman & & D & & & \\
\hline 14L601น94010026 & INSPFET/TRANSPORT TASTE & OTHER & $02 / 29 / 985$ & He Boynton & & D & & & \\
\hline 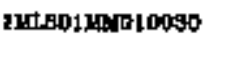 & ANSEGET/TRANSPORT TASTE & OOTHER & 109/20/\%5 & Wh Boystan & & & & & \\
\hline 24L80128N0 L0095 & $\mid \begin{array}{l}\text { INSFECT/RUANSPORT TATE } \\
-\mathrm{APE}\end{array}$ & OTHER & $10 / 30 / 90$ & HC Boynlon & & & - & & \\
\hline IMIS01205010040 & INSPECT/TRKNSPOKT TASTE & ontizs & $06 / 51 / 96$ & HC Boynten & & & P & & \\
\hline WESOLW101004S & ANSPECT/MRANSPDAI TISTE & OAHiRs & 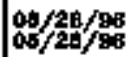 & Hc Boynton & & & & & \\
\hline 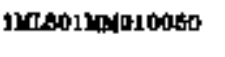 & INSTEGT/TRANSPORT TLSTE & OTHER & $07 / 31 / 98$ & He Boynlon & & & & - & \\
\hline 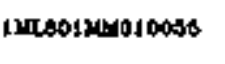 & INSPECT/TRMNGPOKT TASTE & OTHER & $\log _{00} / 30 / 30 / 6$ & HC Boysion & & & & D & \\
\hline 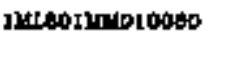 & PNSPECT/TRUNSPORT TLSTE & onien & gag/30/98 & He Boymon & & & & D & \\
\hline 1)LONXMA2 & STITS Fite/gA & & $10 / 02 / 23$ & TXSK PEG & & & & & \\
\hline & |Tranamiltal & & $00 / 50 / 96$ & & & & & & \\
\hline 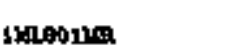 & Wartu Vortflestion & & $10 / 02 / 05$ & Fogx PXG & & & & & \\
\hline & & & $00 / 50 / \% \mathrm{~B}$ & & & & & & \\
\hline IMtsaleria & Perrosta sadom & & 1.0/60/95 & TLAS FEG & & & & & \\
\hline 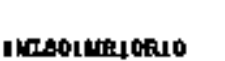 & 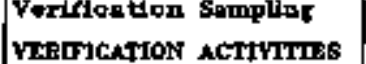 & & $\mid \begin{array}{l}007 / 30 \% / 06 \\
10 / 00 / 06\end{array}$ & Lo IRONS & & & & & \\
\hline 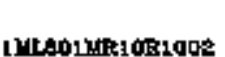 & Yeetry bx of Thets & & $\begin{array}{l}00 / 30 / 95 \\
10 / 31 / 98\end{array}$ & Lo nons & & & & & \\
\hline & AEcatreb - ber & & $10 \% / 1 / \%$ & 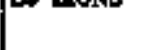 & & & & & \\
\hline 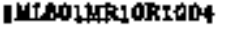 & 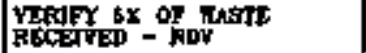 & |OTEKa & $11 / 30 / 95$ & LC IRONS & $\bullet$ & & & & \\
\hline J4LBOHKS1GR19D6 & 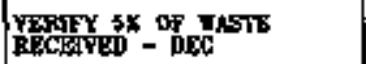 & pries. & $12 / 29 / 65$ & 50 IRONS & & & & & \\
\hline 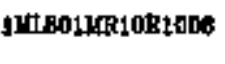 & YERIFY SX OF & DTEAR & $\frac{a 1 / 81 / 98}{01 / 81 / 05}$ & io IRows & & - & & & \\
\hline IWLODLRTORIOIO & Yreary $6 x$ or & OTHER & 102/20/48 & to IRONS & & - & & & \\
\hline 1LLOOMTR1OR1012 & VIRIFY $6 x$ of DAst & OTEERT & Pzas & 10 thows & & & & & \\
\hline 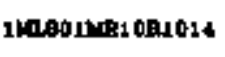 & 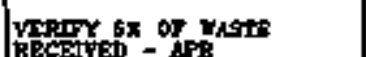 & Orists & 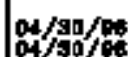 & Io IRONe & & & p & & \\
\hline 1LLOOMR10RIOLE & 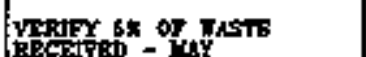 & OTFEar & 105/11/48 & Lo thove & & & - & & \\
\hline & 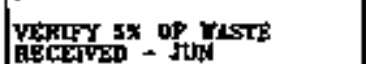 & unisks & 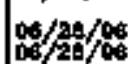 & Is mots & & & & & \\
\hline & & & & - & & & & & \\
\hline
\end{tabular}




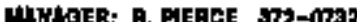

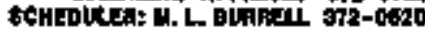

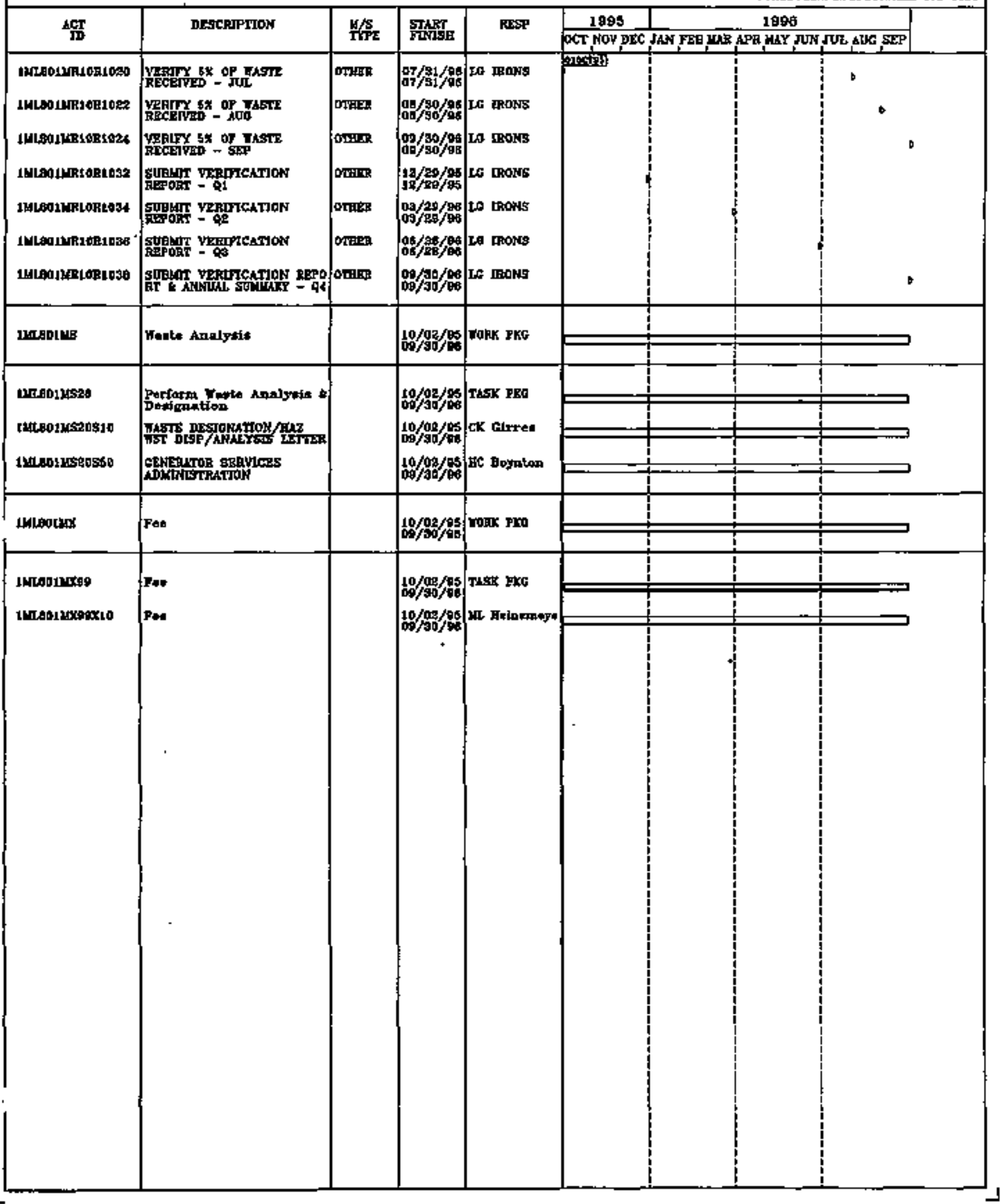




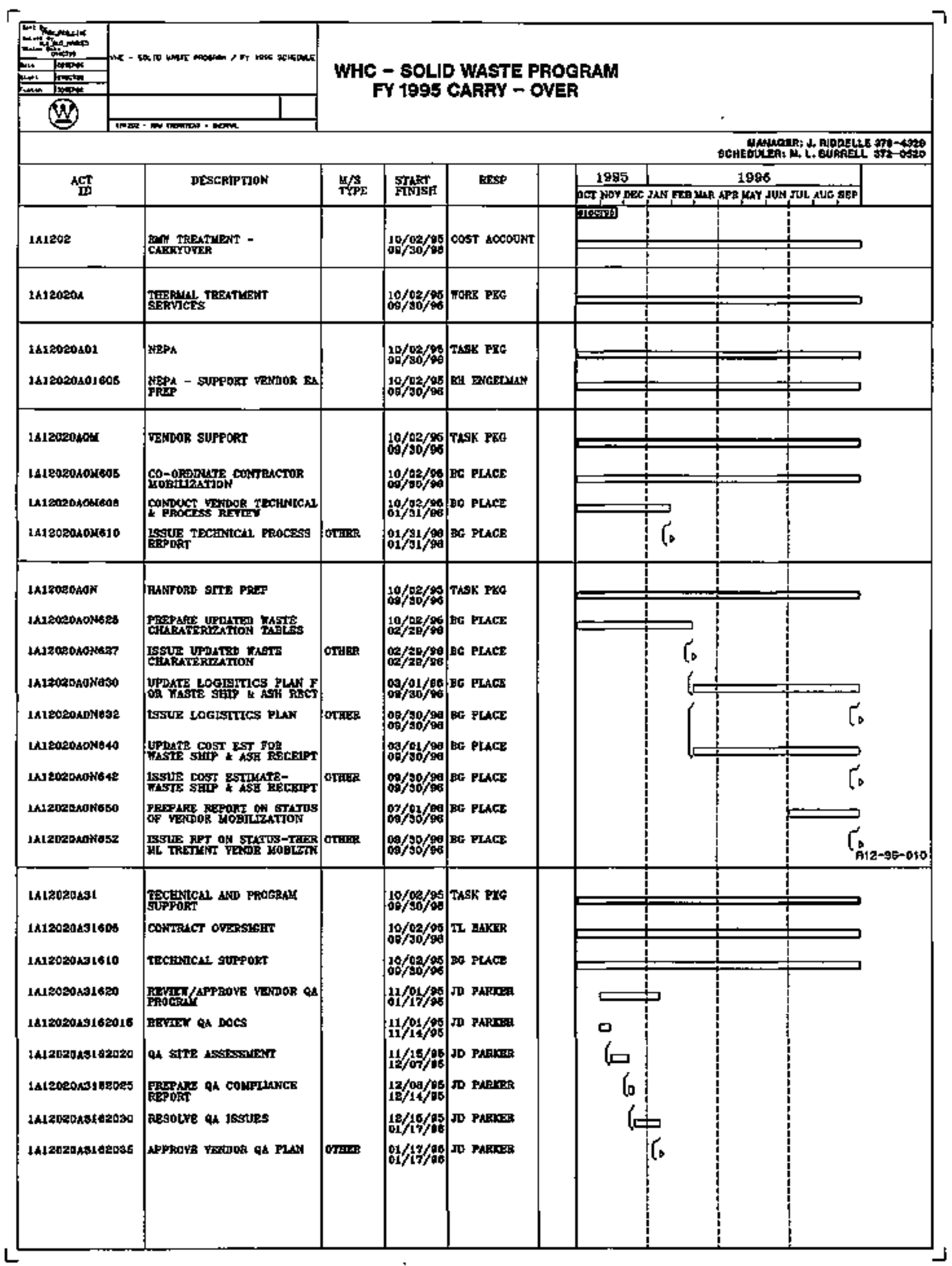



FY 1995 GARRY - OVER

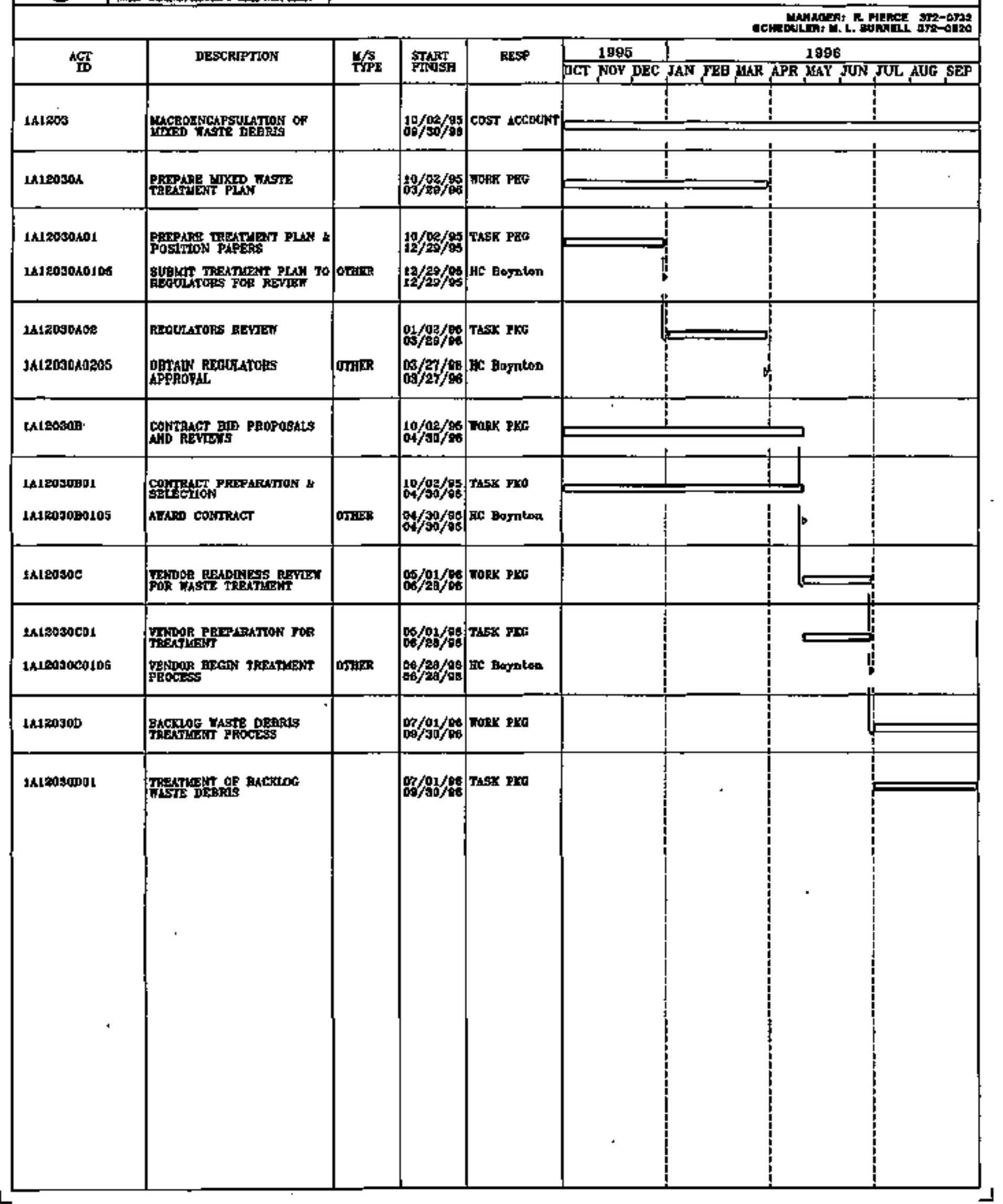




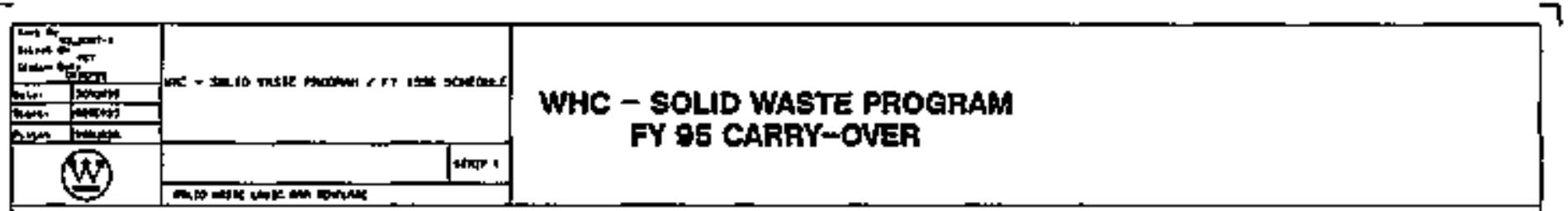

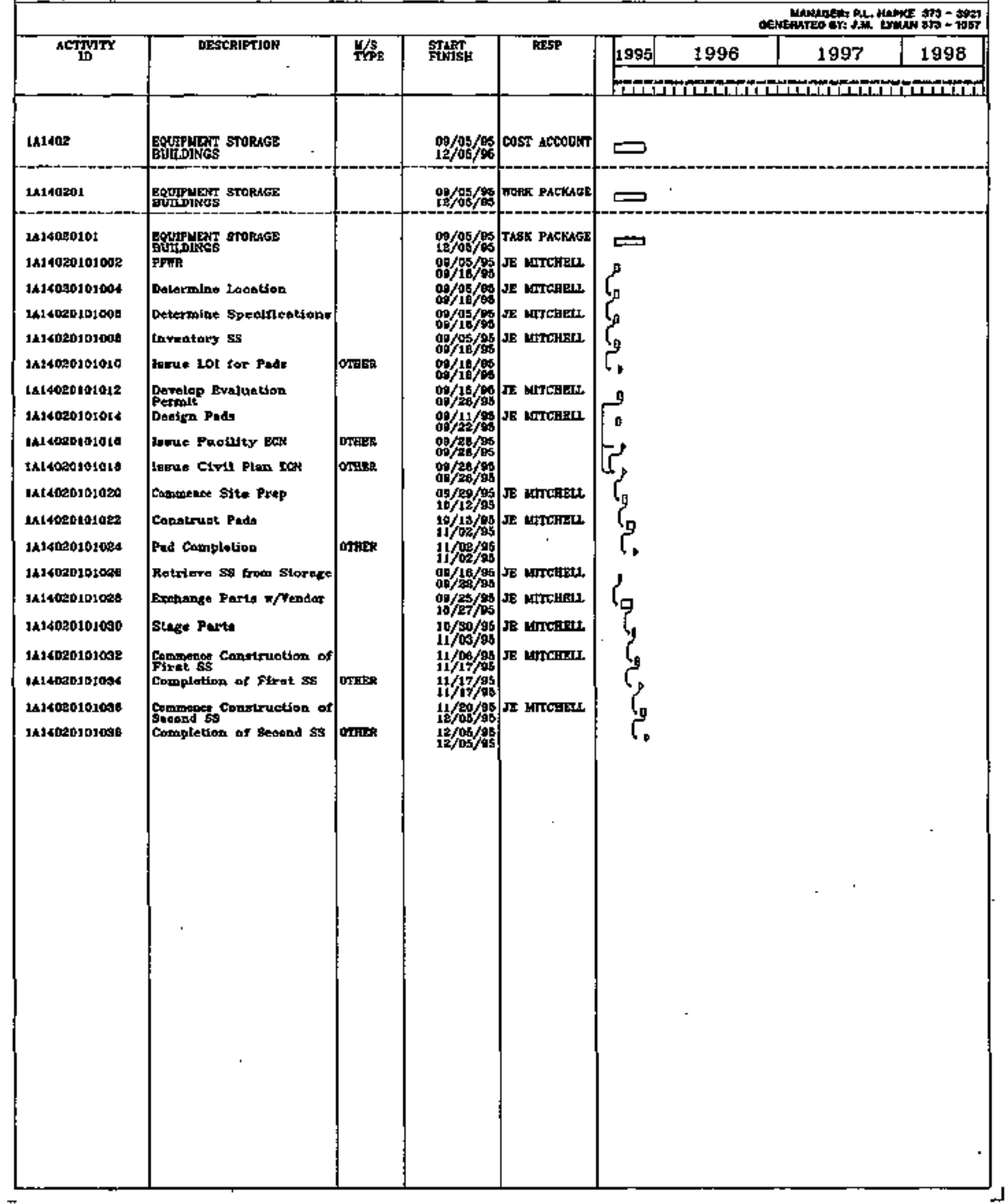


$\Gamma$

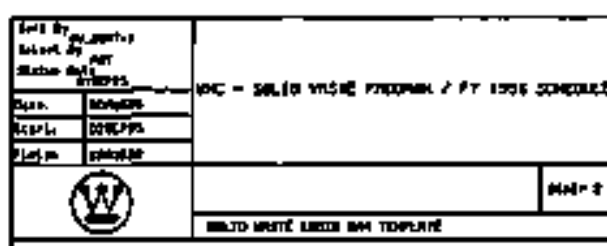

WHC - SOLD WASTE PROQRAM

FY 95 CARRY-OVEA

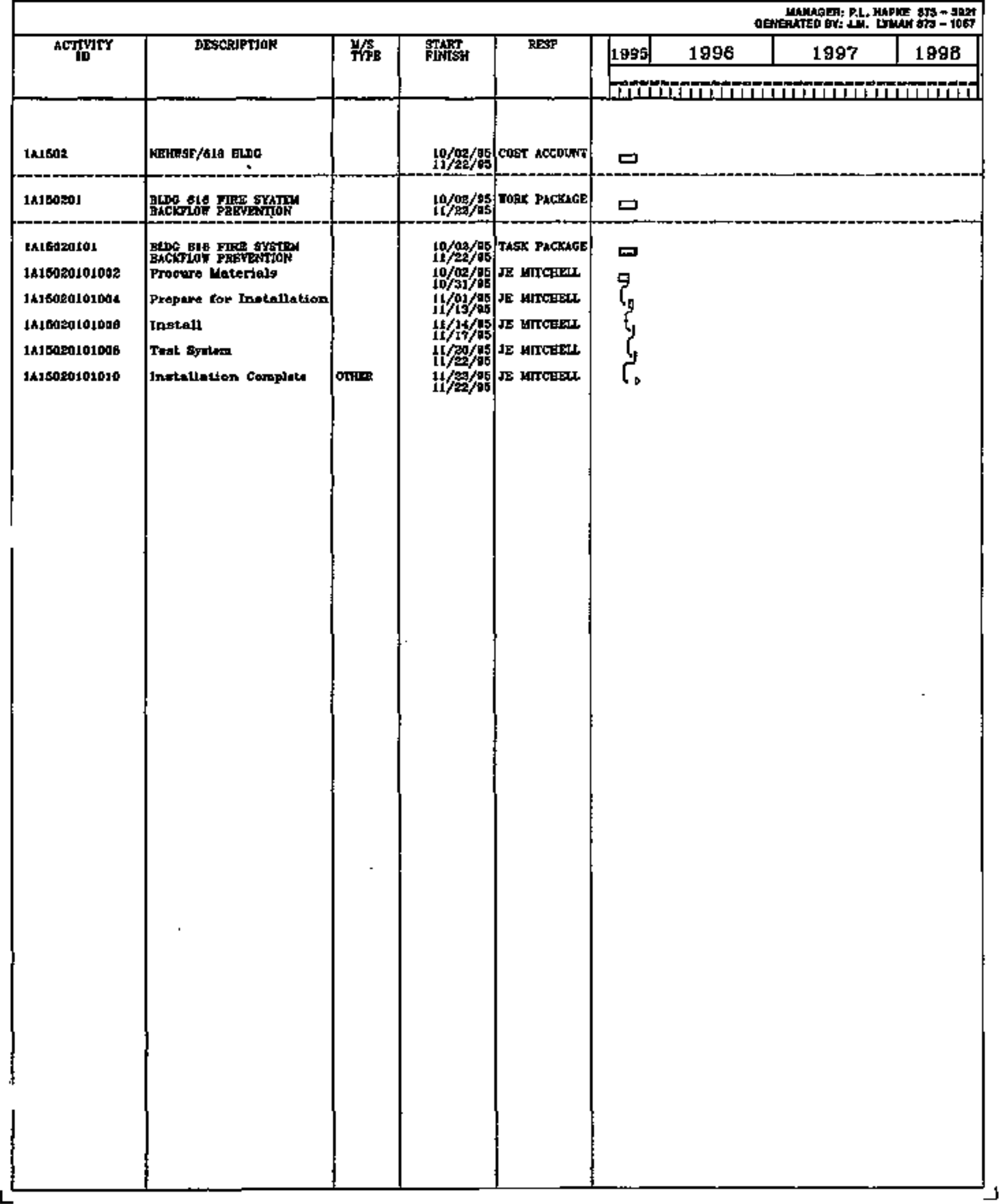




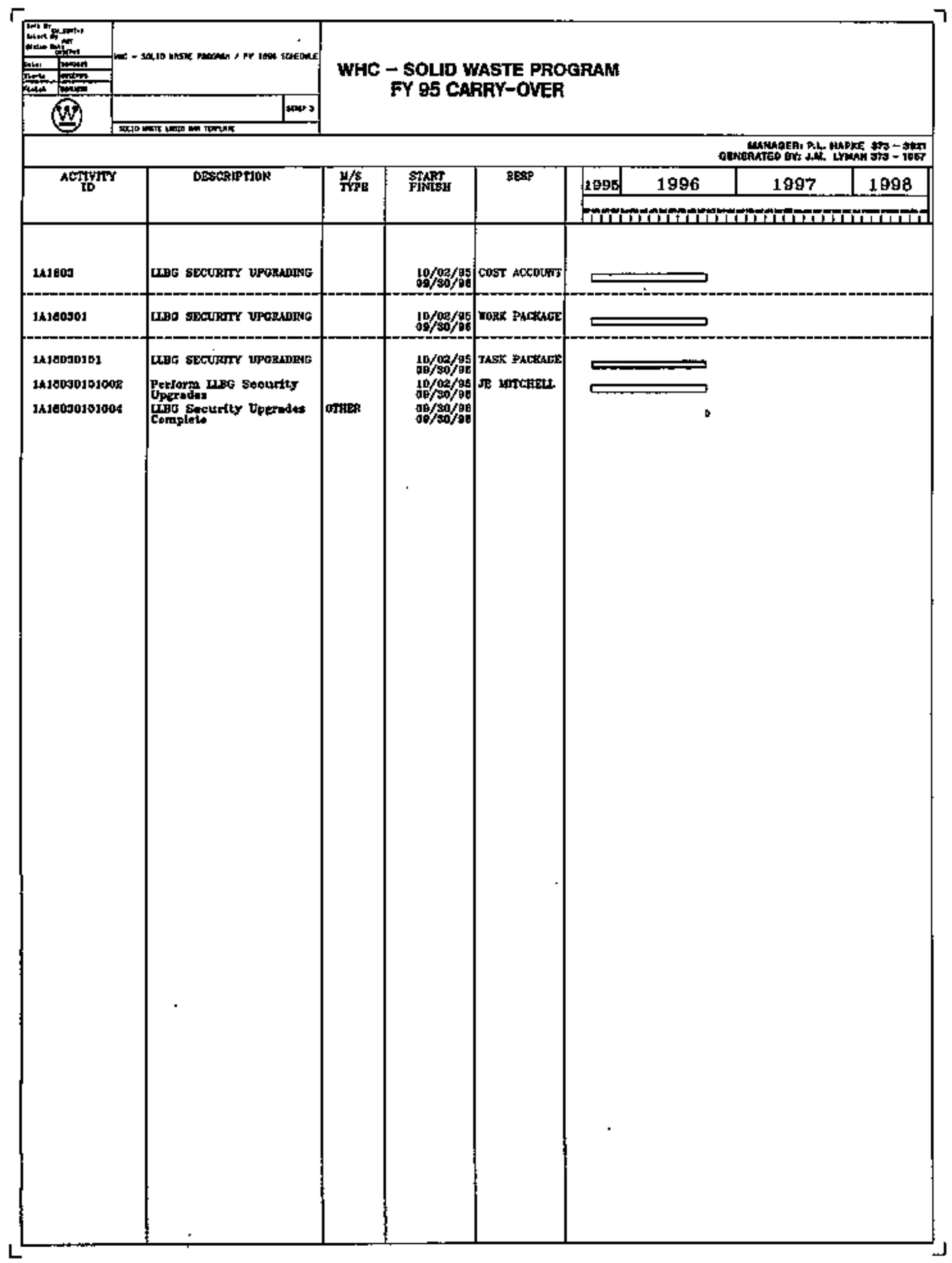




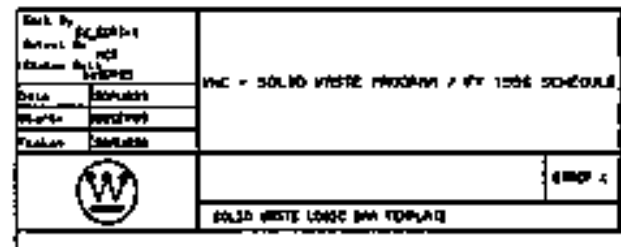

\section{WHC - SOLID WASTE PROGRAM FY 93 CARRY-OVER}

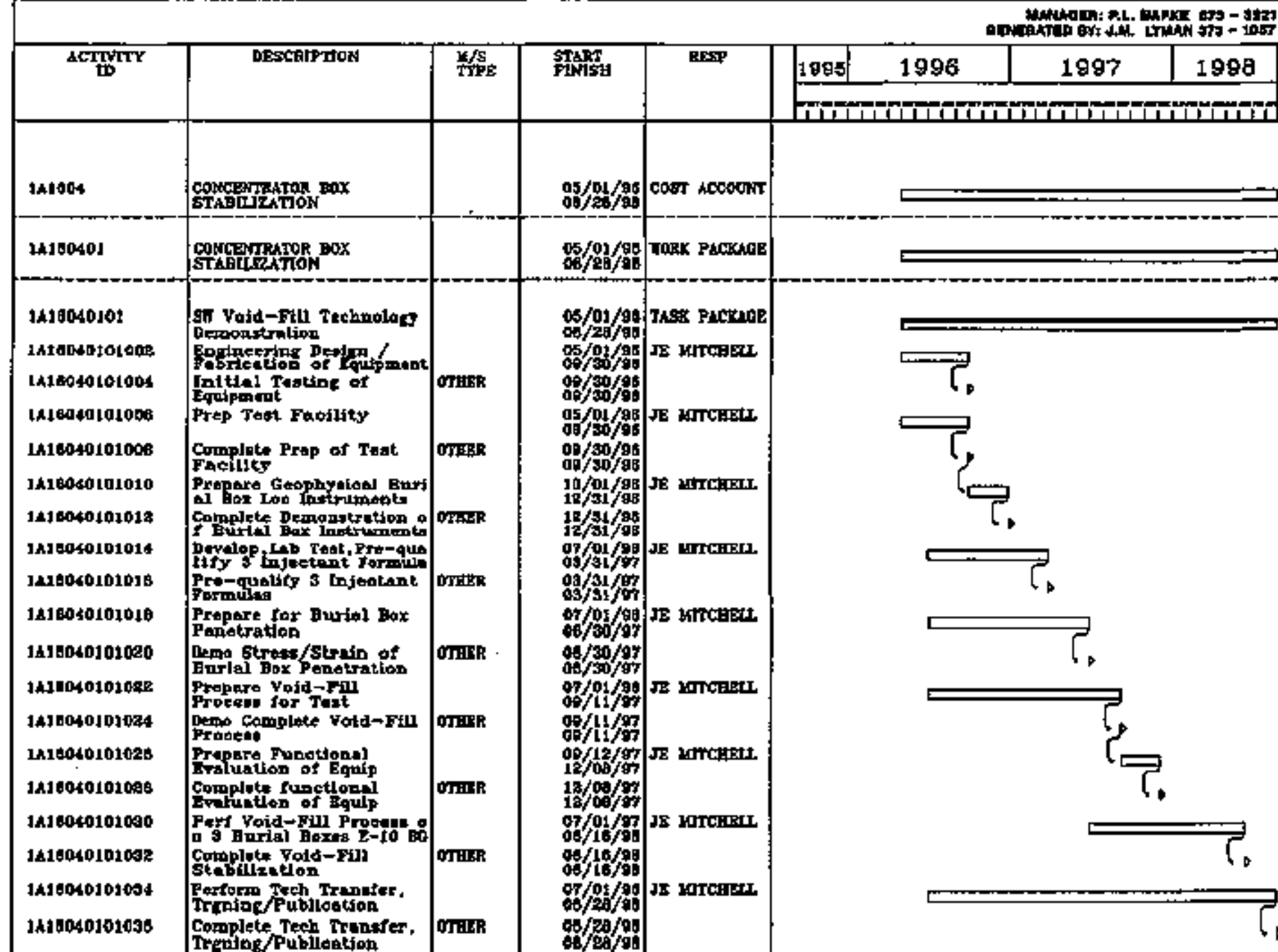




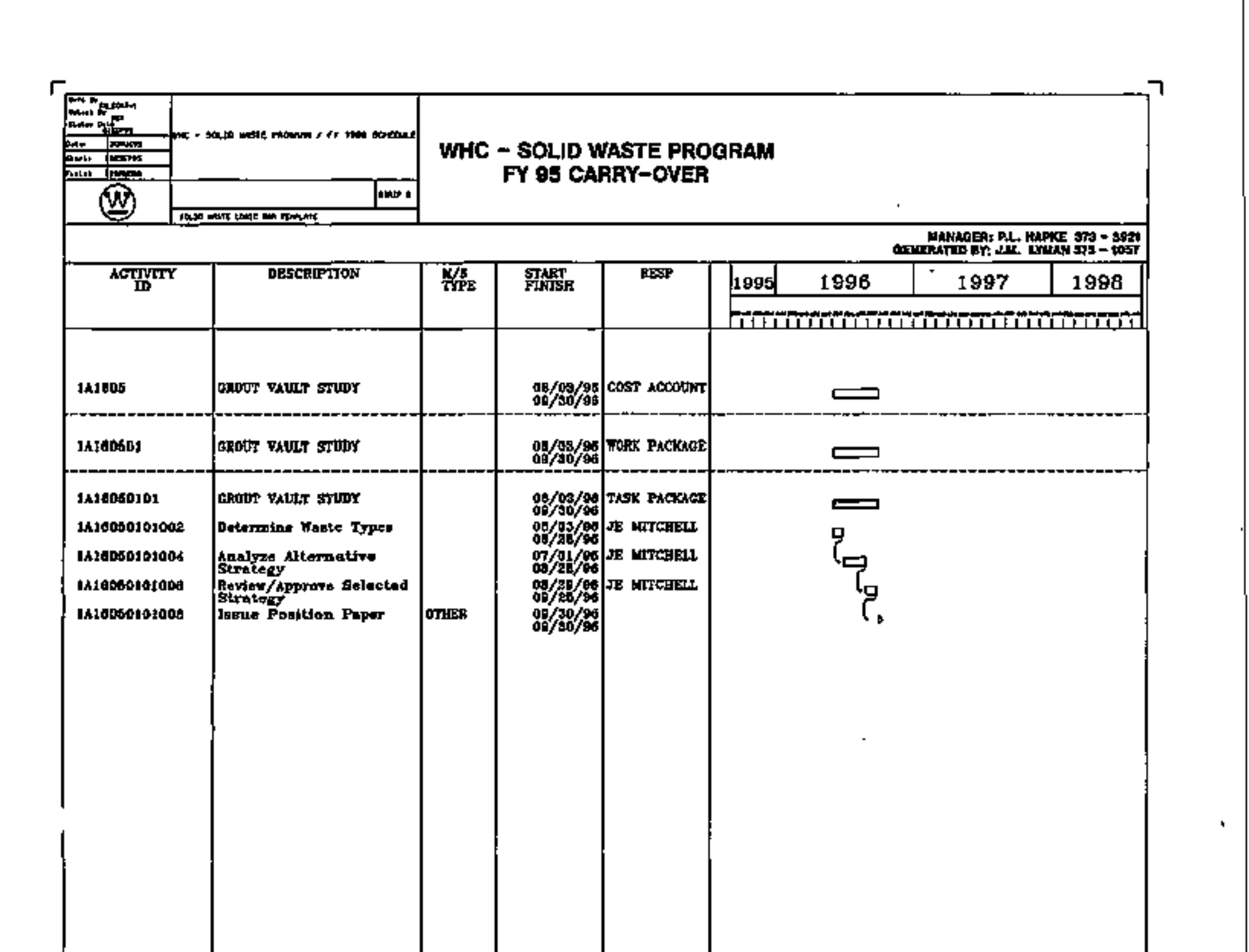


广

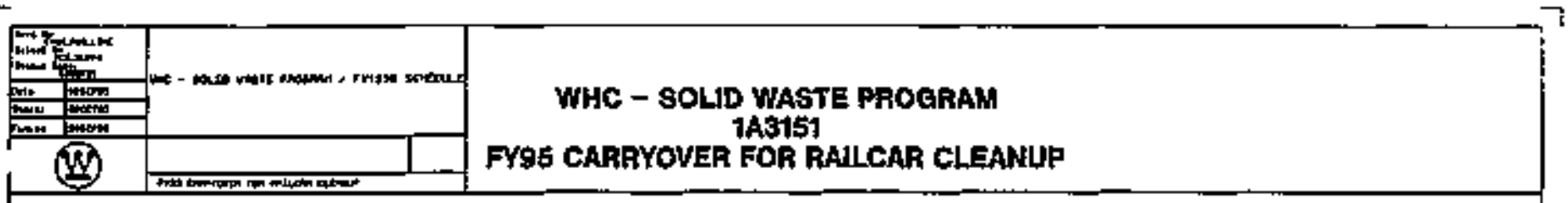

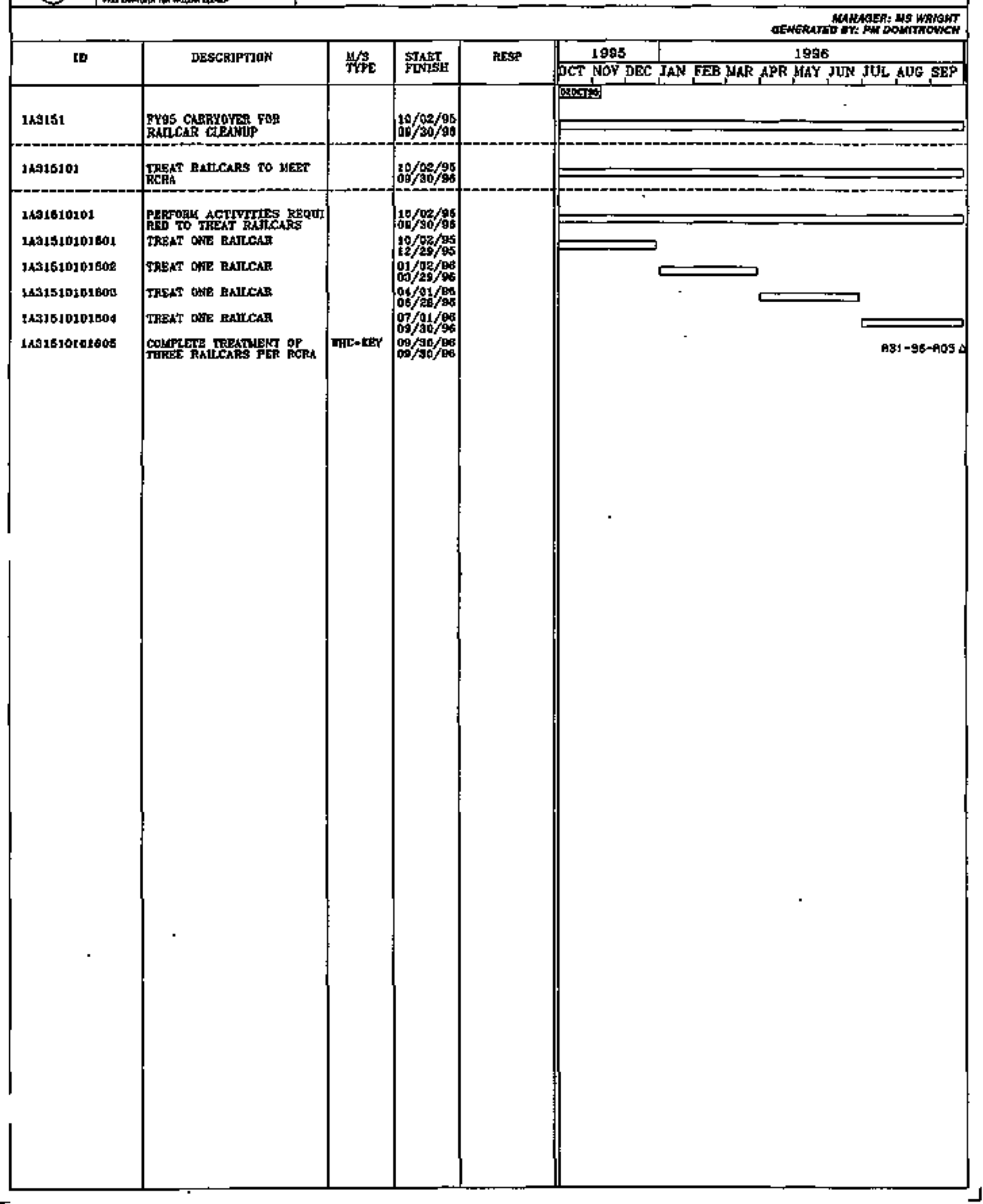


WHC - SOLLD WASTE PROCAAM

143252

FY95 CAFRYOVER FOR PAVING BEHIND 2706-T

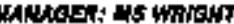

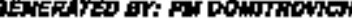

\begin{tabular}{|c|c|c|c|c|c|c|}
\hline ID & DESCRRPTIAN & U/S & THNT & RLSP & $\frac{1995}{\text { OCT NOF DEC }}$ & $\frac{1968}{\text { JAN FEB MAR APR MGY JUN JUL AUG SEP }}$ \\
\hline 1łazsa & 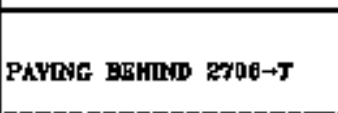 & & o6/01/98 & & 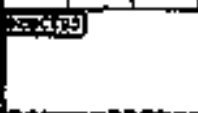 & \\
\hline LA325201 & FAYNAG ERHIND RPAG-T & & $\operatorname{os} / 01 / 98$ & & & $\Longrightarrow$ \\
\hline $\begin{array}{l}\text { 14J285t0101 } \\
\text { 14525R0101605 }\end{array}$ & 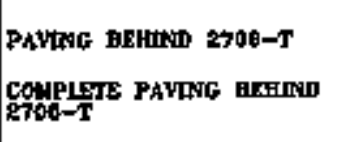 & nic-keY & $\begin{array}{l}04 / 01 / 06 \\
07 / 01 / 06 \\
07 / 01 / 00 \\
07 / 01 / 05\end{array}$ & & & A3e-se-R03 \\
\hline
\end{tabular}


$\Gamma$

WHC - SOUD WABTE PROERAW

143053

FYSS CARRYOVER FOR NEW AOLL-UP DOOR AT 2ZJ-T

MAMAOEF: WS WANtWr

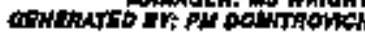

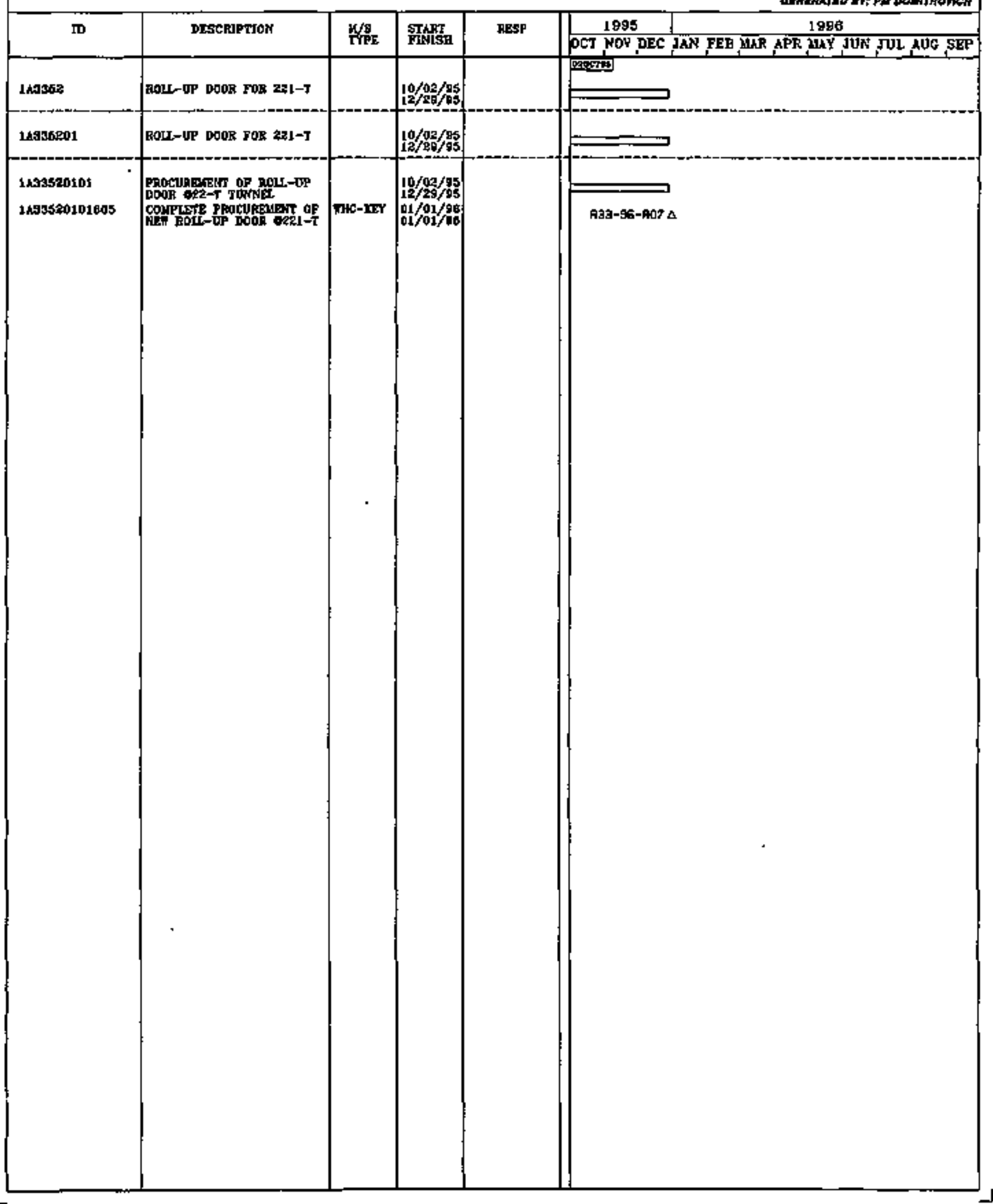




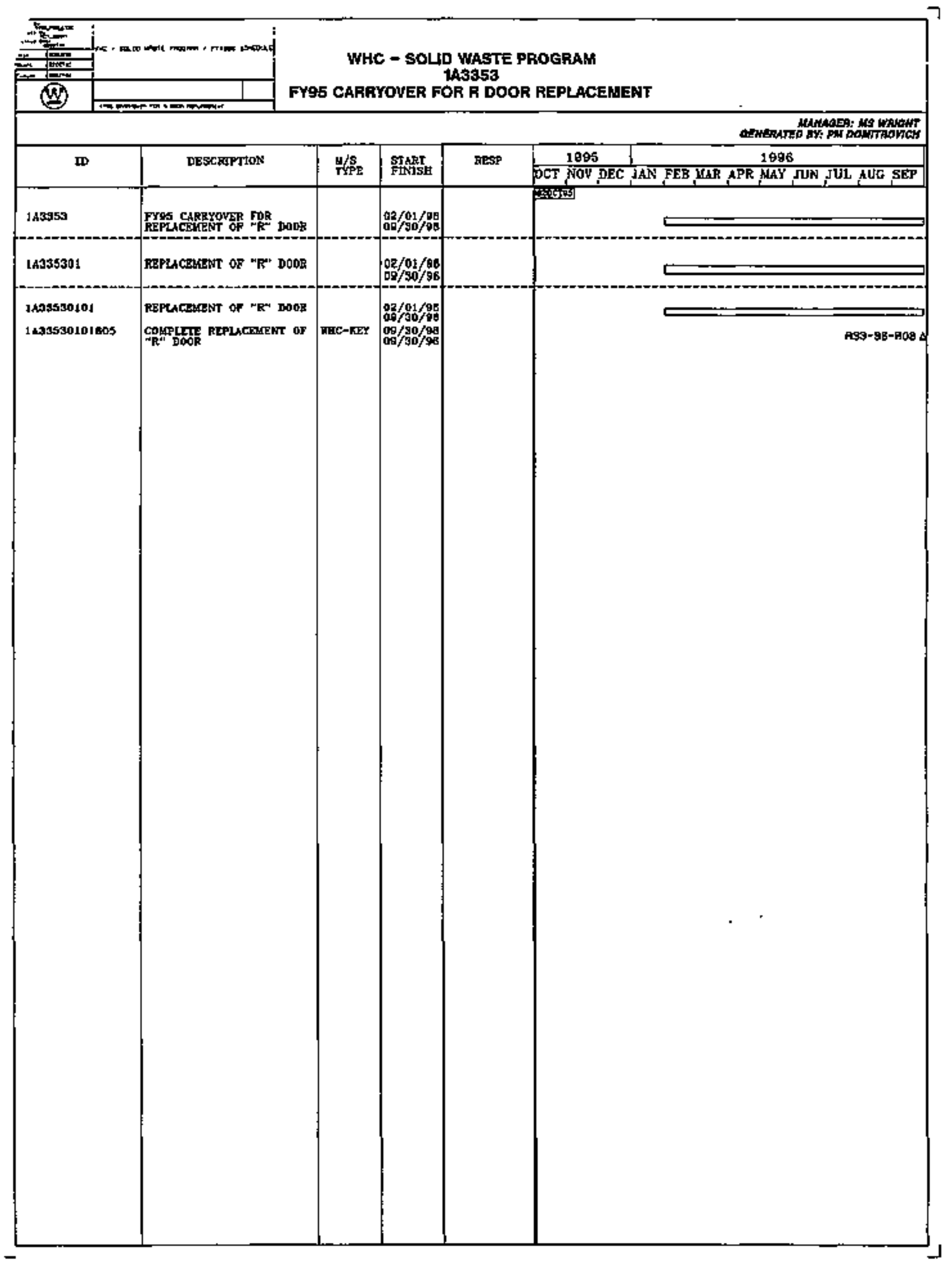


$\Gamma$ WHC - SOLID WASTE PROGAAM

(Qi) F⿻十 FY95 CAARYOVER FOR SANITARY WATER LINE

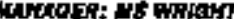

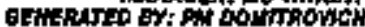

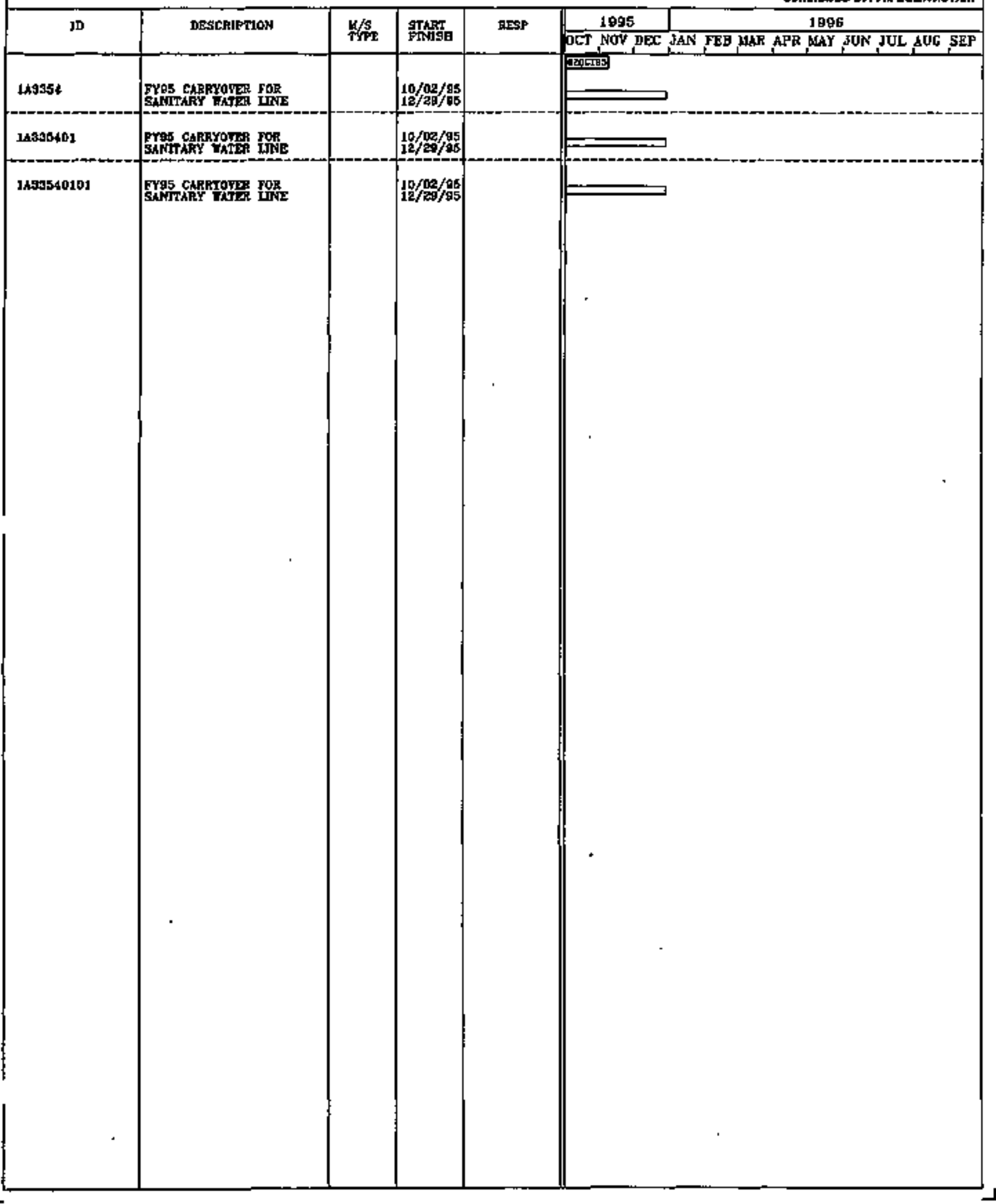




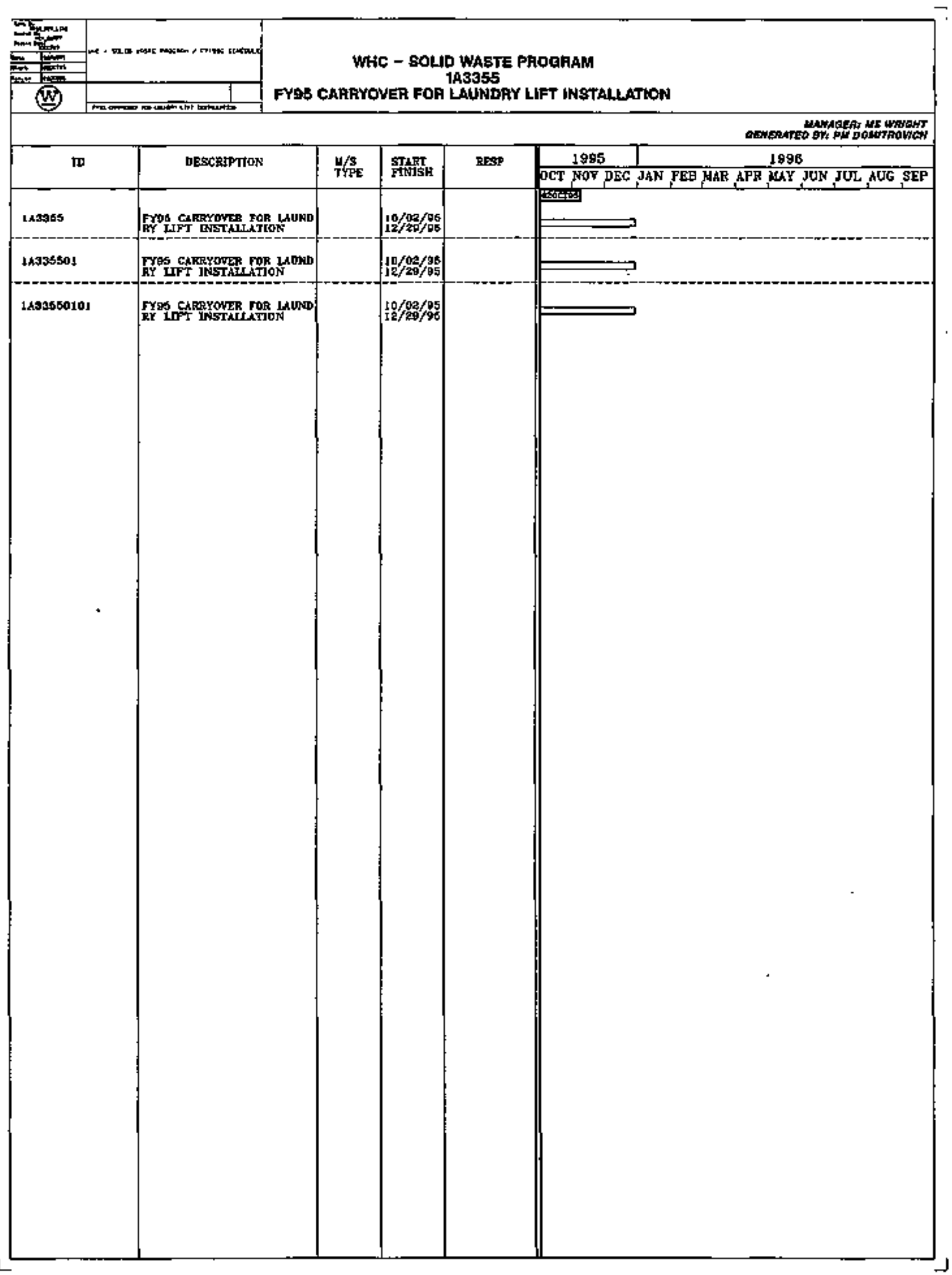




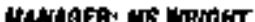

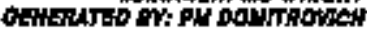

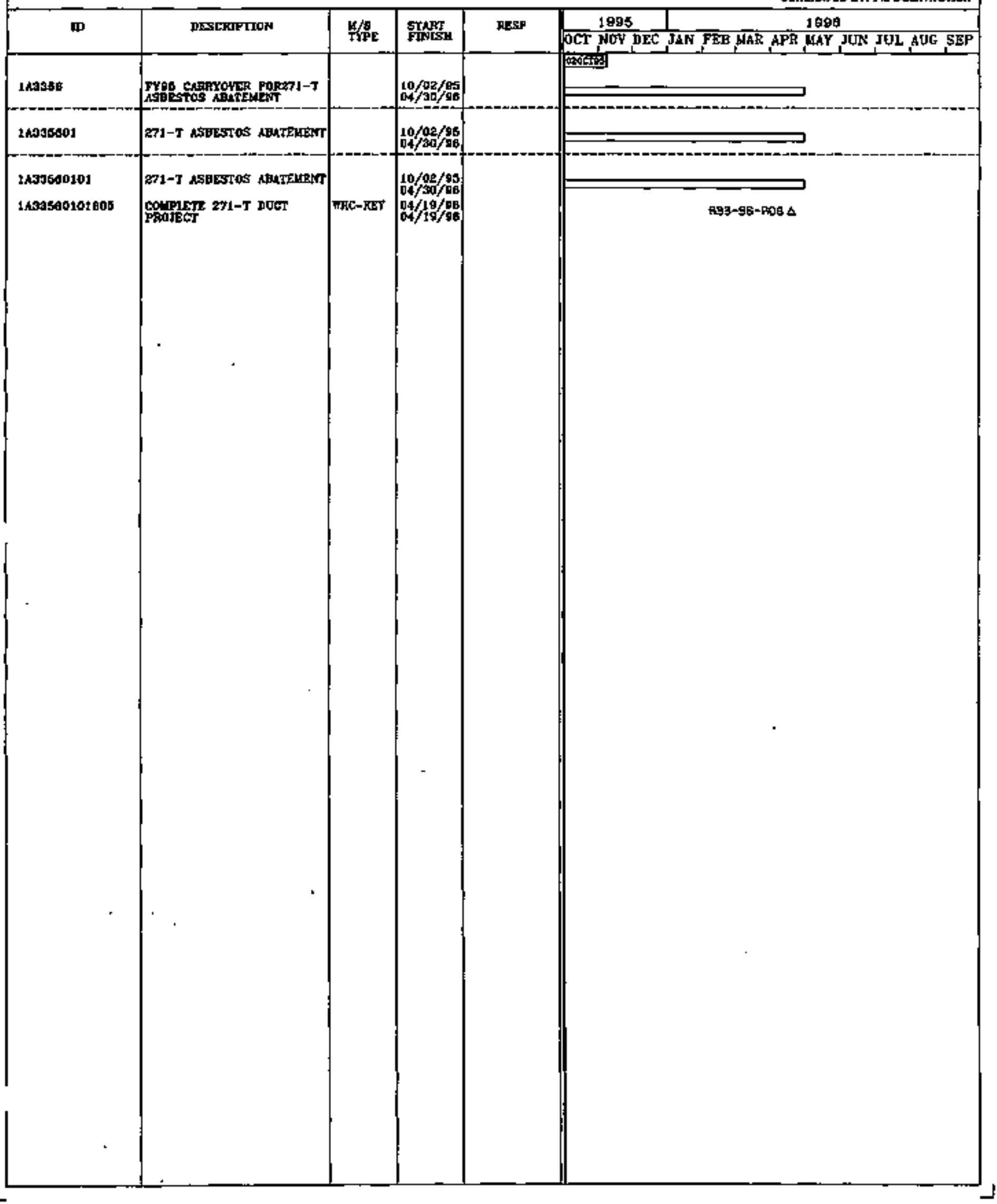




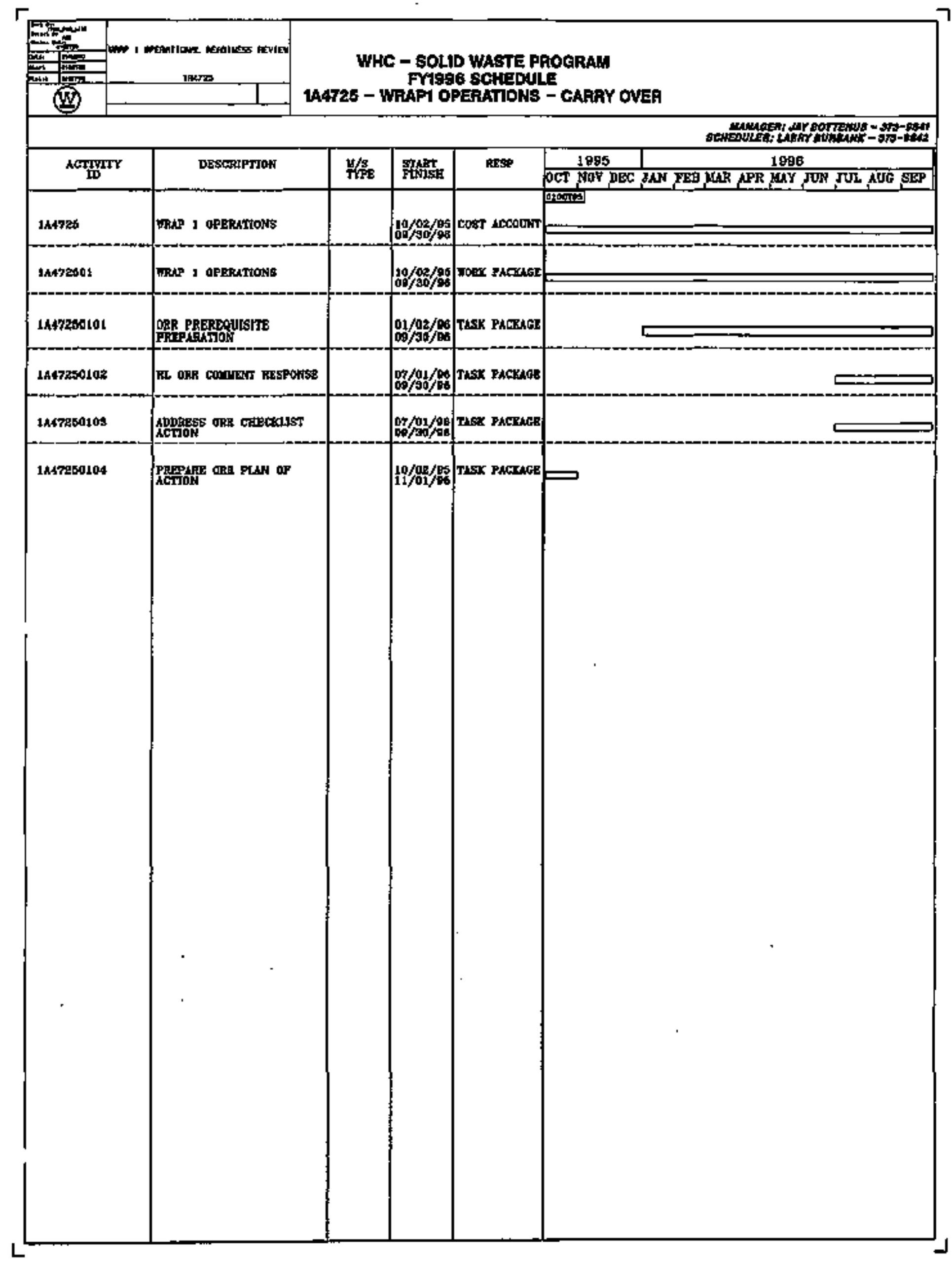




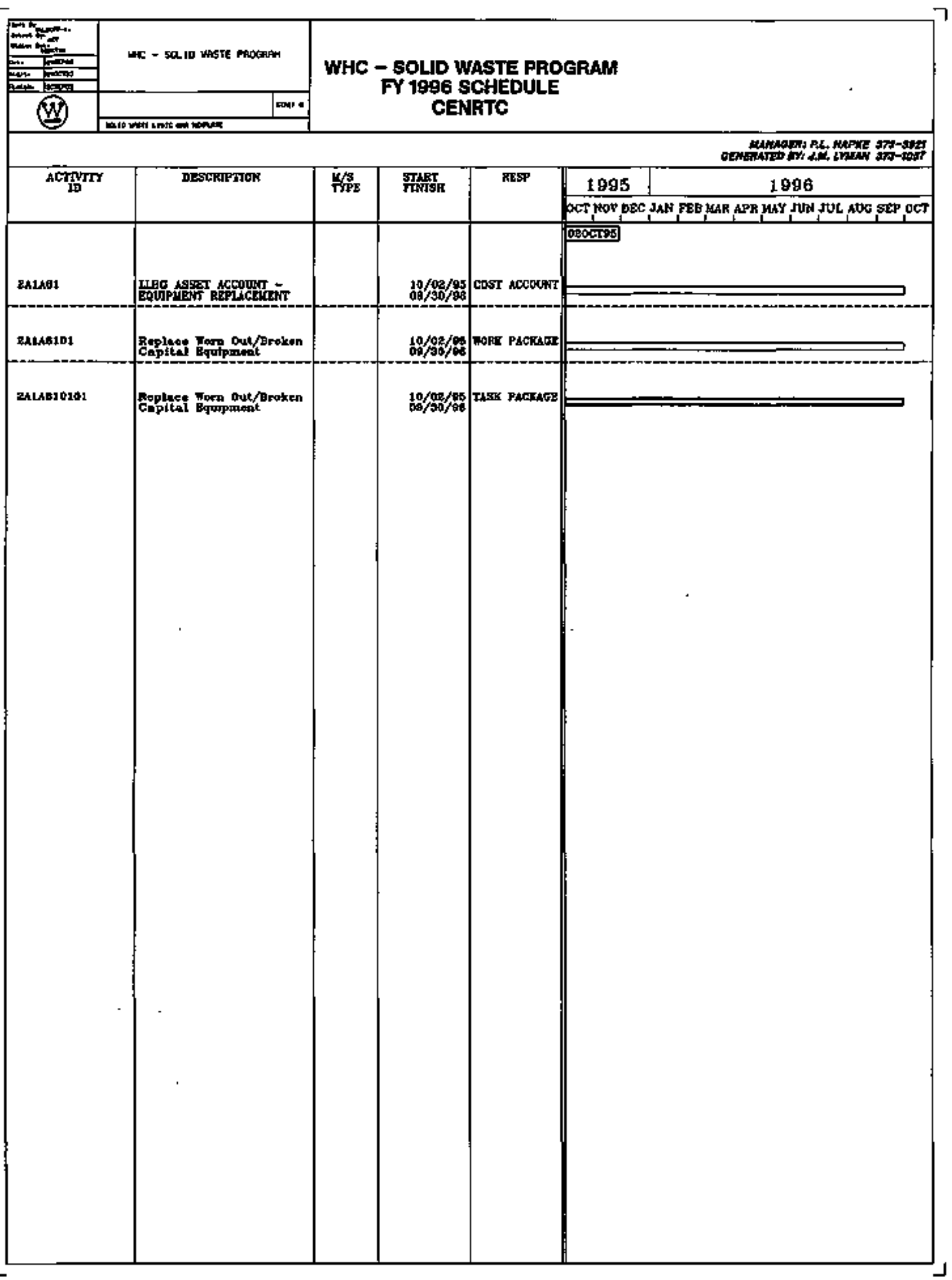


WHC - SOLID WASTE PROCRAM

FY 1996 SCHEDULE CENRTC

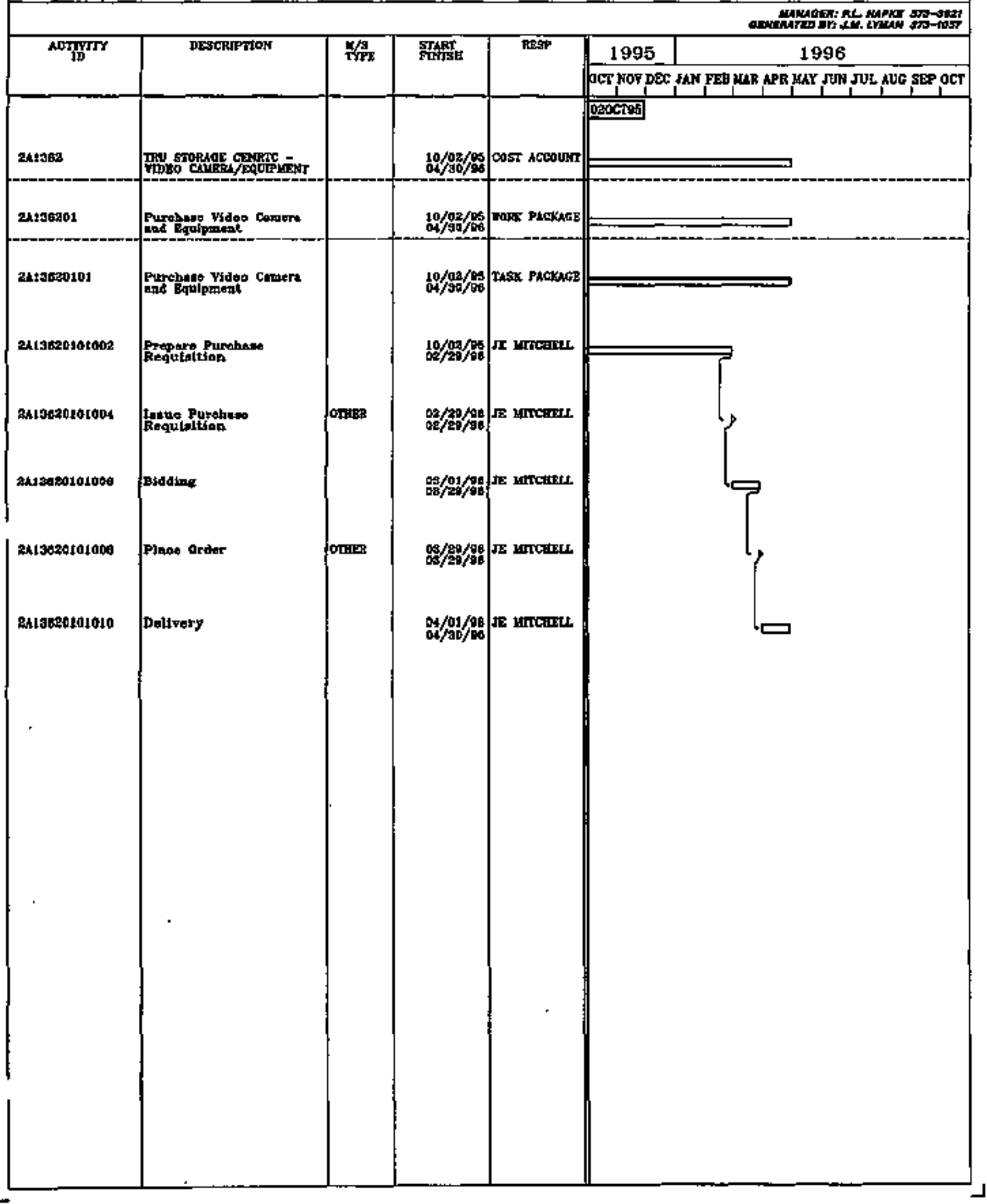


WHC - SOLID WASTE PROCRAM

FY 1996 SCHEDULE

CENRTC

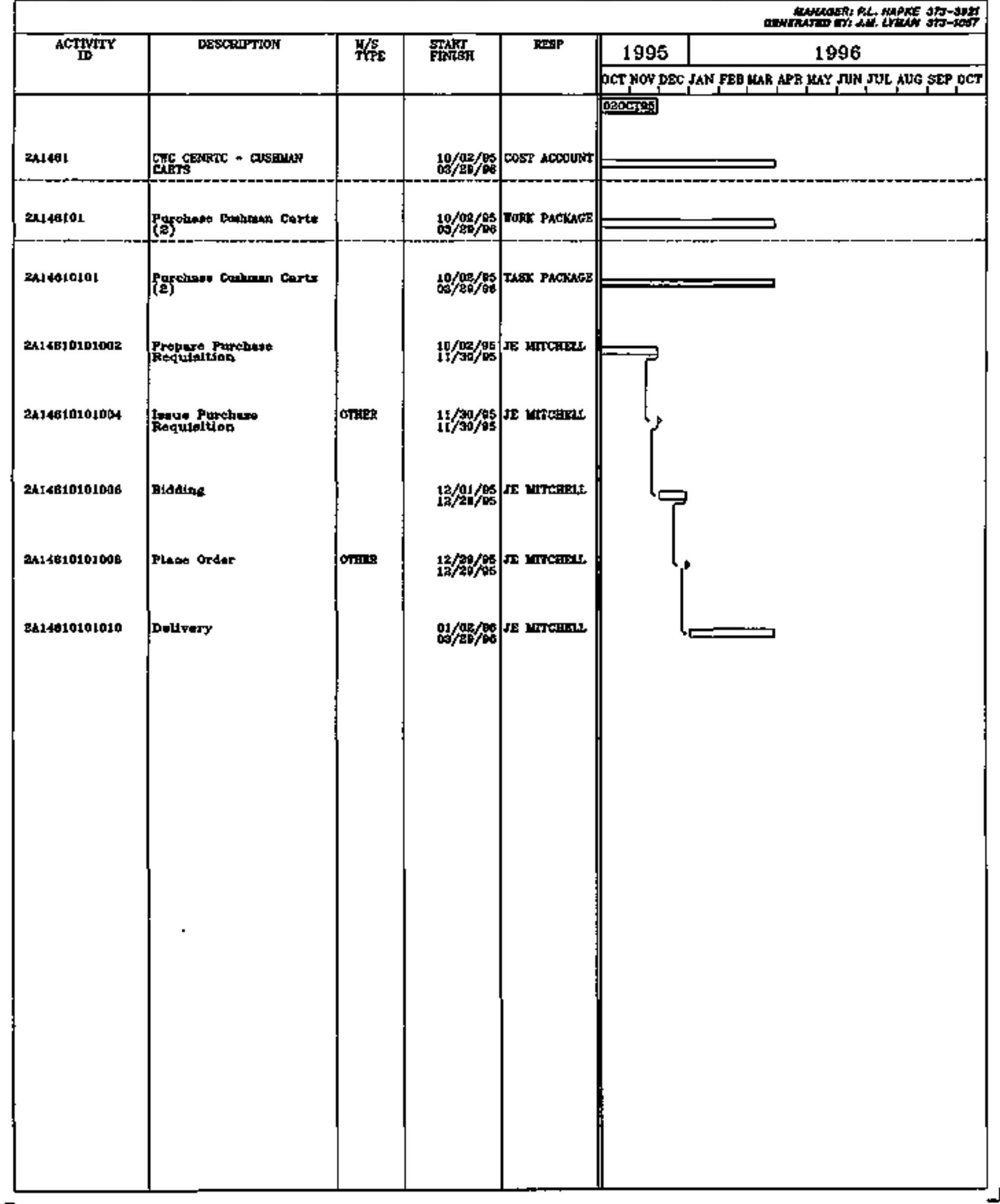


$\Gamma$

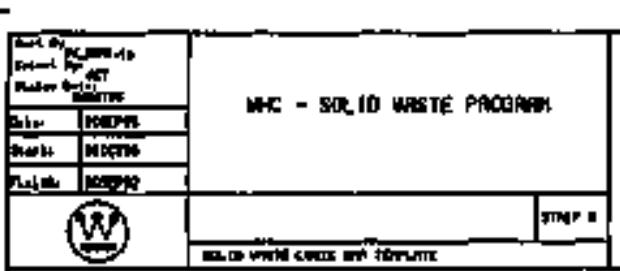

WHC - SOLID WASTE PROGRAN

FY 1996 SCHEDULE

CENRTC

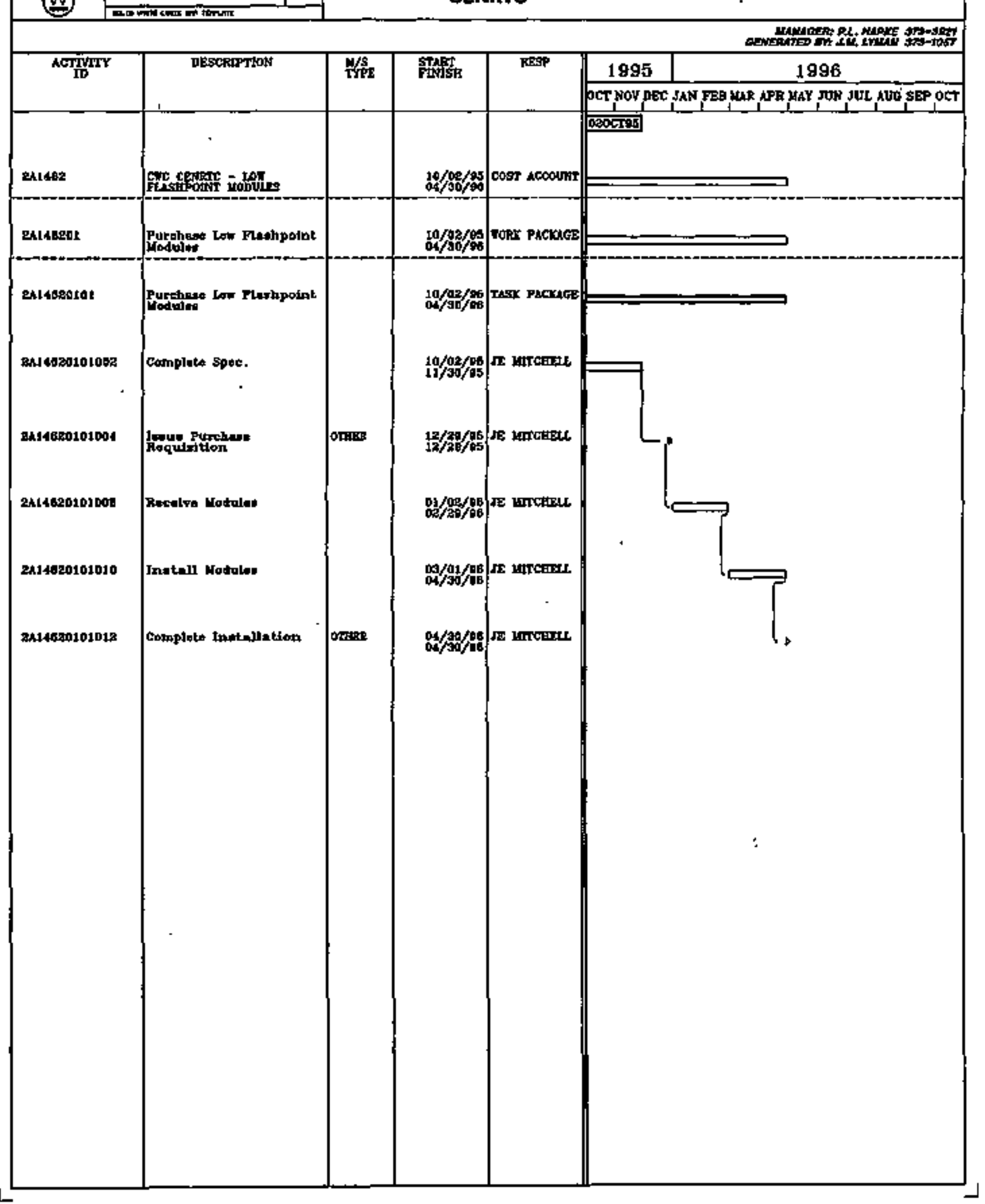


WHC - SOLID WASTE PROERAM

FY 1996 SCHEDULE CENRTC

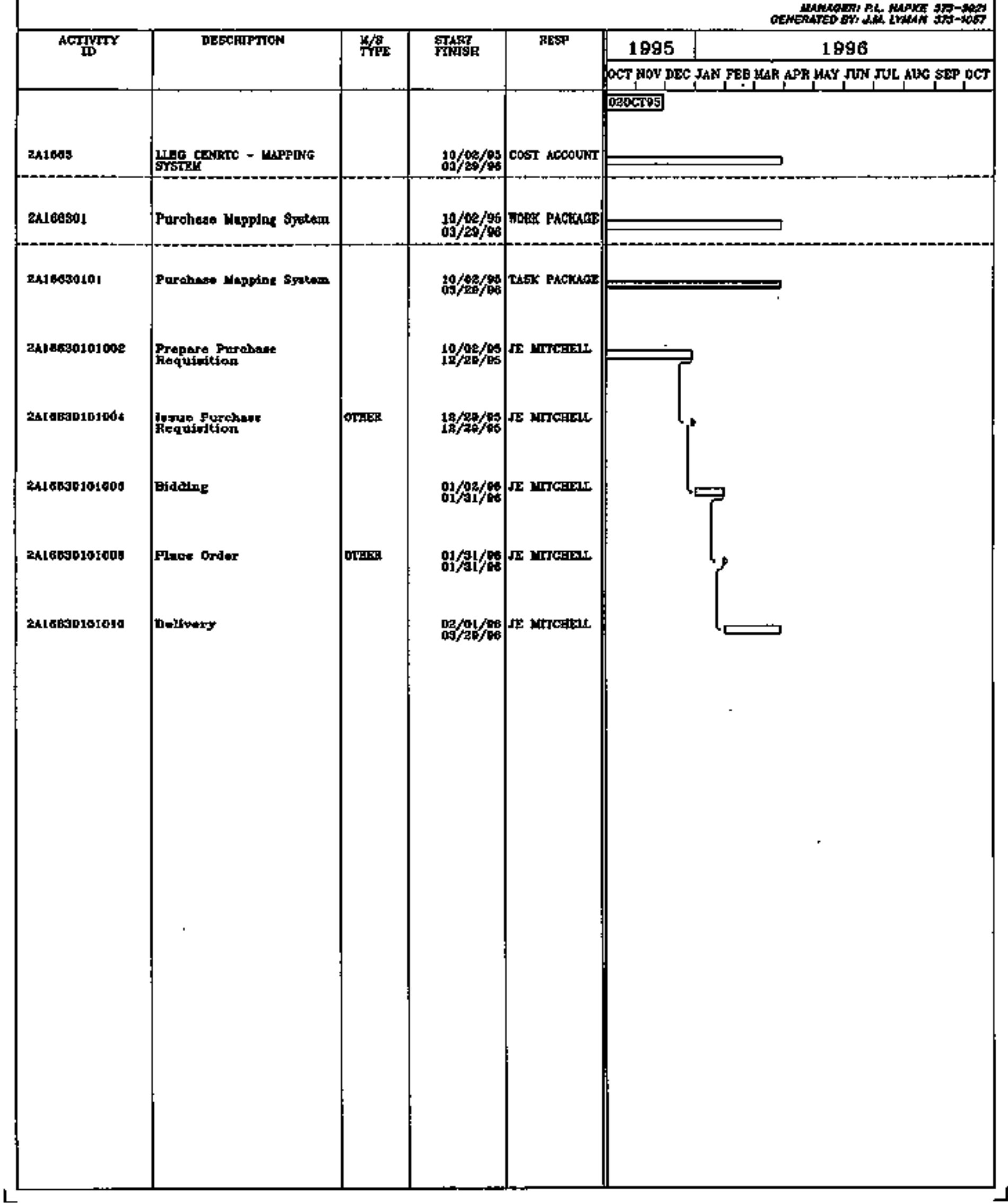




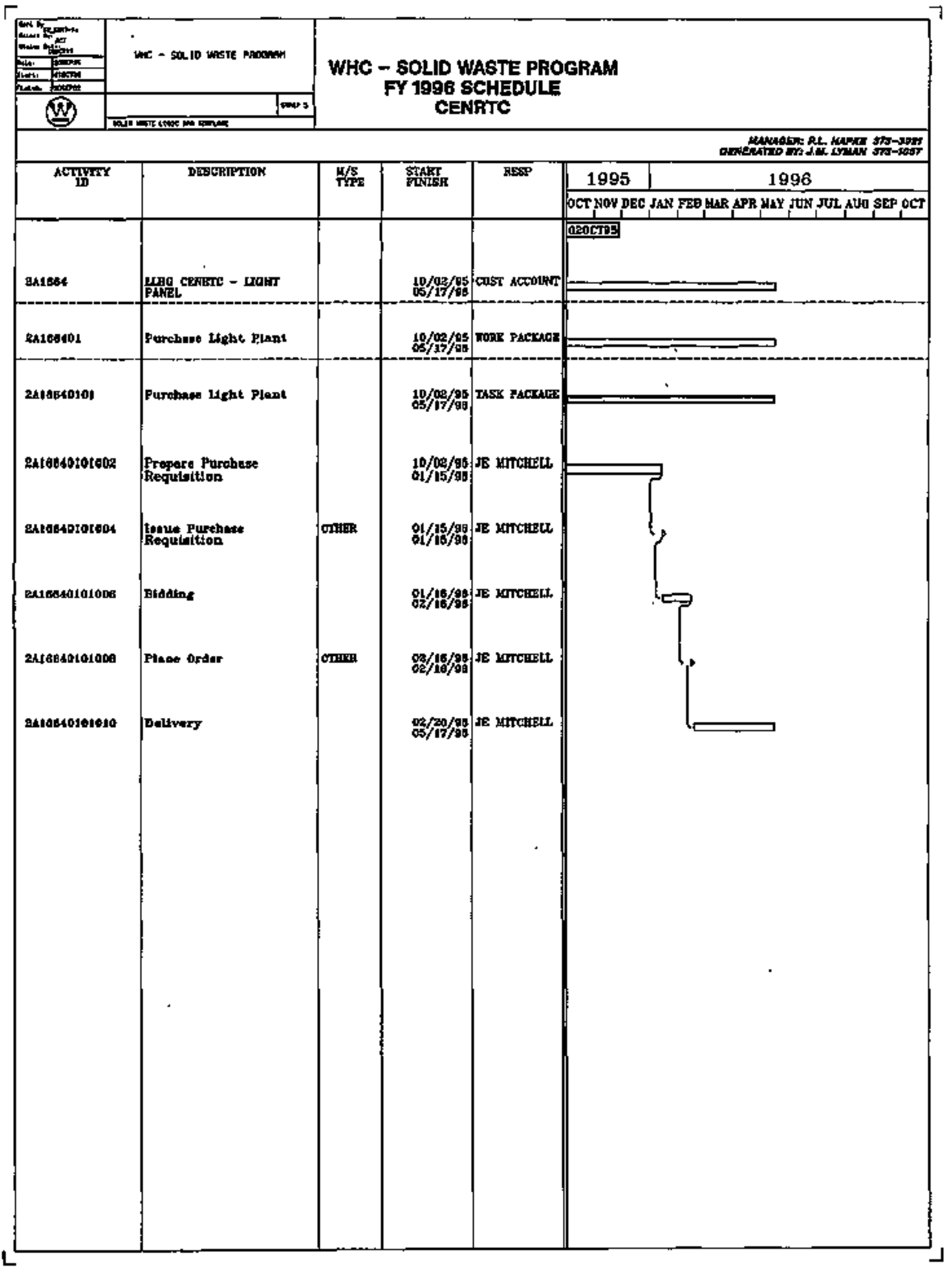



FY 1996 SCHEDULE CENRTC

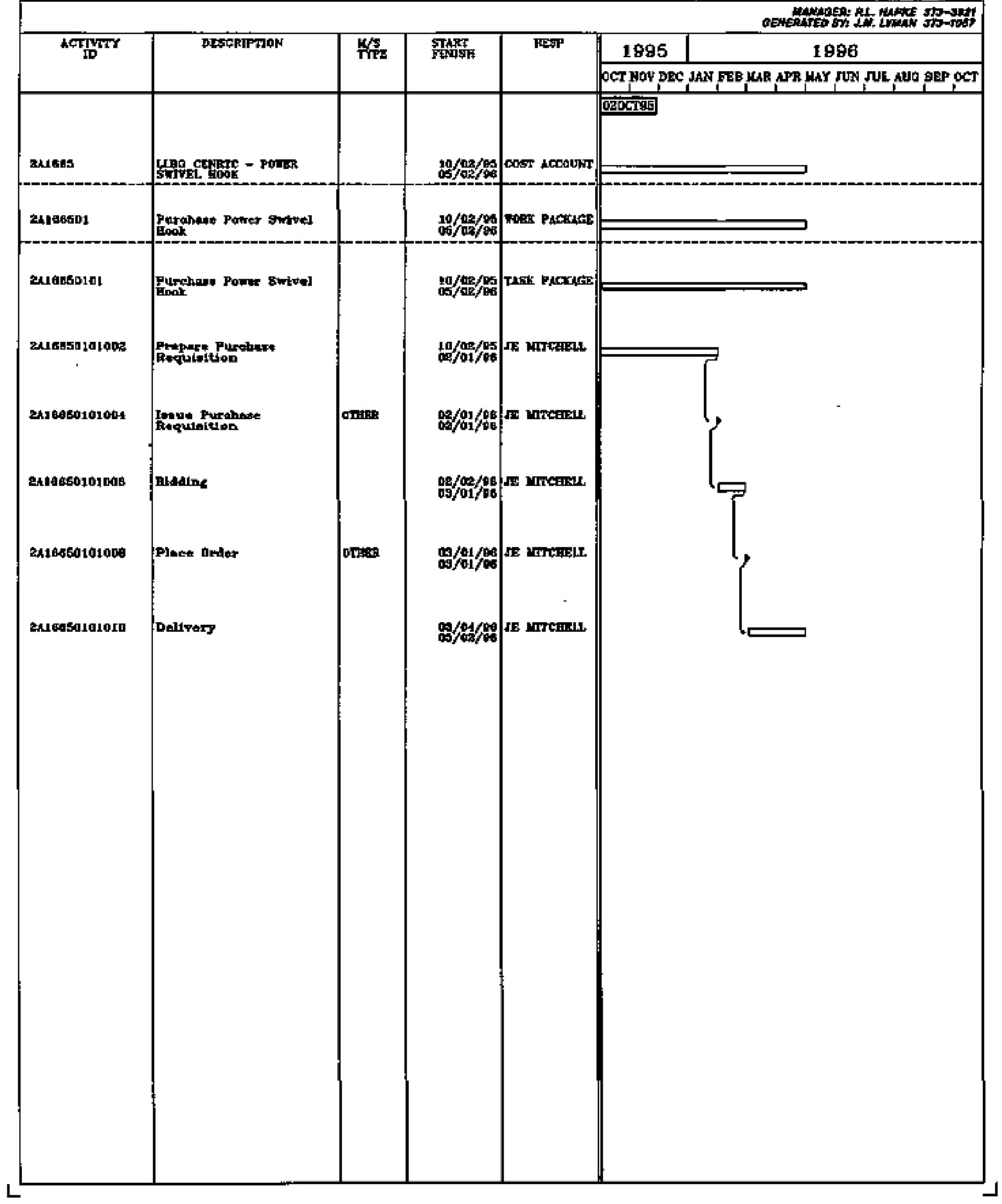




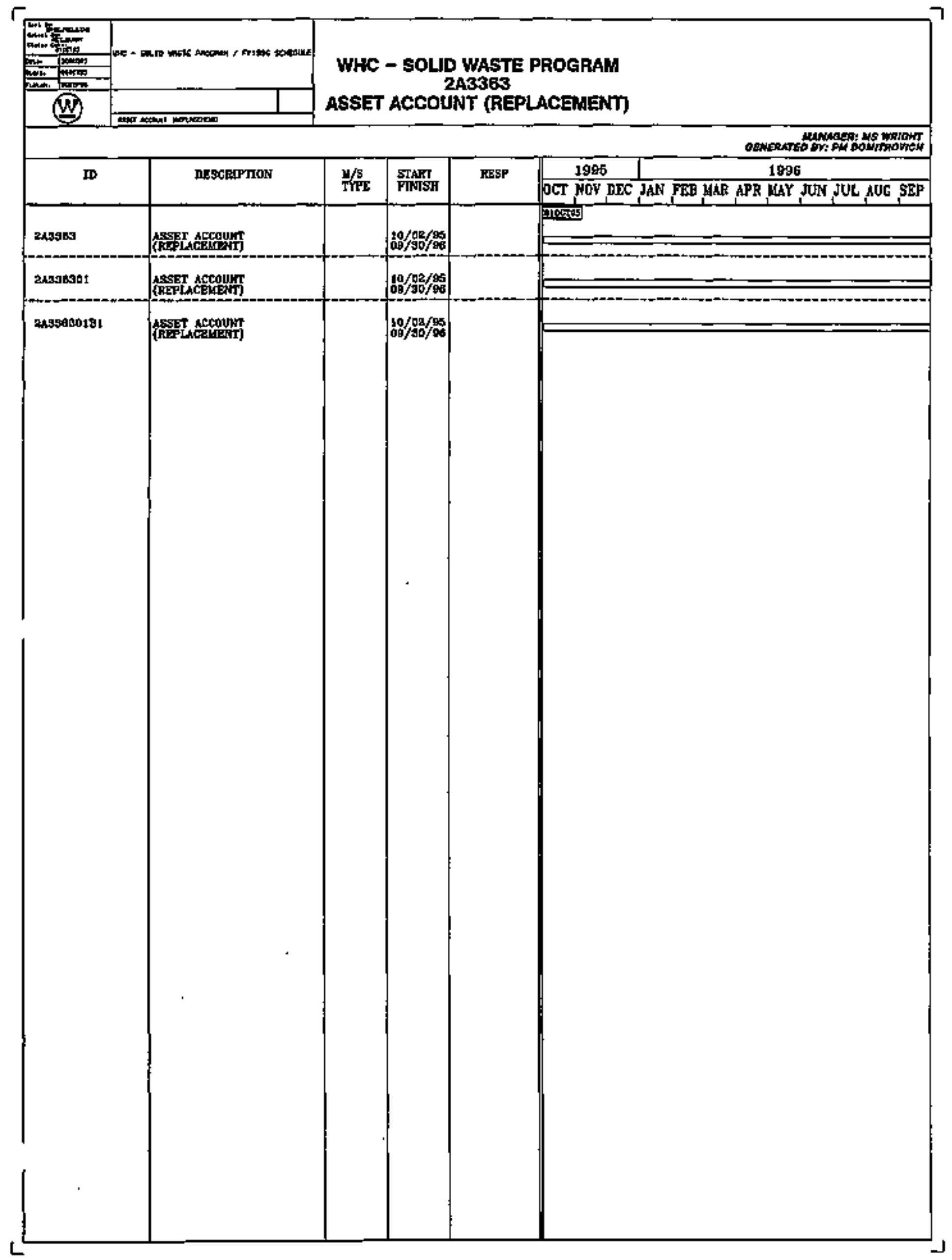


$\Gamma$

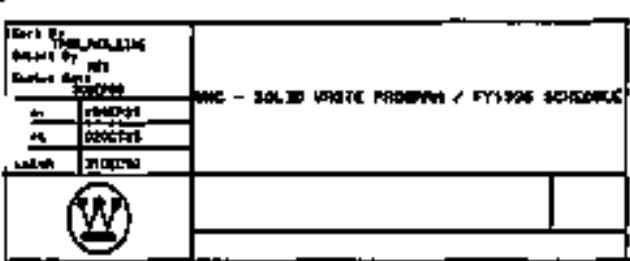

WHC - SOLID WASTE PROGRAM

FY1996 SCHEDULE

3A7BO1 - W-112 - LINE ITEM

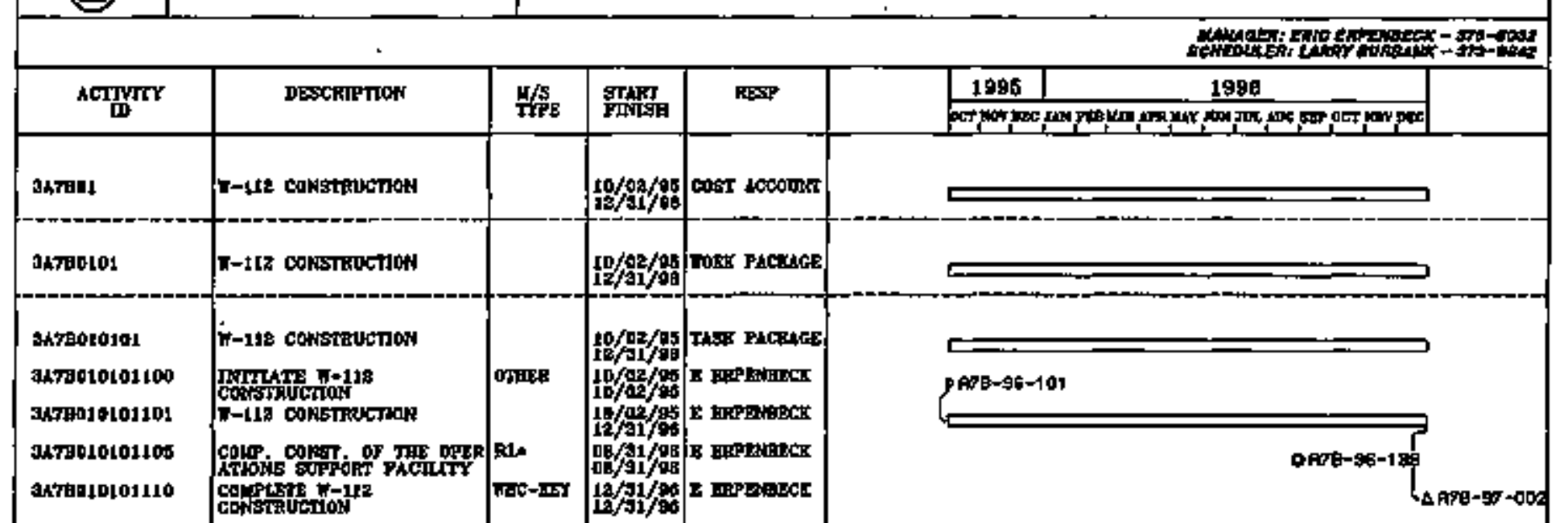

akthapol01110

cantrine t-142

तreter.

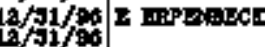




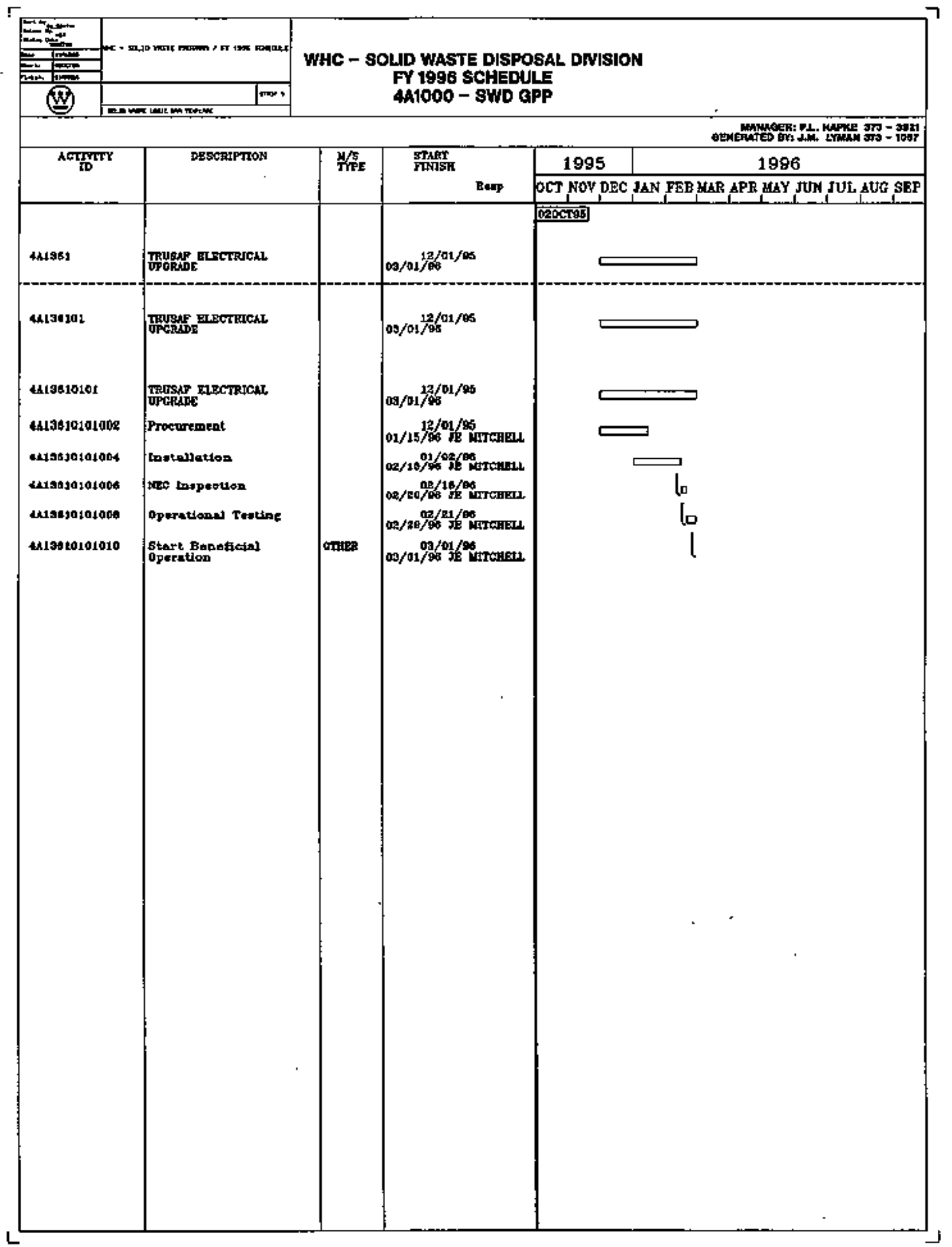


$4 A 1000$ - SWD EPP

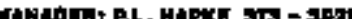

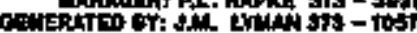

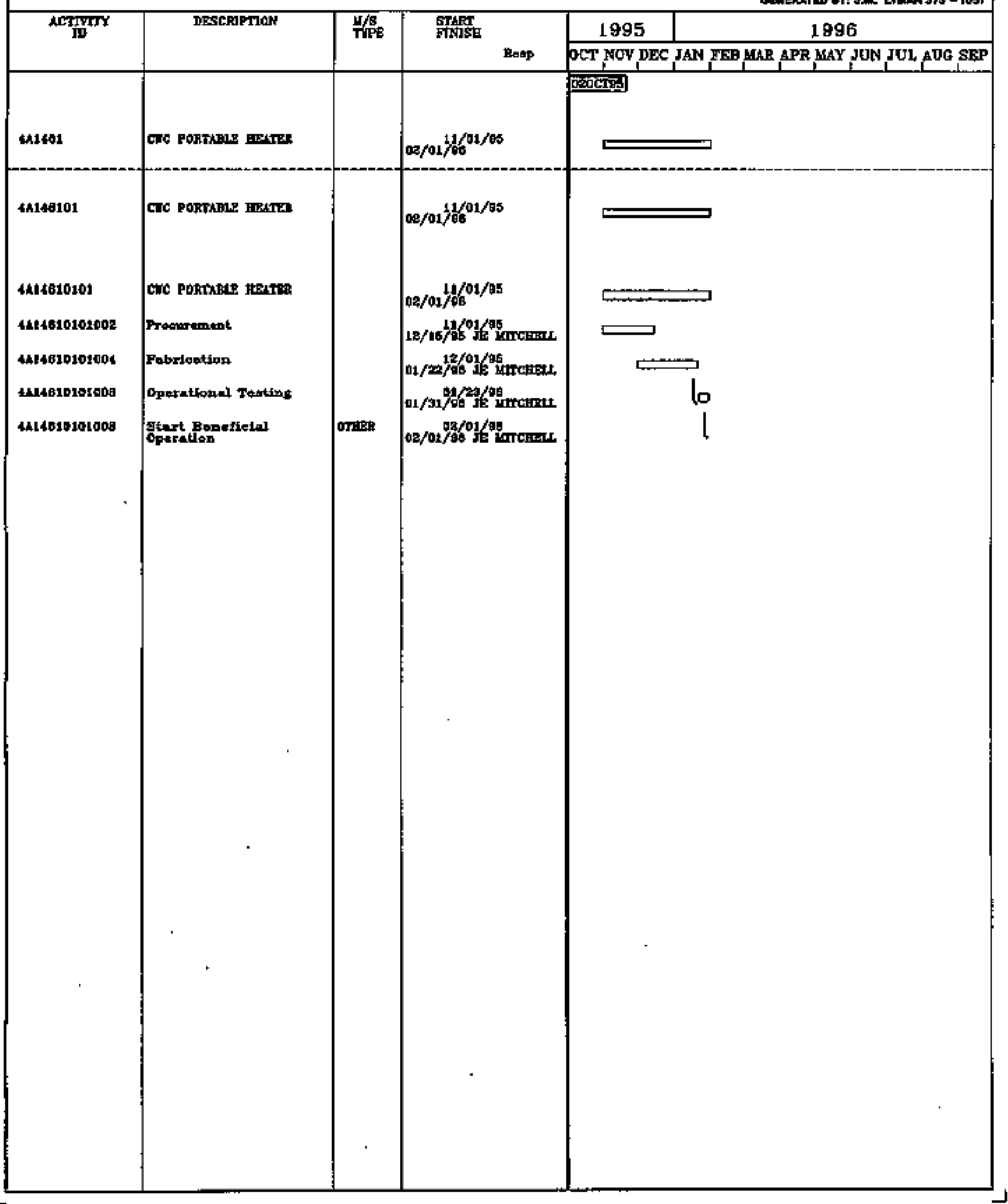




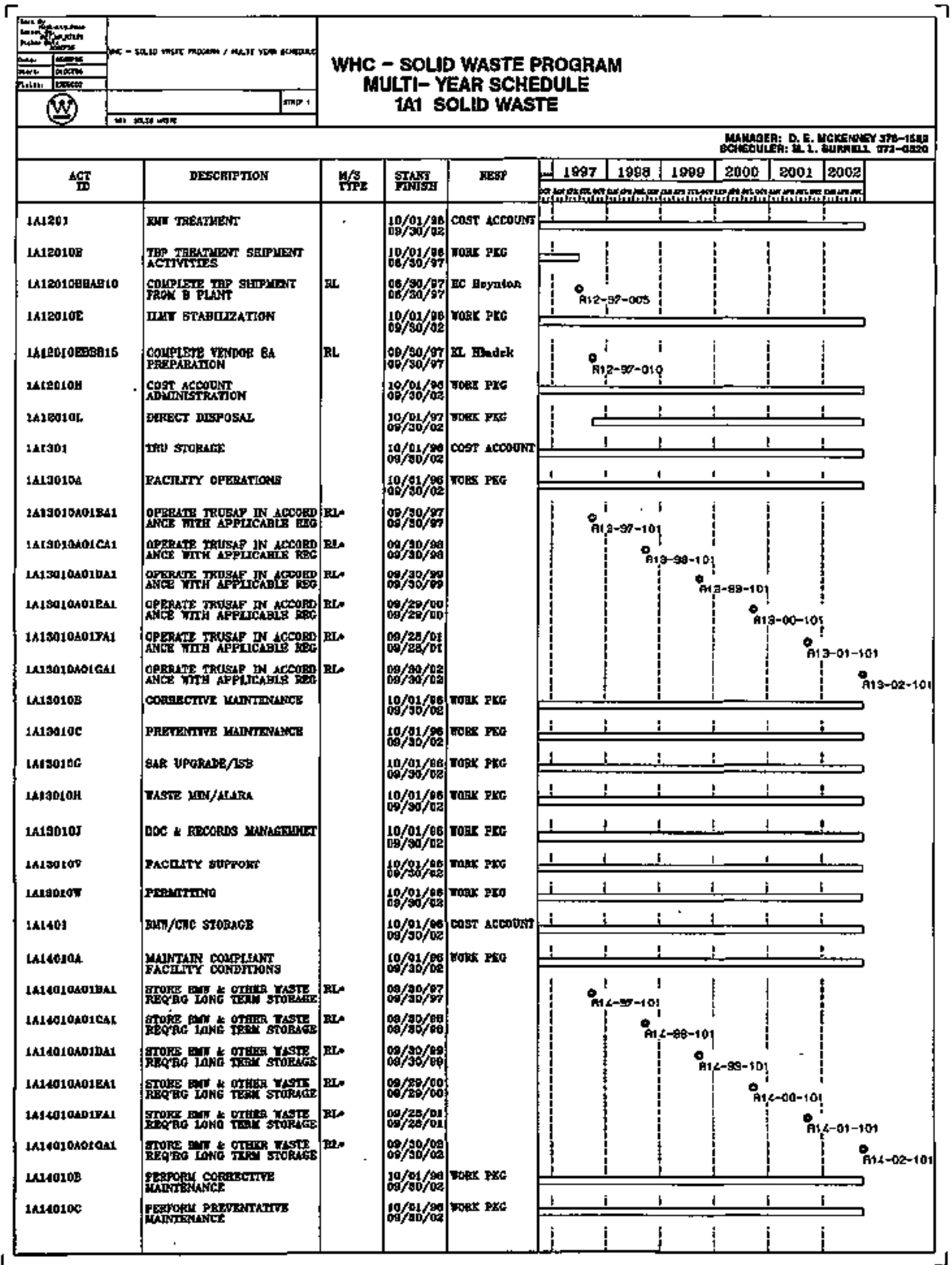




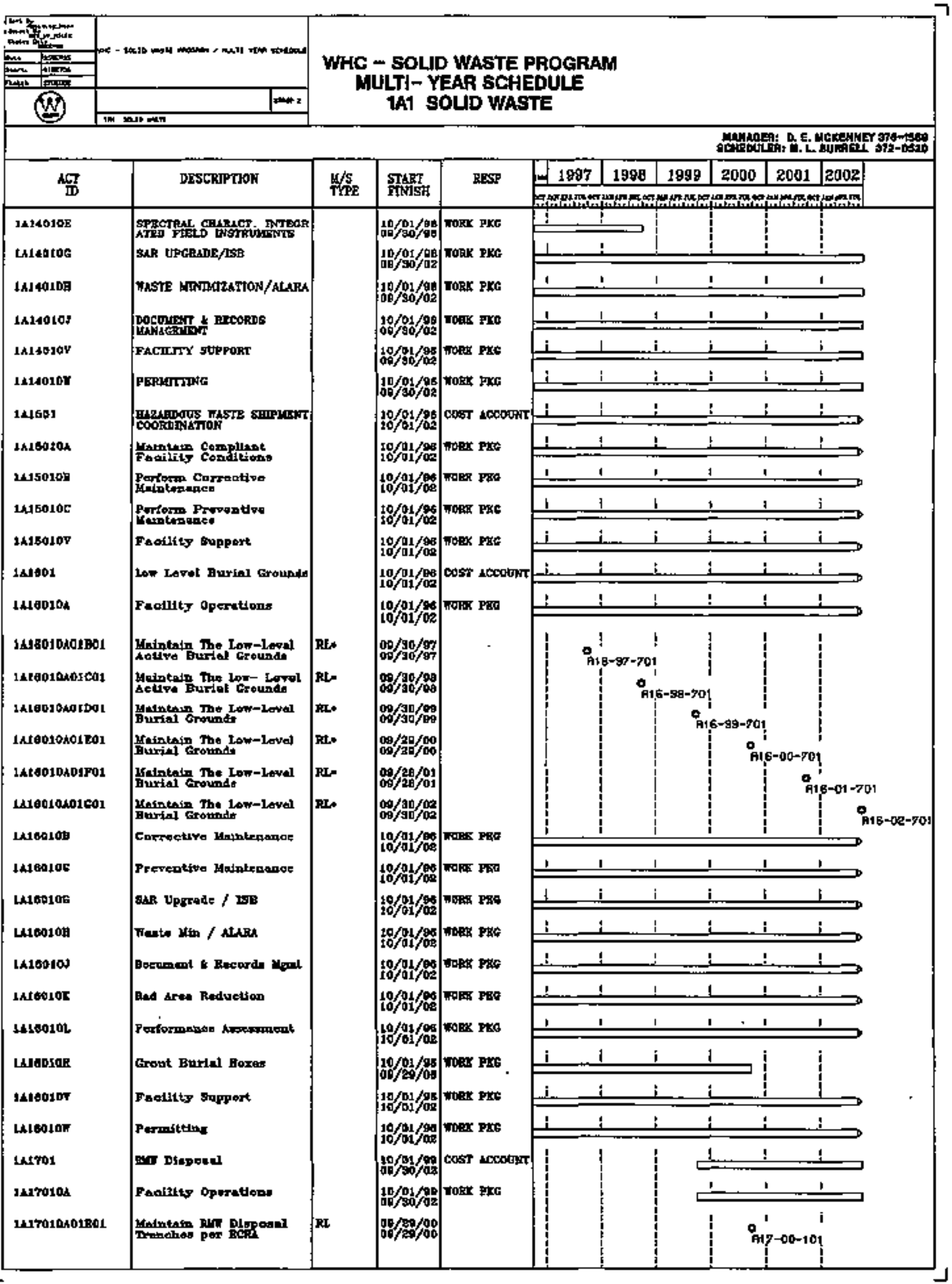




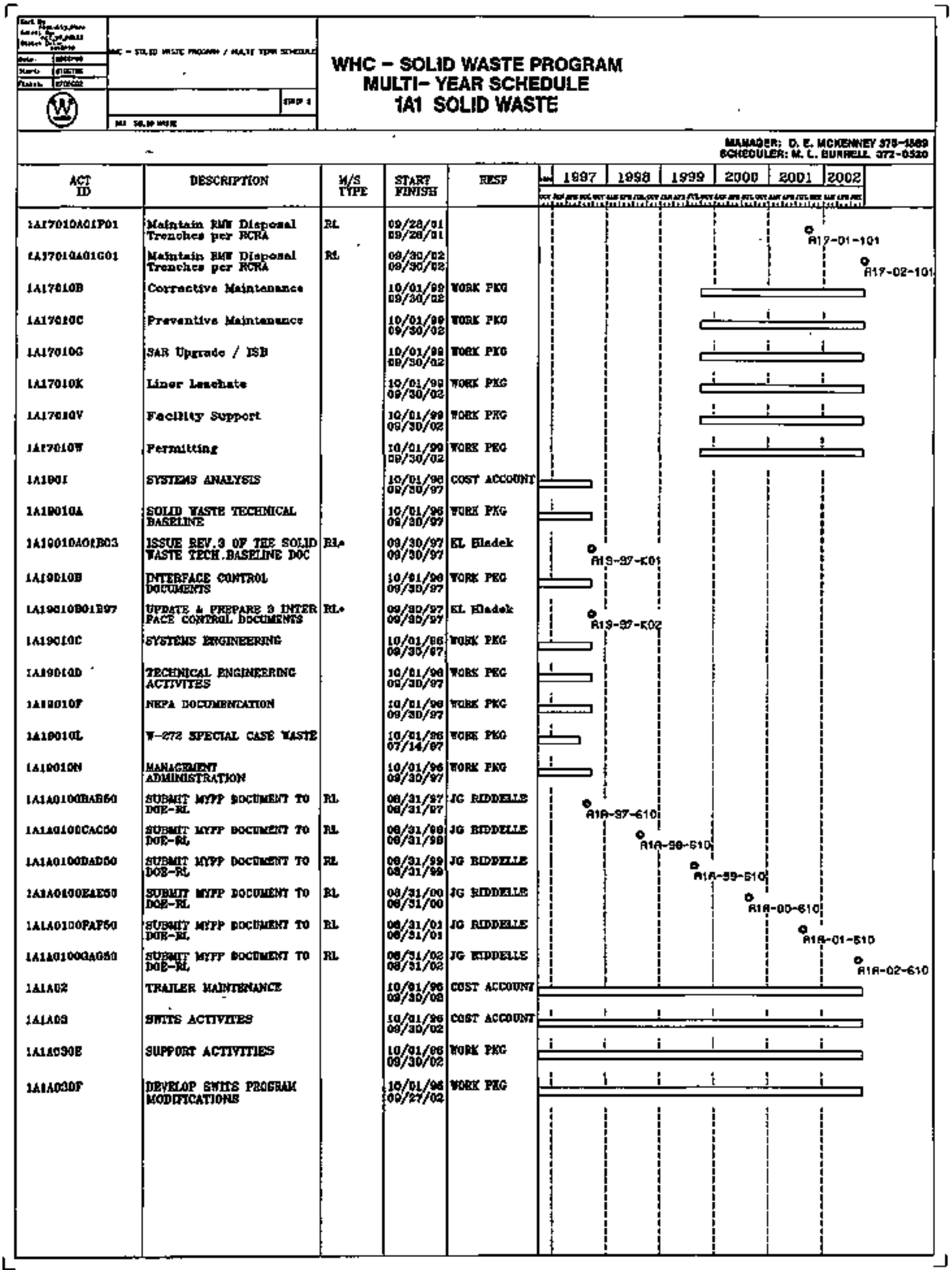




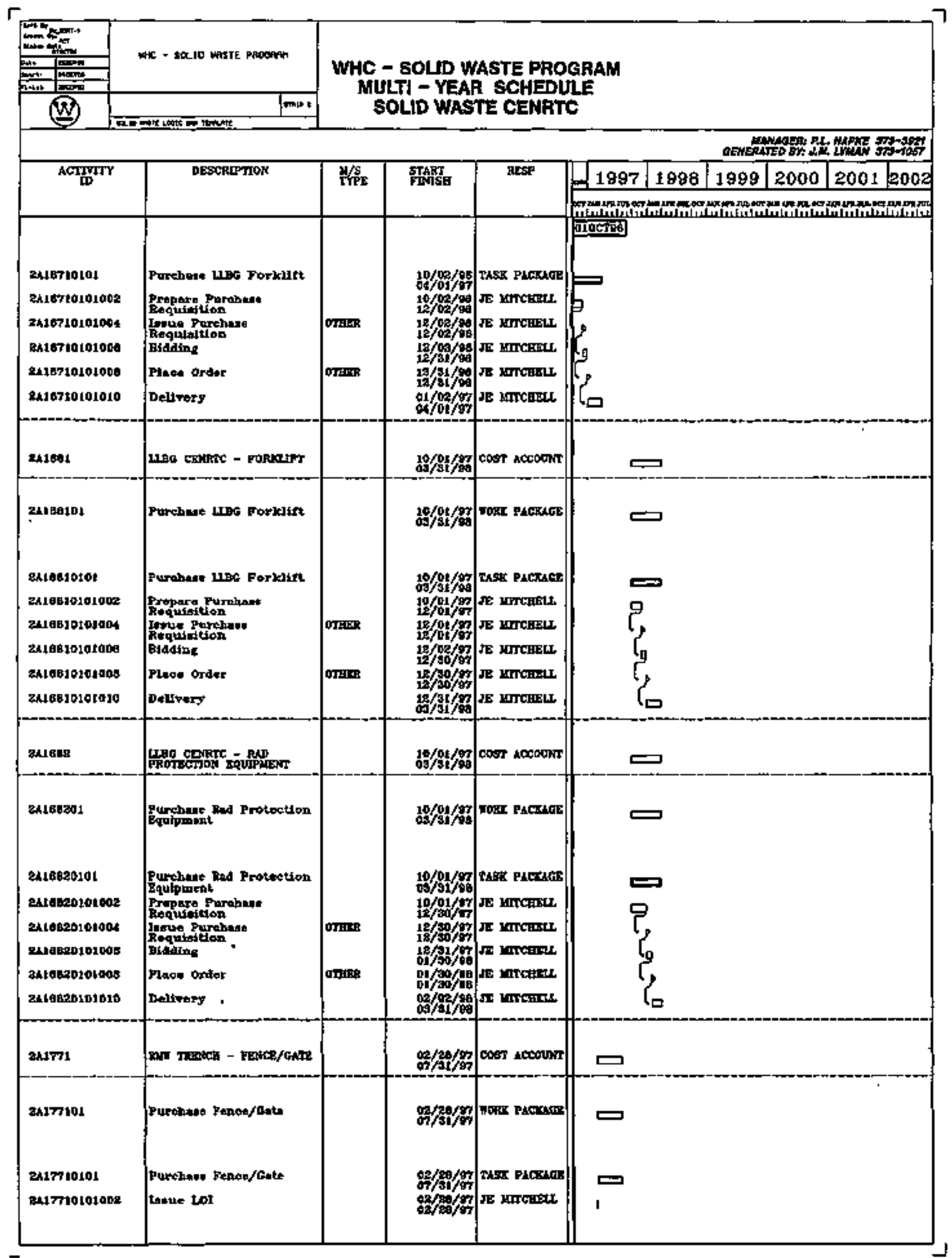



SOLID WASTE CENRTC

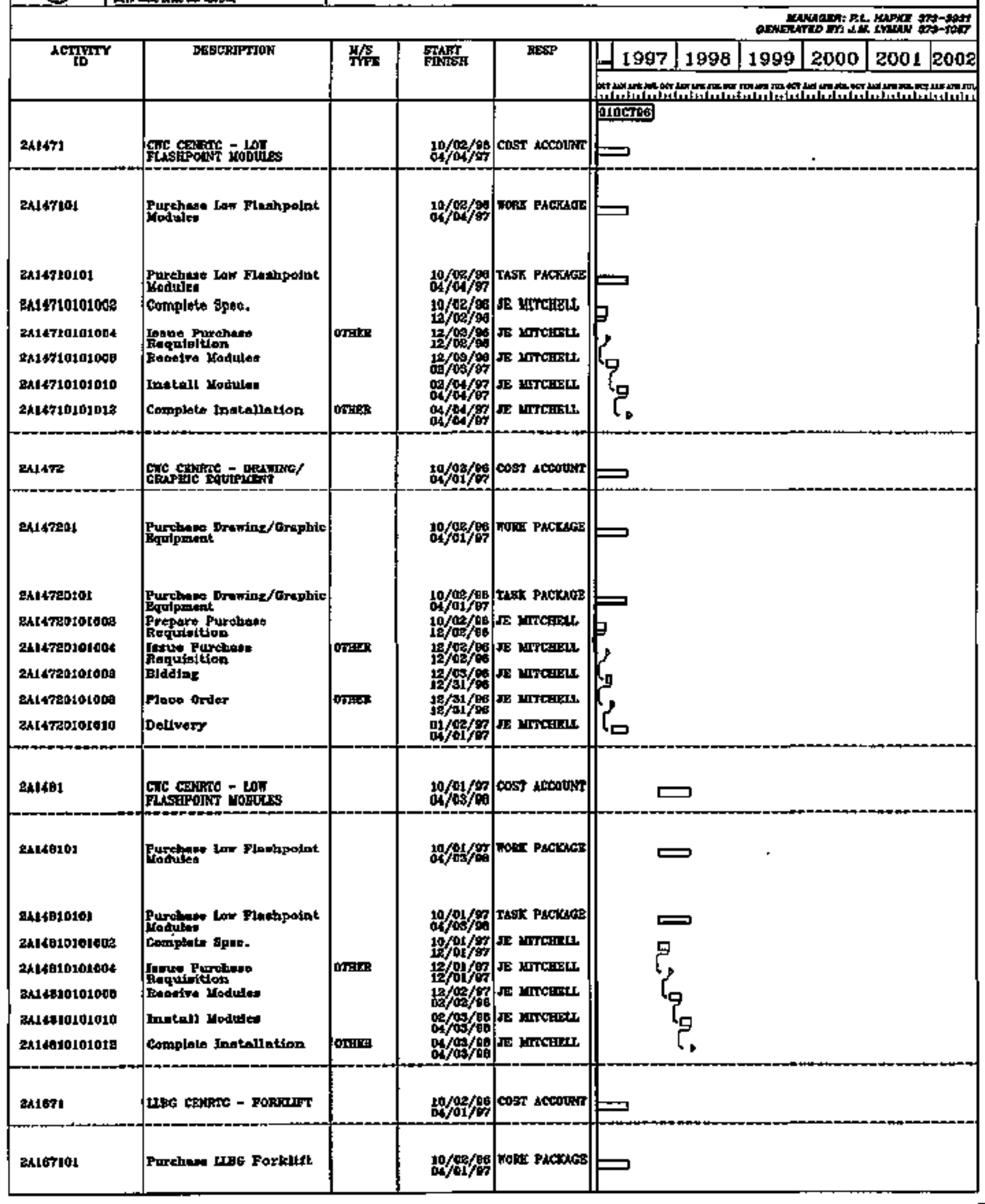




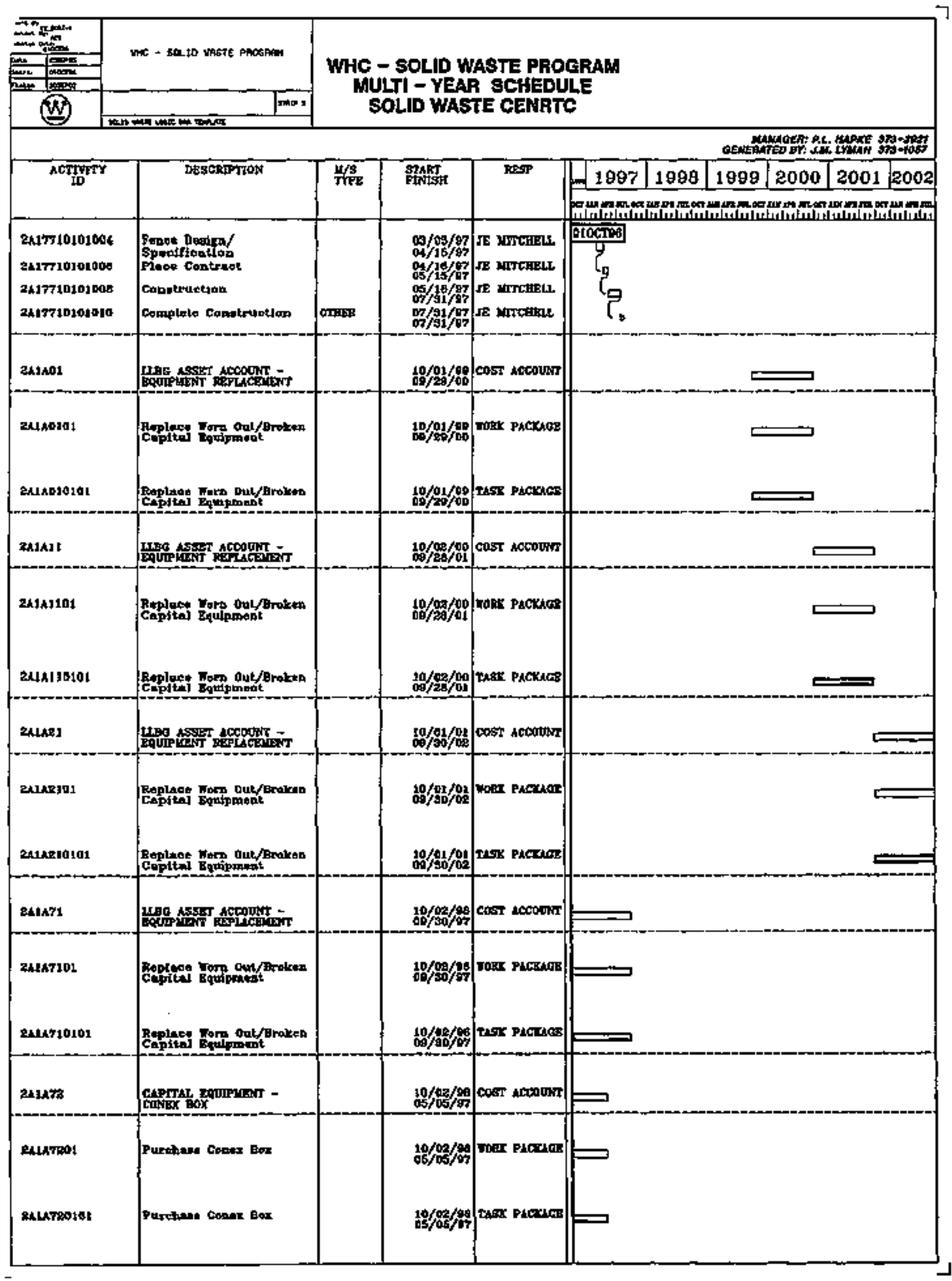




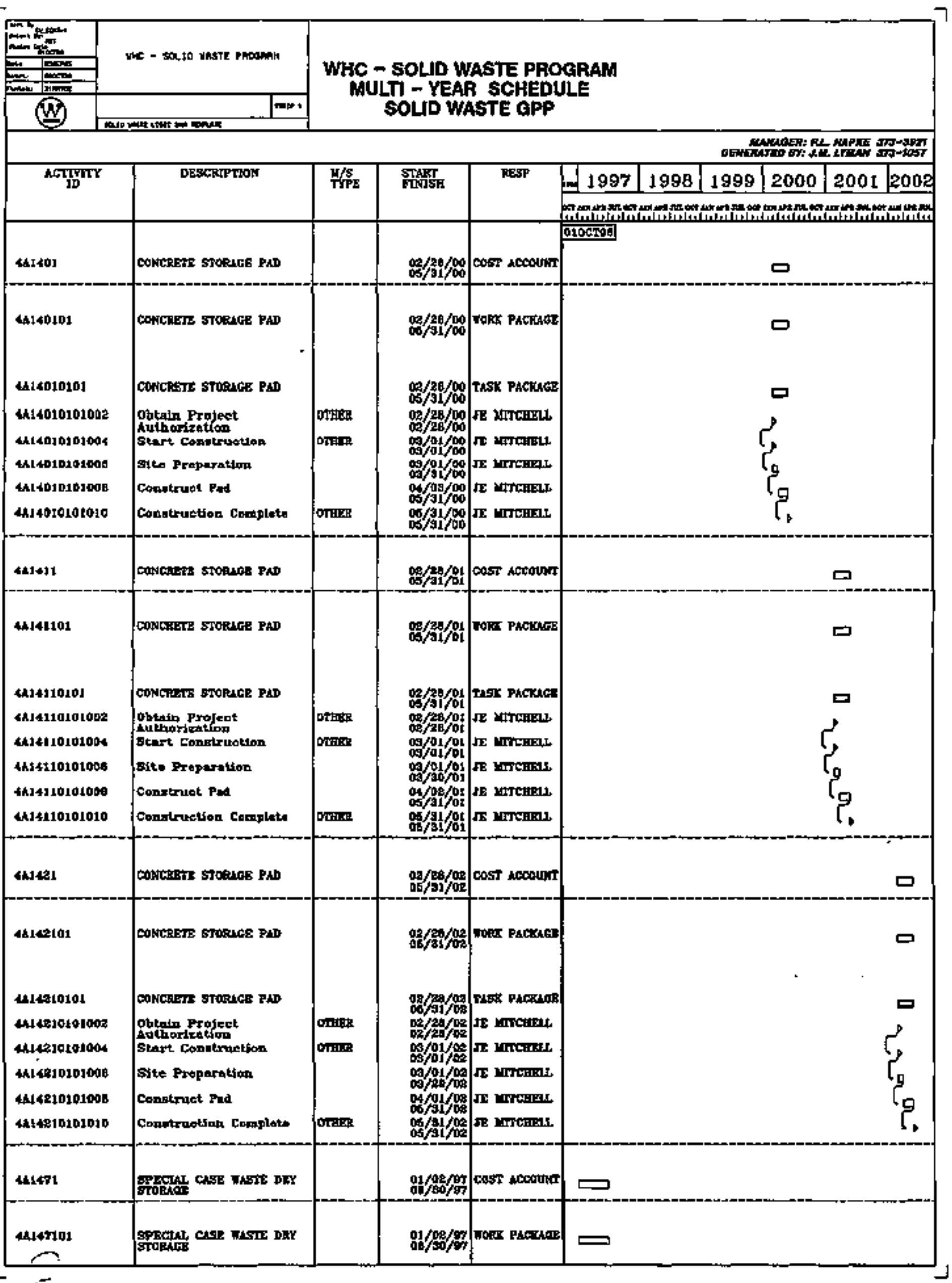




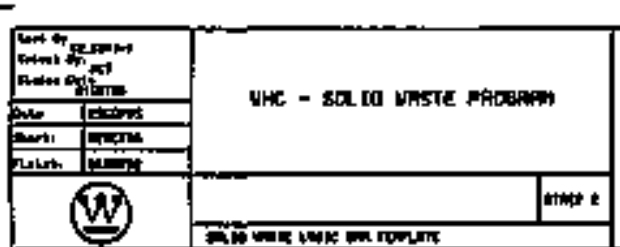

WHC - SOLID WASTE PROGRAM

MULT - YEAR SCHEDULE SOL1D WASTE GPP

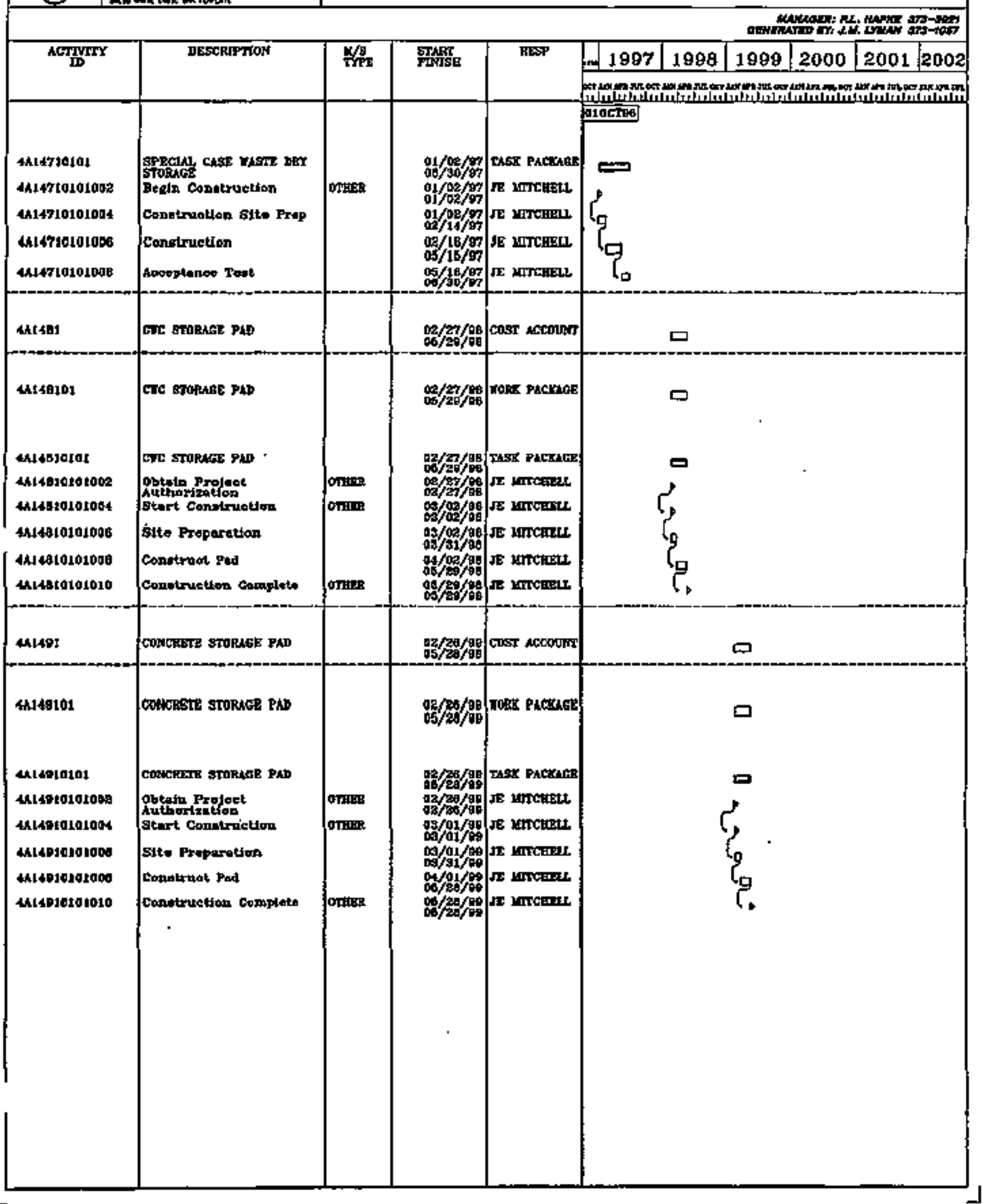




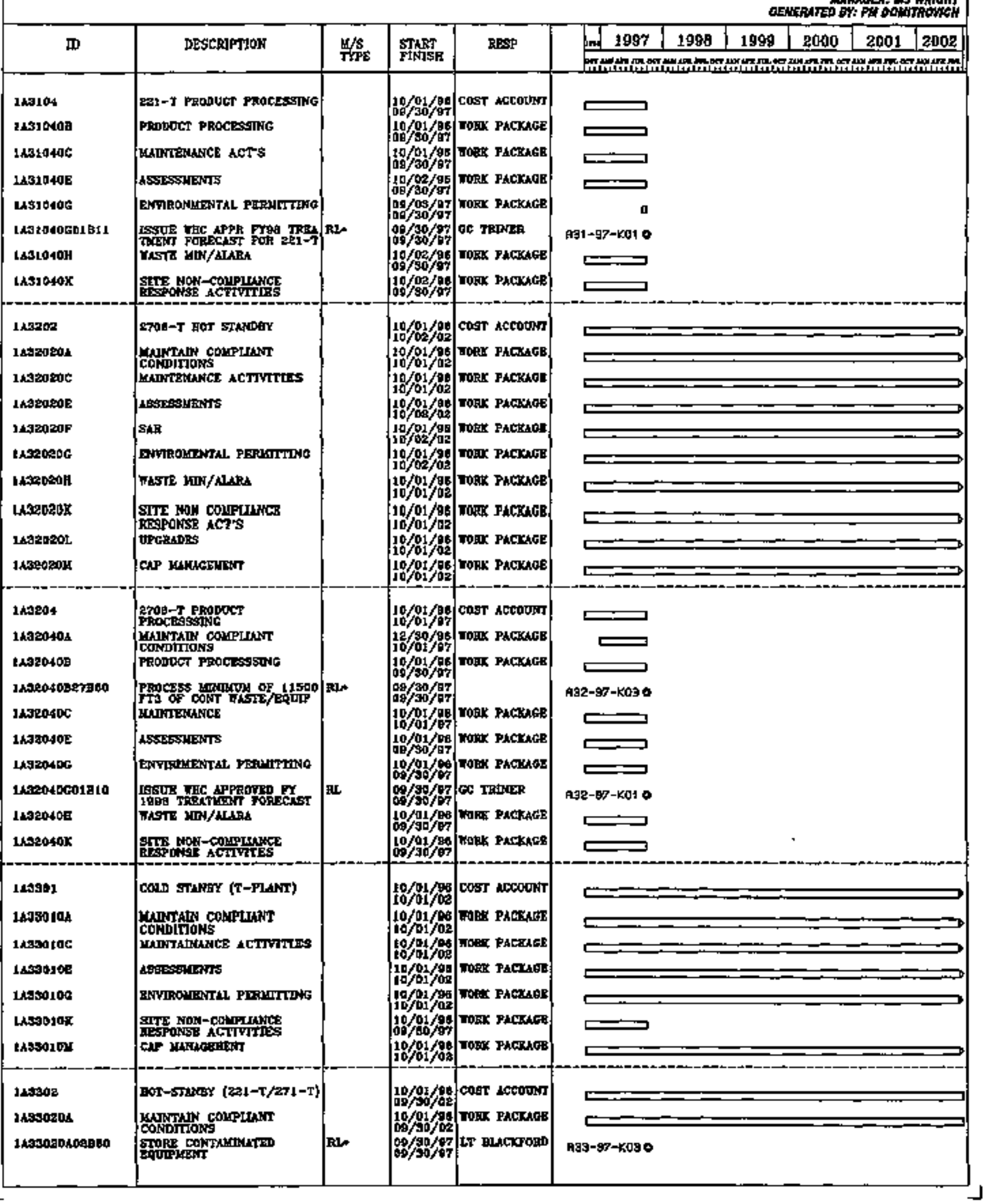




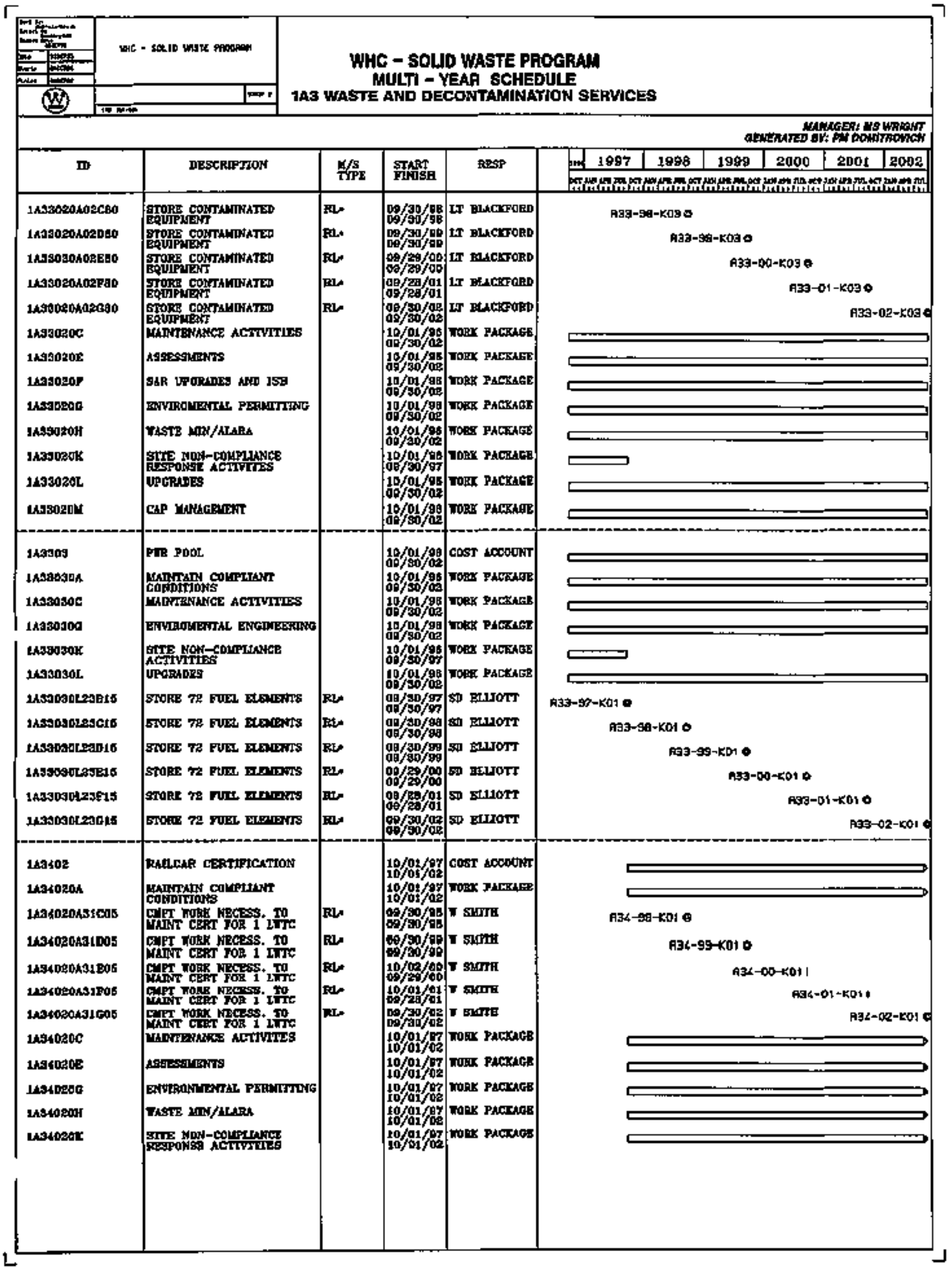




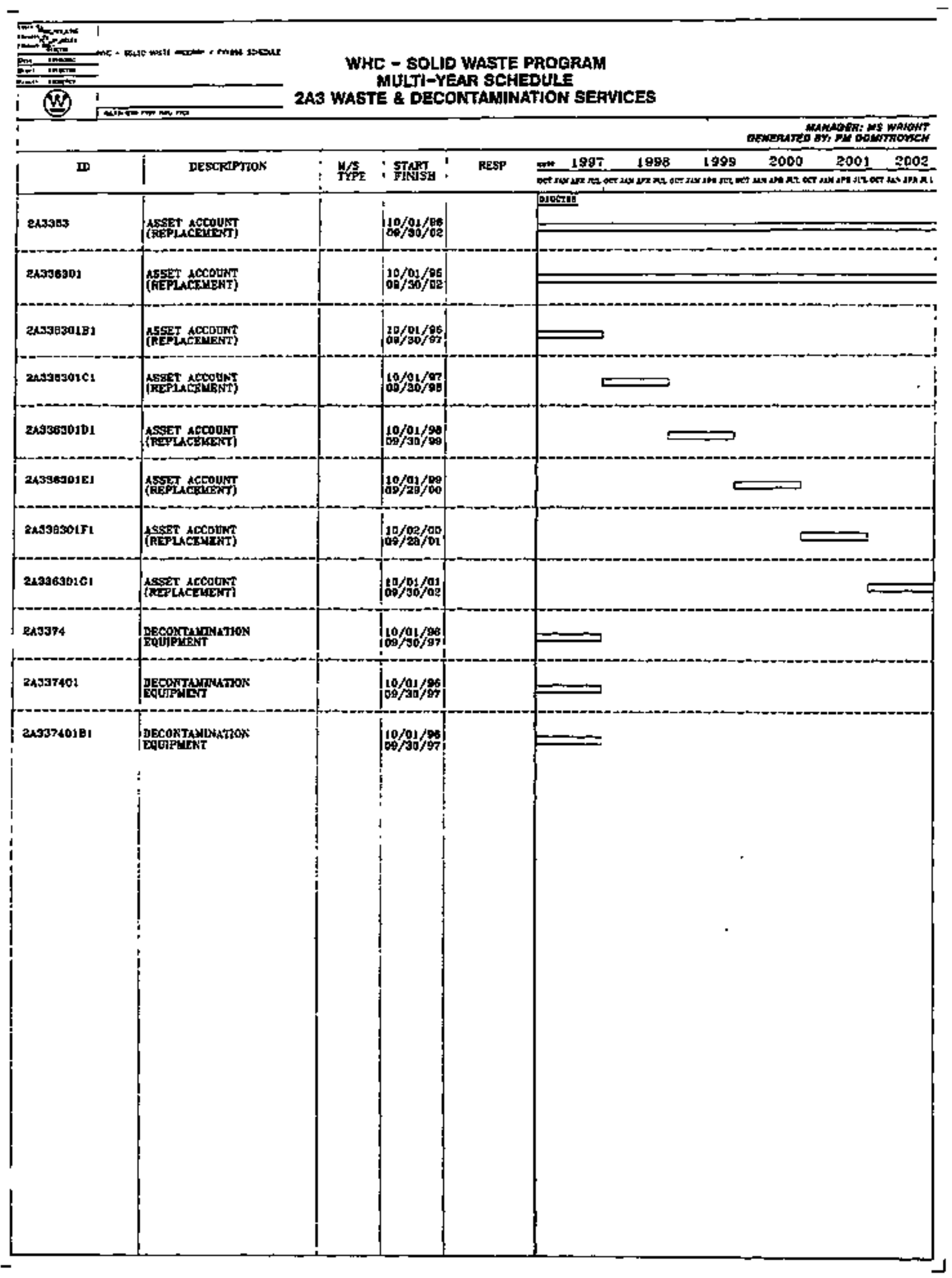




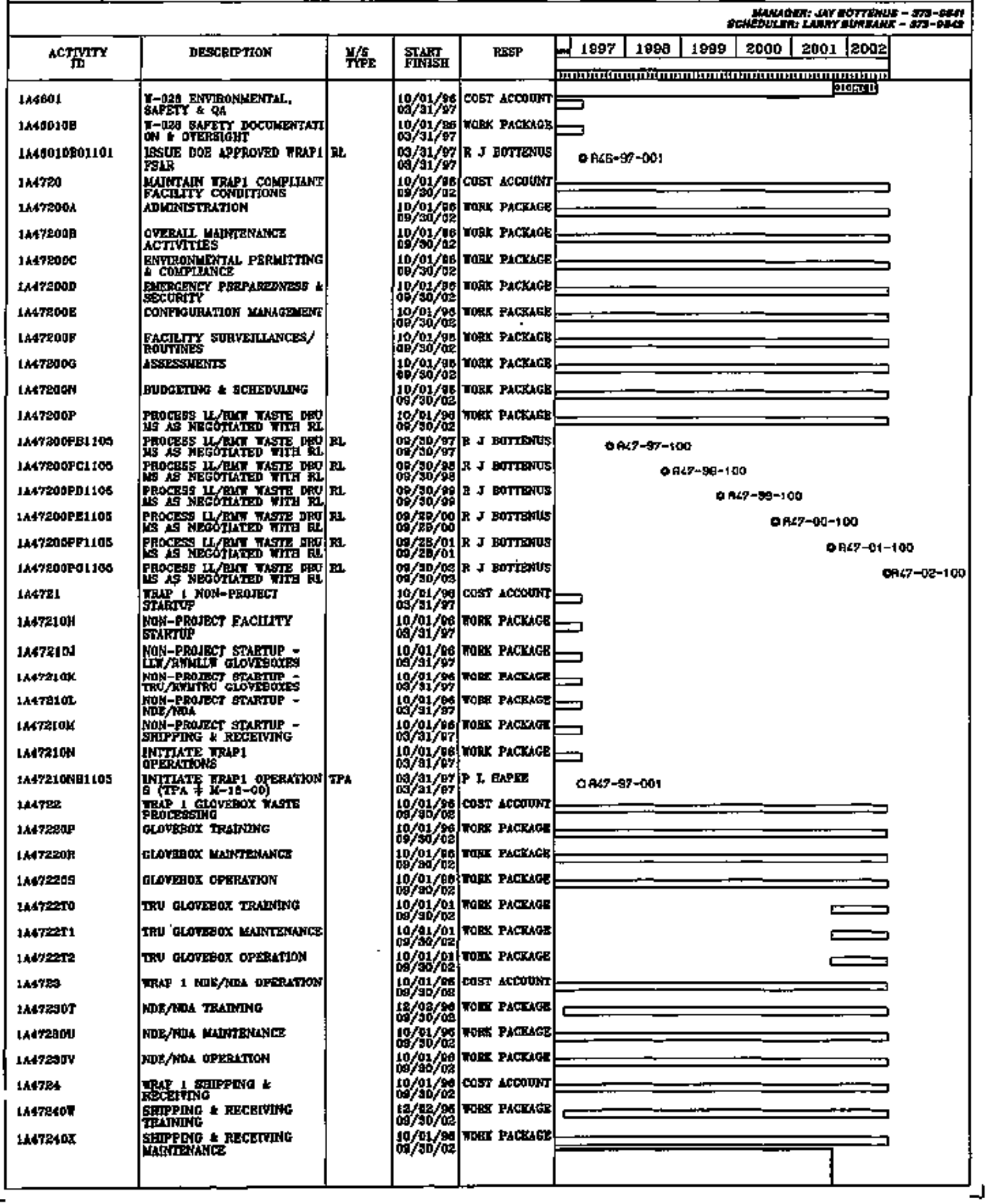


$\Gamma$

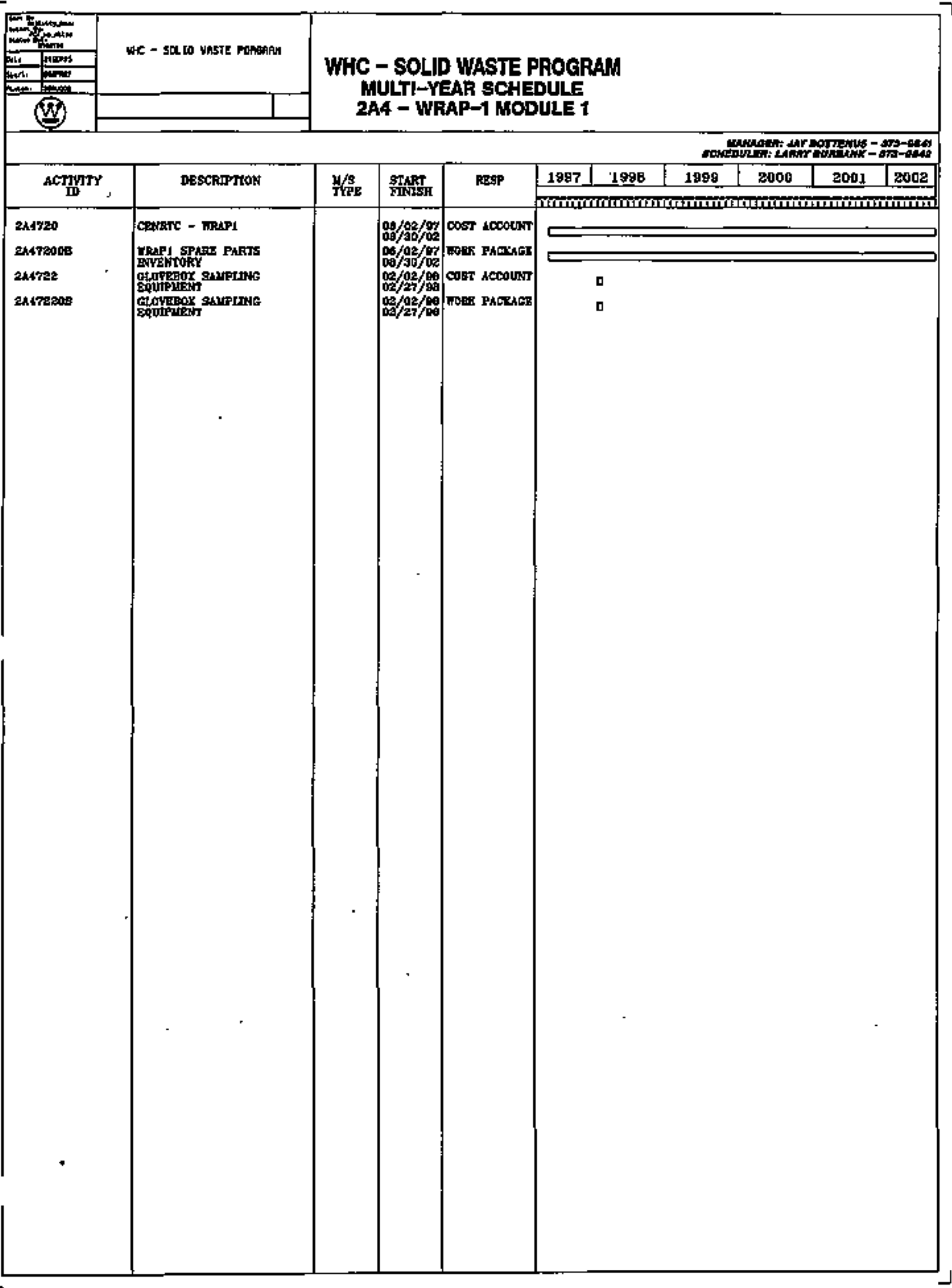




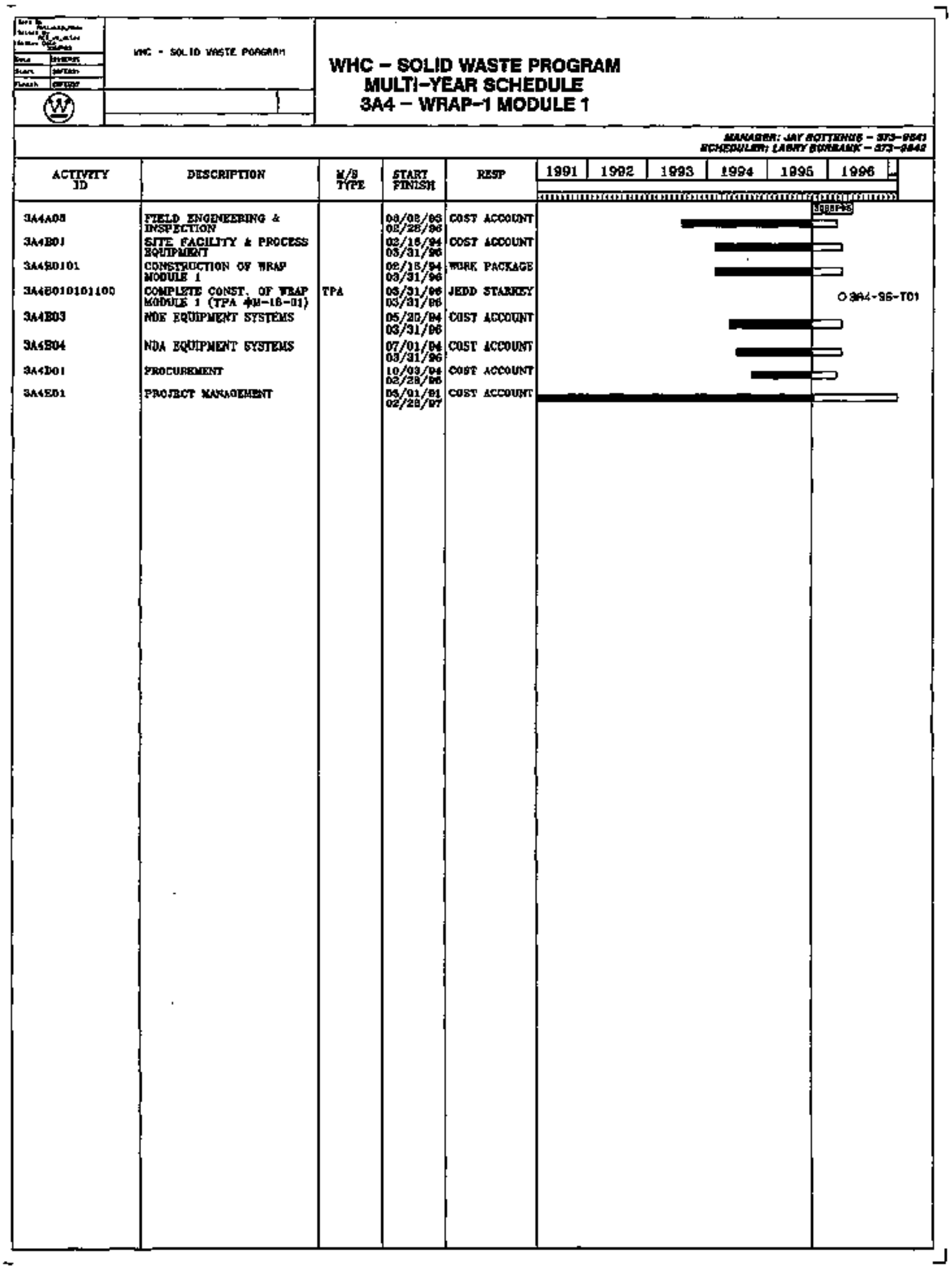




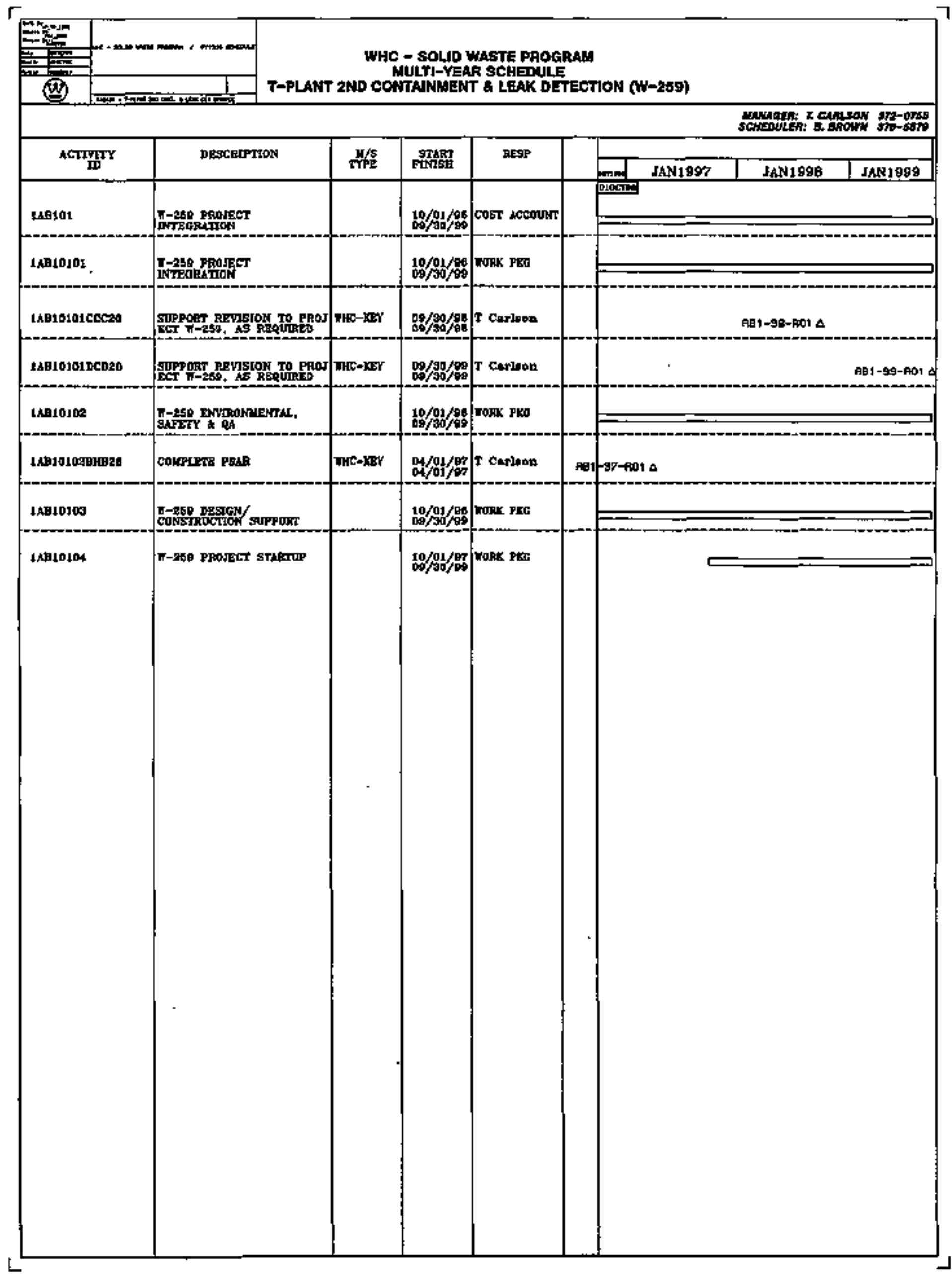




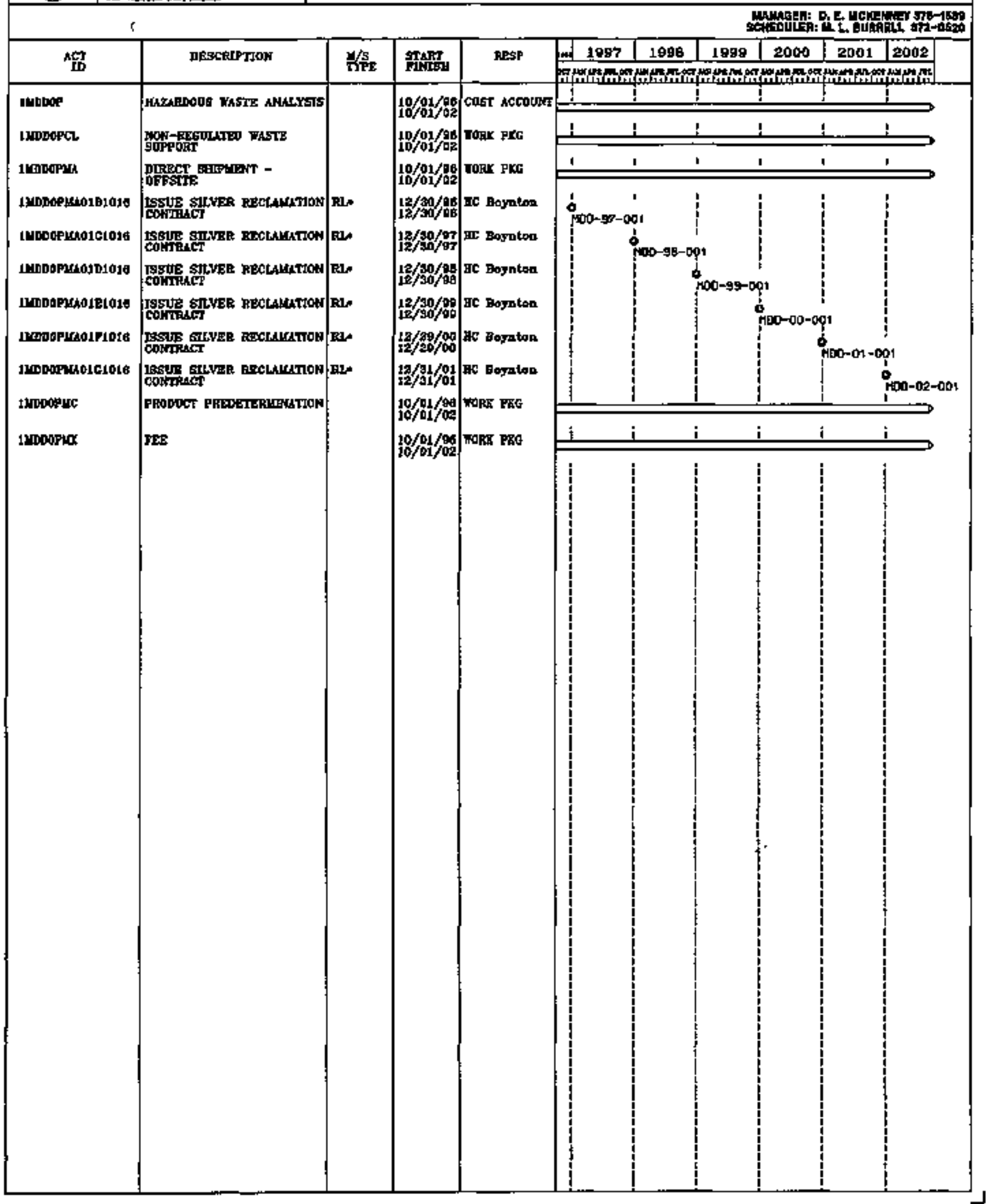




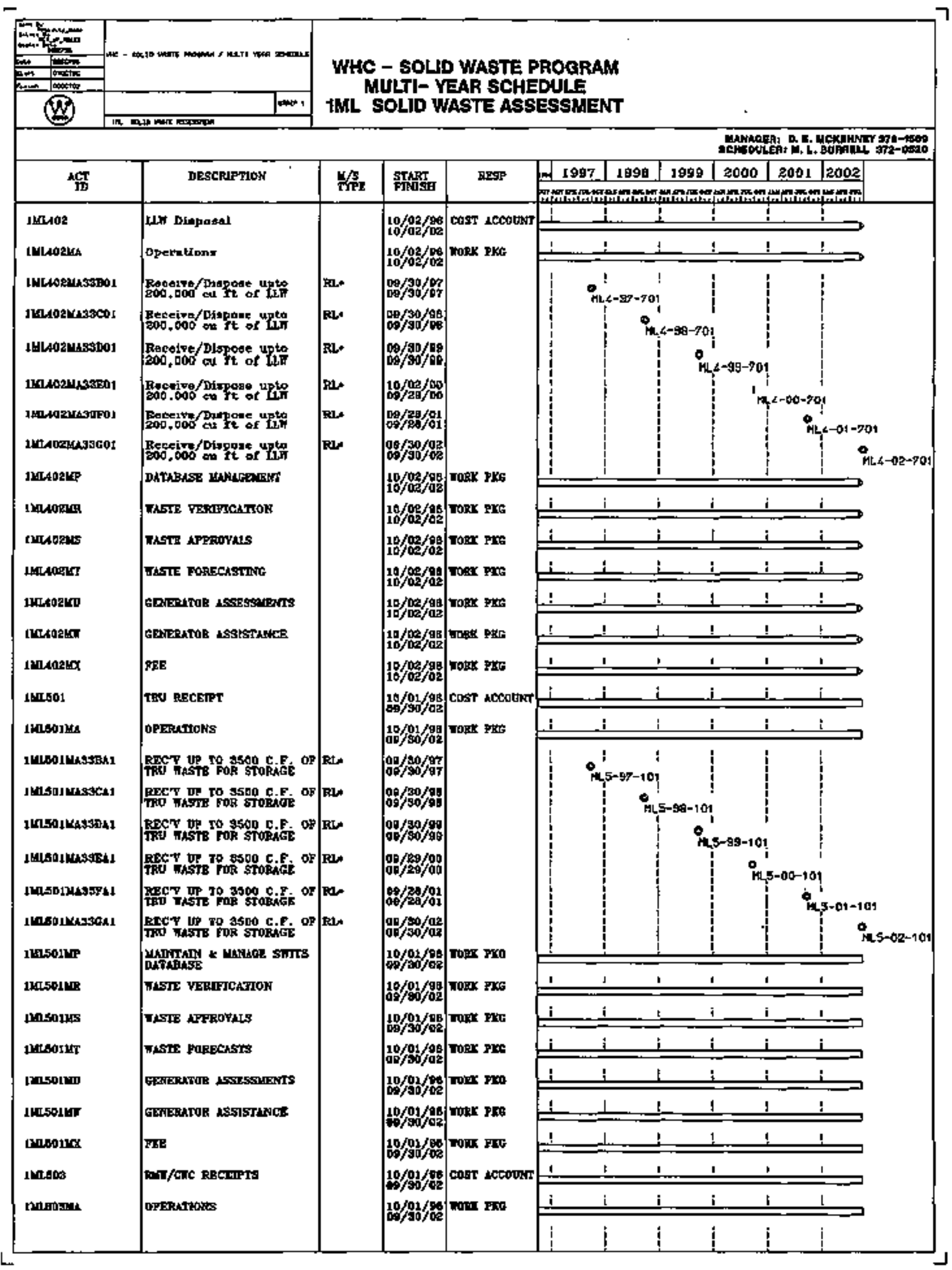




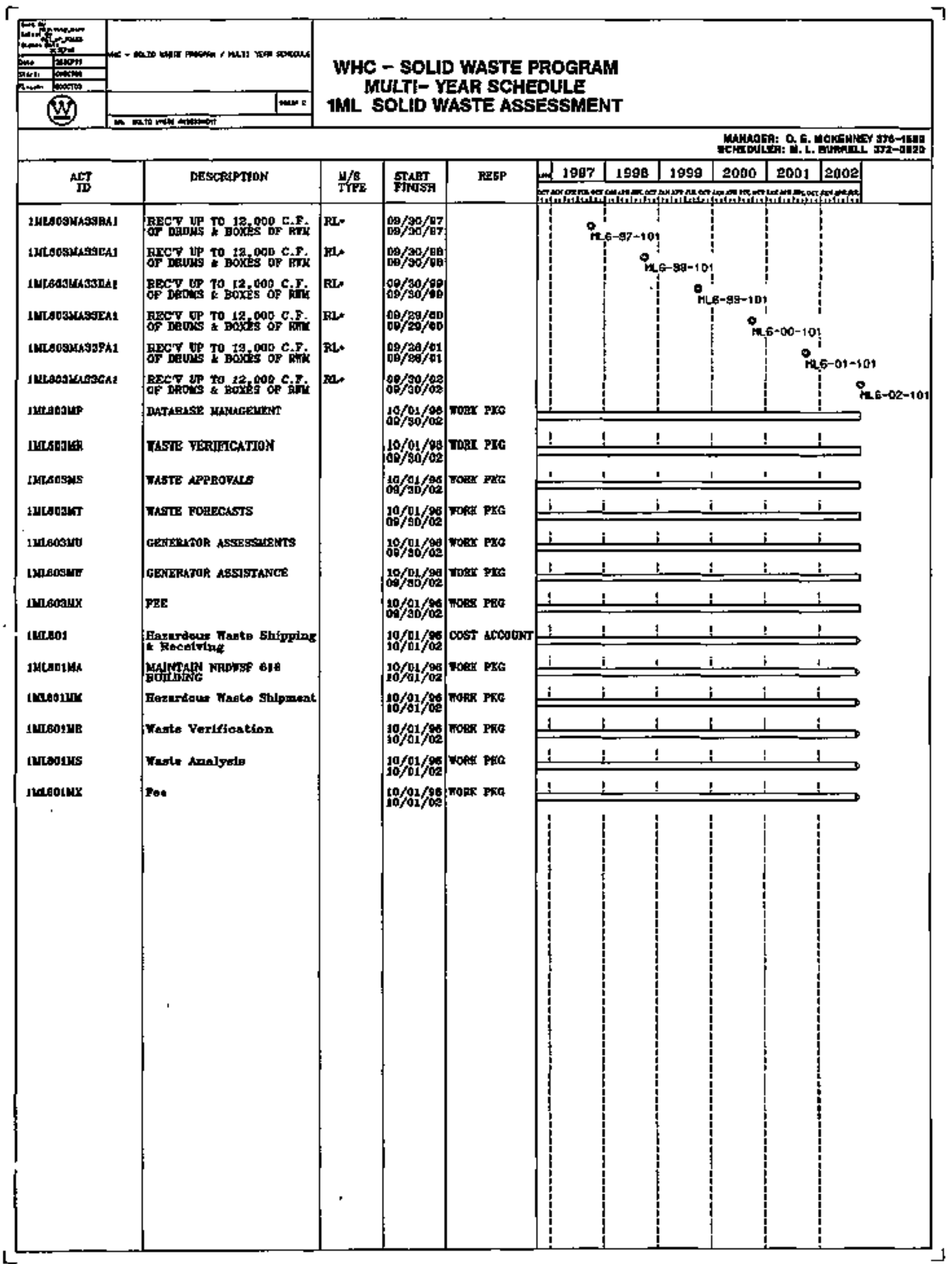




\begin{tabular}{|c|c|c|c|c|c|c|c|c|c|c|c|c|c|}
\hline \multicolumn{14}{|c|}{ 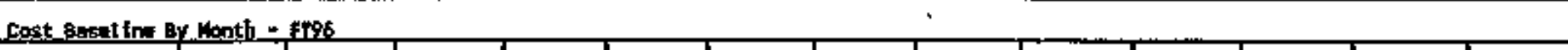 } \\
\hline $\begin{array}{c}\text { Act Ivity/tost } \\
\text { Atcount }\end{array}$ & OCI & HOW & DEC & JAM & EEB & MAR & APR & MAT & NUN & JUL & AUG & SEP & IDTAL \\
\hline \multicolumn{14}{|l|}{ 1A1Zn1 } \\
\hline Lubor $\$$ & 160.2 & 140.3 & 176.3 & 206.2 & 189.7 & 248.0 & 175,0 & 97.1 & 104.6 & 87.6 & 98.7 & 123.6 & 18007.3 \\
\hline nther $\$$ & 1116.7 & 900.9 & 164. & 194.1 & 178.1 & 359.1 & 281.7 & 242.9 & 168.8 & 121.9 & 138,3 & 135.6 & 3990,6 \\
\hline $\begin{array}{l}\text { Nonthly } \$ \\
\text { Totels }\end{array}$ & 1276.9 & 1049,2 & $34,1.1$ & 400.3 & 367.8 & 587.1. & 456.7 & 340,0 & 273.4 & 209.5 & 237,0 & 59.2 & 5777.9 \\
\hline pies & 20,8 & 20.3 & 20.8 & 29.0 & 26.2 & 25.9 & $\mathbf{3 2 . 4}$ & 12.9 & 11.4 & 13.5 & 13.5 & 13.0 & 19.2 \\
\hline
\end{tabular}

\begin{tabular}{|c|c|c|c|c|c|c|c|c|c|c|c|c|c|}
\hline $\begin{array}{l}\text { Activity/cost } \\
\text { Aecount }\end{array}$ & oct & nov & OEEC & JAL & FEB & MAR & $A P R$ & mar & JuW & JK & Alls & SEP & TOTAL \\
\hline \multicolumn{14}{|l|}{1,1202} \\
\hline Lebor $s$ & 17.3 & 17,3 & 22,3 & 17.7 & 15.3 & 18,0 & 16.1 & $t 4.1$ & 16.7 & 14.1 & 15.6 & 19.5 & 202.1 \\
\hline otter $s$ & 113,2 & 102.9 & 131.8 & 108.4 & 107.0 & 140.5 & 112.4 & 112.4 & 134.8 & 101.1 & 112,4 & 140.5 & 1417,4 \\
\hline $\begin{array}{l}\text { Monthly s } \\
\text { Totels }\end{array}$ & 130.5 & 120.3 & 154.1 & 126.1 & 223.3 & 158,5 & 126.5 & 126.5 & 151.7 & 115.2 & 128,0 & 160.0 & 1619.5 \\
\hline FTEs & 2.3 & 2.5 & 2,6 & 2.4 & 2.1 & 1.8 & 1,9 & 1.9 & 1.9 & 2.2 & 2.2 & $2 . t$ & 2.2 \\
\hline
\end{tabular}

\begin{tabular}{|c|c|c|c|c|c|c|c|c|c|c|c|c|c|}
\hline 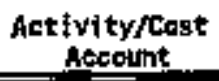 & OCT & now & DEC & JAH & FEB & MAR & APR & MAV & JuH & $\mathrm{J12}$ & AUG & SEP & TOTAL \\
\hline \multicolumn{14}{|l|}{$1 A 1203$} \\
\hline \multicolumn{14}{|l|}{ Lubor $\$$} \\
\hline Other \$ & 32.3 & 29.1 & 37.1 & 9.0 & 9.0 & 11.9 & 9.5 & 67.2 & 80.4 & 285.2 & 316.9 & 396.2 & 1292,0 \\
\hline $\begin{array}{l}\text { Monthly s } \\
\text { Totals }\end{array}$ & 32.3 & 29.1 & 37,1 & 9.0 & 9.0 & 11.9 & 9.5 & 67.2 & BE. 4 & 285.2 & 316,9 & 396.2 & 1292.0 \\
\hline FTES & & & & & & & & & & & & & \\
\hline
\end{tabular}




\begin{tabular}{|c|c|c|c|c|c|c|c|c|c|c|c|c|c|}
\hline$\underset{\text { Account }}{\text { Aetivi tw/cost }}$ & काт & HOY & DEC & SAH & FER & MAR & $A P R$ & MAY & תIm & . & AWG & $\operatorname{SE}^{*}$ & TOTAL \\
\hline \multicolumn{14}{|l|}{$1 \mathrm{A1301}$} \\
\hline Lenor 8 & 203,9 & 183.0 & 233.8 & 193.4 & 193,4 & 254.3 & 202.4 & 202,4 & 240.5 & 180.3 & 200.9 & 250.5 & 2538.9 \\
\hline other $\$$ & 36.0 & 32.4 & 41.4 & 34.4 & 34.4 & 45.10 & 36.0 & 36.0 & 43.3 & 32.3 & 36.1 & 45,1 & 452,3 \\
\hline $\begin{array}{l}\text { Monthly } \\
\text { Iotal } \\
\text { Iots }\end{array}$ & 299.9 & 215.4 & $2 \pi .2$ & 227.8 & 227.8 & 299.3 & 258.4 & 238,4 & 283,8 & 212.6 & 237.0 & 295.6 & 2991,2 \\
\hline FTEs & 27.5 & 27,4 & 28.6 & 27,3 & 27,0 & 27.1 & 27,5 & 27,8 & 27.2 & 28.8 & 28.4 & 27.3 & 27.6 \\
\hline
\end{tabular}

\begin{tabular}{|c|c|c|c|c|c|c|c|c|c|c|c|c|c|}
\hline $\begin{array}{c}\text { Activity/Cost } \\
\text { Actolut }\end{array}$ & oCT & Hor & DEC & JAN & FEB & MAR & APK & MAY & 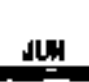 & Lي & AUG & SEP & TOTAL, \\
\hline \multicolumn{14}{|l|}{$1 \mathrm{~A} 1401$} \\
\hline Labbor $s$ & 338,5 & 304.2 & 388.9 & 321,3 & 321.3 & 422.6 & 359.5 & 338.5 & $405 . B$ & 304.2 & 338.5 & 422,6 & 43449 \\
\hline other 5 & 92.1 & 82.8 & 106,0 & 87.6 & 87.6 & 195.2 & 92.1 & 92.1 & 110.6 & 82.8 & 92.1 & 195.2 & 1156,4 \\
\hline $\begin{array}{l}\text { Wonthly } 5 \\
\text { Iotels }\end{array}$ & 430,6 & 387.0 & 496.9 & 409.9 & 409.9 & 537.8 & 430.6 & 430.6 & 516.4 & 387,0 & 430.6 & 537.8 & 5401.3 \\
\hline FTEs & 45.4 & 45.5 & 47,4 & 45.3 & 44.6 & 44.8 & 65.7 & 46.4 & 45.7 & 48.6 & 47.7 & 45.8 & 46. \\
\hline
\end{tabular}

\begin{tabular}{|c|c|c|c|c|c|c|c|c|c|c|c|c|c|}
\hline \multicolumn{14}{|c|}{ cossts Butel ine By wonth - Frgs } \\
\hline $\begin{array}{c}\text { Act ivity/Cost } \\
\text { Account }\end{array}$ & $\boldsymbol{O C T}_{\mathrm{I}}$ & HOW & Dex & JEAN & FEB & MAR & APR & MAY & Nend & JUL & AMG & SEP & TOTAL \\
\hline \multicolumn{14}{|l|}{$14: 402$} \\
\hline \multicolumn{14}{|l|}{ Lepor 8} \\
\hline other $s$ & 58,5 & 52.6 & 35.1 & & & & & & & & & & 146.2 \\
\hline $\begin{array}{l}\text { Wonthly } 5 \\
\text { Totals }\end{array}$ & 58.5 & 52.6 & 35.1 & & & & & & & & & & 146.2 \\
\hline FTEA & & & & & & & & & & & & & \\
\hline
\end{tabular}




\begin{tabular}{|c|c|c|c|c|c|c|c|c|c|c|c|c|c|}
\hline $\begin{array}{c}\text { Activity/Cost } \\
\text { Account }\end{array}$ & OCT & How & DEC & swㅆ & FEB & MaR & APR & HAY & МH & $\mathrm{Ju}$ & $A N_{G}$ & SEP & TOTAL \\
\hline \multicolumn{14}{|l|}{ 1AJ50? } \\
\hline talpes & 141.0 & 126.7 & 165,4 & 140.4 & .337 .5 & 1000 & 144.4 & 204.4 & 172.9 & 125,7 & 139.9 & 174.3 & 1792.6 \\
\hline Othes $\$$ & 34.9 & 31.5 & 40.5 & 33.1 & 33,2 & 4.5.8 & 35.2 & 35.2 & 42.1 & 31.6 & 35.1 & 43.9 & 440.0 \\
\hline $\begin{array}{l}\text { Monthly } \% \\
\text { Totals }\end{array}$ & 175.9 & 158.2 & 205,9 & 173.5 & 170.7 & 223.8 & 179.6 & 179.6 & 215.0 & 157.3 & 175.0 & 218,2 & 2232.6 \\
\hline FTEs & 19.0 & 19.1 & 20.2 & 19.9 & 19.2 & 19.2 & 19.6 & 19.9 & 19.6 & 20,1 & 19.8 & 19.0 & 19.6 \\
\hline
\end{tabular}

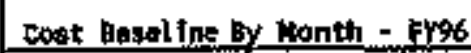

\begin{tabular}{|c|c|c|c|c|c|c|c|c|c|c|c|c|c|}
\hline $\begin{array}{c}\text { Activity/cost } \\
\text { A Accplnt }\end{array}$ & OCT & mow & DEC & JAH & F看 & mak & APR & MAP & JW & Nal. & NWG & SEP & TOTAL \\
\hline 1A1601 & \multicolumn{13}{|c|}{ " } \\
\hline Leboor \& & 300.2 & 277.9 & 355.1 & 291,9 & 291.9 & 394.5 & 305.8 & 305.8 & 366.3 & 275.7 & 306.8 & 363.2 & 3854.2 \\
\hline other : & 164.5 & $14 \mathrm{~B}, 0$ & 189.2 & 156,4 & 156,4 & 205,7 & 154.1 & 154.1 & 184.9 & 139.6 & 154.1 & 192.6 & 1998,4 \\
\hline $\begin{array}{l}\text { Wonthly } \\
\text { Iotals }\end{array}$ & $4 \pi 3$ & 425.9 & 546,3 & 448,3 & 448.3 & 590.2 & 459.9 & 459.9 & 551.2 & 414,3 & 460.9 & 575.8 & 5852.6 \\
\hline FTEB & 42.4 & 42.5 & 44.2 & 42.0 & 41.5 & 41.7 & 42.2 & 42.8 & 42.2 & 44.9 & 44,2 & 42.5 & 42.7 \\
\hline
\end{tabular}




\begin{tabular}{|c|c|c|c|c|c|c|c|c|c|c|c|c|c|}
\hline \multicolumn{14}{|c|}{ 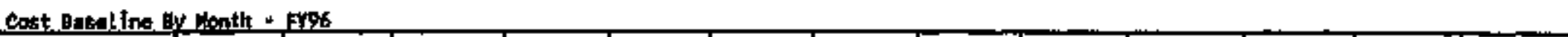 } \\
\hline $\begin{array}{c}\text { Aetivity/Cost } \\
\text { Actalunt }\end{array}$ & DCT & MOV & DEC & JAM & FEB & MAR & APR & MAY & JUN & Juth & Atts & sep & TOTAL \\
\hline \multicolumn{14}{|l|}{$1 \mathrm{~A} 1602$} \\
\hline Lebor $s$ & 6.2 & 5.7 & 7.2 & 6.0 & 5.7 & 6.2 & 4.9 & 4.5 & & & & & 46.4 \\
\hline Other 8 & 41.1 & 36.9 & 47,2 & 39.0 & 17.9 & 2.4 & 2.0 & $\$ .7$ & & & & & 188.1 \\
\hline $\begin{array}{l}\text { Monthly } \\
\text { Iotedls }\end{array}$ & 47,3 & 42,6 & 54.4 & 45.0 & 23.6 & 8.6 & 6,9 & 6.2 & & & & & 234.5 \\
\hline FEs & .8 & .8 & .9 & .8 & .8 & .6 & .7 & .6 & & & & & .5 \\
\hline
\end{tabular}

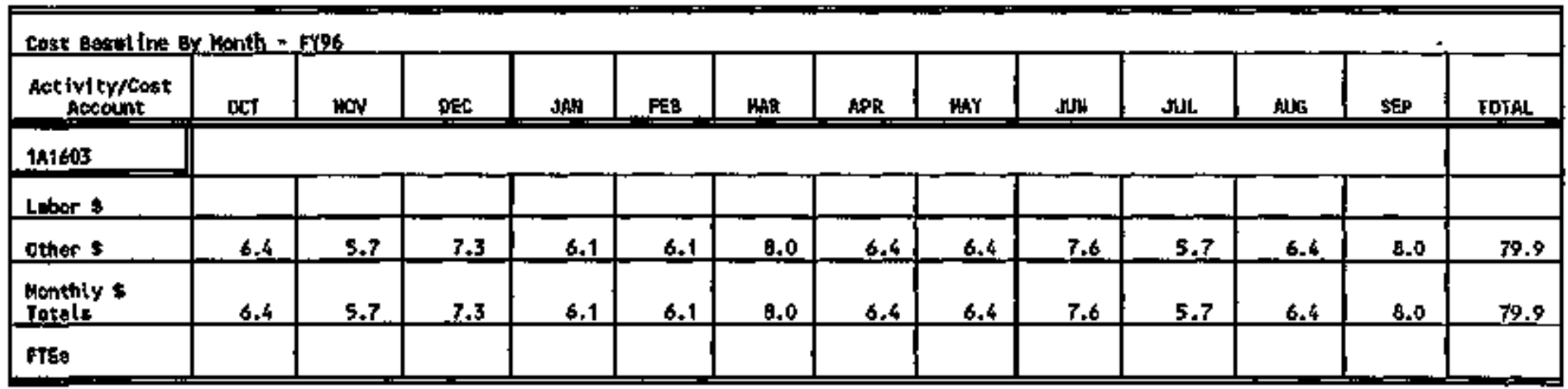

\begin{tabular}{|c|c|c|c|c|c|c|c|c|c|c|c|c|c|}
\hline \multicolumn{14}{|c|}{ cost Gaseline gy honth - Fy9s } \\
\hline $\begin{array}{c}\text { Activity/coot } \\
\text { Aecaunt }\end{array}$ & OCT & nor & DEC & Jall & FEE & N䖵 & APR & MAY & wh & Jut & 此 & SEP & TOTAL \\
\hline 1A1606 & \multicolumn{12}{|c|}{. } & \\
\hline \multicolumn{14}{|l|}{ Leber $\$$} \\
\hline Other $\$$ & & & & & & & & 33.5 & 44.7 & 33.5 & 37.3 & 46.6 & 15,6 \\
\hline $\begin{array}{l}\text { Nonthly } 5 \\
\text { Totals. }\end{array}$ & & & & & & & & 33,5 & 4.7 & 33.5 & 37.3 & 46.6 & 195.6 \\
\hline FJEs & & & & & & & & & & & & & \\
\hline
\end{tabular}




\begin{tabular}{|c|c|c|c|c|c|c|c|c|c|c|c|c|c|}
\hline \multicolumn{14}{|c|}{ 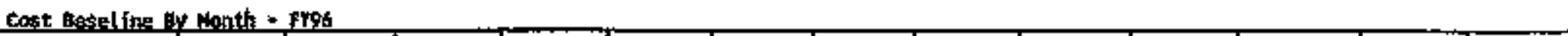 } \\
\hline $\begin{array}{c}\text { Aet ivity/Cost } \\
\text { Account }\end{array}$ & aCT & now & DEC & SAM & FEB & MAR & APR & MAY & NM & Ntht & AUE & SEF & TOTAL \\
\hline \multicolumn{14}{|l|}{ 1A1605 } \\
\hline Labers & & & & & & & & & 26,2 & 23.5 & 26.2 & 32,7 & 108.7 \\
\hline \multicolumn{14}{|l|}{ Other $s$} \\
\hline $\begin{array}{l}\text { Nonthly } \\
\text { Iotsils }\end{array}$ & & & & & & & & & 26.2 & 23.5 & 26.2 & 32.7 & 108.7 \\
\hline FTES & & & & & & & & & 2.9 & 3.7 & 3.6 & 3.5 & 1.1 \\
\hline
\end{tabular}

\begin{tabular}{|c|c|c|c|c|c|c|c|c|c|c|c|c|c|}
\hline \multicolumn{14}{|c|}{ cost Bgogline Hy wenth n FYg } \\
\hline $\begin{array}{c}\text { Act ivity/Cost } \\
\text { Axoount } \\
\end{array}$ & OCT & Hoy & DEC. & JAN & FEB & $M R$ & $A P R$ & Mar & $\mathrm{d}$ & . Nي & AUSG & SEP & TOTAL \\
\hline \multicolumn{14}{|l|}{181701} \\
\hline Lebor? & 57,1 & $\$ 1.3$ & 65.5. & 54.4 & 54.4 & 71.4 & 54.6 & 54.6 & 65.6 & 49.1 & 54.6 & 68,3 & 700.7 \\
\hline Other $\$$ & 7,8 & 7.1 & 0.1 & 7,5 & 7.5 & 9.9 & 7.8 & 7,8 & 9.4 & 7.0 & 7.8 & 9.8 & 唪 \\
\hline $\begin{array}{l}\text { Nonthly } \\
\text { Yofals }\end{array}$ & 64,9 & 58,4 & 74,6 & 61.9 & 61.9 & 81.3 & 62.4 & 62,4 & 75.0 & 56.1 & 62.4 & 78.1 & 799.6 \\
\hline FTES & 7,6 & 7.6 & 7.9 & 7.6 & 7.5 & 7.5 & 7.3 & 7.4 & 3.3 & 7.7 & 7.6 & 7.3 & 7.5 \\
\hline
\end{tabular}

\begin{tabular}{|c|c|c|c|c|c|c|c|c|c|c|c|c|c|}
\hline $\begin{array}{c}\text { Activity/cost } \\
\text { Account }\end{array}$ & OCT & Hov & DEC & JaW & FEB & MAR & APR & MAY & 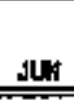 & UL & AUg & SEP & IOTAL \\
\hline \multicolumn{14}{|l|}{ 181702 } \\
\hline Lebors & .5 & .5 & 6 & 5 & .5 & .7 & 5 & 3 & & & & & 4.1 \\
\hline okther $s$ & 18.9 & 17,0 & 21.8 & 19.0 & 18.0 & 23.6 & 18.9 & 9.4 & & & & & 145.7 \\
\hline $\begin{array}{l}\text { Monthly } \\
\text { Tatsels }\end{array}$ & 19.4 & 17.5 & 22.4 & 18.5 & 18,5 & 24.3 & 19.4 & 9.7 & & & & & 149.8 \\
\hline FTEs & .1 & .1 & .1 & .1 & 1 & .1 & . 1 & & & & & & \\
\hline
\end{tabular}




\begin{tabular}{|c|c|c|c|c|c|c|c|c|c|c|c|c|c|}
\hline $\begin{array}{l}\text { Act }[v 1 t y / C o s t \\
\text { Account }\end{array}$ & DCT & Nov & DEC & JAM & FEB & Math & APR & MAY & JU: & JUL & AlJf & SEP & TOTAL \\
\hline \multicolumn{14}{|l|}{ 1A1901 } \\
\hline L L labor 5 & 168.0 & 147.2 & 172.1 & 159.0 & 158.7 & 224.7 & 154.2 & 148,0 & $138+8$ & 90.0 & 84.8 & 914 & 1730.4 \\
\hline other 5 & 147.6 & 167.6 & 231.3 & 187.8 & 172.7 & 233.4 & 112.7 & 9.5 & 125.7 & 501.1 & 112.1 & 139.4 & $182 B .2$ \\
\hline $\begin{array}{l}\text { Monthly } 5 \\
\text { Totals }\end{array}$ & 3.15 .0 & 394.8 & 403.4 & 346.8 & 331.4 & 458,1 & 266.9 & 238.5 & 264.5 & 191.1 & 196.9 & 230.8 & 3559.1 \\
\hline FTES & 26.7 & 24.2 & 22.4 & 2z. 3 & 22.0 & 23.7 & 20.9 & 19.6 & 15.9 & 14.5 & 12.3 & 10.5 & 19.4 \\
\hline
\end{tabular}

\begin{tabular}{|c|c|c|c|c|c|c|c|c|c|c|c|c|c|}
\hline $\begin{array}{l}\text { Activity/Cost } \\
\text { Atcount }\end{array}$ & oct & $\mathrm{NOW}$ & DEE & JAM & FER & nhe & APR & MaY & Jut & . & ANG & SEP & TOIAL \\
\hline \multicolumn{14}{|l|}{ 1A1,01 } \\
\hline Laber $*$ & B6.4 & 76.1 & 137. 호 & 125,2 & 123 & 169.1 & 144.9 & 150.4 & 178.9 & 136.3 & 149.3 & 145.5 & 1623.7 \\
\hline Dother $\$$ & $336-9$ & 304,6 & 389.4 & 322.5 & 336.5 & 454,2 & 344.1 & $34,2.2$ & 410.6 & 307.9 & 342.2 & 417.6 & 4308,8 \\
\hline $\begin{array}{l}\text { Konthly } \$ \\
\text { Iotals }\end{array}$ & 423.3 & 380.7 & 527.2 & 447.7 & 460.3 & 623.3 & 489,0 & 492.6 & 589.5 & 444.2 & 491.5 & 563.1 & 5932.5 \\
\hline Ft勘 & 11.6 & 11.3 & 15.8 & 16,6 & 26.1 & 17.1 & 19.0 & 20.2 & 19.8 & 21.3 & 20.6 & 15.9 & 17.1 \\
\hline
\end{tabular}

\begin{tabular}{|c|c|c|c|c|c|c|c|c|c|c|c|c|c|}
\hline $\begin{array}{c}\text { Activity/cost } \\
\text { Mccoint }\end{array}$ & OCT & Hov & DEC & Jي & FEB & MaR & APR & KAY & JuW & $\mathrm{JUL}$ & AU, & SEP & TOTAL \\
\hline \multicolumn{14}{|l|}{$1 A 1,02$} \\
\hline Labor $s$ & 38,8 & 36.8 & 47.4 & 39.2 & 39.2 & 51.8 & 36.7 & 36.1 & 48.4 & 37.0 & 41.2 & 46.4 & 498.5 \\
\hline other \$ & 39.6 & 36.9 & 47.4 & 39.1 & 39.1 & 51.5 & 39.7 & 39.7 & 47.6 & 35.7 & 39.7 & 49.6 & 505.5 \\
\hline $\begin{array}{l}\text { Nanthly } \\
\text { Tatats }\end{array}$ & 79,4 & 73.7 & 94.8 & 78.3 & 78,3 & 103.3 & 75.8 & 7.8 & 96.0 & 72.7 & $\Delta 0,9$ & 96,0 & 1004,0 \\
\hline FTEs & 5.9 & 5.9 & 6.2 & 5.9 & 5.8 & 5.9 & 8.0 & 6.0 & 6.5 & 7.0 & 6.9 & 6.1 & 6.2 \\
\hline
\end{tabular}




\begin{tabular}{|c|c|c|c|c|c|c|c|c|c|c|c|c|c|}
\hline \multicolumn{14}{|c|}{ 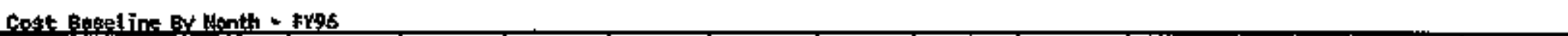 } \\
\hline $\begin{array}{c}\text { Activity/Cost } \\
\text { Actount }\end{array}$ & oct & WON & bec & JAM & Fea & MAR & APk & MAY & JUT & Lال & W/B & SEP & TOTAL, \\
\hline \multicolumn{14}{|l|}{$1 A 310$} \\
\hline 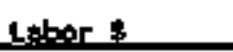 & 163.0 & 146,6 & 147.1 & 207.6 & 117,5 & 139.9 & 157,0 & 154.7 & 170,8 & 132.8 & 140.1 & 182,3 & 1059.2 \\
\hline other $s$ & 17.0 & 15.3 & 19.4 & 16.1 & 16.1 & 21.2 & 17.0 & 16.9 & 20.3 & 15.2 & 16,9 & 81.6 & $2 \pi 3.2$ \\
\hline $\begin{array}{l}\text { Nonthly s } \\
\text { Totals }\end{array}$ & 1800,0 & 161.9 & 166.5 & 223.7 & 133.6 & 161.1 & 174,0 & 171.6 & 191.1 & 148.0 & 157.0 & 263.9 & 2132,4 \\
\hline FTES & 20.2 & 20.3 & 17.1 & 27.6 & 15.6 & 14.3 & 20.3 & 19.9 & 18.1 & 19.8 & 18.5 & 18.7 & 19.2 \\
\hline
\end{tabular}

cose angal the By Month - Eros

Activity/Cost Atcount

$\left.\right|^{2}$

(1)

\begin{tabular}{|c|c|c|c|c|c|c|c|c|c|c|c|c|c|}
\hline 3151 & & & & & & & & & & & & & \\
\hline Letor $\$$ & 20.8 & 18.7 & 23.9 & 19.8 & 19.8 & 26,0 & 20.8 & 20.8 & 24.9 & 18.7 & 200.8 & 26,0 & 260.9 \\
\hline other 5 & 3.4 & 3.1 & 4.0 & 3.2 & 3.2 & 4.3 & 3.4 & 3.4 & 4.2 & 3.1 & 3.4 & 4.3 & 43.3 \\
\hline $\begin{array}{l}\text { Wanthly } \\
\text { Totals }\end{array}$ & 24.2 & 21.日 & 27.9 & 23.0 & 24.0 & 30.3 & 24.7 & 24.2 & 29,1 & 21,8 & 24.2 & 30.3 & 304.2 \\
\hline FTEs & 2.9 & 3.0 & 3.1 & 2.7 & 2.9 & 2.9 & 3.0 & 3.0 & 3.0 & 3.1 & 3.1 & 3.0 & 3.0 \\
\hline
\end{tabular}

\begin{tabular}{|c|c|c|c|c|c|c|c|c|c|c|c|c|c|}
\hline $\begin{array}{c}\text { Act I vity/Cost } \\
\text { Acsoumt }\end{array}$ & oct & Wu & DEC & JNH & FEB & MAR & $A P R$ & Mar & L的 & SUL & AUG & SEP & TOTAL. \\
\hline \multicolumn{14}{|l|}{143202} \\
\hline Lethor $\$$ & 102.8 & 92.5 & 113.5 & 92,0 & 95.0 & 122,5 & 97.5 & 97.5 & 147,8 & 141.7 & 157.6 & 164.8 & 1425.9 \\
\hline other $\$$ & 31.8 & 28.7 & 36.6 & 30.4 & 30.4 & 39.8 & 31.9 & 31.9 & 46.3 & 36.2 & 40.1 & 40.9 & 425.2 \\
\hline $\begin{array}{l}\text { Nonthly } \\
\text { Totals }\end{array}$ & 134.6 & 121.2 & 150.1 & 123.2 & 125.4 & 162.3 & 129.4 & 129.4 & 194.1 & 177.9 & 197.7 & 205.7 & 1851,1 \\
\hline FTES & 13.3 & 13.4 & 13.3 & 12.4 & 12.8 & 12.5 & 12.7 & 12.9 & 16.7 & 29.2 & 20.7 & 16.7 & 14.9 \\
\hline
\end{tabular}




\begin{tabular}{|c|c|c|c|c|c|c|c|c|c|c|c|c|c|}
\hline \multicolumn{14}{|c|}{ Cost Babel line by Honth - Fyog } \\
\hline$\underset{\substack{\text { Account } \\
\text { Metivity/cost }}}{\text {. }}$ & oct & Har & OEEC & JAN & FEg & MAR & $\mathrm{APR}$ & MaY & MW & NUL & ANG & SEP & TOTAL \\
\hline \multicolumn{14}{|l|}{$1 \mathrm{A3204}$} \\
\hline Lebot: 8 & 131.4 & 146.6 & 146,8 & 121.1 & 127.8 & 197.2 & 132,1 & 167,3 & 155,6 & 142.3 & 135.1 & 195.4 & 1796.7 \\
\hline ather 5 & 30.9 & 22. B & 35.5 & 29.4 & 29,6 & 38.7 & 31.0 & 31.0 & 37.1 & 27.8 & 30.9 & 39,7 & 388.2 \\
\hline $\begin{array}{l}\text { Nanthly } \$ \\
\text { fotala }\end{array}$ & $162, y$ & 174.4 & 1800.3 & 150.5 & 157.2 & 235.9 & 163.1 & 198.3 & 192.7 & 170.1 & 166.0 & 234.1 & 2184.9 \\
\hline FTEQ & 18.1 & 22.5 & 18.2 & 17.5 & 18.4 & 21.4 & $\$ 8.3$ & 23.6 & 98.1 & 23.1 & 19.5 & 21.7 & 20,0 \\
\hline
\end{tabular}

\begin{tabular}{|c|c|c|c|c|c|c|c|c|c|c|c|c|c|}
\hline \multicolumn{14}{|c|}{ 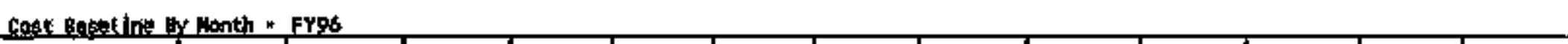 } \\
\hline$\underset{\text { Activity/Cost }}{\text { Acomnt }}$ & OCT & $\mathrm{NOF}$ & DEC & Jah & FEB & $M A R$ & $A P R$ & HAY & N & NL & ANO & SEP & TOTAL \\
\hline \multicolumn{14}{|l|}{$1 \times 3251$} \\
\hline Letors: & 1.8 & 1.7 & 2.0 & 1.7 & 1.7 & 2.2 & 1, & 1.8 & 2.1 & 1.7 & 1.8 & 2.2 & $2 z, 4$ \\
\hline Other : & 6.2 & 5.5 & 7.2 & 5.8 & 5,9 & 7.8 & a.2. & 6.2 & 7.5 & $\mathbf{5 . 5}$ & 6.2 & 7,9 & 78.0 \\
\hline $\begin{array}{l}\text { Wanthly } \$ \\
\text { Totaks }\end{array}$ & 8.0 & 7.2 & 9.2 & 7.6 & 7.6 & 10,0 & e., & 8.0 & 9.6 & 7.2 & $-8,0$ & 10,0 & 100.4 \\
\hline FTES & .3 & .3 & .3 & .2 & .2 & .2 & .3 & 3 & 3 & 3. & .3 & .3 & .3 \\
\hline
\end{tabular}

\begin{tabular}{|c|c|c|c|c|c|c|c|c|c|c|c|c|c|}
\hline $\begin{array}{c}\text { Act Ivity/Cost } \\
\text { Accoount }\end{array}$ & OCT & MON & DEC & JAM & FE日 & MAR & Pes & HAY & duk & בL & MG & SEP & TOTAL \\
\hline 143252 & & & & & & & & & & & & & \\
\hline Lebor $\$$ & & & & & & & 3.0 & 3.0 & 3.5 & .2 & & & 9.5 \\
\hline other $\$$ & & & & & & & 17.1 & 17.1 & 20.5 & 8 & & & 55.7 \\
\hline $\begin{array}{l}\text { Nonthly } \\
\text { Totels }\end{array}$ & & & & & & & 20.1 & 20,7 & 24.0 & 1,0 & & & 65.2 \\
\hline FTEs & & & & & & & .4 & .4 & .4 & & & & .1 \\
\hline
\end{tabular}




\begin{tabular}{|c|c|c|c|c|c|c|c|c|c|c|c|c|c|}
\hline$\underset{\text { Aceount }}{\text { Activitw/cist }}$ & OCT & How & DEC & JAN & FE日 & Has & APR & MAT & JUN & JUL & AUSG & sep & ToTAl \\
\hline 1A330t & . & & & & & & & & & & & & \\
\hline Leror 5 & $10 t .6$ & 207,7 & 117.2 & 오.6 & 96.6 & 126.9 & 139.4 & 101.6 & 121.9 & 91.B & 101.6 & 127.0 & 1419.7 \\
\hline other $\$$ & 94.8 & 26,4 & 110.5 & 91.4 & 91.4 & 120,1 & 96,1 & 94.8 & $113 . \mathrm{B}$ & 85.3 & 94.8 & 118.5 & 1198.0 \\
\hline $\begin{array}{l}\text { Wonthly } \$ \\
\text { Totals }\end{array}$ & 196.4 & 794.1 & 227,7 & 188,0 & 188.0 & 247.0 & 276.5 & 196.4 & 235.7 & 177.1 & 196.4 & 245.5 & $2617+7$ \\
\hline FIES & 13.4 & 23.6 & 14.1 & 13.4 & 13.2 & 13.3 & 17.5 & 13.7 & 13.5 & 14.4 & 14.1 & 13,6 & 14.8 \\
\hline
\end{tabular}

\begin{tabular}{|c|c|c|c|c|c|c|c|c|c|c|c|c|c|}
\hline $\begin{array}{l}\text { Activity/Cost } \\
\text { Aecount }\end{array}$ & OCT & NDY & BEc & Jhin & FEt & $M A R$ & APR & may & لي & JWL & ANO & SEP. & TOTAL \\
\hline \multicolumn{14}{|l|}{$1 \mathrm{A33} 0 \mathrm{C2}$} \\
\hline Labor $\$$ & 646.t & 589.5 & 767.7 & 640.8 & 6 보.z. & 8:18. & 654,0 & 654,0 & 791.1 & 582.8 & 640,5 & 837.2 & 8362.0 \\
\hline other $\$$ & 199.2 & 179,2 & 229.0 & 189.3 & 199.3 & 269.0 & 199.2 & 199.2 & 247.3 & 186.5 & 207,4 & 239.0 & 2333.5 \\
\hline $\begin{array}{l}\text { Wonthly } \\
\text { Totals }\end{array}$ & 848,0 & 767.7 & 996.7 & 830.1 & 827,5 & 1067.3 & 853.2 & 653.2 & 1038.4 & 769.3 & 847.9 & $10 \% .2$ & 10795.5 \\
\hline FE: & ⿱一彑.7.7 & 84.6 & 69.9 & B6.7 & 85.2 & 83.4 & B4. & 86.0 & Bs.6 & Bg.0 & 86.5 & a7.2 & 36.0 \\
\hline
\end{tabular}

\begin{tabular}{|c|c|c|c|c|c|c|c|c|c|c|c|c|c|}
\hline $\begin{array}{c}\text { Activity/cost } \\
\text { Accoxnt }\end{array}$ & OCT & HOV & 听 & sen & FEB & MAR & APR & MAY & JUM & JUL & AWG & sep & TOIAL. \\
\hline \multicolumn{14}{|l|}{$2 A 3303$} \\
\hline Labor $\$$ & 35.4 & 42.7 & 46.1 & 36.4 & 36,4 & 47.8 & 38,4 & 38.4 & 46.0 & 34.4 & 38,4 & 47,8 & 491.1 \\
\hline Other \$ & 23.7 & 21.4 & 27.3 & 22.5 & 22.5 & 29.7 & 23.7 & 23.7 & 28.5 & 21.4 & 23.7 & 29.7 & $2 \% 8.1$ \\
\hline $\begin{array}{l}\text { Monthly s } \\
\text { Iotels }\end{array}$ & 62.1 & 64.1 & 73.4 & 58,9 & 58,9 & $\pi 7.5$ & $\Delta$ & 62.1 & 74.5 & 55,8 & 62.1 & $\pi .5$ & 709.2 \\
\hline $\mathrm{FTE}_{8}$ & 5.1 & 6.4 & 5.6 & 5.1 & 5.0 & 5,0 & 5.1 & 5.2 & 5.2 & 5.4 & 5.4 & 5.1 & 5.3 \\
\hline
\end{tabular}




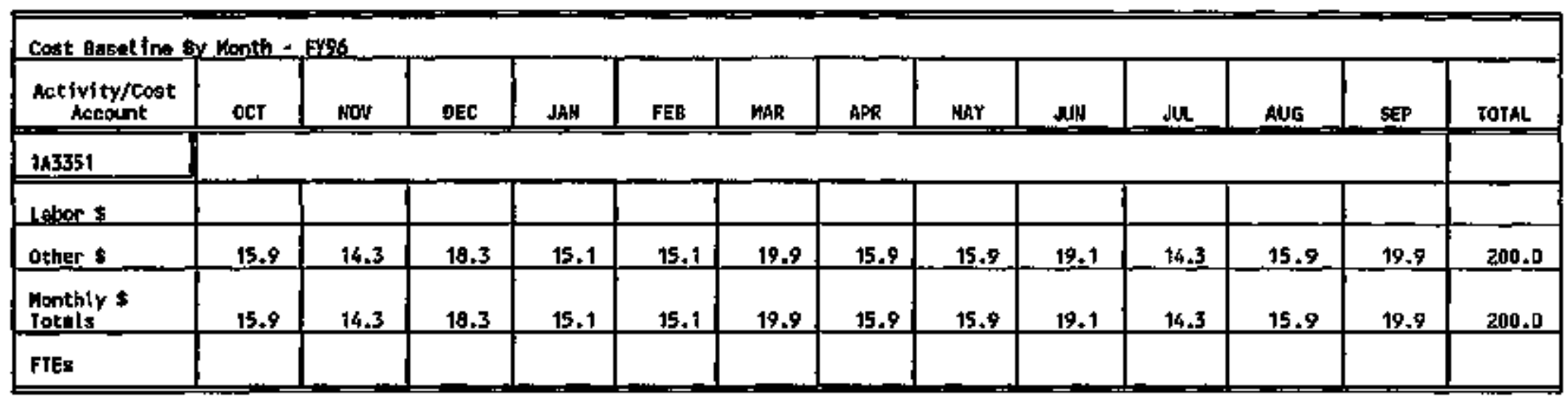

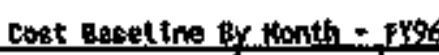

Ast ivity/Cost

Account:

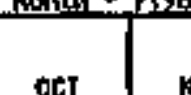

Toc
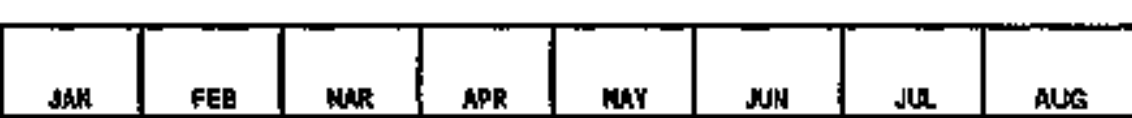

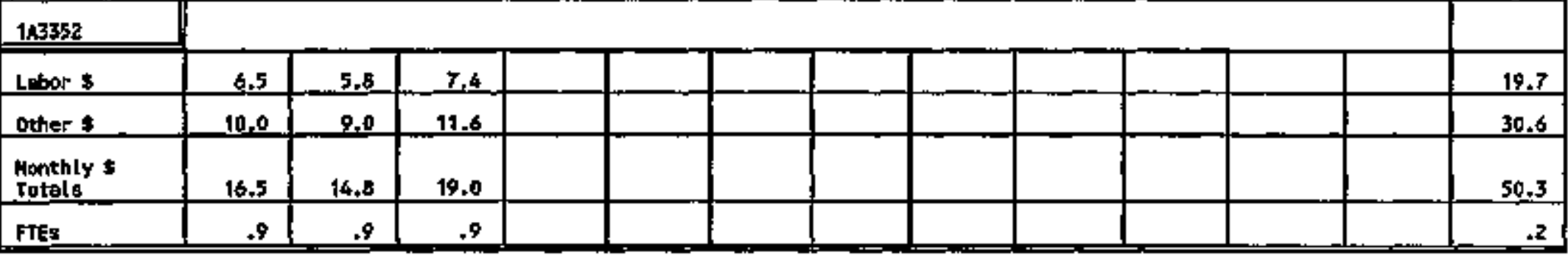

\begin{tabular}{|c|c|c|c|c|c|c|c|c|c|c|c|c|c|}
\hline 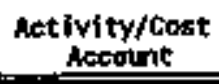 & oct & How & DEC & SAM & FEB & MAR & $A P R$ & nhy & vus & JUL & AUGG & SEP & TOTAL \\
\hline \multicolumn{14}{|l|}{$1 \times 3353$} \\
\hline tabor $\mathbf{s}$ & & & & & 33.1 & 51. $\mathrm{B}$ & 41.4 & 41,4 & 49.7 & 37.2 & 41.4 & 51.8 & 347.7 \\
\hline other 5 & & & & & 22.8 & 35.6 & 28.5 & 28.5 & 34,2 & 25.7 & 28.5 & 35.6 & 239.5 \\
\hline $\begin{array}{l}\text { Wonthity s } \\
\text { Jotnls }\end{array}$ & & & & & 55.9 & 87,4 & 67.2 & 69.9 & 23,9 & 62,9 & 69.9 & 82.4 & 587.2 \\
\hline FTEG & & & & & 4.7 & 5.6 & 5.7 & 5.8 & 5.8 & 6.1 & 6.0 & 5.8 & 3.8 \\
\hline
\end{tabular}




\begin{tabular}{|c|c|c|c|c|c|c|c|c|c|c|c|c|c|}
\hline \multicolumn{14}{|c|}{ 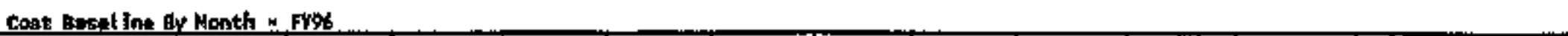 } \\
\hline $\begin{array}{c}\text { Net ivf ty/cost } \\
\text { Account }\end{array}$ & OCT & not & DESC & JM & $\mathbf{F a B}$ & HAR & APR & $\mathbf{M Y}$ & JNH & $\mathbf{u}$ & ANG & SEP & TOTAL \\
\hline \multicolumn{14}{|l|}{$1 \times 3356$} \\
\hline lobors & 2.2 & 1.9 & 2.5 & & & & & & & & & & 6.5 \\
\hline other 5 & 1.1 & 1.1 & 1.3 & & & & & & & & & & 3.6 \\
\hline $\begin{array}{l}\text { Monthly } s \\
\text { Iotals }\end{array}$ & 3.3 & 3.0 & 3.8 & & & & & & & & & & 20.1 \\
\hline FEs & .3 & .3 & .3 & & & & & & & & & & 1 \\
\hline
\end{tabular}

\begin{tabular}{|c|c|c|c|c|c|c|c|c|c|c|c|c|c|}
\hline $\begin{array}{c}\text { Aet } 1 \text { vity/Cost } \\
\text { Accomnt }\end{array}$ & OCT & MON & OEC & NhH & EEB & NAR & APR & may & Jw & ل. & AUG & $\operatorname{sep}$ & TOIAL \\
\hline \multicolumn{14}{|l|}{183355} \\
\hline Lepor 5 & 12.4 & 11.2 & 14.3 & & & & & & & & & & 38.0 \\
\hline Other $\$$ & 20.5 & 19.4 & 23,6 & & & & & & & & & & 62.5 \\
\hline $\begin{array}{l}\text { Manthty : } \\
\text { Totels }\end{array}$ & 32.9 & 29.6 & 37.9 & & & & & & & & & & $t 00.5$ \\
\hline FTES & 1.7 & 1.7 & 1.8 & & & & & & & & & & .4 \\
\hline
\end{tabular}

\begin{tabular}{|c|c|c|c|c|c|c|c|c|c|c|c|c|c|}
\hline \multicolumn{14}{|c|}{ Cost Based line By Honth $n$ FYPG } \\
\hline $\begin{array}{l}\text { Actlvity/cost } \\
\text { Accalunt }\end{array}$ & OT & $\mathrm{ASOH}$ & OEC & נה & FEE & MAR & 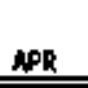 & NAY & JW & _ Lلالد & AUG & SEP & TOTAL \\
\hline \multicolumn{14}{|l|}{$1 A 3356$} \\
\hline \multicolumn{14}{|l|}{ Labors } \\
\hline other $\$$ & 92.3 & 83.1 & 106.2 & 07.7 & B?.7 & 015.4 & 92,3 & 9.2 & & & & & 674.0 \\
\hline $\begin{array}{l}\text { Monthly } 5 \\
\text { Totals }\end{array}$ & 72.3 & 23.1 & 106.2 & 07.7 & B?.7 & 115,4 & $\mathscr{R}, 3$ & 9.2 & & & & & 674.0 \\
\hline FFEs & & & & & & & & & & & & & \\
\hline
\end{tabular}




\begin{tabular}{|c|c|c|c|c|c|c|c|c|c|c|c|c|c|}
\hline $\begin{array}{l}\text { Actlvity/cost } \\
\text { Account }\end{array}$ & OCT & HOW & DEC. & JAM & Feg & MAR & $A P R$ & mar & Jul & $M_{L}$ & NBS & SEP & TOTAL \\
\hline \multicolumn{14}{|l|}{143402} \\
\hline Laster_s & 440 & 31.2 & 19.2 & 15.2 & 15.2 & 22,3 & 20,1 & 20.3 & 34,1 & $1 \mathrm{~B} .2$ & 20.3 & 25.0 & 276.3 \\
\hline other $s$ & 6.9 & 6.4 & Q.9. & 6.8 & 6.8 & B.9 & 7.2 & 7.1 & a.6 & 6.4 & 7,1 & 8.9 & 89.2 \\
\hline $\begin{array}{l}\text { Menthly s } \\
\text { Totals }\end{array}$ & 51.1 & 37.6 & 26.3 & 22,0 & 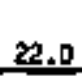 & 31,2 & 27.3 & 27.4 & 32.7 & 24.6 & 27.4 & 33.9 & 363.5 \\
\hline PTE & 5.8 & 6.7 & 2.2 & 2.1 & 2.1 & 2.3 & 2.7 & 2.8 & 2.7 & 2.9 & 2.8 & 2.7 & 3.0 \\
\hline
\end{tabular}

\begin{tabular}{|c|c|c|c|c|c|c|c|c|c|c|c|c|c|}
\hline 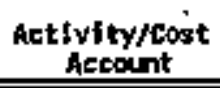 & QCCT & $\mathrm{MON}$ & DEE: & sk & Fe日 & NaR & APR & MAY & لI山M & 쇼 & AUB & SEP & TOTAL \\
\hline \multicolumn{14}{|l|}{$1 \mathrm{A3} 451$} \\
\hline Laber 5 & & 15.8 & 22.8 & 18.8 & 18.8 & 3.0 & & & & & & & 79,2 \\
\hline Other $\$$ & & 4.2 & 5.9 & 4.9 & 4.9 & 8 & & & & & & & 20.8 \\
\hline $\begin{array}{l}\text { Yonthly } \\
\text { Totels }\end{array}$ & & 20,0 & 29.7 & 33.7 & 23.7 & 3.8 & & & & & & & 100.0 \\
\hline FJES & & 2.0 & 2.3 & 2.2 & 2.2 & .3 & & & & & & & 7 \\
\hline
\end{tabular}

\begin{tabular}{|c|c|c|c|c|c|c|c|c|c|c|c|c|c|}
\hline \multicolumn{14}{|c|}{ Cost bassil Ine By Month - Fros } \\
\hline $\begin{array}{c}\text { Act ivity/cost } \\
\text { mocount }\end{array}$ & oct & HOW & DEC & 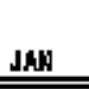 & FEB & MNR & APR & MAY & 些 & sul & No & 8EP & TOTAL \\
\hline \multicolumn{14}{|l|}{1 1A3501 } \\
\hline \multicolumn{14}{|l|}{ Lotoor 5} \\
\hline other $\$$ & $\mathbf{5 1 . 8}$ & 46.6 & 59.5 & 49.2 & 49.2 & 64.7 & 51,8 & 51.8 & 62.1 & 46.6 & 51,8 & 64.7 & 49.8 \\
\hline $\begin{array}{l}\text { Nonthly } \\
\text { Totals }\end{array}$ & 51.8 & 46,6 & 59.5 & 49.2 & 49.2 & 64.7 & 51.8 & 51,8 & $\Delta 2 . t$ & 46.6 & 51,8 & 64.7 & 649.8 \\
\hline VTES & & & & & & & & & & & & & \\
\hline
\end{tabular}




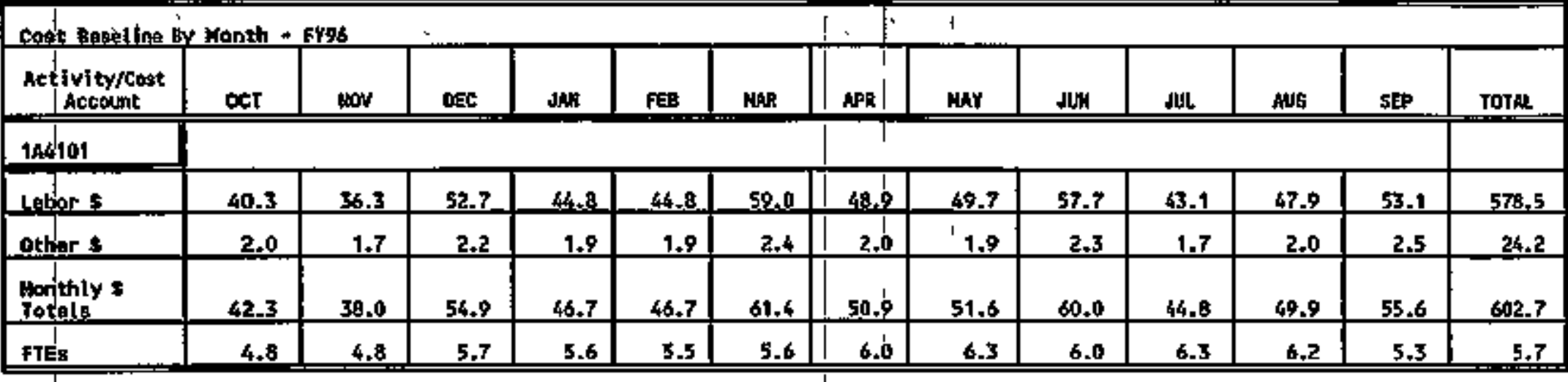

\begin{tabular}{|c|c|c|c|c|c|c|c|c|c|c|c|c|c|}
\hline \multicolumn{14}{|c|}{ cust Heseltine by month - Fros } \\
\hline $\begin{array}{c}\text { Act fulty/cost } \\
\text { Acromnt }\end{array}$ & $\propto \mathbf{C I}$ & nov & DEC & JAL & f比 & MLR & $A P R$ & MAY & WW & $\mathbf{u}$ & Alla & SEP & TOIAL \\
\hline \multicolumn{14}{|l|}{$1 \mathrm{A4200}$} \\
\hline Lettors & 6.9 & 7.7 & 17.4 & 15.6 & 19.0 & 28..8 & 13.5 & 5.6 & 5.4 & 12.8 & 14.4 & 17.3 & 261.1 \\
\hline \multicolumn{14}{|l|}{ other 5} \\
\hline $\begin{array}{l}\text { Monthly } \\
\text { Tatals }\end{array}$ & 6.9 & 7.7 & 17.4 & 15.6 & 19,0 & 25.2 & 13.5 & 5.4 & 5.4 & 12.9 & $\$ 4,4$ & 17.3 & 161,1 \\
\hline FTEO & .9 & 1.3 & 2.2 & 2.3 & 2.7 & 2.8 & 1.8 & .7 & 6 & 2.1 & 2.1 & 1.9 & 1,8 \\
\hline
\end{tabular}

\begin{tabular}{|c|c|c|c|c|c|c|c|c|c|c|c|c|c|}
\hline $\begin{array}{c}\text { Activi ty/Cost } \\
\text { Acoount }\end{array}$ & OCT & wor & DEC & Mall & FEB & Mor & ASR & MAY & AN & JuL & AUIG & \$सP & TOTAL \\
\hline \multicolumn{14}{|l|}{144391} \\
\hline Lebor 5 & 80.7 & 74.8 & 90.8 & 80.7 & 92.7 & 133.9 & 108.7 & 105.6 & 105.0 & 62.8 & 54.2 & 03.3 & 1054.2 \\
\hline other $\$$ & 29.2 & 7.5 & 6.7 & 22.2 & 32.4 & 4.4 & 39.0 & 36.7 & 42.8 & 22.3 & 12.4 & 12.4 & 307.9 \\
\hline $\begin{array}{l}\text { Hornthly } 5 \\
\text { Totels }\end{array}$ & 109.9 & 02.3 & 97.7 & 102.9 & 125.1 & 178.3 & 147.7 & 142,3 & 147.8 & 65.1 & 67.3 & 75.7 & $1360^{2} .1$ \\
\hline FTES & 10.7 & 11.1 & 11.0 & 11.3 & 13.0 & 14.5 & 15.0 & 14.9 & 12.2 & 10.4 & 8.0 & 7.1 & 11.6 \\
\hline
\end{tabular}




\begin{tabular}{|c|c|c|c|c|c|c|c|c|c|c|c|c|c|}
\hline Activit $/ \operatorname{Cos} t$ & OCt & nor & bec & SNM & FEg & 增保 & APR & MAY & JU & JlR & AUG & SEP & TOTAL. \\
\hline \multicolumn{14}{|l|}{2,14501} \\
\hline Lents & 7.1 & 11.0 & 14.1 & 11.6 & 19.5 & 39.7 & 33.5 & 30.2 & 20.1 & 9.0 & 10.0 & 12.2 & 218.2 \\
\hline other 5 & 105,0 & 95,4 & 121,9 & 100.7 & too. 7 & 132.5 & 最, 3 & 84.3 & 101.2 & 75.9 & 34.3 & 105.4 & $\$ 192.4$ \\
\hline $\begin{array}{l}\text { Wonthtr } 5 \\
\text { Totals }\end{array}$ & 113,1 & 106,4 & 135.0 & 112.5 & 120.2 & 17.2 & $117 . \mathrm{B}$ & 114.5 & 121.3 & $84-9$ & 94.3 & 117,6 & 1410.6 \\
\hline FIES & .9 & 1.4 & 1.5 & 1.4 & 2.4 & 3.0 & 4.0 & 3.7 & 2,1 & 1.3 & 1.3 & 1.2 & 2.1 \\
\hline
\end{tabular}

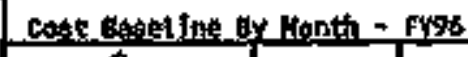

\section{Activity/Cost} Aocolint

\begin{tabular}{|c|c|c|c|c|c|c|c|c|c|c|c|c|c|}
\hline $1 \times 4601$ & & & & & & & & & & & & & \\
\hline Lestots & 57.1 & 51.5 & 65.B & 56.5 & 56.5 & 77.7 & 6.7 & 59.1 & 70.3 & 52.8 & 49.6 & 59.7 & 719,2 \\
\hline other $s$ & 18.1 & 16.3 & 25.2 & 17.2 & 17.2 & 22.6 & 18.0 & 16.6 & 19.8 & 14.8 & 16,4 & 200 & $2 m, 6$ \\
\hline $\begin{array}{l}\text { monthly } \$ \\
\text { Tothle }\end{array}$ & 75.2 & 67,0 & 51.0 & 73.7 & 7.7 & 100.3 & 80.7 & 75.7 & 90,1 & 67.6 & 65.8 & 80,3 & 941.8 \\
\hline FTES & 6.9 & 6.9 & 7.2 & 7.2 & 7.1 & 7.4 & 7.6 & 7.3 & 7.1 & 7.6 & 6.3 & 5.9 & 7.0 \\
\hline
\end{tabular}

\begin{tabular}{|c|c|c|c|c|c|c|c|c|c|c|c|c|c|}
\hline $\begin{array}{c}\text { Activity/Cost } \\
\text { Afecotht }\end{array}$ & 매 & $\mathrm{HOW}$ & OEC & JAM & FEB & MAR & APR & My & 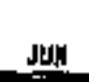 & Lل & AUG & SEP & TOTAL \\
\hline 144720 & & 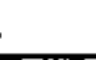 & & & & & & & & & & & \\
\hline Enbor $\$$ & 125,4 & 106.3 & 133.1 & 148.0 & 151,0 & 200 .4 & 212,7 & 239.0 & 291.6 & 210.1 & 233.0 & 286.6 & 2333.2 \\
\hline others: & 26.6 & 23,9 & 30.5 & 25.3 & 조.3 & 46.9 & 78,4 & 애. 7 & 111,6 & 83.6 & - 72.9 & 105.5 & 741.2 \\
\hline $\begin{array}{l}\text { Wonthly } \\
\text { Totals }\end{array}$ & 142.0 & 128.2 & 163,6 & 173.3 & 176.3 & 255.3 & $29 t .1$ & 329.7 & 403.2 & 293.7 & 325.9 & 392.1 & $30,74.4$ \\
\hline FTEY & 16.0 & 16.1 & 16,7 & 21.7 & 21.8 & 23.5 & 31.2 & 35.1 & 35.2 & 35.9 & 35.3 & 33.4 & 26.8 \\
\hline
\end{tabular}




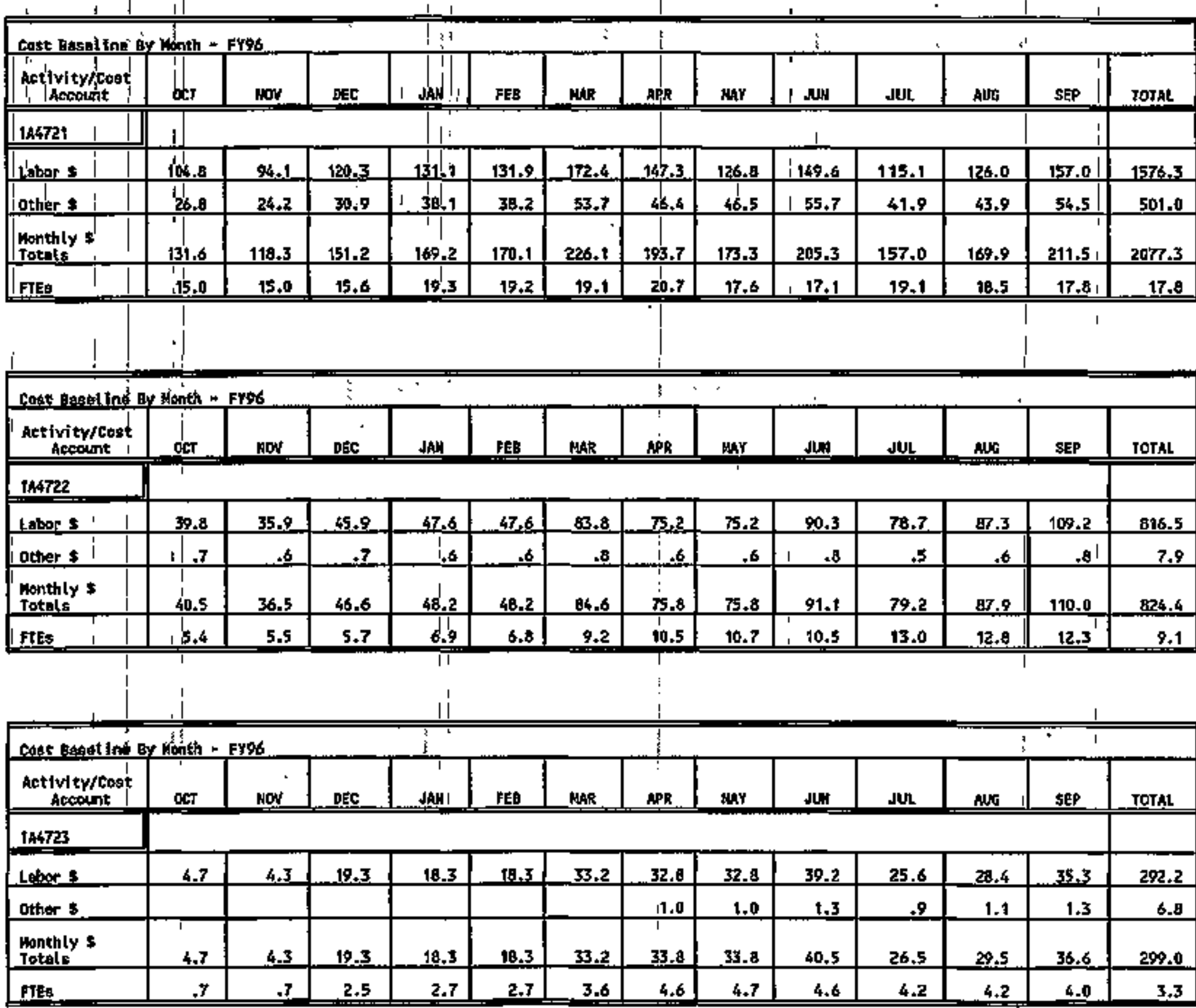




\begin{tabular}{|c|c|c|c|c|c|c|c|c|c|c|c|c|c|}
\hline \multicolumn{14}{|c|}{ 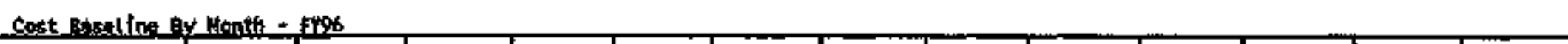 } \\
\hline $\begin{array}{c}\text { Aativity/cost } \\
\text { Acrodnt }\end{array}$ & cct & HOV & bec & JNN & FEB & NAF & APR & MAY & JUH & LIJI & AL'G & sep & TOTAL \\
\hline \multicolumn{14}{|l|}{1,14724} \\
\hline Lehor 5 & 9.7 & 8.8 & 30,8 & 28.8 & $-28,8$ & 62.9 & 59.4 & 59,4 & .71 .2 & 46.5 & 51.2 & 63.9 & 521.4 \\
\hline \multicolumn{14}{|l|}{ other $\$$} \\
\hline $\begin{array}{l}\text { Honthly } 5 \\
\text { Iotols }\end{array}$ & 9.7 & B.B & $30 . \mathrm{B}$ & 28.8 & $2 \mathrm{ZB}, \mathrm{B}$ & 62.9 & 59.4 & 59.4 & 71.2 & 46,5 & 51,2 & 63.9 & 521,4 \\
\hline FTES & 1.4 & 1.4 & 3.7 & 4,2 & 4.2 & 6,9 & 8.4 & e.6 & 8.4 & 7,8 & 7.6 & 7.3 & 5.8 \\
\hline
\end{tabular}

\begin{tabular}{|c|c|c|c|c|c|c|c|c|c|c|c|c|c|}
\hline $\begin{array}{c}\text { Aet fvity/cost } \\
\text { Acfount }\end{array}$ & ㄷT & nov & DEC & 개판 & FEB & MAR & APR & MAY & SNIN & LL & Alls & SEP & TOTAL \\
\hline \multicolumn{14}{|l|}{$1 \wedge 4725$} \\
\hline Lebor $\$$ & 16.8 & 2.5 & & 6.8 & 11.0 & 15.5 & 12.4 & 12.4 & 14.9 & 59.5 & 66.0 & 02.5 & 300,1 \\
\hline \multicolumn{14}{|l|}{ gathers $s$} \\
\hline $\begin{array}{l}\text { Nonthly s } \\
\text { Totale }\end{array}$ & 16.8 & 2.5 & & 6.8 & 11.0 & 19.5 & 12.4 & 12.4 & 14.9 & 59.5 & 66,0 & 82.5 & $300+1$ \\
\hline FTER & 2.2 & .4 & & .9 & 1.5 & 1,6 & 1.6 & 1.7 & 1.6 & 9.2 & 9.1 & g. 7 & 3.2 \\
\hline
\end{tabular}

\begin{tabular}{|c|c|c|c|c|c|c|c|c|c|c|c|c|c|}
\hline \multicolumn{14}{|c|}{ cost Boseline, By Nonith - Frot } \\
\hline Activity/Cost & OCT & $\mathbf{H O N}$ & DEC & JAH & 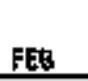 & MAR & Aps & MaY & Jut & & ALE & SEP & TOTAL \\
\hline \multicolumn{14}{|l|}{ 1A7101 } \\
\hline Labor $\$$ & 66.7 & 66.0 & as.z & 68.9 & 51.5 & 65.3 & 52.1 & 52,1 & 63.7 & 49.1 & 54.6 & 70,6 & 746.0 \\
\hline other $\$$ & 28.0 & 25.3 & 32.3 & 25.5 & 4.6 & 2.7 & 2.2 & 2.2 & 37.0 & $3 B .8$ & 42.9 & 53.8 & 295.2 \\
\hline $\begin{array}{l}\text { Monthly \$ } \\
\text { Iottils }\end{array}$ & 94.7 & 21.3 & 117.5 & 94.6 & $56 . t$ & 68.0 & 58,3 & 54.3 & 100.7 & 87.9 & 97.5 & 124,4 & 1041.2 \\
\hline fteg & 7.9 & 8.6 & 9.1 & 8.5 & 6.3 & 6.1 & 6.2 & 6.3 & 6.4 & 6.9 & 6.8 & 6.8 & 7.1 \\
\hline
\end{tabular}




\begin{tabular}{|c|c|c|c|c|c|c|c|c|c|c|c|c|c|}
\hline $\begin{array}{c}\text { Actlylity/cost } \\
\text { Mccount }\end{array}$ & ACT & Hov & DEC & JAH & FER & MAR & APA & whY & JUA & JuL & ANG & SEF & ToTat \\
\hline \multicolumn{14}{|l|}{ IAB 101} \\
\hline Labery $\$$ & 24.5 & 22,0 & $2 \dot{3}, 0$ & 17.9 & 17.9 & 20.3 & 15.9 & 15.9 & 20,8 & 15,0 & 22.1 & 28.9 & 249.2 \\
\hline other $\$$ & 34,8 & 31.4 & 38.3 & .8 & 8 & 1.0 & 8 & 8 & 1.0 & .7 & +8 & 1.1 & 112.3 \\
\hline $\begin{array}{l}\text { Wonthly } \$ \\
\text { Iotala }\end{array}$ & 59.3 & 53.4 & 66.3 & 10.7 & 18.7 & 21.3 & 16.7 & 16.7 & 21.8 & 15.7 & 22.9 & 30.0 & $3 \times 1.5$ \\
\hline FTEE & 2.6 & 2.8 & 2.9 & 2.1 & 2.1 & 1.8 & 1.8 & 1.8 & 2.0 & 2.0 & 2.7 & 2.7 & 2.3 \\
\hline
\end{tabular}

\section{Cost anceltins By Nonth. FrOS}

\section{Actlvity/Cost}

oftemt

1konos

Lebor 5

othar $\$$

montply 5

Totels

FTES

\section{act}

|

\begin{tabular}{|c|c|c|}
\hline DEC & Jan & FEB \\
\hline
\end{tabular}

Mag

MA

Mar

-

ง

\begin{tabular}{|l|l|l|}
\hline AUG & SEP & TaTAL \\
\hline
\end{tabular}

-

\begin{tabular}{|c|c|c|c|c|c|c|c|c|c|c|c|c|}
\hline-16.5 & $14+2$ & 19.0 & 15,5 & 17,0 & 27.8 & 18,2 & 17.6 & 21,1 & 15,8 & -18.2 & 29.5 & 226 \\
\hline 4.7 & 4.2 & 5.4 & 4.5 & 4.5 & 5.9 & 4.7 & 4.7 & 5.6 & 4.2 & 4.7 & 5.8 & \\
\hline
\end{tabular}

4.7 .2

\begin{tabular}{r|r|}
21.2 & 19.1 \\
\hline 2.4 & 2.4 \\
\hline
\end{tabular}

34.4.

\begin{tabular}{l|r|r|r}
2.5 & 2.5 & 2.6 & 2.6 \\
\hline
\end{tabular}

22.9

22.3

7

2.5

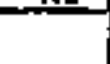

\begin{tabular}{r|r|r|r} 
& & & \\
\hline .0 & 22.9 & 35.4 & 285.3 \\
\hline & 2.8 & 3.5 & 2.7 \\
\hline
\end{tabular}

\begin{tabular}{|c|c|c|c|c|c|c|c|c|c|c|c|c|c|}
\hline $\begin{array}{c}\text { Att } \text { Ivity/cost }_{\text {Aceount }} \\
\end{array}$ & OCT & tow & DEC & dN & F韭 & Hag & $\mathbf{A F R}$ & HaY & Jun & 핀 & AUG & sepp & Total \\
\hline \multicolumn{14}{|l|}{ 14L402 } \\
\hline Labor 5 & 95.4. & 857 & 117.5 & $105 . B$ & 115.6 & 136,5 & 107.9 & 107.8 & 136.5 & 96.8 & 106.2 & 133.1 & 1347,7 \\
\hline other $\$$ & 137.1 & 123.4 & 161.4 & 201.5 . & 221.1 & 20.7 & 200.6 & 197.0 & 234.2 & 176.9 & 474.2 & 653.9 & 3034.1 \\
\hline $\begin{array}{l}\text { Nuthty } 5 \\
\text { Totals }\end{array}$ & 232.5 & 209.1 & 270.9 & 307.3 & 339.7 & 387.2 & 308.5 & 304.8 & 372.7 & 273.7 & 580.4 & 7 묵.0 & 4381.8 \\
\hline FTES & 14.7 & $\$ 4.8$ & 16.5 & 17.2 & 19.5 & 16.7 & 16.8 & 17.0 & 17.7 & 17.7 & 17.2 & 16.6 & 16.9 \\
\hline
\end{tabular}




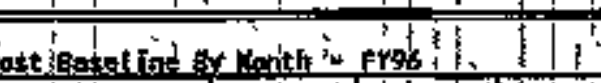

\begin{tabular}{|c|c|c|c|c|c|c|c|c|c|c|c|c|c|}
\hline 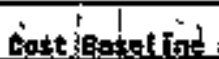 & ittik & 1 & $i$ & $i_{1}$ & & $t$ & "n & & 1 & $1: 3$ & v & & \\
\hline Activity/Cost & of & Har 1 & DEL & Then & Fag & mon & APR & MaY & w & JUL & AUE & SEP & TDIAL \\
\hline 1enLod1 & & & & 1 & & & & & & & & & $T$ \\
\hline Labor & 34,3 & (30, & kolo & 133.2 & 34.9 & $46, \frac{1}{3}$ & $|37,1|$ & 36.6 & 43.7 & 32.8 & 36.6 & $45 \cdot 17$ & 451.8 \\
\hline other $\$$ & .18 .3 & $\mid 16.51$ & 1640 & 21.1 & 25.7 & 35.0 & 28.10 & 17.2 & 19.3 & 14.5 & $16.0^{\prime}$ & 20.0 & 256,8 \\
\hline 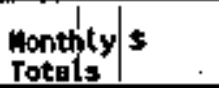 & 52,61 & 473 & ! & 5413 & 60.6 & 61, 3 & $\mid 65.11$ & 53.8 & 63,0 & $47.3^{1}$ & 52.6 & $\begin{array}{r}1 \\
65: 7\end{array}$ & 708.6 \\
\hline fTEa & 5.8 & 5.8 & 6.1 & $5 ! 9$ & 6.1 & 6.1 & $6,3]$ & 6.2 & 6.1 & $6.4^{1}$ & 6.3 & $6: 1$ & 6.1 \\
\hline & & & 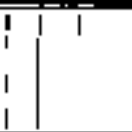 & & & & ! & & & & & & \\
\hline const Basule the & conth. & & & $\therefore$ & & & $\overline{7}$ & & $A^{2}$ & & & & 1 \\
\hline $\begin{array}{c}\text { Act trity/cost } \\
\text { Actount }\end{array}$ & $0<1$ & mon & DEC & JAR & FEE & MAR & ReR & MAY & Suls & لUL & Alls & sep & TOTAL \\
\hline thlogs & & $\begin{array}{ll}1 & 1\end{array}$ & $T$ & & & & & $T$ & $T$ & & & & I \\
\hline Laboor 8 & 98,3 & 183 & 113.5 & $93 ! 9$ & 97.5 & 230,0 & 102,4 & 101,8 & 121.7 & 99,4 & 요. & 123,3 & 1261.6 \\
\hline other $\$$ & 142,4 & 129.9 & $167.4^{\circ}$ & 139.0 & 143.6 & 290.1 & 143,3 & $\frac{1}{132.5}$ & 157,6 & 118,2 & 131,4 & $164: 2$ & 1757.9 \\
\hline $\begin{array}{l}\text { Monthety } \\
\text { Totals }\end{array}$ & 240.7 & 216.4 & 290.9 & $232^{1} g$ & 241.5 & 320.1 & $\begin{array}{c}1 \\
1245.7 \\
\end{array}$ & 234.3 & 279.3 & 209,6 & 230.6 & 287.5 & 3019.5 \\
\hline PTEs & 14.5 & 14.5 & 1. 15.2 & 14.6 & 15.0 & $15 . \frac{1}{2}$ & 125.2 & 15.3 & 15.0 & $15.9^{1}$ & 15.3 & $14 ! 6$ & 15.0 \\
\hline & & & I & 1 & & & 111 & & & & & & \\
\hline 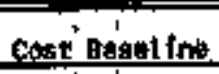 & Nenth & 206:3: & $1 ;$ & & & & T & & $T$ & $\overline{1}$ & & 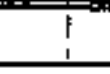 & 1 \\
\hline act $\left.\right|_{\substack{\text { vityount } \\
\text { cecoust }}}$ & aCt & $\begin{array}{c}1 \\
1 \\
\text { Ind }\end{array}$ & TEE & JALI & FEB & $\begin{array}{l} \\
\text { IMAR }\end{array}$ & \begin{tabular}{l|l|}
1 \\
1 Apk
\end{tabular} & may & bur & JUL & ANG & SEP & Torkl \\
\hline $\begin{array}{l}1 \\
H\end{array}$ & & & & & & & & & & & & & \\
\hline Labor 5 & 60.5 & 54.5 & 69.6 & 57.5 & 57.5 & 73.7 & 60.5 & 60.5 & 72.6 & 54.5 & 60.5 & 75.7 & 39.5 \\
\hline Other 5 & 9.9 & 8.9 & 11.4 & 9.5 & 9.5 & 12.4 & 9.9 & 19.9 & 12.0 & 8.9 & 9.9 & 12,4 & 124.9 \\
\hline $\begin{array}{l}\text { Wonthly } \\
\text { Totate }\end{array}$ & 70.4 & 63,4 & 81.0 & 67,0 & 67.0 & $8 \mathrm{~g} .1$ & 30.4 & 70.4 & 84,6 & 63.4 & 70,4 & 88.1 & 834.4 \\
\hline FIEE & 9.6 & 9.6 & 10.0 & 9.6 & 9.5 & 9.5 & 9.7 & 9.8 & 9.7 & 10.3 & 10.1 & 9.7 & 9.7 \\
\hline
\end{tabular}




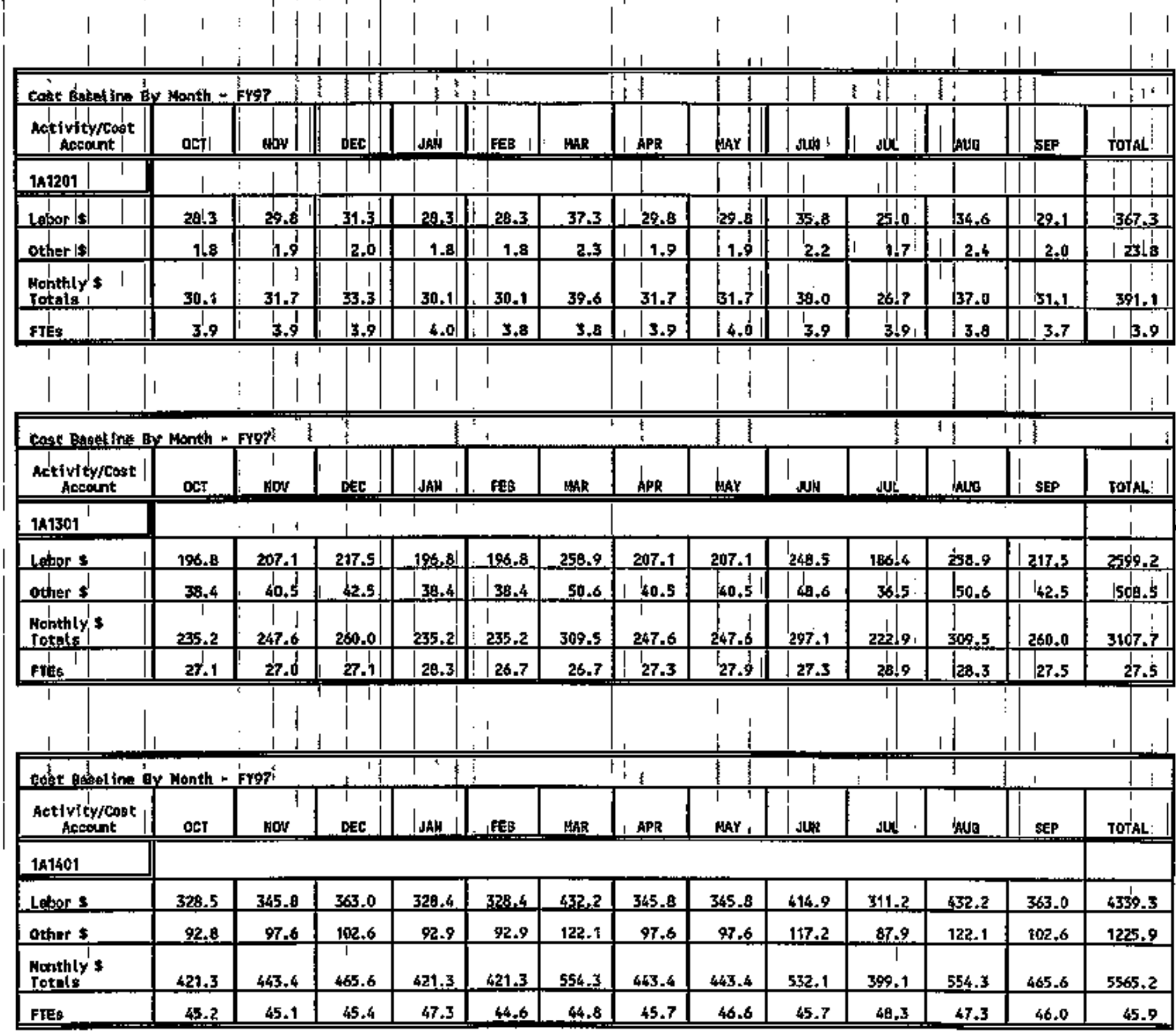




\begin{tabular}{|c|c|c|c|c|c|c|c|c|c|c|c|c|c|}
\hline $\begin{array}{c}\text { Act ivity/Cost } \\
\text { Aceount }\end{array}$ & AcT & $\mathrm{How}$ & DEC & JAN & $\mathrm{FEB}$ & $\mathbf{M R}$ & APR & MAY & JuW & עUL & Atso & SEP & TOTAL \\
\hline \multicolumn{14}{|l|}{ IA1501 } \\
\hline Leteos & 29.9 & 31.5 & 33.1 & 29.9 & 29.9 & 39.4 & 31.5 & 31.5 & 37.8 & 28,6 & 30.4 & 33.1 & 395.3 \\
\hline other 5 & 9.1 & 6.5 & g.9 & e.1. & $B, 1$ & 10.6 & B. 5 & 8.5 & 10.2 & 7.6 & 10,6 & 8.9 & 106.2 \\
\hline $\begin{array}{l}\text { Nonthly } 8 \\
\text { fatals }\end{array}$ & 3B.0 & 40.0 & 42.0 & 38.0 & 3.8.0 & 50.0 & 40.0 & 40.0 & $4 B .0$ & 3.0 & 50.0 & 42.0 & 501.5 \\
\hline FTES & 4.5 & 4.5 & 4.6 & 4.7 & 4.5 & 4.5 & 4.6 & 4.7 & 4.6 & 4.9 & 4.8 & 4,6 & 4.6 \\
\hline
\end{tabular}

\begin{tabular}{|c|c|c|c|c|c|c|c|c|c|c|c|c|c|}
\hline \multicolumn{14}{|c|}{ 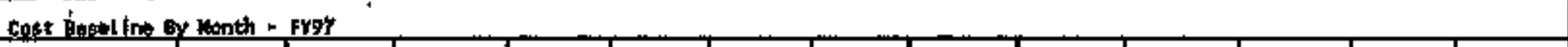 } \\
\hline $\begin{array}{c}\text { Aotivity/Cost } \\
\text { Acconnt }\end{array}$ & OCT & sov & DEC & Jat & FE甘 & $M A R$ & $\mathrm{APR}$ & Mar & Nall & Nul & AUfO & SEP & TOTAL \\
\hline \multicolumn{14}{|l|}{$1 \mathrm{~A} 1601$} \\
\hline Lepor 5 & 278.9 & 294.7 & 300,4 & 279.9 & 279.9 & 360,3 & 292.8 & 292.6 & 351.1 & 263.3 & 365.7 & 307.2 & 6684.9 \\
\hline other : & $10 \mathrm{~B}, 3$ & $1 \$ 4.5$ & $12,0.3$ & 108.9 & 108.9 & 143.2 & 104,9 & $103 . B$ & 124.5 & 93.5 & $129, B$ & 109.0 & 1369.3 \\
\hline $\begin{array}{l}\text { Nonthly } \$ \\
\text { Totala }\end{array}$ & 39.9.1 & 400.2 & 429.7 & 389.8 & 368.0 & 511.5 & 397.7 & 396.4 & 475.6 & 386.7 & 495.5 & 416.2 & 50.54 .2 \\
\hline FTES & 39.3 & 39.1 & 39.5 & 41.1 & $3 \mathrm{e} . \mathrm{B}$ & 38.9 & 39.4 & 40.2 & 39.4 & 41.7 & 40.9 & 39.7 & 39.8 \\
\hline
\end{tabular}

\begin{tabular}{|c|c|c|c|c|c|c|c|c|c|c|c|c|c|}
\hline \multicolumn{14}{|c|}{ cost besellog sy Honth - frot } \\
\hline $\begin{array}{c}\text { Ackivity/Cost } \\
\text { Aceont }\end{array}$ & OL:T & How & DEC & IAN & FEB & MaF & APR & MAY & . & JUL & ANG & SEP & TOTAL \\
\hline \multicolumn{14}{|l|}{ 1A1901 } \\
\hline Lobor $\$$ & 155.6 & 216.4 & 103.0 & 119.9 & 318.0 & 152.0 & 147.2 & 128.3 & $105-8$ & 72.2 & 54.3 & 52.4 & 1325.5 \\
\hline Dther $s$ & 125.7 & 144.5 & 137.0 & 162.4 & 121.7 & 150,7 & 104.7 & 83.6 & 72.5 & 75.3 & 75.0 & 70.5 & 1323.7 \\
\hline $\begin{array}{l}\text { Monthly } \\
\text { Totelo }\end{array}$ & 281.3 & 360.8 & 240.0 & 282.3 & 239.7 & 302.7 & 251.9 & 211.9 & 178,3 & 148.0 & 129.3 & 122.9 & 2649.2 \\
\hline Hes & 21.2 & $\$ 5.1$ & 13.0 & 17.5 & $\$ 5.9$ & 15.6 & 19.4 & 17.3 & 11.7 & 11.7 & 6.4 & 7.1 & 14.1 \\
\hline
\end{tabular}




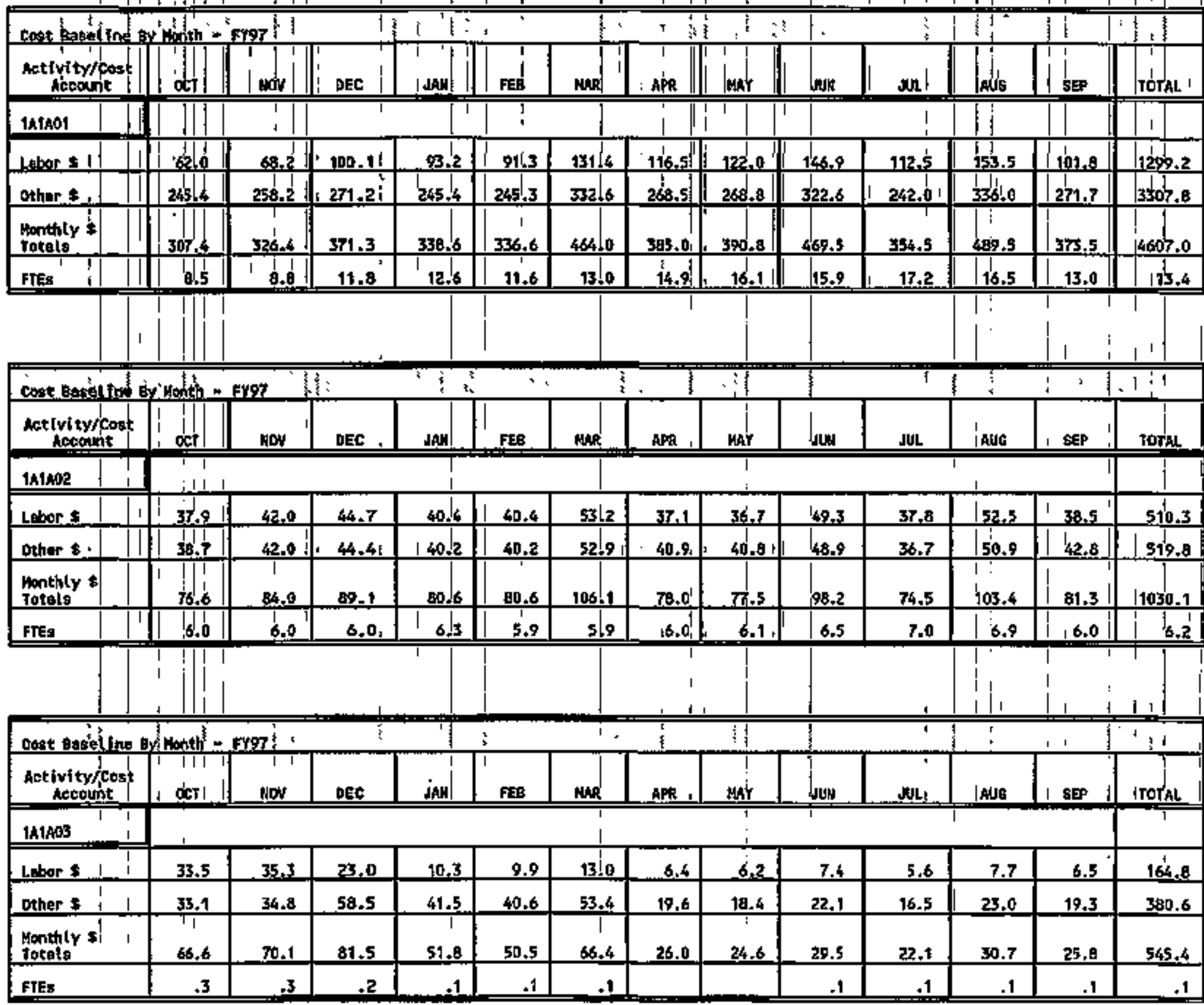




\begin{tabular}{|c|c|c|c|c|c|c|c|c|c|c|c|c|c|}
\hline \multicolumn{14}{|c|}{ 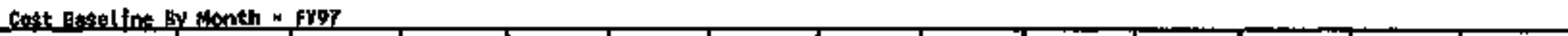 } \\
\hline$\underset{\substack{\text { Aet }|v| t y / \cos t \\
\text { Aecolnt }}}{ }$ & oct & Moy & QEeC & Jak & FEB & whe & APR & mar & NH & JUL & Als & SEP & TOTAL \\
\hline \multicolumn{14}{|l|}{$1 \mathrm{A3} 3104$} \\
\hline Lubar $s$ & 51.4 & 43.4 & 66.2 & 50,10 & 43.2 & 47.7 & 49.8 & 47.6 & 47.1 & 47.5 & 47.5 & 57.6 & $579 . \pm$ \\
\hline other 5 & $\mathbf{3 . 3}$ & 2.9 & 3.0 & 3.3 & 2.8 & 3.2 & 3.4 & 3.1 & 3.2 & 3.2 & 3.2 & 58.1 & 92.7 \\
\hline $\begin{array}{l}\text { Honthly } 5 \\
\text { Totale }\end{array}$ & 54.7 & 46,3 & 49.2 & 53.3 & 46.0 & 50.8 & 53.2 & 50.7 & 50,3 & 50,7 & 50.7 & 115,7 & 671.8 \\
\hline FIES & 7,1 & 5.7 & 5.8 & 7.2 & 5.9 & 4.9 & 6.6 & 6,4 & 5.2 & 7.4 & 5.2 & 7.3 & 6.1 \\
\hline
\end{tabular}

\begin{tabular}{|c|c|c|c|c|c|c|c|c|c|c|c|c|c|}
\hline \multicolumn{14}{|c|}{ Cost Bogshline Hy Whanth " Frgt } \\
\hline $\begin{array}{l}\text { Aet I vit ty/Cost } \\
\text { Accouint }\end{array}$ & $\underline{\underline{O}}$ & Nov & 听 & JAH & FEB & 神 & APR & MaY & 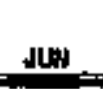 & 亗 & הNي & SEP & TOTAL \\
\hline \multicolumn{14}{|l|}{185202} \\
\hline Lotors & $120-1$ & 99.2 & 99.2. & $108,4$. & $96,3$. & 103.6 & 100.4 & 103.4 & 136.6 & 168.7 & 964,4 & 139.5 & 1446.9 \\
\hline Qthers: & 46.5 & 38,4 & 40,4 & $44-2$ & 38.2 & 23,0 & 23.1 & 2.1 & 30.8 & 30.8 & 29.6 & 22.1 & 389 \\
\hline $\begin{array}{l}\text { Monthly } \\
\text { Totale }\end{array}$ & 166.6 & 137.6 & 139.6 & 252.6 & 134.5 & 126.6 & 131.5 & 129.5 & 167,4 & 199.5 & 194,0 & 160.6 & 1836,0 \\
\hline FEES & 16.1 & 12,6 & 22.0 & 15.1 & 12.7 & 10.4 & 13.8 & 13,5 & 14.6 & 24.6 & 16.9 & 16.4 & 14.7 \\
\hline
\end{tabular}

\begin{tabular}{|c|c|c|c|c|c|c|c|c|c|c|c|c|c|}
\hline \multicolumn{14}{|c|}{ 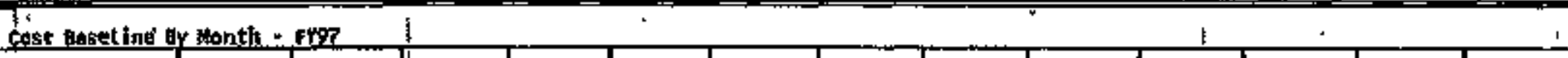 } \\
\hline $\begin{array}{l}\text { Aet IvIty/Cost } \\
\text { AcEolut }\end{array}$ & OCI & MON & DEC & $\begin{array}{l}\mathrm{JAR} \\
\end{array}$ & FEg & MAR & $\mathrm{APR}$ & MaY & 亗政 & _البر & $A N_{G}$ & SEP & TOTAL \\
\hline \multicolumn{14}{|l|}{$1 \times 33204$} \\
\hline Lbor $:$ & 09.9 & 95,7 & $79 . t$ & 96.6 & 85.5 & 100.8 & 94,0 & 112.6 & 85.5 & 97.7 & 84.9 & 104.9 & 1145.2 \\
\hline other 51 & 36.7 & 30.3 & 31.9 & 35.2 & 30.4 & 33.5 & 35.2 & 33.3 & $35: 6$ & 33.5 & 33.5 & 33.6 & 400.9 \\
\hline $\begin{array}{l}\text { Monthlys } \\
\text { Itale }\end{array}$ & 126.6 & 126.0 & $\$ 11.0$ & 131.8 & 115.9 & $\$ 2,3$ & 129,2 & 146.1 & 119.1 & 133.2 & 129.4 & 138.9 & 1546.1 \\
\hline FTES & 12.9 & 13.0 & 10.3 & 14.4 & 12.2 & 11.6 & 12.8 & 15.6 & 9.8 & 15.7 & 10.7 & 13.8 & 12.6 \\
\hline
\end{tabular}




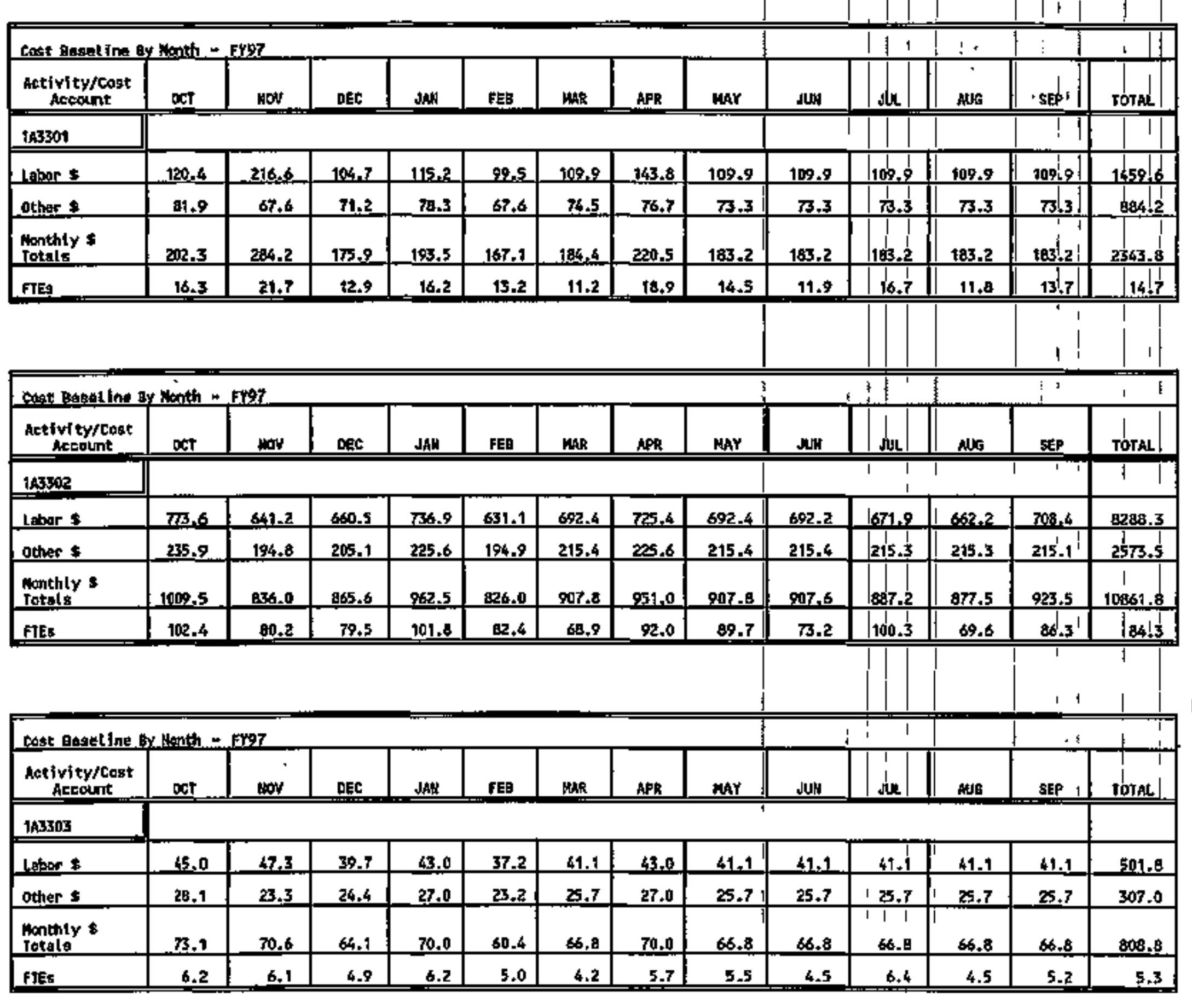




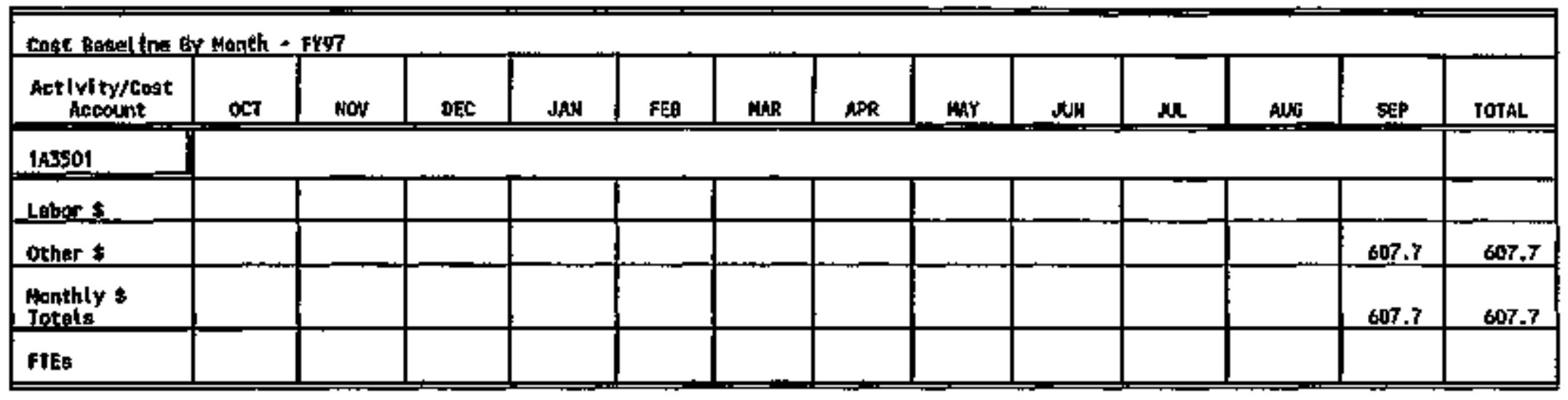

\begin{tabular}{|c|c|c|c|c|c|c|c|c|c|c|c|c|c|}
\hline $\begin{array}{c}\text { Activity/Cost } \\
\text { Anetount }\end{array}$ & DLT & wor & DEC & JAH & FEB & $M R$ & APR & HAY & suk & 펜 & $M H_{G}$ & SEP & Torkl. \\
\hline \multicolumn{14}{|l|}{146401} \\
\hline Labor $\$$ & 19.6 & 30.6 & 21.7 & 19,6 & -19.6 & 25.8 & 1.0 & & & & & & 128.0 \\
\hline \multicolumn{14}{|l|}{ other \& } \\
\hline $\begin{array}{l}\text { Monthly s } \\
\text { Totals }\end{array}$ & 19.6 & 20.6 & 21.7 & 19.6 & 19.6 & 25.8 & 1.0 & & & & & & 128.0 \\
\hline mes & 2.3 & 2.3 & 2.3 & 2.4 & 2.3 & 2.3 & .1 & & & & & & 1.2 \\
\hline
\end{tabular}

\begin{tabular}{|c|c|c|c|c|c|c|c|c|c|c|c|c|c|}
\hline \multicolumn{14}{|c|}{ Cogat losast ine By Honth + Frgy } \\
\hline $\begin{array}{l}\text { At I Ifity/Cost } \\
\text { Account }\end{array}$ & OEY & $\mathrm{HOF}$ & DEC & JAH & FEB & MAR & APR & MAY & Nan & JUL & $M$ & SEP & ToTht \\
\hline \multicolumn{14}{|l|}{$1 \mathrm{~A} 4720$} \\
\hline Enbor $\$$ & 214.7 & 226.0 & 237.3 & 218.4 & 217.4 & 285.5 & 230.7 & 234,2 & 281,0 & 210.7 & 292.7 & 241.4 & 289.8 \\
\hline other: & 148,2 & 155.0 & 163.8 & 148.2 & 148.3 & 195.0 & 156.0 & 155,0 & 187.3 & 160.5 & 195.1 & 163.8 & 1958.5 \\
\hline $\begin{array}{l}\text { Wonthly * } \\
\text { Iotals? }\end{array}$ & 362.9 & 382.0 & 401,1 & 366.6 & 365.7 & 480.5 & 担 6 . & 390.2 & 468.3 & 351,2 & 487.8 & 405,2 & 4840.3 \\
\hline FYE5 & 32.1 & 32.0 & 32.3 & 34.1 & 32.0 & 32.1 & 33.0 & 34.2 & 33.5 & 35.5 & 34.7 & 33.2 & 33.2 \\
\hline
\end{tabular}




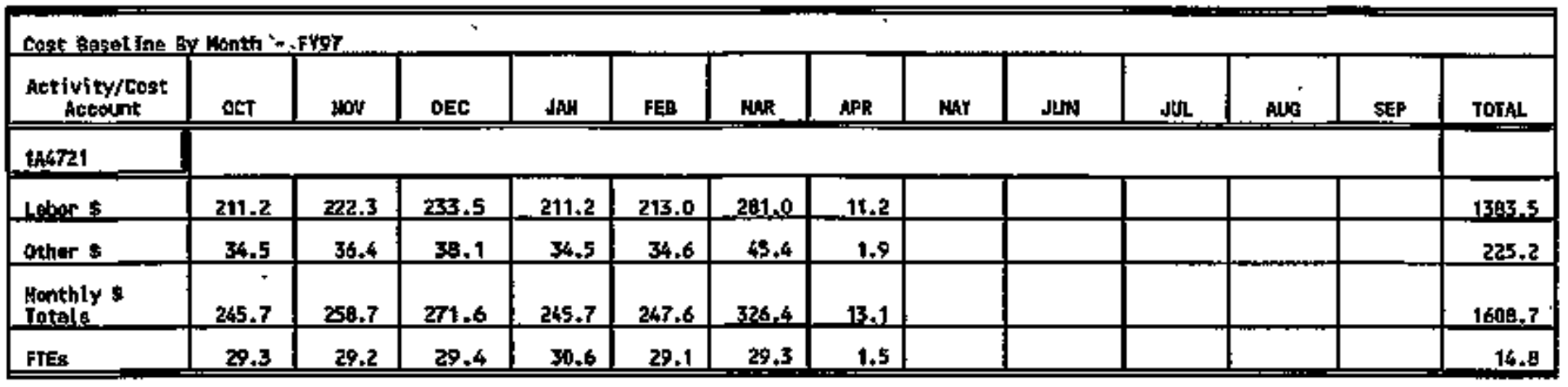

Cost Busellint By Manth n fYg?

\begin{tabular}{|c|c|c|c|c|c|c|c|c|c|c|c|c|c|}
\hline $\begin{array}{c}\text { Act lvity/Cost } \\
\text { Accoint }\end{array}$ & OCT & NON & DEC. & 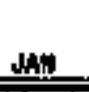 & FEB & MAR & APR & Mrr & JUN & لUL & AUB & SEP & TOTAL \\
\hline \multicolumn{14}{|l|}{144722} \\
\hline Lahar $s$ & 74.3 & 78,4 & 82,3 & 74.5 & 74,5 & 118,8 & 104.1 & 1043 & $125 \cdot 2$ & 90.3 & 124.8 & 104.8 & 1156.2 \\
\hline Other & 2.8 & 3.0 & 3.2 & 2.8 & 2.9 & 3.8 & 3.0 & 3.1 & 3.6 & 2.7 & 3.8 & 3.2 & 39.2 \\
\hline $\begin{array}{l}\text { Nonthly } \\
\text { totals }\end{array}$ & $\pi .1$ & 81,4 & 85.5 & 77.4 & $\pi .4$ & 122,6 & 107.1 & 107.4 & 128.8 & 93.0 & 128,6 & 108.0 & 1194,4 \\
\hline FTES & to.B & 10.7 & 10. $\theta$ & 11.3 & 10.6 & 12.9 & 14.4 & 14.8 & 14.5 & 14.7 & 14.3 & 14,0 & 12.9 \\
\hline
\end{tabular}

\begin{tabular}{|c|c|c|c|c|c|c|c|c|c|c|c|c|c|}
\hline \multicolumn{14}{|c|}{ Cost sos lins by Honth - FY97 } \\
\hline $\begin{array}{l}\text { Activi ty/cost } \\
\text { Accoulnt }\end{array}$ & OCT & $\mathrm{kg}$ & DEC & JAA & FEE & MAR & APR & Mar & JUN & 此 & $M W_{5}$ & SEP & IOIAL, \\
\hline \multicolumn{14}{|l|}{144723} \\
\hline Letors's & 18.3 & 19.2 & 33.7 & 32.5 & 32.5 & 50.5 & 46.6 & 46.9 & 56.2 & 39,3 & 52.9 & 44,5 & 472.0 \\
\hline Dther $\$$ & 1.5 & 1.6 & 1.7 & 1.5 & 1.5 & 2.0 & 2.6 & 2.6 & 3.3 & 2.5 & 3.4 & 2.8 & 27.0 \\
\hline $\begin{array}{l}\text { Monthly s } \\
\text { Totsls }\end{array}$ & 19.8 & 20.8 & 35.4 & 34.0 & 34,4 & 52.5 & 49.2 & 49.5 & 59.5 & 40.8 & 56.3 & 47,3 & 499.0 \\
\hline FTEa & 2.7 & 2.7 & 4.5 & 5.0 & 4.7 & 5.5 & 6.5 & 6.6 & 6.5 & 6.3 & 6.1 & 5.9 & 5.3 \\
\hline
\end{tabular}




\begin{tabular}{|c|c|c|c|c|c|c|c|c|c|c|c|c|c|}
\hline 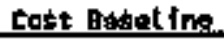 & & & & & & & & & & & & & \\
\hline $\begin{array}{c}\text { Activity/cost } \\
\text { Account }\end{array}$ & OCT & MON & DEC & Ja & FEg & HAR & APR & MuY & JuW & $\mathrm{NOL}$ & All15 & sEe & TOTAL \\
\hline \multicolumn{14}{|l|}{144724} \\
\hline Labor $s$ & 30.5 & 32.1 & 52,8 & 50.7 & 50.7 & 89.1 & 80.8 & 8t.1 & 97.3 & 66.2 & 90.8 & 76,3 & 798.3 \\
\hline Other $\$$ & 1.7 & 1.8 & 1.9 & 1.7 & 1.7 & 2.3 & 1.9 & 1.8 & 2.2 & 1.7 & 2.3 & 1.9 & 23.1 \\
\hline $\begin{array}{l}\text { Monthly } \\
\text { Iotelis }\end{array}$ & 32.2 & 33.9 & 54.7 & .52 .4 & $\$ 2.4$ & 91.4 & 82.7 & 82.9 & 99.5 & 67.9 & 93.1 & 78.2 & 921.4 \\
\hline FTES & 4.6 & 4.6 & 7,1 & 7.8 & 7.4 & 9.8 & 11,4 & 11,6 & 11.6 & $1 t .0$ & 10.6 & 10.3 & 9.0 \\
\hline
\end{tabular}

\begin{tabular}{|c|c|c|c|c|c|c|c|c|c|c|c|c|c|}
\hline $\begin{array}{l}\text { Activity/cost } \\
\text { Atecount }\end{array}$ & aCT & May & DEC & 14. & FEE & MAR & APR & MAY & עلالد & ditit & AUG & sitp & TORAL \\
\hline \multicolumn{14}{|l|}{ 1AGFEE } \\
\hline \multicolumn{14}{|l|}{ Lebor $\&$} \\
\hline other 5 & 22.7 & 23.9 & 25.1 & 22.7 & 22.7 & 29.9 & 23.9 & 23.9 & 28.7 & 21.5 & 29.9 & 25.1 & 300,0 \\
\hline $\begin{array}{l}\text { Nonthly } y \\
\text { Totals }\end{array}$ & 22.7 & 23.9 & $\mathbf{2 5 . 1}$ & 22.7 & 22.7 & 29.9 & 23.9 & 23.9 & 29.7 & 21.5 & 29.9 & 25.1 & 300,0 \\
\hline FTes & & & & & & & & & & & & & \\
\hline
\end{tabular}

\begin{tabular}{|c|c|c|c|c|c|c|c|c|c|c|c|c|c|}
\hline \multicolumn{14}{|c|}{ cost Baseling Gy Honsh - FY97 } \\
\hline$\underset{\text { Actint }}{\text { Actity }}$ & act & HON & $\mathrm{DEC}$ & JAN & fEB & MAR & $M P R$ & MAY & sW & NLL & Allos & SEP & rotAl \\
\hline \multicolumn{14}{|l|}{ 1AB101. } \\
\hline Labor * & 19.1 & 20,1 & 21.1 & 19.1 & 19.1 & 21.7 & 46.0 & 15.0 & 17.1 & 11.5 & 16.0 & 13.4 & 239,3 \\
\hline othes $\$$ & .3 & .4 & .4 & 3 & .3 &.$\underline{.}$ & 4 & .4 & .5 & .4 & .5 & .4 & 4.7 \\
\hline $\begin{array}{l}\text { Honthly } s \\
\text { Tatals }\end{array}$ & 19,4 & 20,5 & 21.5 & 19.4 & 12.4 & 22.2 & 46.4 & 15.6 & 17,6 & 11.9 & 16.5 & 13.8 & 244,0 \\
\hline ETES & 2.2 & 2.2 & 2.2 & 2.3 & 2.1 & 1.9 & 5.0 & 1.7 & 1.6 & 1.5 & 1.4 & 1.4 & 2.1 \\
\hline
\end{tabular}




\begin{tabular}{|c|c|c|c|c|c|c|c|c|c|c|c|c|c|}
\hline $\begin{array}{c}\text { Aetivity/Cost } \\
\text { Account }\end{array}$ & DCT & nor & DEC & JAN & FEB & MAR & APR & MAY & ل세 & Lال & $A \mathbb{O}^{-}$ & SEP & TOTAL \\
\hline 1Food & & & & & & & & & $\cdot$ & & & & \\
\hline Lolpars & $16 . t$ & $\$ 7.0$ & 17.8 & 26.1 & 17.5 & 23.5 & 18.8 & 19.2 & 21.7 & 16.3 & 23.5 & 26.6 & 233.1 \\
\hline Othar $s$ & 4.7 & 4.9 & 5.1 & 4.6 & 4.6 & 6,1 & 4.9 & 4.8 & 5.8 & 4.3 & 6.1 & 5.1 & 61.0 \\
\hline $\begin{array}{l}\text { Hontbly s } \\
\text { Iotgles }\end{array}$ & 20.8 & 21.9 & 22.9 & 20.7 & 22.1 & 29.6 & 23.7 & 23.0 & 27.5 & 20.6 & 29.6 & $3 t .7$ & 294.1 \\
\hline FTES & 2.4 & 2.4 & 2.4 & 2.5 & 2,6 & 2.7 & 2,7 & 2.7 & 2,6 & 2,8 & 2,8 & 3.7 & 2.7 \\
\hline
\end{tabular}

\begin{tabular}{|c|c|c|c|c|c|c|c|c|c|c|c|c|c|}
\hline \multicolumn{14}{|c|}{ 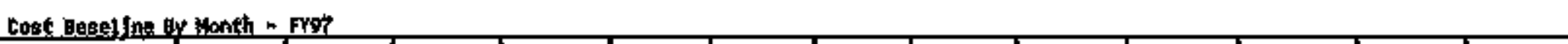 } \\
\hline $\begin{array}{c}\text { Activity/cost } \\
\text { Account }\end{array}$ & QCT & wow & DEC & LAM & FEB & $\operatorname{MaR}$ & AFR & HAY & WN & . Lالال & AUE & SEP & TOTAL \\
\hline \multicolumn{14}{|l|}{ 1AL40R } \\
\hline Labor 5 & $10 t .1$ & 905.6 & arz.3. & 107.6 & $112,1$. & 141,3 & $11 t, 0$ & 111.0 & 132.9 & 94.2 & 125.7 & 104.9 & 1364,7 \\
\hline Other 5 & $\$ 92.2$ & 213.2 & 227.5 & $20,6.4$ & 200.5 & 261,1 & 200, 9 & 205.9 & 245.8 & 133.1 & 164.5 & 138,2 & 2397.2 \\
\hline $\begin{array}{l}\text { Monthly } \$ \\
\text { Totgls }\end{array}$ & 203.3 & 318.8 & 344.8 & 314,0 & 312.6 & 402,4 & 319.9 & 316.0 & 378.7 & 227.3 & 290.2 & 243.1 & 3764.9 \\
\hline FIEO & 16.2 & 16.0 & 17.1 & 18.1 & 17.8 & 17.1 & 17.1 & 17.5 & 17.1 & 17.2 & 16.1 & 15,6 & 16.9 \\
\hline
\end{tabular}

\begin{tabular}{|c|c|c|c|c|c|c|c|c|c|c|c|c|c|}
\hline $\begin{array}{c}\text { Actlivity/tost } \\
\text { decount }\end{array}$ & OCT & $\mathbf{H W}$ & DEC & JAS & FEB & $\operatorname{maR}$ & APR & MAY & suis & Jill & $\mu N_{6}^{\prime}$ & sep & rotal \\
\hline \multicolumn{14}{|l|}{ 1HLSOE } \\
\hline Labor 3 & 33,9 & 35.7 & 38.1 & 34.5 & 36.2 & 48.4 & 38.7 & 39.2 & 45.7 & 34,3 & 47.6 & 40.0 & 471.1 \\
\hline Dther $\$$ & 17.6 & 18.6 & 23.1 & 21.5 & 25.e & 36.0 & 28.8 & 18.2 & 19.6 & 14.6 & 20.4 & 17.1 & 261,6 \\
\hline $\begin{array}{l}\text { Monthly } 5 \\
\text { Totals }\end{array}$ & 51.5 & 54.3 & 61.2 & 56.0 & 62.0 & 84.4 & 67.5 & 56.4 & 65.3 & 48.9 & 68.0 & 57,1 & 732.7 \\
\hline FTES & 5.7 & 5.7 & 5.9 & 6.1 & 6.0 & 6.1 & 6.3 & 6.2 & 6.1 & 6.4 & 6.3 & 6.1 & 6.1 \\
\hline
\end{tabular}




\begin{tabular}{|c|c|c|c|c|c|c|c|c|c|c|c|c|c|}
\hline $\begin{array}{l}\text { Aet fvity/loost } \\
\text { Acoodnt }\end{array}$ & OCt & Noy & DEc & JAB & KE日 & MAR & AFR & MAY & Jum & IUL & Mo & SEP & TOTAL \\
\hline \multicolumn{14}{|l|}{ 14Lons } \\
\hline Lبtor & 949 & 92.9 & 105.5 & 95.5 & 99,3 & 132,5 & 104.2 & 103.5 & 124.1 & 93.0 & 126.0 & 105.3 & $1293 . B$ \\
\hline ather $s$ & 138.6 & 145.9 & 156.B & 142.5 & 146.8 & 195.1 & 147.6 & 136.5 & 161.5 & 121.2 & 168,3 & 141.4 & 1801.9 \\
\hline $\begin{array}{l}\text { Monthity } 5 \\
\text { Totals }\end{array}$ & 735.5 & 245.8 & $262+3$ & 238.0 & 246.1 & 327.6 & 251.8 & 260.0 & 285.6 & 214,2 & 294.3 & 246.7 & 3085.7 \\
\hline FTEs & 14.5 & 14.4 & 14.7 & 15.3 & 15.0 & 15.2 & 15.3 & 15.5 & 15.1 & 16.0 & 15.3 & 14.8 & 15.1 \\
\hline
\end{tabular}

\begin{tabular}{|c|c|c|c|c|c|c|c|c|c|c|c|c|c|}
\hline $\begin{array}{c}\text { Act ivity/cost } \\
\text { Accolnt }\end{array}$ & OCT & $\mathrm{MON}$ & btc & shes & 筙 & MheR & $\mathrm{APR}$ & MaY & $\mathrm{Mn}$ & $3 \mathbf{N L}$ & AUT & SEP & TOTAL \\
\hline \multicolumn{14}{|l|}{$1 \mathrm{Nh}$} \\
\hline Lerbor 5 & 47.9 & 50.4 & 52.9 & 47.9 & 47,9 & 63.0 & 50.4 & 50.4 & 60.5 & 45.4 & 63.0 & 52.9 & 652.6 \\
\hline other $\$$ & 8,3 & 8,7 & 9.2 & 8,3 & 8.3 & 10.9 & 息? & B. 7 & 10.4 & 7.8 & 10.9 & 9.2 & 109.2 \\
\hline $\begin{array}{l}\text { Nunthly } \$ \\
\text { fotals }\end{array}$ & 56.2 & 59.1 & 62.1 & 56.2 & 56.2 & $\pi .9$ & 59.1 & $5 \% .1$ & 70.9 & 53.2 & 7.9 & 62.1 & 741.8 \\
\hline Ftes & 7.8 & 7.8 & 7.8 & 8.1 & 7.7 & 7.7 & 7.9 & B.0 & 7.9 & B.3 & E.1 & 7.9 & 7.9 \\
\hline
\end{tabular}

NOTE: The FY97 cost information is based upon Financial Data system rates as of September 12, 1995. 


\begin{tabular}{|c|c|c|c|c|c|c|c|c|c|c|c|c|c|}
\hline $\begin{array}{c}\text { Activity/Cost } \\
\mid \text { Aecount }\end{array}$ & OCT & NOW & DEC & JAK & FEB & MaR & APR & MAY & $d \mathrm{dL}_{\mathrm{t}}$ & NUL & ANG & SEP & TOEAL \\
\hline \multicolumn{14}{|l|}{ 1A1201 } \\
\hline Lebor & 84.5 & 78.5 & 61.5 & 82,9 & 98.8 & 58,6 & 31.4 & 37.7 & 31.6 & 48,1 & 70.3 & 61.9 & 722,4 \\
\hline other $/ \mathrm{s}$ & 9.7 & 43.0 & 42,4 & 6.9 & 70.0 & 19.6 & 1.7 & 2.3 & 1.9 & 1.8 & 2.4 & 2.1 & 254.4 \\
\hline $\begin{array}{l}\text { Monthly } \\
\text { Intsels }\end{array}$ & 63.2 & 121.5 & 103.9 & 147,8 & 168,8 & 78.2 & 33.3 & 40.0 & 33.3 & 46.9 & T2., & 64.0 & 976.9 \\
\hline FTEN । & 6.5 & 8.5 & B. 7 & 10.9 & 13.0 & 5.7 & 3.8 & 3,8 & 3.9 & 7.2 & 7.4 & 7,2 & 7.3 \\
\hline
\end{tabular}

\begin{tabular}{|c|c|c|c|c|c|c|c|c|c|c|c|c|c|}
\hline \multicolumn{14}{|c|}{ 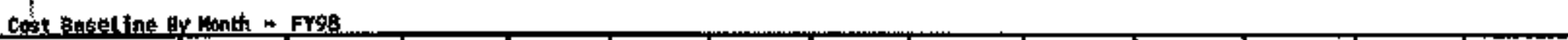 } \\
\hline $\begin{array}{c}\text { Activit } w / \cos t \\
\text { Afseount }\end{array}$ & OCT & HOW & DEC & stpr & FEB & Mas & ApR & MAY & N & JLL & Aus & SEP & TOTAR \\
\hline \multicolumn{14}{|l|}{ 1A1301 } \\
\hline Lebar's. & 17.5 & 226.8 & 77.5 & 187.3 & 187.3 & 246.5 & 197.2 & 236.6 & 197.2 & 177,5 & 236.5 & 216,9 & 2474.6 \\
\hline other $\$$ & 30.0 & 38,4 & 30.0 & 31.0 & 31,8 & 41.7 & 33,4 & 40.1 & 33.4 & 30.0 & 41.7 & 36.8 & 419.4 \\
\hline $\begin{array}{l}\text { Wointhly } \\
\text { Totale }\end{array}$ & 307,5 & 265.2 & 207.5 & 219.1 & 219.1 & 288.2 & 230.6 & 276.7 & 230,6 & 207.5 & 288.2 & 253.7 & 2899,0 \\
\hline Fues & 25.3 & 25.5 & 25.9 & 25.2 & 24.9 & 24.9 & $x .5$ & 23.4 & 25,8 & 26.9 & 26.3 & 25.7 & 25.6 \\
\hline
\end{tabular}

\begin{tabular}{|c|c|c|c|c|c|c|c|c|c|c|c|c|c|}
\hline \multicolumn{14}{|c|}{ Goot foral In Hy Henth - FY98 } \\
\hline $\begin{array}{c}\text { Activity/cost } \\
\text { Aceosint }\end{array}$ & or T & Hovi & DEC & sam & FEB & MAR & APR & MAY & JuA & . & AtVG & SEP & TOTAL \\
\hline \multicolumn{14}{|l|}{$1 \mathrm{A1} 601$} \\
\hline Labor $\$$ & 310.5 & 396.8 & 310.5 & 327.8 & 327.8 & 431.3 & 345.1 & $414-1$ & 345.1 & 310.5 & 431.3 & 379.6 & 4330.4 \\
\hline other $s$ & 79.3 & 101.3 & 79.3 & 83.6 & 85 & 110.1 & 89.0 & 105.6 & 88.0 & 78.3 & 110.1 & 96.8 & 1104.8 \\
\hline $\begin{array}{l}\text { Monthly } 5 \\
\text { Totenle }\end{array}$ & $389 . \mathrm{a}$ & 4 媳.1 & 309 & $\$ 11.4$ & 411,4 & 541.4 & 433.1 & 519.7 & 433.1 & 399.8 & 547.4 & 476.4 & 5455.2 \\
\hline fTES & 44.3 & 44.7 & 45.3 & 44.1 & 43.5 & 43.6 & 44.5 & 44.5 & 45.1 & 47.1 & 46.3 & 44,9 & 44,8 \\
\hline
\end{tabular}




\begin{tabular}{|c|c|c|c|c|c|c|c|c|c|c|c|c|c|}
\hline \multicolumn{14}{|c|}{ Cost endellnegy inth - mog } \\
\hline $\begin{array}{c}\text { Act I vit ty/Cost } \\
\text { Aecount }\end{array}$ & OCr & 104 & OEC & JAH & fer & MAR & APR & MAY & JuW & dit: & Allí & SEP & TOTAL \\
\hline \multicolumn{14}{|l|}{ 1.1501 } \\
\hline Laber 5 & 29.2. & 37.3 & 29.2 & 30,8 & $30 . \mathrm{B}$ & 40.6 & 32.4 & 39.9 & 32.4 & 29.2 & 40.6 & 35.7 & 401,2 \\
\hline Other $\$$ & 7,8 & 10.0 & $7 . \mathrm{B}$ & 8.3 & 8.3 & 10.9 & 0.8 & 19.5 & B.8 & 7.8 & 10.9 & 9.6 & 109.3 \\
\hline $\begin{array}{l}\text { Monthly } \$ \\
\text { Tot:le }\end{array}$ & 37.0 & 67.3. & 37,0 & 39,1 & 39.1 & $\$ 1.5$ & 41,2 & 49.4 & 41.2 & 37.0 & 51.5 & 45.3 & $\$ 16.5$ \\
\hline FTES & 4.6 & 4.6 & 4.7 & 4.5 & 4.5 & 4,5 & 4.6 & 4.6 & 4.7 & 4.9 & 4,8 & 4.6 & 4.6 \\
\hline
\end{tabular}

\begin{tabular}{|c|c|c|c|c|c|c|c|c|c|c|c|c|c|}
\hline 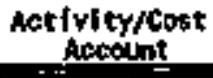 & oct & mon & DEC & JAN & FEB & MAR & $A P R$ & wir & 昰 & JUL & NUG & s巴 & fotal \\
\hline \multicolumn{14}{|l|}{ 181601 } \\
\hline Lobor & $265 . ?$ & 339.1 & 265.4 & 280,1 & 280,1 & 368.5 & 293.0 & 351,2 & 292,7 & 20304 & 365.9 & 322.9 & 3686.7 \\
\hline other 5 & 83.2 & 107.0 & 83.7 & 88.4 & 88.4 & 1196.4 & 83.6 & 90.4 & 81,9 & 73 & 102.4 & 90.1 & 1097.2 \\
\hline $\begin{array}{l}\text { Howthly } 5 \\
\text { Iotels }\end{array}$ & 348.4 & 446.1 & 349.1 & 368,5 & 368,5 & 484.9 & 376.6 & 449.6 & 374.6 & 337.2 & 468.3 & 412.1 & 4783.9 \\
\hline FTE & 38.5 & 38.9 & 39.4 & 38.4 & 37.8 & 38.0 & 38.4 & 38.4 & 38.9 & 40.7 & 39.9 & 38,8 & 38.8 \\
\hline
\end{tabular}

\begin{tabular}{|c|c|c|c|c|c|c|c|c|c|c|c|c|c|}
\hline $\begin{array}{c}\text { Act IYisy/cost } \\
\text { Acepount }\end{array}$ & oct & How & DEE & $\triangle A A$ & FEB & MaR & APR & 流Y & JU: & & Alto & SEP & TOTAL \\
\hline \multicolumn{14}{|l|}{ 1ATA01 } \\
\hline Lebor $\$$ & 51.8 & 83.8 & 92.2 & 97.3 & 95.5 & 136,5 & 121,4 & 152.4 & 127.5 & 197.1 & 160.0 & 110.5 & 1355.8 \\
\hline other $\$$ & 213.0 & $2 \pi 2.2$ & 213.0 & 224,9 & 224.9 & 305.4 & 247.2 & 297.1 & 247.6 & 222.8 & 309.5 & 261.4 & 3039,1 \\
\hline $\begin{array}{l}\text { Nonthly } \\
\text { Tatals }\end{array}$ & 274.8 & 356.0 & 305.2 & 322.2 & 320,4 & 441.9 & 368,6 & 449.5 & 375.1 & 339.9 & 469.5 & 371.9 & 4394.8 \\
\hline TTEs & B.7 & 9.3 & 12.5 & 12.2 & 11.8 & 13.1 & 15,0 & 16.0 & 16.2 & 17.3 & 16.7 & 13.1 & 13,5 \\
\hline
\end{tabular}




\begin{tabular}{|c|c|c|c|c|c|c|c|c|c|c|c|c|c|}
\hline \multicolumn{14}{|c|}{ cost Hasel ins By Honth * FYsg } \\
\hline $\begin{array}{l}\text { Metivity/Cost } \\
\text { Accoumt }\end{array}$ & at & kito & DEF & JAN & FeB & MAR & APR & MAY & JWN & JUL & ANS & SEP & TOTAL \\
\hline \multicolumn{14}{|l|}{ 1A1k02 } \\
\hline Labor 5 & 36.6 & 49.2 & 39.1 & 41.2 & 41,2 & 54.3 & 38.5 & 45.3 & 43.0 & 38.9 & 54,0 & 418 & $5 \times 3.2$ \\
\hline ather s & 37.7 & 49.7 & 39.2 & 41.4 & 41.4 & 54.4 & 42.1 & 50.3 & 41.9 & 37.7. & 52.4 & 46.9 & 534.4 \\
\hline $\begin{array}{l}\text { Honthly } \$ \\
\text { Iotale. }\end{array}$ & 74.3 & 98.9 & 38,3 & 82.6 & 82.6 & 108.7 & 80.6 & 05.6 & 84.9 & 76.6 & 106.4 & 87.9 & 1057.6 \\
\hline FTEs: & 6.0 & 6.0 & 6.1 & 5.9 & 5.9 & 5.9 & 6.0 & 6.0 & 6.7 & 7.0 & 6.9 & 6.0 & 6.2 \\
\hline
\end{tabular}

\begin{tabular}{|c|c|c|c|c|c|c|c|c|c|c|c|c|c|}
\hline $\begin{array}{c}\text { Netivity/Cost } \\
\text { iccoutint }\end{array}$ & DCT & Mav & DEC & JNAN & 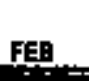 & MAR & $A P R$ & maY & 政 & 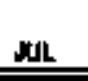 & AUE & SEP & TOTAL \\
\hline 1A1A03 & \multicolumn{12}{|c|}{. } & \\
\hline Leseber $s$ & 32.7 & 41.8 & 19.0 & 14.1 & 20.2 & 13.4 & 6.8 & 7,7 & 6.4 & 5.7 & A, 0 & 7.0 & 169.8 \\
\hline other \& & 32.3 & 41.2 & 93.7 & 43.6 & 41.8 & 55.0 & 21.4 & 22.7 & 18.9 & 17.1 & 23.6 & 20.8 & 392.0 \\
\hline $\begin{array}{l}\text { Nonthly } 8 \\
\text { Tokels }\end{array}$ & 65.0 & $\mathrm{~B}, 0$ & 72.7 & 54.7 & 52,0 & 68.4 & 28,2 & 30.4 & 25,3 & 22.8 & 31.6 & $27, \theta$ & 561.8 \\
\hline FTES & .5 & .3 & .2 & 1 & .1 & .1 & .1 & .1 & & .1 & .1 & .1 & .1 \\
\hline
\end{tabular}

\begin{tabular}{|c|c|c|c|c|c|c|c|c|c|c|c|c|c|}
\hline $\begin{array}{c}\text { Activity/cost } \\
\text { Accolint }\end{array}$ & OCT & HON & DEC & JAB & FEB & HAR & APR & MAY & ل & $\sqrt{\mathrm{N}} \mathrm{C}$ & Allo & SEP & TOTAL, \\
\hline \multicolumn{14}{|l|}{$1 \times 3202$} \\
\hline Letbor $\$$ & $12 x^{\circ}$ & 5.9 & 107,6 & 106.6 & 99.2 & 111.8 & 111,6 & 101.5 & 145.7 & 173.8 & 169.4 & 142.7 & 1490,4 \\
\hline ather 8 & 47.8 & 37.4 & 43.7 & 43.4 & 39.4 & 25.8 & 23.9 & 21.6 & 32.9 & 31.8 & 30.4 & 22.7 & 400.8 \\
\hline $\begin{array}{l}\text { Monthty } \\
\text { Totals }\end{array}$ & 171.6 & 134.3 & 151.3 & 150.0 & 1338,6 & 137.6 & 135.4 & 123.1 & 178.6 & 205,6 & 199.8 & 165,4 & 1891.2 \\
\hline FTEx & 17,0 & 10.5 & 15.1 & 13.8 & 12.7 & 10.9 & 13.8 & 10.5 & 18.4 & 24.6 & 16.5 & 15.7 & 14.7 \\
\hline
\end{tabular}




\begin{tabular}{|c|c|c|c|c|c|c|c|c|c|c|c|c|c|}
\hline $\begin{array}{l}\text { Aetivity/Cost } \\
\text { Aecolnt }\end{array}$ & OCT & Hov & DEt & NAM & FEB & mak & APR & may & Jup & UL & $M G$ & SEP & TotAl \\
\hline \multicolumn{14}{|l|}{143301} \\
\hline tabor $\&$ & 86.2 & 151.8 & 78.7 & 76,7 & 71.2 & 82,4 & 103.0 & 74.9 & 88,4 & 78.7 & 78,7 & 78,7 & 1045.3 \\
\hline other $\$$ & $59 . \mathrm{B}$ & 46.9 & 54.6 & 54.6 & 49.4 & 57.1 & 56.2 & 51.0 & 56.1 & 53.5 & 53.5 & 53.5 & 646.6 \\
\hline Honthty 5 & 146.0 & 198. 8 & 133.3 & 133.3 & 120.6 & 139.5 & 159.2 & 125.7 & 138.5 & 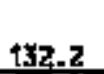 & 432.2 & 132.2 & 1691.9 \\
\hline FTEB & 12.1 & 13,1 & 11,3 & 10.4 & 9.3 & 0.2 & 13.2 & 3.9 & 10.6 & 11.7 & B.3 & 9.2 & 10.3 \\
\hline
\end{tabular}

\begin{tabular}{|c|c|c|c|c|c|c|c|c|c|c|c|c|c|}
\hline $\begin{array}{c}\text { Attivity/cost } \\
\text { Accosint }\end{array}$ & OSI & now & $\mathrm{DEC}$ & JAB & EEB & MAR & $\mathrm{APR}$ & MAY & $\sin$ & JUL & Avis & SEP & TOTAL \\
\hline \multicolumn{14}{|l|}{$1 \mathrm{~A} 330 \mathrm{Z}$} \\
\hline Lotor 5 & 714.0 & 563.2 & 669.9 & 690.4 & 613,4 & 730,7 & 725,3 & 637.2 & 704.1 & 647.9 & 637.8 & 665,4 & 8000,4 \\
\hline Other $\$$ & 242.2 & 189.5 & $2 \geq 1,1$ & $2 \geq 1.1$ & 200.9 & 231.7 & 231.7 & 210.6 & 231.7 & 221.1 & 221.2 & 220.9 & 2643.0 \\
\hline $\begin{array}{l}\text { Monthly : } \\
\text { Totals }\end{array}$ & 956.2 & 752.7 & 891.0 & 901.5 & 813.5 & 962.4 & 957.0 & 84.8 & 935.8 & Bsq.0 0 & 859.1 & 906,3 & 10652,4 \\
\hline FTES & 98,0 & 61,1 & 94.1 & 89.0 & 78.2 & 71.0 & 89.7 & 65.9 & 88.5 & 94.6 & 65.6 & 78.0 & 74.6 \\
\hline
\end{tabular}

\begin{tabular}{|c|c|c|c|c|c|c|c|c|c|c|c|c|c|}
\hline \multicolumn{14}{|c|}{ 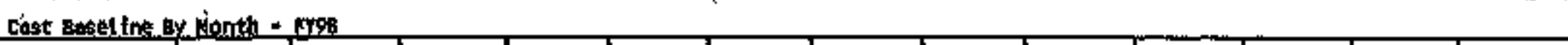 } \\
\hline $\begin{array}{c}\text { Act ivf ty/Cost } \\
\text { Aceoolnt }\end{array}$ & oct & mow & DeC & JAN & FEE & MAR & APR & MYY & Jut & J亗 & ANG & SEP & TOTAL \\
\hline \multicolumn{14}{|l|}{1,3303} \\
\hline Lepor 5 & 46,4 & 46.2 & 43.4 & 42.3 & 38.3 & 44.3 & 44.3 & 40.3 & 44,3 & 42,3 & 42.3 & 42,3 & 516.9 \\
\hline Dther $s$ & 28.9 & 2,7 & 26,5 & 26,5 & 23.9 & $z 7 . \mathrm{B}$ & 27.8 & 25.2 & 27.8 & 26,5 & 26.5 & 26.5 & 316.2 \\
\hline $\begin{array}{l}\text { Wonthly } \\
\text { Totels }\end{array}$ & 73.3 & 68.9 & 69.9 & 68.8 & 62.2 & $\pi 2.1$ & 72.1 & 65.5 & 72.1 & 68,8 & 68.8 & 6 6.8 & 然3.1 \\
\hline ftes & 6.5 & 5.1 & 6.3 & 5.6 & 5.0 & 4.4 & 5.7 & 4.3. & 5.7 & 6,4 & 4.5 & 5.0 & 5.3 \\
\hline
\end{tabular}




\begin{tabular}{|c|c|c|c|c|c|c|c|c|c|c|c|c|c|}
\hline \multicolumn{14}{|c|}{ 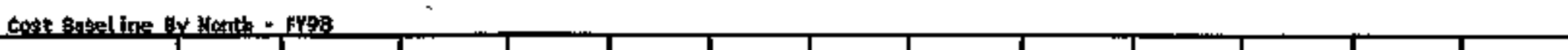 } \\
\hline $\begin{array}{l}\text { Aetivity/Cost } \\
\text { Accouth }\end{array}$ & OCT & MOW & DEC & JAH & FEB & MAR & APR & MAY & $\mathrm{sh}$ & JLL_. & AUS & SEP & TDIAL \\
\hline \multicolumn{14}{|l|}{103402} \\
\hline Lupper if & 58.2 & 32.3 & 23.2 & 22.2 & 20,0 & 23.2 & 21.3 & 15,8 & 18.3 & 17.5 & 17.5 & 17.5 & 288.8 \\
\hline other $\$$ & 8.7 & $6 . B$ & 7.9 & 7.9 & 7.2 & e.3 & 8.3 & 7.5 & 8,3 & 7.9 & 7.9 & 7.9 & 94.6 \\
\hline $\begin{array}{l}\text { Yanthly } \\
\text { Totals }\end{array}$ & 66.9 & 32.1 & 30.1 & 30.1 & 27,2 & 31.5 & 29,6 & 24.3 & 26.6 & $2.25,4$ & 25.4 & 25.4 & 381.4 \\
\hline FTE & 0.3 & 3.7 & 3,2 & 3,0 & 2.7 & 2,3 & 2.7 & 1.8 & 2.3 & 2.6 & 1,8 & 2.0 & 2.8 \\
\hline
\end{tabular}

\begin{tabular}{|c|c|c|c|c|c|c|c|c|c|c|c|c|c|}
\hline $\begin{array}{c}\text { Aetiyity/cost } \\
\text { Metoulink }\end{array}$ & act & Nov & DEC & JNW & FEB & MAR & APR & MAY & ل & JUL & MNG & 祭 & TOTAL \\
\hline \multicolumn{14}{|l|}{113500} \\
\hline lebor & & & & 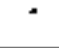 & & & & & & & & & \\
\hline Othor \& & & & & & & & & & & & & 400,0 & 400,0 \\
\hline $\begin{array}{l}\text { Monthly } s \\
\text { totals }\end{array}$ & & & & & & & & - & & & & 400.0 & 600,0 \\
\hline FTEa & & & & & & & & & & & & & \\
\hline
\end{tabular}

\begin{tabular}{|c|c|c|c|c|c|c|c|c|c|c|c|c|c|}
\hline $\begin{array}{l}\text { Act ivity/cost } \\
\text { Actounts }\end{array}$ & D.T & Nov & DEC & JM & FEB & NARR & $A P R$ & $\mathrm{mar}$ & Nath & JUE & AUG & SEP & TOTAL. \\
\hline \multicolumn{14}{|l|}{$1 \mathrm{A4720}$} \\
\hline Lebor 5 & 272.8 & 349.6 & 272.8 & 289.4 & 293.2 & 3 sg. 9 & 306.3 & 375.4 & 314.1 & 282.7 & 392.6 & 345.5 & 3876.5 \\
\hline other 5 & $18 B, 4$ & 240.7 & 189,4 & 198.9 & 188,2 & 261.7 & 209,4 & 251.2 & 209.3 & 188,4 & 261.7 & 230.3 & 2627.0 \\
\hline $\begin{array}{l}\text { Honthly } \$ \\
\text { Totals }\end{array}$ & 661.2 & 5 a 9.3 & 461,2 & 488,3 & 492,1 & 오44,6 & 515.7 & 626,6 & 523,4 & 471.1 & 654.3 & 575.8 & 6503.5 \\
\hline FTE $B$ & 42,0 & 42,5 & 43.0 & 42.0 & 41.9 & 41.8 & 42,6 & 43.5 & 44.2 & 46.2 & 45.2 & 44.0 & 43.2 \\
\hline
\end{tabular}




\begin{tabular}{|c|c|c|c|c|c|c|c|c|c|c|c|c|c|}
\hline $\begin{array}{c}\text { Activity/Cost } \\
\text { Acoourts }\end{array}$ & OCT & Hov & DEC & JAN & F孯 & MAR & APR & MrY & 니배 & anc & ANG & 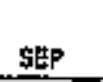 & TOTAL \\
\hline \multicolumn{14}{|l|}{144722} \\
\hline Labar $s$ & 111.2 & 142,1 & 1112 & 117,4 & 112.4 & 154,5 & $122,6$. & $14 \mathrm{~g} .3$ & 123.6 & \pm 11.2 & 154.5 & 135.9 & 1550,9 \\
\hline other $\$$ & 3.7 & 4.7 & 3.7 & 3.9 & 3.9 & 5.1 & 4.1 & 4.9 & 4.1 & 3.7 & 5.1 & 4.5 & 51.3 \\
\hline $\begin{array}{l}\text { Monthly } \\
\text { Totels }\end{array}$ & 114.9 & 146.8 & 124.9 & 521,3 & 121.3 & 159.6 & 127.7 & 153,2 & 127.7 & 114.9 & 159.6 & 140.4 & 1602.2 \\
\hline FTES & 16.6 & 16.7 & 16.9 & 16,5 & 16.3 & 16.3 & 16.6 & 16.7 & 16.9 & 17.6 & 17.3 & 16.8 & 16.7 \\
\hline
\end{tabular}

\begin{tabular}{|c|c|c|c|c|c|c|c|c|c|c|c|c|c|}
\hline \multicolumn{14}{|c|}{ 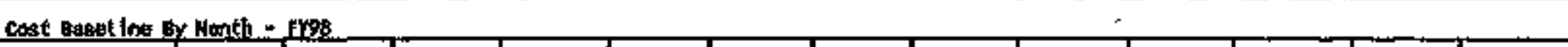 } \\
\hline $\begin{array}{l}\text { Aet ivity/toont } \\
\text { socount }\end{array}$ & oct & HDV & DEC & JaW & FEB & MAR & APR & mar & 山W & لآل & ANG & SEP & IOIAL \\
\hline \multicolumn{14}{|l|}{184723} \\
\hline Llbor \& & 45,4 & 58.0 & 45.4 & 47.9 & 47.8 & 63.1 & 50.5 & 60.5 & 50.5 & 45,4 & 63.1 & 55.5 & 633.2 \\
\hline ather $s$ & 2.6 & 3,3 & 2.6 & 2.8 & 2,8 & 3.6 & 2.8 & 3.5 & 2,8 & 2.6 & 3.6 & 3.2 & 36.2 \\
\hline $\begin{array}{l}\text { Honthly } \$ \\
\text { Torals }\end{array}$ & 48.0 & 61.3 & 40.0 & 50.7 & 50.7 & 66.7 & 53.3 & 64,0 & 53.3 & 48.0 & 66.7 & 58.7 & 669.4 \\
\hline ATE: & 6.8 & 6.9 & 7.0 & 6.8 & 6.7 & 6.7 & 6.8 & 6.8 & 6.9 & 7.2 & 7.1 & 6.9 & 6.9 \\
\hline
\end{tabular}

\begin{tabular}{|c|c|c|c|c|c|c|c|c|c|c|c|c|c|}
\hline $\begin{array}{l}\text { Activity/Cost } \\
\text { Aecoulnt }\end{array}$ & OCT & $\mathrm{HON}$ & DEE & SAH. & FEE & MAR & APR & MAY & Int & $M R$ & ADL5 & SEP & TOTAL \\
\hline \multicolumn{14}{|l|}{144724} \\
\hline Labor 5 & 74.4 & 95.0 & 74.4 & 78.5 & 78. & 107.3 & 87.2 & 104.7 & 87,3 & 78.5 & 109.1 & 98.0 & 1070.9 \\
\hline Other & 2.2 & 2.9 & 2.2 & 2.4 & 2.4 & 3.0 & 2.5 & 3.0 & 2.4 & 2.3 & 3.1 & 2.7 & 31.0 \\
\hline $\begin{array}{l}\text { Monthly } \$ \\
\text { Totals }\end{array}$ & 76.6 & 97.9 & 76.6 & 80.9 & 0.9 & 110.3 & 89.7 & 107.7 & 89.7 & 80,8 & 112,2 & 98.7 & 1101.9 \\
\hline FTEs & 11.3 & 11.4 & 11.6 & 11,3 & 11.1 & 11.5 & 11.9 & 11.9 & 12,0 & 12.6 & 12.3 & 12.0 & 11.8 \\
\hline
\end{tabular}




\begin{tabular}{|c|c|c|c|c|c|c|c|c|c|c|c|c|c|}
\hline & & & & & & & & ! & & 1 & 1 & & $\cdot$ \\
\hline \multicolumn{14}{|c|}{ 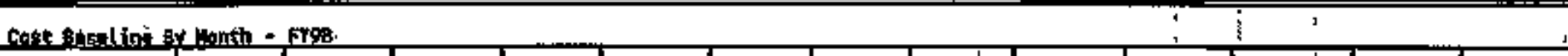 } \\
\hline $\begin{array}{c}\text { Act|rity/Coss } \\
\text { hecount }\end{array}$ & oct & Non & DEC & JAN & Feg & tuR & APR & HAY & העل & 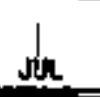 & AUSG & SEP & TOTAL \\
\hline \multicolumn{14}{|l|}{ 1A4FEE } \\
\hline \multicolumn{14}{|l|}{ Laborts } \\
\hline other $\$$ & \$3.9. & 45.1 & 35.9 & 37.8 & 37.8 & 49.8 & 39.8 & 4718 & 39.9 & 35.2 & 49.8 & 43,8 & 590,0 \\
\hline $\begin{array}{l}\text { Honthly } \$ \\
\text { Totels }\end{array}$ & 35.9 & 45.8 & 35.9 & 37. & 37.8 & 49.8 & 39.8 & 47.8 & 39.8 & 35.9 & 49. B & 43,8 & 500.0 \\
\hline \multicolumn{14}{|l|}{ FTEs } \\
\hline \multicolumn{14}{|c|}{$!$} \\
\hline \multicolumn{14}{|c|}{ 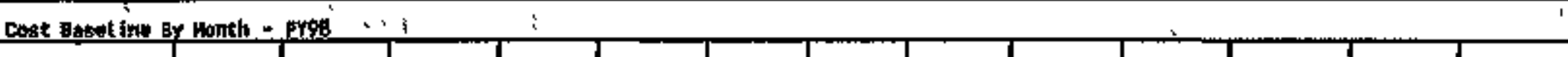 } \\
\hline $\begin{array}{c}\text { Act ivity/Cost } \\
\text { Account }\end{array}$ & at & HON & DEC & JhH & FEE & MAR & APf & $\mathrm{MrY}$ & Jun & JUL & $A W_{G}$ & SEP & TOTAL \\
\hline \multicolumn{14}{|l|}{1 18101 } \\
\hline Lebor 5 & 23,6 & 30.1 & 23.6 & 24.9 & 26.9 & 29.4 & 22. & 27.4 & $2 \geq .0$ & 18.5 & 25,6 & 22.6 & 295.2 \\
\hline other s & 3 & 4 & 3 & .3 & 3 & . & .4 & $\frac{1}{4}$ & .4 & 1.3 & 5 & .4 & $4.8^{1}$ \\
\hline $\begin{array}{l}\text { Montbsy * } \\
\text { Totals }\end{array}$ & 25.9 & 30.5 & 23.9 & 8.2 & 25.2. & 29.8 & 23.2 & 27.8 & 22.4 & 18.8 & 26.1 & 23.0 & 390,0 \\
\hline \multirow[t]{2}{*}{ FTEe } & 2.8 & 2.9 & 2.9 & 2.8 & 2.8 & 2,5 & 2.5 & 2,5 & 2.5 & 2.4 & 2.3 & 2.3 & 2.6 \\
\hline & & & & & & $\cdot$ & & & & 1 & 1 & & \\
\hline \multicolumn{14}{|c|}{ coost lasel fine oy Month - FYgt } \\
\hline $\begin{array}{c}\text { Act ivity/cost } \\
\text { Aceount } \\
\end{array}$ & oct & nor & DEC & J 새 & FEg & WAR & APR & MAY & لي & ILL & AlNG & SEP & TOTAL \\
\hline \multicolumn{14}{|l|}{ 1hopos } \\
\hline Lebor \$ & 15.8 & 20.1 & 15.8 & 16.5 & 17.9 & 26.2 & 19,4 & 27.5 & 18,6 & 16.8 & 24.2 & 28.3 & 240.1 \\
\hline other: 5 & 4.5 & S.B & 4.5 & 4.8 & 4.8 & 6.3 & 5.0 & 6.0 & 5.0 & 4.5 & 6.3 & 5.5 & 62.8 \\
\hline $\begin{array}{l}\text { Nonthly \$ } \\
\text { Iotegls }\end{array}$ & 20.3 & 25.9 & 20,3 & 21.3 & 22.7 & 30.5 & 24.4 & 28.5 & 73.6 & 21.3 & 30.5 & 33.8 & 3029 \\
\hline FTES & 2.4 & 2.5 & 2.5 & 2.4 & 2.6 & 2,7 & 2.7 & 2,6 & 2.6 & 2.8 & 2.8 & 3.7 & 2.7 \\
\hline
\end{tabular}




\begin{tabular}{|c|c|c|c|c|c|c|c|c|c|c|c|c|c|}
\hline $\begin{array}{c}\text { Aet lyity/cost } \\
\text { Account }\end{array}$ & OCI & Hov & DEC & JAH & FEP & HAR & APR & mur & JuH & Juk & All6 & SEP & TOTAL \\
\hline \multicolumn{14}{|l|}{34402} \\
\hline Labor 5 & 103.3 & 12.5 .3 & 304.2 & 110.9 & 115.5 & 146,0 & $+14,3$ & 136.9 & 114.1 & 97.5 & 129.5 & 113.2 & 1410.7 \\
\hline ather $\$$ & 192,2 & 251.1 & 2000.3 & 211.4 & 206.2 & 267.4 & 213.9 & 252.3 & 209.7 & 139.7 & 167.9 & 147.8 & 2459.9 \\
\hline $\begin{array}{l}\text { Monthly }= \\
\text { Ioregls }\end{array}$ & 295.5 & 376.4 & 306.5 & 322,3 & 321.7 & 413,4 & 328,2 & 389.2 & 323.8 & 237,2 & 297.4 & 261,0 & 3870.6 \\
\hline PIEs & 17,0 & 16.4 & 17.7 & 17.3 & 17.8 & 17.1 & 17.1 & 17.1 & 17.3 & 17.2 & 16.1 & 15.6 & 16.9 \\
\hline
\end{tabular}

\begin{tabular}{|c|c|c|c|c|c|c|c|c|c|c|c|c|c|}
\hline $\begin{array}{l}\text { Activity/cost } \\
\text { Afcownt }\end{array}$ & OCT & Nor & DEC & $\sqrt{4} 4$ & FEB & PAR & $A P R$ & BAY & Jut & ast. & ANG & SEP & TOThL \\
\hline \multicolumn{14}{|l|}{ 1) 501} \\
\hline lebers & 33.1 & 42.3 & 33.7 & 35.6 & 37.2 & 49.7 & 39.8 & 47.2 & 39.2 & 39.3 & 49.0 & 43.1 & 495.3 \\
\hline Othar 3 & 17.1 & 21.B & 20,8 & 22.0 & 26. 7 & 36,5 & 29.2 & 22.4 & 16,6 & 14.9 & 20.7 & 18.3 & 266.5 \\
\hline $\begin{array}{l}\text { Nonthly } \\
\text { Totalo }\end{array}$ & $50 . ?$ & 69,1 & 54.5 & 57.6 & 63.4 & 86.2 & 69.0 & 69.6 & 55.8 & 50,2 & 69.7 & 61.4 & $7 \$ 1.8$ \\
\hline FIEs & 5.8 & 5.8 & 6,0 & 5.9 & 6.0 & 6.1 & 6.2 & 6,1 & 6.2 & 6.4 & 6.3 & 8.1 & 6.1 \\
\hline
\end{tabular}

\begin{tabular}{|c|c|c|c|c|c|c|c|c|c|c|c|c|c|}
\hline \multicolumn{14}{|c|}{ 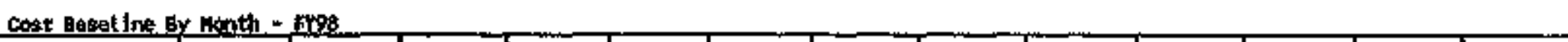 } \\
\hline $\begin{array}{l}\text { Act }|v| \text { ty/cost } \\
\text { Accolnt }\end{array}$ & OCT & now & Dec & dell & fEE & MAR & APR & mar & 步些 & MK & AUS & SEP & FOTfL, \\
\hline \multicolumn{14}{|l|}{ tNL603 } \\
\hline Enbor $\$$ & 92.6 & 118,4 & 93,3 & 98.4 & 102.2 & 136,3 & 107.3 & 127.9 & 106.5 & $95, B$ & 428.9 & 113.7 & 1322,4 \\
\hline Other $\leqslant$ & 134.7 & 172.0 & 138.3 & 146.1 & 150.4 & 199.9 & 151.6 & 168.1 & 137.9 & 134.2 & 172.5 & 151.7 & 1847.4 \\
\hline $\begin{array}{l}\text { Wonthly } \$ \\
\text { Iotals }\end{array}$ & 227.3 & 299,4 & 231.6 & 244.5 & $\underline{252.6}$ & 336.2 & $25 \mathrm{~B} .9$ & $28 \%, 0$ & 244,4 & 220,0 & 302,4 & 265.4 & 3169.8 \\
\hline ftes & 15.2 & 15.3 & 15.6 & 15.2 & 15.5 & 15.8 & 15.9 & 15.7 & 15.9 & 16.6 & 15.9 & 15.4 & 15.7 \\
\hline
\end{tabular}




\begin{tabular}{|c|c|c|c|c|c|c|c|c|c|c|c|c|c|}
\hline $\begin{array}{c}\text { Act Ivitì/Cost } \\
\text { Account }\end{array}$ & DCT & nov & DEC & JAH & fen & MAR & $M P R$ & way & Jorin & Jux & ANB & SEP & TOTAL \\
\hline \multicolumn{14}{|l|}{ 1kapt } \\
\hline Llpor s & 66.7 & 59.7 & 46.7 & 48,3 & 49.3 & 64.9 & 51.9 & 62.3 & 51.2 & 46.7 & 64.8 & 57.7 & 651.7 \\
\hline other $\$$ & 8.1 & 10.3 & 1.1 & 9.5 & 0.5 & 11.2 & 9.0 & 10.8 & 9.0 & $B, 1$ & 11.2 & 9.9 & 112.5 \\
\hline $\begin{array}{l}\text { Wonthly } \\
\text { Total } 8\end{array}$ & 54.8 & 70.0 & 54.8 & $57, a$ & 57,8 & 76.1 & 60,9 & 73. & 60.9 & 54.8 & 76.1 & 67.0 & 764.2 \\
\hline FTES & 7.8 & 7.9 & 8.0 & 7.8 & 7.7 & 7.7 & 7.9 & 7.9 & 8.0 & 8,3 & B.1 & 7.9 & 7.9 \\
\hline
\end{tabular}

NOTE: The FY9B cost Information is based upon Financial Data System rates as of September $12,1995$. 
Futal Ycer 1005

\begin{tabular}{|c|c|c|c|c|c|c|c|c|c|c|c|c|c|c|}
\hline AD:STHES & Tundrye & Qtober & Howember & antepanthos & Leauary I & Ectriany & Wareh & Exill & Mer & fline & $5+1$ & Augtit & Sopitmber & ToTALS \\
\hline $22000-41$ & 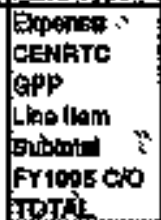 & 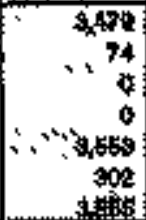 & 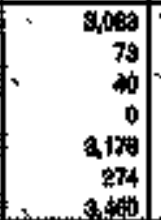 & 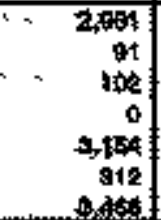 & 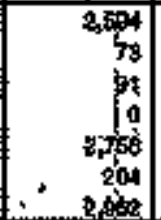 & 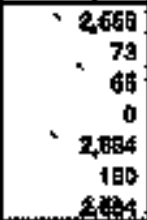 & 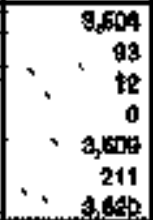 & 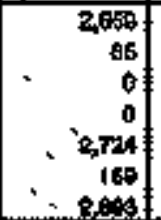 & 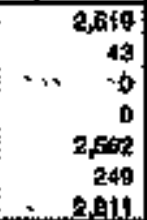 & 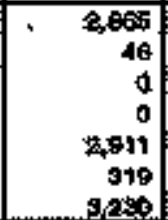 & $\begin{array}{r}2746 \\
37 \\
0 \\
0 \\
0 \\
2,589 \\
464 \\
8,617\end{array}$ & 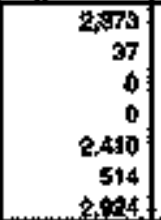 & $\begin{array}{r}2,858 \\
40 \\
0 \\
0 \\
0 \\
2005 \\
043 \\
8016\end{array}$ & 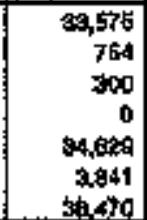 \\
\hline 2020000015 & 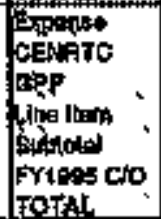 & 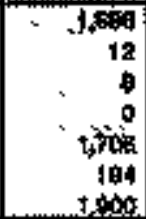 & 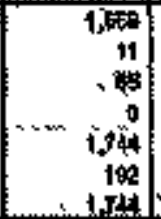 & 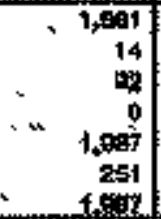 & 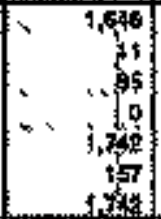 & 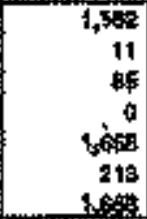 & 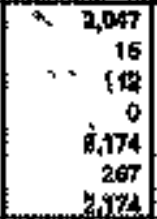 & 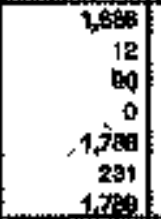 & $\begin{array}{r}1,600 \\
12 \\
-90 \\
0 \\
i, 792 \\
118 \\
5,714\end{array}$ & $\begin{array}{r}2,07 \\
14 \\
107 \\
0 \\
2,142 \\
169 \\
209\end{array}$ & 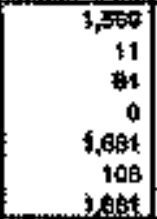 & 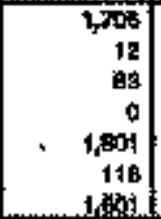 & 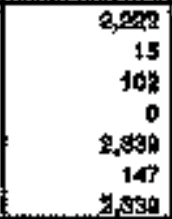 & $\begin{array}{r}21,394 \\
1,90 \\
1,000 \\
0 \\
0 \\
22,584 \\
2,102 \\
22,720\end{array}$ \\
\hline 2520-1/A4 & 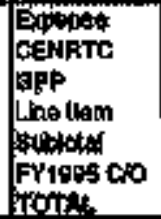 & 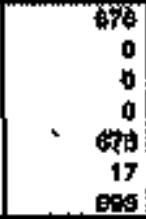 & \begin{tabular}{r|}
130 \\
0 \\
0 \\
0 \\
309 \\
3 \\
3 \\
302
\end{tabular} & $\begin{array}{r}0 \\
0 \\
0 \\
0 \\
0 \\
0 \\
0\end{array}$ & $\begin{array}{r}6 \\
0 \\
4 \\
0 \\
769 \\
7 \\
706\end{array}$ & $\begin{array}{r}2 \\
0 \\
0 \\
0 \\
0 \\
51 \\
54\end{array}$ & $\begin{array}{r}1,50: \\
0 \\
0 \\
0 \\
3,001 \\
10 \\
4,217\end{array}$ & 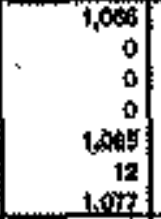 & $\begin{array}{r}r \\
0 \\
0 \\
0 \\
1,40 \\
12 \\
1,2024 \\
\end{array}$ & $\begin{array}{r}1,250 \\
0 \\
0 \\
0 \\
1,80 \\
15 \\
1,45\end{array}$ & $\begin{array}{r}097 \\
0 \\
0 \\
0 \\
096 \\
59 \\
057\end{array}$ & $\begin{array}{r}585 \\
0 \\
0 \\
0 \\
045 \\
56 \\
1,021 \\
, 02\end{array}$ & $\begin{array}{r}189 \\
0 \\
0 \\
0 \\
1,469 \\
82 \\
1,241 \\
\end{array}$ & $\begin{array}{r}11,275 \\
0 \\
0 \\
0 \\
0 \\
14,278 \\
300 \\
13,676\end{array}$ \\
\hline $2500-1 / N$ & 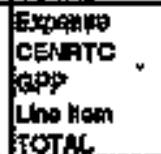 & $\begin{array}{r}130 \\
0 \\
0 \\
0 \\
1 \leqslant 0\end{array}$ & \begin{tabular}{r|}
0 \\
0 \\
0 \\
0 \\
0 \\
579
\end{tabular} & $\begin{array}{r}15 \\
0 \\
0 \\
0 \\
0\end{array}$ & 130 & 19 & $\begin{array}{r}108 \\
0\end{array}$ & \begin{tabular}{r|}
0 \\
0 \\
0 \\
0 \\
0 \\
0
\end{tabular} & $\begin{array}{c}0 \\
0 \\
0 \\
0 \\
0\end{array}$ & $\begin{array}{r}138 \\
0 \\
0 \\
0 \\
0\end{array}$ & & $\begin{array}{r}55 \\
0 \\
0 \\
0 \\
136\end{array}$ & & $\begin{array}{r}1,494 \\
0 \\
0 \\
0\end{array}$ \\
\hline $2026-0.1 / 5$ & 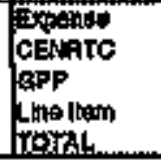 & 6 & \begin{tabular}{r|}
0 \\
0 \\
0 \\
0 \\
4
\end{tabular} & $\begin{array}{r}0 \\
0 \\
0 \\
0 \\
0\end{array}$ & $\mid \begin{array}{l}0 \\
0 \\
0 \\
0\end{array}$ & $\begin{array}{r}0 \\
0 \\
0 \\
0 \\
.18 \\
\end{array}$ & $\begin{array}{r}21 \\
0 \\
0 \\
0 \\
y\end{array}$ & $\begin{array}{r}17 \\
0 \\
0 \\
0 \\
17\end{array}$ & $\begin{array}{c}77 \\
0 \\
0 \\
0 \\
12\end{array}$ & $\begin{array}{l}20 \\
0 \\
0 \\
0\end{array}$ & $\begin{array}{r}r \\
0 \\
0\end{array}$ & $\begin{array}{r}0 \\
0 \\
0 \\
0 \\
3\end{array}$ & $\begin{array}{r}50 \\
0 \\
0 \\
0 \\
0\end{array}$ & $\begin{array}{r}30 \\
0 \\
0 \\
0 \\
0 \\
302\end{array}$ \\
\hline Dovarst & & & & & & & & & & & & & & \\
\hline 7OTALs & $\begin{array}{l}\text { Ponso } \\
\text { ENRTC }\end{array}$ & $\frac{0.03}{89}$ & $\frac{5.592}{89}$ & $\frac{6,875}{105}$ & $\frac{5.178}{84}$ & $\frac{5,067}{89}$ & $\frac{\theta .681}{106}$ & $\frac{5.519}{77}$ & $\frac{6.3 n}{66}$ & $\frac{16200}{80}$ & 475 & $\frac{5,192}{49}$ & $\frac{\theta, 430}{84}$ & $\frac{69}{6090}$ \\
\hline & & & 106 & 104 & 178 & 14 & 124 & 10 & 0 & 10 & 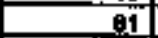 & 08 & 102 & 1,3000 \\
\hline & & & & 00 & 0 & & a & $\underline{w}$ & o & & & 마 & & \\
\hline & & 6.177 & 6.701 & 0,174 & EASB & B,2B1 & 7,113 & 5,6685 & E, E202 & B, 147 & 4896; & $5 \$ 24$ & 0,506 & 70 阳 \\
\hline & & 613 & 469 & 55 & 368 & 409 & बबा & 412 & 409 & 500 & 631 & 698 & 672 & \\
\hline & ㄴ. & 5,840 & 6,170 & 8.737 & $x, 005$ & 5,665 & 7007 & $\mathrm{E}, 090$ & 8931 & 8,097 & 3,516 & 8,020 & 7怕自 & 78,627 \\
\hline
\end{tabular}




$$
\text { WHC-SP-1114, Rev. } 1
$$

September 26, 1995

\section{DISTRIAUTION}

Number

of Copies

MSIH

\section{OFFSITE}

2 Unitod States Department of Energy. Headquarters

Rob Martinez, EW-361

12800 Middlebrook Road

Germantown, MD , 20874

1 Hashington State Department of Ecology

Hoses Jaraysi

1315 W. 4th Ave.

Keñnewick, WA 99336

1 United States Environmental Protection Agency

D. Sherwood

712 Swfft Boulevard, MSIN B5-01

Rich] and, WA 99352

\section{엔ITE}

7 U.S. Department of Energy. Richland Field office

M. S. French

A. K. Crowell

K. D. Bazzell

$57-55$

$57-55$

R. M. Gordon

$\$ 7-55$

R. F. Guercia

S7-55

T. K. Teynor

S. L. Jones

$57-55$

$57+55$

$\$ 7-55$

48 Westinghouse_Hanford_Company

L. T. BTackford

L. E. Bolan

T2-08

S. K. Barnard

B3-54

R. J. Bottenus 
WHC-SP-1114, Rev. 1

Westinghouse Hanford Company (con't)

H. C. Boynton

T3-04

T. A. [ar]son

(-3)-15

E. A. Cruz

D. C. Durban

T3-01

S. D. Elliott

T3-0I

T3-28

N. P. Emerson

T4-03

T. L. Erickson

T3-28

E. G. Erpenbeck

G3-15

ป. K. Fish

R. J. Giroir

B3-62

C. K. Girres

T4-05

P. L. Hapke

T3-05

M. L. Hejneneyer

T4-05

K. L. HTadek

T3-01

T4-02

J. D. Hookf in

$63-15$

J. A. Hunt

D. B. Jordan

T3-04

H8-64

D. B. Powel 1, Jr.

T4-03

W. C. Mallory, Jr.

T4-03

L. L. Kowatski

G2-02

J. J. Lang

T3-01

D. R. Lucas

63-15

C. Ma]stroal

G3-15

D. E. McKenney

T3-01

S. H. Norton

$\mathrm{T3}-28$

R. D. Pierce

T3-04

K. M. Quigley

T3-01

R. W. Reddinger

T4-05

G. C. Ríce

G3-15

J. G. RiddelTe

T3-01

C. A. Rinne

63-10

B. A. Rotlbwel1

T3-28

R. R. 5laybaugh

T3-0]

H. 5mith

6. C. Triner

M. S. Wright

Central Files

Office of Science and Technology

Information (2)

T3-20

T3-28

T3-28

A3-88

A3-36 